\title{
Propriedades de algumas classes de relações racionais
}

Rodrigo Nonamor Pereira Mariano de Souza

\author{
DisSERTAÇÃO APRESENTADA AO \\ Instituto DE MATEMÁticA E \\ EsTATÍSTICA DA \\ Universidade de SÃo Paulo \\ COMO PARTE DOS REQUISITOS \\ PARA OBTENÇÃO DO GRAU DE \\ Mestre em CiÊnCia da ComputaÇÃo
}

Orientadora: Profa. Dra. Nami Kobayashi

— São Paulo, junho de 2004 -

- Durante o desenvolvimento deste trabalho, o aluno recebeu apoio financeiro do CNPq - 


\section{Propriedades de algumas classes de relações racionais}

Este exemplar corresponde à redação final da dissertação devidamente corrigida e defendida por Rodrigo Nonamor Pereira Mariano de Souza e aprovada pela comissão julgadora.

São Paulo, junho de 2004

Banca examinadora:

- Profa. Dra. Nami Kobayashi (IME - USP)

- Prof. Dr. Imre Simon (IME - USP)

- Prof. Dr. Arnaldo Vieira Moura (IC - Unicamp)

Suplentes:

- Prof. Dr. Arnaldo Mandel (IME - USP)

- Prof. Dr. Jorge Stolfi (IC - Unicamp) 
Beauty is our business

Dijkstra 


\section{Agradecimentos}

Ao longo dos três anos em que este trabalho foi realizado, pude contar com a generosidade de muitas pessoas que se interessaram por mim, apoiando-me e facultando-me concluí-lo. Os parágrafos que se seguem são uma das pequenas formas de que disponho para tentar agradecê-las.

Mais do que uma honra, foi para mim uma felicidade ser orientado pela professora Nami Kobayashi. Com relação ao seu acompanhamento deste trabalho, devo dizer que sou muito grato pela sua imensa dedicação, suas idéias excelentes, sua paciência em ler, discutir e melhorar cada parte do texto, e sua tolerância constante com minhas faltas. Mas sou imensamente grato também pela sua amizade, pelo seu interesse em meu crescimento como pesquisador, e por ela estar sempre disposta a me ouvir e me auxiliar, no assunto que eu lhe apresentasse. Essa gratidão espero saber expressar honrando sua orientação, com um bom uso dos seus ensinamentos.

No que se refere ao meu aprimoramento acadêmico, agradeço ainda aos professores Jacques Sakarovitch, Imre Simon e Arnaldo Mandel, pelas oportunidades que me facultaram, e que também espero saber honrar, e aos grandes professores com quem tive a oportunidade de conviver e estudar desde a época da graduação, em particular os professores Paulo Feofiloff, Carlos Eduardo Ferreira, José Coelho de Pina, Yoshiko Wakabayashi e Yoshiharu Kohayakawa. Também agradeço ao professor Arnaldo Vieira Moura, pela leitura do texto e participação na banca examinadora. Finalmente, agradeço ao CNPq pelo auxílio financeiro, que foi imprescindível para a realização deste trabalho.

Sou imensamente grato aos grandes amigos que me acompanharam e me auxiliaram fielmente desde a época da graduação, e felizmente os tive muitos. Em São Roque, agradeço em particular a Ivan (um velho irmão de Ciência), Ricardo, Rodrigo Salvetti, José Miguel, Vinícius e Fábio. Em São Bernardo, agradeço a Cristina, Karen, Ana Lúcia e Thiago, pelo auxílio e compreensão, sempre que não pude exercer corretamente meu trabalho de professor, a Maristela, e a Elói, pela confiança imperecível. Em São Paulo, foi inestimável minha convivência com tantos amigos que apareceram no início da graduação, e partilharam comigo todas as circunstâncias felizes e difíceis desde então. Em particular, agradeço a Flavio Regis de Arruda, pela sua amizade superior, e cujo tesouro de incentivo, consideração e dedicação sinceros a mim sempre estiveram muito acima do meu mérito, bem como o estão da possibilidade de qualquer menção; a Luanna Marques da Silva, pela amizade que me dedicou junto ao Flavio; a Alexey Villas Bôas, a quem aqui chamo de grande amigo e professor, em virtude de seu companheirismo, de sua paciência infindável para me ouvir e me aconselhar, e dos belos ensinamentos que me dispensou; a Livio Baldini Soares, um grande irmão de Ciência, pelo grande incentivo ao meu trabalho, bem como seu exemplo e disposição de fazer Ciência no Brasil; a Caetano Jimenez Carezzato, que me honrou com sua amizade, e cujas grandes idéias sempre me trouxeram luzes novas; a Edgard Pevidor de Miranda, pela sinceridade e simplicidade de sua amizade; a Leandro Farina dos Santos, pela amizade e consideração; e a Silvio Rodrigues de Faria Junior, pela tolerância e pelas muitas vezes que me ajudou. Finalmente, agradeço aos novos bons amigos que apareceram no Mestrado, em particular Said, Danielle, Fábio Viduani e Cláudia, e a Mitsue e Alessandra, por iluminarem meu caminho com seu carinho e afeto.

Agora, não tenho recursos para agradecer à minha família, e em particular aos meus avós maternos, Maria e Vicente, e aos meus pais, Aparecida e Maximino, a quem devo tudo. À eles, cujas vidas foram dedicadas aos filhos, e que sempre velaram e se sacrificaram por mim, não é com este trabalho ou uma palavra que posso retribuir, mas sim pedindo a Deus que o faça por mim. 


\section{Resumo}

Neste trabalho, estudamos aspectos teóricos e algorítmicos de algumas classes de relações racionais: as relações racionais finitamente valoradas, as relações racionais $k$-valoradas, para todo inteiro positivo $k$, as funções racionais, as funções seqüenciais, e as funções subseqüenciais.

Inicialmente, apresentamos alguns resultados clássicos para as relações racionais, a representação de relações racionais por transdutores e por matrizes, e algumas propriedades de fechamento.

Weber provou que toda relação racional $k$-valorada pode ser decomposta numa união de $k$ funções racionais. Apresentamos uma prova para esse resultado, que utiliza $k$ aplicações do Teorema Cross-section de Eilenberg, e parece ser mais simples que a de Weber. Utilizando essa decomposição, escrevemos uma outra prova para mostrar que o problema da equivalência de relações racionais $k$ valoradas é decidível.

Incluímos também uma prova de Griffiths da indecidibilidade da equivalência de relações racionais finitamente valoradas.

Generalizamos para as relações racionais $k$-valoradas, para todo inteiro positivo $k$, uma propriedade de Schützenberger para as funções racionais. Como conseqüência dessa generalização, temos um algoritmo (não-polinomial) para decidir se um transdutor realiza uma relação racional $k$-valorada, para um dado inteiro positivo $k$.

Descrevemos um algoritmo eficiente de Béal, Carton, Prieur e Sakarovitch para decidir se uma relação racional é uma função, e um algoritmo eficiente dos mesmos autores para decidir se uma função racional é subseqüencial. A nossa descrição utiliza uma propriedade simples de simetria, que permitiu uma economia nos consumos de tempo e espaço desses dois algoritmos (na constante multiplicativa).

Apresentamos uma caracterização de Choffrut das funções subseqüenciais palavra-palavra, e um algoritmo para a determinização de um transdutor, que utiliza explicitamente essa caracterização.

Estudamos a minimização de transdutores subseqüenciais, utilizando uma família de monóides que chamamos de monóides com mdc. Provamos a existência de um transdutor minimal para funções subseqüenciais $\Sigma^{*} \rightarrow M$, onde $M$ é um monóide cancelativo com mdc único. Esse resultado inclui diversos monóides de interesse, como os monóides livres, e o monóide aditivo dos números reais não-negativos. Também apresentamos uma caracterização das funções subseqüenciais $\Sigma^{*} \rightarrow M$, onde $\mathrm{M}$ é um monóide cancelativo com mdc, utilizando a congruência à direita de uma função.

Finalmente, descrevemos um algoritmo eficiente para a minimização de um transdutor subseqüencial. Esse algoritmo tem duas etapas, sendo que a primeira é o algoritmo de Béal e Carton para a construção do prefixo de um transdutor, e a segunda é a minimização de um transdutor visto como um autômato finito determinístico, utilizando o algoritmo de Hopcroft, versão de Gries. 


\begin{abstract}
We study the theoretical and algorithmic aspects of some classes of rational relations: the finite valued rational relations, the $k$-valued rational relations, for every positive integer $k$, the sequential functions, and the subsequential functions.

At first, we study some classical results concerning the rational relations, the representations of rational relations by transducers and matrices, and some closure properties.

Weber proved that every $k$-valued rational relation can be decomposed into a union of $k$ rational functions. We present a proof for this result by using $k$ applications of Eilengerg's Cross-section Theorem, and it seems to be simpler than that of Weber. By using this decomposition, we give another proof for deciding the equivalence of $k$-valued rational relations.

We also include the proof of Griffiths for the undecidability of the equivalence problem for finitely valued rational relations.

We extend a Schützenberger's property for rational functions to $k$-valued rational relations. As a consequence, we obtain a (non-polynomial) algorithm to decide if a transducer realizes a $k$-valued rational relation, for a given positive integer $k$.

We describe two efficient algorithms of Béal, Carton, Prieur and Sakarovitch: an algorithm for deciding if a rational relation is a function and another for deciding if a rational function is subsequential. Our description employs a simple symmetry property that leads to slightly more efficient algorithms in terms of time and space (the improvement is in the multiplicative constant).

We present Choffrut's characterization of subsequential functions, and an algorithm for the determinization of a transducer based on this characterization.

We study the minimization of subsequential transducers by using a class of monoids that we call monoids with gcd (where gcd stands by greatest common divisor). We prove the existence of a minimal subsequential transducer for a subsequential function $\Sigma^{*} \rightarrow M$, where $M$ is a cancellative monoid such that every non-empty subset has a single gcd. This result includes the minimization for functions whose images are in a free monoid or in the additive monoid of the non-negative real numbers.

We also show a characterization of the subsequential functions $\Sigma^{*} \rightarrow M$, where $M$ is a cancellative monoid with gcd, by using the right congruence of a function.

Finally, we describe an efficient minimization algorithm for subsequential transducers. This algorithm has two phases. The first phase is an algorithm of Béal and Carton for the construction of the prefix of a transducer, and the second is the minimization of the resulting transducer viewed as a deterministic automaton, by using the algorithm of Hopcroft-Gries.
\end{abstract}




\section{ÍNDICE}

Introdução $\quad$ iii

1 Preliminares 1

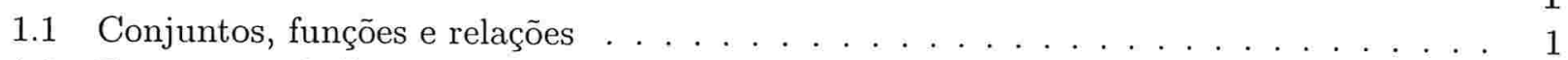

1.2 Estruturas algébricas . . . . . . . . . . . . . . . . . . . . 4

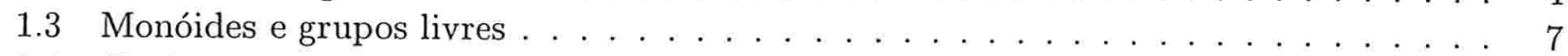

1.4 Grafos . . . . . . . . . . . . . . . . . . . . . . . 10

1.5 Subconjuntos racionais e reconhecíveis de um monóide . . . . . . . . . . . . . 12

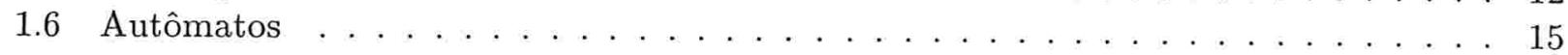

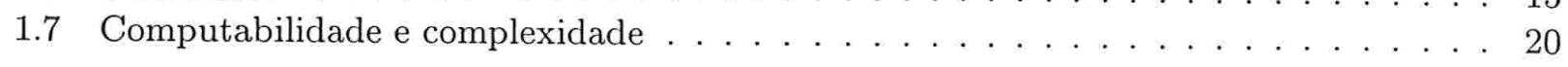

2 Relações racionais 23

2.1 Definições . . . . . . . . . . . . . . . . . . . . . . . . . 23

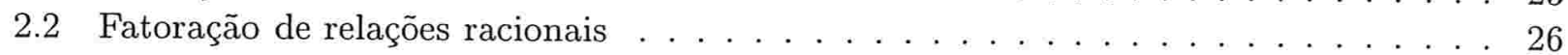

2.3 Transdutores . . . . . . . . . . . . . . . . . . . . . . . . . 31

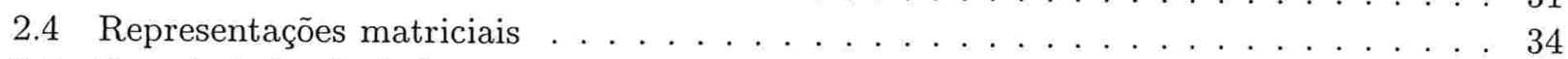

2.5 Propriedades de fechamento . . . . . . . . . . . . . . . . . 40

3 Relações racionais finitamente valoradas $\quad 45$

3.1 Definições e propriedades básicas . . . . . . . . . . . . . . . . . . 45

3.2 Transdutores letra-saída e representações matriciais . . . . . . . . . . . . . . . . . 51

3.3 Decidibilidade de se uma relação racional é finitamente valorada . . . . . . . . . . 56

3.4 Decidibilidade de se uma relação racional é $k$-valorada para um dado $k \ldots \ldots 6$

3.5 Equivalência de relações racionais finitamente valoradas . . . . . . . . . . . . . 65

3.6 Métricas e diferenças . . . . . . . . . . . . . . . . . . . . . . . 67 . . . . . . . .

3.7 Decomposição de uma relação racional $k$-valorada . . . . . . . . . . . . . . . . . . . 72

3.8 Equivalência de relações racionais $k$-valoradas . . . . . . . . . . . . . . . . 85

3.9 Ambigüidade . . . . . . . . . . . . . . . . . . . . . . 88

4 Funções racionais $\quad 95$

4.1 Decisão de se uma relação racional finitamente valorada é uma função . . . . . . . . 95

4.2 Funções seqüenciais e subseqüenciais . . . . . . . . . . . . . . . . . 105 
4.3 Decomposição de funções racionais em funções seqüenciais . . . . . . . . . . . . . . 122

4.4 Caracterização de funções seqüenciais e de funções subseqüenciais . . . . . . . . . . 125

4.5 Decidibilidade de se uma função racional é subseqüencial . . . . . . . . . . . . . 135

4.6 Determinização de um transdutor . . . . . . . . . . . . . . . . . . . . 152

4.7 Monóides com mdc . . . . . . . . . . . . . . . . . . . . . . . . 161

4.8 Forma prefixo e morfismos de transdutores subseqüenciais . . . . . . . . . . . 167

4.9 Transdutores subseqüenciais minimais . . . . . . . . . . . . . . 173

4.10 Congruência de uma função e redução . . . . . . . . . . . . . . . . . . 182

4.11 Construção do prefixo de um transdutor subseqüencial . . . . . . . . . . . . . . . 195

4.12 Construção do transdutor minimal . . . . . . . . . . . . . . . . . . 204

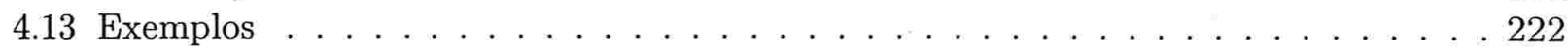

$\begin{array}{lr}\text { Conclusão } & 229\end{array}$

Referências Bibliográficas $\quad 233$

$\begin{array}{ll}\text { Índice Remissivo } & 237\end{array}$ 


\section{INTRODUÇÃO}

Uma das ramificações da Teoria dos Autômatos, área muito extensa e fundamental em Ciência da Computação, é o estudo de relações entre monóides que podem ser realizadas por máquinas de estados finitos. Essas relações são denominadas relações racionais, e essas máquinas transdutores.

A origem do estudo dos transdutores confunde-se com as primeiras pesquisas em Teoria dos Autômatos, e remonta à década de cinqüenta, com a introdução das máquinas de Mealy e de Moore. Posteriormente, o desenvolvimento de transdutores mais gerais que essas máquinas demonstrou a riqueza das relações racionais, fazendo com que seu estudo ocupasse uma posição de destaque.

Assim, verificou-se ao longo das décadas de sessenta e setenta o surgimento de muitos resultados teóricos sobre relações racionais, relacionados a problemas de decisão, caracterizações, e aplicações na pesquisa de linguagens formais. Uma parte importante desse desenvolvimento é o livro de Eilenberg [Eil74] de 1974, que apresenta com rigor grande parte de todo o conhecimento existente na época sobre a área.

Paralelamente ao estudo teórico, verificou-se nas décadas de oitenta e noventa um grande esforço no desenvolvimento de algoritmos eficientes para transdutores. Na década de noventa, ocorreu também a aplicação de transdutores e de alguns desses algoritmos em sistemas para processamento de linguagem natural. Outras áreas também foram beneficiadas com as técnicas desenvolvidas.

Um aspecto interessante desses algoritmos é a utilização direta de resultados teóricos, alguns dos quais bastante complexos. Como exemplo, podemos citar dois algoritmos: um para a determinização de um transdutor funcional, e outro para decidir se um transdutor funcional realiza uma função subseqüencial, ambos estudados neste trabalho, e que utilizam explicitamente uma caracterização muito forte das funções subseqüenciais obtida na década de setenta por Choffrut [Cho79, Cho78].

Neste trabalho, estudamos aspectos teóricos e algorítmicos de algumas classes de relações racionais: as relações racionais finitamente valoradas, as relações racionais $k$-valoradas, para todo inteiro positivo $k$, as funções seqüenciais e as funções subseqüenciais.

Dizemos que uma relação $\tau: M \rightarrow N$, onde $\mathrm{M}$ e $\mathrm{N}$ são monóides, é uma relação racional, se o seu gráfico for um subconjunto racional do monóide produto $\mathrm{M} \times \mathrm{N}$. Um transdutor sobre $\mathrm{M}$ e $\mathrm{N}$ é uma máquina cujas transições são rotuladas por um elemento de $\mathrm{M}$, representando uma entrada, e por um elemento de $\mathrm{N}$, representando uma saída.

Inicialmente, apresentamos as demonstrações de alguns resultados clássicos sobre as relações 
racionais, sendo que os mais importantes são o Teorema de Nivat (Teorema 2.2.1), que fornece uma fatoração muito útil das relações racionais, e o Teorema de Elgot e Mezei (Teorema 2.5.1), que prova o fechamento de determinadas relações racionais pela operação de composição. Também definimos e estudamos algumas propriedades de transdutores e representações matriciais, que são duas maneiras equivalentes de representar as relações racionais (seções 2.3 e 2.4).

Em seguida, estudamos as relações racionais finitamente valoradas, e as $k$-valoradas, para todo inteiro positivo $k$. Dizemos que uma relação racional $\tau: \mathrm{M} \rightarrow \mathrm{N}$ é finitamente valorada se

$$
\operatorname{card}(x \tau)<\infty, \quad \forall x \in \mathrm{M},
$$

e $k$ valorada, onde $k$ é um inteiro positivo, se

$$
\operatorname{card}(x \tau) \leq k, \quad \forall x \in \mathrm{M} .
$$

Primeiramente, apresentamos um algoritmo eficiente para decidir se um transdutor realiza uma relação finitamente valorada (Teorema 3.3.1), e uma caracterização das relações racionais $k$-valoradas $\Sigma^{*} \rightarrow \Gamma^{*}$, onde $\Sigma$ e $\Gamma$ são alfabetos, para todo inteiro positivo $k$. Essa caracterização estende uma caracterização de Schützenberger das relações racionais 1-valoradas, ou funções racionais, entre monóides livres. Provamos que, dada uma representação matricial $\mathbf{M}=(Q, \lambda, \mu, \rho)$ de dimensão $n$ sobre $\Sigma$ e $\Gamma$, se a condição card $\left(u \mu_{p q}\right) \leq k$ for satisfeita para todo par de estados $p$ e $q$ e toda palavra $u$ em $\Sigma^{*}$ de comprimento limitado por uma função que depende apenas de $n$ e $k$, então essa condição é satisfeita para toda palavra $u$ em $\Sigma^{*}$ (Teorema 3.4.1). Esse resultado fornece um algoritmo (não-eficiente) para decidir se um transdutor realiza uma relação racional $k$-valorada, para um dado inteiro positivo $k$.

Em 1992, Weber [Web92] provou que toda relação racional $k$-valorada $\tau: \Sigma^{*} \rightarrow \Gamma^{*}$ pode ser decomposta numa união

$$
\tau=f_{1} \cup \cdots \cup f_{k},
$$

onde cada $f_{i}$ é uma função racional. A prova de Weber é longa e complicada, e utiliza intensivamente construções com transdutores. Neste trabalho, apresentamos uma prova mais algébrica para esse resultado (Teorema 3.7.2), que utiliza $k$ aplicações do Teorema Cross-section de Eilenberg. Essa prova parece ser mais simples que a de Weber.

Utilizando essa decomposição, apresentamos uma prova para a decidibilidade da equivalência de relações racionais $k$-valoradas (Teorema 3.8.1). Esse resultado já havia sido provado por Culik e Karhumaki [CK86] em 1986, e por Weber [Web88] em 1988. Por outro lado, Griffiths [Gri68] provou que o problema da equivalência para as relações racionais finitamente valoradas é indecidível. Neste trabalho, incluímos também a prova de Griffiths (Teorema 3.5.1).

Com relação às funções racionais, apresentamos um algoritmo eficiente de Sakarovitch et al. [BCPS03] para decidir se um transdutor realiza uma função. Observamos uma propriedade simples de simetria, que permitiu a diminuição do tempo e espaço consumidos por esse algoritmo (Seção 4.1).

Finalmente, estudamos as funções subseqüenciais e as seqüenciais. Dizemos que uma função racional é subseqüencial (seqüencial) se puder ser realizada por um transdutor subseqüencial (seqüen- 
cial). Um transdutor subseqüencial é um transdutor determinístico na entrada, mais geral que os transdutores seqüenciais, e que foi proposto por Schützenberger [Sch77].

Apresentamos uma prova de Elgot e Mezei da representação de uma função racional $\Sigma^{*} \rightarrow \Gamma^{*}$ como uma composição de uma função seqüencial à esquerda com uma função seqüencial à direita (Teorema 4.3.1).

Estudamos uma caracterização de Choffrut [Cho79] das funções subseqüenciais $\Sigma^{*} \rightarrow \Gamma^{*}$ (Teorema 4.4.2), que generaliza uma caracterização de Ginsburg e Rose [GR66] das funções seqüenciais (Teorema 4.4.1). Essa caracterização é utilizada explicitamente em um algoritmo de Sakarovitch et al. [BCPS03] para decidir se uma função racional é subseqüencial, e também em um algoritmo para a determinização de um transdutor letra-palavra que realiza uma função subseqüencial [RS97, BC00b, Moh95]. Os dois algoritmos estão descritos neste trabalho (seções 4.5 e 4.6 , respectivamente), sendo que os consumos de tempo e espaço do algoritmo de Sakarovitch et al. puderam ser melhorados, utilizando uma propriedade simples de simetria.

Em seguida, estudamos a minimização de transdutores subseqüenciais. O estudo de um transdutor minimal que realiza uma função subseqüencial aparece implicitamente em trabalhos da década de setenta de Choffrut [Cho78, Cho79], e explicitamente em trabalhos mais recentes de Reutenauer [Reu90] e Choffrut [Cho03]. Nesses dois últimos trabalhos, são consideradas funções com emissão em um monóide livre. Ademais, um conceito de transdutor minimal para funções subseqüenciais com emissões em monóides que não sejam um monóide livre, como o monóide dos números reais com a operação de adição, também foi estudado [Moh95, Moh97]. Em nosso trabalho, definimos uma família de monóides, que chamamos de monóides com mdc, e que abstrai as propriedades tanto de monóides livres, quanto de outros monóides de interesse, que sejam essenciais para a definição de um transdutor minimal. A teoria de minimização é desenvolvida utilizando essa classe de monóides (Seção 4.9).

Dessa forma, provamos que, para toda função subseqüencial $f: \Sigma^{*} \rightarrow \mathrm{M}$, onde $\mathrm{M}$ é um monóide cancelativo e com mdc único, existe um $\Sigma$-M-transdutor bi-acessível $\mathcal{T}_{f}$ que realiza $f$ e tal que, para todo $\Sigma$-M-transdutor $\mathcal{T}$ que realiza $f$, existe um morfismo próprio $\mathcal{T} \rightarrow \mathcal{T}_{f}$. O transdutor $\mathcal{T}_{f}$ é denominado transdutor minimal para a função $f$, e segue da definição de morfismo de transdutores que esse transdutor é único a menos de isomorfismo, e tem o menor número de estados dentre os transdutores que realizam $f$. A construção de $\mathcal{T}_{f}$ é feita diretamente a partir de $f$, utilizando as idéias desenvolvidas nas seções III.3 e XII.5 do livro de Eilenberg [Ei174].

Ainda com relação à minimização de transdutores subseqüenciais, definimos um $\Sigma$-M-transdutor subseqüencial reduzido, e provamos que a redução de um $\Sigma$-M-transdutor que realiza uma função $f$ produz o transdutor minimal $\mathcal{T}_{f}$ (Teorema 4.10.2).

Também estudamos uma caracterização das funções subseqüenciais $\Sigma^{*} \rightarrow M$, onde $M$ é um monóide cancelativo com mdc (sem a necessidade da propriedade do mdc único), que reforça um resultado semelhante de Choffrut para o caso de emissões em um monóide livre. Esse resultado utiliza o conceito de congruência sintática $\sim_{f}$ de uma função $f: \Sigma^{*} \rightarrow \mathrm{M}$, que é semelhante ao conceito de congruência sintática de um subconjunto de um monóide livre (Teorema 4.10.3).

Por fim, descrevemos um algoritmo para a minimização de um transdutor subseqüencial com 
emissões em um monóide livre. Esse algoritmo tem duas etapas, sendo que a primeira é a construção de uma forma normalizada intermediária de transdutor subseqüencial, denominada forma prefixo, e a segunda é a redução do transdutor resultante visto como um autômato finito. Para a construção do prefixo de um transdutor, descrevemos o algoritmo de Béal e Carton [BC00a] (Seção 4.11), e para a redução de um autômato, descrevemos o algoritmo de Hopcroft, versão de Gries [Hop71, Gri73] (Seção 4.12). Ambos os algoritmos são eficientes.

Dessa forma, este trabalho está organizado como segue.

No Capítulo 1, introduzimos os conceitos básicos de Álgebra e Teoria dos Autômatos que são utilizados ao longo de todo o texto, e fixamos a notação.

No Capítulo 2, definimos e estudamos algumas propriedades clássicas das relações racionais, bem como dos transdutores e das representações matriciais.

O Capítulo 3 é dedicado ao estudo das relações racionais finitamente valoradas, e das $k$ valoradas, para todo inteiro positivo $k$. Neste capítulo, apresentamos a decomposição de uma relação racional $k$-valorada em uma união de $k$ funções racionais, além de outros resultados para essas relações.

No Capítulo 4, estudamos as funções racionais, as funções seqüenciais, e as funções subseqüenciais. Destacamos os estudos da caracterização de Choffrut das funções subseqüenciais, da minimização de transdutores subseqüenciais, e de alguns algoritmos.

Por fim, apresentamos a Conclusão, a Bibliografia, e o Índice Remissivo. 


\section{PRELiminaRES}

Neste capítulo, fixamos a notação e apresentamos um resumo da teoria básica utilizada em todo o texto. As referências que seguimos são [Eil74, Ber79, CLRS01].

\subsection{Conjuntos, funções e relações}

Denotamos por $\mathbb{Z}$ o conjunto dos números inteiros, por $\mathbb{N}$ o conjunto dos números inteiros nãonegativos, ou naturais, por $\mathbb{Q}$ o conjunto dos números racionais, e por $\mathbb{R}$ o conjunto dos números reais. O conjunto dos números reais não-negativos será denotado por $\mathbb{R}_{+}$.

O conjunto vazio será denotado por $\varnothing$.

Dado um inteiro positivo $k$, vamos denotar por [ $k$ ] o conjunto de todos os inteiros de 1 até $k$.

Um conjunto unitário pode ser identificado com seu único elemento. Assim, podemos escrever simplesmente $u$ para o conjunto unitário $\{u\}$.

A cardinalidade de um conjunto $A$ será denotada por card $(A)$. Se $A$ for infinito, então escrevemos card $(A)=\infty$. O conjunto de todos os subconjuntos de um conjunto $A$, denominado conjunto das partes de $A$, será denotado por $2^{A}$.

Uma partição de $A$ é uma família

$$
\mathcal{P} \subseteq 2^{A}
$$

de subconjuntos não-vazios e dois a dois disjuntos de $A$ tal que

$$
\bigcup_{X \in \mathcal{P}} X=A \text {. }
$$

Dizemos que uma partição $\mathcal{P}^{\prime}$ de $A$ refina $\mathcal{P}$, ou é um refinamento de $\mathcal{P}$, se todo conjunto em $\mathcal{P}^{\prime}$ estiver contido em um conjunto em $\mathcal{P}$.

O produto cartesiano de dois conjuntos $A$ e $B$ é o conjunto $A \times B$ de todos os pares $(a, b)$ tais que $a$ pertence a $A$ e $b$ pertence a $B$.

Uma função parcial, ou simplesmente função, de $A$ em $B$, é um subconjunto

$$
f \subseteq A \times B
$$

tal que, para todo $a$ em $A$, se

$$
(a, b),\left(a, b^{\prime}\right) \in f
$$

então

$$
b=b^{\prime} \text {. }
$$


Escrevemos

$$
f: A \rightarrow B
$$

para denotar que $f$ é uma função de $A$ em $B$.

Dado um par $(a, b)$ em $f$, a imagem de $a$ por $f$ é

$$
a f=b .
$$

A notação $f(a)$ para a imagem de $a$ também poderá ser usada, embora a notação acima seja preferida.

Se não existir nenhum par da forma $(a, b)$ em $f$, então convencionamos que $a f=\varnothing$. Se $a f \neq \varnothing$ para todo $a \mathrm{em} A$, dizemos que $f$ é uma função total. Se $f=\varnothing$, dizemos que $f$ é a função vazia.

Se

$$
a f \neq b f, \quad \forall a, b \in A \text { tais que } a \neq b,
$$

dizemos que $f$ é uma função injetora. Se

$$
\text { para todo } b \in B \text {, existe } a \in A \text { tal que } a f=b \text {, }
$$

dizemos que $f$ é uma função sobrejetora. Se $f$ for uma função total injetora e sobrejetora, dizemos que $f$ é uma função bijetora, ou é uma bijeção.

É comum definir uma relação de $A$ em $B$ como um subconjunto de $A \times B$. Uma relação $r$ de $A$ em $B$ também pode ser vista como uma função

$$
r: A \rightarrow 2^{B},
$$

que associa todo elemento $a$ em $A$ ao conjunto dos elementos $b$ em $B$ tais que existe um par $(a, b)$ em $r$.

Na maior parte deste trabalho, vamos preferir a segunda definição, que é seguida em [Eil74]. Mas, um subconjunto de $A \times B$ também poderá ser chamado de relação, se não houver possibilidade de confusão.

Vamos usar as notações

$$
A \stackrel{r}{\rightarrow} B \quad \text { e } \quad r: A \rightarrow B
$$

para indicar que $r$ é uma relação de $A$ em $B$. Se $A=B$, dizemos que $r$ é uma relação sobre $A$.

Podemos estender $r$ para uma função $f: 2^{A} \rightarrow 2^{B}$ definindo

$$
X f=\bigcup_{a \in X} a r, \forall X \in 2^{A} .
$$

O domínio e a imagem de $r$ são respectivamente os conjuntos

$$
\operatorname{dom} r=\{a \in A: a r \neq \varnothing\}
$$

$\mathrm{e}$

$$
\operatorname{im} r=A r=(\operatorname{dom} r) r .
$$

O conjunto $A$ é denominado conjunto de definição de $r$, ou entrada, e $B$ conjunto de saída de $r$, ou simplesmente saída. 
O gráfico de $r$ é o subconjunto de $A \times B$ definido por

$$
\# r=\{(a, b) \in A \times B: b \in a r\} .
$$

A inversa de $r$ é a relação $r^{-1}: B \rightarrow A$ definida por

$$
b r^{-1}=\{a: b \in a r\}, \forall b \in B .
$$

A restrição de $r$ a um subconjunto $X$ de $A$ é a relação $r \mid X: A \rightarrow B$ definida por

$$
a(r \mid X)= \begin{cases}a r & \text { se } a \in X \\ \varnothing & \text { caso contrário. }\end{cases}
$$

A união de relações $r: A \rightarrow B$ e $s: A \rightarrow B$ é a relação $r \cup s: A \rightarrow B$ definida por

$$
a(r \cup s)=a r \cup a s, \forall a \in A .
$$

A composição de relações $r: A \rightarrow B$ e $s: B \rightarrow C$ é a relação $r s: A \rightarrow C$ definida por

$$
a(r s)=(a r) s, \quad \forall a \in A \text {. }
$$

Também podemos denotar uma composição por

$$
A \stackrel{r}{\rightarrow} B \stackrel{s}{\rightarrow} C .
$$

Dada outra relação $t: C \rightarrow D$, demonstra-se facilmente a associatividade da composição:

$$
(r s) t=r(s t) .
$$

Demonstra-se também facilmente que, para todo subconjunto $X$ de $A$,

$$
X(r s)=(X r) s .
$$

Dizemos que uma relação $r \subseteq A \times B$ é simétrica se satisfizer

$$
\text { se }(a, b) \in r \text {, então }(b, a) \in r \text {, }
$$

anti-simétrica se satisfizer

$$
\text { se }(a, b) \in r \text { e }(b, a) \in r \text {, então } a=b \text {, }
$$

e transitiva se satisfizer

$$
\text { se }(a, b) \in r \text { e }(b, c) \in r \text {, então }(a, c) \in r \text {. }
$$

Dizemos que uma relação $r \subseteq A \times A$ é reflexiva se satisfizer

$$
(a, a) \in r, \quad \forall a \in A \text {. }
$$

Dada uma relação $r$ sobre $A$, escrevemos $r^{(k)}, k \geq 0$, para denotar a relação $r \ldots r$ sobre $A$ obtida com $k$ composições de $r$. A notação $r^{(0)}$ representa a função identidade $A \rightarrow A$, ou seja, 
a função cujo gráfico é $\{(a, a): a \in A\}$. O fecho reflexivo e transitivo de $r$ é a relação sobre $A$ definida por

$$
r^{(\infty)}=\bigcup_{k \geq 0} r^{(k)}
$$

É claro que o fecho reflexivo e transitivo de uma relação é uma relação reflexiva e transitiva.

Uma relação de ordem (parcial) sobre um conjunto $A$ é uma relação sobre $A$ anti-simétrica e transitiva.

Uma relação de equivalência sobre um conjunto $A$ é uma relação $\sim$ sobre $A$ reflexiva, simétrica e transitiva. Também escrevemos

$$
a \sim b
$$

para denotar que $(a, b)$ pertence a $\sim$.

Para todo elemento $a$ em $A$, o conjunto

$$
[a]=\{x: x \sim a\}
$$

é denominado classe de equivalência de $a$. Um representante de $[a]$ é um elemento qualquer desse conjunto.

Uma relação de equivalência induz uma partição

$$
A / \sim=\{[a]: a \in A\}
$$

em $A$, denominada quociente de $A$ por $\sim$. Por outro lado, toda partição de $A$ é o quociente de $A$ por alguma relação de equivalência.

\subsection{Estruturas algébricas}

Um semigrupo é um par $(\mathrm{S}, \cdot \mathrm{s})$, onde $\mathrm{S}$ é um conjunto munido de uma operação associativa $\cdot \mathrm{S}$, que podemos denominar produto. Por comodidade, podemos simplesmente dizer que o conjunto $\mathrm{S}$ é um semigrupo. Não havendo possibilidade de confusão, podemos denotar 's por ', ou mesmo omitir esse símbolo em um produto de dois elementos de $\mathrm{S}$.

Se $\cdot s$ tiver uma identidade $1_{S}$ (ou 1, se não houver possibilidade de confusão), dizemos que $S$ é um monóide.

$\mathrm{O}$ produto de $\mathrm{S}$ pode ser estendido para quaisquer subconjuntos $A$ e $B$ de $\mathrm{S}$ como segue:

$$
A \cdot B=\{a \cdot b: a \in A, b \in B\} .
$$

Com essa extensão, $2^{\mathrm{S}}$ é um semigrupo. Se $\mathrm{S}$ for um monóide, então $2^{\mathrm{S}}$ é um monóide, cuja identidade é $\left\{1_{\mathrm{S}}\right\}$.

Dizemos que $\mathrm{M}$ é um monóide comutativo se, para quaisquer $u$ e $v$ em $\mathrm{M}$,

$$
u v=v u .
$$

Dizemos que um elemento $a$ de um monóide $\mathrm{M}$ é inversível se existir um elemento $a^{-1} \mathrm{em} \mathrm{M}$, chamado de inverso de $a$, tal que

$$
a a^{-1}=a^{-1} a=1 .
$$


Um grupo é um monóide tal que todo elemento é inversível.

Dado um subconjunto $A$ de um monóide $\mathrm{M}$, definimos o conjunto $A^{+}$como

$$
A^{+}=\bigcup_{k \geq 1} A^{k}
$$

onde $A^{k}$ é o produto de $A$ por $A, k$ vezes. De forma equivalente,

$$
A^{+}=\left\{x_{1} \ldots x_{k}: k \geq 1 \text { e } x_{i} \in A \text { para } 1 \leq i \leq k\right\} .
$$

A estrela de $A$ é o conjunto

$$
A^{*}=A^{+} \cup 1
$$

Claramente,

$$
A^{+}=A^{*} A=A A^{*} .
$$

Vamos fazer a convenção de que $A^{0}$ é o unitário $\{1\}$.

Dizemos que um semigrupo (monóide) $\mathrm{S}$ é gerado por um subconjunto $A$ de $\mathrm{S}$ se $\mathrm{S}=A^{+}$ $\left(\mathrm{S}=A^{*}\right)$. Dizemos que $A$ é um conjunto gerador para $\mathrm{S}$. Se $A$ for um conjunto finito, dizemos que $\mathrm{S}$ é finitamente gerado por $A$.

Seja $\mathrm{M}$ um monóide. Dizemos que um subconjunto $\mathrm{S}$ de $\mathrm{M}$ é um submonóide de $\mathrm{M}$ se

$$
1_{\mathrm{M}} \in \mathrm{S} \quad \text { e } \quad \mathrm{S}^{2} \subseteq \mathrm{S}
$$

Demonstra-se facilmente que, para qualquer subconjunto $A$ de $\mathrm{M}, A^{*}$ é um submonóide de $\mathrm{M}$.

Um morfismo de semigrupos $\mathrm{M}$ e $\mathrm{N}$ é uma função

$$
f: \mathrm{M} \rightarrow \mathrm{N}
$$

que satisfaz

$$
(a b) f=(a f)(b f), \quad \forall a, b \in \mathrm{M} .
$$

Se $M$ e $N$ forem monóides e $1 f=1$, então $f$ é um morfismo de monóides. Demonstra-se facilmente que a composição de dois morfismos de semigrupos é um morfismo de semigrupos, e que a composição de dois morfismos de monóides é um morfismo de monóides.

Um semi-anel é um conjunto $K$ munido de duas operações, denotadas por $+\mathrm{e} \cdot$, e denominadas respectivamente adição e produto, e que possuem as seguintes propriedades:

- A adição é associativa, comutativa, e possui uma identidade, denotada por 0, e denominada zero;

- O produto é associativo e possui uma identidade, denotada por 1, e denominada unidade;

- O produto é distributivo com respeito à adição: $a(b+c)=a b+a c$ e $(b+c) a=b a+c a$, para quaisquer $a, b$ e $c$ em $K$;

- O produto de qualquer elemento por 0 , e também o produto de 0 por qualquer elemento, é igual a 0 . 
A última condição não é conseqüência das anteriores.

Vamos denotar por $K^{n \times n}$ o conjunto das matrizes quadradas de ordem $n$ com entradas no semi-anel $K$. Esse conjunto, com as operações de adição e produto de matrizes, é um semi-anel.

Uma ação à direita, ou simplesmente ação, de um monóide $\mathrm{M}$ sobre um conjunto $Q$, é uma função parcial

$$
\delta: Q \times \mathrm{M} \rightarrow Q
$$

que respeita as propriedades a seguir. Para quaisquer $q$ em $Q$, e $m$ e $n$ em $\mathrm{M}$,

$$
\begin{aligned}
& (q, 1) \delta=q ; \\
& (q, m n) \delta=((q, m) \delta, n) \delta .
\end{aligned}
$$

Para simplificar, podemos escrever $q \cdot m$, ou ainda $q m$, ao invés de $(q, m) \delta$. Então, as condições (1.2.1a) e (1.2.1b) podem ser escritas como

$$
\begin{aligned}
& q \cdot 1=q ; \\
& q \cdot(m n)=(q \cdot m) \cdot n .
\end{aligned}
$$

Uma definição simétrica pode ser feita. Uma ação à esquerda de um monóide $\mathrm{M}$ sobre um conjunto $Q$ é uma função parcial

$$
\delta: \mathrm{M} \times Q \rightarrow Q
$$

que satisfaz

$$
\begin{aligned}
& 1 \cdot q=q \\
& (m n) \cdot q=m \cdot(n \cdot q), \quad \forall q \in Q, \forall m, n \in \mathrm{M},
\end{aligned}
$$

onde $m \cdot q$ representa $(m, q) \delta$.

Uma congruência sobre um monóide $\mathrm{M}$ é uma relação de equivalência $\sim$ sobre $\mathrm{M}$ que é consistente com o produto desse monóide. Formalmente, para todo par de elementos $m_{1}$ e $m_{2}$ de M,

$$
\text { se } m_{1} \sim m_{2} \text {, então } m m_{1} \sim m m_{2} \text { e } m_{1} m \sim m_{2} m, \quad \forall m \in \mathrm{M} \text {. }
$$

Uma formulação equivalente é

$$
\text { se } m_{1} \sim m_{1}{ }^{\prime} \text { e } m_{2} \sim m_{2}{ }^{\prime} \text {, então } m_{1} m_{2} \sim m_{1}{ }^{\prime} m_{2}{ }^{\prime} \text {. }
$$

$\mathrm{O}$ quociente $\mathrm{M} / \sim$ é um monóide com a operação

$$
[m][n]=[m n], \quad \forall m, n \in \mathrm{M} .
$$

Pela propriedade (1.2.5), essa definição não depende da escolha dos representantes $m$ e $n$. Portanto, está bem definida. A propriedade associativa é uma conseqüência da propriedade associativa do produto de $\mathrm{M}$, e a identidade é $\left[1_{\mathrm{M}}\right]$.

$\mathrm{Se} \sim$ satisfizer apenas

$$
\text { se } m_{1} \sim m_{2} \text {, então } m_{1} m \sim m_{2} m, \forall m \in \mathrm{M} \text {, }
$$

dizemos que $\sim$ é uma congruência à direita. 


\subsection{Monóides e grupos livres}

Um alfabeto é um conjunto finito e não-vazio, cujos elementos chamamos de letras. O semigrupo livre $\Sigma^{+}$gerado por um alfabeto $\Sigma$, ou semigrupo livre sobre $\Sigma$, é o conjunto das seqüências de elementos de $\Sigma$, chamadas de palavras. Acrescentando-se uma identidade 1 a $\Sigma^{+}$, obtemos um monóide, que denominamos monóide livre gerado por $\Sigma$, e denotamos por $\Sigma^{*}$. Essa identidade é chamada de palavra vazia. Vamos dizer simplesmente monóide livre para significar monóide livre gerado por um alfabeto.

O produto de elementos de um monóide livre também é denominado concatenação.

Subconjuntos de um monóide livre são denominados linguagens. Dizemos também que uma linguagem $L$ em $\Sigma^{*}$ é uma linguagem sobre $\Sigma$.

Uma palavra $u=\left(\sigma_{1}, \ldots, \sigma_{n}\right)$ pode ser escrita como $\sigma_{1} \ldots \sigma_{n}, \sigma_{i} \in \Sigma$ para todo $i$. O comprimento de $u$ é o inteiro $|u|=n$. Se $u=1$, então $|u|=0$. Evidentemente, $|u v|=|u|+|v|$, para palavras $u$ e $v$ quaisquer.

O número de ocorrências de uma letra $\sigma$ em uma palavra $u$ é denotado por $|u|_{\sigma}$.

O reverso de uma palavra $u=\sigma_{1} \ldots \sigma_{k}$ é a palavra $u^{\varrho}=\sigma_{k} \ldots \sigma_{1}$. Se $u=1$, então $1^{\varrho}=1$.

Dado um inteiro positivo $l$, denotamos o conjunto das palavras de $\Sigma^{*}$ de comprimento no máximo $l$ por $\Sigma \leq l$. Formalmente,

$$
\Sigma^{\leq l}=1 \cup \Sigma^{1} \cup \cdots \cup \Sigma^{l}
$$

Um fator de uma palavra $u$ é uma palavra $w$ tal que $u$ pode ser fatorada como $x w y$. Se $x=1$ $(y=1)$, dizemos que $w$ é um prefixo (sufixo) de $u$. Utilizamos o adjetivo próprio para $w$ quando $w \neq u$.

O conjunto de todos os fatores de $u$ é denotado por fat $(u)$. O conjunto de todos os prefixos (sufixos) de $u$ é denotado por $\operatorname{pref}(u)(\operatorname{suf}(u))$. Essas notações podem ser estendidas para linguagens. Dada uma linguagem não-vazia $A \mathrm{em} \Sigma^{*}$, denotamos por pref $(A)$ o conjunto dos prefixos de todas as palavras em $A$, e por $\operatorname{suf}(A)$ o conjunto dos sufixos de todas as palavras em $A$.

O fator com início em uma posição $i$ e término em uma posição $j$ de $u$ é denotado por $u[i \ldots j]$. Se $i=j$, então esse fator é a letra que ocorre em $u$ na posição $i$, e pode ser escrito como $u[i]$. Se $i>j$, definimos $u[i \ldots j]$ como a palavra vazia.

Dadas palavras $u$ e $v$, escrevemos $u \succ v$ para denotar que $u$ é prefixo de $v$, e $u \prec v$ para denotar que $u$ é sufixo de $v$. As relações $\succ \mathrm{e} \prec$ são ordens parciais sobre $\Sigma^{*}$. Quando duas palavras forem comparáveis por $\succ$, dizemos simplesmente que são comparáveis.

Outra relação de ordem sobre $\Sigma^{*}$ é a ordem lexicográfica, que denotamos por $<_{l}$. Essa ordem supõe uma ordenação $\sigma_{1}, \ldots, \sigma_{n}$ das letras de $\Sigma$. Dadas palavras $u$ e $v$ de $\Sigma^{*}, u<_{l} v$ quando uma das condições abaixo for satisfeita:

- $u \succ v$, ou

- $u$ e $v$ podem ser fatorados como $u=x \sigma_{i} u^{\prime}$ e $v=x \sigma_{j} v^{\prime}$, onde $\sigma_{i}$ e $\sigma_{j}$ são letras tais que $i<j$.

Verifica-se facilmente que $<_{l}$ é uma ordem total.

Dadas palavras $s$ e $t$, se existir uma palavra $t^{\prime}$ tal que

$$
t=s t^{\prime}
$$


então definimos

$$
s^{-1} t=t^{\prime}
$$

Se $s$ não for prefixo de $t$, fazemos a convenção de que $s^{-1} t=\varnothing$. De forma simétrica, definimos $t s^{-1}$ como a palavra $t^{\prime}$ tal que $t=t^{\prime} s$, ou $\varnothing$, caso essa palavra não exista.

Essas notações podem ser estendidas para linguagens como segue. Dadas uma linguagem $A$ sobre $\Sigma$ e uma palavra $s$ em $\Sigma^{*}$, definimos

$$
s^{-1} A=\left\{s^{-1} x: x \in A\right\} \quad \text { e } \quad A s^{-1}=\left\{x s^{-1}: x \in A\right\} .
$$

Dizemos que um morfismo de monóides livres $f: \Sigma^{*} \rightarrow \Gamma^{*}$ é fino se $\Sigma f \subseteq \Gamma \cup 1$, e muito fino se $\Sigma f \subseteq \Gamma$.

Proposição 1.3.1 Seja $f: \Sigma^{*} \rightarrow \Gamma^{*}$ um morfismo fino de monóides. Então, $f^{-1}$ é um morfismo de semigrupos de $\Gamma^{*}$ no monóide multiplicativo $2^{\Sigma^{*}}$.

Demonstração. Vamos mostrar que, para todo par de palavras $u$ e $v$ em $\Gamma^{*}$,

$$
(u v) f^{-1}=\left(u f^{-1}\right)\left(v f^{-1}\right) .
$$

Sejam $x$ e $y$ palavras em $u f^{-1}$ e $v f^{-1}$, respectivamente. Então, como $f$ é um morfismo, $x y$ pertence a $(u v) f^{-1}$. Assim,

$$
\left(u f^{-1}\right)\left(v f^{-1}\right) \subseteq(u v) f^{-1} .
$$

Seja agora $w$ uma palavra em $(u v) f^{-1}$. Como $f$ é um morfismo fino, deve existir uma fatoração $x y$ de $w$ tal que $x f=u$ e $y f=v$. Portanto, $w$ pertence a $\left(u f^{-1}\right)\left(v f^{-1}\right)$. Assim,

$$
(u v) f^{-1} \subseteq\left(u f^{-1}\right)\left(v f^{-1}\right) .
$$

Uma substituição é uma função $h: \Sigma^{*} \rightarrow 2^{\mathrm{M}}$ que satisfaz

$$
1 h=1 \text { e }(u v) h=(u h)(v h), \quad \forall u, v \in \Sigma^{*} .
$$

Ou seja, uma substituição é um morfismo de monóides de $\Sigma^{*}$ no monóide dos subconjuntos de M com a operação de produto de conjuntos. Dizemos que $h$ é uma substituição racional se uh for um subconjunto racional de $\mathrm{M}$ para toda palavra $u$. A definição de subconjunto racional é apresentada na Seção 1.5 (Definição 1.5.2).

Podemos estender $h$ a um morfismo de monóides $2^{\Sigma^{*}} \rightarrow 2^{\mathrm{M}}$ devido à propriedade

$$
(A B) h=(A h)(B h), \forall A, B \in 2^{\Sigma^{*}} .
$$

Se $f: \Sigma^{*} \rightarrow \Gamma^{*}$ for um morfismo muito fino de monóides, então $1 f^{-1}=1$. Da Proposição 1.3.1, segue que $f^{-1}$ é um morfismo de monóides. Ademais, para toda palavra $u$ em $\Gamma^{*}, u f^{-1}$ é um conjunto finito. Portanto, $f^{-1}$ é uma substituição racional.

As seguintes propriedades de morfismos de monóides livres são fundamentais. 
Proposição 1.3.2 Sejam $f: \Sigma^{*} \rightarrow \mathrm{M}$ e $g: \Sigma^{*} \rightarrow \mathrm{M}$ morfismos de semigrupos. Se $f$ e $g$ coincidirem em $\Sigma \cup 1$, então $f=g$.

Demonstração. Basta provar que, para toda palavra $s=\sigma_{1} \ldots \sigma_{n}$ em $\Sigma^{+}$, onde $\sigma_{i}$ é uma letra para todo índice $i, s f=s g$. Vamos usar indução em $|s|$ :

- $|s|=1$ : segue da hipótese de que $f$ e $g$ coincidem em $\Sigma$.

- $|s|>1$ : fatore $s$ como $t \sigma$, onde $\sigma$ é uma letra. Pela hipótese de indução, $t f=t g$. Como $f$ e $g$ coincidem em $\Sigma, \sigma f=\sigma g$. Portanto, $s f=(t f)(\sigma f)=(t g)(\sigma g)=s g$.

Proposição 1.3.3 Sejam $\Sigma$ um alfabeto e $\mathrm{M}$ um monóide. Uma função $f: \Sigma \rightarrow \mathrm{M}$ admite uma única extensão a um morfismo de monóides $\Sigma^{*} \rightarrow \mathrm{M}$.

Demonstração. É evidente que a função $f^{\prime}: \Sigma^{*} \rightarrow$ M definida por

$$
\left(\sigma_{1} \ldots \sigma_{n}\right) f^{\prime}=\left(\sigma_{1} f\right) \ldots\left(\sigma_{n} f\right)
$$

para toda palavra $\sigma_{1} \ldots \sigma_{n}$ em $\Sigma^{*}$, onde $n \geq 0$ e cada $\sigma_{i}$ é uma letra, é uma extensão de $f$ a um morfismo de monóides.

Para provar a unicidade de $f^{\prime}$, seja $g: \Sigma^{*} \rightarrow$ M um morfismo de monóides que coincide com $f$ em $\Sigma$. Então, $g$ coincide com $f^{\prime}$ em $\Sigma \cup 1$. Da Proposição 1.3.2, segue então que $g=f^{\prime}$.

Pela Proposição 1.3.3, um morfismo de monóides $\Sigma^{*} \rightarrow$ M fica completamente determinado pelas imagens de $f$ nas letras de $\Sigma$.

A cópia de um alfabeto $\Sigma$ é uma bijeção $b: \Sigma \rightarrow \Sigma^{\prime}$, onde $\Sigma^{\prime}$ é um alfabeto disjunto de $\Sigma$. A imagem $\sigma b$ de uma letra $\sigma$ pode ser denotada por $\sigma^{\prime}$. A bijeção $b$ pode ser estendida para um morfismo, que denotamos também por $b$.

Vamos freqüentemente identificar o monóide aditivo $\mathbb{N}$ com o monóide livre gerado por uma única letra $\sigma$. Ou seja, um número natural $n$ será visto como a palavra $\sigma^{n}$.

Seja $Q$ um conjunto. Uma função parcial

$$
Q \times \Sigma \rightarrow Q
$$

pode ser estendida a uma ação de $\Sigma^{*}$ sobre $Q$ indutivamente como segue:

$$
\begin{aligned}
& q \cdot 1=q ; \\
& q \cdot(u \sigma)=(q \cdot u) \cdot \sigma, \quad \forall u \in \Sigma^{*}, \forall \sigma \in \Sigma .
\end{aligned}
$$

Demonstra-se facilmente por indução que · de fato é uma ação.

Vamos agora introduzir o conceito de grupo livre gerado por um alfabeto $\Sigma$.

Seja $b: \Sigma \rightarrow \Sigma^{\prime}$ uma cópia de $\Sigma$. A imagem por $b$ ou por $b^{-1}$ de uma letra $\sigma$ em $\Sigma \cup \Sigma^{\prime}$ é denotada por $\sigma^{-1}$. Seja $\sim$ a relação no monóide livre gerado pelo alfabeto $\Gamma=\Sigma \cup \Sigma^{\prime}$ definida como segue. Dadas palavras $u$ e $v$ em $\Gamma^{*}, u \sim v$ se, e somente se, existem uma letra $\sigma$ em $\Gamma$ e palavras $s$ e $t$ em $\Gamma^{*}$ tais que

$$
u=s \sigma \sigma^{-1} t \quad \text { e } \quad v=s t, \quad \text { ou } \quad v=s \sigma \sigma^{-1} t \quad \text { e } \quad u=s t .
$$


É evidente que a relação $\sim$ é simétrica.

Seja $\approx$ o fecho reflexivo e transitivo de $\sim$. Informalmente, $u \approx v$ significa que $u$ pode ser transformada em $v$ através da aplicação de um número finito de cancelamentos ou inclusões de pares de letras da forma $\sigma \sigma^{-1}$.

Afirmamos que $\approx$ é uma congruência em $\Gamma^{*}$. De fato, $\approx$ é reflexiva e transitiva pela definição de fecho, e simétrica porque $\sim$ é simétrica. Para mostrar que essa relação é uma congruência, considere palavras $s, t, u$ e $v$ em $\Gamma^{*}$ tais que $s \approx t$ e $u \approx v$. Então, as transformações $s u \approx t u \approx t v$ mostram que $s u \approx t v$.

O grupo livre gerado por $\Sigma$ é o monóide quociente $\Gamma^{*} / \approx$. Denotamos esse grupo por $\Sigma^{(*)}$.

Para provar que $\Sigma^{(*)}$ de fato é um grupo, seja $u=\sigma_{1} \ldots \sigma_{n}$ uma palavra em $\Gamma^{*}$, onde $n>0$ e cada $\sigma_{i}$ é uma letra, e defina o inverso formal de $u$ como a palavra $u^{-1}=\sigma_{n}{ }^{-1} \ldots \sigma_{1}{ }^{-1}$. Então, pela definição de $\approx$,

$$
u u^{-1} \approx 1 \text { e } u^{-1} u \approx 1 .
$$

Portanto, $\left[u^{-1}\right]$ é o inverso da classe $[u]$.

Será conveniente representar uma classe $[u]$ simplesmente pela palavra $u$. Nesse caso, podemos fazer um abuso de notação e dizer que $u$ é uma palavra em $\Sigma^{(*)}$. Podemos então reescrever (1.3.4) como

$$
u u^{-1}=u^{-1} u=1,
$$

e dizer que o inverso formal $u^{-1}$ é o inverso da palavra $u$ no grupo $\Sigma^{(*)}$.

Usualmente, cada classe é representada por uma palavra que não pode ser reduzida por cancelamento. Formalmente, dizemos que uma palavra $u$ em $\Sigma^{(*)}$ é reduzida se $u$ não puder ser fatorada como $x s s^{-1} y$, onde $s$ é uma palavra diferente de 1. Equivalentemente, $u$ é reduzida se puder ser escrita como $\sigma_{1} \ldots \sigma_{n}$, onde cada $\sigma_{i}$ é uma letra, e duas letras $\sigma$ e $\sigma^{-1}$ não aparecem consecutivamente nessa palavra.

Um fato básico sobre grupos livres é a unicidade da existência de uma palavra reduzida em cada classe de $\Sigma^{(*)}$. Em outras palavras, duas palavras equivalentes $s$ e $t$ são equivalentes a exatamente uma palavra reduzida. Esse fato intuitivo tem uma demonstração construtiva simples, que não apresentamos aqui, mas que pode ser vista em [MKS66].

\subsection{Grafos}

Em alguns algoritmos descritos neste trabalho, um autômato é visto simplesmente como um grafo dirigido. Nesta seção, resumimos os conceitos da Teoria dos Grafos necessários para esses algoritmos.

Definição 1.4.1 Um grafo (dirigido) é um par $G=(Q, E)$ composto de

- um conjunto finito $Q$ de vértices;

- um subconjunto $E \subseteq Q \times Q$ de arcos.

Um arco $e=(p, q)$ pode ser representado como

$$
e: p \rightarrow q
$$


Um passeio $c$ é uma seqüência de arcos

$$
c:\left(p_{1} \rightarrow q_{1}\right)\left(p_{2} \rightarrow q_{2}\right) \ldots\left(p_{n} \rightarrow q_{n}\right)
$$

tal que

O comprimento de $c$ é

$$
q_{i}=p_{i+1}, \quad \forall i, 1 \leq i<n .
$$

Podemos representar $c$ como

$$
\|c\|=n .
$$

$$
c: p_{1} \rightarrow p_{2} \rightarrow \cdots \rightarrow p_{n}
$$

ou

$$
c: p_{1} \rightarrow p_{n},
$$

e dizer que $c$ é um passeio de $p_{1}$ a $p_{n}$. Dizemos que $p_{1}$ é a origem de $c$, que $p_{n}$ é o término de $c$, e que $c$ passa pelos vértices $p_{1}, \ldots, p_{n}$.

Dizemos que $c$ é um passeio fechado se $p_{1}=p_{n}$, que é um circuito se $p_{1}=p_{n}$ e, para todo par de vértices $p_{i}$ e $p_{j}$ tais que $i \neq j$ e $1<j<n, p_{i} \neq p_{j}$, e que é um caminho se, para todo par de vértices $p_{i}$ e $p_{j}$ tais que $i \neq j, p_{i} \neq p_{j}$.

Dizemos que um grafo é acíclico se não possuir nenhum passeio fechado.

Dizemos que um passeio é vazio se não possuir nenhum arco. O comprimento de um passeio vazio é 0 .

Dados passeios $c: p \rightarrow q^{\prime}$ e $d: q^{\prime} \rightarrow q$, a concatenação de $c$ e $d$ é o passeio de $p$ a $q$

$$
c d:\left(p \rightarrow q^{\prime}\right)\left(q^{\prime} \rightarrow q\right) .
$$

Se $c(d)$ for um passeio vazio, então $c d=d(=c)$.

Dados vértices $p$ e $q$, dizemos que $q$ é acessivel a partir de $p$ e que $p$ é co-acessivel a $q$ se existir um passeio de $p$ a $q$.

O território de um conjunto de vértices $Q^{\prime}$ é o conjunto dos vértices acessíveis a partir de algum vértice em $Q^{\prime}$.

Às vezes será conveniente permitir arcos múltiplos, ou seja, arcos com a mesma origem e o mesmo término. Nesse caso, $E$ pode ser definido como uma função

$$
E: Q \times Q \rightarrow \mathbb{N}
$$

que associa cada par $(p, q)$ ao número de arcos com origem igual a $p$ e término igual a $q$.

Seja $Q^{\prime}$ um subconjunto de $Q$. A restrição de $G$ a $Q^{\prime}$ é o grafo $G\left[Q^{\prime}\right]=\left(Q^{\prime}, E^{\prime}\right)$, onde $E^{\prime}$ contém exatamente os arcos com origem e término em $Q^{\prime}$.

Seja $X$ um subconjunto de $E$. A restrição de $G$ a $X$ é o grafo $G[X]=\left(Q^{\prime}, X\right)$, onde $Q^{\prime}$ contém exatamente os vértices que são origem ou término de algum arco em $X$.

Considere a relação de equivalência em $Q$ definida a seguir:

$$
p \sim q \text { se, e somente se, existem passeios } p \rightarrow q \text { e } q \rightarrow p .
$$

Cada classe de equivalência em $Q / \sim$ é denominada um componente fortemente conexo de $G$. A classe de um vértice $p$ é denotada por $C(p)$. Definimos o grafo quociente por $\sim$ como o grafo $G / \sim=\left(Q / \sim, E^{\prime}\right)$, onde $E^{\prime}$ contém um arco

$$
C(p) \rightarrow C(q)
$$


se, e somente se, $E$ contém um arco

$$
p \rightarrow q
$$

Demonstra-se facilmente que $G / \sim$ é acíclico.

Dizemos que uma função $\psi$ que associa cada vértice de $G$ a um inteiro positivo é uma numeração topológica se satisfizer

$$
q \psi<p \psi \text { para todo arco } p \rightarrow q .
$$

O seguinte resultado, cuja demonstração pode ser vista em [CLRS01], é clássico:

Proposição 1.4.1 Se G for um grafo acíclico, então existe uma numeração topológica dos vértices de $G$.

\subsection{Subconjuntos racionais e reconhecíveis de um monóide}

Nesta seção, vamos estudar os subconjuntos racionais e reconhecíveis de um monóide qualquer. Esse estudo independe do uso de máquinas de estados finitos. As conexões dessas famílias com máquinas de estados finitos serão apresentadas na Seção 1.6.

Ao longo desta seção, $\mathrm{M}$ e $\mathrm{N}$ representarão monóides, e $\Sigma$ um alfabeto.

Definição 1.5.1 Dizemos que um subconjunto $R$ de $\mathrm{M}$ é reconhecível se existirem um monóide finito $\mathrm{N}$, um morfismo $f: \mathrm{M} \rightarrow \mathrm{N}$ e um subconjunto $P$ de $\mathrm{N}$ tais que $R=P f^{-1}$. A família dos conjuntos reconhecíveis de $\mathrm{M}$ é denotada por $\operatorname{Rec}(\mathrm{M})$.

Definição 1.5.2 A família dos subconjuntos racionais de $\mathrm{M}$, denotada por $\operatorname{Rac}(\mathrm{M})$, é a menor família de subconjuntos de $\mathrm{M}$ que contém o conjunto vazio, os conjuntos unitários, e é fechada pelas operações de produto, união $e+$.

Segue dessas condições que Rac(M) também é fechada por $*$. Dizemos que um subconjunto $R$ de M é racional se $R \in \operatorname{Rac}(\mathrm{M})$.

As operações de produto, união, $+\mathrm{e}$ * são denominadas operações racionais. Uma expressão racional é uma expressão envolvendo operações racionais e subconjuntos unitários de $\mathrm{M}$, podendo ser definida indutivamente como segue:

- $\varnothing$ e $m$, para todo $m$ em $\mathrm{M}$, são expressões racionais;

- se $e$ e $e^{\prime}$ forem expressões racionais, então $(e), e \cup e^{\prime}, e e^{\prime}, e^{+}$e $e^{*}$ são expressões racionais.

Uma expressão racional corresponde a um subconjunto de M. Utilizando a definição de $\operatorname{Rac}(\mathrm{M})$, e indução no número de operadores de uma expressão racional, demonstra-se facilmente que

Proposição 1.5.1 A família $\operatorname{Rac}(\mathrm{M})$ é exatamente a família dos subconjuntos de $\mathrm{M}$ obtidos com expressões racionais.

Um dos resultados principais da Teoria dos Autômatos, e rico em conseqüências, é o Teorema de Kleene:

Teorema 1.5.1 (Kleene 1956) Seja $\Sigma$ um alfabeto. Então, $\operatorname{Rec}\left(\Sigma^{*}\right)=\operatorname{Rac}\left(\Sigma^{*}\right)$. 
Essa igualdade não é verdadeira para qualquer monóide. Um contra-exemplo pode ser visto no Exemplo 2.1.3.

As propriedades

$$
A(B \cup C)=A B \cup A C, \quad(B \cup C) A=B A \cup C A, \quad \forall A, B, C \in \operatorname{Rac}(\mathrm{M})
$$

e

$$
A 1=1 A=A, \quad A \cup \varnothing=\varnothing \cup A=A, \quad \forall A \in \operatorname{Rac}(\mathrm{M}),
$$

mostram que $\operatorname{Rac}(\mathrm{M})$ é um semi-anel, cuja adição é a operação de união de conjuntos, e o produto é a operação de produto de conjuntos.

A seguir, listamos algumas propriedades fundamentais dos subconjuntos racionais e reconhecíveis de um monóide. As provas não apresentadas neste texto podem ser vista no livro de Berstel [Ber79].

Proposição 1.5.2 Sejam $f: \mathrm{M} \rightarrow \mathrm{N}$ um morfismo de monóides e A um subconjunto racional de M. Então, Af é um subconjunto racional de N.

Proposição 1.5.3 Sejam $A$ e $B$ subconjuntos reconhecíveis de um monóide $\mathrm{M}, C$ um subconjunto reconhecivel de um monóide $\mathrm{N}$, e $f: \mathrm{M} \rightarrow \mathrm{N}$ um morfismo de monóides. Então,

$$
C f^{-1} \in \operatorname{Rec}(\mathrm{M}), \quad A \cup B \in \operatorname{Rec}(\mathrm{M}), \quad A \cap B \in \operatorname{Rec}(\mathrm{M}) \text { e } \mathrm{M}-A \in \operatorname{Rec}(\mathrm{M}) .
$$

Proposição 1.5.4 Seja $R$ um subconjunto de um monóide M. Então, $R$ é um conjunto racional se, e somente se, existem um alfabeto $\Sigma$, uma linguagem racional $L \subseteq \Sigma^{*}$ e um morfismo $f: \Sigma^{*} \rightarrow \mathrm{M}$ tais que $R=L f$.

Demonstração. Se $R=L f$ para uma linguagem racional $L$ e um morfismo $f: \Sigma^{*} \rightarrow \mathrm{M}$, então, pela Proposicao 1.5.2, $R$ é um subconjunto racional de $M$.

Para provar a implicação contrária, seja $F$ a família dos subconjuntos $A$ de $\mathrm{M}$ tais que existem um alfabeto $\Sigma$, uma linguagem racional $L \subseteq \Sigma^{*}$ e um morfismo $f: \Sigma^{*} \rightarrow$ M satisfazendo $L f=A$. Vamos provar que $\operatorname{Rac}(\mathrm{M}) \subseteq F$.

Claramente, $\varnothing$ e todo subconjunto finito de $M$ pertencem a $F$. Suponha que $A$ e $B$ sejam subconjuntos de $M$ tais que existem alfabetos $\Sigma$ e $\Gamma$, morfismos de monóides $f: \Sigma^{*} \rightarrow \mathrm{M}$ e $g: \Gamma^{*} \rightarrow \mathrm{M}$, e linguagens racionais $X \subseteq \Sigma^{*}$ e $Y \subseteq \Gamma^{*}$ tais que $A=X f$ e $B=Y g$. Podemos supor que $\Sigma$ e $\Gamma$ são disjuntos. Seja $h:(\Sigma \cup \Gamma)^{*} \rightarrow \mathrm{M}$ o morfismo de monóides gerado por

$$
\sigma h=\sigma f, \gamma h=\gamma g, \quad \forall \sigma \in \Sigma, \forall \gamma \in \Gamma .
$$

Verifica-se facilmente que

$$
A \cup B=(X \cup Y) h, A B=(X Y) h \text { e } A^{+}=X^{+} h .
$$

Assim, $A \cup B, A B$ e $A^{+}$são imagens por $h$ de linguagens racionais sobre o alfabeto $\Sigma \cup \Gamma$.

Concluímos então que $F$ contém $\varnothing$ e os subconjuntos unitários de $\mathrm{M}$, e é fechada pelas operações racionais. Pela definição de $\operatorname{Rac}(\mathrm{M})$, obtemos $\operatorname{Rac}(\mathrm{M}) \subseteq F$.

A demonstração da proposição a seguir é semelhante à demonstração de uma das implicações da Proposição 1.5.4. 
Proposição 1.5.5 Sejam $\mathrm{M}$ e $\mathrm{N}$ monóides, e $f: \mathrm{M} \rightarrow \mathrm{N}$ um morfismo sobrejetor. Então, para todo subconjunto racional $A$ de $\mathrm{N}$, existe um subconjunto racional $B$ de $\mathrm{M}$ tal que $A=B f$.

Proposição 1.5.6 Sejam $\mathrm{M}$ um monóide, e $\mathrm{S}_{1}$ e $\mathrm{S}_{2}$ subconjuntos de $\mathrm{M}$. Então, para quaisquer $A_{1} \in \operatorname{Rac}\left(\mathrm{S}_{1}^{*}\right)$ e $A_{2} \in \operatorname{Rac}\left(\mathrm{S}_{2}^{*}\right)$,

$$
A_{1} \cup A_{2} \in \operatorname{Rac}\left(\left(\mathrm{S}_{1} \cup \mathrm{S}_{2}\right)^{*}\right), A_{1} A_{2} \in \operatorname{Rac}\left(\left(\mathrm{S}_{1} \cup \mathrm{S}_{2}\right)^{*}\right) \text { e } A_{1}^{+} \in \operatorname{Rac}\left(\left(\mathrm{S}_{1} \cup \mathrm{S}_{2}\right)^{*}\right) .
$$

Demonstração. Vamos primeiro demonstrar que, para qualquer subconjunto racional $A$ de $\mathrm{S}_{1}^{*}, A$ é um subconjunto racional de $\left(\mathrm{S}_{1} \cup \mathrm{S}_{2}\right)^{*}$.

Seja $F$ a família de subconjuntos de $\mathrm{S}_{1}^{*}$ que são subconjuntos racionais de $\left(\mathrm{S}_{1} \cup \mathrm{S}_{2}\right)^{*}$. Vamos provar que $\operatorname{Rac}\left(\mathrm{S}_{1}^{*}\right) \subseteq F$.

Claramente, $\varnothing$ e os unitários em $\mathrm{S}_{1}^{*}$ pertencem a $F$. Sejam $A$ e $B$ dois conjuntos em $F$. Como $A \cup B, A B$ e $A^{+}$são subconjuntos de $\mathrm{S}_{1}^{*}$, e $\operatorname{Rac}\left(\left(\mathrm{S}_{1} \cup \mathrm{S}_{2}\right)^{*}\right)$ é fechada pelas operações racionais, temos que

$$
A \cup B \in F, A B \in F \text { e } A^{+} \in F .
$$

Logo, $F$ contém $\varnothing$ e os unitários em $\mathrm{S}_{1}^{*}$, e é fechada pelas operações racionais. Portanto, $\operatorname{Rac}\left(\mathrm{S}_{1}^{*}\right) \subseteq$ $F$.

Vamos agora provar (1.5.1). Temos que

$$
A_{1} \in \operatorname{Rac}\left(\left(\mathrm{S}_{1} \cup \mathrm{S}_{2}\right)^{*}\right) \text { e } A_{2} \in \operatorname{Rac}\left(\left(\mathrm{S}_{1} \cup \mathrm{S}_{2}\right)^{*}\right) .
$$

Como $\operatorname{Rac}\left(\left(S_{1} \cup S_{2}\right)^{*}\right)$ é fechada pelas operações racionais, obtemos (1.5.1).

Proposição 1.5.7 Sejam $\mathrm{M}$ um monóide, $\mathrm{S}$ um subconjunto de $\mathrm{M}, R \in \operatorname{Rac}\left(\mathrm{S}^{*}\right)$, e $x$ um elemento em M. Então,

$$
R x \in \operatorname{Rac}\left((\mathrm{S} \cup \mathrm{Sx})^{*}\right) \quad e \quad x R \in \operatorname{Rac}\left((\mathrm{S} \cup \mathrm{xS})^{*}\right) .
$$

Demonstração. Vamos provar que $R x \in \operatorname{Rac}\left((\mathrm{S} \cup \mathrm{Sx})^{*}\right)$.

Seja $F$ a família dos subconjuntos de $S^{*}$ definida como segue. Um conjunto $A$ pertence a $F$ se, e somente se,

- $A \in \operatorname{Rac}\left(\mathrm{S}^{*}\right), \mathrm{e}$

- $A x \in \operatorname{Rac}\left((\mathrm{S} \cup \mathrm{Sx})^{*}\right)$.

Vamos mostrar que $\operatorname{Rac}\left(\mathrm{S}^{*}\right) \subseteq F$.

Claramente, $\varnothing$ e os unitários em $\mathrm{S}^{*}$ pertencem a $F$. Sejam $A$ e $B$ dois conjuntos em $F$. Vamos provar que $A \cup B, A B$ e $A^{+}$pertencem a $F$.

- $A \cup B \in F$ : temos que $A, B \in \operatorname{Rac}\left(\mathrm{S}^{*}\right)$ e $A x, B x \in \operatorname{Rac}\left((\mathrm{S} \cup \mathrm{Sx})^{*}\right)$. Portanto, $A \cup B \in \operatorname{Rac}\left(\mathrm{S}^{*}\right)$ e $(A \cup B) x=A x \cup B x \in \operatorname{Rac}\left((\mathrm{S} \cup \mathrm{Sx})^{*}\right)$. Portanto, $A \cup B \in F$. 
- $A B \in F:$ como $A, B \in \operatorname{Rac}\left(\mathrm{S}^{*}\right), A B \in \operatorname{Rac}\left(\mathrm{S}^{*}\right)$. Como $B x \in \operatorname{Rac}\left((\mathrm{S} \cup \mathrm{Sx})^{*}\right)$, segue da Proposição 1.5.6 que $A(B x) \in \operatorname{Rac}\left((\mathrm{S} \cup \mathrm{Sx})^{*}\right)$. Portanto, $A B \in F$.

- $A^{+} \in F$ : temos que $A \in \operatorname{Rac}\left(\mathrm{S}^{*}\right)$, e $A x \in \operatorname{Rac}\left((\mathrm{S} \cup \mathrm{Sx})^{*}\right)$. Como $A^{+}=A^{*} A$, e $A^{*} \in \operatorname{Rac}\left(\mathrm{S}^{*}\right)$, segue da Proposição 1.5.6 que $A^{+} x=A^{*}(A x) \in \operatorname{Rac}\left((\mathrm{S} \cup \mathrm{Sx})^{*}\right)$. Como $A^{+} \in \operatorname{Rac}\left(\mathrm{S}^{*}\right)$, concluímos que $A^{+} \in F$.

Como $F$ contém $\varnothing$ e os unitários em S, e é fechada pelas operações racionais, concluímos que $\operatorname{Rac}\left(\mathrm{S}^{*}\right) \subseteq F$.

A demonstração de $x R \in \operatorname{Rac}\left((\mathrm{S} \cup \mathrm{xS})^{*}\right)$ é análoga.

Proposição 1.5.8 Seja $R$ um subconjunto racional de um monóide M. Então, existe um submonóide $\mathrm{N} \subseteq \mathrm{M}$ finitamente gerado tal que $R \in \operatorname{Rac}(\mathrm{N})$.

Demonstração. Seja $F$ a família dos subconjuntos de $\mathrm{M}$ que são subconjuntos racionais de algum submonóide finitamente gerado de $M$. Vamos mostrar que $\operatorname{Rac}(\mathrm{M}) \subseteq F$.

Claramente, $\varnothing$ e os unitários pertencem a $F$. Sejam $A$ e $B$ conjuntos em $F$, e $\mathrm{S}_{1}$ e $\mathrm{S}_{2}$ subconjuntos finitos de $\mathrm{M}$ tais que $A \in \operatorname{Rac}\left(\mathrm{S}_{1}^{*}\right)$ e $B \in \operatorname{Rac}\left(\mathrm{S}_{2}^{*}\right)$. Pela Proposição 1.5.6,

$$
A \cup B \in \operatorname{Rac}\left(\left(\mathrm{S}_{1} \cup \mathrm{S}_{2}\right)^{*}\right), \quad A B \in \operatorname{Rac}\left(\left(\mathrm{S}_{1} \cup \mathrm{S}_{2}\right)^{*}\right) \text { e } A^{+} \in \operatorname{Rac}\left(\left(\mathrm{S}_{1} \cup \mathrm{S}_{2}\right)^{*}\right) \text {. }
$$

Portanto, $A \cup B, A B$ e $A^{+}$pertencem a $F$. Concluímos que $F$ é fechada pelas operações racionais. Portanto, $\operatorname{Rac}(\mathrm{M}) \subseteq F$.

Exemplo 1.5.1 O conjunto $\mathbb{Q}$ não é um subconjunto racional do monóide aditivo $\mathbb{Q}$, porque esse monóide não é finitamente gerado.

\subsection{Autômatos}

Nesta seção, fazemos um resumo de alguns conceitos fundamentais sobre autômatos finitos. As referências que seguimos são [Eil74, How91, HMU01].

Começamos considerando um tipo de autômato definido por Eilenberg [Eil74], cujas transições são rotuladas por elementos de um monóide qualquer. Nosso objetivo é mostrar que algumas propriedades dos transdutores, que estudamos no Capítulo 2, são propriedades desse tipo mais geral de máquina.

Definição 1.6.1 Seja M um monóide. Um autômato sobre M, ou M-autômato, é uma máquina $\mathcal{A}=(Q, E, I, T)$ composta de

- um conjunto finito $Q$ de estados;

- subconjuntos $I, T \subseteq Q$ de estados iniciais e finais, respectivamente;

- um subconjunto finito $E \subseteq Q \times \mathrm{M} \times Q$ de transições. 
Um M-autômato $\mathcal{A}=(Q, E, I, T)$ pode ser visto como um grafo, cujos arcos são rotulados por elementos de M. Assim, o conteúdo da Seção 1.4 aplica-se para M-autômatos sem alteração. O grafo obtido eliminando-se os rótulos das transições de $\mathcal{A}$ será denominado grafo de $\mathcal{A}$.

Uma transição

$$
e=(p, m, q)
$$

pode ser representada como

$$
e: p \stackrel{m}{\longrightarrow} q
$$

Um passeio

$$
c: p_{0} \stackrel{m_{1}}{\longrightarrow} p_{1} \stackrel{m_{2}}{\longrightarrow} \cdots \stackrel{m_{n}}{\longrightarrow} p_{n}
$$

pode ser representado como

$$
c: p_{0} \stackrel{m_{1} \ldots m_{n}}{\longrightarrow} p_{n}
$$

O rótulo de $c$ é o produto

$$
|c|=m_{1} \ldots m_{n}
$$

$\mathrm{O}$ rótulo do passeio vazio é a identidade de $\mathrm{M}$.

Dizemos que $c$ é um passeio bem-sucedido se $p_{0} \in I$ e $p_{n} \in T$. O comportamento de $\mathcal{A}$ é o subconjunto $|\mathcal{A}|$ de $\mathrm{M}$ dos rótulos dos passeios bem-sucedidos nesse autômato. Dizemos também que $\mathcal{A}$ realiza ou reconhece $|\mathcal{A}|$.

Dizemos que $\mathrm{M}$-autômatos $\mathcal{A}$ e $\mathcal{B}$ são equivalentes se

$$
|\mathcal{A}|=|\mathcal{B}|
$$

Um estado $q$ de $\mathcal{A}$ é acessível (co-acessivel) se existir um passeio com origem em um estado inicial (em $q$ ) e término em $q$ (em um estado final). Se todos os estados forem acessíveis (co-acessíveis), dizemos que $\mathcal{A}$ é um autômato acessível (autômato co-acessível). Um autômato bi-acessível é um autômato acessível e co-acessível. Equivalentemente, podemos dizer que um autômato é bi-acessível se todo estado pertencer a um passeio bem-sucedido.

Todo autômato pode ser transformado em um autômato bi-acessível, bastando remover os estados não-acessíveis e não-co-acessíveis.

Dizemos que $\mathcal{A}$ é normalizado se $I$ e $T$ forem unitários distintos $i$ e $t$, respectivamente, $i$ não for o término de nenhuma transição, e $t$ não for a origem de nenhuma transição. Um autômato $\mathcal{A}$ pode ser transformado em um autômato normalizado como segue. Primeiro, são acrescentados novos estados $i$ e $t$, transições rotuladas por 1 de $i$ para todos os estados iniciais de $\mathcal{A}$, e transições rotuladas por 1 de todos os estados finais de $\mathcal{A}$ para $t$. No autômato obtido, $i$ é o único estado inicial, e $t$ é o único final. Demonstra-se facilmente que esse autômato é normalizado, e é equivalente a $\mathcal{A}$.

Seja $\Sigma$ um alfabeto. Um autômato sobre $\Sigma$, ou $\Sigma$-autômato, é um autômato sobre o monóide livre $\Sigma^{*}$, e cujas transições são rotuladas por letras em $\Sigma$. Dizemos que um passeio $c$ em um $\Sigma$-autômato soletra a palavra $|c|$.

Os subconjuntos de um monóide livre $\Sigma^{*}$ reconhecidos por um $\Sigma$-autômato são exatamente os subconjuntos reconhecíveis de $\Sigma^{*}$. A demonstração dessa propriedade pode ser vista na Seção III.10 de [Eil74]. Na literatura, é usual definir um subconjunto reconhecível de um monóide livre como sendo o comportamento de algum $\Sigma$-autômato, ao invés de utilizar a Definição 1.5.1, que é mais geral.

O seguinte teorema é uma versão particular do Teorema VII.10.1 de [Eil74]. 
Teorema 1.6.1 Um subconjunto de um monóide $\mathrm{M}$ é racional se, e somente se, for o comportamento de um M-autômato.

Demonstração. Seja $R$ um subconjunto racional de M. Sejam $\Sigma, L$ e $f$ como na Proposição 1.5.4. Pelo Teorema de Kleene, $L$ é uma linguagem reconhecível. Seja $\mathcal{A}=(Q, E, I, T)$ um $\Sigma$-autômato que reconhece $L$.

Construa um M-autômato $\mathcal{A}^{\prime}=\left(Q, E^{\prime}, I, T\right)$ a partir de $\mathcal{A}$, substituindo cada transição

$$
(p, \sigma, q) \in E
$$

pela transição

$$
(p, \sigma f, q) \text {. }
$$

Como $f$ é um morfismo, o rótulo de um passeio em $\mathcal{A}^{\prime}$ é a imagem do rótulo do passeio correspondente em $\mathcal{A}$. Assim,

$$
\left|\mathcal{A}^{\prime}\right|=|\mathcal{A}| f=R .
$$

Suponha agora que $R=|\mathcal{A}|$, onde $\mathcal{A}=(Q, E, I, T)$ é um M-autômato. Construa um $E$ autômato $\mathcal{A}^{\prime}=\left(Q, E^{\prime}, I, T\right)$ a partir de $\mathcal{A}$, substituindo cada transição

$$
e=(p, m, q) \in E
$$

pela transição

$$
(p, e, q) \text {. }
$$

Seja $f: E^{*} \rightarrow \mathrm{M}$ o morfismo gerado por

$$
e f=|e|, \quad \forall e \in E .
$$

Então $\left|\mathcal{A}^{\prime}\right| f=|\mathcal{A}|$. Pelo Teorema de Kleene, $\left|\mathcal{A}^{\prime}\right|$ é um subconjunto racional de $E^{*}$. Segue então da Proposição 1.5.2 que $R$ é um subconjunto racional de $M$.

Utilizando as transformações de um M-autômato em um M-autômato bi-acessível e normalizado, obtemos também

Corolário 1.6.1 Todo subconjunto racional não-vazio de um monóide $\mathrm{M}$ é o comportamento de um M-autômato bi-acessível e normalizado.

Dizemos que um $\Sigma$-autômato é deterministico se contiver exatamente um estado inicial, e se transições com origens idênticas tiverem rótulos distintos. É conveniente definir um $\Sigma$-autômato determinístico de forma mais algébrica como segue.

Definição 1.6.2 Seja $\Sigma$ um alfabeto. Um $\Sigma$-autômato determinístico é uma máquina $\mathcal{A}=(Q, i, T)$ composta de

- um conjunto finito $Q$ de estados;

- um estado inicial $i$;

- um subconjunto $T \subseteq Q$ de estados finais; 
- uma função parcial $: Q \times \Sigma \rightarrow Q$.

A função - é denominada função de transição de $\mathcal{A}$. Utilizando (1.3.3), essa função pode ser estendida para uma ação de $\Sigma^{*}$ sobre $Q$ satisfazendo (1.2.2).

O comportamento de $\mathcal{A}$ pode ser escrito como

$$
|\mathcal{A}|=\left\{s: s \in \Sigma^{*} \text { e } i s \in T\right\} .
$$

Dizemos que $\mathcal{A}$ é completo se, para todo estado $q$ e toda letra $\sigma, q \sigma \neq \varnothing$. Um procedimento simples de completamento de um $\Sigma$-autômato determinístico, que consiste em acrescentar um novo estado e transições convenientes, permite a construção de um $\Sigma$-autômato determinístico completo e acessível a partir de um $\Sigma$-autômato determinístico acessível e não-completo. Esse procedimento é descrito na Seção 4.12.

O algoritmo clássico da construção dos subconjuntos permite a construção de um $\Sigma$-autômato determinístico equivalente a partir de um $\Sigma$-autômato qualquer. Esse algoritmo é descrito em todos os textos fundamentais sobre Teoria dos Autômatos.

Dessa discussão e do Teorema 1.6.1, segue

Teorema 1.6.2 Todo subconjunto racional de um monóide livre $\Sigma^{*}$ é o comportamento de um $\Sigma$-autômato determinístico acessivel e completo.

Outro conceito fundamental é o de $\Sigma$-autômato minimal reconhecendo um subconjunto racional de $\Sigma^{*}$. Esse conceito será discutido apenas na Seção 4.12, como consequiência de uma teoria de minimização de transdutores desenvolvida no Capítulo 4.

Será útil em algumas demonstrações considerar passeios em um autômato como palavras sobre o conjunto de transições. Essa técnica leva ao conceito de linguagem local.

Definição 1.6.3 Dizemos que um subconjunto $L$ de um monóide livre $\Sigma^{*}$ é local se existirem subconjuntos $A, B \subseteq \Sigma e C \subseteq \Sigma^{2}$ tais que

$$
L=A \Sigma^{*} \cap \Sigma^{*} B-\Sigma^{*} C \Sigma^{*}
$$

ou

$$
L=1 \cup A \Sigma^{*} \cap \Sigma^{*} B-\Sigma^{*} C \Sigma^{*} .
$$

Claramente, toda linguagem local é racional.

Sejam $\mathcal{A}=(Q, E, I, T)$ um M-autômato, e

$$
A=\{p \rightarrow q \in E: p \in I\}, \quad B=\{p \rightarrow q \in E: q \in T\} \text { e } C=\left\{(p \rightarrow q)\left(p^{\prime} \rightarrow q^{\prime}\right) \in E^{2}: q \neq p^{\prime}\right\} .
$$

A relação entre linguagens locais e passeios em $\mathcal{A}$ aparece ao considerarmos a linguagem local

$$
\# \mathcal{A}=A E^{*} \cap E^{*} B-E^{*} C E^{*}
$$

se $I \cap T=\varnothing$, ou

$$
\# \mathcal{A}=1 \cup A E^{*} \cap E^{*} B-E^{*} C E^{*},
$$

caso contrário. 
Claramente, $\# \mathcal{A}$ é o conjunto de todos os passeios bem-sucedidos em $\mathcal{A}$. O passeio vazio é representado pela palavra vazia.

Outra linguagem local útil é o conjunto dos passeios com origem em um estado $p$ e término em um estado $q$. Se $p$ e $q$ forem distintos, então esse conjunto é igual a

$$
L_{p q}=A_{p} E^{*} \cap E^{*} B_{q}-E^{*} C E^{*}
$$

onde

$$
A_{p}=\{r \rightarrow s \in E: r=p\} \quad \text { e } \quad B_{q}=\{r \rightarrow s \in E: s=q\} .
$$

Se $p=q$, esse conjunto é igual a

$$
L_{p q}=1 \cup A_{p} E^{*} \cap E^{*} B_{q}-E^{*} C E^{*} .
$$

As seguintes propriedades são de fácil verificação:

$$
\begin{aligned}
& \# \mathcal{A}=\bigcup_{\substack{i \in I \\
t \in T}} L_{i t}, \\
& L_{p q}=\bigcup_{r \in Q} L_{p r} L_{r q} .
\end{aligned}
$$

Seja $f: E^{*} \rightarrow \mathrm{M}$ o morfismo que associa todo passeio ao seu rótulo. Então, $(\# \mathcal{A}) f$ é o conjunto dos rótulos dos passeios bem-sucedidos de $\mathcal{A}$. Ou seja,

$$
|\mathcal{A}|=(\# \mathcal{A}) f .
$$

Essa propriedade mostra que todo subconjunto racional de $\mathrm{M}$ é a imagem de uma linguagem local por um morfismo de monóides. Ademais, se $\mathrm{M}$ for um monóide livre gerado por um alfabeto $\Sigma$, então podemos supor que $\mathcal{A}$ é um $\Sigma$-autômato, ou seja, que as transições desse autômato são rotuladas por letras. Nesse caso, $f$ é um morfismo muito fino. Fortalecemos então a Proposição 1.5.4 como segue.

Teorema 1.6.3 Seja $R$ um subconjunto de um monóide $M$. Então, $R$ é um conjunto racional se, e somente se, existem um alfabeto $\Sigma$, uma linguagem local $L \subseteq \Sigma^{*}$ e um morfismo de monóides $f: \Sigma^{*} \rightarrow \mathrm{M}$ tais que $R=L f$. Ademais, se M for um monóide livre, podemos supor que $f$ é um morfismo muito fino.

Seja $\mathcal{A}_{1}=\left(Q_{1}, i_{1}, T_{1}\right), \ldots, \mathcal{A}_{k}=\left(Q_{k}, i_{k}, T_{k}\right)$ uma seqüência de $k$-autômatos determinísticos completos. O produto

$$
\mathcal{A}_{1} \times \cdots \times \mathcal{A}_{k}
$$

é o $\Sigma$-autômato $\mathcal{A}=(Q, i, T)$ definido como segue. O conjunto de estados de $\mathcal{A}$ é

$$
Q=Q_{1} \times \cdots \times Q_{k} .
$$

O estado inicial de $\mathcal{A}$ é $i=\left(i_{1}, \ldots, i_{k}\right)$. A transição de um estado $\left(q_{1}, \ldots, q_{k}\right)$ com uma letra $\sigma$ é definida por

$$
\left(q_{1}, \ldots, q_{k}\right) \sigma=\left(q_{1} \sigma, \ldots, q_{k} \sigma\right)
$$


Para toda palavra $s$ em $\Sigma^{*}$, segue por indução em $|s|$ que

$$
\left(q_{1}, \ldots, q_{k}\right) s=\left(q_{1} s, \ldots, q_{k} s\right) .
$$

A escolha do conjunto de estados finais de $\mathcal{A}$ depende do uso a ser feito desse autômato. Se considerarmos que um estado $\left(q_{1}, \ldots, q_{k}\right)$ é final quando pelo menos um dos estados $q_{1}, \ldots, q_{k}$ for final no autômato correspondente, então

$$
|\mathcal{A}|=\bigcup_{1 \leq i \leq k}\left|\mathcal{A}_{i}\right|
$$

Se considerarmos que esse estado é final quando todos os estados $q_{1}, \ldots, q_{k}$ forem finais, então

$$
|\mathcal{A}|=\bigcap_{1 \leq i \leq k}\left|\mathcal{A}_{i}\right|
$$

O seguinte resultado fundamental, denominado Lema da Iteração, é utilizado freqüentemente para demonstrar que uma linguagem não é reconhecível.

Lema da Iteração Seja $A$ um subconjunto reconhecível de um monóide livre $\Sigma^{*}$. Então, existe um inteiro positivo $n$ tal que, para toda palavra $s$ em $A$ com $|s| \geq n$, existe uma fatoração $s=u w v$ tal que $w \neq 1,|u w| \leq n$ e $u w^{*} v \subseteq A$.

Por exemplo, utilizando o Lema da Iteração, podemos provar que os subconjuntos descritos a seguir não são reconhecíveis:

$$
\left\{u \in\{\sigma, \gamma\}^{*}:|u|_{\sigma}=|u|_{\gamma}\right\} \quad \text { e } \quad\left\{\sigma^{n} \gamma^{n}: n \geq 1\right\} .
$$

O fato de que esses conjuntos não são reconhecíveis será utilizado livremente ao longo do texto.

\subsection{Computabilidade e complexidade}

Dizemos que um problema de decisão $P$ é decidivel se existir um algoritmo que resolve $P$ (ou seja, decide qualquer instância do problema), e indecidível caso contrário. Da mesma forma, dizemos que uma construção pode ser feita efetivamente se existir um algoritmo que realiza essa construção.

O seguinte problema clássico é indecidível, e esse fato será utilizado livremente neste texto. Uma demonstação pode ser vista em [HMU01].

Teorema 1.7.1 (Problema da Correspondência de Post) Dados alfabetos $\Sigma$ e $\Gamma$ e morfismos $f, g: \Sigma^{*} \rightarrow \Gamma^{*}$, é indecidivel se existe uma palavra $s$ em $\Sigma^{+}$tal que $s f=s g$.

Neste trabalho, alguns algoritmos serão escritos utilizando uma linguagem definida em [CLRS01]. Nessa linguagem, cada algoritmo começa com um nome e uma lista de parâmetros, e pode ou não devolver algum valor. Algoritmos que não devolvem valores realizam modificações nos parâmetros.

As estrutras de controle dessa linguagem são

- se (condição) então ... 
- para (intervalo) faça ...

- para cada (conjunto) faça ...

- enquanto (condição) faça ...

Uma condição pode ser qualquer expressão matemática, envolvendo um número qualquer de variáveis e operadores, e que possa assumir o valor verdadeiro ou falso. A construção para é executada para cada inteiro de um intervalo especificado. A construção para cada é executada para cada elemento de um conjunto especificado.

A atribuição de um valor $x$ a uma variável $a$ é feita com a construção

$$
a \leftarrow x \text {. }
$$

Conjuntos e vetores são tipos de dados complexos que serão utilizados com freqüência. Um vetor pode ser indexado por um número, mas outros tipos de dados, como os estados de um autômato, também serão utilizados como índices. O valor na posição $i$ de um vetor $V$ será representado por $V[i]$.

Na manipulação de conjuntos, a linguagem matemática será utilizada livremente. Por exemplo, a inserção de um elemento $x$ em um conjunto $L$ pode ser denotada por

$$
L \leftarrow L \cup x .
$$

Nessa operação, o conjunto $L$ é atualizado, e a letra $L$ passa a representar o novo conjunto.

Outra operação com conjuntos que utilizamos é a retirada de um elemento de um conjunto $L$, representada como segue:

$$
a \leftarrow \operatorname{Extrai}(L) .
$$

Essa operação retira um elemento qualquer de $L$, e o atribui à variável $a$. Novamente, o conjunto $L$ é atualizado, e a letra $L$ passa a representar o novo conjunto.

Vamos supor que os tipos de dados vetor e conjunto são implementados de forma que as operações de acesso a uma posição de um vetor, e inserção e remoção de elementos em um conjunto, possam ser feitas em complexidade de tempo constante.

Em nossas análises de complexidade de tempo, vamos utilizar o critério de custo uniforme, segundo o qual operações aritméticas e comparações numéricas são feitas em complexidade de tempo constante. Dessa forma, nossas análises de complexidade de tempo apenas contam o número de operações essenciais realizadas.

Nossa preocupação com as estruturas de dados e os detalhes de implementação é superficial. No entanto, esses detalhes são discutidos com a profundidade necessária para justificar as complexidades calculadas para cada algoritmo. Discussões sobre detalhes de implementação podem ser vistas em [CLRS01, CR94].

Algumas de nossas análises de complexidade são superficiais. Um tratamento mais preciso é feito para os algoritmos de complexidades polinomiais.

Neste texto, vamos utilizar livremente dois algoritmos clássicos, um para autômatos e outro para grafos:

- construção da parte bi-acessível de um $\Sigma$-autômato, e

- construção do conjunto dos componentes fortemente conexos de um grafo. 
Esses dois algoritmos têm complexidades de tempo e espaço $\Theta(m+n)$, onde denotamos por $n$ o número de vértices de um grafo, ou estados de um autômato, e por $m$ o número de arcos de um grafo, ou transições de um autômato. 


\section{RELAÇÕES RACIONAIS}

Neste capítulo, introduzimos os conceitos de relação racional, transdutor e representação matricial. Toda a teoria desenvolvida neste trabalho está fundamentada nesses conceitos.

A maior parte do material deste capítulo é clássica, e pode ser encontrada nos livros de Eilenberg [Eil74] e Berstel [Ber79].

Ao longo de todo o capítulo, $\Sigma$ e $\Gamma$ representam alfabetos, e $M$ e $\mathrm{N}$ representam monóides.

\subsection{Definições}

O produto cartesiano $\mathrm{M} \times \mathrm{N}$ equipado com a operação

$$
(a, b) \cdot(c, d)=(a \cdot \mathrm{M} c, b \cdot \mathrm{N} d), \quad \forall(a, b),(c, d) \in \mathrm{M} \times \mathrm{N},
$$

é um monóide com identidade $\left(1_{\mathrm{M}}, 1_{\mathrm{N}}\right)$, denominado monóide produto.

A projeção na primeira coordenada e a projeção na segunda coordenada de $\mathrm{M} \times \mathrm{N}$ são, respectivamente, as funções $\pi_{1}: \mathrm{M} \times \mathrm{N} \rightarrow \mathrm{M}$ e $\pi_{2}: \mathrm{M} \times \mathrm{N} \rightarrow \mathrm{N}$ definidas por

$$
(m, n) \pi_{1}=m \quad \text { e } \quad(m, n) \pi_{2}=n, \quad \forall(m, n) \in \mathrm{M} \times \mathrm{N} .
$$

Essas funções são morfismos de monóides.

Sejam $\mathrm{S}$ um monóide, e $f: \mathrm{S} \rightarrow \mathrm{M} \times \mathrm{N}$ um morfismo de monóides. As funções $f_{1}: \mathrm{S} \rightarrow \mathrm{M}$ e $f_{2}: \mathrm{S} \rightarrow \mathrm{N}$ definidas por

$$
s f_{1}=s\left(f \pi_{1}\right) \quad \text { e } \quad s f_{2}=s\left(f \pi_{2}\right), \quad \forall s \in \mathrm{S}
$$

são morfismos de monóides. Dizemos que esses morfismos são os desmembramentos de $f$. Claramente, para todo subconjunto $X$ de $\mathrm{S}$,

$$
X f=\bigcup_{x \in X}\left(x f_{1}, x f_{2}\right) .
$$

Sejam $f: \mathrm{S} \rightarrow \mathrm{M}$ e $g: \mathrm{S} \rightarrow \mathrm{N}$ morfismos de monóides. A função $(f, g): \mathrm{S} \rightarrow \mathrm{M} \times \mathrm{N}$ definida por

$$
s(f, g)=(s f, s g), \quad \forall s \in \mathrm{S}
$$

é um morfismo de monóides. Um par $(f, g)$ de morfismos é denominado bimorfismo. 
As operações racionais para um monóide produto $\mathrm{M} \times \mathrm{N}$ podem ser definidas de maneira mais explícita como segue. Sejam $r$ e $r^{\prime}$ subconjuntos de $\mathrm{M} \times \mathrm{N}$. Então,

$$
\begin{aligned}
& r \cup r^{\prime}=\left\{(a, b):(a, b) \in r \text { ou }(a, b) \in r^{\prime}\right\} \\
& r \cdot r^{\prime}=\left\{(a c, b d):(a, b) \in r \text { e }(c, d) \in r^{\prime}\right\} \\
& r^{+}=\left\{\left(a_{1} \ldots a_{k}, b_{1} \ldots b_{k}\right): k \geq 1 \text { e }\left(a_{i}, b_{i}\right) \in r \text { para } 1 \leq i \leq k\right\} \\
& r^{*}=r^{+} \cup\left(1_{\mathrm{M}}, 1_{\mathrm{N}}\right) .
\end{aligned}
$$

Considerando $r$ e $r^{\prime}$ como relações $\mathrm{M} \rightarrow \mathrm{N}$, podemos escrever essas operações como segue. Para todo elemento $w$ em M,

$$
\begin{aligned}
& w\left(r \cup r^{\prime}\right)=w r \cup w r^{\prime} ; \\
& w\left(r \cdot r^{\prime}\right)=\bigcup_{x y=w}(x r)\left(y r^{\prime}\right) ; \\
& w r^{+}=\bigcup_{x_{1} \ldots x_{k}=w}\left(x_{1} r\right) \ldots\left(x_{k} r\right) ; \\
& w r^{*}=w r^{+} \cup 1_{\mathrm{N}} .
\end{aligned}
$$

Definição 2.1.1 Sejam $\mathrm{M}$ e $\mathrm{N}$ monóides. Dizemos que uma relação $\tau: \mathrm{M} \rightarrow \mathrm{N}$ é racional (reconhecível) se o seu gráfico for um subconjunto racional (reconhecível) do monóide produto $\mathrm{M} \times \mathrm{N}$.

O monóide $\mathrm{M}$ será denominado monóide de entrada, e $\mathrm{N}$, monóide de saída. Se $\mathrm{M}$ for um monóide livre sobre um alfabeto $\Sigma$, chamamos $\Sigma$ de alfabeto de entrada. Se $\mathrm{N}$ for um monóide livre sobre um alfabeto $\Gamma$, chamamos $\Gamma$ de alfabeto de saída.

Se $\mathrm{M}$ e $\mathrm{N}$ forem monóides livres, dizemos que $\tau$ é uma relação racional palavra-palavra.

Exemplo 2.1.1 Seja $\mathbb{N}$ o monóide aditivo dos números naturais. O gráfico da relação $\tau: \mathbb{N} \rightarrow \mathbb{N}$ definida por

$$
n \tau=\{k \in \mathbb{N}: k \geq n\}, \forall n \in \mathbb{N}
$$

é

$$
(1,1)^{*}(0,1)^{*}
$$

Portanto, $\tau$ é uma relação racional.

Exemplo 2.1.2 Seja M um monóide finitamente gerado por um conjunto $S$. Então, a função identidade $\tau: \mathrm{M} \rightarrow \mathrm{M}$ é uma relação racional. De fato, seu gráfico é o conjunto racional

$$
\# \tau=\{(s, s): s \in S\}^{*}
$$

Se M não for um monóide finitamente gerado, então a identidade não é uma relação racional. De fato, suponha que \# $\tau$ seja um subconjunto racional de $\mathrm{M} \times \mathrm{M}$. Então, segue da Proposição 1.5.8 que \# $\boldsymbol{\tau}$ está contido em um submonóide de $\mathrm{M} \times \mathrm{M}$ gerado por um subconjunto finito $S$. Observe 
que im $\tau$ está contida no submonóide de $\mathrm{M}$ gerado pelo conjunto finito $S \pi_{2}$. Mas, $\operatorname{im} \tau=\mathrm{M}$, o que contradiz a hipótese de que $M$ não é um monóide finitamente gerado.

Por exemplo, a identidade $\mathbb{Q} \rightarrow \mathbb{Q}$, onde $\mathbb{Q}$ é o monóide aditivo dos números racionais, não é uma relação racional.

Em geral, não é verdade que $\operatorname{Rac}(M \times N)=\operatorname{Rec}(M \times N)$, como mostra o exemplo a seguir.

Exemplo 2.1.3 Sejam $\Sigma=\{\sigma\}, \Gamma=\{\gamma\}, \Omega=\{\sigma, \gamma\}$ e $f: \Omega^{*} \rightarrow \Sigma^{*} \times \Gamma^{*}$ o morfismo definido por

$$
\sigma f=(\sigma, 1) \text { e } \gamma f=(1, \gamma) .
$$

O subconjunto $(\sigma, \gamma)^{*}$ de $\Sigma^{*} \times \Gamma^{*}$ é racional, mas $(\sigma, \gamma)^{*} f^{-1}=\left\{u \in \Omega^{*}:|u|_{\sigma}=|u|_{\gamma}\right\}$ não é reconhecível. Então, pela Proposição $1.5 .3,(\sigma, \gamma)^{*}$ não é reconhecível.

Neste texto, vamos estudar apenas as relações racionais. Sobre as relações reconhecíveis, vamos apenas citar uma caracterização interessante obtida por Mezei, que não foi publicada. Uma demonstração pode ser vista no livro de Berstel [Ber79].

Teorema 2.1.1 (Mezei) Seja $r$ um subconjunto do produto $\mathrm{M} \times \mathrm{N}$. Então, $r$ é reconhecível se, $e$ somente se, $r$ é uma união finita de conjuntos da forma $A \times B$, onde $A$ é um subconjunto reconhecivel de $\mathrm{M}$, e $B$ é um subconjunto reconhecivel de $\mathrm{N}$.

Essa caracterização não vale para relações racionais, como mostra o exemplo a seguir.

Exemplo 2.1.4 Vamos mostrar que $\operatorname{Rac}(\mathbb{N} \times \mathbb{N}) \neq \operatorname{Rec}(\mathbb{N} \times \mathbb{N})$. O monóide aditivo $\mathbb{N}$ será identificado com $\sigma^{*}$, onde $\sigma$ é uma letra.

O conjunto $r=(\sigma, \sigma)^{*}$ é um subconjunto racional de $\mathbb{N} \times \mathbb{N}$. Mas, a única união de produtos de subconjuntos reconhecíveis de $\mathbb{N}$ igual a $r$ é $\bigcup_{n \geq 0}\left(\sigma^{n}, \sigma^{n}\right)$. Do Teorema 2.1.1, segue que $r$ não é reconhecível.

No entanto, o produto de dois conjuntos racionais é um conjunto racional, conforme demonstramos abaixo.

Proposição 2.1.1 Sejam $A$ e $B$ subconjuntos racionais dos monóides $\mathrm{M}$ e $\mathrm{N}$, respectivamente. Então, $A \times B$ é um subconjunto racional do monóide produto $\mathrm{M} \times \mathrm{N}$.

Demonstração. Sejam $f: M \rightarrow M \times N$ e $g: N \rightarrow M \times N$ as funções definidas por

$$
m f=(m, 1) \quad \text { e } \quad n g=(1, n), \quad \forall m \in \mathrm{M}, \forall n \in \mathrm{N} .
$$

Claramente, $f$ e $g$ são morfismos de monóides. Logo, pela Proposição 1.5.2, $A f$ e $B g$ são subconjuntos racionais de $\mathrm{M} \times \mathrm{N}$. Portanto, $(A f)(B g)$ é um subconjunto racional de $\mathrm{M} \times \mathrm{N}$. Basta agora notar que $A \times B=(A f)(B g)$.

Exemplo 2.1.5 Sejam $\Sigma$ um alfabeto e $h: \Sigma^{*} \rightarrow \mathrm{M}$ uma substituição racional. Então, o gráfico de $h$ é

$$
\left(\bigcup_{\sigma \in \Sigma} \sigma \times \sigma h\right)^{*} .
$$


Portanto, $h$ é uma relação racional.

\subsection{Fatoração de relações racionais}

Nesta seção, vamos apresentar um resultado clássico de Nivat, que fornece uma fatoração das relações racionais [Niv68]. Esse resultado é muito útil na demonstração de propriedades dessas relações.

Será conveniente representar a intersecção de conjuntos como uma relação. Formalmente, dados um conjunto $A$ e um subconjunto $X$ de $A$, definimos a interseç̧ão com $X$ como a relação

$$
A \stackrel{\cap X}{\longrightarrow} A
$$

definida por

$$
Y(\cap X)=X \cap Y, \quad \forall Y \subseteq A .
$$

Vamos começar com um lema.

Lema 2.2.1 Sejam $A, B$ e $C$ conjuntos, $r: C \rightarrow A$ e $s: C \rightarrow B$ relações, $X$ um subconjunto de $C$, e $\tau$ a relação $A \stackrel{r^{-1}}{\longrightarrow} C \stackrel{\cap X}{\longrightarrow} C \stackrel{s}{\rightarrow} B$. Então,

$$
\# \tau=\bigcup_{x \in X} x r \times x s
$$

Demonstração. Seja $(u, v)$ um par em \# $\tau$. Pela definição de $\tau$, existe um elemento $x$ de $X$ que pertence a $u r^{-1}$ e a $v s^{-1}$. Assim, $(u, v)$ pertence ao produto $x r \times x s$, e obtemos

$$
\# \tau \subseteq \bigcup_{x \in X} x r \times x s
$$

Seja agora $(u, v)$ um par em $x r \times x s$ para algum elemento $x$ de $X$. Então, $x$ pertence a $u r^{-1}$, e $v$ pertence a $x s$. Portanto, $v$ pertence a $\left(u r^{-1}(\cap X)\right) s=u \tau$. Assim,

$$
\bigcup_{x \in X} x r \times x s \subseteq \# \tau
$$

Teorema 2.2.1 (Nivat 1968) Uma relação $\tau: \mathrm{M} \rightarrow \mathrm{N}$ é racional se, e somente se, existem um alfabeto $\Omega$, uma linguagem local $L$ sobre $\Omega$, e morfismos de monóides $f: \Omega^{*} \rightarrow \mathrm{M}$ e $g: \Omega^{*} \rightarrow \mathrm{N}$ tais que

$$
\tau=\mathrm{M} \stackrel{f^{-1}}{\longrightarrow} \Omega^{*} \stackrel{\cap L}{\longrightarrow} \Omega^{*} \stackrel{g}{\rightarrow} \mathrm{N} .
$$

Ademais, se $\mathrm{M}(\mathrm{N})$ for um monóide livre, então $f(g)$ pode ser escolhido como um morfismo fino, e, se $\mathrm{M}$ e $\mathrm{N}$ forem monóides livres, então $f$ e g podem ser escolhidos como mofismos finos. 
Demonstração. Suponha que $\tau$ seja uma composição da forma $f^{-1}(\cap L) g$. Por (2.2.1), seu gráfico é o conjunto

$$
\# \tau=L(f, g) .
$$

Como $(f, g)$ é um morfismo, segue da Proposição 1.5.2 que esse conjunto é racional. Assim, $\tau$ é uma relação racional.

Suponha que o gráfico de $\tau$ seja um subconjunto racional de $\mathrm{M} \times \mathrm{N}$. Então, pelo Teorema 1.6.3, existem um alfabeto $\Omega$, uma linguagem local $L$ sobre $\Omega$ e um morfismo de monóides $\varphi: \Omega^{*} \rightarrow \mathrm{M} \times \mathrm{N}$ tais que $\# \tau=L \varphi$.

Sejam $f: \Omega^{*} \rightarrow \mathrm{M}$ e $g: \Omega^{*} \rightarrow \mathrm{N}$ os desmembramentos de $\varphi$. Então, \# $\tau=L(f, g)$. Utilizando o Lema 2.2.1, obtemos

$$
\tau=f^{-1}(\cap L) g .
$$

Suponha agora que $M$ seja um monóide livre sobre um alfabeto $\Sigma$. Sejam $b: \Sigma \rightarrow \Sigma^{\prime}$ uma cópia de $\Sigma$, com $\Sigma^{\prime}$ distinto de $\Omega, \Omega^{\prime}=\Sigma^{\prime} \cup \Omega, h: \Omega^{*} \rightarrow \Omega^{\prime *}$ o morfismo definido por

$$
\sigma h=(\sigma f b) \sigma, \quad \forall \sigma \in \Omega,
$$

e $f^{\prime}: \Omega^{*} \rightarrow \Sigma^{*}$ e $g^{\prime}: \Omega^{\prime *} \rightarrow \mathrm{N}$ os morfismos definidos por

$$
\sigma f^{\prime}=\sigma b^{-1}, \sigma g^{\prime}=1, \gamma f^{\prime}=1, \gamma g^{\prime}=\gamma g, \forall \sigma \in \Sigma^{\prime}, \forall \gamma \in \Omega .
$$

Observe que $f^{\prime}$ é um morfismo fino, e $L h$ é uma linguagem racional (não necessariamente local) em $\Omega^{\prime *}$.

Seja $u=\sigma_{1} \ldots \sigma_{n}$ uma palavra em $\Omega^{+}$, onde $\sigma_{i}$ é uma letra para todo índice $i$. Então,

$$
(u h) f^{\prime}=\left(\left(\sigma_{1} f b\right) \sigma_{1} \ldots\left(\sigma_{n} f b\right) \sigma_{n}\right) f^{\prime}=\left(\sigma_{1} f\right) \ldots\left(\sigma_{n} f\right)=u f
$$

$\mathrm{e}$

$$
(u h) g^{\prime}=\left(\left(\sigma_{1} f b\right) \sigma_{1} \ldots\left(\sigma_{n} f b\right) \sigma_{n}\right) g^{\prime}=\left(\sigma_{1} g\right) \ldots\left(\sigma_{n} g\right)=u g .
$$

Assim, $u(f, g)=(u h)\left(f^{\prime}, g^{\prime}\right)$. Portanto,

$$
L(f, g)=(L h)\left(f^{\prime}, g^{\prime}\right) .
$$

Pelo Teorema 1.6.3, existem um alfabeto $\Xi$, uma linguagem local $L^{\prime}$ sobre $\Xi$ e um morfismo muito fino $\varphi^{\prime}: \Xi^{*} \rightarrow \Omega^{\prime *}$ tais que $L h=L^{\prime} \varphi^{\prime}$. Assim, $\varphi^{\prime} f^{\prime}$ é um morfismo fino, $\varphi^{\prime} g^{\prime}$ é um morfismo, e, para toda palavra $v$ em $L^{\prime}, v\left(\varphi^{\prime} f^{\prime}, \varphi^{\prime} g^{\prime}\right)=\left(v \varphi^{\prime}\right)\left(f^{\prime}, g^{\prime}\right)$. Portanto,

$$
L^{\prime}\left(\varphi^{\prime} f^{\prime}, \varphi^{\prime} g^{\prime}\right)=\left(L^{\prime} \varphi^{\prime}\right)\left(f^{\prime}, g^{\prime}\right)=(L h)\left(f^{\prime}, g^{\prime}\right)
$$

e temos que

$$
\tau=\left(\varphi^{\prime} f^{\prime}\right)^{-1}\left(\cap L^{\prime}\right)\left(\varphi^{\prime} g^{\prime}\right) .
$$

De forma análoga, demonstra-se que $g$ pode ser escolhido como um morfismo fino, se $\mathrm{N}$ for um monóide livre.

Suponha finalmente que $\mathrm{M}=\Sigma^{*}$ e $N=\Gamma^{*}$, onde $\Sigma$ e $\Gamma$ são alfabetos. Sejam $b_{1}: \Sigma \rightarrow \Sigma^{\prime}$ e $b_{2}: \Gamma \rightarrow \Gamma^{\prime}$ cópias tais que $\Sigma^{\prime} \cap \Gamma^{\prime}=\varnothing, h: \Omega^{*} \rightarrow\left(\Sigma^{\prime} \cup \Gamma^{\prime}\right)^{*}$ o morfismo definido por

$$
\sigma h=\left(\sigma f b_{1}\right)\left(\sigma g b_{2}\right), \quad \forall \sigma \in \Omega,
$$


e $f^{\prime}:\left(\Sigma^{\prime} \cup \Gamma^{\prime}\right)^{*} \rightarrow \Sigma^{*}$ e $g^{\prime}:\left(\Sigma^{\prime} \cup \Gamma^{\prime}\right)^{*} \rightarrow \Gamma^{*}$ os morfismos finos definidos por

$$
\sigma f^{\prime}=\sigma b_{1}^{-1}, \sigma g^{\prime}=1, \gamma f^{\prime}=1, \gamma g^{\prime}=\gamma b_{2}^{-1}, \forall \sigma \in \Sigma^{\prime}, \forall \gamma \in \Gamma^{\prime} .
$$

Seja $u=\sigma_{1} \ldots \sigma_{n}$ uma palavra em $\Omega^{+}$, onde $\sigma_{i}$ é uma letra para todo índice $i$. Então,

$$
(u h) f^{\prime}=\left(\left(\sigma_{1} f b_{1}\right)\left(\sigma_{1} g b_{2}\right) \ldots\left(\sigma_{n} f b_{1}\right)\left(\sigma_{n} g b_{2}\right)\right) f^{\prime}=\left(\sigma_{1} f\right) \ldots\left(\sigma_{n} f\right)=u f
$$

$\mathrm{e}$

$$
(u h) g^{\prime}=\left(\left(\sigma_{1} f b_{1}\right)\left(\sigma_{1} g b_{2}\right) \ldots\left(\sigma_{n} f b_{1}\right)\left(\sigma_{n} g b_{2}\right)\right) g^{\prime}=\left(\sigma_{1} g\right) \ldots\left(\sigma_{n} g\right)=u g .
$$

Assim, $u(f, g)=(u h)\left(f^{\prime}, g^{\prime}\right)$. Portanto,

$$
L(f, g)=(L h)\left(f^{\prime}, g^{\prime}\right) .
$$

Utilizando o Teorema 1.6.3, obtemos um alfabeto $\Xi$, uma linguagem local $L^{\prime}$ sobre $\Xi$ e um morfismo muito fino $\varphi^{\prime}: \Xi^{*} \rightarrow\left(\Sigma^{\prime} \cup \Gamma^{\prime}\right)^{*}$ tais que

$$
(L h)\left(f^{\prime}, g^{\prime}\right)=L^{\prime}\left(\varphi^{\prime} f^{\prime}, \varphi^{\prime} g^{\prime}\right) .
$$

Portanto,

$$
\tau=\left(\varphi^{\prime} f^{\prime}\right)^{-1}\left(\cap L^{\prime}\right)\left(\varphi^{\prime} g^{\prime}\right)
$$

Como $\varphi^{\prime} f^{\prime}$ e $\varphi^{\prime} g^{\prime}$ são morfismos finos, a demonstração está concluída.

Os morfismos $f$ e $g$ em (2.2.2) são denominados morfismo de entrada e morfismo de saída da relação $\tau$, respectivamente.

Observamos que a demonstração do Teorema 2.2.1 também permite concluir que, se $\mathrm{M}$ e $\mathrm{N}$ forem monóides livres sobre alfabetos $\Sigma$ e $\Gamma$, respectivamente, então $\tau$ pode ser fatorada como

$$
\Sigma^{*} \stackrel{f^{-1}}{\longrightarrow}\left(\Sigma^{\prime} \cup \Gamma^{\prime}\right)^{*} \stackrel{\cap L}{\longrightarrow}\left(\Sigma^{\prime} \cup \Gamma^{\prime}\right)^{*} \stackrel{g}{\longrightarrow} \Gamma^{*},
$$

onde $\Sigma^{\prime}$ e $\Gamma^{\prime}$ são cópias de $\Sigma$ e $\Gamma$, respectivamente, tais que $\Sigma^{\prime} \cap \Gamma^{\prime}=\varnothing, L$ é uma linguagem racional (não necessariamente local) sobre $\Sigma^{\prime} \cup \Gamma^{\prime}$, e $f:\left(\Sigma^{\prime} \cup \Gamma^{\prime}\right)^{*} \rightarrow \Sigma^{*}$ e $g:\left(\Sigma^{\prime} \cup \Gamma^{\prime}\right)^{*} \rightarrow \Gamma^{*}$ são os morfismos finos $f^{\prime}$ e $g^{\prime}$, respectivamente, definidos em (2.2.3).

Vamos estudar algumas aplicações do Teorema 2.2.1.

Proposição 2.2.1 Seja $\tau: \mathrm{M} \rightarrow \mathrm{N}$ uma relação racional. Então, $\operatorname{dom} \tau$ é um subconjunto racional de $\mathrm{M}, e \operatorname{im} \tau$ é um subconjunto racional de $\mathrm{N}$.

Demonstração. O Teorema de Nivat fornece uma fatoração $\tau=\mathrm{M} \stackrel{f^{-1}}{\longrightarrow} \Omega^{*} \stackrel{\cap L}{\longrightarrow} \Omega^{*} \stackrel{g}{\longrightarrow} \mathrm{N}$, onde $f$ e $g$ são morfismos, e $L$ é um conjunto racional. Claramente, $\operatorname{dom} \tau=L f$ e im $\tau=L g$. Pela Proposição 1.5.2, temos então que $\operatorname{dom} \tau$ e $\operatorname{im} \tau$ são conjuntos racionais.

O Exemplo 2.1.2 pode ser generalizado para a propriedade a seguir:

Corolário 2.2.1 Um morfismo de monóides $f: \mathrm{M} \rightarrow \mathrm{N}$ é uma relação racional se, e somente se, $\mathrm{M}$ é um monóide finitamente gerado. 
Demonstração. Suponha que $f$ seja uma relação racional. Como $\operatorname{dom} f=\mathrm{M}$, segue da Proposição 2.2.1 que M é um conjunto racional. Logo, pela Proposição 1.5.8, M deve ser finitamente gerado.

Suponha que $\mathrm{M}=S^{*}$, onde $S$ é um conjunto finito. Então, o gráfico de $f$ é o conjunto racional

$$
\# f=\left(\bigcup_{s \in S}(s, s f)\right)^{*}
$$

o que mostra que $f$ é uma relação racional.

Proposição 2.2.2 Sejam $\Sigma$ um alfabeto, $\tau: \Sigma^{*} \rightarrow \mathrm{N}$ uma relação racional, e $A$ um subconjunto

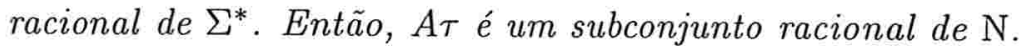

Demonstração. O Teorema de Nivat fornece uma fatoração $\tau=\Sigma^{*} \stackrel{f^{-1}}{\longrightarrow} \Omega^{*} \stackrel{\cap L}{\longrightarrow} \Omega^{*} \stackrel{g}{\longrightarrow} \mathrm{N}$ de $\tau$, onde $f$ e $g$ são morfismos, e $L$ é um conjunto racional. Observe que $A \tau=A f^{-1}(\cap L) g$. Pelo Teorema de Kleene, $A$ e $L$ são reconhecíveis. Pela Proposição 1.5.3, $A f^{-1} \cap L$ é reconhecível. Pelo Teorema de Kleene, esse conjunto é racional. Finalmente, pela Proposição 1.5.2, $A f^{-1}(\cap L) g$ é racional.

Na proposição acima, a hipótese de que o monóide de entrada é um monóide livre é fundamental, como mostra o contra-exemplo a seguir.

Exemplo 2.2.1 Sejam $\Sigma=\{\sigma, \gamma\}$ e $f: \Sigma^{*} \rightarrow \Sigma^{*} \times \Sigma^{*}$ o morfismo definido por

$$
\sigma f=(\sigma, 1) \quad \text { e } \quad \gamma f=(1, \gamma)
$$

Pelo Teorema de Nivat, o morfismo inverso $f^{-1}: \Sigma^{*} \times \Sigma^{*} \rightarrow \Sigma^{*}$ é uma relação racional. No entanto, essa relação não preserva conjuntos racionais, pois

$$
(\sigma, \gamma)^{*} f^{-1}=\left\{u \in \Sigma^{*}:|u|_{\sigma}=|u|_{\gamma}\right\} .
$$

O teorema a seguir é o correspondente para relações racionais do Lema da Iteração para linguagens reconhecíveis.

Teorema 2.2.2 Seja $\tau: \Sigma^{*} \rightarrow \Gamma^{*}$ uma relação racional. Então, existe um inteiro positivo $n$ tal que todo par $(u, v)$ em \#r com $|u|+|v| \geq n$ admite uma fatoração

$$
\left(u_{1}, u_{2}\right)\left(w_{1}, w_{2}\right)\left(v_{1}, v_{2}\right)
$$

satisfazendo

i. $\left(w_{1}, w_{2}\right) \neq(1,1)$,

ii. $\left|u_{1}\right|+\left|u_{2}\right|+\left|w_{1}\right|+\left|w_{2}\right| \leq n e$ 
iii. $\left(u_{1}, u_{2}\right)\left(w_{1}, w_{2}\right)^{*}\left(v_{1}, v_{2}\right) \subseteq \# \tau$.

Demonstração. Seja $\Sigma^{*} \stackrel{f^{-1}}{\longrightarrow}\left(\Sigma^{\prime} \cup \Gamma^{\prime}\right)^{*} \stackrel{\cap L}{\longrightarrow}\left(\Sigma^{\prime} \cup \Gamma^{\prime}\right)^{*} \stackrel{g}{\longrightarrow} \Gamma^{*}$ uma fatoração de $\tau$ como em (2.2.4). Pelo Lema 2.2.1.

$$
\# \tau=L(f, g) .
$$

Vamos utilizar a seguinte propriedade de $f$ e $g$, que segue diretamente da definição desses morfismos. Para toda palavra $w$ sobre $\Sigma^{\prime} \cup \Gamma^{\prime}$,

$$
|w|=|w f|+|w g| \text {. }
$$

Como $L$ é racional, segue do Teorema de Kleene que também é reconhecível. Então, pelo Lema da Iteração para linguagens reconhecíveis, existe um inteiro positivo $n$ tal que toda palavra $w$ em $L$ com $|w| \geq n$ admite uma fatoração da forma $x y z$ satisfazendo $y \neq 1,|x y| \leq n$, e $x y^{*} z \subseteq L$.

Sejam $(u, v)$ um par em \# $\tau$ tal que $|u|+|v| \geq n$, e $w$ uma palavra em $L$ tal que $w(f, g)=(u, v)$. Por (2.2.5), $|w| \geq n$. Seja $x y z$ a fatoração de $w$ dada pelo Lema da Iteração. Então, $(y f, y g) \neq$ $(1,1)$, e de $|x y| \leq n$ e (2.2.5) segue que $|x f|+|x g|+|y f|+|y g| \leq n$. Como $x y^{*} z \subseteq L$, temos que $(x f, x g)(y f, y g)^{*}(z f, z g) \subseteq \# \tau$. Portanto, $(x f, x g)(y f, y g)(z f, z g)$ é uma fatoração de $(u, v)$ como queríamos.

Exemplo 2.2.2 Sejam $\Sigma$ um alfabeto com pelo menos duas letras e $\varrho: \Sigma^{*} \rightarrow \Sigma^{*}$ a função que associa cada palavra ao seu reverso. Vamos mostrar que essa relação não é racional.

Suponha o contrário, e seja $n$ como no Teorema 2.2.2. Sejam $\sigma$ e $\gamma$ letras distintas de $\Sigma$, e considere o $\operatorname{par}\left(\sigma^{n} \gamma^{n}, \gamma^{n} \sigma^{n}\right)$ em \# $\varrho$. Então, esse par adimite uma fatoração da forma

$$
\left(u_{1}, u_{2}\right)\left(w_{1}, w_{2}\right)\left(v_{1}, v_{2}\right)
$$

satisfazendo as condições $i$, ii e $i i i$ do Teorema 2.2.2.

Como $\left|u_{1}\right|+\left|u_{2}\right|+\left|w_{1}\right|+\left|w_{2}\right| \leq n,\left(u_{1}, u_{2}\right)$ é da forma $\left(\sigma^{k_{1}}, \gamma^{k_{2}}\right)$ e $\left(w_{1}, w_{2}\right)$ é da forma $\left(\sigma^{l_{1}}, \gamma^{l_{2}}\right)$. Como $\left(w_{1}, w_{2}\right) \neq(1,1), l_{1}$ e $l_{2}$ não são ambos iguais a 0 . Suponha que $l_{1}>0$, e seja $\left(s_{1}, s_{2}\right)=\left(\sigma^{k_{1}}, \gamma^{k_{2}}\right)\left(\sigma^{l_{1}}, \gamma^{l_{2}}\right)^{2}\left(v_{1}, v_{2}\right)$. Então, $\left|s_{1}\right|_{\sigma}>\left|s_{1}\right|_{\gamma}$, mas $\left|s_{2}\right|_{\sigma} \leq\left|s_{2}\right|_{\gamma}$. Então, $s_{1}$ não pode ser o reverso de $s_{2}$, e $\left(s_{1}, s_{2}\right)$ não pertence a \# $\#$, o que contraria a condição iii. O caso $l_{2}>0$ é análogo. Da contradição, segue que $\varrho$ não é uma relação racional.

Exemplo 2.2.3 Vamos identificar o monóide aditivo $\mathbb{N}$ com o monóide livre gerado por uma única letra $\sigma$.

Vamos provar que a relação $\tau: \mathbb{N} \rightarrow \mathbb{N}$ que associa cada número natural ao seu quadrado não é racional. Suponha o contrário, e seja $n$ o inteiro fornecido pelo Teorema 2.2.2. Então, $\left(n, n^{2}\right)$ pode ser fatorado como $\left(n_{1}, n_{1}^{\prime}\right)\left(n_{2}, n_{2}^{\prime}\right)\left(n_{3}, n_{3}^{\prime}\right)$, e

$$
\left(n_{1}, n_{1}^{\prime}\right)\left(n_{2}, n_{2}^{\prime}\right)^{k}\left(n_{3}, n_{3}^{\prime}\right) \in \# \tau, \quad \forall k \geq 0 .
$$

Mas, isso significa que

$$
\left(\left(n_{1}+n_{3}\right)+k n_{2}\right) \tau=\left(n_{1}^{\prime}+n_{3}^{\prime}\right)+k n_{2}^{\prime}, \quad \forall k \geq 0,
$$


o que é um absurdo. Portanto, $\tau$ não é uma relação racional.

O resultado mais forte de que a função quadrado não é racional independente da base utilizada na representação numérica foi provado por Eilenberg [Eil74].

\subsection{Transdutores}

Transdutores são as máquinas de estados finitos que realizam as relações racionais. Sua definição explícita é

Definição 2.3.1 Um transdutor é um autômato sobre um monóide produto $\mathrm{M} \times \mathrm{N}$.

O Teorema 1.6.1 implica em

Teorema 2.3.1 Uma relação $\tau: \mathrm{M} \rightarrow \mathrm{N}$ é racional se, e somente se, seu gráfico é o comportamento de um transdutor sobre o monóide produto $\mathrm{M} \times \mathrm{N}$.

Pelo Corolário 1.6.1, podemos dizer que toda relação racional é o comportamento de um transdutor bi-acessível e normalizado.

Dizemos que um transdutor $\mathcal{T}$ sobre um produto $\mathrm{M} \times \mathrm{N}$ é um $\mathrm{M}-\mathrm{N}$-transdutor. Se $\mathrm{M}$ for um monóide livre sobre um alfabeto $\Sigma$, podemos dizer que $\mathcal{T}$ é um $\Sigma$-N-transdutor. Se $N$ for um monóide livre sobre um alfabeto $\Gamma$, podemos dizer que $\mathcal{T}$ é um $\mathrm{M}$ - $\Gamma$-transdutor.

O rótulo de um passeio

$$
c: p \stackrel{(m, n)}{\longrightarrow} q
$$

em $\mathcal{T}$ é um par no produto $\mathrm{M} \times \mathrm{N}$. Será conveniente dizer que $m$ é a entrada de $c$, e $n$ a saída ou emissão de $c$. Se M for um monóide livre, dizemos também que $c$ soletra $m$.

A seguinte notação para um passeio será preferida:

$$
c: p \stackrel{m / n}{\longrightarrow} q
$$

Ademais, vamos denotar uma transição $(p,(m, n), q)$ por $(p, m, n, q)$.

É conveniente definir o comportamento de $\mathcal{T}$ como uma relação $|\mathcal{T}|: M \rightarrow N$, ao invés de um subconjunto de pares de $\mathrm{M} \times \mathrm{N}$. Dizemos que $\mathcal{T}$ realiza a relação $|\mathcal{T}|$.

$\mathrm{O}$ autômato de entrada (autômato de saída) de $\mathcal{T}$ é o M-autômato ( $N$-autômato) obtido a partir de $\mathcal{T}$ substituindo cada transição $(p, m, n, q)$ pela transição $(p, m, q)((p, n, q))$. O comportamento do autômato de entrada (saída) de $\mathcal{T}$ é $\operatorname{dom}|\mathcal{T}|(\mathrm{im}|\mathcal{T}|)$.

Exemplo 2.3.1 Sejam $\Sigma=\Gamma=\{\sigma, \gamma\}$. A figura a seguir é a representação pictórica de um $\Sigma-\Gamma$ transdutor:

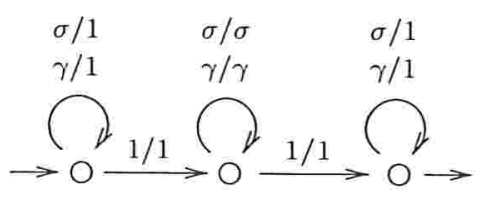


Seu comportamento é a relação fat $: \Sigma^{*} \rightarrow \Gamma^{*}$ que associa a toda entrada o conjunto dos seus fatores.

Sejam $\mathcal{T}=(Q, E, I, T)$ um M-N-transdutor, $L$ o conjunto local dos passeios bem-sucedidos de $\mathcal{T}$, e $\alpha: E^{*} \rightarrow \mathrm{M}$ e $\beta: E^{*} \rightarrow \mathrm{N}$ os morfismos definidos por

$$
(p, m, n, q) \alpha=m \quad \text { e } \quad(p, m, n, q) \beta=n, \quad \forall(p, m, n, q) \in E .
$$

Então,

$$
\#|\mathcal{T}|=L(\alpha, \beta)
$$

Pelo Lema 2.2.1, temos então que

$$
|\mathcal{T}|=\alpha^{-1}(\cap L) \beta
$$

Dizemos que o par $(\alpha, \beta)$ é o bimorfismo de $\mathcal{T}$. Como no Teorema de Nivat, chamamos $\alpha$ e $\beta$ de morfismo de entrada e morfismo de saída de $\mathcal{T}$, respectivamente.

Um transdutor pode ser facilmente construído a partir de uma fatoração de uma relação racional.

Seja $\tau: M \stackrel{f^{-1}}{\longrightarrow} \Omega^{*} \stackrel{\cap L}{\longrightarrow} \Omega^{*} \stackrel{g}{\rightarrow} \mathrm{N}$ uma fatoração onde $\Omega$ é um alfabeto, $f$ e $g$ são morfismos, e $L$ é uma linguagem racional sobre $\Omega$. Sejam $\mathcal{A}=(Q, E, I, T)$ um $\Omega$-autômato bi-acessível e normalizado que reconhece $L$, e $\mathcal{T}$ o $\mathrm{M}$-N-transdutor $\left(Q, E^{\prime}, I, T\right)$ obtido substituindo cada transição

$$
(p, \sigma, q)
$$

de $\mathcal{A}$ pela transição

$$
(p, \sigma f, \sigma g, q)
$$

Assim, cada passeio bem-sucedido $i \stackrel{u}{\rightarrow} t$ em $\mathcal{A}$ corresponde a um passeio bem-sucedido $i \stackrel{u f / u g}{\longrightarrow} t$ em $\mathcal{T}$. Portanto,

$$
\#|\mathcal{T}|=L(f, g)
$$

Do Lema 2.2.1, segue então que

$$
|\mathcal{T}|=\tau
$$

Essa construção e o Teorema 2.2.1 implicam na proposição a seguir:

Proposição 2.3.1 Sejam $\Sigma$ e $\Gamma$ alfabetos.

- Toda relação racional $\Sigma^{*} \rightarrow \mathrm{M}$ é o comportamento de um $\Sigma$-M-transdutor bi-acessível $e$ normalizado com transições rotuladas por pares em $(\Sigma \cup 1) \times \mathrm{M}$.

- Toda relação racional $\mathrm{M} \rightarrow \Sigma^{*}$ é o comportamento de um $\mathrm{M}-\Sigma$-transdutor bi-acessivel $e$ normalizado com transições rotuladas por pares em $\mathrm{M} \times(\Sigma \cup 1)$.

- Toda relação racional $\Sigma^{*} \rightarrow \Gamma^{*}$ é o comportamento de um $\Sigma$ - $\Gamma$-transdutor bi-acessivel $e$ normalizado com transições rotuladas por pares em $(\Sigma \cup 1) \times(\Gamma \cup 1)$. 
Dizemos que um $\Sigma$-M-transdutor $\mathcal{T}=(Q, E, I, T)$ é fino se $E \subseteq Q \times(\Sigma \cup 1) \times \mathrm{M} \times Q$.

Exemplo 2.3.2 Sejam $\Gamma=\{\sigma, \gamma, \xi\}$ um alfabeto e $X$ a linguagem das palavras de $\Gamma^{+}$tais que $\sigma$ e $\gamma$ não apareçam consecutivamente. Formalmente,

$$
X=\sigma^{+} \cup \gamma^{+} \cup\left(\left(\sigma^{*} \cup \gamma^{*}\right) \xi\right)^{+}\left(\sigma^{*} \cup \gamma^{*}\right) .
$$

Dado um outro alfabeto $\Sigma=\{a, b\}$, considere o morfismo $f: \Gamma^{*} \rightarrow \Sigma^{*}$ definido por

$$
\sigma f=a, \quad \gamma f=1 \quad \text { e } \quad \xi f=b .
$$

Vamos construir um transdutor que realiza a relação $\tau=f^{-1}(\cap X): \Sigma^{*} \rightarrow \Gamma^{*}$.

Primeiro, escrevemos $\tau=f^{-1}(\cap X) g$, onde $g: \Gamma^{*} \rightarrow \Gamma^{*}$ é a identidade. Utilizando (2.3.2), um $\Sigma$ - $\Gamma$-transdutor que realiza $\tau$ pode ser construído a partir do $\Gamma$-autômato abaixo, que reconhece $X$ :

A :

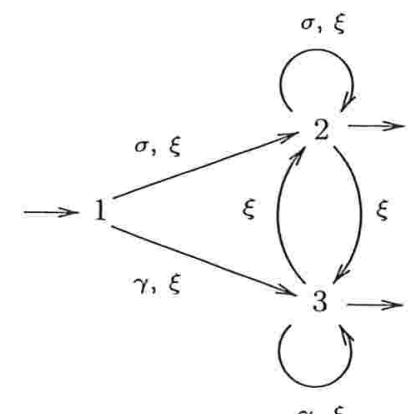

$\gamma, \xi$

Após as substituições, o transdutor resultante é

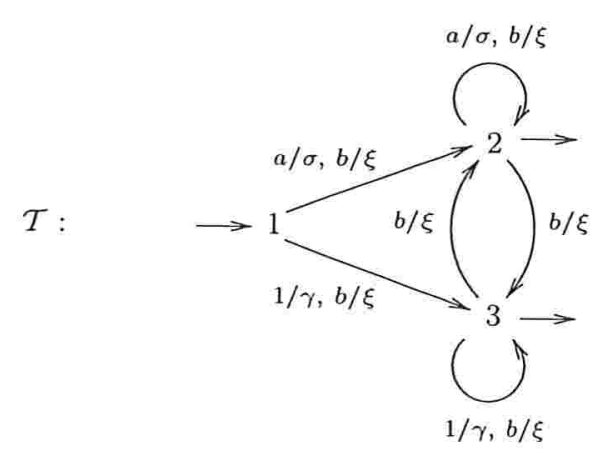

Concluímos a seção com uma observação útil sobre o bimorfismo de um transdutor.

Sejam $p$ e $q$ estados de um M-N-transdutor $\mathcal{T}=(Q, E, I, T)$, e $L_{p q}$ o conjunto local dos passeios em $\mathcal{T}$ de $p$ a $q$. Dado um elemento $m$ em $\mathrm{M}$, o conjunto dos passeios em $\mathcal{T}$ de $p$ a $q$ e com entrada $m$ é

$$
\left(m \alpha^{-1}\right) \cap L_{p q}
$$

Portanto,

$$
\left(\left(m \alpha^{-1}\right) \cap L_{p q}\right) \beta=\{n \text { : existe um passeio } p \stackrel{m / n}{\longrightarrow} q \text { em } \mathcal{T}\} .
$$


Assim,

$$
m|\mathcal{T}|=\bigcup_{\substack{i \in I \\ t \in T}}\left(\left(m \alpha^{-1}\right) \cap L_{i t}\right) \beta
$$

Se considerarmos $I$ e $T$ como as funções $Q \rightarrow \mathrm{M}$ definidas por

$$
\begin{aligned}
q I & = \begin{cases}1 & \text { se } q \text { é um estado inicial, } \\
\varnothing & \text { caso contrário; }\end{cases} \\
q T & = \begin{cases}1 & \text { se } q \text { é um estado final, } \\
\varnothing & \text { caso contrário }\end{cases}
\end{aligned}
$$

para todo estado $q$, podemos também escrever

$$
m|\mathcal{T}|=\bigcup_{p, q \in Q}(p I)\left(\left(\left(m \alpha^{-1}\right) \cap L_{p q}\right) \beta\right)(q T)
$$

\subsection{Representações matriciais}

As representações matriciais são representações de relações racionais em termos de matrizes e morfismos bastante compactas, onde as operações matriciais correspondem a operações com passeios em transdutores.

Nesta seção, vamos definir essas representações, e mostrar sua relação com as relações racionais. A seguinte notação será utilizada para a indexação de matrizes e vetores: o elemento na linha $i$ e coluna $j$ de uma matriz $a$ será denotado por $a_{i j}$, e o elemento na posição $i$ de um vetor $v$ será denotado por $v_{i}$.

Definição 2.4.1 Seja $n$ um inteiro positivo. Uma representação matricial de dimensão $n$ sobre um alfabeto $\Sigma$ e um monóide $\mathrm{M}$ é uma quádrupla $\mathbf{M}=(Q, \lambda, \mu, \rho)$ onde

- $Q$ é um conjunto de cardinalidade $n$;

- $\lambda$ é um vetor linha de dimensão $n$ sobre $\operatorname{Rac}(\mathrm{M})$;

- $\rho$ é um vetor coluna de dimensão $n$ sobre $\operatorname{Rac}(\mathrm{M})$;

- $\mu: \Sigma^{*} \rightarrow \operatorname{Rac}(\mathrm{M})^{Q \times Q}$ é um morfismo de semigrupos de $\Sigma^{*}$ no monóide multiplicativo das matrizes quadradas de dimensão $n$ sobre o semi-anel $\operatorname{Rac}(\mathrm{M})$.

$O$ comportamento de $\mathbf{M}$ é a relação $|\mathbf{M}|: \Sigma^{*} \rightarrow \mathrm{M}$ definida por

$$
u|\mathbf{M}|=\lambda(u \mu) \rho,
$$

ou, equivalentemente,

$$
u|\mathbf{M}|=\bigcup_{p, q \in Q} \lambda_{p}\left(u \mu_{p q}\right) \rho_{q},
$$

para toda palavra u em $\Sigma^{*}$. 
$\mathrm{Na}$ definição acima, se $\mathrm{M}$ for um monóide livre gerado por um alfabeto $\Gamma$, podemos dizer que $M$ é uma representação matricial sobre os alfabetos $\Sigma$ e $\Gamma$.

Vamos mostrar que as representações matriciais realizam exatamente a classe das relações racionais com domínio em um monóide livre.

Teorema 2.4.1 Uma relação $\tau: \Sigma^{*} \rightarrow \mathrm{M}$ é racional se, e somente se, é o comportamento de uma representação matricial.

Demonstração. Seja $\mathrm{M}=(Q, \lambda, \mu, \rho)$ uma representação matricial sobre $\Sigma$ e $\mathrm{M}$. Considere o alfabeto $\Omega=Q \times \Sigma \times Q$, o morfismo muito fino $f: \Omega^{*} \rightarrow \Sigma^{*}$ definido por

$$
(p, \sigma, q) f=\sigma, \quad \forall(p, \sigma, q) \in \Omega,
$$

a substituição racional $h: \Omega^{*} \rightarrow \mathrm{M}$ definida por

$$
(p, \sigma, q) h=\sigma \mu_{p q}, \quad \forall(p, \sigma, q) \in \Omega
$$

e a linguagem local

$$
L=\Omega^{+}-\Omega^{*} C \Omega^{*},
$$

onde $C=\left\{(p, \sigma, q)(r, \gamma, s) \in \Omega^{2}: q \neq r\right\}$.

Para todo elemento $q$ em $Q$, vamos representar por $A_{q}$ o conjunto das triplas de $\Omega$ cuja primeira coordenada seja igual a $q$, e por $B_{q}$ o análogo para a terceira coordenada. Para todo par $p$ e $q$ em $Q$, seja

$$
L_{p q}=\left(A_{p} \Omega^{*} \cap \Omega^{*} B_{q}\right)-\Omega^{*} C \Omega^{*} .
$$

Segue diretamente da definição desses conjuntos que

$$
L_{p q}=\left(A_{p} \cap B_{q}\right) \cup\left(\bigcup_{r \in Q} L_{p r} L_{r q}\right)
$$

$\mathrm{e}$

$$
L=\bigcup_{p, q \in Q} L_{p q}
$$

Vamos mostrar que, para toda palavra $u$ de $\Sigma^{+}$,

$$
u \mu_{p q}=\left(\left(u f^{-1}\right) \cap L_{p q}\right) h, \quad \forall p, q \in Q .
$$

Vamos usar indução em $|u|$ :

- $|u|=1$ : então $\left(u f^{-1}\right) \cap L_{p q}=(p, u, q)$, e (2.4.7) segue da definição de $h$. 
- $|u|>1$ : fatore $u$ como $v \sigma$, onde $\sigma$ é uma letra. Então,

$$
\begin{gathered}
u \mu_{p q}=((v \mu)(\sigma \mu))_{p q}=\bigcup_{r \in Q}\left(v \mu_{p r}\right)\left(\sigma \mu_{r q}\right) \stackrel{\text { h.i. }}{=} \bigcup_{r \in Q}\left(\left(\left(v f^{-1}\right) \cap L_{p r}\right) h\right)\left(\left(\left(\sigma f^{-1}\right) \cap L_{r q}\right) h\right) \\
\stackrel{(1.3 .2)}{=}\left(\bigcup_{r \in Q}\left(\left(v f^{-1}\right) \cap L_{p r}\right)\left(\left(\sigma f^{-1}\right) \cap L_{r q}\right)\right) h .
\end{gathered}
$$

De (2.4.5) e do fato de que $v \neq 1$ e $\sigma \neq 1$, segue que

$$
\bigcup_{r \in Q}\left(\left(v f^{-1}\right) \cap L_{p r}\right)\left(\left(\sigma f^{-1}\right) \cap L_{r q}\right)=\left(v f^{-1}\right)\left(\sigma f^{-1}\right) \cap L_{p q}
$$

Substituindo em (2.4.8), obtemos

$$
u \mu_{p q}=\left(\left(v f^{-1}\right)\left(\sigma f^{-1}\right) \cap L_{p q}\right) h
$$

Como $f$ é muito fino, segue da Proposição 1.3.1 que

$$
\left(\left(\left(v f^{-1}\right)\left(\sigma f^{-1}\right)\right) \cap L_{p q}\right) h=\left(\left(u f^{-1}\right) \cap L_{p q}\right) h .
$$

Obtemos então (2.4.7).

Considere agora a composição

$$
\psi: \Sigma^{*} \stackrel{f^{-1}}{\longrightarrow} \Omega^{*} \stackrel{\cap L}{\longrightarrow} \Omega^{*} \stackrel{g}{\longrightarrow} \Xi^{*} \stackrel{h^{\prime}}{\longrightarrow} \mathrm{M}
$$

onde $\Xi$ é o conjunto $\Omega \cup Q \cup Q^{\prime}, Q^{\prime}$ é uma cópia de $Q$ disjunta de $\Omega$ por uma bijeção $b, g$ é a relação racional definida por

$$
\left(\left(p_{0}, \sigma_{1}, p_{1}\right) \ldots\left(p_{n-1}, \sigma_{n}, p_{n}\right)\right) g=p_{0}\left(p_{0}, \sigma_{1}, p_{1}\right) \ldots\left(p_{n-1}, \sigma_{n}, p_{n}\right)\left(p_{n} b\right),
$$

e $h^{\prime}$ é uma substituição racional que coincide com $h$ nas triplas em $\Omega$, e nas demais letras é definida por

$$
q h^{\prime}=q \lambda \quad \text { e } \quad q^{\prime} h^{\prime}=\left(q^{\prime} b^{-1}\right) \rho, \quad \forall q \in Q, \forall q^{\prime} \in Q^{\prime} .
$$

Pela definição de $L, 1 \psi=\varnothing$. Ademais, para toda palavra $u$ em $\Sigma^{+}$,

$$
\begin{aligned}
u \psi & =\left(\left(u f^{-1}\right) \cap L\right) g h^{\prime} \stackrel{(2.4 .6)}{=}\left(\bigcup_{p, q \in Q}\left(u f^{-1}\right) \cap L_{p q}\right) g h^{\prime}=\left(\bigcup_{p, q \in Q}\left(\left(u f^{-1}\right) \cap L_{p q}\right) g\right) h^{\prime} \\
& =\left(\bigcup_{p, q \in Q} p\left(\left(u f^{-1}\right) \cap L_{p q}\right)(q b)\right) h^{\prime} \stackrel{(1.3 .2)}{=} \bigcup_{p, q \in Q}\left(p h^{\prime}\right)\left(\left(\left(u f^{-1}\right) \cap L_{p q}\right) h\right)\left((q b) h^{\prime}\right) \\
& \stackrel{(2.4 .7)}{=} \bigcup_{p, q \in Q} \lambda_{p}\left(u \mu_{p q}\right) \rho_{q}=\lambda(u \mu) \rho=u|\mathbf{M}| .
\end{aligned}
$$


Assim, $\psi$ é o comportamento de $\mathrm{M}$ restrito a $\Sigma^{+}$.

$\mathrm{Na}$ Seção 2.5, provamos que uma composição $\mathrm{S} \rightarrow \mathrm{N} \rightarrow \mathrm{S}$ de relações racionais tal que o monóide $\mathrm{N}$ seja um monóide livre é uma relação racional (Teorema 2.5.1). Como as relações $f^{-1}, \cap L, g$ e $h^{\prime}$ são racionais, temos que $|M| \mid \Sigma^{+}$que é uma relação racional.

Como $X=1|\mathbf{M}|$ é um conjunto racional, segue da Proposição 2.1.1 que $|\mathbf{M}| \mid 1=1 \times X$ é uma relação racional.

Como $|\mathbf{M}|=|\mathbf{M}|\left|\Sigma^{+} \cup\right| \mathbf{M}|| 1$, concluímos que $|\mathbf{M}|$ é uma relação racional.

Suponha agora que $\tau$ seja uma relação racional. Sejam $\mathcal{T}=(Q, E, I, T)$ um $\Sigma$-M-transdutor fino com $n$ estados que realiza $\tau$, e $(\alpha, \beta)$ o bimorfismo de $\mathcal{T}$. A partir desse transdutor, vamos construir uma representação matricial $\mathbf{M}_{\mathcal{T}}=(Q, \lambda, \mu, \rho)$ que realiza $\tau$.

Para definir os vetores $\lambda$ e $\rho$, vamos considerar $I$ e $T$ como as funções características definidas em (2.3.5). Para todo estado $q$, definimos

$$
\lambda_{q}=q I \quad \text { e } \quad \rho_{q}=q T .
$$

Seja $\nu: \Sigma^{*} \rightarrow \operatorname{Rac}(\mathrm{M})^{Q \times Q}$ o morfismo de monóides definido por

$$
\sigma \nu_{p q}=\left(\left(\sigma \alpha^{-1}\right) \cap L_{p q}\right) \beta, \quad \forall \sigma \in \Sigma, \forall p, q \in Q,
$$

onde $L_{p q}$ é o conjunto local dos passeios de $p$ a $q$ em $\mathcal{T}$. Definimos $\mu$ como

$$
u \mu_{p q}=\left\{\begin{array}{ll}
\left(\left(1 \alpha^{-1}\right) \cap L_{p q}\right) \beta & \text { se } u=1, \\
u \nu & \text { se } u \neq 1,
\end{array} \quad \forall u \in \Sigma^{*}, \forall p, q \in Q .\right.
$$

Vamos mostrar que $\mu$ é um morfismo de semigrupos. Como $\nu$ é um morfismo, isso segue das igualdades

$$
u \mu=(1 \mu)(u \mu)=(u \mu)(1 \mu), \quad \forall u \in \Sigma^{*} .
$$

Vamos mostrar que $u \mu=(1 \mu)(u \mu)$. A demonstração da outra igualdade é análoga.

Sejam $p$ e $q$ estados quaisquer, e $u$ uma palavra de $\Sigma^{*}$. Recorde que, por (1.6.1), 1 pertence a $L_{p p}$ (ou seja, o passeio vazio pertence a esse conjunto). Assim, 1 pertence a $1 \mu_{p p}$. Então,

$$
u \mu_{p q} \subseteq \bigcup_{r \in Q}\left(1 \mu_{p r}\right)\left(u \mu_{r q}\right)=((1 \mu)(u \mu))_{p q} .
$$

Para mostrar que $((1 \mu)(u \mu))_{p q} \subseteq u \mu_{p q}$, vamos primeiro provar que

$$
u \mu_{p q}=\left(\left(u \alpha^{-1}\right) \cap L_{p q}\right) \beta, \quad \forall u \in \Sigma^{*}, \forall p, q \in Q,
$$

ou seja, $u \mu_{p q}$ é o conjunto das saídas dos passeios em $\mathcal{T}$ de $p$ a $q$ com entrada $u$. Vamos usar indução em $|u|$ :

- $|u|=0$ e $|u|=1$ : segue de (2.4.11) e (2.4.10).

- $|u|>1$ : fatore $u$ como $v \sigma$, onde $\sigma$ é uma letra. Então,

$$
\begin{gathered}
u \mu_{p q}=\bigcup_{r \in Q}\left(v \mu_{p r}\right)\left(\sigma \mu_{r q}\right) \stackrel{\text { h.i. }}{=} \bigcup_{r \in Q}\left(\left(\left(v \alpha^{-1}\right) \cap L_{p r}\right) \beta\right)\left(\left(\left(\sigma \alpha^{-1}\right) \cap L_{r q}\right) \beta\right) \\
\stackrel{(1.3 .2)}{=}\left(\bigcup_{r \in Q}\left(\left(v \alpha^{-1}\right) \cap L_{p r}\right)\left(\left(\sigma \alpha^{-1}\right) \cap L_{r q}\right)\right) \beta .
\end{gathered}
$$


Da definição de $L_{p q}$ e de $\alpha$ segue diretamente que

$$
\bigcup_{r \in Q}\left(\left(v \alpha^{-1}\right) \cap L_{p r}\right)\left(\left(\sigma \alpha^{-1}\right) \cap L_{r q}\right)=\left(\left(v \alpha^{-1}\right)\left(\sigma \alpha^{-1}\right)\right) \cap L_{p q} .
$$

Substituindo em (2.4.13), obtemos

$$
u \mu_{p q}=\left(\left(\left(v \alpha^{-1}\right)\left(\sigma \alpha^{-1}\right)\right) \cap L_{p q}\right) \beta .
$$

Como $\alpha$ é fino, segue da Proposição 1.3.1 que

$$
\left(\left(\left(v \alpha^{-1}\right)\left(\sigma \alpha^{-1}\right)\right) \cap L_{p q}\right) \beta=\left(\left(u \alpha^{-1}\right) \cap L_{p q}\right) \beta,
$$

o que completa a prova de (2.4.12).

Seja $x$ um elemento em $((1 \mu)(u \mu))_{p q}$. Então, existe uma fatoração $y z$ de $x$ e um estado $r$ tal que $y$ pertence a $1 \mu_{p r}$ e $z$ pertence a $u \mu_{r q}$. De (2.4.12) e (2.3.3), segue que existem passeios

$$
c: p \stackrel{1 / y}{\longrightarrow} r \quad \text { e } \quad d: r \stackrel{u / z}{\longrightarrow} q
$$

em $\mathcal{T}$. Assim, $c d$ é um passeio em $\mathcal{T}$ de $p$ a $q$ com entrada $u$ e saída $x$. Segue então novamente de (2.4.12) e (2.3.3) que $x$ pertence a $u \mu_{p q}$. Portanto,

$$
((1 \mu)(u \mu))_{p q} \subseteq u \mu_{p q} .
$$

Como $p$ e $q$ são estados quaisquer, obtemos

$$
(1 \mu)(u \mu)=u \mu \text {. }
$$

Concluímos então que $\mu$ é um morfismo de semigrupos. Portanto, $\mathbf{M}_{\mathcal{T}}$ é uma representação matricial.

Finalmente, provamos que $\left|\mathbf{M}_{\mathcal{T}}\right|$ e $\tau$ coincidem em toda palavra $u$ de $\Sigma^{*}$ como segue:

$$
\begin{gathered}
u\left|\mathbf{M}_{\mathcal{T}}\right|=\bigcup_{p, q \in Q} \lambda_{p}\left(u \mu_{p q}\right) \rho_{q} \stackrel{(2.4 .12)}{=} \bigcup_{p, q \in Q} \lambda_{p}\left(\left(\left(u \alpha^{-1}\right) \cap L_{p q}\right) \beta\right) \rho_{q} \\
\stackrel{(2.4 .9)}{=} \bigcup_{p, q \in Q}(p I)\left(\left(\left(u \alpha^{-1}\right) \cap L_{p q}\right) \beta\right)(q T) \stackrel{(2.3 .6)}{=} u|\mathcal{T}| .
\end{gathered}
$$

Concluímos então que $\tau=\left|\mathbf{M}_{\mathcal{T}}\right|$.

Dizemos que a representação matricial $\mathbf{M}_{\mathcal{T}}$ construída na demonstração do Teorema 2.4.1 é a representação matricial do transdutor $\mathcal{T}$. Enfatizamos que, por (2.4.12) e (2.3.3), para toda palavra $u$ em $\Sigma^{*}, u \mu_{p q}$ é o conjunto das saídas dos passeios em $\mathcal{T}$ de $p$ a $q$ com entrada $u$.

Devido à analogia entre transdutores e representações matriciais, chamamos os elementos do conjunto $Q$ de uma representação matricial de estados. 
Definição 2.4.2 Dizemos que uma representação matricial $\mathrm{M}=(Q, \lambda, \mu, \rho)$ é bi-acessível se os vetores $\lambda$ e $\rho$ tiverem entradas iguais a $\varnothing$ ou 1 e, para todo estado $q$, existirem palavras $u$ e $v$ tais que

$$
u \mu_{i q} \neq \varnothing \quad e \quad v \mu_{q t} \neq \varnothing,
$$

onde $i$ e $t$ são estados tais que $\lambda_{i}=1$ e $\rho_{t}=1$. Nesse caso, dizemos que $i$ é um estado inicial, et é um estado final de $\mathbf{M}$.

Dizemos que $\mathbf{M}$ é normalizada se $\lambda$ possuir exatamente uma coordenada não-nula $i, \operatorname{com} \lambda_{i}=1$, e $\rho$ possuir exatamente uma coordenada não-nula $t$, distinta de $i$, com $\rho_{t}=1$.

Claramente, a representação matricial de um transdutor bi-acessível e normalizadado é biacessível e normalizada. Logo, segue da Proposição 2.3.1 que

Corolário 2.4.1 Toda relação racional é o comportamento de uma representação matricial biacessivel e normalizada.

A demonstração do Teorema 2.4.1 fornece uma fatoração de relações racionais diferente da obtida no Teorema 2.2.1. Esse resultado, que demonstramos a seguir, é o Teorema IX.5.1 do livro de Eilenberg [Eil74].

Teorema 2.4.2 Sejam $\Sigma$ um alfabeto, e $\tau: \Sigma^{*} \rightarrow \mathrm{M}$ uma relação tal que $1 \tau=\varnothing$ ou $1 \tau=1$. Então, $\tau$ é uma relação racional se, e somente se, existem um alfabeto $\Omega$, um conjunto local $L$ sobre $\Omega$, um morfismo muito fino $f: \Omega^{*} \rightarrow \Sigma^{*}$, e uma substituição racional $h: \Omega^{*} \rightarrow$ M tais que

$$
\tau=\Sigma^{*} \stackrel{f^{-1}}{\longrightarrow} \Omega^{*} \stackrel{\cap L}{\longrightarrow} \Omega^{*} \stackrel{h}{\rightarrow} \mathrm{M} .
$$

Demonstração. Se $\tau$ for uma composição $f^{-1}(\cap L) h$, então segue do Teorema 2.5.1 (Seção 2.5) que $\tau$ é uma relação racional. Como $f$ é muito fino, temos também que $1 \tau=1$ ou $1 \tau=\varnothing$, dependendo de se a palavra vazia pertencer ou não a $L$.

Suponha agora que $\tau$ seja uma relação racional. Vamos considerar primeiro o caso $1 \tau=\varnothing$. Seja $\mathbf{M}=(Q, \lambda, \mu, \rho)$ uma representação matricial bi-acessível que realiza $\tau$. Então, cada coordenada dos vetores $\lambda$ e $\rho$ é igual a $\varnothing$ ou 1 . Considere a composição

$$
\Sigma^{*} \stackrel{f^{-1}}{\longrightarrow} \Omega^{*} \stackrel{\cap L}{\longrightarrow} \Omega^{*} \stackrel{h}{\rightarrow} \mathrm{M},
$$

onde $\Omega=Q \times \Sigma \times Q, f$ e $h$ são o morfismo muito fino e a substituição racional definidos em (2.4.1) e (2.4.2), respectivamente, $L$ é o conjunto local

$$
L=\bigcup_{\substack{i, t \in Q \\ \lambda_{i}=1, \rho_{t}=1}} L_{i t}
$$

e cada $L_{i t}$ é o conjunto local definido em (2.4.4).

Como 1 não pertence a $L, 1 f^{-1}(\cap L) h=\varnothing$. Seja $u$ uma palavra em $\Sigma^{+}$. Então,

$$
u f^{-1}(\cap L) h=\bigcup_{\substack{i, t \in Q \\ \lambda_{i}=1, \rho_{t}=1}}\left(\left(u f^{-1}\right) \cap L_{i t}\right) h \stackrel{(2.4 .7)}{=} \bigcup_{\substack{i, t \in Q \\ \lambda_{i}=1, \rho_{t}=1}} u \mu_{i t}=u|\mathbf{M}| .
$$


Portanto, $\tau=f^{-1}(\cap L) h$.

Se $1 \tau=1$, basta fazer a mesma construção, incluindo $1 \mathrm{em} L$. Como $f$ é muito fino, $1 f^{-1}=1$, e $\operatorname{assim} 1 f^{-1}(\cap L) h=1=1 \tau$.

Exemplo 2.4.1 Seguindo a demonstração do Teorema 2.4.1, a representação matricial $\mathrm{M}_{\mathcal{T}}$ construída para o transdutor do Exemplo 2.3.2 é

$$
\lambda=\left(\begin{array}{lll}
1 & 0 & 0
\end{array}\right) \quad \rho=\left(\begin{array}{l}
0 \\
1 \\
1
\end{array}\right) \quad 1 \mu=\left(\begin{array}{ccc}
1 & 0 & \gamma^{+} \\
0 & 1 & 0 \\
0 & 0 & \gamma^{*}
\end{array}\right) \quad a \mu=\left(\begin{array}{ccc}
0 & \sigma & 0 \\
0 & \sigma & 0 \\
0 & 0 & 0
\end{array}\right) \quad b \mu=\left(\begin{array}{ccc}
0 & \gamma^{*} \xi & \gamma^{*} \xi \gamma^{*} \\
0 & \xi & \xi \gamma^{*} \\
0 & \gamma^{*} \xi & \gamma^{*} \xi \gamma^{*}
\end{array}\right)
$$

O conjunto vazio é representado por 0 . As linhas e colunas são indexadas pelos estados do transdutor.

\subsection{Propriedades de fechamento}

Nesta seção, vamos estudar o fechamento da classe das relações racionais por algumas operações.

Proposição 2.5.1 Sejam $\Sigma$ e $\Gamma$ alfabetos tais que um deles tem pelo menos duas letras. Então, $\operatorname{Rac}\left(\Sigma^{*} \times \Gamma^{*}\right)$ não é fechada por intersecção e nem por complementação.

Demonstração. Considere os alfabetos $\Sigma=\{a, b\}$ e $\Gamma=\{\sigma\}$, e os subconjuntos racionais $A=(a, \sigma \sigma)^{+}(b, \sigma)^{+}$e $B=(a, \sigma)^{+}(b, \sigma \sigma)^{+}$de $\Sigma^{*} \times \Gamma^{*}$. Então, o domínio da intersecção $A \cap B$ é $\left\{a^{n} b^{n}: n \geq 1\right\}$, que não é um conjunto racional. Portanto, $A \cap B$ não é um conjunto racional. $\mathrm{O}$ caso em que $\Gamma$ tem mais de uma letra é análogo.

Como $\operatorname{Rac}\left(\Sigma^{*} \times \Gamma^{*}\right)$ é fechada por união e não é fechada por intersecção, essa classe não pode ser fechada por complementação.

Proposição 2.5.2 Seja $\tau: \mathrm{M} \rightarrow \mathrm{N}$ uma relação racional. Então, a inversa $\tau^{-1}: \mathrm{N} \rightarrow \mathrm{M}$ é uma relação racional.

Demonstração. Seja $\mathcal{T}=(Q, E, I, T)$ um transdutor que realiza $\tau$, e $\mathcal{T}^{-1}=\left(Q, E^{\prime}, I, T\right)$ o transdutor obtido substituindo cada transição

$$
(p, m, n, q) \in E
$$

pela transição

$$
(p, n, m, q)
$$

Então,

$$
(m, n) \in \#|\mathcal{T}| \text { se, e somente se, }(n, m) \in \#\left|\mathcal{T}^{-1}\right| .
$$

Assim, $\left|\mathcal{T}^{-1}\right|=\tau^{-1}$. 
Proposição 2.5.3 Sejam $\Sigma$ e $\Gamma$ alfabetos, e $\tau: \Sigma^{*} \rightarrow \Gamma^{*}$ uma relação racional. Então, a relação $\tau^{\varrho}: \Sigma^{*} \rightarrow \Gamma^{*}$ definida por

$$
\# \tau^{\varrho}=\left\{\left(u^{\varrho}, v^{\varrho}\right):(u, v) \in \# \tau\right\}
$$

é racional.

Demonstração. Sejam $\mathcal{T}=(Q, E, I, T)$ um $\Sigma$ - $\Gamma$-transdutor que realiza $\tau$, e $\mathcal{T} \varrho=\left(Q, E^{\prime}, T, I\right)$ o transdutor obtido substituindo-se cada transição

$$
(p, u, v, q) \in E
$$

pela transição

$$
\left(q, u^{\varrho}, v^{\varrho}, p\right)
$$

Demonstra-se facilmente que existe um passeio bem-sucedido

$$
i \stackrel{(u, v)}{\longrightarrow} t
$$

em $\mathcal{T}$, e somente se, existe um passeio bem-sucedido

$$
t \stackrel{\left(u^{e}, v^{e}\right)}{\longrightarrow} i
$$

em $\mathcal{T}$. Assim,

$$
(u, v) \in \#|\mathcal{T}| \text { se, e somente se, }\left(u^{\varrho}, v^{\varrho}\right) \in \#\left|\mathcal{T}^{\varrho}\right| .
$$

Portanto, $\left|\mathcal{T}^{\varrho}\right|=\tau^{\varrho}$.

Proposição 2.5.4 Sejam $\tau: \mathrm{M} \rightarrow \mathrm{N}$ uma relação racional e $A$ um subconjunto reconhecível de $\mathrm{M}$. Então, a restrição $\tau \mid A$ é uma relação racional.

Demonstração. O Teorema 2.2 .1 fornece uma fatoração $M \stackrel{f^{-1}}{\longrightarrow} \Omega^{*} \stackrel{\cap L}{\longrightarrow} \Omega^{*} \stackrel{g}{\longrightarrow} \mathrm{N}$ de $\tau$, onde $L$ é uma linguagem racional, e $f$ e $g$ são morfismos. Pela Proposição 1.5.3, $A f^{-1}$ é um subconjunto reconhecível de $\Omega^{*}$. Pelo Teorema de Kleene e pela Proposição 1.5.2, $L^{\prime}=L \cap A f^{-1}$ é um conjunto racional. Basta agora observar que

$$
\tau \mid A=\mathrm{M} \stackrel{f^{-1}}{\longrightarrow} \Omega^{*} \stackrel{\cap L^{\prime}}{\longrightarrow} \Omega^{*} \stackrel{f}{\longrightarrow} \mathrm{N} .
$$

Se $M$ for um monóide livre, então segue do Teorema de Kleene que a restrição de $\tau$ a um subconjunto racional de $M$ é uma relação racional.

Proposição 2.5.5 Seja $\Sigma$ um alfabeto com pelo menos duas letras. Então, $\operatorname{Rac}\left(\Sigma^{*} \times \Sigma^{*}\right)$ não é fechada pela operação de fecho reflexivo e transitivo.

Demonstração. Sejam $\sigma$ e $\gamma$ letras distintas de $\Sigma$, e $\tau: \Sigma^{*} \rightarrow \Sigma^{*}$ a relação definida como segue. O domínio de $\tau$ é o conjunto de todas as palavras sobre $\{\sigma, \gamma\}$ que contenham pelo menos uma ocorrência de $\gamma$. Para toda palavra $u$ nesse domínio, seja $x y$ a fatoração de $u$ tal que a letra $\gamma$ não ocorra em $x$, e a primeira letra de $y$ seja $\gamma$. Definimos então

$$
u \tau=x \sigma \gamma y .
$$

Essa relação é o comportamento do transdutor 


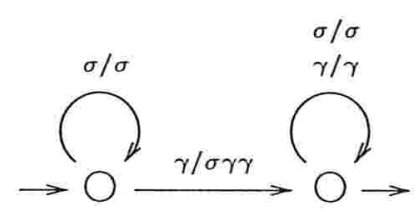

Portanto, \# $\tau$ é um subconjunto racional de $\Sigma^{*} \times \Sigma^{*}$.

Pela definição de $\tau$, a imagem de $\sigma \gamma$ pelo fecho reflexivo e transitivo $\tau^{(\infty)}$ é

$$
(\sigma \gamma) \tau^{(\infty)}=\left\{\sigma^{n} \gamma^{n}: n \geq 1\right\}
$$

Esse conjunto não é racional. Pela Proposição 2.2 .1 , temos então que $\# \tau^{(\infty)}$ não é um subconjunto racional de $\Sigma^{*} \times \Sigma^{*}$.

A composição de duas relações racionais não é em geral uma relação racional, como mostra o exemplo a seguir.

Exemplo 2.5.1 Considere o morfismo inverso definido no Exemplo 2.2.1, e o morfismo $g: \sigma^{*} \rightarrow$ $\Sigma^{*} \times \Sigma^{*}$ definido por

$$
\sigma g=(\sigma, \gamma)
$$

Então,

$$
\operatorname{im}\left(g f^{-1}\right)=\left\{u \in \Sigma^{*}:|u|_{\sigma}=|u|_{\gamma}\right\} .
$$

Como a imagem da composição $g f^{-1}$ não é um conjunto racional, segue da Proposição 2.2.1 que essa relação não é racional.

No entanto, o fechamento se verifica se o monóide intermediário na composição for um monóide livre. Esse resultado foi obtido em 1965 por Elgot e Mezei [EM65], e será demonstrado a seguir. Nossa demonstração segue a de Eilenberg [Eil74].

Começamos com um lema.

Lema 2.5.1 Sejam $A$ e $B$ conjuntos, $f: B \rightarrow A$ uma função, e $X$ um subconjunto de $A$. Então,

$$
A \stackrel{\cap X}{\longrightarrow} A \stackrel{f^{-1}}{\longrightarrow} B=A \stackrel{f^{-1}}{\longrightarrow} B \stackrel{\cap\left(X f^{-1}\right)}{\longrightarrow} B
$$

$e$

$$
B \stackrel{f}{\rightarrow} A \stackrel{\cap X}{\longrightarrow} A=B \stackrel{\cap\left(X f^{-1}\right)}{\longrightarrow} B \stackrel{f}{\rightarrow} A .
$$

Demonstração. Para mostrar (2.5.1), sejam $\tau=(\cap X)\left(f^{-1}\right)$ e $\tau^{\prime}=\left(f^{-1}\right)\left(\cap\left(X f^{-1}\right)\right)$. Vamos mostrar que $x \tau=x \tau^{\prime}$ para todo elemento $x$ em $A$. Vamos considerar dois casos:

- $x \in X$ : então, $x \tau=x f^{-1}$. Pela definição da relação $\cap, x \tau^{\prime}=\left(x f^{-1}\right) \cap\left(X f^{-1}\right)$. Como $x \in X$, o conjunto $\left(x f^{-1}\right) \cap\left(X f^{-1}\right)$ é igual a $x f^{-1}$. Assim, $x \tau=x \tau^{\prime}$.

- $x \notin X$ : então, $x \cap X=\varnothing$, e portanto $x \tau=\varnothing$. Como $f$ é uma função, para todo $y$ em $\left(x f^{-1}\right) \cap\left(X f^{-1}\right), y f=x$ e $y f \in X$. Como $x \notin X,\left(x f^{-1}\right) \cap\left(X f^{-1}\right)=\varnothing$. Portanto, $x \tau^{\prime}=\varnothing$, e obtemos $x \tau=x \tau^{\prime}$. 
Em seguida, vamos mostrar que ambos os lados de (2.5.2) coincidem para todo elemento $x$ em $B$. Vamos considerar dois casos para $x f$ :

- $x f \in X$ : então $x f(\cap X)=x f$, e, como $x$ pertence a $X f^{-1}, x\left(\cap\left(X f^{-1}\right)\right) f=x f$.

- $x f \notin X$ : então $x f(\cap X)=\varnothing$, e, como $x$ não pertence a $X f^{-1}, x\left(\cap\left(X f^{-1}\right)\right) f=\varnothing f=\varnothing$.

Teorema 2.5.1 (Elgot e Mezei 1965) Sejam $\Sigma$ um alfabeto, $\mathrm{M}$ e $\mathrm{N}$ monóides, e $\tau_{1}: \mathrm{M} \rightarrow \Sigma^{*}$ e $\tau_{2}: \Sigma^{*} \rightarrow \mathrm{N}$ relações racionais. Então, a composiçãoo $\tau_{1} \tau_{2}: \mathrm{M} \rightarrow \mathrm{N}$ é uma relação racional.

Demonstração. Aplicando o Teorema 2.2.1 em $\tau_{1}$ e $\tau_{2}$, obtemos uma fatoração de $\tau_{1} \tau_{2}$ da forma

$$
\mathrm{M} \stackrel{f^{-1}}{\longrightarrow} \Gamma^{*} \stackrel{n X}{\longrightarrow} \Gamma^{*} \stackrel{g}{\rightarrow} \Sigma^{*} \stackrel{r^{-1}}{\longrightarrow} \Omega^{*} \stackrel{\cap Y}{\longrightarrow} \Omega^{*} \stackrel{s}{\rightarrow} \mathrm{N},
$$

onde $\Gamma$ e $\Omega$ são alfabetos, $f, g, r$ e $s$ são mofismos de monóides, $g$ e $r$ são morfismos finos, $X$ é um subconjunto racional de $\Gamma^{*}$, e $Y$ é um subconjunto racional de $\Omega^{*}$.

Afirmamos que existe um alfabeto $\Xi$ e morfismos finos $k: \Xi^{*} \rightarrow \Gamma^{*}$ e $l: \Xi^{*} \rightarrow \Omega^{*}$ tais que

$$
\Gamma^{*} \stackrel{g}{\rightarrow} \Sigma^{*} \stackrel{r^{-1}}{\longrightarrow} \Omega^{*}=\Gamma^{*} \stackrel{k^{-1}}{\rightarrow} \Xi^{*} \stackrel{l}{\rightarrow} \Omega^{*} .
$$

Esse alfabeto e esses morfismos serão construídos posteriormente. Assumindo que a afirmação seja verdadeira, vamos completar a demonstração.

Temos então que $\tau_{1} \tau_{2}$ pode ser fatorado como

$$
\mathrm{M} \stackrel{f^{-1}}{\longrightarrow} \Gamma^{*} \stackrel{\cap X}{\longrightarrow} \Gamma^{*} \stackrel{k^{-1}}{\longrightarrow} \Xi^{*} \stackrel{l}{\rightarrow} \Omega^{*} \stackrel{\cap Y}{\longrightarrow} \Omega^{*} \stackrel{s}{\rightarrow} \mathrm{N} .
$$

Pelo Lema 2.5.1, essa fatoração é igual a

$$
\mathrm{M} \stackrel{f^{-1}}{\longrightarrow} \Gamma^{*} \stackrel{k^{-1}}{\longrightarrow} \Xi^{*} \stackrel{\cap\left(X k^{-1}\right)}{\longrightarrow} \Xi^{*} \stackrel{\cap\left(Y l^{-1}\right)}{\longrightarrow} \Xi^{*} \stackrel{l}{\rightarrow} \Omega^{*} \stackrel{s}{\rightarrow} \mathrm{N} .
$$

Sejam $f^{\prime}$ o morfismo $k f, g^{\prime}$ o morfismo $l s$, e $Z$ a linguagem racional $X k^{-1} \cap Y l^{-1}$. Então,

$$
\tau_{1} \tau_{2}=\mathrm{M} \stackrel{f^{\prime-1}}{\longrightarrow} \Xi^{*} \stackrel{\cap Z}{\longrightarrow} \Xi^{*} \stackrel{g^{\prime}}{\longrightarrow} \mathrm{N} .
$$

Pelo Teorema 2.2.1, temos que $\tau_{1} \tau_{2}$ é uma relação racional.

Vamos mostrar (2.5.3).

Sejam $\Xi$ a união dos conjuntos de pares

$A=\{(\gamma, \omega) \in \Gamma \times \Omega: \gamma g=\omega r \neq 1\}, B=\{(\gamma, 1) \in \Gamma \times 1: \gamma g=1\}$ e $C=\{(1, \omega) \in 1 \times \Omega: \omega r=1\}$, e $k: \Xi^{*} \rightarrow \Gamma^{*}, l: \Xi^{*} \rightarrow \Omega^{*}$ os morfismos finos definidos por

$$
(a, b) k=a,(a, b) l=b, \forall(a, b) \in \Xi .
$$

Vamos demonstrar que $g r^{-1}=k^{-1} l$. 
Como $k$ e $r$ são morfismos finos, segue da Proposição 1.3.1 que as inversas $r^{-1}: \Sigma^{*} \rightarrow 2^{\Omega^{*}}$ e $k^{-1}$ : $\Gamma^{*} \rightarrow 2^{\Xi^{*}}$ são morfismos de semigrupos. Então, $g r^{-1}$ é um morfismo de semigrupos. Estendendo $l$ para um morfismo $l: 2^{\Xi^{*}} \rightarrow 2^{\Omega^{*}}$, temos também que $k^{-1} l$ é um morfismo de semigrupos. Então, pela Proposição 1.3.2, basta mostrar que essas funções coincidem em $\Gamma \cup 1$.

Vamos considerar primeiro a palavra vazia. Das definições de $k$ e $l$, segue que

$$
1 k^{-1} l=C^{*} l=\{w \in \Omega: \omega r=1\}^{*}=1 r^{-1}=1 g r^{-1} .
$$

Seja agora $\gamma$ uma letra em $\Gamma$. Vamos considerar dois casos para $\gamma g$ :

- $\gamma g$ é a palavra vazia: então

$$
\gamma k^{-1} l=\left(C^{*}(\gamma, 1) C^{*}\right) l \stackrel{(\gamma, 1) l=1}{=} C^{*} l=\{w \in \Omega: \omega r=1\}^{*}=1 r^{-1}=\gamma g r^{-1} .
$$

- $\gamma g$ é uma letra: então

$$
\begin{aligned}
\gamma k^{-1} l & =\left(C^{*}\{(\gamma, \omega): \omega r=\gamma g\} C^{*}\right) l \\
& =\{w \in \Omega: \omega r=1\}^{*}\{w \in \Omega: w r=\gamma g\}\{w \in \Omega: \omega r=1\}^{*}=\gamma g r^{-1} .
\end{aligned}
$$




\section{RELAÇÕES RACIONAIS FINITAMENTE VALORADAS}

Neste capítulo, estudamos a classe das relações cuja imagem de toda entrada é um conjunto finito. Essa restrição traz algumas conseqüências bastante interessantes sobre problemas de decisão.

O resultado principal deste capítulo é uma decomposição de relações $k$-valoradas em $k$ funções racionais.

Ao longo deste capítulo, $\Sigma$ e $\Gamma$ representam alfabetos, e $\mathrm{M}$ e $\mathrm{N}$ monóides.

\subsection{Definições e propriedades básicas}

Definição 3.1.1 Dizemos que uma relação $\tau: \mathrm{M} \rightarrow \mathrm{N}$ é finitamente valorada se

$$
\operatorname{card}(m \tau)<\infty, \quad \forall m \in \mathrm{M} \text {. }
$$

Dizemos que $\tau$ é $k$-valorada, onde $k$ é um inteiro positivo, se

$$
\operatorname{card}(m \tau) \leq k, \quad \forall m \in \mathrm{M}
$$

A classe das relações racionais $k$-valoradas está contida propriamente na classe das relações racionais finitamente valoradas. Um exemplo de relação racional que é finitamente valorada, mas não é $k$-valorada para nenhum $k$, é a relação fat : $\Sigma^{*} \rightarrow \Sigma^{*}$, que associa a toda entrada o conjunto dos seus fatores (Exemplo 2.3.1).

Uma relação racional 1-valorada é também denominada função racional. O Capítulo 4 é dedicado ao estudo de propriedades dessas funções.

Exemplo 3.1.1 Seja $\Sigma=\{\sigma\}$. Vamos identificar o monóide aditivo $\mathbb{N}$ com $\Sigma^{*}$.

O gráfico da relação $r: \mathbb{N} \rightarrow \mathbb{N}$ definida por

$$
n r=[n], \quad \forall n \in \mathbb{N}
$$

é

$$
(\sigma, \sigma)(\sigma \times\{1, \sigma\})^{*}
$$


Portanto, $\tau$ é uma relação racional finitamente valorada.

Exemplo 3.1.2 A relação $\tau_{\Sigma}: \Sigma^{*} \rightarrow \Sigma^{*}$ definida por

$$
u \tau_{\Sigma}=\left\{\sigma^{|u|_{\sigma}}: \sigma \in \Sigma \text { e } \sigma \text { ocorre em } u\right\}, \quad \forall u \in \Sigma^{*},
$$

é $k$-valorada, para $k=\operatorname{card}(\Sigma)$.

Essa relação é o comportamento do $\Sigma$ - $\Sigma$-transdutor $\mathcal{T}_{\Sigma}$ definido como segue. O conjunto de estados de $\mathcal{T}_{\Sigma}$ é $i \cup \Sigma \cup \Sigma^{\prime}$, onde $\Sigma^{\prime}$ é uma cópia de $\Sigma$, e $i$ é um elemento que não pertence a $\Sigma \cup \Sigma^{\prime}$. $\mathrm{O}$ único estado inicial é $i$, e o conjunto de estados finais de $\mathcal{T}_{\Sigma}$ é $\Sigma$. Para cada letra $\sigma$ em $\Sigma, \mathcal{T}_{\Sigma}$ tem as transições

$$
i \stackrel{\sigma / \sigma}{\longrightarrow} \sigma, \quad \sigma \stackrel{\sigma / \sigma}{\longrightarrow} \sigma \quad \text { e } \quad \sigma^{\prime} \stackrel{\sigma / \sigma}{\longrightarrow} \sigma .
$$

Para cada par de letras distintas $\sigma$ e $\gamma$ em $\Sigma, \mathcal{T}_{\Sigma}$ tem as transições

$$
i \stackrel{\sigma / 1}{\longrightarrow} \gamma^{\prime}, \quad \gamma^{\prime} \stackrel{\sigma / 1}{\longrightarrow} \gamma^{\prime} \quad \text { e } \quad \gamma \stackrel{\sigma / 1}{\longrightarrow} \gamma
$$

Portanto, $\tau_{\Sigma}$ é uma relação racional $k$-valorada.

Exemplo 3.1.3 Sejam $\Sigma=\{\sigma\}$ e $\Gamma=\{\sigma, \gamma\}$. A relação 2-valorada $\tau: \Sigma^{*} \rightarrow \Gamma^{*}$ definida por

$$
\sigma^{n} \tau=\left\{\sigma^{n}, \gamma^{n}\right\}, \quad \forall n \geq 0,
$$

é claramente a união de dois morfismos muito finos. Portanto, essa relação é racional.

Se $\tau$ for vista como uma função $\Sigma^{*} \rightarrow 2^{\Gamma^{*}}$, então $\tau$ não é uma função racional. De fato, para todo inteiro $n>1$, o conjunto $\left\{\sigma^{n}, \gamma^{n}\right\}$ não admite uma fatoração

tal que

$$
A, B \subseteq \Gamma^{*}, \quad A \neq\{1\} \quad \text { e } \quad B \neq\{1\} .
$$

Assim, im $\tau$ não pertence a nenhum submonóide finitamente gerado de $2^{\Gamma^{*}}$. Pela Proposição 1.5.8, essa imagem não é um conjunto racional. Da Proposição 2.2.1, segue então que $\tau$ não é uma função racional.

A seguir, vamos discutir algumas propriedades de fechamento para as relações racionais finitamente valoradas e para as $k$-valoradas.

Proposição 3.1.1 Sejam $\tau: \mathrm{M} \rightarrow \Sigma^{*}$ e $v: \Sigma^{*} \rightarrow \mathrm{N}$ relações racionais finitamente valoradas. Então, a composição $\tau v: \mathrm{M} \rightarrow \mathrm{N}$ é uma relação racional finitamente valorada.

Demonstração. Pelo Teorema 2.5.1, $\tau v$ é uma relação racional. Como $\tau$ e $v$ são finitamente valoradas

$$
\operatorname{card}(m(\tau v))=\operatorname{card}((m \tau) v)<\infty, \quad \forall m \in \mathrm{M} .
$$


Portanto, $\tau v$ é uma relação racional finitamente valorada.

A hipótese de que o monóide intermediário nessa composição é um monóide livre é fundamental. Por exemplo, as relações racionais $g$ e $f^{-1}$ no Exemplo 2.5.1 são finitamente valoradas, mas a composição $g f^{-1}$ não é uma relação racional.

Proposição 3.1.2 Sejam $\tau: \mathrm{M} \rightarrow \Sigma^{*}$ uma relação racional $k$-valorada e $v: \Sigma^{*} \rightarrow \mathrm{N}$ uma relação racional l-valorada. Então, a composição $\tau v: \mathrm{M} \rightarrow \mathrm{N}$ é uma relação racional kl-valorada.

Demonstração. Pelo Teorema 2.5.1, $\tau v$ é uma relação racional. Observando que

$$
\operatorname{card}(m(\tau v)) \leq k l, \quad \forall m \in \mathrm{M},
$$

concluímos que $\tau v$ é uma relação racional $k l$-valorada.

Novamente, a hipótese de que o monóide intermediário na composição é um monóide livre é fundamental. Para construir um contra-exemplo, vamos utilizar as funções definidas nos exemplos 2.2.1 e 2.5.1. Pelo Teorema 2.5.1, a composição

$$
h=\left(\Sigma^{*} \times \Sigma^{*}\right) \stackrel{f^{-1}}{\longrightarrow} \Sigma^{*} \stackrel{\cap\left(\sigma^{*} \gamma^{*}\right)}{\longrightarrow} \Sigma^{*}
$$

é uma relação racional. Ademais, $h$ é uma função, porque

$$
\operatorname{dom}\left(f^{-1}\right)=\sigma^{*} \times \gamma^{*} \quad \text { e } \quad(s, t) h=\sigma^{|s|} \gamma^{|t|}, \quad \forall(s, t) \in \sigma^{*} \times \gamma^{*} .
$$

No entanto, como

$$
\operatorname{im}(g h)=\left\{\sigma^{n} \gamma^{n}: n \geq 0\right\}
$$

segue da Proposição 2.2.1 que a composição $g h$ não é uma relação racional.

A proposição a seguir é uma conseqüência direta do fechamento da classe das relações racionais pela união.

Proposição 3.1.3 Sejam $\tau: \mathrm{M} \rightarrow \mathrm{N}$ e $v: \mathrm{M} \rightarrow \mathrm{N}$ relações racionais finitamente valoradas. Então, $\tau \cup v$ é uma relação racional finitamente valorada.

Sejam $\tau: \mathrm{M} \rightarrow \mathrm{N}$ uma relação racional $k$-valorada, e $v: \mathrm{M} \rightarrow \mathrm{N}$ uma relação racional $l$ valorada. Então, $\tau \cup v$ é uma relação racional $(k+l)$-valorada.

$\mathrm{O}$ exemplo a seguir mostra que a classe das funções racionais $\mathrm{M} \rightarrow \mathrm{N}$, onde $\mathrm{M}$ e $\mathrm{N}$ são monóides arbitrários, não é fechada pela operação de produto. O exemplo também mostra que a classe das relações racionais finitamente valoradas não é fechada por essa operação.

Exemplo 3.1.4 Considere a função racional $A=(1,1)^{*} \cup(-1,0)^{*}$ sobre o monóide $\mathbb{Z}$ com a operação de adição. O produto $A^{2}$ não é uma relação finitamente valorada, porque, para todo inteiro $n$ positivo,

$$
n A^{2}=\{k: k \geq n\}
$$

Mas, quando o monóide de entrada é um monóide livre, temos as seguintes propriedades de fechamento. 
Proposição 3.1.4 Sejam $\tau: \Sigma^{*} \rightarrow \mathrm{M}$ e $v: \Sigma^{*} \rightarrow \mathrm{M}$ relações racionais finitamente valoradas. Então

- o produto $\tau \cdot v$ é uma relação racional finitamente valorada;

- se $1 \tau=\varnothing$ ou $1 \tau=1$, então $\tau^{+}$é uma relação racional finitamente valorada.

Demonstração. Vamos demonstrar o fechamento pelo produto. Pela definição do produto de relações, para todo inteiro positivo $k$,

$$
u(\tau \cdot v)=\bigcup_{x y=u}(x \tau)(y v), \quad \forall u \in \Sigma^{*}
$$

Como o número de fatorações de $u$ é finito, e os conjuntos $x \tau$ e $y v$ são finitos, segue que $\operatorname{card}(u(\tau \cdot v))<\infty$. Portanto, $\tau v$ é uma relação finitamente valorada.

Suponha agora que $1 \tau=\varnothing$ ou $1 \tau=1$. Pela definição do produto de relações,

$$
u \tau^{k}=\bigcup_{x_{1} \ldots x_{k}=u}\left(x_{1} \tau\right) \ldots\left(x_{k} \tau\right), \quad \forall u \in \Sigma^{*} .
$$

No máximo $|u|$ fatores na fatoração $x_{1} \ldots x_{k}$ podem ser diferentes da palavra vazia. Assim, para todo $k>|u|$, se $1 \tau=\varnothing$, então

$$
u \tau^{k}=\varnothing,
$$

$\mathrm{e}$, se $1 \tau=1$,

$$
u \tau^{k}=\bigcup_{1 \leq l \leq|u|} u \tau^{l}
$$

Portanto,

$$
u \tau^{+}=\bigcup_{1 \leq l \leq|u|} u \tau^{l}
$$

Do fechamento pelo produto, segue que $\tau^{l}$ é uma relação finitamente valorada, para todo $l \geq 2$. Portanto, $\tau^{+}$é uma relação finitamente valorada.

O exemplo a seguir mostra que o produto e a estrela de relações racionais $k$-valoradas não são, em geral, $j$-valoradas algum inteiro $j \geq 0$, mesmo com a restrição de a imagem da palavra vazia ser o conjunto vazio.

Exemplo 3.1.5 Considere os alfabetos $\Sigma=\{\sigma\}$ e $\Gamma=\{a, b\}$, e a função $\tau: \Sigma^{+} \rightarrow \Gamma^{+}$definida por

$$
\sigma^{n} \tau= \begin{cases}a^{n} & \text { se } n \text { for par; } \\ b^{n} & \text { se } n \text { for ímpar. }\end{cases}
$$

Essa função é racional, porque seu gráfico é

$$
\left(\sigma^{2}, a^{2}\right)^{+} \cup(\sigma, b)\left(\sigma^{2}, b^{2}\right)^{*}
$$


Seja $n>1$ um inteiro ímpar. Então, em toda fatoração $x y$ de $\sigma^{n}$, os comprimentos $|x|$ e $|y|$ têm paridades distintas. Portanto,

$$
\sigma^{n} \tau^{2}=\left\{a^{i} b^{j}, b^{j} a^{i}: i \text { é par e positivo, } j \text { é ímpar, e } i+j=n\right\} .
$$

Dessa forma, para todo inteiro ímpar $n>1$,

$$
\operatorname{card}\left(\sigma^{n} \tau^{2}\right)=n-1 .
$$

Portanto, $\tau^{2}$ não é $k$-valorada para nenhum $k$.

Concluímos a seção como uma versão da Proposição 1.5.8 para relações racionais finitamente valoradas palavra-palavra. Nesse resultado, vamos denotar uma relação racional e seu gráfico por uma mesma letra, por comodidade.

Proposição 3.1.5 Seja $r \subseteq \Sigma^{*} \times \Gamma^{*}$ uma relação racional finitamente valorada. Então, existe um subconjunto finito $\mathrm{X}$ de $\Sigma \times \Gamma^{*}$ tal que

$$
r \mid \Sigma^{+} \in \operatorname{Rac}\left(\mathrm{X}^{*}\right)
$$

Demonstração. Vamos usar indução no número de operadores de uma expressão racional $e$ que representa $r$. Vamos denotar esse número por $n(e)$ :

- $n(e)=0$ : então $r=\varnothing$ ou $r$ é um conjunto unitário. Em ambos os casos, temos trivialmente (3.1.1).

- $n(e)>0$ : vamos considerar três casos:

- $e=e_{1} \cup e_{2}$ : sejam $r_{1}$ e $r_{2}$ os conjuntos representados por $e_{1}$ e $e_{2}$, respectivamente. Como $r$ é uma relação racional finitamente valorada, $r_{1}$ e $r_{2}$ devem ser relações racionais finitamente valoradas. Como $n\left(e_{1}\right)<n(e)$ e $n\left(e_{2}\right)<n(e)$, segue da hipótese de indução que existem subconjuntos finitos $\mathrm{Y}$ e $\mathrm{Z}$ de $\Sigma \times \Gamma^{*}$ tais que

$$
r_{1} \mid \Sigma^{+} \in \operatorname{Rac}\left(\mathrm{Y}^{*}\right) \quad \text { e } \quad r_{2} \mid \Sigma^{+} \in \operatorname{Rac}\left(\mathrm{Z}^{*}\right) .
$$

Observe que

$$
r\left|\Sigma^{+}=r_{1}\right| \Sigma^{+} \cup r_{2} \mid \Sigma^{+}
$$

Seja $\mathrm{X}=\mathrm{Y} \cup \mathrm{Z}$. Então, $\mathrm{X}$ é um subconjunto finito de $\Sigma \times \Gamma^{*}$. Ademais, segue da Proposição 1.5.6 que

$$
r \mid \Sigma^{+} \in \operatorname{Rac}\left(\mathrm{X}^{*}\right) .
$$

- $e=e_{1} e_{2}$ : sejam $r_{1}$ e $r_{2}$ os conjuntos representados por $e_{1}$ e $e_{2}$, respectivamente. Basta considerar o caso em que $r_{1} \neq \varnothing$ e $r_{2} \neq \varnothing$. Como $r$ é uma relação racional finitamente valorada, $r_{1}$ e $r_{2}$ devem ser relações racionais finitamente valoradas. Como $n\left(e_{1}\right)<n(e)$ e $n\left(e_{2}\right)<n(e)$, segue da hipótese de indução que existem subconjuntos finitos $\mathrm{Y}$ e Z de $\Sigma \times \Gamma^{*}$ tais que

$$
r_{1} \mid \Sigma^{+} \in \operatorname{Rac}\left(\mathrm{Y}^{*}\right) \quad \text { e } r_{2} \mid \Sigma^{+} \in \operatorname{Rac}\left(\mathrm{Z}^{*}\right) .
$$


Observe que

$$
r \mid \Sigma^{+}=\left(r_{1} \mid \Sigma^{+}\right)\left(r_{2} \mid \Sigma^{+}\right) \cup\left(r_{1} \mid \Sigma^{+}\right)\left(r_{2} \mid 1\right) \cup\left(r_{1} \mid 1\right)\left(r_{2} \mid \Sigma^{+}\right) .
$$

Para cada elemento $x$ em $\left(r_{1} \mid 1\right)$ e cada elemento $y$ em $\left(r_{2} \mid 1\right)$, segue da Proposição 1.5.7 que

$$
\left(r_{1} \mid \Sigma^{+}\right) y \in \operatorname{Rac}\left((\mathrm{Y} \cup \mathrm{Yy})^{*}\right) \quad \text { e } \quad x\left(r_{2} \mid \Sigma^{+}\right) \in \operatorname{Rac}\left((\mathrm{Z} \cup \mathrm{xZ})^{*}\right) .
$$

Como $r_{1}$ e $r_{2}$ são relações finitamente valoradas, $\left(r_{1} \mid 1\right)$ e $\left(r_{2} \mid 1\right)$ devem ser conjuntos finitos. Utilizando a Proposição 1.5.6 para a união dos conjuntos da forma $\left(r_{1} \mid \Sigma^{+}\right) y$, e novamente para a união dos conjuntos da forma $x\left(r_{2} \mid \Sigma^{+}\right)$, obtemos

$$
\left(r_{1} \mid \Sigma^{+}\right)\left(r_{2} \mid 1\right) \in \operatorname{Rac}\left(\left(\mathrm{Y} \cup \mathrm{Y}\left(\mathrm{r}_{2} \mid 1\right)\right)^{*}\right) \quad \text { e } \quad\left(r_{1} \mid 1\right)\left(r_{2} \mid \Sigma^{+}\right) \in \operatorname{Rac}\left(\left(\mathrm{Z} \cup\left(\mathrm{r}_{1} \mid 1\right) \mathrm{Z}\right)^{*}\right) .
$$

Ademais, pela Proposição 1.5.6,

$$
\left(r_{1} \mid \Sigma^{+}\right)\left(r_{2} \mid \Sigma^{+}\right) \in \operatorname{Rac}\left((\mathrm{Y} \cup \mathrm{Z})^{*}\right) .
$$

Seja

$$
X^{\prime}=Y \cup Z \cup\left(Y \cup Y\left(r_{2} \mid 1\right)\right) \cup\left(Z \cup\left(r_{1} \mid 1\right) Z\right) .
$$

Pela Proposição 1.5.6,

$$
r \mid \Sigma^{+} \in \operatorname{Rac}\left(\mathrm{X}^{\prime *}\right) .
$$

Resta observar que $X^{\prime}$ é um subconjunto finito de $\Sigma \times \Gamma^{*}$.

$-e=\left(e^{\prime}\right)^{+}$: seja $r^{\prime}$ o conjunto representado por $e^{\prime}$. Como $n\left(e^{\prime}\right)<n(e)$, segue da hipótese de indução que existe um subconjunto finito $\mathrm{X}^{\prime}$ de $\Sigma \times \Gamma^{*}$ tal que

$$
r^{\prime} \mid \Sigma^{+} \in \operatorname{Rac}\left(\mathrm{X}^{*}\right) \text {. }
$$

Como $r$ é uma relação finitamente valorada, $1 r^{\prime}=\varnothing$ ou $1 r^{\prime}=1$ necessariamente. Portanto,

$$
r^{\prime} \in \operatorname{Rac}\left(\mathrm{X}^{\prime *}\right) .
$$

Dessa forma, $r=\left(r^{\prime}\right)^{+} \in \operatorname{Rac}\left(\mathrm{X}^{*}\right)$.

Corolário 3.1.1 Seja $\tau: \Sigma^{*} \rightarrow \Gamma^{*}$ uma relação racional tal que $1 \tau=\varnothing$ ou $1 \tau=1$. Então, $\tau$ é finitamente valorada se, e somente se, existe um subconjunto finito $X$ de $\Sigma \times \Gamma^{*}$ tal que $\# \tau \in \operatorname{Rac}\left(\mathrm{X}^{*}\right)$.

Demonstração. Suponha que exista um subconjunto finito $X$ de $\Sigma \times \Gamma^{*}$ tal que $\# \tau \in \operatorname{Rac}\left(\mathrm{X}^{*}\right)$. Observe que $X$ é uma relação racional finitamente valorada, cuja imagem da palavra vazia é $\varnothing$. Então, pela Proposição 3.1.4, $X^{*}$ é uma relação racional finitamente valorada. De $\# \tau \subseteq X^{*}$, segue que $\tau$ é uma relação finitamente valorada.

Suponha que $\tau$ seja uma relação finitamente valorada tal que $1 \tau=\varnothing$ ou $1 \tau=1$. Pela Proposição 3.1.5, existe um subconjunto finito $X$ de $\Sigma \times \Gamma^{*}$ tal que $\#\left(\tau \mid \Sigma^{+}\right) \in \operatorname{Rac}\left(\mathrm{X}^{*}\right)$. Como $1 \tau=\varnothing$ ou $1 \tau=1$, temos que $\# \tau \in \operatorname{Rac}\left(\mathrm{X}^{*}\right)$. 


\subsection{Transdutores letra-saída e representações matriciais}

Nesta seção, vamos estudar classes especiais de representações matriciais e transdutores que realizam as relações racionais finitamente valoradas palavra-palavra, e as $k$-valoradas palavra-palavra.

Proposição 3.2.1 Seja $\mathrm{M}=(Q, \lambda, \mu, \rho)$ uma representação matricial bi-acessível sobre alfabetos $\Sigma$ e $\Gamma$. Então, a relaçấo racional $|\mathrm{M}|$ é finitamente valorada se, e somente se,

$$
\operatorname{card}\left(u \mu_{p q}\right)<\infty, \quad \forall u \in \Sigma^{*}, \forall p, q \in Q \text {. }
$$

Demonstração. Suponha que $|\mathbf{M}|$ seja uma relação finitamente valorada.

Sejam $p$ e $q$ estados quaisquer, e $u$ uma palavra em $\Sigma^{*}$. Como $\mathbf{M}$ é bi-acessível, existem palavras $v$ e $w$, um estado inicial $i$, e um estado final $t$, tais que $v \mu_{i p} \neq \varnothing$ e $w \mu_{q t} \neq \varnothing$. De $(v u w) \mu=(v \mu)(u \mu)(w \mu)$, segue que

$$
\left(v \mu_{i p}\right)\left(u \mu_{p q}\right)\left(w \mu_{q t}\right) \subseteq(v u w) \mu_{i t} .
$$

Como $\lambda_{i}=1$ e $\rho_{t}=1$,

$$
(\text { vuw }) \mu_{i t} \subseteq \lambda((v u w) \mu) \rho=(\text { vuw })|\mathbf{M}| .
$$

Como $|\mathbf{M}|$ é finitamente valorada, card $\left((v u w) \mu_{i t}\right)<\infty$. Portanto, card $\left(u \mu_{p q}\right)<\infty$, necessariamente.

Suponha agora que M satisfaça (3.2.1). Então, para toda palavra $u$ em $\Sigma^{*}, \operatorname{card}(\lambda(u \mu) \rho)<\infty$. Portanto, $|\mathbf{M}|$ é uma relação finitamente valorada.

Corolário 3.2.1 Seja $\mathrm{M}=(Q, \lambda, \mu, \rho)$ uma representação matricial bi-acessivel sobre alfabetos $\Sigma$ e $\Gamma$. Então, a relação racional $|\mathrm{M}|$ é finitamente valorada se, e somente se,

$$
\operatorname{card}\left(u \mu_{p q}\right)<\infty, \quad \forall u \in \Sigma \cup 1, \forall p, q \in Q \text {. }
$$

Demonstração. Se $|\mathbf{M}|$ for finitamente valorada, então a Proposição 3.2.1 implica em (3.2.2).

Para provar a recíproca, seja

$$
w=\sigma_{1} \ldots \sigma_{n}
$$

uma palavra em $\Sigma^{+}$, onde cada $\sigma_{i}$ é uma letra. Como $w \mu=\left(\sigma_{1} \mu\right) \ldots\left(\sigma_{n} \mu\right)$, a hipótese de que as entradas das matrizes $\sigma_{i} \mu$ são finitas, para toda letra $\sigma_{i}$, implica em

$$
\operatorname{card}\left(w \mu_{p q}\right) \leq \infty
$$

Pela Proposição 3.2.1, $|\mathbf{M}|$ é uma relação racional finitamente valorada.

Um estudo semelhante pode ser feito para as relações $k$-valoradas. 
Proposição 3.2.2 Sejam $\mathbf{M}=(Q, \lambda, \mu, \rho)$ uma representação matricial bi-acessivel e normalizada sobre alfabetos $\Sigma$ e $\Gamma$, e $k$ um inteiro positivo. Então, a relação racional $|\mathbf{M}|$ é $k$-valorada se, e somente se,

$$
\operatorname{card}\left(u \mu_{p q}\right) \leq k, \quad \forall u \in \Sigma^{*}, \forall p, q \in Q
$$

Demonstração. Suponha que $|\mathrm{M}|$ seja uma relação $k$-valorada para algum inteiro positivo $k$.

Sejam $p$ e $q$ estados quaisquer, e $u$ uma palavra em $\Sigma^{*}$. Como $\mathbf{M}$ é bi-acessível, existem palavras $v$ e $w$ tais que $v \mu_{i p} \neq \varnothing$ e $w \mu_{q t} \neq \varnothing$, onde $i$ é o estado inicial de $\mathbf{M}$, e $t$ o final. De $(v u w) \mu=(v \mu)(u \mu)(w \mu)$, segue que

$$
\left(v \mu_{i p}\right)\left(u \mu_{p q}\right)\left(w \mu_{q t}\right) \subseteq(v u w) \mu_{i t}=\lambda_{i}\left((v u w) \mu_{i t}\right) \rho_{t}=(v u w)|\mathbf{M}| .
$$

Como $|\mathbf{M}|$ é $k$-valorada, concluímos que card $\left(u \mu_{p q}\right) \leq k$.

Suponha agora que $\mathbf{M}$ satisfaça (3.2.3). Então, para toda palavra $u$ em $\Sigma^{*}$, $\operatorname{card}(\lambda(u \mu) \rho)=$ $\operatorname{card}\left(\lambda_{i}\left(u \mu_{i t}\right) \rho_{t}\right) \leq k$. Portanto, $|\mathbf{M}|$ é uma relação $k$-valorada.

Definição 3.2.1 Dizemos que um $\Sigma$-M-transdutor é letra-saída se a entrada de toda transição for uma letra. Se M for um monóide livre, dizemos que o transdutor é letra-palavra.

O transdutor definido no Exemplo 3.1.2 é letra-palavra.

Dizemos que um transdutor letra-saída é funcional se o seu comportamento for uma função racional.

As conexões entre as relações racionais palavra-palavra finitamente valoradas e os transdutores letra-palavra estão estabelecidas a seguir.

Teorema 3.2.1 Seja $\tau: \Sigma^{*} \rightarrow \Gamma^{*}$ uma relação palavra-palavra. As seguintes condições são equivalentes:

i. $\tau$ é uma relação racional finitamente valorada $e 1 \tau=\varnothing$ ou $1 \tau=1$;

ii. $\tau$ é o comportamento de um transdutor letra-palavra;

iii. $\tau$ pode ser fatorada como $\Sigma^{*} \stackrel{f^{-1}}{\longrightarrow} \Omega^{*} \stackrel{\cap L}{\longrightarrow} \Omega^{*} \stackrel{g}{\rightarrow} \Gamma^{*}$, onde $\Omega$ é um alfabeto, $f$ é um morfismo muito fino, $g$ é um morfismo, e $L$ é uma linguagem local sobre $\Omega$.

\section{Demonstração.}

- $i \Rightarrow i i$ : pelo Corolario 3.1.1, existe um subconjunto finito $X$ de $\Sigma \times \Gamma^{*}$ tal que \# $\tau \in \operatorname{Rac}\left(\mathrm{X}^{*}\right)$. Sejam $\Omega$ o conjunto $X, \Omega^{*}$ o monóide livre gerado por esse alfabeto, e $f: \Omega^{*} \rightarrow \Sigma^{*}$ e $g: \Omega^{*} \rightarrow \Gamma^{*}$ os morfismos definidos por

$$
(\sigma, u) f=\sigma \quad \text { e } \quad(\sigma, u) g=u, \quad \forall(\sigma, u) \in \Omega .
$$

Assim, o bimorfismo $(f, g): \Omega^{*} \rightarrow \Sigma^{*} \times \Gamma^{*}$ associa cada letra $(\sigma, u)$ de $\Omega$ ao par de $X$ correspondente. Portanto, $(f, g)$ é um bimorfismo sobrejetor de $\Omega^{*}$ no submonóide $X^{*}$ de $\Sigma^{*} \times \Gamma^{*}$. 
Pela Proposição 1.5.5, existe um subconjunto racional $A$ de $\Omega^{*}$ tal que $A(f, g)=\# \tau$. Seja $\mathcal{A}$ um $\Omega$-autômato que reconhece $A$, e $\mathcal{T}$ o $\Sigma$ - $\Gamma$-transdutor obtido de $\mathcal{A}$ com a aplicação da substituição descrita em (2.3.2). Então, $\mathcal{T}$ é um transdutor letra-palavra que realiza $\tau$.

- $i i \Rightarrow i i i$ : seja $\mathcal{T}$ um transdutor letra-palavra que realiza $\tau,(\alpha, \beta)$ o bimorfismo de $\mathcal{T}$, e $L$ a linguagem local de todos os passeios bem-sucedidos de $\mathcal{T}$. Então, $\alpha$ é um morfismo muito fino, e de (2.3.1) segue que $\tau=\alpha^{-1}(\cap L) \beta$.

- $i i i \Rightarrow i$ : segue da observação de que, como $f$ é um morfismo muito fino, $1 f^{-1}=1$ e card $\left(u f^{-1}\right)<\infty$, para toda palavra $u$ em $\Sigma^{+}$.

A proposição a seguir mostra a construção da representação matricial de um transdutor letrapalavra, e também a construção de um transdutor letra-palavra a partir de uma representação matricial de um relação racional palavra-palavra finitamente valorada. Essa demonstração mostra que os transdutores letra-palavra e suas representações matriciais são facilmente intercambiáveis.

Proposição 3.2.3 Seja $\tau: \Sigma^{*} \rightarrow \Gamma^{*}$ uma relação palavra-palavra tal que $1 \tau=1$ ou $1 \tau=\varnothing$. As duas condiçôes a seguir são equivalentes:

i. $\tau$ é uma relaçẫo racional finitamente valorada;

ii. $\tau$ é o comportamento de uma representação matricial $\mathbf{M}=(Q, \lambda, \mu, \rho)$ sobre $\Sigma$ e $\Gamma$ tal que $\lambda$ e $\rho$ são vetores com entradas iguais a $\varnothing$ ou 1 , e $\mu$ é um morfismo de monóides que satisfaz

$$
\operatorname{card}\left(u \mu_{p q}\right)<\infty, \quad \forall u \in \Sigma^{*}, \forall p, q \in Q .
$$

\section{Demonstração.}

- $i \Rightarrow i$ : Pelo Teorema 3.2.1, existe um transdutor letra-palavra $\mathcal{T}$ que realiza $\tau$. Vamos mostrar uma construção efetiva da representação matricial de $\mathcal{T}$, e mostrar que essa representação matricial satisfaz as condições desejadas.

Seja $\mathbf{M}=(Q, \lambda, \mu, \rho)$ a representação matricial definida como segue. Os vetores $\lambda$ e $\rho$ são definidos como em (2.4.9). O morfismo $\mu$ é o morfismo de monóides definido por:

$$
\sigma \mu_{p q}=\{x: \exists p \stackrel{\sigma / x}{\longrightarrow} q \in E\}, \quad \forall p, q \in Q, \forall \sigma \in \Sigma .
$$

Como o número de transições de $\mathcal{T}$ é finito, temos que as entradas das matrizes $\sigma \mu$, para toda letra $\sigma$ de $\Sigma$, são finitas. Portanto, para toda palavra $u$, as entradas da matriz $u \mu$ são finitas.

Vamos provar que $\mathbf{M}$ é a representação matricial de $\mathcal{T}$. Pela definição dos vetores $\lambda$ e $\rho$, resta demonstrar que $\mu$ é o morfismo construído na demonstração do Teorema 2.4.1.

Seja $\nu$ o morfismo de monóides definido em (2.4.10). Sejam $\sigma$ uma letra em $\Sigma$, e $p$ e $q$ estados de $\mathcal{T}$. Segue da hipótese de que esse transdutor é letra-palavra que o conjunto dos passeios de $p$ a $q$ com entrada $\sigma$ é o conjunto das transições de $p$ a $q$ com entrada $\sigma$. Portanto, $\mu$ e $\nu$ coincidem em $\Sigma$. Como $\mu$ e $\nu$ são morfismos de monóides, também coincidem em 1 . Da Proposição 1.3 .2 , segue que $\mu=\nu$. 
Como $\mathcal{T}$ não tem transições com entrada 1 , segue que a matriz da palavra vazia da representação matricial de $\mathcal{T}$, definida em (2.4.11), é igual a $1 \mu$ (a matriz identidade). Portanto, $\mu$ é o morfismo da representação matricial de $\mathcal{T}$.

- $i i \Rightarrow i$ : Seja $\mathbf{M}=(Q, \lambda, \mu, \rho)$ uma representação matricial que satisfaz as hipóteses em ii. Vamos mostrar uma construção efetiva de um transdutor letra-palavra com o mesmo comportamento de $\mathbf{M}$.

Seja $\mathcal{T}=(Q, E, I, T)$ o $\Sigma$ - $\Gamma$-transdutor definido por

- $I$ é o conjunto das coordenadas não-nulas de $\lambda$, e $T$ é o conjunto das coordenadas nãonulas de $\rho$;

- $E$ contém uma transição $p \stackrel{\sigma / x}{\longrightarrow} q$ para cada par de estados $p$ e $q$, cada letra $\sigma$, e cada palavra $x$ em $\sigma \mu_{p q}$.

Pelas hipóteses sobre $\mathbf{M}$, temos que $E$ é finito, e por construção, $\mathcal{T}$ é um transdutor letrapalavra. Basta agora observar que $\mathbf{M}$ é a representação matricial de $\mathcal{T}$ para concluir que $\tau=|\mathcal{T}|$.

Exemplo 3.2.1 Considere o Exemplo 2.3.2. Altere o morfismo $f$ para

$$
\sigma f=a, \quad \gamma f=a \quad \text { e } \quad \xi f=b,
$$

e defina $\tau$ como a relação $f^{-1}(\cap X)$.

Como $f$ é um morfismo muito fino, $\tau$ é uma relação racional finitamente valorada. Como $1 \tau=\varnothing$, essa relação pode ser realizada por um transdutor letra-palavra.

Repetindo a construção feita no Exemplo 2.3.2, obtemos o transdutor letra-palavra que realiza $\tau$ ilustrado a seguir:

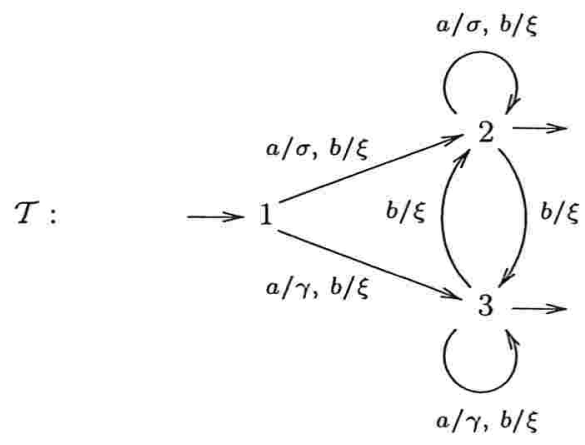

A representação matricial desse transdutor está definida a seguir. O conjunto vazio é representado por 0 , e as linhas e colunas são indexadas pelos números dos estados do transdutor.

$$
\lambda=\left(\begin{array}{lll}
1 & 0 & 0
\end{array}\right), \quad \rho=\left(\begin{array}{l}
0 \\
1 \\
1
\end{array}\right), \quad a \mu=\left(\begin{array}{lll}
0 & \sigma & \gamma \\
0 & \sigma & 0 \\
0 & 0 & \gamma
\end{array}\right), \quad b \mu=\left(\begin{array}{lll}
0 & \xi & \xi \\
0 & \xi & \xi \\
0 & \xi & \xi
\end{array}\right) .
$$


A matriz da palavra vazia é a matriz identidade (ou seja, $\mu$ é um morfismo de monóides).

Concluímos esta seção com um procedimento de normalização para transdutores letra-palavra.

Proposição 3.2.4 Seja $\tau: \Sigma^{*} \rightarrow \Gamma^{*}$ uma relação racional finitamente valorada com $1 \tau=\varnothing$. Então, $\tau$ pode ser realizada por um $\Sigma$-Г-transdutor letra-palavra bi-acessivel e normalizado.

Demonstração. Pelo Teorema 3.2.1, $\tau$ é o comportamento de um $\Sigma$ - $\Gamma$-transdutor letra-palavra $\mathcal{T}=(Q, E, I, T)$. Após eliminar estados não-acessíveis e não-co-acessíveis, podemos supor que esse transdutor é bi-acessível. A partir de $\mathcal{T}$, um $\Sigma$ - $\Gamma$-transdutor letra-palavra bi-acessível e normalizado $\mathcal{T}^{\prime}=\left(Q^{\prime}, E^{\prime}, i, t\right)$ pode ser construído como segue.

O conjunto de estados de $\mathcal{T}^{\prime}$ é $Q^{\prime}=Q \cup i \cup t$, onde $i$ e $t$ são estados distintos e que não pertencem a $Q$. O conjunto de transições $E^{\prime}$ é a união de $E$ com as transições descritas a seguir. Para cada transição da forma

$$
p \stackrel{\sigma / x}{\longrightarrow} q, \quad p \in I
$$

$E^{\prime}$ tem uma transição

$$
i \stackrel{\sigma / x}{\longrightarrow} q
$$

Para cada transição da forma

$$
p \stackrel{\sigma / x}{\longrightarrow} q, \quad q \in T
$$

$E^{\prime}$ tem uma transição

$$
p \stackrel{\sigma / x}{\longrightarrow} t
$$

Finalmente, para cada transição da forma

$$
p \stackrel{\sigma / x}{\longrightarrow} q, \quad p \in I, q \in T,
$$

$E^{\prime}$ tem uma transição

$$
i \stackrel{\sigma / x}{\longrightarrow} t .
$$

Segue facilmente dessa construção que, para todo par $(u, x)$ em $\Sigma^{*} \times \Gamma^{*}$, existe um passeio bem-sucedido em $\mathcal{T}$ com rótulo igual a $(u, x)$ se, e somente se, existe um passeio bem-sucedido rotulado por esse par em $\mathcal{T}^{\prime}$. Assim, esses transdutores são equivalentes, e a demonstração está concluída.

Exemplo 3.2.2 Sejam $\Sigma=\{\sigma\}$ e $\Gamma=\{a\}$. Aplicando a normalização no transdutor

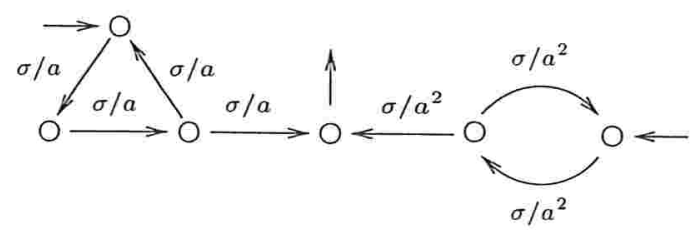

e mantendo apenas os estados acessíveis e co-acessíveis, obtemos 


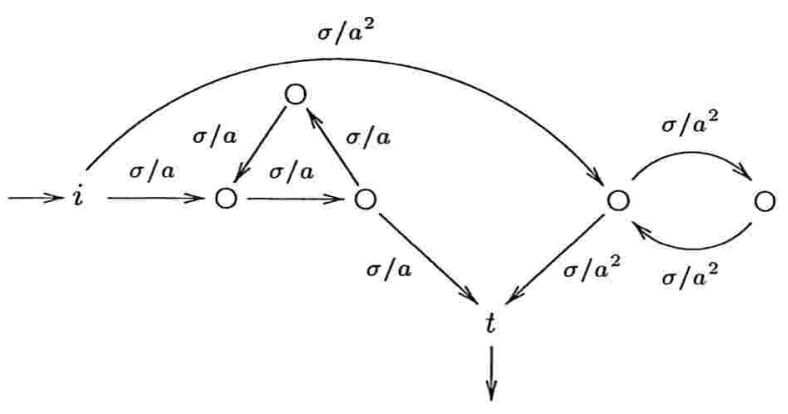

\subsection{Decidibilidade de se uma relação racional é finitamente valo- rada}

Nesta seção, vamos estudar o problema de decidir se uma relação racional $\tau: \Sigma^{*} \rightarrow \Gamma^{*}$ é finitamente valorada. Vamos supor que $\tau$ é representada por um $\Sigma$ - $\Gamma$-transdutor fino bi-acessível e normalizado, o que é possível pela Proposição 2.3.1.

Em seguida, vamos estudar a construção de um transdutor letra-palavra equivalente a um transdutor fino que realiza uma relação racional finitamente valorada.

Proposição 3.3.1 Seja $\mathcal{T}=(Q, E, I, T)$ um $\Sigma$-Г-transdutor fino bi-acessivel. Então, $|\mathcal{T}|$ é uma relação finitamente valorada se, e somente se,

$$
\operatorname{card}\left(1 \mu_{p q}\right)<\infty, \quad \forall p, q \in Q,
$$

onde $\mu$ é o morfismo da representação matricial de $\mathcal{T}$.

Demonstração. Suponha que $|\mathcal{T}|$ seja finitamente valorada. Como $\mathcal{T}$ é bi-acessível, segue da Proposição 3.2.1 que a condição (3.3.1) é satisfeita.

Para provar a implicação contrária, afirmamos que (3.3.1) implica em

$$
\operatorname{card}\left(\sigma \mu_{p q}\right)<\infty, \forall \sigma \in \Sigma, \forall p, q \in Q .
$$

De fato, todo passeio em $\mathcal{T}$ da forma

$$
p \stackrel{\sigma / v}{\longrightarrow} q, \quad \sigma \in \Sigma
$$

pode ser fatorado como

$$
p \stackrel{1 / x}{\longrightarrow} r \stackrel{\sigma / y}{\longrightarrow} s \stackrel{1 / z}{\longrightarrow} q
$$

onde $r \stackrel{\sigma / y}{\longrightarrow} s$ é uma transição e $x y z=v$. Então,

$$
\operatorname{card}\left(\sigma \mu_{p q}\right) \leq \sum_{r, s \in Q} \operatorname{card}\left(1 \mu_{p r}\right)+\operatorname{card}(E)+\operatorname{card}\left(1 \mu_{s q}\right)<\infty .
$$

Pelo Corolário 3.2.1, as propriedades (3.3.1) e (3.3.2) implicam que $|\mathcal{T}|$ é uma relação finitamente valorada. 
Teorema 3.3.1 É decidivel se uma relação racional $\tau: \Sigma^{*} \rightarrow \Gamma^{*}$ é finitamente valorada.

Demonstração. Sejam $\mathcal{T}=(Q, E, I, T)$ um $\Sigma$ - $\Gamma$-transdutor fino, bi-acessível e normalizado que realiza $\tau$, e $\mu$ o morfismo de sua representação matricial. Primeiro, vamos mostrar que a condição (3.3.1) é equivalente a todos os passeios fechados de $\mathcal{T}$ com entrada 1 terem saída 1.

Suponha que $\mathcal{T}$ verifique a condição (3.3.1). Seja $c: q \stackrel{1 / x}{\longrightarrow} q$ um passeio fechado. Como card $\left(1 \mu_{q q}\right)<\infty, x=1$ necessariamente.

Para provar a recíproca, sejam $x$ uma palavra em $1 \mu_{p q}$, e $c: p \stackrel{1 / x}{\longrightarrow} q$ um passeio de comprimento mínimo de $p$ a $q$ com rótulo $(1, x)$. Afirmamos que $c$ é um caminho. Caso contrário, $c$ pode ser fatorado como $c: c_{1} c_{2} c_{3}$, onde $c_{2}$ é um passeio fechado não-trivial. Mas, por hipótese, a saída de $c_{2}$ é 1 , e portanto $c_{1} c_{3}$ é um passeio de $p$ a $q$ com rótulo $(1, x)$, o que contraria a minimalidade de c. Como o número de caminhos de $\mathcal{T}$ é finito, obtemos (3.3.1).

Agora, vamos mostrar como essa propriedade pode ser verificada algoritmicamente. Seja $\mathcal{T}_{0}$ o grafo com conjunto de vértices igual a $Q$, e cujos arcos correspondem às transições de $\mathcal{T}$ com entrada igual a 1 . O primeiro passo é construir os componentes fortemente conexos desse grafo. Esses componentes são gerados pela relação $\sim$ sobre $Q$ definida por

$$
p \sim q \text { se, e somente se, existem passeios } p \stackrel{1 / x}{\longrightarrow} q \text { e } q \stackrel{1 / y}{\longrightarrow} p \text { em } \mathcal{T} \text {. }
$$

O componente a que um estado $p$ pertence será denotado por $C(p)$.

Segue da definição de $\sim$ que $\mathcal{T}$ tem um passeio fechado com entrada igual a 1 e saída diferente de 1 se, e somente se, $\mathcal{T}$ tem uma transição da forma

$$
p \stackrel{1 / u}{\longrightarrow} q
$$

tal que

$$
C(p)=C(q) \quad \text { e } \quad u \neq 1 .
$$

Assim, percorrendo os arcos de $\mathcal{T}_{0}$, podemos decidir a condição (3.3.1).

Sejam $n$ o número de estados e $m$ o número de transições de $\mathcal{T}$. As complexidades de tempo e espaço do algoritmo descrito na demonstração do Teorema 3.3.1 dependem apenas da construção dos componentes fortemente conexos de $\mathcal{T}_{0}$, e de uma visita às transições de $\mathcal{T}$. Portanto, essas complexidades são

$$
\Theta(n+m) .
$$

Agora, vamos estudar a construção de um transdutor letra-palavra equivalente a um transdutor fino que realiza uma relação racional palavra-palavra finitamente valorada. Essa construção está descrita em [RS97], para o caso em que a restrição do transdutor às transições com entrada igual a 1 é um grafo acíclico.

Teorema 3.3.2 Seja $\mathcal{T}$ um $\Sigma$-Г-transdutor fino, bi-acessivel e normalizado que realiza uma relação finitamente valorada. Esse transdutor pode ser efetivamente transformado em uma união

$$
\mathcal{T}_{1} \cup \mathcal{T}_{2},
$$


onde $\mathcal{T}_{1}$ é um $\Sigma$-Г-transdutor letra-palavra bi-acessivel e normalizado tal que

$$
\left|\mathcal{T}_{1}\right|=|\mathcal{T}| \mid \Sigma^{+}
$$

e $\mathcal{T}_{2}$ é um $\Sigma$-Г-transdutor tal que

$$
\left|\mathcal{T}_{2}\right|=|\mathcal{T}| \mid 1
$$

Demonstração. Sejam $\mathcal{T}=(Q, E, i, t), \tau=|\mathcal{T}|$, e $\mu$ o morfismo da representação matricial de $\mathcal{T}$.

Claramente, a restrição de $\mathcal{T}$ às transições com entrada igual a 1 realiza exatamente a relação $\tau \mid 1$. Portanto, podemos definir $\mathcal{T}_{2}$ como essa restrição.

Vamos apresentar uma construção efetiva de $\mathcal{T}_{1}$ que utiliza a matriz $1 \mu$. Uma construção efetiva dessa matriz será descrita no final da demonstração.

Seja $\mathcal{T}^{\prime}=\left(Q, E^{\prime}, i, t\right)$ o $\Sigma$ - $\Gamma$-transdutor letra-palavra cujo conjunto de transições é definido como segue. Para cada transição

$$
p \stackrel{\sigma / x}{\longrightarrow} q \in E
$$

e cada par de estados $p^{\prime}$ e $q^{\prime}$ em $Q$ tais que $1 \mu_{p^{\prime} p} \neq \varnothing$ e $1 \mu_{q q^{\prime}} \neq \varnothing, E^{\prime}$ tem as transições

$$
p^{\prime} \stackrel{\sigma / u x v}{\longrightarrow} q^{\prime}, \quad \forall u \in 1 \mu_{p^{\prime} p}, \forall v \in 1 \mu_{q q^{\prime}} .
$$

Observe que, como $1 \in \mu_{q q}$ para todo estado $q$, as transições em $E$ com entrada diferente da palavra vazia pertencem a $E^{\prime}$.

Vamos provar que $\left|\mathcal{T}^{\prime}\right|=\tau \mid \Sigma^{+}$. Primeiro, observe que, como $\mathcal{T}^{\prime}$ não tem nenhuma transição com entrada 1 , e $i \neq t, 1\left|\mathcal{T}^{\prime}\right|=\varnothing$. Resta provar que $\left|\mathcal{T}^{\prime}\right|$ e $|\mathcal{T}|$ coincidem em $\Sigma^{+}$.

Seja $w=\sigma_{1} \ldots \sigma_{l}$ uma palavra em $\Sigma^{+}$, onde cada $\sigma_{i}$ é uma letra em $\Sigma$. Segue diretamente da construção de $\mathcal{T}^{\prime}$ que esse transdutor tem um passeio bem-sucedido com entrada $w$

$$
c: c_{1} \ldots c_{l}, \quad c_{i} \in E^{\prime} \quad \forall i, 1 \leq i \leq l,
$$

se, e somente se, existe um passeio bem-sucedido

$$
d: d_{1} \ldots d_{l}
$$

em $\mathcal{T}$ tal que, para todo $i, 1 \leq i \leq l, d_{i}$ é da forma

$$
p^{\prime} \stackrel{1 / x_{i}}{\longrightarrow} p \stackrel{\sigma_{i} / y_{i}}{\longrightarrow} q \stackrel{1 / z_{i}}{\longrightarrow} q^{\prime},
$$

$\mathrm{e}$

$$
c_{i}=p^{\prime} \stackrel{\sigma_{i} / x_{i} y_{i} z_{i}}{\longrightarrow} q^{\prime}
$$

Portanto, $w\left|\mathcal{T}^{\prime}\right|=w|\mathcal{T}|$.

Definindo $\mathcal{T}_{1}$ como a parte bi-acessível de $\mathcal{T}^{\prime}$, obtemos um transdutor letra-palavra bi-acessível e normalizado que realiza $\tau \mid \Sigma^{+}$. 
Passamos à descrição de como a matriz $1 \mu$ pode ser construída. Sejam $\mathbb{N}$ o monóide aditivo dos números naturais, $\left(2^{\Gamma^{*}}\right)^{Q \times Q}$ o monóide multiplicativo das matrizes quadradas indexadas por $Q \times Q$ sobre o semi-anel $\left(2^{\Gamma^{*}}, \cup, \cdot\right)$, e

$$
f: \mathbb{N} \rightarrow\left(2^{\Gamma^{*}}\right)^{Q \times Q}
$$

o morfismo de monóides definido por

$$
1 f_{p q}=\{u: \exists p \stackrel{1 / u}{\longrightarrow} q \in E\}, \quad \forall p, q \in Q .
$$

A propriedade

$$
k f_{p q}=\{u: \text { existe um passeio } p \stackrel{1 / u}{\longrightarrow} q \text { de comprimento } k \text { em } \mathcal{T}\}, \quad \forall p, q \in Q, \forall k \in \mathbb{N}
$$

pode ser demonstrada facilmente por indução em $k$.

Recordando a demonstração do Teorema 3.3.1, temos que, para todo par de estados $p$ e $q$ e todo $x$ em $1 \mu_{p q}$, existe um caminho de $p$ a $q$ com entrada 1 e saída $x$. Assim

$$
1 \mu=0 f+\cdots+(n-1) f
$$

onde $n=\operatorname{card}(Q)$. Portanto, $1 \mu$ pode ser efetivamente construída.

Não vamos analisar as complexidades de tempo e espaço do algoritmo descrito na desmonstração do Teorema 3.3.2. Observamos apenas que esse algoritmo pode criar muitas transições redundantes no transdutor intermediário $\mathcal{T}^{\prime}$, ou seja, que podem ser eliminadas sem alterar o comportamento desse transdutor. Um exemplo está ilustrado a seguir. Essa observação sugere que o algoritmo pode ser melhorado.

Exemplo 3.3.1 Sejam $\Sigma=\Gamma=\{\sigma, \gamma\}$ e $\mathcal{T}$ o $\Sigma$ - $\Gamma$-transdutor

$$
\longrightarrow 1 \stackrel{\substack{1 / \sigma \\ 1 / \gamma}}{\longrightarrow} 2 \stackrel{\substack{1 / \sigma \\ 1 / \gamma}}{\longrightarrow} 3 \stackrel{\substack{1 / \sigma \\ \sigma / \sigma}}{\longrightarrow} 4 \stackrel{1 / \sigma}{\longrightarrow} 5 \longrightarrow
$$

Utilizando a demonstração do Teorema 3.3.2, vamos construir um $\Sigma$ - $\Gamma$-transdutor $\mathcal{T}_{2}$ e um $\Sigma$ - $\Gamma$-transdutor letra-palavra $\mathcal{T}_{1}$ com comportamentos iguais a $\mid \mathcal{T} \| 1$ e $\mid \mathcal{T} \| \Sigma^{+}$, respectivamente.

$\mathrm{O}$ transdutor $\mathcal{T}_{2}$ é a restrição

$$
\longrightarrow 1 \stackrel{1 / \sigma}{1 / \gamma} \longrightarrow 2 \stackrel{1 / \sigma}{\longrightarrow} 3 \stackrel{1 / \sigma}{\longrightarrow} 4 \stackrel{1 / \sigma}{\longrightarrow} 5 \longrightarrow
$$

Para construir $\mathcal{T}_{1}$, vamos calcular a matriz $1 \mu$.

A matriz $1 f$ está ilustrada a seguir. O conjunto vazio é denotado por 0 . As linhas e colunas são indexadas pelos estados do transdutor:

$$
1 f=\left(\begin{array}{ccccc}
0 & \{\sigma, \gamma\} & 0 & 0 & 0 \\
0 & 0 & \{\sigma, \gamma\} & 0 & 0 \\
0 & 0 & 0 & \{\sigma\} & 0 \\
0 & 0 & 0 & 0 & \{\sigma\} \\
0 & 0 & 0 & 0 & 0
\end{array}\right) .
$$


O comprimento máximo de um caminho com entrada 1 em $\mathcal{T}$ é 4 . Portanto, $1 \mu$ é a soma das matrizes $0 f, 1 f$,

$$
\begin{gathered}
2 f=\left(\begin{array}{ccccc}
0 & 0 & \{\sigma, \gamma\}^{2} & 0 & 0 \\
0 & 0 & 0 & \{\sigma, \gamma\} \sigma & 0 \\
0 & 0 & 0 & 0 & \left\{\sigma^{2}\right\} \\
0 & 0 & 0 & 0 & 0 \\
0 & 0 & 0 & 0 & 0
\end{array}\right), 3 f=\left(\begin{array}{ccccccc}
0 & 0 & 0 & \{\sigma, \gamma\}^{2} \sigma & 0 \\
0 & 0 & 0 & 0 & \{\sigma, \gamma\} \sigma^{2} \\
0 & 0 & 0 & 0 & 0 \\
0 & 0 & 0 & 0 & 0
\end{array}\right), \mathrm{e} \\
4 f=\left(\begin{array}{ccccc}
0 & 0 & 0 & 0 & \{\sigma, \gamma\}^{2} \sigma^{2} \\
0 & 0 & 0 & 0 & 0 \\
0 & 0 & 0 & 0 & 0 \\
0 & 0 & 0 & 0 & 0
\end{array}\right) .
\end{gathered}
$$

Essa soma é

$$
1 \mu=\left(\begin{array}{ccccc}
\{1\} & \{\sigma, \gamma\} & \{\sigma, \gamma\}^{2} & \{\sigma, \gamma\}^{2} \sigma & \{\sigma, \gamma\}^{2} \sigma^{2} \\
0 & \{1\} & \{\sigma, \gamma\} & \{\sigma, \gamma\} \sigma & \{\sigma, \gamma\} \sigma^{2} \\
0 & 0 & \{1\} & \{\sigma\} & \left\{\sigma^{2}\right\} \\
0 & 0 & 0 & \{1\} & \{\sigma\} \\
0 & 0 & 0 & 0 & \{1\}
\end{array}\right) .
$$

O transdutor $\mathcal{T}^{\prime}$ é

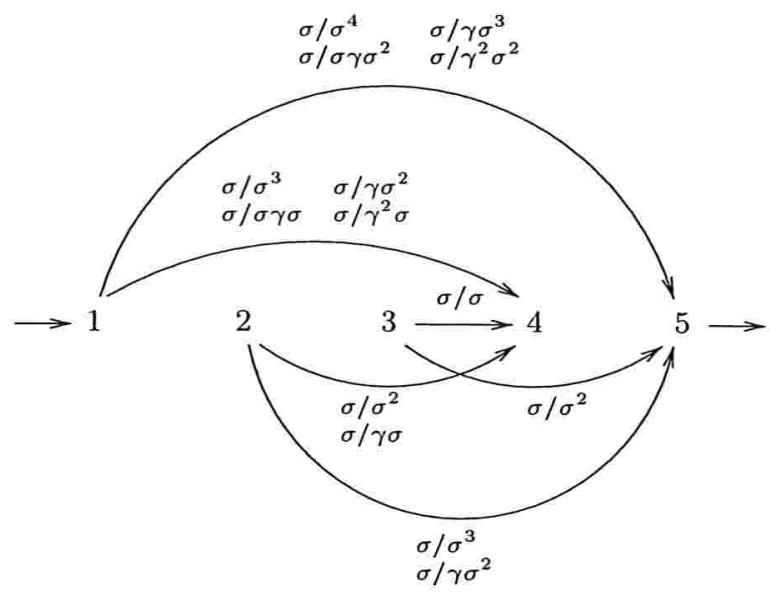

A parte bi-acessível de $\mathcal{T}^{\prime}$ é o transdutor $\mathcal{T}_{1}$, e está ilustrada a seguir:

$$
\longrightarrow 1 \stackrel{\begin{array}{ll}
\sigma / \sigma^{4} \\
\sigma / \sigma \gamma \sigma^{2}
\end{array} \stackrel{\sigma / \gamma \sigma^{3}}{\sigma / \gamma^{2} \sigma^{2}}}{\longrightarrow} 5 \longrightarrow
$$

\subsection{Decidibilidade de se uma relação racional é $k$-valorada para um dado $k$}

Nesta seção, vamos estudar o problema de decidir se uma relação racional $\Sigma^{*} \rightarrow \Gamma^{*}$ é $k$-valorada para um dado inteiro positivo $k$. 
Em 1983, Gurari e Ibarra mostraram que esse problema é decidível em complexidade de tempo polinomial [GI83]. Esses autores não apresentam um algoritmo explícito para o problema, mas utilizam uma redução a outro problema, para outro tipo de máquinas, cuja decidibilidade em complexidade de tempo polinomial já era conhecida.

Não vamos apresentar a prova desses autores. Nossa abordagem para o problema será combinatória, e consiste em estender um pequeno resultado sobre transdutores letra-palavra que Schützenberger publicou em 1975, e que fornece um algoritmo (não-eficiente) para decidir se um transdutor letra-palavra realiza uma função [Sch75]:

Teorema 3.4.1 (Schützenberger 1975) Sejam $\mathcal{T}=(Q, E, I, T)$ um $\Sigma$ - $\Gamma$-transdutor letra-palavra, $\mu$ o morfismo de sua representação matricial, e $n=\operatorname{card}(Q)$. Se a condiçãa

$$
\operatorname{card}\left(u \mu_{p q}\right) \leq 1
$$

for satisfeita para todo par de estados $p$ e q e toda palavra $u$ em $\Sigma^{*}$ tal que

$$
|u| \leq 1+2 n(n-1)
$$

então essa condição é satisfeita para toda palavra u em $\Sigma^{*}$.

Nossa extensão é

Teorema 3.4.2 Sejam $k$ um inteiro positivo, $\mathcal{T}=(Q, E, I, T)$ um $\Sigma$ - $\Gamma$-transdutor letra-palavra, $\mu$ o morfismo de sua representação matricial, e $n=\operatorname{card}(Q)$. Se a condição

$$
\operatorname{card}\left(u \mu_{p q}\right) \leq k
$$

for satisfeita para todo par de estados $p$ e $q$ e toda palavra $u$ em $\Sigma^{*}$ tal que

$$
|u| \leq n^{k+1} T(k(k+1) / 2)+1,
$$

onde $T$ é a função definida por

$$
T(l)=l !\left(1+\sum_{1 \leq i \leq l} \frac{1}{i !}\right), \quad \forall l \in \mathbb{N}, l>0,
$$

então essa condição é satisfeita para toda palavra u em $\Sigma^{*}$.

No Teorema 3.4.2, ao fazer $k=1$, não obtemos a expressão de Schützenberger. Isso ocorre devido a algumas particularidades do caso $k=1$ que permitem obter um limitante mais justo. No entanto, nossa demonstração consiste numa generalização dos argumentos de Schützenberger. A demonstração para o caso $k=1$ pode ser vista no livro de Berstel [Ber79].

Vamos precisar de três lemas.

Lema 3.4.1 Sejam $\Sigma$ um alfabeto e $s=\operatorname{card}(\Sigma)$. Então, para todo inteiro positivo l, toda palavra $u$ em $\Sigma^{*}$ com $|u| \geq s(l-1)+1$ contém pelo menos l ocorrências de uma mesma letra. 
Demonstração. Se toda letra ocorrer no máximo $l-1$ vezes em $u$, então

$$
|u|=\sum_{\sigma \in \Sigma}|u|_{\sigma} \leq s(l-1)
$$

contrariando a escolha de $u$.

Lema 3.4.2 (Schützenberger) Sejam $x_{1}, x_{2}, x_{3}, x_{4}, y_{1}, y_{2}, y_{3}, y_{4}$ palavras sobre um alfabeto $\Sigma$ tais que
i. $x_{1} x_{4}=y_{1} y_{4}$
ii. $x_{1} x_{2} x_{4}=y_{1} y_{2} y_{4}$,
iii. $x_{1} x_{3} x_{4}=y_{1} y_{3} y_{4}$.

Então,

$$
x_{1} x_{2} x_{3} x_{4}=y_{1} y_{2} y_{3} y_{4}
$$

Demonstração. De $i$, segue que $x_{1}$ e $y_{1}$ são comparáveis pela ordem do prefixo. Sem perda de generalidade, suponha que $x_{1} \succ y_{1}$. Então, podemos fatorar

$$
y_{1}=x_{1} y_{1}^{\prime} \quad \text { e } \quad x_{4}=y_{1}^{\prime} y_{4} .
$$

De $i i$ e $i i i$, obtemos então

$$
x_{2} y_{1}^{\prime}=y_{1}^{\prime} y_{2} \quad \text { e } \quad x_{3} y_{1}^{\prime}=y_{1}^{\prime} y_{3} .
$$

Assim,

$$
x_{1} x_{2} x_{3} x_{4}=x_{1} x_{2} x_{3} y_{1}^{\prime} y_{4}=x_{1} x_{2} y_{1}^{\prime} y_{3} y_{4}=x_{1} y_{1}^{\prime} y_{2} y_{3} y_{4}=y_{1} y_{2} y_{3} y_{4} .
$$

Vamos fazer duas considerações antes de apresentar o terceiro lema.

A primeira, é explicitar que a função (3.4.3) é a solução da recorrência

$$
\begin{aligned}
& T(1)=2, \\
& T(l)=l T(l-1)+1, \quad \forall l>1 .
\end{aligned}
$$

Essa afirmação é comprovada facilmente por indução em $l$.

A segunda é uma definição. Sejam $\theta$ um inteiro positivo, e

$$
\mathcal{C}_{\theta}=\{(r, s): r, s \in[\theta] \text { e } r \leq s\} .
$$

Dada uma função $f: \mathcal{C}_{\theta} \rightarrow \Omega$, onde $\Omega$ é um alfabeto, um encaixamento de $f$ é uma tripla de pares de $\mathcal{C}_{\theta}$ da forma

$$
((i, j),(i, k),(k+1, j))
$$

satisfazendo

$$
i \leq k<j \quad \text { e } \quad(i, j) f=(i, k) f=(k+1, j) f .
$$


Lema 3.4.3 Sejam $\theta$ um inteiro positivo, $\Omega$ um alfabeto, e $f: \mathcal{C}_{\theta} \rightarrow \Omega$ uma função. Se $\theta \geq$ $T(\operatorname{card}(\Omega))$, então $f$ tem um encaixamento.

Demonstração. Vamos usar indução em $s=\operatorname{card}(\Omega)$.

- $s=1$ : Evidente.

- $s>1$ : Seja $x \in \Omega^{*}$ a palavra de comprimento $\theta$ definida como

$$
x[i]=(1, i) f, \quad \forall i, 1 \leq i \leq \theta .
$$

Como $T(s)=s((T(s-1)+1)-1)+1$, segue do Lema 3.4.1 que existe uma letra $\sigma$ de $\Omega$ que ocorre pelo menos $T(s-1)+1$ vezes em $x$. Podemos assim fatorar $x$ como $w x^{\prime}$, onde $x^{\prime} \in \Omega^{*}$,

$$
w=x_{0} \sigma x_{1} \sigma \ldots x_{T(s-1)} \sigma
$$

e

$$
x_{i} \in(\Omega-\sigma)^{*}, \quad \forall i, 0 \leq i \leq T(s-1) .
$$

Para cada $i, 0 \leq i \leq T(s-1)$, vamos denotar por $w_{i}$ um prefixo de $w$ da forma $x_{0} \ldots x_{i}$.

Suponha que existam inteiros $i$ e $j$, com $i \geq 0$ e $i<j$, tais que $\left(\left|w_{i} \sigma\right|+1,\left|w_{j} \sigma\right|\right) f=\sigma$. Então, $\left(\left(1,\left|w_{j} \sigma\right|\right),\left(1,\left|w_{i} \sigma\right|\right),\left(\left|w_{i} \sigma\right|+1,\left|w_{j} \sigma\right|\right)\right)$ é um encaixamento de $f$. Caso contrário, considere a função $g: \mathcal{C}_{T(s-1)} \rightarrow(\Omega-\sigma)$ definida por

$$
(i, j) g=\left(\left|w_{i-1} \sigma\right|+1,\left|w_{j} \sigma\right|\right) f, \quad \forall(i, j) \in \mathcal{C}_{T(s-1)} .
$$

A definição é consistente pois, por hipótese, $\left(\left|w_{i-1} \sigma\right|+1,\left|w_{j} \sigma\right|\right) f \neq \sigma$, para todo $(i, j) \in$ $\mathcal{C}_{T(s-1)}$. Pela hipótese de indução, $g$ tem um encaixamento $((i, j),(i, k),(k+1, j))$. Assim,

$$
\left(\left(\left|w_{i-1} \sigma\right|+1,\left|w_{j} \sigma\right|\right),\left(\left|w_{i-1} \sigma\right|+1,\left|w_{k} \sigma\right|\right),\left(\left|w_{k} \sigma\right|+1,\left|w_{j} \sigma\right|\right)\right)
$$

é um encaixamento de $f$.

Demonstração do Teorema 3.4.2. Suponha, por absurdo, que a condição (3.4.1) seja satisfeita para toda palavra satisfazendo (3.4.2), mas não seja satisfeita para todas as palavras em $\Sigma^{*}$.

Considere então estados $p$ e $q$ e uma palavra de comprimento mínimo $u=\sigma_{1} \ldots \sigma_{l}$, com $\sigma_{i}$ em $\Sigma$ para $1 \leq i \leq l$, tais que card $\left(u \mu_{p q}\right)>k$. Então, existem $k+1$ passeios $c_{1}, \ldots, c_{k+1}$ em $\mathcal{T}$ da forma

$$
c_{j}: p \stackrel{\sigma_{1} / v_{j_{1}}}{\longrightarrow} q_{j_{1}} \ldots q_{j_{l-1}} \stackrel{\sigma_{l} / v_{j_{l}}}{\longrightarrow} q, \quad \forall j, 1 \leq j \leq k+1,
$$

e cujas saídas são duas a duas distintas.

Considere a palavra $\xi=\xi_{1} \ldots \xi_{l-1}$ sobre o alfabeto $Q^{k+1}$ dada por $\xi_{i}=\left(q_{1_{i}}, \ldots, q_{k+1_{i}}\right)$, para todo $i, 1 \leq i<|u|=l$. Pela escolha de $u$,

$$
l-1 \geq n^{k+1}((T(k(k+1) / 2)+1)-1)+1 .
$$


Como card $\left(Q^{k+1}\right)=n^{k+1}$, segue do Lema 3.4.1 que algum $\xi_{i}$ ocorre pelo menos $T(k(k+1) / 2)+1$ vezes em $\xi$. Podemos então fatorar os passeios $c_{1}, \ldots, c_{k+1}$ como

$$
\begin{aligned}
& c_{1}: \quad p \stackrel{u_{0} / x_{1_{0}}}{\longrightarrow} q_{1_{i}} \stackrel{u_{1} / x_{1_{1}}}{\longrightarrow} \ldots \stackrel{u_{\theta} / x_{1_{\theta}}}{\longrightarrow} q_{1_{i}} \stackrel{u_{\theta+1} / x_{1_{\theta+1}}}{\longrightarrow} q, \\
& \vdots \\
& c_{k+1}: \quad p \stackrel{u_{0} / x_{k+1_{0}}}{\longrightarrow} q_{k+1_{i}} \stackrel{u_{1} / x_{k+1_{1}}}{\longrightarrow} \ldots \stackrel{u_{\theta} / x_{k+1_{\theta}}}{\longrightarrow} q_{k+1_{i}} \stackrel{u_{\theta+1} / x_{k+1_{\theta+1}}}{\longrightarrow} q,
\end{aligned}
$$

onde $\theta=T(k(k+1) / 2)$.

Sejam $\Omega=\{\{i, j\}: i, j \in[k+1], i \neq j\}$ e $f: \mathcal{C}_{\theta} \rightarrow \Omega$ a função definida como segue. Para cada par $(a, b)$ em $\mathcal{C}_{\theta}$, considere os passeios

$$
\begin{aligned}
& c_{1}^{(a, b)}: \quad p \stackrel{u_{0} \ldots u_{a-1} / x_{1_{0}} \ldots x_{1_{a-1}}}{\longrightarrow} q_{1_{i}} \stackrel{u_{b+1} \ldots u_{\theta+1} / x_{1_{b+1}} \ldots x_{1_{\theta+1}}}{\longrightarrow} q, \\
& \vdots \\
& c_{k+1}^{(a, b)}: \quad p \stackrel{u_{0} \ldots u_{a-1} / x_{k+1_{0}} \ldots x_{k+1_{a-1}}}{\longrightarrow} q_{k+1_{i}} \stackrel{u_{b+1} \ldots u_{\theta+1} / x_{k+1_{b+1}} \ldots x_{k+1_{\theta+1}}}{\longrightarrow} q,
\end{aligned}
$$

obtidos omitindo-se o passeio fechado da forma $q_{1_{j}} \stackrel{u_{a} \ldots u_{b} / x_{j_{a}} \ldots x_{j_{b}}}{\longrightarrow} q_{1_{j}}$ do passeio $c_{j}$, para $1 \leq j \leq$ $k+1$. Pela minimalidade de $u$, devem existir dois passeios distintos $c_{r}^{(a, b)}$ e $c_{s}^{(a, b)}$ com a mesma saída. Defina então $(a, b) f$ como $\{r, s\}$.

Como $\operatorname{card}(\Omega)=k(k+1) / 2$, temos que $\theta=T(\operatorname{card}(\Omega))$. Segue então do Lema 3.4.3 que $f$ tem um encaixamento $((a, b),(a, e),(e+1, b))$, ou seja, existem índices $r$ e $s$ tais que $(a, b) f=$ $(a, e) f=(e+1, b) f=\{r, s\}$. Pela definição de $f$, obtemos as igualdades

$$
\begin{aligned}
\left(x_{r_{0}} \ldots x_{r_{a-1}}\right)\left(x_{r_{b+1}} \ldots x_{r_{\theta+1}}\right) & =\left(x_{s_{0}} \ldots x_{s_{a-1}}\right)\left(x_{s_{b+1}} \ldots x_{s_{\theta+1}}\right), \\
\left(x_{r_{0}} \ldots x_{r_{a-1}}\right)\left(x_{r_{e+1}} \ldots x_{r_{b}}\right)\left(x_{r_{b+1}} \ldots x_{r_{\theta+1}}\right) & =\left(x_{s_{0}} \ldots x_{s_{a-1}}\right)\left(x_{s_{e+1}} \ldots x_{s_{b}}\right)\left(x_{s_{b+1}} \ldots x_{s_{\theta+1}}\right), \\
\left(x_{r_{0}} \ldots x_{r_{a-1}}\right)\left(x_{r_{a}} \ldots x_{r_{e}}\right)\left(x_{r_{b+1}} \ldots x_{r_{\theta+1}}\right) & =\left(x_{s_{0}} \ldots x_{s_{a-1}}\right)\left(x_{s_{a}} \ldots x_{s_{e}}\right)\left(x_{s_{b+1}} \ldots x_{s_{\theta+1}}\right) .
\end{aligned}
$$

Pelo Lema 3.4.2, concluímos que

$$
c_{r} \beta=x_{r_{0}} \ldots x_{r_{\theta+1}}=x_{s_{0}} \ldots x_{s_{\theta+1}}=c_{s} \beta,
$$

contrariando a hipótese sobre $u$. Logo, $\operatorname{card}\left(u \mu_{p q}\right) \leq k$ para todo par de estados $p$ e $q$, e toda palavra $u$ em $\Sigma^{*}$.

Corolário 3.4.1 Dado um inteiro positivo $k$, é decidivel se uma relação racional $\tau: \Sigma^{*} \rightarrow \Gamma^{*}$ é $k$-valorada.

Demonstração. Seja $\mathcal{T}$ um $\Sigma$ - $\Gamma$-transdutor fino, bi-acessível e normalizado que realiza $\tau$. Vamos descrever como decidir se $\tau$ é $k$-valorada em vários passos.

O primeiro passo é verificar se $\tau$ é finitamente valorada. Pelo Teorema 3.3.1, esse problema é decidível.

Em seguida, utilizando o Teorema 3.3.2, construímos um $\Sigma$ - $\Gamma$-transdutor $\mathcal{T}_{1}$ letra-palavra biacessível e normalizado que realiza a restrição $\tau \mid \Sigma^{+}$, e um $\Sigma$ - $\Gamma$-transdutor $\mathcal{T}_{2}$ que realiza a restrição 
$\tau \mid 1$. Utilizando esses transdutores, vamos estudar a valoração da palavra vazia e das demais palavras em separado.

Testar se card $(1 \tau) \leq k$ consiste em verificar se o comportamento do autômato de saída $\mathcal{A}$ de $\mathcal{T}_{2}$ tem no máximo $k$ palavras. Uma possibilidade para essa verificação é como segue. Primeiro, $\mathcal{A}$ é transformado em um $\Gamma$-autômato equivalente $\mathcal{B}$, onde cada transição é rotulada por uma letra (observe que $\mathcal{A}$ tem transições rotuladas por palavras quaisquer). Essa transformação é clássica, e está descrita por exemplo em [HMU01]. Em seguida, utilizamos a propriedade simples de que o comportamento de $\mathcal{B}$ é finito se, e somente se, para toda palavra $s$ em $|\mathcal{B}|,|s|<n$, onde $n$ é o número de estados de $\mathcal{B}$. Com essa propriedade, podemos decidir não apenas se $|\mathcal{B}|$ é finito, como também se esse conjunto tem no máximo $k$ palavras.

Para estudar a valoração do transdutor $\mathcal{T}_{1}$, utilizamos o Teorema 3.4.2. Após verificar a condição (3.4.1) para um número finito de palavras, podemos decidir se card $\left(u \mu_{p q}\right) \leq k$ para toda palavra $u$ em $\Sigma^{+}$, e todo par de estados $p$ e $q$. Pela Proposição 3.2 .2 , essa condição é equivalente a $\left|\mathcal{T}_{2}\right|$ ser $k$-valorada.

Por outro lado, o problema de se uma relação racional palavra-palavra é exatamente $k$-valorada (ou seja, a imagem de toda entrada tem exatamente $k$ palavras) é indecidível, conforme observado por Gurari e Ibarra [GI83].

Teorema 3.4.3 (Gurari e Ibarra 1983) Dado um inteiro positivo k, é indecidivel se uma relação racional $\tau: \Sigma^{*} \rightarrow \Gamma^{*}$ é exatamente $k$-valorada.

Demonstração. Basta provar que o caso $k=2$ é indecidível. Vamos reduzir o Problema da Correspondência de Post para esse caso.

Sejam $f: \Sigma^{*} \rightarrow \Gamma^{*}$ e $g: \Sigma^{*} \rightarrow \Gamma^{*}$ morfismos. Então, $f \cup g$ é uma relação racional 2-valorada. Ademais, a restrição de $f \cup g$ a $\Sigma^{+}$é exatamente 2-valorada se, e somente se, não existe nenhuma palavra $s$ em $\Sigma^{+}$tal que $s f=s g$. Portanto, a decidibilidade de se $(f \cup g) \mid \Sigma^{+}$é exatamente 2valorada implica na decidibilidade do Problema da Correspondência de Post.

\subsection{Equivalência de relações racionais finitamente valoradas}

Em 1968, Griffiths mostrou que o problema da equivalência de relações racionais finitamente valoradas é indecidível [Gri68]. Esse resultado foi reforçado por Ibarra em 1978, que demonstrou que a equivalência permanece indecidível mesmo quando o alfabeto de entrada ou o de saída for unitário [Iba78]. Nesta seção, vamos apresentar o resultado de Griffiths.

Griffiths considera uma classe de relações racionais mais restrita que a das relações racionais finitamente valoradas, a saber, a das relações que podem ser realizadas por transdutores letrapalavra em que todos os estados são finais, e a saída de toda transição é diferente da palavra vazia. Como essa restrição não é necessária para nosso trabalho, vamos considerar o caso mais geral. Dessa forma, a demonstração se torna mais simples, mas ainda segue o raciocínio original de Griffiths.

Teorema 3.5.1 (Griffiths 1968) O problema da equivalência de relações racionais $\Sigma^{*} \rightarrow \Gamma^{*}$ finitamente valoradas é indecidivel.

Demonstração. Vamos reduzir o Problema da Correspondência de Post ao problema da equivalência de relações racionais finitamente valoradas. 
Sejam $f: \Sigma^{*} \rightarrow \Gamma^{*}$ e $g: \Sigma^{*} \rightarrow \Gamma^{*}$ morfismos. Defina

$$
M=\max \{|\sigma f|,|\sigma g|: \sigma \in \Sigma\} .
$$

Sejam $\tau: \Sigma^{*} \rightarrow \Gamma^{*}$ a relação definida por

$$
u \tau=\left\{v \in \Gamma^{*}: 0 \leq|v| \leq M|u|\right\}, \quad \forall u \in \Sigma^{*},
$$

e $\tau_{f}: \Sigma^{*} \rightarrow \Gamma^{*}$ e $\tau_{g}: \Sigma^{*} \rightarrow \Gamma^{*}$ as relações definidas por

$$
\begin{gathered}
1 \tau_{f}=1 \tau_{g}=1, \\
u \tau_{f}=u \tau-u f \quad \text { e } u \tau_{g}=u \tau-u g, \quad \forall u \in \Sigma^{+} .
\end{gathered}
$$

Claramente, $\tau, \tau_{f}$ e $\tau_{g}$ são relações finitamente valoradas. Afirmamos que são relações racionais. De posse desse resultado, temos que $\tau_{f} \cup \tau_{g}$ é uma relação racional finitamente valorada. Basta então observar que

$$
\tau_{f} \cup \tau_{g} \neq \tau \text { se, e somente se, existe uma palavra } s \in \Sigma^{+} \text {tal que } s f=s g
$$

para concluir que, se o problema da equivalência de relações racionais finitamente valoradas fosse decidível, então o Problema da Correspondência de Post seria decidível.

Resta então demonstrar que as relações $\tau, \tau_{f}$ e $\tau_{g}$ são racionais.

A relação $\tau$ é o comportamento de um transdutor letra-palavra que tem apenas um estado $i$, inicial e final, e, para cada letra $\sigma$ de $\Sigma$ e cada palavra $x$ de $\Gamma^{*}$ com comprimento entre 0 e $M$, uma transição $i \stackrel{\sigma / x}{\longrightarrow} i$.

Vamos construir um $\Sigma$ - $\Gamma$-transdutor letra-palavra $\mathcal{T}_{f}$ que realiza $\tau_{f}$. Esse transdutor tem cinco estados, que denotamos por $i, q_{<}, q_{>}, q_{=}$e $q_{\neq}$. O estado inicial é $i$, e os estados finais são $i, q_{<}, q_{>}$ e $q_{\neq}$. O conjunto de transições está descrito a seguir:

- para toda letra $\sigma$ em $\Sigma$, toda palavra $x$ em $\Gamma^{*}$ tal que $|x|<|\sigma f|$, toda palavra $y$ em $\Gamma^{*}$ tal que $|\sigma f|<|y| \leq M$ e toda palavra $z$ em $\Gamma^{*}$ tal que $|z|=|\sigma f|$ e $z \neq \sigma f, \mathcal{T}_{f}$ tem as transições

$$
\begin{array}{lll}
i \stackrel{\sigma / \sigma f}{\longrightarrow} q_{=} & q_{=} \stackrel{\sigma / \sigma f}{\longrightarrow} q_{=} & q_{\neq} \stackrel{\sigma / \sigma f}{\longrightarrow} q_{\neq} \\
i \stackrel{\sigma / x}{\longrightarrow} q_{<} & q_{=} \stackrel{\sigma / x}{\longrightarrow} q_{<} & q_{\neq} \stackrel{\sigma / x}{\longrightarrow} q_{<} \\
i \stackrel{\sigma / y}{\longrightarrow} q_{>} & q_{=} \stackrel{\sigma / y}{\longrightarrow} q_{>} & q_{\neq} \stackrel{\sigma / y}{\longrightarrow} q_{>} \\
i \stackrel{\sigma / z}{\longrightarrow} q_{\neq} & q_{=} \stackrel{\sigma / z}{\longrightarrow} q_{\neq} & q_{\neq} \stackrel{\sigma / z}{\longrightarrow} q_{\neq}
\end{array}
$$

- para toda letra $\sigma$ em $\Sigma$ e toda palavra $x$ em $\Gamma^{*}$ tal que $|x| \leq|\sigma f|, \mathcal{T}_{f}$ tem a transição

$$
q_{<} \stackrel{\sigma / x}{\longrightarrow} q_{<}
$$

- para toda letra $\sigma$ em $\Sigma$ e toda palavra $x$ em $\Gamma^{*}$ tal que $|\sigma f| \leq|x| \leq M, \mathcal{T}_{f}$ tem a transição

$$
q_{>} \stackrel{\sigma / x}{\longrightarrow} q_{>}
$$


Seja $\mu$ o morfismo da representação matricial de $\mathcal{T}_{f}$. Segue diretamente da construção desse transdutor que $u \mu_{i i}$ é igual a 1 , se $u=1$, ou igual a $\varnothing$, caso contrário. Vamos mostrar que, para toda palavra $u$ em $\Sigma^{+}$,

$$
\begin{aligned}
u \mu_{i q_{=}} & =u f, \\
u \mu_{i q_{<}} & =\left\{x \in \Gamma^{*}:|x|<|u f|\right\}, \\
u \mu_{i q_{>}} & =\left\{x \in \Gamma^{*}:|u f|<|x| \leq M|u|\right\}, \\
\text { e } u \mu_{i q_{\neq}} & =\left\{x \in \Gamma^{*}:|x|=|u f| \text { e } x \neq u f\right\} .
\end{aligned}
$$

Claramente, essas propriedades implicam em $\tau_{f}=\left|\mathcal{T}_{f}\right|$.

Vamos usar indução em $|u|$.

- $|u|=1$ : segue diretamente da definição das transições com origem em $i$.

- $|u|>1$ : fatore $u$ como $v \sigma$, onde $\sigma$ é uma letra. Utilizando a definição de $\mathcal{T}_{f}$ e a hipótese de indução, temos que

$$
\begin{aligned}
u \mu_{i q_{=}=}= & v \mu_{i q_{=}}(\sigma f)=(v f)(\sigma f)=u f ; \\
u \mu_{i q_{<}}= & \left(v \mu_{i q_{=}}\right)\left(\sigma \mu_{q_{=} q_{<}}\right) \cup\left(v \mu_{i q_{\neq}}\right)\left(\sigma \mu_{q_{\neq} q_{<}}\right) \cup\left(v \mu_{i q_{<}}\right)\left(\sigma \mu_{q_{<} q_{<}}\right) \\
= & (v f)\left\{x \in \Gamma^{*}:|x|<|\sigma f|\right\} \\
& \cup\left\{x \in \Gamma^{*}:|x|=|v f| \text { e } x \neq v f\right\}\left\{x \in \Gamma^{*}:|x|<|\sigma f|\right\} \\
& \cup\left\{x \in \Gamma^{*}:|x|<|v f|\right\}\left\{x \in \Gamma^{*}:|x| \leq|\sigma f|\right\} \\
= & \left\{x \in \Gamma^{*}:|x|<|u f|\right\} \\
u \mu_{i q_{>}}= & \left(v \mu_{i q_{=}}\right)\left(\sigma \mu_{q_{=} q_{>}}\right) \cup\left(v \mu_{i q_{\neq}}\right)\left(\sigma \mu_{q_{\neq} q_{>}}\right) \cup\left(v \mu_{i q_{>}}\right)\left(\sigma \mu_{q_{>} q_{>}}\right)= \\
= & (v f)\left\{x \in \Gamma^{*}:|\sigma f|<|x| \leq M\right\} \\
& \cup\left\{x \in \Gamma^{*}:|x|=|v f| \mathrm{e} x \neq v f\right\}\left\{x \in \Gamma^{*}:|\sigma f|<|x| \leq M\right\} \\
& \cup\left\{x \in \Gamma^{*}:|v f|<|x| \leq M|v|\right\}\left\{x \in \Gamma^{*}:|\sigma f| \leq|x| \leq M\right\} \\
= & \left\{x \in \Gamma^{*}:|u f|<|x| \leq M|u|\right\} \\
u \mu_{i q_{\neq}}= & \left(v \mu_{i q_{=}}\right)\left(\sigma \mu_{q_{=} q_{\neq}}\right) \cup\left(v \mu_{i q_{\neq}}\right)\left(\sigma \mu_{q_{\neq} q_{\neq}}\right) \\
= & (v f)\left\{x \in \Gamma^{*}:|x|=|\sigma f| \mathrm{e} x \neq \sigma f\right\} \\
& \cup\left\{x \in \Gamma^{*}:|x|=|v f| \text { e } x \neq v f\right\}\left\{x \in \Gamma^{*}:|x|=|\sigma f| \text { e } x \neq \sigma f\right\} \\
= & \left\{x \in \Gamma^{*}:|x|=|u f| \text { e } x \neq u f\right\} .
\end{aligned}
$$

A construção de um transdutor letra-palavra que realiza $\tau_{g}$ é similar.

\subsection{Métricas e diferenças}

Nesta seção, apresentamos alguns conceitos que serão utilizados em diversas partes da teoria subseqüente. Em particular, o material desta seção é um pré-requisito para a demonstração do teorema da decomposição de Weber, que será feita na próxima seção.

A notação definida em (1.3.1) será utilizada livremente.

Definição 3.6.1 Seja $U$ um subconjunto não-vazio de $\Sigma^{*}$. O prefixo de $U$, que denotamos por $\wedge U$, é o prefixo comum de maior comprimento das palavras de $U$. 
Se $U$ for um conjunto finito $\left\{x_{1}, \ldots, x_{n}\right\}$, podemos também escrever $\wedge U=x_{1} \wedge \cdots \wedge x_{n}$. Dada uma palavra $u$ em $U$, é fácil ver que $\wedge U$ é a palavra de menor comprimento do conjunto

$$
\{u \wedge v: v \in U\}
$$

Definição 3.6.2 A diferença de um par de palavras é uma função $\Delta: \Sigma^{*} \times \Sigma^{*} \rightarrow \Sigma^{*} \times \Sigma^{*}$, definida por

$$
(x, y) \Delta=\left((x \wedge y)^{-1} x,(x \wedge y)^{-1} y\right), \quad \forall(x, y) \in \Sigma^{*} \times \Sigma^{*}
$$

Dizemos que um par de palavras $(x, y)$ é uma diferença se $x \wedge y=1$.

Vamos também utilizar o conjunto

$$
\Delta_{\Sigma^{*}}=\left\{(x, y) \in \Sigma^{*} \times \Sigma^{*}: x \wedge y=1\right\}
$$

dos pares de palavras em $\Sigma^{*} \times \Sigma^{*}$ que são diferenças, e uma função

$$
\cdot \Delta_{\Sigma^{*}} \times\left(\Sigma^{*} \times \Sigma^{*}\right) \rightarrow \Delta_{\Sigma^{*}}
$$

definida por

$$
((u, v),(x, y)) \cdot=(u x, v y) \Delta, \quad \forall(u, v) \in \Delta_{\Sigma^{*}}, \forall(x, y) \in \Sigma^{*} \times \Sigma^{*} .
$$

Podemos escrever $((u, v),(x, y)) \cdot$ como $(u, v) \cdot(x, y)$. Essa notação se justifica porque - é uma ação de $\Sigma^{*} \times \Sigma^{*}$ no conjunto $\Delta_{\Sigma^{*}}$, conforme demonstrado a seguir.

Proposição 3.6.1 A função - é uma ação do monóide $\Sigma^{*} \times \Sigma^{*}$ no conjunto $\Delta_{\Sigma^{*}}$, ou seja, satisfaz

$$
\begin{gathered}
(u, v) \cdot(1,1)=(u, v) \\
(u, v) \cdot((x, y)(z, w))=((u, v) \cdot(x, y)) \cdot(z, w) .
\end{gathered}
$$

Demonstração. A primeira propriedade é imediata. Para demonstrar a segunda, vamos considerar três casos para $t=u x \wedge v y$ :

- $t \neq u x$ e $t \neq v y$ : então podemos fatorar $u x$ e $v y$ como $t \sigma x^{\prime}$ e $t \gamma y^{\prime}$, respectivamente, onde $\sigma$ e $\gamma$ são letras distintas. Assim,

$$
\begin{aligned}
\begin{aligned}
(u, v) \cdot(x z, y w)=\left(t \sigma x^{\prime} z, t \gamma y^{\prime} w\right) \Delta=\left(\sigma x^{\prime} z,\right. & \left.\gamma y^{\prime} w\right)= \\
& \left(\sigma x^{\prime}, \gamma y^{\prime}\right) \cdot(z, w)=((u, v) \cdot(x, y)) \cdot(z, w) .
\end{aligned}
\end{aligned}
$$

- $t=u x$ : então $u x \succ v y$, e podemos fatorar $v y$ como $u x x^{\prime}$. Assim,

$$
(u, v) \cdot(x z, y w)=\left(u x z, u x x^{\prime} w\right) \Delta=\left(z, x^{\prime} w\right) \Delta=\left(1, x^{\prime}\right) \cdot(z, w)=((u, v) \cdot(x, y)) \cdot(z, w) .
$$


- $t=v y$ : análogo ao caso anterior.

Definição 3.6.3 Vamos denotar por \|\|$: \Sigma^{*} \times \Sigma^{*} \rightarrow \mathbb{N}$ a função que associa cada par de palavras $(u, v)$ ao inteiro

$$
\|u, v\|=|u|+|v|-2|u \wedge v| .
$$

Em outras palavras, $\|u, v\|$ é a soma dos comprimentos dos sufixos de $u$ e $v$ obtidos após retirar-se o prefixo $u \wedge v$.

Podemos também escrever

$$
\|u, v\|=\left|u^{-1} v\right|
$$

onde consideramos $u$ e $v$ como elementos do grupo livre gerado por $\Sigma$.

Algumas propriedades dessa função, de fácil demonstração, são

$$
\begin{aligned}
\|u, u\| & =0, \\
\|u, v\| & =\|v, u\|, \\
\|u, v\| & \leq\|u, z\|+\|z, v\|, \quad \forall z \in \Sigma^{*}, \\
\|u, v\| & >0, \text { se } u \neq v, \\
\text { e }\|u, v\| & =\|z u, z v\|, \quad \forall z \in \Sigma^{*} .
\end{aligned}
$$

As quatro primeiras propriedades mostram que \|\| é uma métrica em $\Sigma^{*}$ (veja [Lim03] para uma definição). Essa métrica foi introduzida por Choffrut em 1978 [Cho78], em sua caracterização das funções subseqüenciais. Esse assunto será estudado no Capítulo 4.

Definição 3.6.4 Dado um morfismo de monóides $g: \Sigma^{*} \rightarrow \Gamma^{*}$, vamos denotar por \|\|$_{g}: \Sigma^{*} \times \Sigma^{*} \rightarrow$ $\mathbb{N} \cup \infty$ a função definida por

$$
\|u, v\|_{g}= \begin{cases}\max \{\|x g, y g\|: x \in \operatorname{pref}(u), y \in \operatorname{pref}(v) \text { e }|x|=|y|\} & \text { se ug=vg} \\ \infty & \text { caso contrário }\end{cases}
$$

As propriedades

$$
\begin{aligned}
\|u, u\|_{g} & =0, \\
\|u, v\|_{g} & =\|v, u\|_{g}, \\
\text { e }\|u, v\|_{g} & \leq\|u, z\|_{g}+\|z, v\|_{g}, \quad \forall z \in \Sigma^{*},
\end{aligned}
$$

também são de fácil verificação. Em (3.6.8c), estendemos a operação de adição em $\mathbb{N}$ para $\mathbb{N} \cup \infty$ da seguinte forma: $a+\infty=\infty+a=\infty$, para todo $a$ em $\mathbb{N} \cup \infty$. Também estendemos a ordem de $\mathbb{N}$ definindo $a \leq \infty$, para todo $a$ em $\mathbb{N} \cup \infty$. 
Será conveniente chamar $\|u, v\|_{g}$ de distância de $u$ a $v$.

A esparsidade com relação ao morfismo $g$ de um subconjunto $A$ de $\Sigma^{*}$ tal que card $(A)>1$ é definida como

$$
\|A\|_{g}=\min \left\{\|u, v\|_{g}: u, v \in A \text { e } u \neq v\right\} .
$$

Listamos a seguir algumas propriedades de fácil demonstração dos conceitos acima definidos, e que serão utilizadas livremente.

(3.6.9a) Para palavras $u$ e $v$ em $\Sigma^{*}, u=v$ se, e somente se, $(u, v) \Delta=(1,1)$.

(3.6.9b) Dados pares $(u, v)$ e $(x, y)$ em $\Sigma^{*} \times \Sigma^{*},(u, v) \Delta \cdot(x, y)=(u x, v y) \Delta$.

(3.6.9c) Sejam $u$ e $v$ palavras em $\Sigma^{*}$, e $(x, y)=(u, v) \Delta$. Então, $\|u, v\|=|x|+|y|$. Portanto, se $(u, v) \Delta \cdot(z, w)=(u, v) \Delta$, então $\|u, v\|=\|u z, v w\|$.

(3.6.9d) Sejam $u$ e $v$ palavras comparáveis por prefixo. Então, $\|u, v\|=\|u|-| v\|$.

Dados uma diferença $(u, v)$ em $\Delta_{\Sigma^{*}}$ e um par $(x, y)$ em $\Sigma^{*} \times \Sigma^{*}$, se $(u, v) \cdot(x, y)=$ $(u, v)$, então, para todo inteiro positivo $l,(u, v) \cdot(x, y)^{l}=(u, v)$.

Dadas diferenças $(u, v)$ e $(x, y)$ em $\Delta_{\Sigma^{*}}$, se $(u, v) \neq(x, y)$, então, para todo $(w, z)$ em $\Sigma^{*} \times \Sigma^{*},(u, v) \cdot(w, z) \neq(x, y) \cdot(w, z)$.

Dados uma diferença $(u, v)$ em $\Delta_{\Sigma^{*}}$ e um par $(x, y)$ em $\Sigma^{*} \times \Sigma^{*}$, se $(u, v) \cdot(x, y) \in$ $\left(\Sigma^{*} \times 1\right) \cup\left(1 \times \Sigma^{*}\right)$ então $(u, v) \in\left(\Sigma^{*} \times 1\right) \cup\left(1 \times \Sigma^{*}\right)$.

Concluímos a seção com duas propriedades que estão em um trabalho de Sakarovitch et al. [BCPS03].

Proposição 3.6.2 Sejam $(u, v)$ uma diferença em $\Delta_{\Sigma^{*}}$, e $(x, y)$ um par em $\Sigma^{*} \times \Sigma^{*}$ diferente de $(1,1)$. Então, $(u, v) \cdot(x, y)=(u, v)$ se, e somente se, uma das condições abaixo é satisfeita:

i. $u=1$, e existem palavras $r$ e $s$ e um inteiro $k$ tais que $x=r s, y=s r$, e $v=x^{k} r$

ii. $v=1$, e existem palavras $r$ e $s$ e um inteiro $k$ tais que $y=r s, x=s r$, e $u=y^{k} r$

Demonstração. Se a condição $i$ ou a $i$ for satisfeita, então a igualdade $(u, v) \cdot(x, y)=(u, v)$ é evidentemente verdadeira.

Reciprocamente, suponha que $(u, v) \cdot(x, y)=(u, v)$. Então, $|x|=|y|$, e, como $u \wedge v=1 \mathrm{e}$ $(x, y) \neq(1,1), u$ ou $v$ deve ser igual à palavra vazia.

Suponha que $u=1$. Seja $\nu$ um inteiro tal que $\left|x^{\nu}\right|>|v y|$. Então, por $(3.6 .9 \mathrm{e}),(1, v)=$ $(1, v) \cdot(x, y)^{\nu}$, ou seja $(1, v)=\left(x^{\nu}, v y^{\nu}\right) \Delta$. Logo, $v y \succ x^{\nu}$, o que implica nas fatorações em $i$.

O caso $v=1$ é análogo. 
Proposição 3.6.3 Sejam $(u, v)$ uma diferença em $\Delta_{\Sigma^{*}}, e(x, y)$ um par em $\Sigma^{*} \times \Sigma^{*}$ diferente de $(1,1)$. Se $(u, v) \cdot(x, y) \neq(u, v)$, então, para todo par de inteiros positivos distintos $k$ e $l$,

$$
(u, v) \cdot(x, y)^{k} \neq(u, v) \cdot(x, y)^{l} .
$$

Demonstração. Vamos considerar três possibilidades para as palavras $x, y, u$ e $v$.

- $|x| \neq|y|$ : sem perda de generalidade, suponha que $|y|>|x|$. Sejam $d=|y|-|x|$ e $\left(u^{\prime}, v^{\prime}\right)=$ $(u, v) \cdot(x, y)^{l}$. Então,

$$
\left|v^{\prime}\right|-\left|u^{\prime}\right|=|v|-|u|+l d,
$$

o que implica claramente em (3.6.10).

- $|x|=|y|, u \neq 1$ e $v \neq 1$ : como $u \wedge v=1$, podemos fatorar $u=\sigma u^{\prime}$ e $v=\gamma v^{\prime}$, onde $\sigma$ e $\gamma$ são letras distintas. Assim, para todo inteiro $l>0,(u, v) \cdot(x, y)^{l}=\left(u x^{l}, v y^{l}\right)$. Como $(x, y) \neq(1,1)$, obtemos $(3.6 .10)$.

- $|x|=|y|$ e $u=1$ : seja $\nu$ um inteiro tal que $\left|x^{\nu}\right|>|v y|$. Afirmamos que vy não é um prefixo de $x^{\nu}$. De fato, o contrário implica nas fatorações da condição $i$ da Proposição 3.6.2. Mas, essa condição contradiz a hipótese de que $(u, v) \cdot(x, y) \neq(u, v)$.

Portanto, existem uma letra $\sigma$ e um inteiro $\zeta$ tal que $v y$ pode ser fatorado como $x^{\zeta} x^{\prime} \sigma z$, onde $x^{\prime}$ é um prefixo próprio de $x$, e $x^{\prime} \sigma$ não é um prefixo de $x$.

Sem perda de generalidade, suponha que $k<l$. Vamos considerar três casos para esses inteiros.

$-k<\zeta$ e $l \leq \zeta$ : então,

$$
(u, v) \cdot(x, y)^{k}=\left(x^{k}, v y^{k}\right) \Delta=\left(1, x^{\zeta-k} x^{\prime} \sigma z y^{k-1}\right)
$$

$\mathrm{e}$

$$
(u, v) \cdot(x, y)^{l}=\left(x^{l}, v y^{l}\right) \Delta=\left(1, x^{\zeta-l} x^{\prime} \sigma z y^{l-1}\right) .
$$

Como $k<l$, podemos fatorar $x^{\zeta-k} \operatorname{como} x^{\zeta-l} x^{\zeta^{\prime}}$, onde $\zeta^{\prime}>0$. Como $x^{\prime} \sigma$ não é prefixo de $x$, segue que $x^{\zeta-k} x^{\prime} \sigma z y^{k-1} \neq x^{\zeta-l} x^{\prime} \sigma z y^{l-1}$, o que implica em (3.6.10).

- $k \leq \zeta$ e $l>\zeta$ : então, $(u, v) \cdot(x, y)^{k}$ é um par em $1 \times \Sigma^{*}$, e $(u, v) \cdot(x, y)^{l}$ é um par em $\Sigma^{+} \times \Sigma^{+}$, o que implica em (3.6.10).

$-k>\zeta$ e $l>\zeta$ : então, $(u, v) \cdot(x, y)^{k}=\left(x^{\prime-1} x x^{k-\zeta-1}, \sigma z y^{k-1}\right)$ e $(u, v) \cdot(x, y)^{l}=$ $\left(x^{\prime-1} x x^{l-\zeta-1}, \sigma z y^{l-1}\right)$. Como $k \neq l$, obtemos (3.6.10).

- $|x|=|y|$ e $v=1$ : análogo ao caso anterior. 


\subsection{Decomposição de uma relação racional $k$-valorada}

Nesta seção, demonstramos que uma relação racional $k$-valorada $\tau: \Sigma^{*} \rightarrow \Gamma^{*}$, para qualquer inteiro positivo $k$, pode ser decomposta numa união de $k$ funções racionais.

Esse problema começou a ser estudado em 1976 por Schützenberger [Sch76], mas uma demonstração completa só foi obtida por Weber em 1992 [Web92, Web93, Web96].

Nós apresentamos aqui uma demonstração diferente da de Weber, e que parece ser mais simples que a desse autor. Em linhas gerais, Weber utiliza em sua demonstração propriedades estruturais de transdutores. Nossa abordagem é mais algébrica.

Nossa estratégia será a seguinte.

Primeiro, vamos demonstrar o resultado para o caso em que $1 \tau=\varnothing$. Em seguida, observamos que esse caso implica facilmente no caso geral.

Feita essa hipótese, utilizamos o Teorema 3.2.1, e consideramos uma fatoração

$$
\tau: \Sigma^{*} \stackrel{f^{-1}}{\longrightarrow} \Omega^{*} \stackrel{\cap L}{\longrightarrow} \Omega^{*} \stackrel{g}{\longrightarrow} \Gamma^{*},
$$

onde $f$ é um morfismo muito fino, $L$ é uma linguagem reconhecível sobre um alfabeto $\Omega$, e $g$ é um morfismo.

Definimos então uma família $L_{\nu}, \nu \geq 0$, de subconjuntos reconhecíveis de $L$, cada uma satisfazendo

$$
f^{-1}(\cap L) g=f^{-1}\left(\cap L_{\nu}\right) g .
$$

Finalmente, obtemos uma decomposição aplicando $k$ vezes um teorema de Eilenberg em uma dessas linguagens, que possua determinadas propriedades especiais. Mais precisamente, mostramos que existe um índice $\nu$ tal que, a partir de uma linguagem reconhecível $L_{\nu^{\prime}}$, com $\nu^{\prime} \geq \nu$, é possível obter $k$ subconjuntos reconhecíveis especiais, que constituiem uma partição de $L_{\nu^{\prime}}$. A cada subconjunto assim obtido associamos uma função racional, e a união dessas funções é $\tau$.

Veremos também que autômatos reconhecendo as linguagens $L_{\nu}$ podem ser construídos efetivamente. Essa construção implica numa construção efetiva de uma decomposição de $\tau$ em funções racionais.

Toda a nossa discussão supõe fixada uma relação racional $k$-valorada $\tau$, uma fatoração de $\tau$ como em (3.7.1), e uma ordem lexicográfica $<_{l}$ em $\Omega^{*}$.

Se $r: X \rightarrow Y$ for uma função definida em um conjunto $X$, vamos denotar por

$$
X / r
$$

o quociente de $X$ pela relação de equivalência $\sim$ sobre $X$ definida por

$$
x \sim y \text { se, e somente se, } x r=y r .
$$

Ao longo desta seção, a notação introduzida na Seção 3.6 será utilizada livremente.

Começamos definindo a família $L_{\nu}, \nu \geq 0$. Observamos que a definição dessas linguagens, bem como os resultados preliminares antes do teorema principal, não exigem a hipótese de $\tau$ ser $k$-valorada, mas apenas finitamente valorada.

Definição 3.7.1 Para cada inteiro $\nu \geq 0$, vamos denotar por $L_{(f, g), \nu}$ o subconjunto de $L$ definido como segue. Uma palavra $u$ de $L$ pertence a $L_{(f, g), \nu}$ se, e somente se, para toda palavra $v$ em $L$ tal que $u(f, g)=v(f, g)$ e $v<_{l} u,\|u, v\|_{g} \geq \nu$. 
Enfatizamos que essa definição supõe fixados uma linguagem reconhecível $L$ em $\Omega^{*}$, uma ordem lexicográfica em $\Omega^{*}$, e um bimorfismo $(f, g)$, com $f$ muito fino. Quando não houver possibilidade de confusão, podemos escrever $L_{\nu}$ ao invés de $L_{(f, g), \nu}$.

Intuitivamente, palavras de $L_{\nu}$ com a mesma imagem pelo bimorfismo $(f, g)$ são "distantes" pela função \|\|$_{g}$, e um limitante inferior para essa distância é o índice $\nu$. Dizemos que as palavras com a mesma imagem por esse bimorfismo são redundantes.

Vemos da definição que, quanto maior o índice $\nu$, mais palavras redundantes de $L$ não pertencem a $L_{\nu}$. Assim, essas linguagens satisfazem

$$
L=L_{0} \supseteq L_{1} \supseteq \cdots \supseteq L_{\infty}
$$

onde $L_{\infty}$ denota o conjunto das palavras mínimas, segundo a ordem $<_{l}$, dos conjuntos do quociente $L /(f, g)$. Assim, $(f, g)$ restrito a $L_{\infty}$ é injetor. Isso não ocorre para uma linguagem $L_{\nu}$ se $L_{\nu} \neq L_{\infty}$.

Demonstramos a seguir algumas propriedades dessas linguagens. Vamos denotar por $\tau_{\nu}$ a composição

$$
\tau_{\nu}: \Sigma^{*} \stackrel{f^{-1}}{\longrightarrow} \Omega^{*} \stackrel{\cap L_{\nu}}{\longrightarrow} \Omega^{*} \stackrel{g}{\rightarrow} \Gamma^{*}
$$

Proposição 3.7.1 Para todo $\nu \in \mathbb{N} \cup \infty$,

$$
\tau=\tau_{\nu} .
$$

Demonstração. Seja $u$ uma palavra em $\Sigma^{*}$. Como $L_{\nu} \subseteq L$, segue que $u f^{-1}\left(\cap L_{\nu}\right) \subseteq u f^{-1}(\cap L)$. Portanto,

$$
u \tau_{\nu} \subseteq u \tau .
$$

Sejam agora $x$ uma palavra em $u \tau$, e $y$ a palavra mínima do conjunto $(u, x)(f, g)^{-1}$ pela ordem $<_{l}$. Então, pela definição de $L_{\nu}, y \in L_{\nu}$. Logo, $x \in u \tau_{\nu}$. Portanto,

$$
u \tau \subseteq u \tau_{\nu} .
$$

Assim, $\tau=\tau_{\nu}$.

Nem sempre $L_{\infty}$ é reconhecível, como mostra o exemplo a seguir.

Exemplo 3.7.1 Seja $M>1$ um inteiro. Considere os alfabetos

$$
\Sigma=\{a, b\}, \quad \Omega=\{\sigma, \gamma, \eta, \xi\}, \quad \Gamma=\{\theta\}
$$

a linguagem

$$
L=\sigma^{+} \gamma^{+} \cup \eta^{+} \xi^{+},
$$

o bimorfismo $(f, g): \Omega^{*} \rightarrow \Sigma^{*} \times \Gamma^{*}$ definido por

$$
\sigma(f, g)=(a, \theta), \quad \eta(f, g)=\left(a, \theta^{M}\right), \quad \gamma(f, g)=\left(b, \theta^{M}\right), \quad \xi(f, g)=(b, \theta)
$$


e a ordem lexicográfica

$$
\sigma<_{l} \gamma<_{l} \eta<_{l} \xi
$$

O domínio da relação $\tau: \Sigma^{*} \stackrel{f^{-1}}{\longrightarrow} \Omega^{*} \stackrel{\cap L}{\longrightarrow} \Omega^{*} \stackrel{g}{\rightarrow} \Gamma^{*}$ é

$$
L f=a^{+} b^{+} \text {. }
$$

Para todo $a^{i} b^{j} \in \operatorname{dom} \tau$

$$
a^{i} b^{j}\left(f^{-1}(\cap L) g\right)=\left\{\sigma^{i} \gamma^{j}, \eta^{i} \xi^{j}\right\} g=\left\{\theta^{i+j M}, \theta^{i M+j}\right\} .
$$

Portanto, $\tau$ é 2-valorada.

Se $i=j$, então

$$
\theta^{i+j M}=\theta^{i M+j}
$$

senão,

$$
\theta^{i+j M} \neq \theta^{i M+j} .
$$

Assim, para todo $i>0,\left(\sigma^{i} \gamma^{i}\right) g=\left(\eta^{i} \xi^{i}\right) g$, e, para $j \neq i,\left(\sigma^{i} \gamma^{j}\right) g \neq\left(\eta^{i} \xi^{j}\right) g$. Portanto, $L /(f, g)$ é composto de conjuntos da forma $\left\{\sigma^{i} \gamma^{i}, \eta^{i} \xi^{i}\right\}$ e de unitários das formas $\left\{\sigma^{i} \gamma^{j}\right\}$ e $\left\{\eta^{i} \xi^{j}\right\}$, com $i \neq j$. Considerando as palavras mínimas desses conjuntos segundo $<_{l}$, obtemos

$$
L_{\infty}=\left\{\sigma^{i} \gamma^{j}: i>0 \text { e } j>0\right\} \cup\left\{\eta^{i} \xi^{j}: i>0, j>0 \text { e } i \neq j\right\}
$$

que não é reconhecível.

Vamos mostrar que, para todo inteiro $\nu \geq 1$, a linguagem $L_{\nu}$ é reconhecível. A demonstração consiste numa construção efetiva de um autômato que reconhece $L_{\nu}$ a partir de um autômato reconhecendo $L$.

Proposição 3.7.2 Para todo inteiro $\nu \geq 1$, o conjunto $L_{\nu}$ é reconhecivel.

Demonstração. Seja $\mathcal{A}=(Q, E, i, T)$ um $\Omega$-autômato determinístico e bi-acessível, reconhecendo $L$. Para cada inteiro $\nu \geq 1$, definimos um $\Omega$-autômato determinístico acessível $\mathcal{A}_{\nu}=$ $\left(Q_{\nu}, E_{\nu}, i_{\nu}, T_{\nu}\right)$ como segue.

- Cada estado de $Q_{\nu}$ é a união de um estado de $Q$ com um subconjunto (possivelmente vazio) de $(Q \times \Omega \leq \nu-1) \cup(\Omega \leq \nu-1 \times Q)$.

- $i_{\nu}$ é o unitário $i$.

- $T_{\nu}$ é o conjunto dos estados que contêm um estado de $T$, e que não contêm nenhum par em $T \times 1$.

- As transições com origem em um estado

$$
P=\left\{q,\left(p_{1}, u_{1}\right), \ldots,\left(p_{r}, u_{r}\right),\left(v_{1}, q_{1}\right), \ldots,\left(v_{s}, q_{s}\right)\right\},
$$

onde

$$
\left(p_{j}, u_{j}\right) \in\left(Q \times \Omega^{\leq \nu-1}\right) \text { e }\left(v_{l}, q_{l}\right) \in\left(\Omega^{\leq \nu-1} \times Q\right), \quad \forall j, 1 \leq j \leq r, \forall l, 1 \leq l \leq s,
$$

são definidas como segue. Para cada letra $\sigma \operatorname{em} \Omega$, se $q \sigma=\varnothing$, então $P \sigma=\varnothing$. Caso contrário, $P \sigma$ é a união de $q \sigma$ com os pares descritos a seguir. 
- Para cada $\gamma \in \Omega$ tal que $q \gamma \neq \varnothing, \gamma<_{l} \sigma$ e $\sigma f=\gamma f$, se

$$
\sigma g \succ \gamma g \quad \text { e } \quad|\gamma g|-|\sigma g|<\nu,
$$

então

$$
\left(q \gamma,(\sigma g)^{-1}(\gamma g)\right) \in P \sigma
$$

se

$$
\gamma g \succ \sigma g \quad \text { e } \quad 0<|\sigma g|-|\gamma g|<\nu
$$

então

$$
\left((\gamma g)^{-1}(\sigma g), q \gamma\right) \in P \sigma .
$$

- Para cada $\left(p_{j}, u_{j}\right) \in P$ e cada $\gamma \in \Omega$ tal que $p_{j} \gamma \neq \varnothing$ e $\sigma f=\gamma f$, se

$$
\sigma g \succ u_{j}(\gamma g) \text { e }\left|u_{j}(\gamma g)\right|-|\sigma g|<\nu,
$$

então

$$
\left(p_{j} \gamma,(\sigma g)^{-1}\left(u_{j}(\gamma g)\right)\right) \in P \sigma
$$

se

$$
u_{j}(\gamma g) \succ \sigma g \quad \text { e } \quad 0<|\sigma g|-\left|u_{j}(\gamma g)\right|<\nu,
$$

então

$$
\left(\left(u_{j}(\gamma g)\right)^{-1}(\sigma g), p_{j} \gamma\right) \in P \sigma .
$$

- Para cada $\left(v_{l}, q_{l}\right) \in P$ e cada $\gamma \in \Omega$ tal que $q_{l} \gamma \neq \varnothing$ e $\sigma f=\gamma f$, se

$$
v_{l}(\sigma g) \succ \gamma g \text { e }|\gamma g|-\left|v_{l}(\sigma g)\right|<\nu
$$

então

$$
\left(q_{l} \gamma,\left(v_{l}(\sigma g)\right)^{-1}(\gamma g)\right) \in P \sigma
$$

se

$$
\gamma g \succ v_{l}(\sigma g) \text { e } 0<\left|v_{l}(\sigma g)\right|-|\gamma g|<\nu,
$$

então

$$
\left((\gamma g)^{-1}\left(v_{l}(\sigma g)\right), q_{l} \gamma\right) \in P \sigma .
$$

Vamos explicar o funcionamento de $\mathcal{A}_{\nu}$ descrevendo as informações contidas em um estado acessível $i_{\nu} u$ desse autômato, onde $u$ é uma palavra qualquer de $\Omega^{*}$. A demonstração de que $i_{\nu} u$ de fato contém essas informações pode ser feita por indução em $|u|$. Vamos omitir essa demonstração porque ela é puramente técnica, e utiliza apenas as definições das transições de $\mathcal{A}_{\nu}$.

Os pares em $\left(Q \times \Omega^{\leq \nu-1}\right) \cup\left(\Omega^{\leq \nu-1} \times Q\right)$ representam diferenças entre $u g$ e as imagens por $g$ de determinadas palavras de $\Omega^{*}$ cujas imagens por $f$ são iguais a $u f$. Mais precisamente, o estado $i_{\nu} u$ é a união do estado $i u$ de $\mathcal{A}$ com os seguintes pares. Para toda palavra $v$ em $\Omega^{*}$ satisfazendo

$$
\begin{aligned}
& i v \neq \varnothing, \\
& v<_{l} u, \\
& u f=v f, \\
& \quad u g \succ v g \text { ou } v g \succ u g \\
& \text { e }\|u, v\|_{g}<\nu,
\end{aligned}
$$


se $u g \succ v g$, então $i_{\nu} u$ tem um par

$$
\left(i v,(u g)^{-1}(v g)\right),
$$

e se $v g \succ u g$ e $v g \neq u g$, então $i_{\nu} u$ tem um par

$$
\left((v g)^{-1}(u g), i v\right) .
$$

Pela definição de $T_{\nu}, i_{\nu} u$ é final se, e somente se, $u$ pertence a $L$ (ou seja, iu pertence a $T$ ), e não existe uma palavra $v$ tal que

$$
\begin{aligned}
& v \in L, \\
& v<_{l} u, \\
& \\
& u(f, g)=v(f, g) \\
& \text { e }\|u, v\|_{g}<\nu .
\end{aligned}
$$

Da definição de $L_{\nu}$, segue então que

$$
\left|\mathcal{A}_{\nu}\right|=L_{\nu}
$$

Utilizando as linguagens $L_{\nu}$, vamos demonstrar uma versão mais fraca do seguinte resultado de Eilenberg [Eil74], conhecido como Teorema Cross-section:

Teorema 3.7.1 (Eilenberg 1974) Sejam $f: \Sigma^{*} \rightarrow \Gamma^{*}$ um morfismo e $L$ uma linguagem reconhecível sobre $\Sigma$. Então, existe um subconjunto reconhecivel $L^{\prime}$ de $L$ tal que $L^{\prime} f=L f$, ef restrito a $L^{\prime}$ é injetor.

O Teorema 3.7.1, ainda que importante, não será demonstrado aqui, porque nossa versão será suficiente para demonstrar o teorema da decomposição. Uma outra referência sobre o Teorema Cross-section é o livro de Berstel [Ber79].

Proposição 3.7.3 Sejam $f: \Sigma^{*} \rightarrow \Gamma^{*}$ um morfismo muito fino e $L$ uma linguagem reconhecível sobre $\Sigma$. Então, existe um subconjunto reconhecivel $L^{\prime}$ de $L$ tal que $L^{\prime} f=L f$, e $f$ restrito a $L^{\prime}$ é injetor.

Demonstração. Sejam $<_{l}$ uma ordem lexicográfica em $\Sigma^{*}$ e $L^{\prime}$ a linguagem contendo as palavras mínimas, segundo $<_{l}$, dos conjuntos no quociente $L / f$. Então, $L^{\prime} f=L f$, e $f$ restrito a $L^{\prime}$ é injetor. Vamos mostrar que $L^{\prime}$ é reconhecível.

Sejam $a$ uma letra e $h: \Sigma^{*} \rightarrow a^{*}$ o morfismo definido por

$$
\sigma h=a, \quad \forall \sigma \in \Sigma .
$$

Da definição de $h$ e da hipótese de que $f$ é um morfismo muito fino, segue que $L / f=L /(h, f)$. Sejam $C$ uma classe nesse quociente, $x$ a palavra mínima em $C$ segundo a ordem $<_{l}$, e $y$ uma palavra qualquer em $C$, e diferente de $x$. Como $f$ é um morfismo muito fino, $\|x, y\|_{f}=0$. Logo, $y$ não pertence a $L_{(h, f), 1}$. Portanto,

$$
L^{\prime}=L_{(h, f), 1}
$$


Utilizando a Proposição 3.7.2, concluímos que $L^{\prime}$ é reconhecível.

Dizemos que o conjunto $L^{\prime}$ é uma cross-section de $L$ com $f$. Um exemplo da construção efetiva de uma cross-section será apresentado na Seção 3.9.

Sempre que a Proposição 3.7.3 for utilizada, vamos supor que $L^{\prime}$ é a linguagem construída na demonstração (ou seja, a linguagem contendo as palavras mínimas, segundo $<_{l}$, dos conjuntos do quociente $L / f$ ).

Através de um exemplo, vamos ilustrar como obter uma decomposição de uma relação racional $k$-valorada em $k$ funções racionais aplicando os conceitos introduzidos nesta seção.

Exemplo 3.7.2 Dados inteiros $M \geq 1$ e $t \geq 2$, considere os alfabetos

$$
\Omega=\{\sigma, \gamma, \xi\}, \Sigma=\{a\} \text { e } \Gamma=\{\theta\}
$$

a linguagem

$$
\begin{aligned}
& L=\left(\sigma^{2 t}\right)^{+} \cup\left(\gamma^{t} \xi^{t}\right)^{+} \cup\left(\xi^{t} \gamma^{t}\right)^{+} \\
& \quad \cup\left(\sigma^{t} \gamma^{t}\right)^{+} \sigma \cup\left(\gamma^{t} \sigma^{t}\right)^{+} \sigma \cup\left(\xi^{2 t}\right)^{+} \xi \\
& \quad \cup\left(\sigma^{t} \xi^{t}\right)^{+} \xi^{2} \cup\left(\gamma^{2 t}\right)^{+} \gamma^{2} \cup\left(\xi^{t} \sigma^{t}\right)^{+} \xi^{2},
\end{aligned}
$$

o morfismo muito fino $f: \Omega^{*} \rightarrow \Sigma^{*}$, e o morfismo $g: \Omega^{*} \rightarrow \Gamma^{*}$, definidos por

$$
\begin{gathered}
\sigma f=\gamma f=\xi f=a, \\
\sigma g=1, \gamma g=\theta^{M}, \xi g=\theta^{2 M} .
\end{gathered}
$$

O domínio da relação

$$
\tau: \Sigma^{*} \stackrel{f^{-1}}{\longrightarrow} \Omega^{*} \stackrel{\cap L}{\longrightarrow} \Omega^{*} \stackrel{g}{\longrightarrow} \Gamma^{*}
$$

é

$$
L f=\left(a^{2 t}\right)^{+}\left(1 \cup a \cup a^{2}\right),
$$

e as imagens são

$$
\begin{aligned}
& a^{2 t l} \tau=a^{2 t l}\left(f^{-1}(\cap L) g\right)=\left\{\sigma^{2 t l},\left(\gamma^{t} \xi^{t}\right)^{l},\left(\xi^{t} \gamma^{t}\right)^{l}\right\} g=\left\{1, \theta^{3 M t l}\right\}, \\
& a^{2 t l+1} \tau=\left\{\left(\sigma^{t} \gamma^{t}\right)^{l} \sigma,\left(\gamma^{t} \sigma^{t}\right)^{l} \sigma, \xi^{2 t l+1}\right\} g=\left\{\theta^{M t l}, \theta^{2 M(2 t l+1)}\right\}, \\
& a^{2 t l+2} \tau=\left\{\left(\xi^{t} \sigma^{t}\right)^{l} \xi^{2},\left(\sigma^{t} \xi^{t}\right)^{l} \xi^{2}, \gamma^{2 t l+2}\right\} g=\left\{\theta^{2 M(t l+2)}, \theta^{M(2 t l+2)}\right\},
\end{aligned}
$$

para todo $l \geq 1$. Portanto, $\tau$ é 2 -valorada.

A tabela a seguir exibe as imagens por $f$ e por $g$ de alguns subconjuntos de $L$ em separado, tornando mais clara a estrutura de $\tau$.

$$
\begin{array}{l|l|l}
\left(a^{2 t}\right)^{+} \stackrel{f}{\leftarrow}\left(\sigma^{2 t}\right)^{+} \stackrel{g}{\rightarrow} 1 & \left(a^{2 t}\right)^{+} a \stackrel{f}{\leftarrow}\left(\sigma^{t} \gamma^{t}\right)^{+} \sigma \stackrel{g}{\rightarrow}\left(\theta^{M t}\right)^{+} & \left(a^{2 t}\right)^{+} a^{2} \stackrel{f}{\leftarrow}\left(\sigma^{t} \xi^{t}\right)^{+} \xi^{2} \stackrel{g}{\rightarrow}\left(\theta^{2 M t}\right)^{+} \theta^{4 M} \\
\left(a^{2 t}\right)^{+} \stackrel{f}{\leftarrow}\left(\gamma^{t} \xi^{t}\right)^{+} \stackrel{g}{\rightarrow}\left(\theta^{3 M t}\right)^{+} & \left(a^{2 t}\right)^{+} a \stackrel{f}{\leftarrow}\left(\gamma^{t} \sigma^{t}\right)^{+} \sigma \stackrel{g}{\rightarrow}\left(\theta^{M t}\right)^{+} & \left(a^{2 t}\right)^{+} a^{2} \stackrel{f}{\leftarrow}\left(\gamma^{2 t}\right)^{+} \gamma^{2} \stackrel{g}{\rightarrow}\left(\theta^{2 M t}\right)^{+} \theta^{2 M} \\
\left(a^{2 t}\right)^{+} \stackrel{f}{\leftarrow}\left(\xi^{t} \gamma^{t}\right)^{+} \stackrel{g}{\rightarrow}\left(\theta^{3 M t}\right)^{+} & \left(a^{2 t}\right)^{+} a \stackrel{f}{\leftarrow}\left(\xi^{2 t}\right)^{+} \xi \stackrel{g}{\rightarrow}\left(\theta^{4 M t}\right)^{+} \theta^{2 M} & \left(a^{2 t}\right)^{+} a^{2} \stackrel{f}{\leftarrow}\left(\xi^{t} \sigma^{t}\right) \xi^{2} \stackrel{g}{\rightarrow}\left(\theta^{2 M t}\right)^{+} \theta^{4 M}
\end{array}
$$

A idéia básica da decomposição é aplicar a Proposição 3.7 .3 repetidamente a partir de um determinado subconjunto reconhecível de $L$, utilizando o morfismo $f$, de modo a obter crosssections duas a duas disjuntas. Cada cross-section corresponderá a uma função. 
Vamos primeiro tentar obter uma decomposição de $\tau$ aplicando a Proposição $3.7 .3 \mathrm{em} L$ com $f$. Considere a seguinte ordem lexicográfica:

$$
\sigma<_{l} \gamma<_{l} \xi
$$

Observando (3.7.4), temos que a primeira cross-section é

$$
A_{1}=\left(\sigma^{2 t}\right)^{+} \cup\left(\sigma^{t} \gamma^{t}\right)^{+} \sigma \cup\left(\sigma^{t} \xi^{t}\right)^{+} \xi^{2} .
$$

Como $f$ restrito a $A_{1}$ é injetor, segue que $\tau_{1}: \Sigma^{*} \stackrel{f^{-1}}{\longrightarrow} \Omega^{*} \stackrel{\cap A_{1}}{\longrightarrow} \Omega^{*} \stackrel{g}{\longrightarrow} \Gamma^{*}$ é uma função. Ao aplicar a Proposição 3.7 .3 no conjunto $L-A_{1}$, utilizando $f$, obtemos a cross-section

$$
A_{2}=\left(\gamma^{t} \xi^{t}\right)^{+} \cup\left(\gamma^{t} \sigma^{t}\right)^{+} \sigma \cup\left(\gamma^{2 t}\right)^{+} \gamma^{2}
$$

e a função $\tau_{2}: \Sigma^{*} \stackrel{f^{-1}}{\longrightarrow} \Omega^{*} \stackrel{\cap A_{2}}{\longrightarrow} \Omega^{*} \stackrel{g}{\longrightarrow} \Gamma^{*}$. Mas, $\tau \neq \tau_{1} \cup \tau_{2}$, pois

$$
a^{2 t l+1}\left(\tau_{1} \cup \tau_{2}\right)=\left\{\left(\sigma^{t} \gamma^{t}\right)^{l} \sigma,\left(\gamma^{t} \sigma^{t}\right)^{l} \sigma\right\} g=\left\{\theta^{M t l}\right\} \neq a^{2 t l+1} \tau,
$$

para todo $l \geq 1$.

Esse problema ocorre porque as cross-sections escolhidas podem conter palavras redundantes, ou seja, com a mesma imagem por $f$ e por $g$, mas não conter outras essenciais. Na tentativa acima, os subconjuntos

$$
B_{1}=\left(\sigma^{t} \gamma^{t}\right)^{+} \sigma \subsetneq A_{1} \quad \text { e } \quad B_{2}=\left(\gamma^{t} \sigma^{t}\right)^{+} \sigma \subsetneq A_{2}
$$

são tais que

$$
B_{1} f=B_{2} f=\left(a^{2 t}\right)^{+} a \quad \text { e } \quad B_{1} g=B_{2} g=\left(\theta^{M t}\right)^{+} .
$$

O subconjunto $\left(\theta^{4 M t}\right)^{+} \theta^{2 M} \subseteq\left(\left(a^{2 t}\right)^{+} a\right) \tau$ só pode ser obtido como imagem por $g$ do subconjunto $\left(\xi^{2 t}\right)^{+} \xi$, que não é considerado nem em $A_{1}$, e nem em $A_{2}$. Ou seja,

$$
\left(\xi^{2 t}\right)^{+} \xi \cap\left(A_{1} \cup A_{2}\right)=\varnothing .
$$

Ademais, esse problema persiste mesmo alterando a ordem lexicográfica das letras de $\Omega$.

A observação central da nossa demonstração é que um dos subconjuntos $B_{1}$ ou $B_{2}$ pode ser eliminado antes do cálculo das cross-sections. Para isso, utilizamos os conjuntos $L_{\nu}$. A idéia é que, para $\nu$ suficientemente grande, um dos subconjuntos redundantes é eliminado, porque palavras de $B_{1}$ e $B_{2}$ com a mesma imagem por $f$ são "próximas" pela função \|\|$_{g}$.

De fato, para todo $l \geq 1$,

$$
\left\|\left(\sigma^{t} \gamma^{t}\right)^{l} \sigma,\left(\gamma^{t} \sigma^{t}\right)^{l} \sigma\right\|_{g}=\left\|\left(\sigma^{t} \gamma^{t}\right) \sigma,\left(\gamma^{t} \sigma^{t}\right) \sigma\right\|_{g}=t M .
$$

Isso sugere considerar o conjunto

$$
\begin{aligned}
L_{t M+1} & =\left(\sigma^{2 t}\right)^{+} \cup\left(\gamma^{t} \xi^{t}\right)^{+} \\
& \cup\left(\sigma^{t} \gamma^{t}\right)^{+} \sigma \cup\left(\xi^{2 t}\right)^{+} \xi \\
& \cup\left(\sigma^{t} \xi^{t}\right)^{+} \xi^{2} \cup\left(\gamma^{2 t}\right)^{+} \gamma^{2} \cup\left(\xi^{t} \sigma^{t}\right)^{+} \xi^{2},
\end{aligned}
$$

que não contém nenhuma palavra de $B_{2}$. Aplicando a Proposição 3.7.3, obtemos então as crosssections

$$
A_{1}=\left(\sigma^{2 t}\right)^{+} \cup\left(\sigma^{t} \gamma^{t}\right)^{+} \sigma \cup\left(\sigma^{t} \xi^{t}\right)^{+} \xi^{2} \text { e } A_{2}^{\prime}=\left(\gamma^{t} \xi^{t}\right)^{+} \cup\left(\xi^{2 t}\right)^{+} \xi \cup\left(\gamma^{2 t}\right)^{+} \gamma^{2}
$$


e a união das funções $\tau_{1}: \Sigma^{*} \stackrel{f^{-1}}{\longrightarrow} \Omega^{*} \stackrel{\cap A_{1}}{\longrightarrow} \Omega^{*} \stackrel{g}{\longrightarrow} \Gamma^{*}$ e $\tau_{2}^{\prime}: \Sigma^{*} \stackrel{f^{-1}}{\longrightarrow} \Omega^{*} \stackrel{\cap A_{2}^{\prime}}{\longrightarrow} \Omega^{*} \stackrel{g}{\longrightarrow} \Gamma^{*}$ é $\tau$.

Podemos observar também neste exemplo que a família $L_{\nu}, \nu \geq 0$, consiste apenas de três linguagens:

$$
L_{0}=L, \quad L_{t M+1} \quad \text { e } \quad L_{2 t M+1}=L_{t M+1}-\left(\xi^{t} \sigma^{t}\right)^{+} \xi^{2}=L_{\infty} .
$$

Vamos demonstrar dois lemas técnicos antes do resultado principal.

Lema 3.7.1 Seja $g: \Omega^{*} \rightarrow \Gamma^{*}$ um morfismo de monóides livres. Então, para todo par de palavras $u$ e $v$ de $\Omega^{*}$, e todas as fatoraçóes xyz de $u$ e rst de $v$ com $|x|=|r|$ e $|y|=|s|$,

i. se $u g=v g, e(x g, r g) \Delta=(x g, r g) \Delta \cdot(y g, s g)$, então, para todo $l \geq 1,\left(x y^{l} z\right) g=\left(r s^{l} t\right) g$ e $\left\|x y^{l} z, r s^{l} t\right\|_{g}=\|u, v\|_{g}$

ii. se $u g \neq v g$, então existe um inteiro $l_{0}$ tal que, para todo $l \geq l_{0},\left(x y^{l} z\right) g \neq\left(r s^{l} t\right) g$.

Demonstração. Suponha as hipóteses em $i$. Então, para todo $l \geq 1$,

$$
(x g, r g) \Delta=(x g, r g) \Delta \cdot(y g, s g)^{l}=\left(x g(y g)^{l}, r g(s g)^{l}\right) \Delta=\left(\left(x y^{l}\right) g,\left(r s^{l}\right) g\right) \Delta .
$$

Como $u g=v g,((x y) g,(r s) g) \Delta \cdot(z g, t g)=(1,1)$. Portanto,

$$
\left(\left(x y^{l}\right) g,\left(r s^{l}\right) g\right) \Delta \cdot(z g, t g)=(1,1)
$$

o que implica em $\left(\left(x y^{l} z\right) g,\left(r s^{l} t\right) g\right) \Delta=(1,1)$. Obtemos assim que $\left(x y^{l} z\right) g=\left(r s^{l} t\right) g$.

Para mostrar que $\left\|x y^{l} z, r s^{l} t\right\|_{g}=\|u, v\|_{g}$, vamos mostrar que as distâncias pela métrica \|\| entre as imagens por $g$ dos prefixos $w_{1} \succ x y^{l} z$ e $w_{2} \succ r s^{l} t$ de mesmo comprimento correspondem a distâncias entre as imagens por $g$ de determinados prefixos de $u$ e $v$. O caso em que $w_{1}$ e $w_{2}$ são prefixos de $x$ e $r$, respectivamente, é evidente. Vamos considerar outros dois casos. que

Tome primeiro prefixos da forma $w_{1}=x y^{l} z^{\prime}$ e $w_{2}=r s^{l} t^{\prime}$, onde $z^{\prime} \succ z, t^{\prime} \succ t$ e $\left|z^{\prime}\right|=\left|t^{\prime}\right|$. Note

$$
\begin{aligned}
& \left(\left(x y^{l} z^{\prime}\right) g,\left(r s^{l} t^{\prime}\right) g\right) \Delta=(x g, r g) \Delta \cdot\left(y^{l} g, s^{l} g\right) \cdot\left(z^{\prime} g, t^{\prime} g\right)= \\
& (x g, r g) \Delta \cdot(y g, s g) \cdot\left(z^{\prime} g, t^{\prime} g\right)=\left(\left(x y z^{\prime}\right) g,\left(r s t^{\prime}\right) g\right) \Delta .
\end{aligned}
$$

De (3.6.9c), segue então que

$$
\left\|w_{1} g, w_{2} g\right\|=\left\|\left(x y^{l} z^{\prime}\right) g,\left(r s^{l} t^{\prime}\right) g\right\|=\left\|\left(x y z^{\prime}\right) g,\left(r s t^{\prime}\right) g\right\| .
$$

Tome agora prefixos da forma $w_{1}=x y^{l^{\prime}} y^{\prime}$ e $w_{2}=r s^{l^{\prime}} s^{\prime}$, onde $l^{\prime}<l, y^{\prime} \succ y, s^{\prime} \succ s$ e $\left|y^{\prime}\right|=\left|s^{\prime}\right|$. Notando que $\left(\left(x y^{l^{\prime}} y^{\prime}\right) g,\left(r s^{l^{\prime}} s^{\prime}\right) g\right) \Delta=\left(\left(x y^{\prime}\right) g,\left(r s^{\prime}\right) g\right) \Delta$ e repetindo o argumento anterior, obtemos

$$
\left\|w_{1} g, w_{2} g\right\|=\left\|\left(x y^{\prime}\right) g,\left(r s^{\prime}\right) g\right\| .
$$

Temos então a igualdade

$\left\{\left\|w_{1} g, w_{2} g\right\|: w_{1} \succ u, w_{2} \succ v\right.$ e $\left.\left|w_{1}\right|=\left|w_{2}\right|\right\}=\left\{\left\|w_{1} g, w_{2} g\right\|: w_{1} \succ x y^{l} z, w_{2} \succ r s^{l} t\right.$ e $\left.\left|w_{1}\right|=\left|w_{2}\right|\right\}$, 
que implica em $\left\|x y^{l} z, r s^{l} t\right\|_{g}=\|u, v\|_{g}$.

Vamos agora demonstrar $i$.

Se $(x y) g$ e $(r s) g$ não forem comparáveis pela ordem $\succ$, então evidentemente $\left(x y^{l} z\right) g \neq\left(r s^{l} t\right) g$, para todo $l \geq 1$.

Suponha que essas palavras sejam comparáveis por essa ordem, e que $(x g, r g) \Delta=(x g, r g) \Delta$. $(y g, s g)$. Então, para todo $l \geq 1,\left(\left(x y^{l}\right) g,\left(r s^{l}\right) g\right) \Delta=((x y) g,(r s) g) \Delta$. Como $u g \neq v g$, concluímos que $((x y) g,(r s) g) \Delta \cdot(z g, t g) \neq(1,1)$. Portanto,

$$
\left(\left(x y^{l}\right) g,\left(r s^{l}\right) g\right) \Delta \cdot(z g, t g) \neq(1,1),
$$

o que implica em $\left(x y^{l} z\right) g \neq\left(r s^{l} t\right) g$.

Suponha agora que $(x g, r g) \Delta \neq(x g, r g) \Delta \cdot(y g, s g)$. Vamos considerar dois casos para os comprimentos $|y g|$ e $|s g|$.

- $|y g| \neq|s g|$ : suponha que $|y g|>|s g|$. Então, existe um inteiro $l_{0}$ tal que

$$
\left|\left(x y^{l_{0}} z\right) g\right|>\left|\left(r s^{l_{0}} t\right) g\right| \text {. }
$$

Assim, para todo $l \geq l_{0}$,

$$
\left|\left(x y^{l} z\right) g\right|>\left|\left(r s^{l} t\right) g\right|,
$$

ou seja, $\left(x y^{l} z\right) g \neq\left(r s^{l} t\right) g$. O caso $|s g|>|y g|$ é análogo.

- $|y g|=|s g|:$ suponha que $x g \succ r g$. De $(x g, r g) \Delta \neq(x g, r g) \Delta \cdot(y g, s g)$, segue que $y g$ e $s g$ são diferentes da palavra vazia. Seja então $l_{0}$ o menor inteiro tal que $\left|\left(x y^{l_{0}}\right) g\right| \geq|(r s) g|$. Se as palavras $\left(x y^{l_{0}}\right) g$ e $(r s) g$ forem comparáveis, obtemos as fatorações

$$
r g=(x g)\left(y^{l_{0}-2} g\right) y^{\prime}, s g=y^{\prime \prime} y^{\prime}, y g=y^{\prime} y^{\prime \prime},
$$

o que implica em $(x g, r g) \Delta=(x g, r g) \Delta \cdot(y g, s g)$. Portanto, essas palavras não são comparáveis. Assim, para todo $l \geq l_{0}$,

$$
\left(\left(x y^{l} z\right) g,\left(r s^{l} t\right) g\right) \Delta \neq(1,1),
$$

o que implica em $\left(x y^{l} z\right) g \neq\left(r s^{l} t\right) g$. O caso $r g \succ x g$ é análogo.

Recordando a fatoração (3.7.1), vamos investigar como determinados subconjuntos finitos de $L$ implicam na existência de outros subconjuntos de mesma cardinalidade, mas com um número maior de elementos na imagem por $g$. Vamos denotar

$$
M=\max \{\sigma g: \sigma \in \Omega\} .
$$

Lema 3.7.2 Seja $\mathcal{A}$ um $\Omega$-autômato com $n$ estados que reconhece $L$. Se existir um subconjunto finito $A$ de $L$ tal que

$$
\operatorname{card}(A f)=1 \quad e\|A\|_{g} \geq n^{\operatorname{card}(A)} M,
$$

então existe um subconjunto $B$ de $L$ satisfazendo

$$
\operatorname{card}(B)=\operatorname{card}(A), \operatorname{card}(B f)=1 e \operatorname{card}(B g)=\operatorname{card}(B) .
$$


Demonstração. Observamos primeiro que a condição card $(A f)=1$ implica que todas as palavras de $A$ têm o mesmo comprimento, porque $f$ é um morfismo muito fino. Nossa demonstração utiliza indução em $\theta=\operatorname{card}(A)-\operatorname{card}(A g)$.

- $\theta=0$ : basta tomar $B=A$.

- $\theta>0$ : seja $\zeta=\operatorname{card}(A)$. Como $\theta>0$, existem palavras distintas $u_{a}$ e $u_{b}$ em $A$ tais que $u_{a} g=u_{b} g$. Então, pelas hipóteses sobre $A,\left\|u_{a}, u_{b}\right\|_{g} \geq n^{\zeta} M$, e pela definição de \|\|$_{g}$, existem prefixos $u_{a}^{\prime} \succ u_{a}$ e $u_{b}^{\prime} \succ u_{b}$ de mesmo comprimento satisfazendo

$$
\left\|u_{a}^{\prime} g, u_{b}^{\prime} g\right\| \geq n^{\zeta} M
$$

Como $u_{a} g=u_{b} g$, as imagens $u_{a}^{\prime} g$ e $u_{b}^{\prime} g$ devem ser comparáveis pela ordem do prefixo. Assim,

$$
\left\|u_{a}^{\prime} g, u_{b}^{\prime} g\right\|=\left\|u_{a}^{\prime} g|-| u_{b}^{\prime} g\right\| .
$$

Sem perda de generalidade, suponha que $\left|u_{b}^{\prime} g\right|<\left|u_{a}^{\prime} g\right|$, e seja $l$ o número de inteiros $i$ entre 1 e $\left|u_{a}^{\prime}\right|$ tais que $\left|\left(u_{b}^{\prime}[i]\right) g\right| \leq\left|\left(u_{a}^{\prime}[i]\right) g\right|$. Como $\left|\left(u_{a}^{\prime}[i]\right) g\right|-\left|\left(u_{b}^{\prime}[i]\right) g\right| \leq M$, segue que $l \geq n^{\zeta}$. Portanto,

$$
\left|u_{a}^{\prime}\right| \geq n^{\zeta}
$$

Vamos definir um autômato $\mathcal{A}^{\zeta}$ cujos passeios correspondem a $\zeta$ passeios simultâneos em $\mathcal{A}$. Os estados de $\mathcal{A}^{\zeta}$ são $\zeta$-uplas de estados de $\mathcal{A}$, e os rótulos das transições são $\zeta$-uplas de letras em $\Sigma$. Esse autômato tem uma transição

$$
\left(p_{1}, \ldots, p_{\zeta}\right) \stackrel{\left(\sigma 1, \ldots, \sigma_{\zeta}\right)}{\longrightarrow}\left(q_{1}, \ldots, q_{\zeta}\right)
$$

se, e somente se, existem transições

$$
p_{i} \stackrel{\sigma_{i}}{\rightarrow} q_{i}, \quad \forall i, 1 \leq i \leq \zeta
$$

em $\mathcal{A}$. Um estado $\left(i_{1}, \ldots, i_{\zeta}\right)$ é inicial se, e somente se, cada $i_{j}, 1 \leq j \leq \zeta$, é um estado inicial em $\mathcal{A}$. Um estado $\left(t_{1}, \ldots, t_{\zeta}\right)$ é final se, e somente se, cada $t_{j}, 1 \leq j \leq \zeta$, é um estado final em $\mathcal{A}$. Dessa forma, $\mathcal{A}^{\zeta}$ reconhece o conjunto das $\zeta$-uplas de palavras de mesmo comprimento em $|\mathcal{A}|$.

Considere em $\mathcal{A}^{\zeta}$ um passeio bem-sucedido

$$
p:\left(i_{1}, \ldots, i_{\zeta}\right) \stackrel{\left(u_{1}, \ldots, u_{\zeta}\right)}{\longrightarrow}\left(t_{1}, \ldots, t_{\zeta}\right),
$$

onde as coordenadas do rótulo $\left(u_{1}, \ldots, u_{\zeta}\right)$ são as $\zeta$ palavras de $A$. Fatore $p$ como

$$
p:\left(i_{1}, \ldots, i_{\zeta}\right) \stackrel{\left(u_{1}^{\prime}, \ldots, u_{\zeta}^{\prime}\right)}{\longrightarrow}\left(p_{1}, \ldots, p_{\zeta}\right) \stackrel{\left(u_{1}^{\prime \prime}, \ldots, u_{\zeta}^{\prime \prime}\right)}{\longrightarrow}\left(t_{1}, \ldots, t_{\zeta}\right),
$$

onde as coordenadas de $\left(u_{1}^{\prime}, \ldots, u_{\zeta}^{\prime}\right)$ contém, respectivamente, os prefixos de $u_{1}, \ldots, u_{\zeta}$ de comprimento $\left|u_{a}^{\prime}\right|$. Então, o passeio

$$
c:\left(i_{1}, \ldots, i_{\zeta}\right) \stackrel{\left(u_{1}^{\prime}, \ldots, u_{\zeta}^{\prime}\right)}{\longrightarrow}\left(p_{1}, \ldots, p_{\zeta}\right)
$$


deve passar duas vezes por um mesmo estado, porque o número de estados de $\mathcal{A}^{\zeta}$ é no máximo $n^{\zeta}$.

Fatore $c$ como

$$
c: d_{0} c_{1} d_{1} \ldots c_{j} d_{j}
$$

onde cada $c_{i}$ é o primeiro passeio fechado maximal do segmento $d_{i-1} \ldots d_{j}$, e $d_{j}$ é um caminho. Dessa forma, o passeio

$$
d: d_{0} \ldots d_{j}
$$

obtido retirando-se os passeios fechados $c_{i}$ de $c$ é um caminho. Vamos denotar o rótulo de $d$ por $\left(y_{1}, \ldots, y_{\zeta}\right)$, e utilizar as notações

$$
\begin{gathered}
\left(x_{1_{i}}, \ldots, x_{\zeta_{i}}\right)=\left|c_{i}\right|, \quad \forall i, 1 \leq i \leq j ; \\
\left(y_{1_{i}}, \ldots, y_{\zeta_{i}}\right)=\left|d_{i}\right|, \quad \forall i, 0 \leq i \leq j ; \\
\left(z_{1_{i}}, \ldots, z_{\zeta_{i}}\right)=\left|d_{0} \ldots d_{i-1}\right|, \quad \forall i, 1 \leq i \leq j \\
\left(w_{1_{i}}, \ldots, w_{\zeta_{i}}\right)=\left|d_{i} \ldots d_{j}\right|, \quad \forall i, 0 \leq i \leq j
\end{gathered}
$$

onde $d_{0} \ldots d_{i-1}$ e $d_{i} \ldots d_{j}$ são segmentos iniciais e finais de $c$, respectivamente.

Afirmamos que existe um índice $i \geq 1$ tal que

$$
\left(z_{a_{i}} g, z_{b_{i}} g\right) \Delta \neq\left(z_{a_{i}} g, z_{b_{i}} g\right) \Delta \cdot\left(x_{a_{i}} g, x_{b_{i}} g\right) .
$$

De fato, suponha que $\left(z_{a_{i}} g, z_{b_{i}} g\right) \Delta=\left(z_{a_{i}} g, z_{b_{i}} g\right) \Delta \cdot\left(x_{a_{i}} g, x_{b_{i}} g\right)$ para todo índice $i \geq 1$. Então,

$$
\left(z_{a_{i}} g, z_{b_{i}} g\right) \Delta \cdot\left(x_{a_{i}} g, x_{b_{i}} g\right) \cdot\left(w_{a_{i}} g, w_{b_{i}} g\right)=\left(z_{a_{i}} g, z_{b_{i}} g\right) \Delta \cdot\left(w_{a_{i}} g, w_{b_{i}} g\right) .
$$

Assim,

$$
\begin{aligned}
\left(u_{a}^{\prime} g, u_{b}^{\prime} g\right) \Delta=\left(y_{a_{0}} g, y_{b_{0}} g\right) \Delta & \cdot\left(x_{a_{1}} g, x_{b_{1}} g\right) \cdot\left(y_{a_{1}} g, y_{b_{1}} g\right) \cdot \ldots \cdot\left(x_{a_{j}} g, x_{b_{j}} g\right) \cdot\left(y_{a_{j}} g, y_{b_{j}} g\right)= \\
& \left(y_{a_{0}} g, y_{b_{0}} g\right) \Delta \cdot\left(y_{a_{1}} g, y_{b_{1}} g\right) \cdot \ldots \cdot\left(y_{a_{j}} g, y_{b_{j}} g\right)=\left(y_{a} g, y_{b} g\right) \Delta .
\end{aligned}
$$

Mas, isso implica que $\left\|u_{a}^{\prime} g, u_{b}^{\prime} g\right\|=\left\|y_{a} g, y_{b} g\right\|$, portanto que $\|d\| \geq n^{\zeta}$, em vista de (3.7.5). Como $d$ é um caminho, chegamos numa contradição.

Seja $\left(z_{a_{i}} g, z_{b_{i}} g\right)$ um par satisfazendo (3.7.6). Então, cada palavra $u_{r}$ de $A$ pode ser fatorada como $z_{r_{i}} x_{r_{i}} w_{r_{i}} u_{r}^{\prime \prime}$. Para todo par $u_{r}$ e $u_{s}$ de palavras distintas de $A$, vamos analisar a relação entre as palavras da forma $\left(z_{r_{i}} x_{r_{i}}^{l} w_{r_{i}} u_{r}^{\prime \prime}\right) g$ e $\left(z_{s_{i}} x_{s_{i}}^{l} w_{s_{i}} u_{s}^{\prime \prime}\right) g$, para determinados valores de $l$. Vamos considerar três casos.

- $u_{r} g=u_{s} g$ e $\left(z_{r_{i}} g, z_{s_{i}} g\right) \Delta=\left(z_{r_{i}} g, z_{s_{i}} g\right) \Delta \cdot\left(x_{r_{i}} g, x_{s_{i}} g\right)$ : neste caso, pelo Lema 3.7.1, para todo inteiro $l \geq l_{r s}=1$,

$$
\left(z_{r_{i}} x_{r_{i}}^{l} w_{r_{i}} u_{r}^{\prime \prime}\right) g=\left(z_{s_{i}} x_{s_{i}}^{l} w_{s_{i}} u_{s}^{\prime \prime}\right) g
$$

$\mathrm{e}$

$$
\left\|z_{r_{i}} x_{r_{i}}^{l} w_{r_{i}} u_{r}^{\prime \prime}, z_{s_{i}} x_{s_{i}}^{l} w_{s_{i}} u_{s}^{\prime \prime}\right\|_{g}=\left\|u_{r}, u_{s}\right\|_{g}
$$


- $u_{r} g=u_{s} g$ e $\left(z_{r_{i}} g, z_{s_{i}} g\right) \Delta \neq\left(z_{r_{i}} g, z_{s_{i}} g\right) \Delta \cdot\left(x_{r_{i}} g, x_{s_{i}} g\right)$ : pela Proposição 3.6.3, para todo $l \geq l_{r s}=2$,

$$
\left(z_{r_{i}} g, z_{s_{i}} g\right) \Delta \cdot\left(x_{r_{i}} g, x_{s_{i}} g\right) \neq\left(z_{r_{i}} g, z_{s_{i}} g\right) \Delta \cdot\left(x_{r_{i}} g, x_{s_{i}} g\right)^{l} .
$$

Assim,

$$
\left(\left(z_{r_{i}} x_{r_{i}} w_{r_{i}} u_{r}^{\prime \prime}\right) g,\left(z_{s_{i}} x_{s_{i}} w_{s_{i}} u_{s}^{\prime \prime}\right) g\right) \Delta \neq\left(\left(z_{r_{i}} x_{r_{i}}^{l} w_{r_{i}} u_{r}^{\prime \prime}\right) g,\left(z_{s_{i}} x_{s_{i}}^{l} w_{s_{i}} u_{s}^{\prime \prime}\right) g\right) \Delta .
$$

Como $\left(z_{r_{i}} x_{r_{i}} w_{r_{i}} u_{r}^{\prime \prime}\right) g=\left(z_{s_{i}} x_{s_{i}} w_{s_{i}} u_{s}^{\prime \prime}\right) g$, concluímos que

$$
\left(z_{r_{i}} x_{r_{i}}^{l} w_{r_{i}} u_{r}^{\prime \prime}\right) g \neq\left(z_{s_{i}} x_{s_{i}}^{l} w_{s_{i}} u_{s}^{\prime \prime}\right) g .
$$

- $u_{r} g \neq u_{s} g$ : pelo Lema 3.7.1, existe um inteiro $l_{r s}$ tal que, para todo $l \geq l_{r s}$,

$$
\left(z_{r_{i}} x_{r_{i}}^{l} w_{r_{i}} u_{r}^{\prime \prime}\right) g \neq\left(z_{s_{i}} x_{s_{i}}^{l} w_{s_{i}} u_{s}^{\prime \prime}\right) g .
$$

Sejam $l_{0}=\max \left\{l_{r s}: 1 \leq r<s \leq \zeta\right\}$ e $C$ o conjunto das palavras

$$
\begin{aligned}
& v_{1}=z_{1_{i}} x_{1_{i}}^{l_{0}} w_{1_{i}} u_{1}^{\prime \prime}, \\
& \vdots \\
& v_{\zeta}=z_{\zeta_{i}} x_{\zeta_{i}}^{l_{0}} w_{\zeta_{i}} u_{\zeta}^{\prime \prime} .
\end{aligned}
$$

Dadas palavras distintas $v_{r}$ e $v_{s}$ em $C$, segue da definição de $l_{0}$ que, se $u_{r} g \neq u_{s} g$, então $v_{r} g \neq v_{s} g$, e, se $u_{r} g=u_{s} g$, então ou $v_{r} g=v_{s} g$, ou $v_{r} g \neq v_{s} g$. Então, card $(C g) \geq \operatorname{card}(A g)$. Mas, por (3.7.6), $v_{a} g \neq v_{b} g$. Logo, $\operatorname{card}(C g)>\operatorname{card}(A g)$. Como $\operatorname{card}(C)=\operatorname{card}(A)$, obtemos

$$
\operatorname{card}(C)-\operatorname{card}(C g)<\theta .
$$

No caso em que $v_{r} g=v_{s} g$, segue de (3.7.7) que $\left\|v_{r}, v_{s}\right\|_{g}=\left\|u_{r}, u_{s}\right\|_{g}$. Assim, se $v_{r} g=v_{s} g$, então $\left\|v_{r}, v_{s}\right\|_{g} \geq n^{\operatorname{card}(A)} M=n^{\operatorname{card}(C)} M$. Portanto,

$$
\|C\|_{g} \geq n^{\operatorname{card}(C)} M
$$

Considerando o passeio bem-sucedido

$$
d_{0} \ldots d_{i-1} c_{i}^{l_{0}} d_{i} \ldots d_{j}\left(p_{1}, \ldots, p_{\zeta}\right) \stackrel{\left(u_{1}^{\prime \prime}, \ldots, u_{\zeta}^{\prime \prime}\right)}{\longrightarrow}\left(t_{1}, \ldots, t_{\zeta}\right)
$$

em $\mathcal{A}^{\zeta}$, concluímos que $C \subseteq L$ e card $(C f)=1$. Utilizando a hipótese de indução, temos que existe um conjunto $B$ com $\operatorname{card}(B)=\operatorname{card}(C), \operatorname{card}(B f)=1 \mathrm{e} \operatorname{card}(B g)=\operatorname{card}(B)$. Como card $(A)=\operatorname{card}(C)$, segue que $B$ é como queríamos.

Teorema 3.7.2 (Weber 1992) Seja $k$ um inteiro positivo. Toda relação racional $k$-valorada $\tau$ : $\Sigma^{*} \rightarrow \Gamma^{*}$ pode ser decomposta numa união

$$
\tau=f_{1} \cup \cdots \cup f_{k}
$$

onde cada $f_{i}$ é uma função racional. 
Demonstração. Vamos primeiro considerar o caso $1 \tau=\varnothing$. Feita essa hipótese, considere a fatoração (3.7.1) de $\tau$, e seja $\mathcal{A}$ um $\Omega$-autômato determinístico bi-acessível com $n$ estados reconhecendo $L$.

Afirmamos que o número máximo de palavras em $L_{n^{k+1} M}$ com uma mesma imagem por $f$ é $k$. De fato, suponha que exista um subconjunto $A$ de $L_{n^{k+1} M} \operatorname{com} \operatorname{card}(A)=k+1$ e $\operatorname{card}(A f)=1$. Pelas propriedades de $A$, o Lema 3.7.2 pode ser aplicado, e fornece um subconjunto $B$ de $L$ com $\operatorname{card}(B)=k+1$, $\operatorname{card}(B f)=1$ e $\operatorname{card}(B g)=\operatorname{card}(B)$. Mas, esse conjunto contradiz a hipótese de que $\tau$ é $k$-valorada.

Vamos agora definir indutivamente $k$ subconjuntos reconhecíveis de $L_{n^{k+1} M}$, utilizando a Proposição 3.7.3. Definimos primeiro o conjunto $A_{1}$ como uma cross-section de $L_{n^{k+1} M} \operatorname{com} f$. Para cada $j, 2 \leq j \leq k$, o $j$-ésimo conjunto $A_{j}$ é definido como uma cross-section de

$$
L_{n^{k+1} M}-\bigcup_{1 \leq i<j} A_{i}
$$

com relação a $f$.

Para cada $i, 1 \leq i \leq k$, definimos a relação racional

$$
f_{i}=\Sigma^{*} \stackrel{f^{-1}}{\longrightarrow} \Omega^{*} \stackrel{\cap A_{i}}{\longrightarrow} \Omega^{*} \stackrel{g}{\longrightarrow} \Gamma^{*} .
$$

Da definição de cross-section, segue que cada $f_{i}$ é uma função. Afirmamos que

$$
\tau=f_{1} \cup \cdots \cup f_{k} \text {. }
$$

De fato, como card $\left(u\left(f^{-1}\left(\cap L_{n^{k+1}}\right)\right)\right) \leq k$, para todo $u$ em $\operatorname{dom} \tau$, temos que

$$
L_{n^{k+1} M}=\bigcup_{1 \leq i \leq k} A_{i}
$$

Assim,

$$
\# \tau=L_{n^{k+1} M}(f, g)=\left(\bigcup_{1 \leq i \leq k} A_{i}\right)(f, g)=\bigcup_{1 \leq i \leq k} A_{i}(f, g),
$$

o que prova (3.7.8).

O caso em que $1 \tau \neq \varnothing$ é uma conseqüência trivial dessa construção. O primeiro passo é calcular a restrição e $\tau^{\prime}=\tau \mid \Sigma^{+}$. Em seguida, calcula-se uma decomposição de $\tau^{\prime}$ em $k$ funçōes racionais $f_{1}, \ldots, f_{k}$. Finalmente, cada valor em $1 \tau$ é atribuído à imagem da palavra vazia em uma dessas funções, tomando-se o cuidado de que para valores distintos são utilizadas funções distintas. Como há no máximo $k$ valores distintos em $1 \tau$, as $k$ funções racionais resultantes são uma decomposição de $\tau$.

Exemplo 3.7.3 O Exemplo 3.7.1 mostra que nem sempre é possível obter uma decomposição de uma relação $k$-valorada em $k$ funções duas a duas disjuntas, ou seja, cujos gráficos sejam conjuntos dois a dois disjuntos. 
De fato, sejam $f_{1}$ e $f_{2}$ duas funções racionais tais que $\tau=f_{1} \cup f_{2}$. Como para todo par $i$ e $j$ de inteiros distintos a imagem $\left(a^{i} b^{j}\right) \tau$ tem cardinalidade 2 , temos que $a^{i} b^{j}$ pertence a dom $f_{1} \cap \operatorname{dom} f_{2}$. Assim,

$$
A=\left\{a^{i} b^{j}: i \geq 0, j \geq 0 \text { e } i \neq j\right\} \subseteq \operatorname{dom} f_{1} \cap \operatorname{dom} f_{2} .
$$

Como $A$ não é reconhecível,

$$
\operatorname{dom} f_{1} \cap \operatorname{dom} f_{2} \neq A \text {. }
$$

Ou seja, existe uma palavra da forma $a^{i} b^{i} \operatorname{em} \operatorname{dom} f_{1} \cap \operatorname{dom} f_{2}$.

Como a imagem por $\tau$ das palavras da forma $a^{i} b^{i}$ é um unitário, segue que

$$
\# f_{1} \cap \# f_{2} \neq \varnothing
$$

\subsection{Equivalência de relações racionais $k$-valoradas}

Em 1986, Culik e Karhumaki provaram que o problema da equivalência de relações racionais $k$ valoradas é decidível [CK86]. Em 1988, Weber apresentou outra demonstração desse resultado, utilizando uma decomposição de relações racionais $k$-valoradas em funções racionais [Web88, Web93]. A decomposição utilizada por Weber nessa demonstração é diferente da apresentada na Seção 3.7.

Nesta seção, vamos demonstrar a decidibilidade desse problema, utilizando a decomposição apresentada na Seção 3.7.

Primeiro, vamos demonstrar que é decidível se uma função racional está contida em uma união finita de funções racionais.

Observamos que decidir se uma relação racional $\tau$ está contida em uma relação racional $\tau^{\prime}$ significa decidir se \# $\pi$ está contido em $\# \tau^{\prime}$.

Lema 3.8.1 Sejam $k$ um inteiro positivo, e $f, f_{1}, \ldots, f_{k}$ funções racionais $\Sigma^{*} \rightarrow \Gamma^{*}$ tais que

$$
\operatorname{dom} f \subseteq \operatorname{dom} f_{i}, \quad \forall i, 1 \leq i \leq k .
$$

É decidivel se

$$
f \subseteq \bigcup_{1 \leq i \leq k} f_{i}
$$

Demonstração. Sejam $\mathcal{T}, \mathcal{T}_{1}, \ldots, \mathcal{T}_{k} \Sigma$ - $\Gamma$-transdutores letra-palavra tais que $|\mathcal{T}|=f$ e $\left|\mathcal{T}_{i}\right|=f_{i}$, para todo $i, 1 \leq i \leq k$. Vamos denotar por $n$ o número de estados de $\mathcal{T}$, e por $n_{i}$ o número de estados de $\mathcal{T}_{i}$, para todo índice $i$. Vamos denotar por $Q$ o conjunto de estados de $\mathcal{T}$, e por $Q_{i}$ o conjunto de estados de $\mathcal{T}_{i}$, para todo índice $i$.

Vamos utilizar argumentos semelhantes à demonstração do Teorema 3.4.2. Em particular, a função $T$ definida em (3.4.3) será utilizada.

Afirmamos que, se

$$
u f \in \bigcup_{1 \leq i \leq k} u f_{i}
$$


para toda palavra $u \operatorname{em} \operatorname{dom} f \operatorname{com}|u| \leq n n_{1} \ldots n_{k} T(k)$, então

$$
f \subseteq \bigcup_{1 \leq i \leq k} f_{i}
$$

Suponha o contrário, e considere uma palavra $u$ em $\operatorname{dom} f$ de comprimento mínimo tal que $u f \neq u f_{i}$ para todo $i, 1 \leq i \leq k$. Então, $|u|>n n_{1} \ldots n_{k} T(k)$.

Sejam $c_{0}, c_{1}, \ldots, c_{k}$ passeios bem-sucedidos que soletram $u$ em $\mathcal{T}, \mathcal{T}_{1}, \ldots, \mathcal{T}_{k}$, respectivamente. Os passeios $c_{1}, \ldots, c_{k}$ existem devido à hipótese em (3.8.1). Para cada prefixo $u[1 \ldots j]$ de $u$, vamos denotar por $\xi_{j}$ a $(k+1)$-upla $\left(q_{0}, \ldots, q_{k}\right)$ dos términos dos segmentos iniciais dos passeios $c_{0}, c_{1}, \ldots, c_{k}$, respectivamente, que soletram $u[1 \ldots j]$.

Assim, $\xi_{1} \ldots \xi_{|u|}$ é uma palavra comprimento $|u|$ sobre o alfabeto $Q \times Q_{1} \times \cdots \times Q_{k}$. Do Lema 3.4.1, segue que existe uma $(k+1)$-upla de estados $\left(q_{0}, q_{1}, \ldots, q_{k}\right)$ que ocorre nessa palavra pelo menos $T(k)+1$ vezes.

Podemos então fatorar os passeios $c_{0}, c_{1}, \ldots, c_{k}$ como

$$
\begin{aligned}
& c_{0}: \quad c_{0_{0}} c_{0_{1}} \ldots c_{0_{l}} c_{0_{l+1}}, \\
& \vdots \\
& c_{k}: \quad c_{k_{0}} c_{k_{1}} \ldots c_{k_{l}} c_{k_{l+1}},
\end{aligned}
$$

onde $l \geq T(k)$ e $c_{i_{j}}$ é um passeio fechado não-trivial que vai de $q_{i}$ a $q_{i}$, para todo $i$ entre 0 e $k$, e todo $j$ entre 1 e $l$. Além disso,

$$
c_{0_{j}} \alpha=\cdots=c_{k_{j}} \alpha, \quad \forall j, 0 \leq j \leq l+1 .
$$

Recorde a definição (3.4.4). Seja $g: \mathcal{C}_{l} \rightarrow[k]$ a função definida como segue. Para cada par $(a, b) \in \mathcal{C}_{l}$, considere os passeios bem-sucedidos

$$
\begin{array}{ll}
c_{0}^{(a, b)}: & c_{0_{0}} \ldots c_{0_{a-1}} c_{0_{b+1}} \ldots c_{0_{l+1}}, \\
\vdots & \\
c_{k}^{(a, b)}: & c_{k_{0}} \ldots c_{k_{a-1}} c_{k_{b+1}} \ldots c_{k_{l+1}},
\end{array}
$$

obtidos omitindo-se os passeios fechados da forma $c_{i_{a}} \ldots c_{i_{b}}$. Então, todos esses passeios têm uma mesma entrada $v$, e, como as entradas dos passeios fechados $c_{i_{j}}$ são diferentes da palavra vazia, $|v|<|u|$. Pela minimalidade de $u$, temos que $v f=v f_{i}$ para algum $i$, ou seja

$$
c_{0}^{(a, b)} \beta=c_{i}^{(a, b)} \beta .
$$

Defina então $(a, b) g$ como $i$.

Como $l \geq T(k)$, segue do Lema 3.4.3 que $g$ tem um encaixamento $((a, b),(a, e),(e+1, b))$, ou seja, existe um índice $i$ tal que $(a, b) g=(a, e) g=(e+1, b) g=i$. Pela definição de $g$, obtemos as igualdades

$$
\begin{aligned}
c_{0}^{(a, b)} \beta & =c_{i}^{(a, b)} \beta, \\
c_{0}^{(a, e)} \beta & =c_{i}^{(a, e)} \beta, \\
c_{0}^{(e+1, b)} \beta & =c_{i}^{(e+1, b)} \beta .
\end{aligned}
$$


Pelo Lema 3.4.2, concluímos que

$$
u f=c_{0} \beta=c_{i} \beta=u f_{i},
$$

contrariando a hipótese sobre $u$. Portanto, verifica-se (3.8.2).

Teorema 3.8.1 (Culik e Karhumaki 1986, Weber 1988) Sejam $k$ um inteiro positivo e $\tau$ : $\Sigma^{*} \rightarrow \Gamma^{*}$ e $v: \Sigma^{*} \rightarrow \Gamma^{*}$ relações racionais $k$-valoradas. É decidivel se

$$
\tau \subseteq v
$$

Demonstração. Pelo Teorema 3.7.2, $\tau$ pode ser decomposta numa união finita de funções racionais. Então, é suficiente demonstrar que é decidível se uma função racional $f$ está contida em $v$. Como é decidível se $\operatorname{dom} f \subseteq \operatorname{dom} v$, podemos supor que essa inclusão é verdadeira.

Aplicando o Teorema 3.7.2 em $v$, obtemos uma decomposição dessa relação em $k$ funções racionais $f_{1}, \ldots, f_{k}$. Para cada subconjunto não-vazio $A$ de $[k]$, vamos denotar por $I_{A}$ a linguagem reconhecível

$$
\left(\bigcap_{i \in A} \operatorname{dom} f_{i}\right)-\left(\bigcup_{j \in[k]-A} \operatorname{dom} f_{j}\right) .
$$

Vamos provar que $f \subseteq v$ se, e somente se, para todo subconjunto não-vazio $A$ de $[k]$,

$$
f\left|I_{A} \subseteq \bigcup_{i \in A} f_{i}\right| I_{A}
$$

Suponha que $f \subseteq v$. Seja $A$ um subconjunto não-vazio de $[k]$. Para todo $x$ em $\operatorname{dom}\left(f \mid I_{A}\right)$, segue da definição de $I_{A}$ que $x \in \operatorname{dom} f_{i}$, para todo $i$ em $A$, e $x \notin \operatorname{dom} f_{j}$, para todo $j$ em $[k]-A$. Portanto, deve existir um índice $i$ em $A$ tal que $x f=x f_{i}=x\left(f_{i} \mid I_{A}\right)$. Obtemos assim (3.8.3).

Suponha agora (3.8.3). Dada uma palavra $x$ em $\operatorname{dom} f$, seja $A$ o conjunto dos índices $i$ tais que $x$ pertence a dom $f_{i}$. Esse conjunto não é vazio porque, por hipótese, $\operatorname{dom} f \subseteq \operatorname{dom} v$. Pela definição de $I_{A}$, temos que $x$ pertence a $I_{A}$. Por (3.8.3), temos que $x f_{i}=x f$, para algum $i$ em $A$. Ou seja, $x f$ pertence a $x v$. Portanto, $f \subseteq v$.

Pela Proposição 2.5.4, cada função $f \mid I_{A}$ é uma função racional. Para cada subconjunto nãovazio $A$ de $[k]$, a construção de um transdutor que realiza $f \mid I_{A}$ a partir de um transdutor que realiza $f$ pode ser feita com operações elementares, e vamos apenas descrevê-la em linhas gerais. Primeiro, um autômato realizando o conjunto $L^{\prime}$ da demonstração da Proposição 2.5.4 é construído. A partir desse autômato, um $\Sigma$ - $\Gamma$-trandutor que realiza $f \mid I_{A}$ pode ser construído com as substituições descritas em (2.3.2).

Para todo subconjunto não-vazio $A$ de $[k]$ e todo $i$ em $A$, segue da definição de $I_{A}$ que $I_{A} \subseteq$ $\operatorname{dom} f_{i}$. Portanto, $\operatorname{dom}\left(f \mid I_{A}\right) \subseteq \operatorname{dom}\left(f_{i} \mid I_{A}\right)$. Do Lema 3.8.1, segue então que (3.8.3) é decidível, o que conclui a demonstração. 


\section{$3.9 \quad$ Ambigüidade}

O Teorema Cross-section tem um papel importante no estudo da ambigüidade em autômatos e transdutores. O objetivo desta seção é estudar a construção de um transdutor não-ambiguo utilizando esse resultado.

Definição 3.9.1 Dizemos que um $\Sigma$-autômato $\mathcal{A}$ é não-ambíguo se cada palavra em $\Sigma^{*}$ puder ser soletrada por no máximo um passeio bem-sucedido em $\mathcal{A}$.

Dizemos que um $\Sigma$-M-transdutor letra-saída $\mathcal{T}$ é não-ambíguo se cada entrada puder ser soletrada por no máximo um passeio bem-sucedido em $\mathcal{T}$.

Seja $k$ um inteiro positivo. Dizemos que um $\Sigma$-M-transdutor letra-saída $\mathcal{T}$ é $k$-ambíguo se cada entrada puder ser soletrada por no máximo $k$ passeios bem-sucedidos em $\mathcal{T}$.

Claramente, todo transdutor $k$-ambíguo realiza uma relação racional $k$-valorada, e um transdutor não-ambíguo realiza uma função. Utilizando o Teorema Cross-section, Eilenberg [Eil74] demonstrou que

Teorema 3.9.1 (Eilenberg 1974) Toda função racional $\tau: \Sigma^{*} \rightarrow \mathrm{M}$ pode ser realizada por um

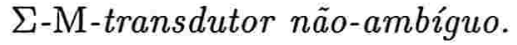

Observe que, nesse resultado, nenhuma restrição é feita no monóide de saída.

Utilizando a decomposição de uma relação racional palavra-palavra $k$-valorada em $k$ funções racionais, Weber [Web92] estendeu esse resultado em 1992:

Teorema 3.9.2 (Weber 1992) Toda relação racional $k$-valorada $\tau: \Sigma^{*} \rightarrow \Gamma^{*}$ pode ser realizada por um $\Sigma$-Г-transdutor k-ambíguo.

A demonstração desse resultado consiste em aplicar o Teorema 3.7.2 para obter uma decomposição de $\tau$ em $k$ funções racionais, e em seguida o Teorema 3.9.1 em cada uma dessas funções.

A seguir, vamos estudar a construção efetiva de uma cross-section de um subconjunto reconhecível com um morfismo muito fino. Em seguida, vamos estudar a construção efetiva de um $\Sigma$-autômato não-ambíguo para uma linguagem reconhecível. Finalmente, vamos demonstrar o Teorema 3.9.1, apresentando uma construção efetiva de um transdutor não-ambíguo a partir de um transdutor letra-saída funcional.

Proposição 3.9.1 Sejam L um subconjunto reconhecível de $\Sigma^{*}$ e $f: \Sigma^{*} \rightarrow \Gamma^{*}$ um morfismo muito fino. É possível construir efetivamente um $\Sigma$-autômato que reconhece uma cross-section de $L$ com $f$.

Demonstração. Sejam $\mathcal{A}=(Q, E, I, T)$ um $\Sigma$-autômato determinístico e bi-acessível que reconhece $L, a$ uma letra, e $h: \Sigma^{*} \rightarrow a^{*}$ o morfismo muito fino definido por

$$
\sigma h=a, \forall \sigma \in \Sigma .
$$

A demonstração da Proposição 3.7 .3 mostra que a linguagem $L_{(h, f), 1}$ é uma cross-section de $L \operatorname{com} f$. A construção de um $\Sigma$-autômato $\mathcal{A}^{\prime}$ reconhecendo essa cross-section a partir de $\mathcal{A}$ pode então ser feita seguindo a demonstração da Proposição 3.7.2. 
A seguir, mostramos um exemplo da construção descrita na Proposição 3.9.1. Observamos que, como $f$ é um morfismo muito fino, todo estado de $\mathcal{A}^{\prime}$ é a união de um estado de $\mathcal{A}$ com um subconjunto de $Q \times 1$.

\section{Exemplo 3.9.1 (Berstel [Ber79] p. 113) Sejam}

$$
\Sigma=\{a, b, c, d\}
$$

$f: \Sigma^{*} \rightarrow a^{*}$ o morfismo definido por

$$
\sigma f=a, \quad \forall \sigma \in \Sigma
$$

$\mathrm{e}$

$$
L=((b d b \cup b c \cup c b) a)^{*} .
$$

Esse conjunto é reconhecido pelo $\Sigma$-autômato determinístico

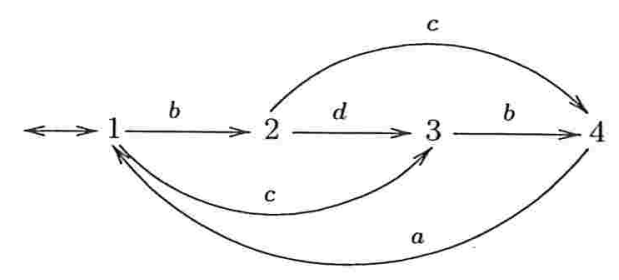

Para construir uma cross-section de $L \operatorname{com} f$, fixamos primeiro uma ordem lexicográfica $<_{l}$ em $\Sigma^{*}$. Em seguida, utilizando a demonstração da Proposição 3.7.2, construímos o autômato $\mathcal{A}_{1}$, que reconhece $L_{(h, f), 1}$, onde $h=f$.

Fixando

$$
a<_{l} b<_{l} c<_{l} d,
$$

obtemos o autômato representado abaixo. Cada estado $q=\left\{p,\left(p_{1}, 1\right), \ldots,\left(p_{r}, 1\right)\right\}$ desse autômato é representado como

$$
p\left(p_{1}, \ldots, p_{r}\right) .
$$

Apenas os estados acessíveis e co-acessíveis são exibidos.

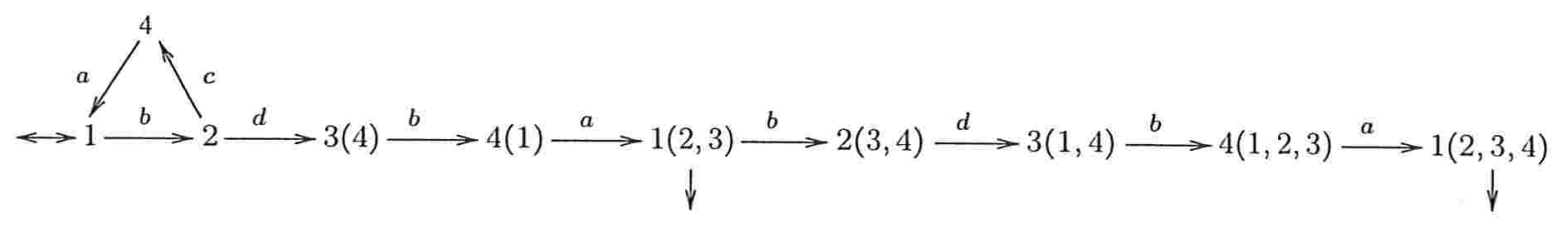

Assim,

$$
L_{1}=(b c a)^{*}\left(1 \cup b d b a \cup(b d b a)^{2}\right)
$$

é uma cross-section de $L$ com $f$.

Observe que todo autômato determinístico é não-ambíguo. Assim, um autômato não-ambíguo para um conjunto reconhecível pode ser obtido com a construção dos subconjuntos. Outra construção, utilizando o Teorema Cross-section, está descrita a seguir. 
Proposição 3.9.2 Dado um $\Sigma$-autômato $\mathcal{A}=(Q, E, I, T)$, um $\Sigma$-autômato não-ambíguo equivalente pode ser construído efetivamente.

Demonstração. Vamos apresentar uma construção em dois passos. Vamos denotar a linguagem local dos passeios bem-sucedidos de $\mathcal{A}$ por $\# \mathcal{A}$, e o morfismo muito fino que associa cada transição de $\mathcal{A}$ ao seu rótulo por $r: E^{*} \rightarrow \Sigma^{*}$.

No primeiro passo, utilizamos a Proposição 3.9.1, e construímos um $E$-autômato determinístico $\mathcal{B}$ que reconhece uma cross-section $B$ de $\# \mathcal{A}$ com $r$.

No segundo, construímos um $\Sigma$-autômato $\mathcal{A}^{\prime}$ a partir de $\mathcal{B}$, substituindo cada transição

$$
p \stackrel{e}{\longrightarrow} q
$$

de $\mathcal{B}$ pela transição

$$
p \stackrel{e r}{\longrightarrow} q .
$$

Das propriedades da cross-section $B$, segue que o comportamento de $\mathcal{A}^{\prime}$ é exatamente $|\mathcal{A}|$, e que passeios bem-sucedidos distintos em $\mathcal{A}^{\prime}$ têm rótulos distintos. Assim, $\mathcal{A}^{\prime}$ é um $\Sigma$-autômato não-ambíguo equivalente a $\mathcal{A}$.

A seguir, mostramos um exemplo dessa construção.

Exemplo 3.9.2 (Leung [Leu98]) Seja $\Sigma=\{\sigma, \gamma\}$. A linguagem

$$
L=\left(\sigma \cup\left(\sigma \gamma^{*}\right)^{2} \sigma\right)^{*}
$$

é reconhecida pelo $\Sigma$-autômato não-determinístido $\mathcal{A}=(Q, E, I, T)$ ilustrado abaixo:

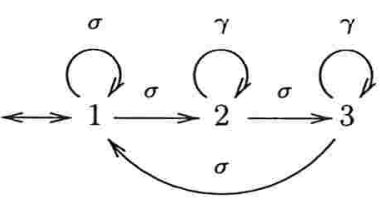

Vamos construir um $\Sigma$-autômato não-ambíguo equivalente a $\mathcal{A}$, utilizando a Proposição 3.9.2.

Associando uma letra a cada transição de $\mathcal{A}$, obtemos um $\Sigma$-autômato determinístico que reconhece $\# \mathcal{A}$, conforme ilustrado a seguir:

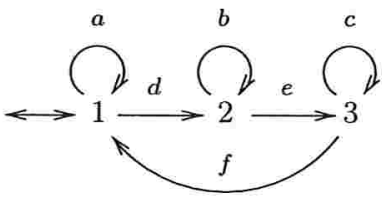

Com essa rotulação,

$$
\# \mathcal{A}=\left(a \cup d b^{*} e c^{*} f\right)^{*} .
$$

Sejam $a$ uma letra, $h: E^{*} \rightarrow a^{*}$ o morfismo muito fino tal que $E h=a, r: E^{*} \rightarrow \Sigma^{*}$ o morfismo muito fino que associa cada transição ao seu rótulo, e $<_{l}$ a ordem lexicográfica em $E^{*}$ definida por

$$
a<_{l} b<_{l} c<_{l} d<_{l} e<_{l} f \text {. }
$$

Pela Proposição 3.7.3, $B=\# \mathcal{A}_{(h, r), 1}$ é uma cross-section de $\# \mathcal{A}$ com $r$. Utilizando a construção da Proposição 3.7.2, obtemos o $E$-autômato determinístico que reconhece $B$ ilustrado abaixo. A notação utilizada é a mesma que a do Exemplo 3.9.1. 


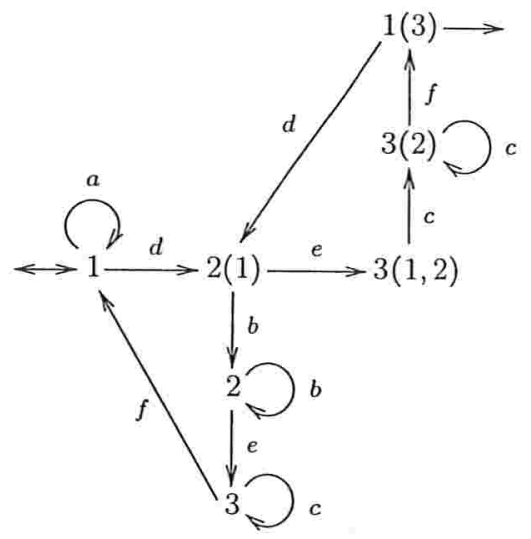

Essa cross-section é a linguagem $\left(a \cup d b^{+} e c^{*} f \cup d e c^{+} f\right)^{*}$.

Substituindo o rótulo de cada transição pela sua imagem por $r$, obtemos o $\Sigma$-autômato nãoambíguo reconhecendo $L$ ilustrado a seguir:

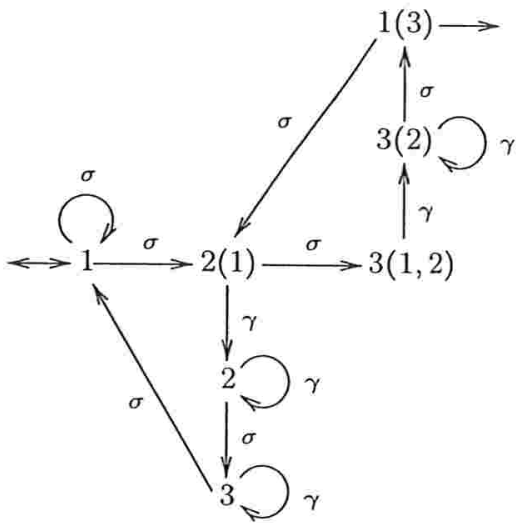

Uma maneira de demonstrar que esse autômato é não-ambíguo é verificar se a parte bi-acessível do seu quadrado é a diagonal principal. Maiores detalhes sobre esse método podem ser vistos em [BP85].

São conhecidos desde 1978 exemplos de autômatos não-determinísticos tais que o número de estados de qualquer autômato não-ambíguo equivalente é exponencialmente maior [Sch78]. Assim, a construção de uma cross-section e a de um autômato não-ambíguo têm complexidade de tempo exponencial, no pior caso. O Exemplo 3.9.2 pertence a uma família de $\Sigma$-autômatos não-determinísticos sobre duas letras com essa propriedade, que foi apresentada em 1998 por Leung [Leu98].

Finalmente, vamos estudar a construção de um transdutor não-ambíguo para uma função racional $\Sigma^{*} \rightarrow \mathrm{M}$, onde $\mathrm{M}$ é um monóide qualquer. Entretanto, uma construção efetiva supõe que $\mathrm{M}$ possa ser implementado.

Demonstração do Teorema 3.9.1. Sejam $\mathcal{T}=(Q, E, I, T)$ um $\Sigma$-M-transdutor letra-saída funcional, e $(\alpha, \beta)$ seu bimorfismo. Vamos denotar por \#T o conjunto local dos passeios bem-sucedidos de $\mathcal{T}$. Vamos descrever uma construção de um transdutor não-ambíguo letra-saída equivalente a $\mathcal{T}$ em dois passos. 
No primeiro passo, utilizando a Proposição 3.9.1, construímos um E-autômato determinístico $\mathcal{B}$ que reconhece uma cross-section $B$ de $\# \mathcal{T} \operatorname{com} \alpha$.

No segundo, construímos um $\Sigma$-M-transdutor $\mathcal{T}^{\prime}$ a partir de $\mathcal{B}$, substituindo cada transição

$$
p \stackrel{e}{\longrightarrow} q
$$

de $\mathcal{B}$ pela transição

$$
p \stackrel{e(\alpha, \beta)}{\longrightarrow} q
$$

Pela definição de cross-section, o domínio de $\left|\mathcal{T}^{\prime}\right|$ é $B \alpha=\operatorname{dom}|\mathcal{T}|$, e cada palavra nesse domínio é soletrada por exatamente um passeio bem-sucedido em $\mathcal{T}^{\prime}$. Assim, $\mathcal{T}^{\prime}$ é não-ambíguo, e, da hipótese de que $\mathcal{T}$ é funcional, segue que $\left|\mathcal{T}^{\prime}\right|=|\mathcal{T}|$.

Apresentamos a seguir um exemplo dessa construção.

Exemplo 3.9.3 Seja $\Sigma=\{\sigma\}$. Considere o $\Sigma$ - $\mathbb{R}$-transdutor funcional $\mathcal{T}=(Q, E, I, T)$ ilustrado a seguir, onde $\mathbb{R}$ é o monóide aditivo dos números reais:

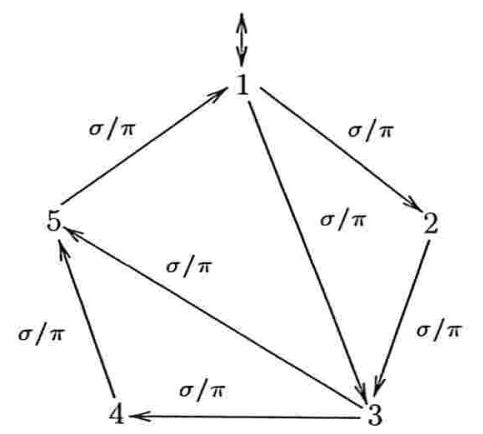

Vamos construir um $\Sigma$ - $\mathbb{R}$-transdutor não-ambíguo equivalente a $\mathcal{T}$.

Associando uma letra a cada transição de $\mathcal{T}$, obtemos um $E$-autômato determinístico que reconhece $\# \mathcal{T}$, conforme ilustrado a seguir:

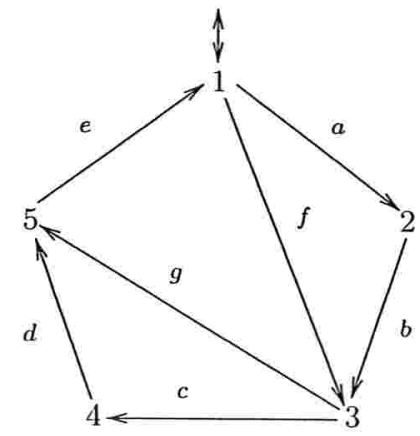

Em seguida, utilizando a Proposição 3.7.2, construímos um E-autômato determinístico que reconhece uma cross-section de $\# \mathcal{T} \operatorname{com} \alpha$. O transdutor obtido está ilustrado a seguir. Utilizamos a mesma notação do Exemplo 3.9.1. 


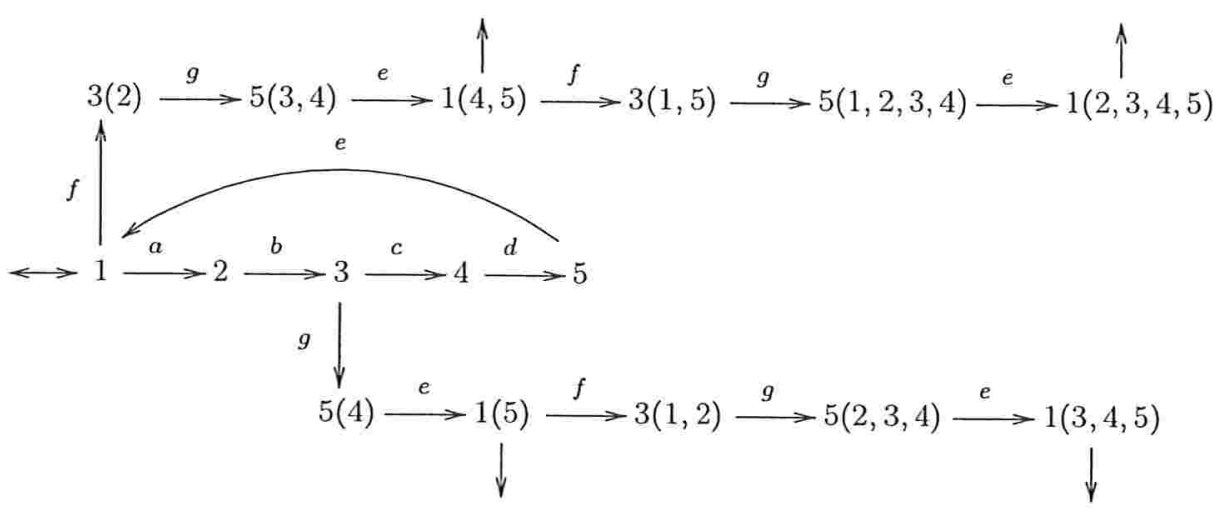

Fazendo as substituições descritas em (3.9.2), obtemos o transdutor não-ambíguo equivalente a $\mathcal{T}$ ilustrado a seguir:

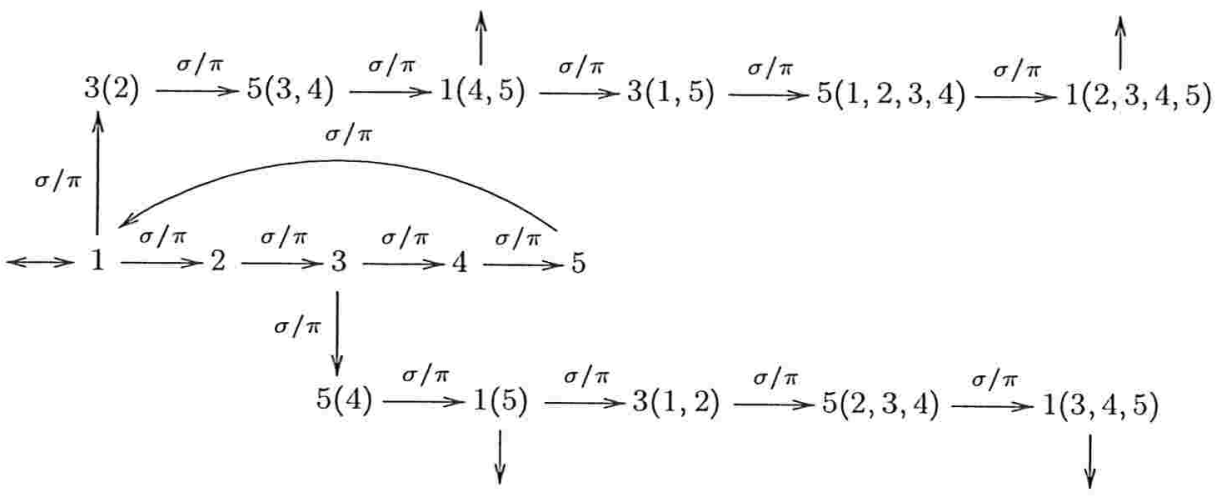

Observamos que esse método não constrói o transdutor mais simples ilustrado a seguir:

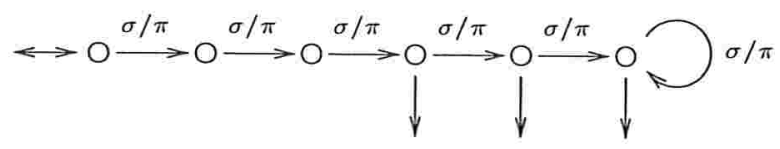





\section{FunÇÕES RACIONAIS}

As funções racionais destacam-se entre as relações racionais devido a algumas propriedades muito interessantes dessa classe. Em particular, é no âmbito das funções racionais que surge o conceito de determinismo para transdutores. Esse conceito é importante não só para a teoria, mas também para as aplicações práticas, já que o determinismo permite obter a imagem de uma palavra soletrando-a seqüencialmente.

Este capítulo tem ênfase nas funções racionais que podem ser realizadas por um transdutor determinístico na entrada, proposto por Schützenberger, denominado transdutor subseqüencial. O objetivo principal do capítulo é apresentar a caracterização de Choffrut das funções subseqüenciais e uma teoria de minimização de transdutores subseqüenciais.

Ao longo deste capítulo, $\Sigma$ e $\Gamma$ representam alfabetos, e $\mathrm{M}$ e $\mathrm{N}$ monóides.

\subsection{Decisão de se uma relação racional finitamente valorada é uma função}

Fixamos um $\Sigma$ - $\Gamma$-transdutor letra-palavra bi-acessível $\mathcal{T}$. Denotamos por $M$ o máximo dentre os comprimentos das saídas de $\mathcal{T}$, por $n$ o número de estados de $\mathcal{T}$, e por $m$ o número de transições de $\mathcal{T}$. Supomos que os estados de $\mathcal{T}$ são numerados de 1 a $n$.

$\mathrm{O}$ algoritmo de Schützenberger para decidir se um transdutor letra-palavra é funcional (Teorema 3.4.1) tem complexidade de tempo exponencial. Um algoritmo bastante eficiente para esse problema foi publicado em 2003 por Béal, Carton, Prieur e Sakarovitch [BCPS03].

Nesta seção, vamos descrever esse algoritmo. Observamos também que a eficiência da versão original do algoritmo pode ser melhorada, utilizando uma propriedade simples de simetria.

Vamos começar com algumas definições. A primeira é um formalismo que permite o estudo simultâneo das saídas de passeios bem-sucedidos com a mesma entrada.

Definição 4.1.1 Seja $\mathcal{T}=(Q, E, I, T)$ um $\Sigma$ - $\Gamma$-transdutor letra-palavra. $O$ quadrado de $\mathcal{T}$ é um $\Sigma$-( $\left.\Gamma^{*} \times \Gamma^{*}\right)$-transdutor letra-palavra $\mathcal{T} \times \mathcal{T}=\left(Q \times Q, E^{\prime}, I \times I, T \times T\right)$, onde

$$
(p, q) \stackrel{\sigma /(x, y)}{\longrightarrow}\left(p^{\prime}, q^{\prime}\right) \in E^{\prime}
$$

se, e somente se,

$$
p \stackrel{\sigma / x}{\longrightarrow} p^{\prime}, q \stackrel{\sigma / y}{\longrightarrow} q^{\prime} \in E .
$$


Claramente, o número de estados de $\mathcal{T} \times \mathcal{T}$ é $n^{2}$, e o número de transições é no máximo $m^{2}$. Segue diretamente dessa definição que existe um passeio

$$
(p, q) \stackrel{u /(x, y)}{\longrightarrow}\left(p^{\prime}, q^{\prime}\right)
$$

em $\mathcal{T} \times \mathcal{T}$ se, e somente se, existem passeios

$$
p \stackrel{u / x}{\longrightarrow} p^{\prime} \quad \text { e } \quad q \stackrel{u / y}{\longrightarrow} q^{\prime}
$$

em $\mathcal{T}$. Portanto,

(4.1.1) $\mathcal{T}$ é funcional se, e somente se, todo passeio bem-sucedido $\left(i, i^{\prime}\right) \stackrel{u /(x, y)}{\longrightarrow}\left(t, t^{\prime}\right)$ em $\mathcal{T} \times \mathcal{T}$ satisfaz $x=y$.

Para cada estado $(p, q)$ de $\mathcal{T} \times \mathcal{T}$, vamos denotar por $\Delta_{p q}$ o conjunto

$$
\Delta_{p q}=\left\{(x, y) \Delta: \text { existe um passeio }\left(i, i^{\prime}\right) \stackrel{u /(x, y)}{\longrightarrow}(p, q) \text { em } \mathcal{T} \times \mathcal{T} \operatorname{com} i, i^{\prime} \in I\right\}
$$

Cada elemento em $\Delta_{p q}$ será denominado uma diferença de $(p, q)$. Podemos reescrever (4.1.1) como

$$
\mathcal{T} \text { é funcional se, e somente se, para todo estado final }\left(t, t^{\prime}\right), \Delta_{t t^{\prime}}=\{(1,1)\} .
$$

A seguinte propriedade será útil. Dados um par $(r, s)$ em $\Delta_{p q}$ e uma transição $e:(p, q) \stackrel{u /(x, y)}{\longrightarrow}$ $\left(p^{\prime}, q^{\prime}\right)$,

$$
(r, s) \cdot(x, y) \in \Delta_{p^{\prime} q^{\prime}}
$$

De fato, seja $c:\left(i, i^{\prime}\right) \stackrel{v /\left(x^{\prime}, y^{\prime}\right)}{\longrightarrow}(p, q)$ um passeio tal que $\left(i, i^{\prime}\right)$ é um estado inicial e $(r, s)=$ $\left(x^{\prime}, y^{\prime}\right) \Delta$. Então

$$
(r, s) \cdot(x, y)=\left(x^{\prime}, y^{\prime}\right) \Delta \cdot(x, y) \stackrel{(3.6 .9 \mathrm{~b})}{=}\left(x^{\prime} x, y^{\prime} y\right) \Delta \text {. }
$$

Resta observar que $\left(x^{\prime} x, y^{\prime} y\right)$ é a saída do passeio ce.

Proposição 4.1.1 Se $\mathcal{T}$ for um $\Sigma$-Г-transdutor letra-palavra funcional, então, para todo estado acessivel e co-acessivel $(p, q)$ de $\mathcal{T} \times \mathcal{T}, \Delta_{p q}$ é unitário, e $\Delta_{p q} \subseteq\left(1 \times \Gamma^{*}\right) \cup\left(\Gamma^{*} \times 1\right)$.

Demonstração. Sejam $(p, q)$ um estado acessível e co-acessível, e

$$
(p, q) \stackrel{v /(r, s)}{\longrightarrow}\left(t, t^{\prime}\right)
$$

um passeio tal que $\left(t, t^{\prime}\right)$ é um estado final.

Sejam

$$
c:\left(i, i^{\prime}\right) \stackrel{u /(x, y)}{\longrightarrow}(p, q) \text { e } \quad c^{\prime}:\left(j, j^{\prime}\right) \stackrel{u^{\prime} /\left(x^{\prime}, y^{\prime}\right)}{\longrightarrow}(p, q)
$$

passeios na parte bi-acessível de $\mathcal{T} \times \mathcal{T}$ tais que $\left(i, i^{\prime}\right)$ e $\left(j, j^{\prime}\right)$ são estados iniciais. Como $\mathcal{T}$ é funcional,

$$
(x r, y s) \Delta=\left(x^{\prime} r, y^{\prime} s\right) \Delta=(1,1)
$$


De (3.6.9f), segue que

$$
(x, y) \Delta=\left(x^{\prime}, y^{\prime}\right) \Delta .
$$

Como $c$ e $c^{\prime}$ são arbitrários, concluímos que $\Delta_{p q}$ é unitário.

Como $(x r, y s) \Delta=(1,1)$, segue de $(3.6 .9 \mathrm{~g})$ que

$$
(x, y) \Delta \in\left(1 \times \Gamma^{*}\right) \cup\left(\Gamma^{*} \times 1\right) .
$$

Na descrição do algoritmo, apenas a parte bi-acessível de $\mathcal{T} \times \mathcal{T}$ será considerada, e será denotada também por $\mathcal{T} \times \mathcal{T}$. Podemos supor que o algoritmo elimina inicialmente os estados não-acessíveis e não-co-acessíveis. Vamos denotar por $F$ o conjunto das transições da parte bi-acessível de $\mathcal{T} \times \mathcal{T}$.

Dado um subconjunto $X$ de transições de $\mathcal{T} \times \mathcal{T}$, recordamos que a restrição de $\mathcal{T} \times \mathcal{T}$ a $X$ é o transdutor $(\mathcal{T} \times \mathcal{T})[X]$ que contém exatamente as transições em $X$ e os estados que são origem ou término de alguma transição nesse conjunto. Nessa restrição, as propriedades de um estado de ser inicial ou final são mantidas.

Informalmente, o algoritmo de Béal, Carton, Prieur e Sakarovitch visita os estados acessíveis e co-acessíveis de $\mathcal{T} \times \mathcal{T}$. Quando um estado $(p, q)$ é visitado, um par no conjunto $\Delta_{p q}$ é construído. A cada novo par construído, as condições da Proposição 4.1.1 são verificadas. Se essas condições forem satisfeitas para todo estado acessível e co-acessível, o algoritmo verifica (4.1.2).

$\mathrm{O}$ algoritmo executa uma seqüência de iterações. Cada iteração começa com um subconjunto $X$ de transições e uma função parcial $\delta: Q \times Q \rightarrow\left(1 \times \Gamma^{*}\right) \cup\left(\Gamma^{*} \times 1\right)$. Vamos denotar a imagem por $\delta$ de um par $(p, q)$ por $\delta_{p q}$.

A primeira iteração começa com $X=\varnothing$ e $\delta_{i i^{\prime}}=(1,1)$, para todo estado inicial e co-acessível $\left(i, i^{\prime}\right)$. Nos demais estados, $\delta$ é indefinido. Cada iteração consiste no seguinte:

Caso $1 X=F$

Se $\delta_{t t^{\prime}}=(1,1)$ para todo estado final $\left(t, t^{\prime}\right)$, responda sim e pare. Senão, responda não e pare.

\section{Caso $2 \quad X \neq F$}

Seja $e:(p, q) \stackrel{u /(x, y)}{\longrightarrow}\left(p^{\prime}, q^{\prime}\right)$ uma transição em $F-X$ tal que $\delta_{p q} \neq \varnothing$.

Se alguma das condições a seguir ocorrer, responda não e pare. Senão, defina $\delta_{p^{\prime} q^{\prime}}=\delta_{p q} \cdot(x, y)$ e insira $e$ em $X$.

- $\delta_{p q} \cdot(x, y) \notin\left(1 \times \Gamma^{*}\right) \cup\left(\Gamma^{*} \times 1\right)$;

- $\delta_{p^{\prime} q^{\prime}} \neq \varnothing$ e $\delta_{p q} \cdot(x, y) \neq \delta_{p^{\prime} q^{\prime}}$.

O seguinte invariante é válido no início de cada iteração:

Invariante 4.1.1 A restrição $(\mathcal{T} \times \mathcal{T})[X]$ é acessivel, e o dominio de $\delta$ é exatamente a união dos estados desse transdutor com os estados iniciais da parte bi-acessivel de $\mathcal{T} \times \mathcal{T}$. Ademais, para todo passeio $\left(i, i^{\prime}\right) \stackrel{u /(x, y)}{\longrightarrow}(p, q)$ em $(\mathcal{T} \times \mathcal{T})[X]$ tal que $\left(i, i^{\prime}\right)$ é um estado inicial, $(x, y) \Delta=\delta_{p q}$. 
Se o Caso 1 for executado, então segue do Invariante 4.1.1 que $\Delta_{p q}=\left\{\delta_{p q}\right\}$, para todo estado acessível e co-acessível $(p, q)$. Portanto, se o algoritmo responder sim, segue de (4.1.2) que $\mathcal{T}$ é funcional.

Se o algoritmo responder não, então ou (4.1.2) ou uma das condições da Proposição 4.1.1 foi contrariada. Em ambos os casos, $\mathcal{T}$ não é funcional.

Uma melhoria pode ser feita considerando que se

$$
c:(p, q) \stackrel{w /(u, v)}{\longrightarrow}\left(p^{\prime}, q^{\prime}\right)
$$

for um passeio em $\mathcal{T} \times \mathcal{T}$, então existe também o passeio

$$
c^{\prime}:(q, p) \stackrel{w /(v, u)}{\longrightarrow}\left(q^{\prime}, p^{\prime}\right)
$$

nesse transdutor. Portanto, para todo estado $(p, q)$, se

$$
(x, y) \in \Delta_{p q},
$$

então

$$
(y, x) \in \Delta_{q p}
$$

Dizemos que o passeio $c^{\prime}$ é o simétrico de $c$, que a diferença $(y, x)$ é a simétrica de $(x, y)$, e que $o$ estado $(q, p)$ é o simétrico de $(p, q)$. Dado um conjunto $X$ de estados, diferenças ou passeios, o conjunto dos simétricos dos elementos em $X$ será denotado por $X^{\prime}$.

Aproveitando a simetria, vamos descrever um algoritmo que constrói o conjunto $\Delta_{p q}$ apenas para os estados $(p, q)$ tais que $p \leq q$. Vamos denominar esses estados essenciais, e denotar por $G$ o subconjunto das transições em $F$ cuja origem seja um estado essencial.

Nesse algoritmo, o conjunto $X$ contém apenas transições cuja origem é um estado essencial. Apenas os valores de $\delta$ para os estados essenciais são definidos explicitamente. No entanto, podemos supor que, para cada estado essencial no domínio de $\delta$, a imagem do seu simétrico também está definida.

Cada iteração é como segue:

Caso $1 X=G$

Se $\delta_{t t^{\prime}}=(1,1)$ para todo estado final $\left(t, t^{\prime}\right)$ tal que $t \leq t^{\prime}$, responda sim e pare. Senão, responda não e pare.

Caso $2 \quad X \neq G$

Seja $e:(p, q) \stackrel{u /(x, y)}{\longrightarrow}\left(p^{\prime}, q^{\prime}\right)$ uma transição em $G-X$ tal que $\delta_{p q} \neq \varnothing$.

Caso 2a $p^{\prime} \leq q^{\prime}$

Se alguma das condições a seguir ocorrer, responda não e pare. Senão, defina $\delta_{p^{\prime} q^{\prime}}=$ $\delta_{p q} \cdot(x, y)$ e insira $e$ em $X$.

- $\delta_{p q} \cdot(x, y) \notin\left(1 \times \Gamma^{*}\right) \cup\left(\Gamma^{*} \times 1\right)$;

- $\delta_{p^{\prime} q^{\prime}} \neq \varnothing$ e $\delta_{p q} \cdot(x, y) \neq \delta_{p^{\prime} q^{\prime}}$.

Caso $2 \mathrm{~b} p^{\prime}>q^{\prime}$

Se alguma das condições a seguir ocorrer, responda não e pare. Senão, defina $\delta_{q^{\prime} p^{\prime}}$ como o simétrico de $\delta_{p q} \cdot(x, y)$ e insira $e$ em $X$. 
- $\delta_{p q} \cdot(x, y) \notin\left(1 \times \Gamma^{*}\right) \cup\left(\Gamma^{*} \times 1\right)$;

- $\delta_{q^{\prime} p^{\prime}} \neq \varnothing$ e o simétrico de $\delta_{p q} \cdot(x, y)$ é diferente de $\delta_{q^{\prime} p^{\prime}}$.

O invariante passa a ser

Invariante 4.1.2 A restrição $(\mathcal{T} \times \mathcal{T})\left[X \cup X^{\prime}\right]$ é acessível, e o dominio de $\delta$ é exatamente a união dos estados desse transdutor com os estados iniciais da parte bi-acessivel de $\mathcal{T} \times \mathcal{T}$. Ademais, para todo passeio $\left(i, i^{\prime}\right) \stackrel{u /(x, y)}{\longrightarrow}(p, q)$ em $(\mathcal{T} \times \mathcal{T})\left[X \cup X^{\prime}\right]$ tal que $\left(i, i^{\prime}\right)$ é um estado inicial, $(x, y) \Delta=\delta_{p q}$.

A demonstração da corretude deste algoritmo é semelhante à do algoritmo anterior, levando-se em consideração a simetria.

Uma descrição mais detalhada pode ser vista no Algoritmo 4.1.1. Nessa descrição, apenas um conjunto $P$ de estados de $\mathcal{T} \times \mathcal{T}$ é mantido. Esse conjunto armazena todos os estados essenciais já visitados, e que são origem de alguma transição ainda não selecionada (ou seja, em $G-X$ ). A cada iteração, um par $(p, q)$ em $P$ é selecionado, e todas as transições com origem em $(p, q)$ são analisadas.

O número de estados e de transições de $\mathcal{T} \times \mathcal{T}$ são no máximo $n^{2}$ e $m^{2}$, respectivamente. Portanto, as complexidades de tempo e espaço para determinar a parte bi-acessível desse transdutor são $O\left(n^{2}+m^{2}\right)$.

Vamos fazer uma análise mais precisa das complexidades de tempo e espaço do Algoritmo 4.1.1. Primeiro, vamos provar que, para todo par $\delta_{p q}=(v, w)$ construído pelo algoritmo,

$$
|v|,|w| \leq M\left(\begin{array}{l}
n \\
2
\end{array}\right) \text {. }
$$

Se $v=w=1$, não há o que demonstrar. Sem perda de generalidade, suponha que $v \neq 1$.

Seja $c:\left(i, i^{\prime}\right) \stackrel{u /(x, y)}{\longrightarrow}(p, q)$ um caminho em $(\mathcal{T} \times \mathcal{T})\left[X \cup X^{\prime}\right]$. Então, pelo Invariante 4.1.2, $(v, w)=(x, y) \Delta$.

Fatore esse caminho como $d d^{\prime}$, onde o término de $d$ é o último estado em c cuja imagem por $\delta$ tem a palavra vazia na primeira coordenada. Note que essa fatoração é possível porque $\delta_{i i^{\prime}}=(1,1)$.

Vamos denotar por $\left(1, w^{\prime}\right)$ a imagem por $\delta$ do primeiro estado de $d^{\prime}$. Por (3.6.9b),

$$
(v, w)=\left(1, w^{\prime}\right) \cdot\left(d^{\prime} \beta\right) .
$$

Sejam $\left(p^{\prime}, q^{\prime}\right) \stackrel{u^{\prime} /\left(x^{\prime}, y^{\prime}\right)}{\longrightarrow}\left(p^{\prime \prime}, q^{\prime \prime}\right)$ uma transição qualquer em $d^{\prime}$, e $r$ e $s$ as palavras nas primeiras coordenadas de $\delta_{p^{\prime} q^{\prime}}$ e $\delta_{p^{\prime \prime}} q^{\prime \prime}$, respectivamente. Claramente,

$$
|s| \leq|r|+M
$$

Portanto,

$$
|v| \leq M\left|d^{\prime}\right|
$$

Para quaisquer estados $\left(p^{\prime}, q^{\prime}\right)$ e $\left(p^{\prime \prime}, q^{\prime \prime}\right)$ que sejam término de alguma transição em $d^{\prime}, \delta_{p^{\prime} q^{\prime}}$ e $\delta_{p^{\prime \prime} q^{\prime \prime}}$ pertencem a $\Gamma^{+} \times 1$. Portanto, esses estados não podem ser simétricos. 


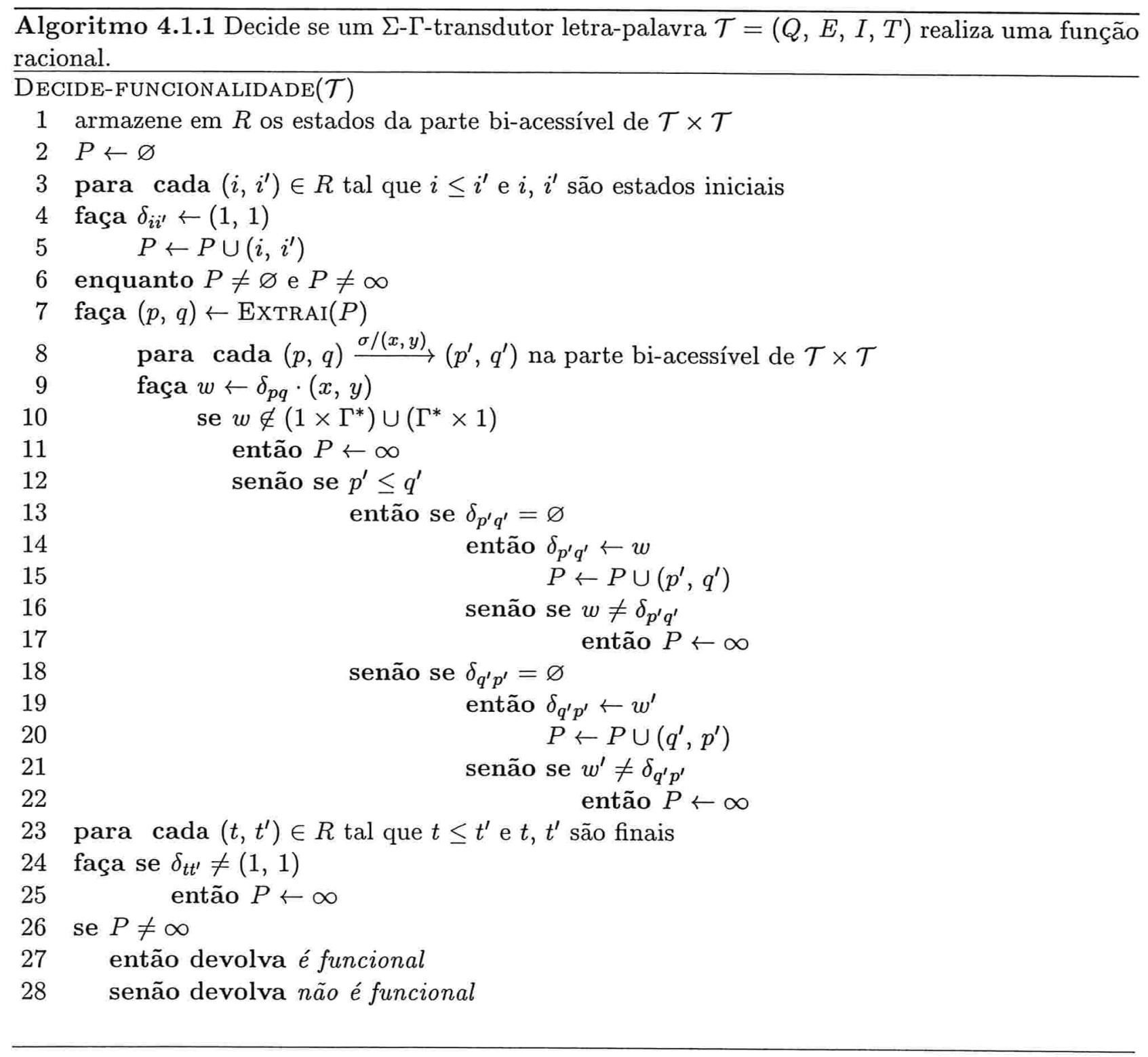


Assim, para todo subconjunto $\left\{p^{\prime}, q^{\prime}\right\}$ de $Q$, no máximo um dos pares $\left(p^{\prime}, q^{\prime}\right)$ ou $\left(q^{\prime}, p^{\prime}\right)$ pode ser o término de uma transição em $d^{\prime}$. Portanto, $\left|d^{\prime}\right|$ é no máximo o número de subconjuntos de dois elementos de $Q$.

Concluímos então que

$$
\left|d^{\prime}\right| \leq\left(\begin{array}{l}
n \\
2
\end{array}\right)
$$

Logo, de (4.1.5), obtemos (4.1.4).

Em seguida, vamos provar que o número de transições com origem em um estado essencial é no máximo $m(m+1) / 2$. Seja $e_{1}, \ldots, e_{m}$ uma enumeração das transições de $\mathcal{T}$ tal que para todo par de transições $e_{i}: p \rightarrow q$ e $e_{j}: p^{\prime} \rightarrow q^{\prime}$, se $p<p^{\prime}$, então $i<j$. Cada transição $(p, q) \rightarrow\left(p^{\prime}, q^{\prime}\right)$ em $\mathcal{T} \times \mathcal{T}$ tal que $(p, q)$ é um estado essencial corresponde então a um par de transições $e_{i}$ e $e_{j}$ tal que $i \leq j$. Basta agora observar que o número de pares de transições com essa propriedade é $m(m+1) / 2$.

A cada transição visitada, o número de operações realizadas para calcular $\delta_{p q} \cdot(x, y)$ é proporcional a $M\left(\begin{array}{l}n \\ 2\end{array}\right)$, no pior caso. Portanto, o número total de operações realizadas pelo Algoritmo 4.1.1 no pior caso é

$$
M \frac{m(m+1) n(n-1)}{4} .
$$

A complexidade de espaço do Algoritmo 4.1.1 depende essencialmente do espaço utilizado para armazenar os pares $\delta_{p q}$. Observando que o número de estados essenciais é no máximo $n(n+1) / 2$, temos que a soma dos comprimentos desses pares é no máximo

$$
M \frac{n^{2}\left(n^{2}-1\right)}{4} .
$$

Concluímos a seção com uma observação simples sobre o quadrado de um transdutor funcional que será útil em alguns exemplos.

Seja $\mathcal{T}$ um transdutor letra-palavra bi-acessível funcional. Segue dessas hipóteses que, para todo par de estados $p$ e $q$ e toda letra $\sigma$, o transdutor $\mathcal{T}$ tem no máximo uma transição da forma

$$
p \stackrel{\sigma / x}{\longrightarrow} q .
$$

Considere a restrição de $\mathcal{T} \times \mathcal{T}$ aos estados da forma $(p, p)$ (ou seja, o transdutor obtido mantendo-se apenas os pares da forma $(p, p)$ e as transições com origem e término nesses pares). De (4.1.6), segue que o autômato de entrada dessa restrição é isomorfo ao autômato de entrada de $\mathcal{T}$ (veja a Seção 4.12 para uma definição formal de isomorfismo de autômatos). Ademais, para todo estado $p$ de $\mathcal{T}, \mathcal{T} \times \mathcal{T}$ tem uma transição da forma

$$
(p, p) \stackrel{\sigma /\left(x, x^{\prime}\right)}{\longrightarrow}(q, q)
$$

se, e somente se, $x=x^{\prime}$ e $\mathcal{T}$ tem uma transição da forma

$$
p \stackrel{\sigma / x}{\longrightarrow} q .
$$

Exemplo 4.1.1 Vamos reproduzir o exemplo ilustrado na Figura 4 de [BCPS03]. O transdutor 


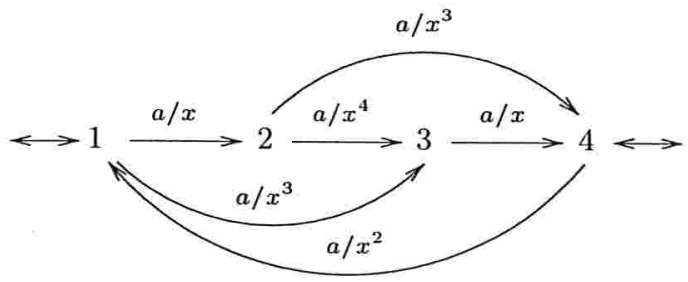

é funcional. Seu quadrado está ilustrado abaixo, com a indicação de $\Delta_{p q}$ para cada estado $(p, q)$.

$\begin{array}{llll}1 & 2 & 3 & 4\end{array}$

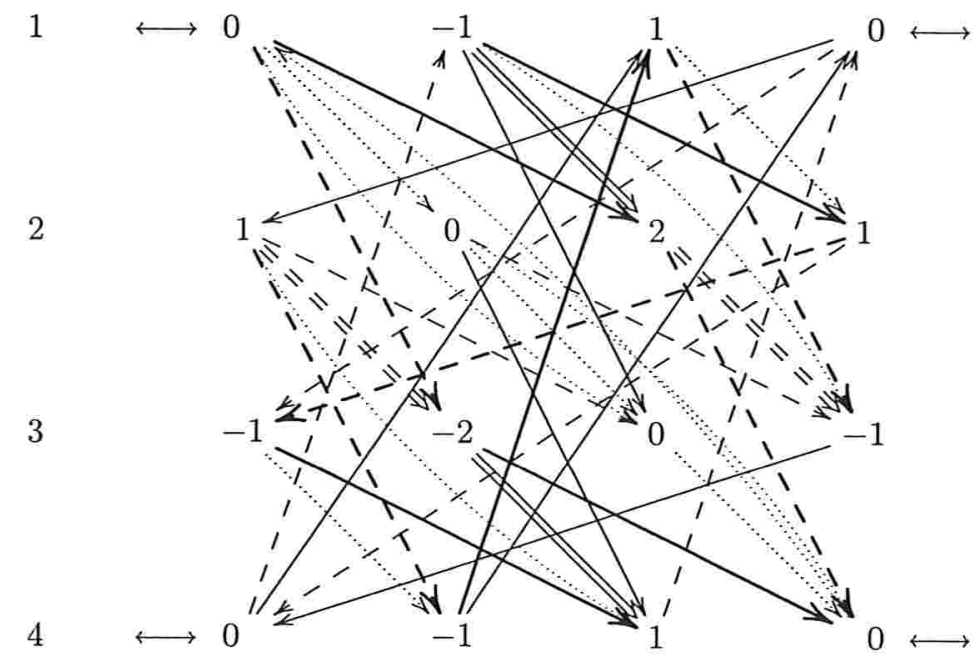

A mesma notação de [BCPS03] é seguida nessa figura. Um par de palavras $(u, v)$ é codificado como o inteiro $|u|-|v|$. A posição na coluna $p$ e linha $q$ corresponde ao estado $(p, q)$, e essa posição contém a codificação da única diferença em $\Delta_{p q}$.

A entrada de toda transição é $a$, e não é indicada. O desenho das transições indica o código da saída como segue: linhas pontilhadas indicam 0 ; linhas contínuas simples indicam +1 ; linhas contínuas escuras indicam +2 , e linhas contínuas duplas indicam +3 . As linhas tracejadas indicam os códigos com os sinais opostos aos códigos das linhas contínuas correspondentes.

A figura a seguir ilustra apenas os pares essenciais e as transições visitadas pelo algoritmo, quando a propriedade da simetria é aproveitada. 


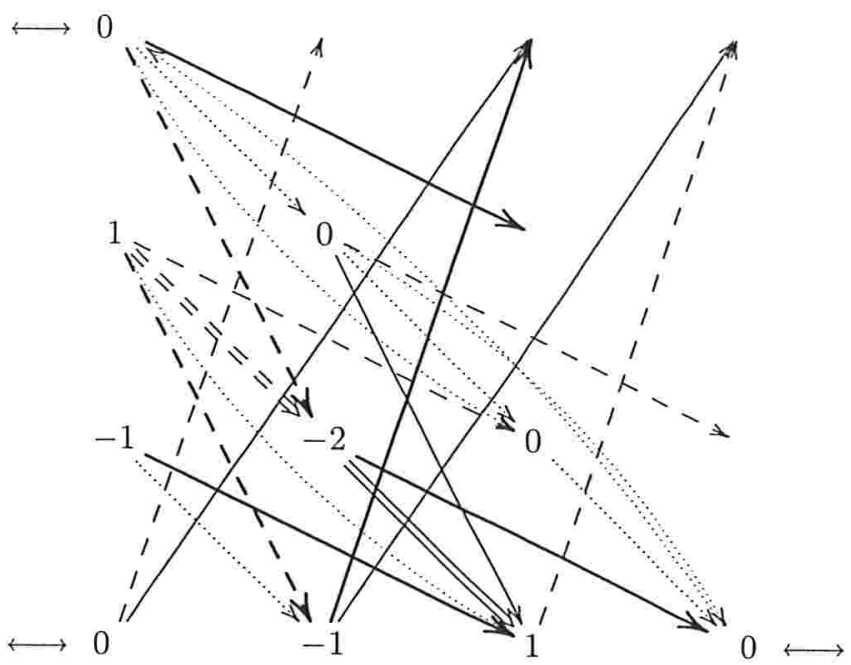

Exemplo 4.1.2 Neste exemplo, vamos definir uma família de transdutores funcionais cujo quadrado contém um par $\delta_{p q}$ que atinge o comprimento máximo provado em (4.1.4).

Sejam $n$ e $M$ inteiros positivos, $\operatorname{com} n>2$. Vamos denotar $\left(\begin{array}{l}n \\ 2\end{array}\right)$ por $k$. Seja

$$
\mathcal{E}=\left(p_{1}, q_{1}\right), \ldots,\left(p_{k}, q_{k}\right)
$$

uma enumeração dos pares $(p, q)$ em $[n] \times[n]$ tais que $p<q$.

Para cada par $\left(p_{i}, q_{i}\right)$ em $\mathcal{E}$ tal que $0<i<k$, defina duas letras $\sigma_{i}$ e $\gamma_{i}$. Sejam

$$
\Sigma=\left\{\sigma_{0}, \sigma_{1}, \ldots, \sigma_{k-1}, \gamma_{1}, \ldots, \gamma_{k-1}, \gamma_{k}\right\} \text { e } \Gamma=\{a\}
$$

onde as letras $\sigma_{0}, \gamma_{k}$ e $a$ são novas.

Utilizando esses dados, e considerando que $\left(p_{0}, q_{0}\right)=(1,1)$, definimos um $\Sigma$ - $\Gamma$-transdutor $\mathcal{T}$ como segue. O conjunto de estados de $\mathcal{T}$ é $[n]$. O único estado inicial é 1 , e esse estado também é o único final. Para cada par $\left(p_{i}, q_{i}\right)$ em $\mathcal{E}$ com $0<i<k, \mathcal{T}$ tem as transições

$$
p_{i} \stackrel{\sigma_{i} / a^{M}}{\longrightarrow} p_{i+1}, \quad q_{i} \stackrel{\sigma_{i} / 1}{\longrightarrow} q_{i+1}, \quad p_{i} \stackrel{\gamma_{i} / 1}{\longrightarrow} p_{i-1} \quad \text { e } \quad q_{i} \stackrel{\gamma_{i} / a^{M}}{\longrightarrow} q_{i-1} .
$$

As únicas transições de $\mathcal{T}$ além dessas são

$$
1 \stackrel{\sigma_{0} / a^{M}}{\longrightarrow} p_{1}, \quad 1 \stackrel{\sigma_{0} / 1}{\longrightarrow} q_{1}, \quad p_{k} \stackrel{\gamma_{k} / 1}{\longrightarrow} p_{k-1} \quad \text { e } q_{k} \stackrel{\gamma_{k} / a^{M}}{\longrightarrow} q_{k-1} .
$$

Para provar que $\mathcal{T}$ é bi-acessível, seja $q$ um estado diferente de 1 e $\left(p_{i}, q_{i}\right)$ o par $(1, q)$. Da definição das transições de $\mathcal{T}$ segue que existe um passeio da forma

$$
1 \stackrel{\sigma_{0} / 1}{\longrightarrow} q_{1} \ldots q_{i-1} \stackrel{\sigma_{i-1} / 1}{\longrightarrow} q_{i} \stackrel{\gamma_{i} / a^{M}}{\longrightarrow} q_{i-1} \ldots q_{1} \stackrel{\gamma_{1} / a^{M}}{\longrightarrow} 1
$$

As seguintes propriedades de $\mathcal{T} \times \mathcal{T}$ são uma conseqüência imediata dessa definição: 
i. As únicas transições com origem em um par $\left(p_{i}, q_{i}\right)$ tal que $0<i<k$ são

$$
\left(p_{i}, q_{i}\right) \stackrel{\sigma_{i} /\left(a^{M}, 1\right)}{\longrightarrow}\left(p_{i+1}, q_{i+1}\right) \text { e }\left(p_{i}, q_{i}\right) \stackrel{\gamma_{i} /\left(1, a^{M}\right)}{\longrightarrow}\left(p_{i-1}, q_{i-1}\right) .
$$

Ademais, há uma única transição com origem em $\left(p_{k}, q_{k}\right)$, a saber,

$$
\left(p_{k}, q_{k}\right) \stackrel{\gamma_{k} /\left(1, a^{M}\right)}{\longrightarrow}\left(p_{k-1}, q_{k-1}\right) .
$$

ii. As únicas transiçōes com origens e entradas idênticas são $1 \stackrel{\sigma_{0} / a^{M}}{\longrightarrow} p_{1}$ e $1 \stackrel{\sigma_{0} / 1}{\longrightarrow} q_{1}$. Assim, para todo estado $p$ diferente de $1, \mathcal{T} \times \mathcal{T}$ tem uma transição da forma

$$
(p, p) \stackrel{\sigma /\left(x, x^{\prime}\right)}{\longrightarrow}\left(q, q^{\prime}\right)
$$

se, e somente se, $q=q^{\prime}, x=x^{\prime}$ e $\mathcal{T}$ tem uma transição da forma

$$
p \stackrel{\sigma / x}{\longrightarrow} q .
$$

A mesma propriedade é válida para $p=1$, com toda letra $\sigma$ em $\Sigma-\sigma_{0}$. Ademais, há quatro transições em $\mathcal{T} \times \mathcal{T}$ com origem em $(1,1)$ e entrada $\sigma_{0}$, ilustradas a seguir:

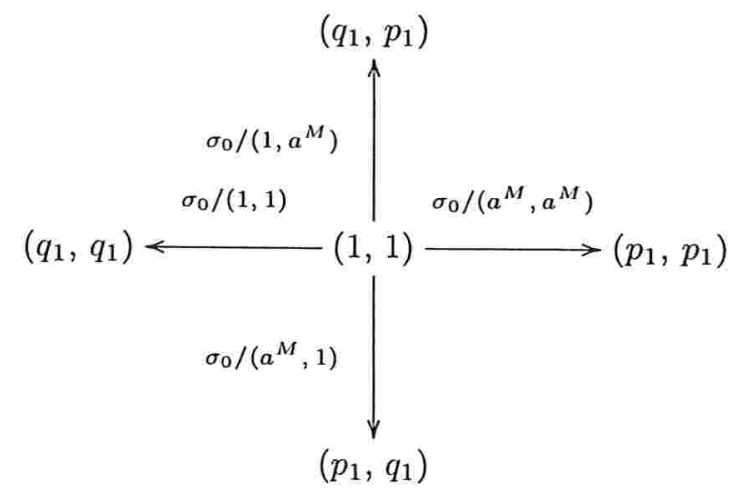

A figura a seguir ilustra $\mathcal{T} \times \mathcal{T}$, sem a indicação dos estados da forma $(p, p)$. Como o autômato de entrada da restrição do quadrado a esses estados é isomorfo ao autômato de entrada de $\mathcal{T}$, segue que esses estados são acessíveis e co-acessíveis. As transições pontilhadas não são visitados pelo Algoritmo 4.1.1.

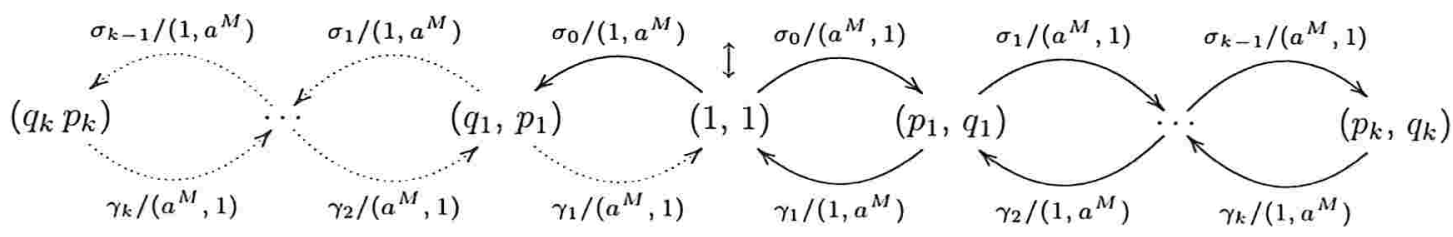

Essa figura deixa claro que $\mathcal{T} \times \mathcal{T}$ é bi-acessível.

Essa discussão mostra que

$$
\Delta_{q q}=\{(1,1)\}, \quad \forall q \in Q,
$$


e

$$
\Delta_{p_{i} q_{i}}=\left\{\left(a^{i M}, 1\right)\right\}, \quad \forall\left(p_{i}, q_{i}\right) \in \mathcal{E} .
$$

De (4.1.8) segue que $\mathcal{T}$ é funcional. De (4.1.9) segue que

$$
\Delta_{p_{k} q_{k}}=\left\{\left(a^{K}, 1\right)\right\}, \text { onde } K=M\left(\begin{array}{l}
n \\
2
\end{array}\right) .
$$

O transdutor para $n=4$ e a enumeração

$$
(1,2),(1,3),(1,4),(2,3),(2,4),(3,4)
$$

está ilustrado a seguir:

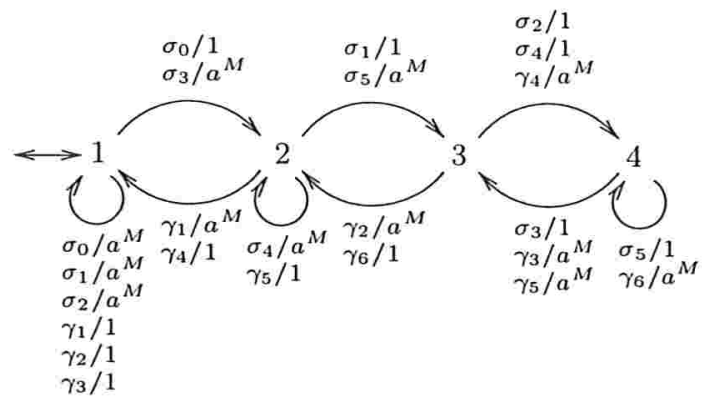

O quadrado desse transdutor está ilustrado na Figura 4.1.1. Ao contrário do Exemplo 4.1.1, um estado $(p, q)$ é representado na linha $p$ e na coluna $q$. Novamente, um par de palavras $(u, v)$ é codificado com o inteiro $|u|-|v|$, e uma posição $(p, q)$ contém a codificação da única diferença em $\Delta_{p q}$.

As saídas são indicadas pelo desenho das transições como segue: as linhas contínuas indicam $\left(a^{M}, 1\right)$; as linhas pontilhadas indicam $\left(1, a^{M}\right)$; as linhas tracejadas correspondem às transições de $\mathcal{T}$, e os rótulos dessas transições podem ser obtidos seguindo (4.1.7).

\subsection{Funções seqüenciais e subseqüenciais}

Na prática, é desejável que uma função racional possa ser computada deterministicamente, ou seja, que a imagem de uma palavra possa ser obtida com uma leitura seqüencial. Essa idéia conduz aos conceitos de transdutor seqüencial e subseqüencial.

Um transdutor seqüencial palavra-palavra é conhecido na literatura como generalized sequential machine ou gsm. Esse modelo de máquina, que é um dos mais antigos na Teoria dos Autômatos, foi introduzido por Mealy [Mea55], e estudado posteriormente por Ginsburg e Rose [GR66]. O termo máquina de Mealy também já foi utilizado para designar esses transdutores.

Outro tipo de máquina determinística existente na literatura é a máquina de Moore. Essa máquina é bastante simples, e suas funções podem ser realizadas por transdutores seqüenciais. Uma discussão a respeito dessas máquinas, bem como a demonstração dessa propriedade, pode ser vista em [Eil74]. 


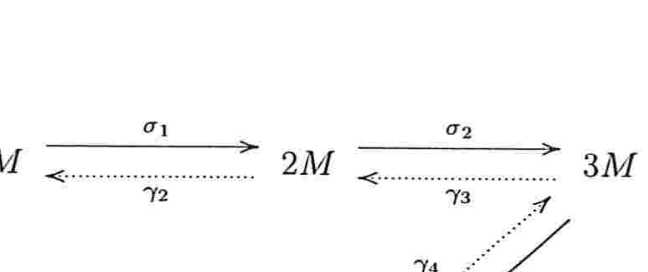

2
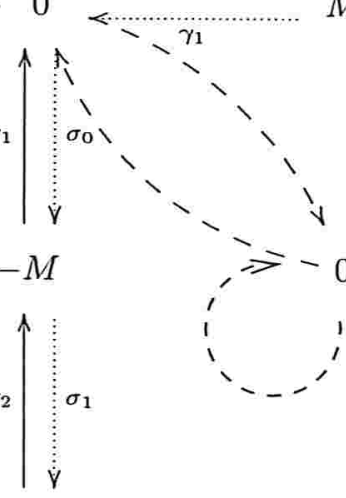

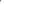

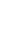

3

$-2 M$

$-4 M$
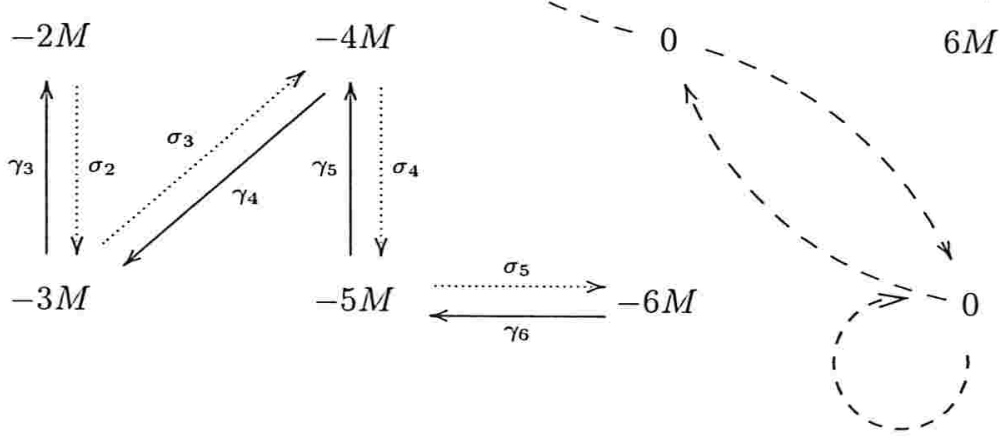

Figura 4.1.1: Quadrado do transdutor do Exemplo 4.1.2

Posteriormente, um modelo de transdutor determinístico mais geral foi proposto por Schützenberger [Sch77]. Esse modelo, conhecido como transdutor subseqüencial, é capaz de realizar diversas funções intuitivamente seqüenciais, mas que não podem ser realizadas por um transdutor seqüencial.

Nesta seção, vamos definir os transdutores seqüenciais e subseqüenciais, e apresentar algumas propriedades simples, para uso nas seções posteriores. O material desta seção é básico, e está contido na maior parte em [Ber79] e [Eil74] (no livro de Eilenberg, apenas os transdutores seqüenciais são estudados).

O determinismo na entrada pode ser visto como uma ação de um monóide livre sobre o conjunto de estados. Assim, é útil definir os transdutores seqüenciais e subseqüenciais com base nesse conceito.

Recordamos que uma ação de um monóide livre $\Sigma^{*}$ sobre um conjunto $Q$ é uma função parcial . : $Q \times \Sigma^{*} \rightarrow Q$ satisfazendo as propriedades em (1.2.2). Dados elementos $q$ em $Q$ e $u$ em $\Sigma^{*}$, podemos omitir o $\cdot$ em $q \cdot u$, e escrever simplesmente $q u$.

Definição 4.2.1 Um $\Sigma$-M-transdutor seqüencial é uma máquina composta de 
- um conjunto finito de estados $Q$;

- um estado inicial $i$;

- uma função parcial : $Q \times \Sigma \rightarrow Q$;

- uma função parcial $*: Q \times \Sigma \rightarrow \mathrm{M}$, com domínio idêntico ao de .

A função - é denominada função de transição, e *, função de emissão, ou simplesmente emissão. Vamos denotar um transdutor seqüencial por $(Q, i)$.

Em [Eil74], o termo máquina seqüencial é utilizado apenas no caso em que toda emissão é uma letra, ao invés de uma palavra qualquer. Vamos chamar um transdutor com essa propriedade de transdutor seqüencial letra-letra.

Utilizando (1.3.3), a função de transição pode ser estendida para uma ação de $\Sigma^{*}$ sobre $Q$ satisfazendo (1.2.2). Também podemos estender $*$ para uma função $*: Q \times \Sigma^{*} \rightarrow \mathrm{M}$ indutivamente como segue:

$$
\begin{aligned}
& q * 1=1 \\
& q *(\sigma u)=(q * \sigma)((q \cdot \sigma) * u), \quad \forall q \in Q, \forall \sigma \in \Sigma, \forall u \in \Sigma^{*} .
\end{aligned}
$$

Essa extensão tem o mesmo domínio de $\cdot$, e satisfaz

$$
q *(u v)=(q * u)((q \cdot u) * v), \quad \forall q \in Q, \forall u, v \in \Sigma^{*} .
$$

Essas propriedades podem ser demonstradas facilmente por indução em $|u|$.

Num transdutor seqüencial, todos os estados são considerados finais.

O comportamento de um $\Sigma$-M-transdutor seqüencial $\mathcal{T}$ é uma função

$$
|\mathcal{T}|: \Sigma^{*} \rightarrow \mathrm{M}
$$

definida por

$$
u|T|=(i * u), \quad \forall u \in \Sigma^{*} .
$$

Note que $i \cdot u$ está definido se, e somente se, $u \in \operatorname{dom}|\mathcal{T}|$.

Definição 4.2.2 Dizemos que uma função $f: \Sigma^{*} \rightarrow M$ é uma função seqüencial se for o comportamento de um $\Sigma$-M-transdutor seqüencial.

A proposição a seguir mostra a construção de um transdutor letra-saída equivalente a um $\Sigma$ M-transdutor seqüencial.

Proposição 4.2.1 Toda função seqüencial é racional.

Demonstração. Sejam $f: \Sigma^{*} \rightarrow M$ uma função seqüencial, e $\mathcal{S}=(Q, i)$ um $\Sigma$-M-transdutor seqüencial que realiza $f$.

Seja $\mathcal{T}=(Q, E, i, Q)$ o $\Sigma$-M-transdutor letra-saída com conjunto de transições definido por

$$
q \stackrel{\sigma / q * \sigma}{\longrightarrow} q \sigma \in E, \forall q \in Q, \forall \sigma \in \Sigma \text {, tal que } q \sigma \neq \varnothing .
$$


Sejam $u$ uma palavra, e $p$ e $q$ estados. Por indução em $|u|$, concluímos que existe um passeio

$$
p \stackrel{u / x}{\longrightarrow} q
$$

em $\mathcal{T}$ se, e somente se,

$$
p u=q \quad \mathrm{e} \quad p * u=x .
$$

Portanto,

$$
|\mathcal{T}|=|\mathcal{S}|,
$$

o que mostra que $f$ é uma função racional.

Uma propriedade simples das funções seqüenciais utiliza a seguinte definição:

Definição 4.2.3 Dizemos que uma função $f: \Sigma^{*} \rightarrow M$ preserva prefixos se $1 f=1$ e $(u \sigma) f \subseteq$ (uf)M, para todo u em $\Sigma^{*}$ e $\sigma$ em $\Sigma$.

Proposição 4.2.2 Toda função seqüencial preserva prefixos.

Demonstração. A demonstração é trivial.

A máquina definida na Definição 4.2.1 é, em algumas referências, denominada transdutor seqüencial à esquerda, pois a leitura da entrada é feita da esquerda para a direita. A função realizada por esse tipo de transdutor também é denominada função seqüencial à esquerda.

Um transdutor seqüencial em que a leitura da entrada, bem como a escrita da saída, são feitas da direita para a esquerda, também pode ser definido. Esse tipo de transdutor será utilizado na Seção 4.3 .

Definição 4.2.4 Um $\Sigma$-M-transdutor seqüencial à direita é uma máquina composta de

- um conjunto finito de estados $Q$;

- um estado inicial $i$;

- uma função parcial $: \Sigma \times Q \rightarrow Q$;

- uma função parcial $*: \Sigma \times Q \rightarrow \mathrm{M}$, com domínio idêntico ao de.

Um transdutor seqüencial à direita também será denotado por $(Q, i)$.

A definição de transdutor seqüencial à direita pode ser vista como a definição de transdutor seqüencial à esquerda revertida. Dessa forma, algumas propriedades dos transdutores seqüenciais à esquerda têm uma versão semelhante para os transdutores seqüenciais à direita.

Assim, podemos denotar as imagens de um par $(\sigma, q)$ pelas funções · e * por $\sigma q$ e $\sigma * q$, respectivamente. Essas funções também podem ser estendidas para $\Sigma^{*} \times Q$ como segue:

$$
\begin{aligned}
& 1 q=1 \quad \text { e } 1 * q=1 \\
& (u \sigma) \cdot q=u \cdot(\sigma \cdot q) \\
& (u \sigma) * q=(u *(\sigma \cdot q))(\sigma * q), \quad \forall \sigma \in \Sigma, \forall u \in \Sigma^{*}, \forall q \in Q .
\end{aligned}
$$


Essas extensões têm as seguintes propriedades, que podem ser demonstradas facilmente por indução em $|v|$ :

$$
\begin{aligned}
& (u v) \cdot q=u \cdot(v \cdot q) ; \\
& (u v) * q=(u *(v \cdot q))(v * q), \quad \forall u, v \in \Sigma^{*}, \forall q \in Q .
\end{aligned}
$$

O comportamento de um $\Sigma$-M-transdutor seqüencial à direita $\mathcal{T}$ é uma função

$$
|\mathcal{T}|: \Sigma^{*} \rightarrow \mathrm{M}
$$

definida por

$$
u|T|=(u * i), \quad \forall u \in \Sigma^{*} .
$$

Definição 4.2.5 Dizemos que uma função $f: \Sigma^{*} \rightarrow \mathrm{M}$ é seqüencial à direita se for o comportamento de um $\Sigma$-M-transdutor seqüencial à direita.

A proposição a seguir mostra a construção de um transdutor letra-saída equivalente a um $\Sigma$ M-transdutor seqüencial à direita.

Proposição 4.2.3 Toda função seqüencial à direita é racional.

Demonstração. Sejam $f: \Sigma^{*} \rightarrow \mathrm{M}$ uma função seqüencial à direita, e $\mathcal{S}=(Q, i)$ um $\Sigma$-Mtransdutor seqüencial à direita que realiza $f$.

Seja $\mathcal{T}=(Q, E, Q, i)$ o $\Sigma$-M-transdutor letra-saída com conjunto de transições definido por

$$
p \stackrel{\sigma / \sigma * q}{\longrightarrow} q \in E, \forall p, q \in Q, \forall \sigma \in \Sigma, \text { tal que } p=\sigma q .
$$

Segue diretamente dessa construção que, para todo estado $q$ e toda palavra $u$,

$$
\text { existe um passeio } q \stackrel{u / x}{\longrightarrow} i \text { em } \mathcal{T} \text { se, e somente se, } q=u i \text { e } x=u * i \text {. }
$$

Pela definição dos estados iniciais e do estado final, concluímos que

$$
|\mathcal{T}|=|\mathcal{S}|
$$

o que mostra que $f$ é uma função racional.

Há uma versão da Proposição 4.2.2 para as funções seqüenciais à direita.

Definição 4.2.6 Dizemos que uma função $f: \Sigma^{*} \rightarrow$ M preserva sufixos se $1 f=1$ e $(\sigma u) f \subseteq$ $\mathrm{M}(u f)$, para todo $u$ em $\Sigma^{*}$ e $\sigma$ em $\Sigma$.

Proposição 4.2.4 Toda função seqüencial à direita preserva sufixos. 
Demonstração. A demonstração é trivial.

Exemplo 4.2.1 Neste exemplo, vamos mostrar que existem funções seqüenciais à direita que não são seqüenciais à esquerda, e vice-versa.

Sejam $\Sigma=\{\sigma, \gamma\}$ e $f: \Sigma^{*} \rightarrow \Sigma^{*}$ a função total definida por

$$
1 f=1,(x \sigma) f=\sigma^{|x|+1} \quad \text { e } \quad(x \gamma) f=\gamma^{|x|+1}, \forall x \in \Sigma^{*} .
$$

Essa função é realizada pelo transdutor seqüencial à direita descrito a seguir:

$$
\begin{aligned}
& Q=\{i, p, q\} ; \\
& \sigma i=\sigma p=\gamma p=p ; \\
& \gamma i=\sigma q=\gamma q=q ; \\
& \sigma * i=\sigma * p=\gamma * p=\sigma ; \\
& \gamma * i=\sigma * q=\gamma * q=\gamma .
\end{aligned}
$$

Mas, essa função não preserva prefixos, pois

$$
\sigma f=\sigma \quad \text { e } \quad(\sigma \gamma) f=\gamma^{2} .
$$

Portanto, $f$ é seqüencial à direita, mas não é seqüencial à esquerda.

Por simetria, também podemos construir uma função seqüencial à esquerda, mas que não preserva sufixos, ou seja, que não é seqüencial à direita.

Os transdutores seqüenciais letra-palavra à esquerda e à direita estão relacionados como segue:

Proposição 4.2.5 Uma função $f: \Sigma^{*} \rightarrow \Gamma^{*}$ é seqüencial à esquerda se, e somente se, a função $f^{\varrho}: \Sigma^{*} \rightarrow \Gamma^{*}$, definida por

$$
u f^{\varrho}=\left(u^{\varrho} f\right)^{\varrho}, \quad \forall u \in \Sigma^{*}
$$

é seqüencial à direita.

Demonstração. Suponha que $f$ seja seqüencial à esquerda. Seja $(Q, i)$ um $\Sigma$ - $\Gamma$-transdutor seqüencial que realiza $f$. Utilizando esse transdutor, definimos um $\Sigma$ - $\Gamma$-transdutor seqüencial à direita $\mathcal{T}=(Q, i)$ por

$$
\sigma q=q \sigma \quad \text { e } \quad \sigma * q=(q * \sigma)^{\varrho}, \quad \forall q \in Q, \forall \sigma \in \Sigma, \text { tal que } q \sigma \neq \varnothing .
$$

Então, para toda palavra $u \operatorname{em} \operatorname{dom} f^{\varrho}$,

$$
u * i=\left(i * u^{\varrho}\right)^{\varrho} .
$$

Portanto, $\mathcal{T}$ realiza $f^{\varrho}$, e concluímos que essa função é seqüencial à direita.

Suponha que $f^{\varrho}$ seja seqüencial à direita. Note que, para toda palavra $u, u f=\left(u^{\varrho} f^{\varrho}\right)^{\varrho}$. Uma construção análoga à feita acima mostra então que $f$ é seqüencial à esquerda. 
Os transdutores seqüenciais são capazes de realizar várias funções entre palavras intuitivamente seqüenciais. Alguns exemplos podem ser vistos na Seção XI.2 de [Eil74]. Um exemplo e dois contra-exemplos são apresentados a seguir.

Exemplo 4.2.2 Um morfismo $f: \Sigma^{*} \rightarrow M$ é uma função seqüencial, e pode ser realizada pelo transdutor seqüencial à esquerda que contém apenas o estado inicial $i$, e funções de transição e de emissão definidas por

$$
i \cdot \sigma=i \quad \text { e } \quad i * \sigma=\sigma f, \quad \forall \sigma \in \Sigma .
$$

De forma semelhante, é possível construir um transdutor seqüencial à direita que realiza $f$.

Exemplo 4.2.3 Parte da notação introduzida nas seções V.2 e V.6 de [Eil74] será utilizada neste e em outros exemplos. Por comodidade, vamos reproduzí-la. Para evitar confusão, a palavra vazia será denotada por $\epsilon$ sempre que essa notação for utilizada.

Dado um inteiro $k>0$, vamos denotar por $\mathrm{k}$ o conjunto

$$
\mathrm{k}=\{0,1, \ldots, k-1\}
$$

A cada palavra $s$ no monóide livre $\mathrm{k}^{*}$, associamos o número natural

$$
\langle s\rangle_{k}=\sum_{i=0}^{n} d_{i} k^{n-i},
$$

onde

$$
s=d_{0} \ldots d_{n} \quad \text { e } \quad d_{i} \in \mathrm{k}, \quad \forall i, 0 \leq i \leq n
$$

Vamos supor que

$$
\langle\epsilon\rangle_{k}=0 .
$$

É um fato básico que, para todo número natural $a$, existe uma palavra $s \mathrm{em} \mathrm{k}^{*}$ tal que $\langle s\rangle_{k}=a$. Ademais, se $a>0$, então esse número pode ser representado por uma única palavra em $\mathrm{k}^{*}-0 \mathrm{k}^{*}$. Vamos denominar essa palavra de representação de a na base $k$. Vamos fazer a convenção de que a representação de 0 é 0 .

A representação reversa também será utilizada. Nessa representação, toda palavra $s=d_{0} \ldots d_{n}$ é associada ao inteiro

$$
\langle s\rangle_{k}^{\varrho}=\sum_{i=0}^{n} d_{i} k^{i} .
$$

Observe que

$$
\langle s\rangle_{k}^{\varrho}=\left\langle s^{\varrho}\right\rangle_{k} .
$$

Não havendo possibilidade de confusão, podemos denotar $\langle s\rangle_{k}$ e $\langle s\rangle_{k}^{\varrho}$ simplesmente por $\langle s\rangle$.

Sejam $k>1$ um inteiro e $\Sigma=\mathrm{k} \times \mathrm{k}$. Considere a função

$$
+: \Sigma^{*} \rightarrow \mathbf{k}^{*}
$$

tal que $\epsilon+=\epsilon$ e que associa a cada palavra

$$
\left(d_{0}, d_{0}^{\prime}\right) \ldots\left(d_{n}, d_{n}^{\prime}\right), \quad\left(d_{i}, d_{i}^{\prime}\right) \in \Sigma, \forall i, 0 \leq i \leq n,
$$


a representação reversa na base $k$ do inteiro

$$
\left\langle d_{n} \ldots d_{0}\right\rangle_{k}+\left\langle d_{n}^{\prime} \ldots d_{0}^{\prime}\right\rangle_{k} .
$$

Essa função não é seqüencial para nenhuma base. Por exemplo, para $k=2$, temos que

$$
(1,1)+=01 \text { e }(1,1)(1,0)+=001,
$$

o que mostra que + não preserva prefixos. Em geral, dados dígitos $d_{1}, d_{2}$ e $d_{3}$ tais que

$$
\left\langle d_{1}\right\rangle+\left\langle d_{2}\right\rangle=k \quad \text { e } \quad\left\langle d_{3}\right\rangle=k-1,
$$

temos que

$$
\left(d_{1}, d_{2}\right)+=01 \quad \text { e } \quad\left(d_{1}, d_{2}\right)\left(d_{3}, 0\right)+=001
$$

Exemplo 4.2.4 Sejam $k>1$ e $c \geq 1$ inteiros. Considere a função

$$
\div: \mathbf{k}^{+} \rightarrow \mathbf{k}^{+}
$$

que associa a toda palavra $u \mathrm{em}^{+}$a representação na base $k$ do quociente inteiro da divisão de $\langle u\rangle_{k}$ por $c$. Vamos denotar a imagem de $u$ por $u \div c$.

Essa função não é seqüencial para $c>1$. Por exemplo, para $k=2$ e $c=2$, temos que

$$
1 \div c=0 \quad \text { e } \quad 10 \div c=1
$$

o que mostra que $\div$ não preserva prefixos. Em geral, se $u$ for a representação de $c-1$ na base $k$, então

$$
u \div c=0 \quad \text { e } \quad u 0 \div c \notin 0 \mathbf{k}^{*}
$$

Conforme visto, a divisão de dois números inteiros não pode ser realizada por um transdutor seqüencial. No entanto, essa função é intuitivamente seqüencial.

Essa deficiência pode ser eliminada fazendo uma distinção entre estados finais e não-finais, e associando a cada estado final um processamento final, entendido como um elemento a ser multiplicado à direita na saída. Essas idéias foram consideradas por Schützenberger, e resultaram no conceito de transdutor subseqüencial, definido em [Sch77].

Definição 4.2.7 Um $\Sigma$-M-transdutor subseqüencial é uma máquina composta de

- um conjunto finito $Q$ de estados;

- um estado inicial $i$;

- uma função parcial $: Q \times \Sigma \rightarrow Q$;

- uma função parcial $*: Q \times \Sigma \rightarrow \mathrm{M}$, com domínio idêntico ao de ·;

- uma função $\lambda: Q \rightarrow \mathrm{M}$ definida apenas em $i$; 
- uma função parcial $\rho: Q \rightarrow \mathrm{M}$.

As considerações feitas para as funções · e *, na definição dos transdutores seqüenciais, aplicamse para os transdutores subseqüenciais.

O elemento $i \lambda$ será denominado emissão inicial. A função $\rho$ será denominada emissão final. Um transdutor subseqüencial será denotado por $(Q, i, \lambda, \rho)$.

Dizemos que os estados $q$ tais que $q \rho \neq \varnothing$ são os estados finais do transdutor.

O comportamento de um $\Sigma$-M-transdutor subseqüencial $\mathcal{T}$ é uma função

$$
|\mathcal{T}|=\Sigma^{*} \rightarrow \mathrm{M},
$$

definida por

$$
u|\mathcal{T}|=(i \lambda)(i * u)((i \cdot u) \rho), \forall u \in \Sigma^{*}
$$

$\mathrm{O}$ elemento $i \lambda$ associado ao estado inicial é multiplicado à esquerda de toda saída, e pode ser considerado como um processamento inicial. Nem todos os autores consideram esse processamento inicial (que está na definição original de Schützenberger), pois para qualquer transdutor subseqüencial é possível construir outro transdutor subseqüencial equivalente sem uma emissão inicial. No entanto, a definição da emissão inicial é fundamental para a minimização de transdutores subseqüenciais, que trataremos posteriormente, e será então mantida.

Definição 4.2.8 Dizemos que uma função $f: \Sigma^{*} \rightarrow \mathrm{M}$ é subseqüencial se for o comportamento de um $\Sigma$-M-transdutor subseqüencial.

Evidentemente, toda função seqüencial à esquerda é subseqüencial.

Desde a década de setenta, funções subseqüenciais têm sido bastante estudadas, e algumas propriedades muito interessantes dessas funções (como o Teorema 4.4.2, que fornece uma caracterização dessas funções) fazem com que elas se destaquem no conjunto das funções racionais. Recentemente, foi constatado que elas são bastante úteis em algumas aplicações em Processamento de Linguagem Natural. Essas aplicações são estudadas, por exemplo, por Mohri [Moh95, Moh97, MR97].

A seguir, vamos estudar algumas propriedades das funções subseqüenciais.

\section{Proposição 4.2.6 Toda função subseqüencial é racional.}

Demonstração. Sejam $f: \Sigma^{*} \rightarrow \mathrm{M}$ uma função subseqüencial, e $\mathcal{T}=(Q, i, \lambda, \rho)$ um $\Sigma$-Mtransdutor subseqüencial que realiza $f$. Utilizando $\mathcal{T}$, vamos construir um $\Sigma$-M-transdutor $\mathcal{T}^{\prime}=$ $\left(Q \cup i^{\prime} \cup t^{\prime}, E, i^{\prime}, t^{\prime}\right)$, onde $i^{\prime}$ e $t^{\prime}$ são estados novos, que realiza $f$.

Parte da definição das transições de $\mathcal{T}^{\prime}$ segue a demonstração da Proposição 4.2.1. Além das construções descritas nessa demonstração, $E$ tem uma transição

$$
i^{\prime} \stackrel{1 / i \lambda}{\longrightarrow} i
$$

e, para cada estado final $t$ de $\mathcal{T}, E$ tem uma transição

$$
t \stackrel{1 / t \rho}{\longrightarrow} t^{\prime}
$$


Segue facilmente dessa construção que

$$
|\mathcal{T}|=\left|\mathcal{T}^{\prime}\right|
$$

o que mostra que $f$ é uma função racional.

Observe que $\mathcal{T}^{\prime}$ não é letra-saída, devido às transições com origem em $i^{\prime}$ e término em $t^{\prime}$.

Os conceitos de transdutor acessível, co-acessível, bi-acessível e completo são definidos para transdutores subseqüenciais da maneira usual. Por clareza, relacionamos esses conceitos a seguir.

Definição 4.2.9 Seja $\mathcal{T}$ um transdutor subseqüencial. Dizemos que $\mathcal{T}$ é acessível se, para todo estado $q$, existe uma palavra u tal que $i u=q$. Dizemos que $\mathcal{T}$ é co-acessível se, para todo estado $q$, existe uma palavra u tal que qu é um estado final. Dizemos que $\mathcal{T}$ é bi-acessivel se $\mathcal{T}$ for acessivel $e$ co-acessível. Dizemos que $\mathcal{T}$ é completo se, para todo estado $q$ e toda letra $\sigma$ do alfabeto de entrada, $q \sigma \neq \varnothing$.

Eliminando estados que não são acessíveis e nem co-acessíveis de um transdutor subseqüencial, podemos provar que

Proposição 4.2.7 Toda função subseqüencial pode ser realizada por um transdutor subseqüencial bi-acessível.

Devido à presença de estados que não são finais na definição de transdutor subseqüencial, existem funções subseqüenciais que não preservam prefixos. Assim, existem funções subseqüenciais que não são seqüenciais. Alguns exemplos serão vistos no final desta seção.

No entanto, as funções subseqüenciais $\Sigma^{*} \rightarrow \Gamma^{*}$ que preservam prefixos são exatamente as funções seqüenciais, conforme demonstrado a seguir.

Proposição 4.2.8 Uma função $f: \Sigma^{*} \rightarrow \Gamma^{*}$ é seqüencial se, e somente se, $f$ é subseqüencial $e$ preserva prefixos.

Demonstração. Se $f$ for uma função seqüencial, então é subseqüencial. Ademais, pela Proposição $4.2 .2, f$ preserva prefixos.

Suponha que $f$ seja uma função subseqüencial que preserva prefixos. Seja $\mathcal{T}=(Q, i, \lambda, \rho)$ um $\Sigma$ - $\Gamma$-transdutor subseqüencial bi-acessível que realiza $f$.

Como $f$ preserva prefixos, todos os estados de $\mathcal{T}$ devem ser finais, $i \lambda=i \rho=1 \mathrm{e}$, para todo estado $q$ e toda letra $\sigma$ tais que $q \sigma \neq \varnothing$,

$$
q \rho \succ(q * \sigma)((q \sigma) \rho) .
$$

Utilizando essas propriedades, definimos um $\Sigma$ - $\Gamma$-transdutor seqüencial $\mathcal{T}^{\prime}=(Q, i)$, com função de transição idêntica à de $\mathcal{T}$, e de emissão definida por

$$
q * \sigma=(q \rho)^{-1}(x((q \sigma) \rho)), \forall q \in Q, \forall \sigma \in \Sigma \text { tal que } q \sigma \neq \varnothing,
$$

onde $x$ é a emissão de $q \operatorname{com} \sigma$ em $\mathcal{T}$. Recorde que $(q \rho)^{-1}(x((q \sigma) \rho))$ representa o sufixo de $(x((q \sigma) \rho))$ resultante após a retirada do prefixo $q \rho$.

Dessa construção, segue por indução em $|u|$ que

$$
u|\mathcal{T}|=u\left|\mathcal{T}^{\prime}\right|, \quad \forall u \in \Sigma^{*}
$$


Portanto, $f$ é seqüencial.

Algumas propriedades de fechamento são apresentadas a seguir.

Proposição 4.2.9 Sejam $f$ e $g$ funções $\Sigma^{*} \rightarrow M$ tais que $f \cup g$ é uma função.

Se $f$ e $g$ forem funções seqüenciais, então $f \cup g$ é uma função seqüencial.

Se $f$ e $g$ forem funções subseqüenciais, então $f \cup g$ não é necessariamente uma função subseqüencial.

Demonstração. Suponha que $f$ e $g$ sejam funções seqüenciais, e sejam $\mathcal{S}=(Q, i)$ e $\mathcal{S}^{\prime}=\left(Q^{\prime}, i^{\prime}\right)$ $\Sigma$-M-transdutores seqüenciais que realizam $f$ e $g$, respectivamente.

Seja $\mathcal{T}=\left(Q \times Q^{\prime} \cup Q \times\{\varnothing\} \cup\{\varnothing\} \times Q^{\prime},\left(i, i^{\prime}\right)\right)$ o transdutor seqüencial definido como segue. Para cada estado $(p, q)$ e cada letra $\sigma$, a transição $(p, q) \sigma$ só é definida se pelo menos uma das transições $p \sigma$ ou $q \sigma$ estiver definida (supondo que $\varnothing \sigma=\varnothing$ ), e $p * \sigma=q * \sigma$, se ambas estiverem definidas. Neste caso,

$$
(p, q) \sigma=(p \sigma, q \sigma) \quad \text { e } \quad(p, q) * \sigma=x
$$

onde $x$ é $p * \sigma$ ou $q * \sigma$, dependendo de se $p \sigma \neq \varnothing$ ou $q \sigma \neq \varnothing$.

Demonstra-se facilmente que $|\mathcal{T}|=f \cup g$.

Vamos apresentar um contra-exemplo para o caso em que $f$ e $g$ são funções subseqüenciais.

Sejam $\Sigma=\{\sigma\}, \Gamma=\{a, b\}$, e $f$ e $g$ as funções $\Sigma^{+} \rightarrow \Gamma^{*}$ definidas por

$$
\sigma^{n} f= \begin{cases}a^{n} & \text { se } n \text { for par } \\ \varnothing & \text { caso contrário. }\end{cases}
$$

$\mathrm{e}$

$$
\sigma^{n} g= \begin{cases}b^{n} & \text { se } n \text { for ímpar } \\ \varnothing & \text { caso contrário. }\end{cases}
$$

Claramente, $f$ e $g$ são disjuntas. Portanto, $f \cup g$ é uma função. Observe que $f \cup g$ é a função definida no Exemplo 3.1.5.

É trivial construir transdutores subseqüenciais que realizam essas funções. Mas, $f \cup g$ não é uma função subseqüencial. Esse fato é provado na Seção 4.4 (Exemplo 4.4.3).

Proposição 4.2.10 A composição de duas funções seqüenciais (subseqüenciais) $f: \Sigma^{*} \rightarrow \Gamma^{*} e$ $g: \Gamma^{*} \rightarrow \Omega^{*}$ é uma função seqüencial (subseqüencial).

A interseç̧ão de duas funções seqüenciais (subseqüenciais) pode não ser uma função seqüencial (subseqüencial).

Demonstração. As demonstrações para as composições de funções seqüenciais e subseqüenciais serão feitas na Seção 4.4 (Proposição 4.4 .1 e Corolário 4.4.2).

Para a intersecção, considere as funções racionais cujos gráficos são $r=(a, \sigma \sigma)^{*}(b, \sigma)^{*} \mathrm{e}$ $s=(a, \sigma)^{*}(b, \sigma \sigma)^{*}$. Essa funções são seqüenciais, mas $r \cap s$ não é nem racional, pois dom $r \cap s=$ $\left\{a^{n} b^{n}: n \geq 0\right\}$ não é reconhecível. 
Proposição 4.2.11 Sejam $f: \Sigma^{*} \rightarrow \mathrm{M}$ uma função subseqüencial, e A um subconjunto racional de $\Sigma^{*}$. Então, a restrição $f \mid A$ é uma função subseqüencial.

Demonstração. Sejam $\mathcal{A}=(P, i, T)$ um $\Sigma$-autômato determinístico que reconhece $A$, e $\mathcal{T}=$ $(Q, j, \lambda, \rho)$ um transdutor subseqüencial que realiza $f$.

Seja $\mathcal{A} \times \mathcal{T}$ o $\Sigma$-M-transdutor subseqüencial definido como segue. O conjunto de estados desse transdutor é $P \times Q$. A função de transição e a de emissão são definidas por

$$
(p, q) \cdot \sigma=(p \sigma, q \sigma) \quad \text { e }(p, q) * \sigma=q * \sigma,
$$

para quaisquer $p \in P, q \in Q$ e $\sigma \in \Sigma$ tais que $p \sigma \neq \varnothing$ e $q \sigma \neq \varnothing$.

O estado inicial desse transdutor é $(i, j)$, e sua emissão inicial é $j \lambda$. Um estado $(p, q)$ é final se, e somente se, $p$ e $q$ são estados finais em $\mathcal{A}$ e $\mathcal{T}$, respectivamente. Neste caso, a emissão final desse estado é $q \rho$.

Segue dessa construção que, para toda entrada $u$,

$$
(i, j) u \neq \varnothing \text { se, e somente se, } i u \neq \varnothing \text { e } j u \neq \varnothing .
$$

Então, pela definição dos estados finais de $\mathcal{A} \times \mathcal{T}$,

$$
\operatorname{dom}(|\mathcal{A} \times \mathcal{T}|)=A \cap \operatorname{dom}|\mathcal{T}|
$$

Ademais, se $(i, j) u \neq \varnothing$, então

$$
(i, j) * u=j * u
$$

Das definições das emissões inicial e final de $\mathcal{A} \times \mathcal{T}$, segue então que $|\mathcal{A} \times \mathcal{T}|=f \mid A$.

As proposições 4.2.9 e 4.2.11 são exercícios do Capítulo IV de [Ber79].

Concluímos esta seção com alguns exemplos de funções seqüenciais e subseqüenciais. Outros exemplos podem ser vistos em [Reu90].

Exemplo 4.2.5 Considere a função racional $\tau$ definida no Exemplo 3.1.5. Essa função não preserva prefixos nem sufixos, portanto não é seqüencial à esquerda e nem à direita. Na Seção 4.4 vamos provar que essa função também não é subseqüencial.

Exemplo 4.2.6 Os transdutores seqüenciais e subseqüenciais podem ser representados de forma pictórica como usual.

No caso dos transdutores seqüenciais, como todos os estados são finais, apenas o estado inicial precisa ser identificado. A figura a seguir mostra um exemplo, com $\Sigma=\{\sigma, \gamma\}$.

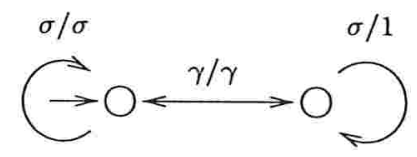

Esse $\Sigma$ - $\Sigma$-transdutor apaga todos os símbolos $\sigma$ que aparecem imediatamente após um número ímpar de ocorrências do símbolo $\gamma$ na entrada.

No caso dos transdutores subseqüenciais, a emissão inicial e as emissões finais podem ser escritas próximas às setas que indicam o estado inicial e os estados finais. A figura a seguir mostra um exemplo com $\Sigma=\{0,1\}$. A palavra vazia é representada por $\epsilon$. 


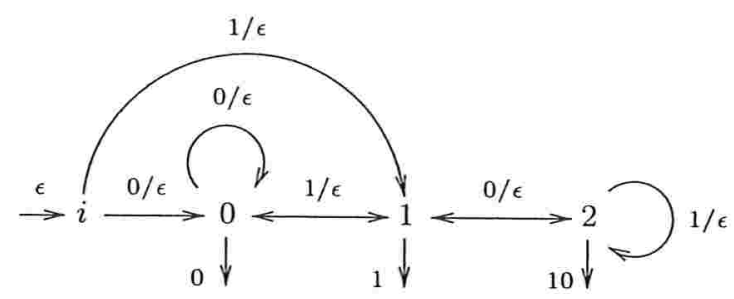

Esse $\Sigma$ - $\Sigma$-transdutor determina, em notação binária, o resto da divisão de números naturais por 3.

Exemplo 4.2.7 Sejam $\Sigma=\{\sigma, \gamma\}$ e $f: \Sigma^{*} \rightarrow \Sigma^{*}$ a função definida por

$$
u f=\sigma u \gamma, \forall u \in \Sigma^{*} .
$$

Essa função é subseqüencial, e é realizada pelo $\Sigma$ - $\Sigma$-transdutor subseqüencial

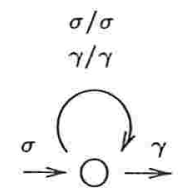

Mas, essa função não é seqüencial à esquerda e nem à direita, pois $1 f \neq 1$.

Exemplo 4.2.8 Seja $\sigma$ uma letra. Vamos representar um número natural $n$ pela palavra $\sigma^{n}$ e a palavra vazia por $\epsilon$.

Seja $f: \mathbb{N} \rightarrow \mathbb{N}$ uma função total e periódica de período $k$. Essa função é subseqüencial, e é realizada pelo transdutor

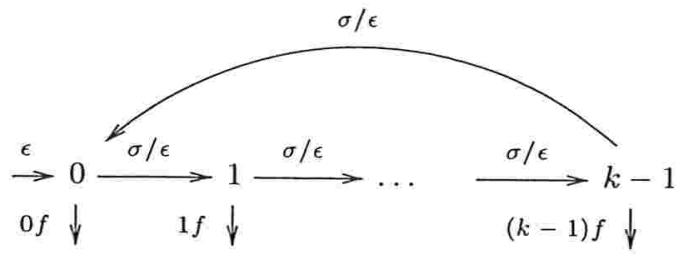

Exemplo 4.2.9 A função de adição definida no Exemplo 4.2 .3 pode ser realizada por um transdutor subseqüencial, representando o vai-um nos estados.

Formalmente, o conjunto de estados é $Q=\{0,1\}$. O estado inicial é 0 , e ambos os estados são finais. As emissões inicial e final de 0 são iguais à palavra vazia, e a emissão final de 1 é o dígito 1 (em k).

As transições e emissões são definidas a seguir. Nessa definição, um dígito $d$ em $\mathrm{k}$ é identificado com o inteiro $\langle d\rangle$, e $\div$ e mod representam o quociente e o resto da divisão euclidiana, 
respectivamente:

$$
\begin{aligned}
& 0 \cdot\left(d, d^{\prime}\right)=\left(d+d^{\prime}\right) \div k, \\
& 0 *\left(d, d^{\prime}\right)=\left(d+d^{\prime}\right) \bmod k, \\
& 1 \cdot\left(d, d^{\prime}\right)=\left(1+d+d^{\prime}\right) \div k, \\
& 1 *\left(d, d^{\prime}\right)=\left(1+d+d^{\prime}\right) \bmod k, \quad \forall\left(d, d^{\prime}\right) \in \mathbf{k} \times \mathbf{k} .
\end{aligned}
$$

Claramente, esse transdutor é o algoritmo de adição tradicional, mas executado da esquerda para a direita sobre os reversos dos números representados (seguidos de eventuais zeros para que tenham o mesmo comprimento). Ou seja, seu comportamento é a função +.

Para $\mathbf{k}=\{0,1\}$, esse transdutor está ilustrado a seguir:

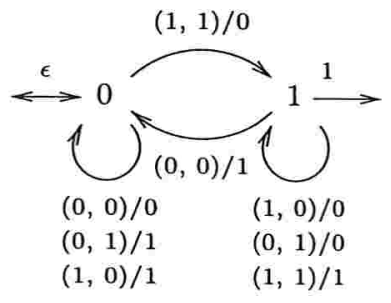

Exemplo 4.2.10 Vamos descrever um transdutor subseqüencial que realiza o algoritmo da divisão inteira, provando assim que a função $\div$ definida no Exemplo 4.2.4 é uma função subseqüencial. Supomos fixos uma base $k>0$ e um divisor $c>1$. Vamos identificar os dígitos em $\mathrm{k}$ com os inteiros representados.

Informalmente, os possíveis restos nessa divisão são representados nos estados. Ademais, são necessários dois tipos de estados para cada resto. Os estados do primeiro tipo são utilizados enquanto o prefixo lido do dividendo é menor que o divisor. Quando o valor desse prefixo se torna maior que o divisor, os estados do segundo tipo são utilizados.

Assim, para cada inteiro $r, 0 \leq r<c$, esse transdutor tem estados

$$
q_{r} \quad \mathrm{e} \quad q_{r}^{\prime} .
$$

Além desses estados, o transdutor tem um estado $i$, que é o inicial. A emissão inicial é a palavra vazia.

Apenas o estado $i$ não é final. As emissões finais dos demais estados são definidas por

$$
q_{r} \rho=\epsilon \quad \text { e } \quad q_{r}^{\prime} \rho=0 .
$$

Para todo dígito $d$, a transição e emissão com origem em $i$ são

$$
i \cdot d=q_{d}^{\prime} \quad \text { e } \quad i * d=\epsilon, \text { se } \mathrm{d}<\mathrm{c},
$$

e

$$
i \cdot d=q_{d \bmod c} \quad \text { e } \quad i * d=d \div c, \text { caso contrário. }
$$

Para todo estado $q_{r}$, definimos

$$
q_{r} \cdot d=q_{(r k+d) \bmod c} \quad \text { e } \quad q_{r} * d=(r k+d) \div c .
$$


Para todo estado $q_{r}^{\prime}$, se $r k+d \geq c$, então definimos

$$
q_{r}^{\prime} \cdot d=q_{(r k+d) \bmod c} \quad \text { e } \quad q_{r}^{\prime} * d=(r k+d) \div c,
$$

senão,

$$
q_{r}^{\prime} \cdot d=q_{(r k+d)}^{\prime} \quad \text { e } \quad q_{r}^{\prime} * d=\epsilon .
$$

Nessa definição, $\div$ e mod são definidos como no Exemplo 4.2.9.

Esse transdutor executa o algoritmo tradicional da divisão. Formalmente, o fato de que esse transdutor realiza a função $\div$ é uma conseqüência da propriedade a seguir. Para toda palavra $u$ em $\mathbf{k}^{+}$, se $\langle u\rangle<c$, então

$$
i u=q_{\langle u\rangle}^{\prime} \quad \mathrm{e} \quad i * u=\epsilon,
$$

senão,

$$
i u=q_{\langle u\rangle \bmod c} \quad \text { e } \quad i * u=\langle u\rangle \div c .
$$

Essa propriedade pode ser demonstrada por indução em $|u|$.

A divisão por 3 na base decimal está ilustrada a seguir:

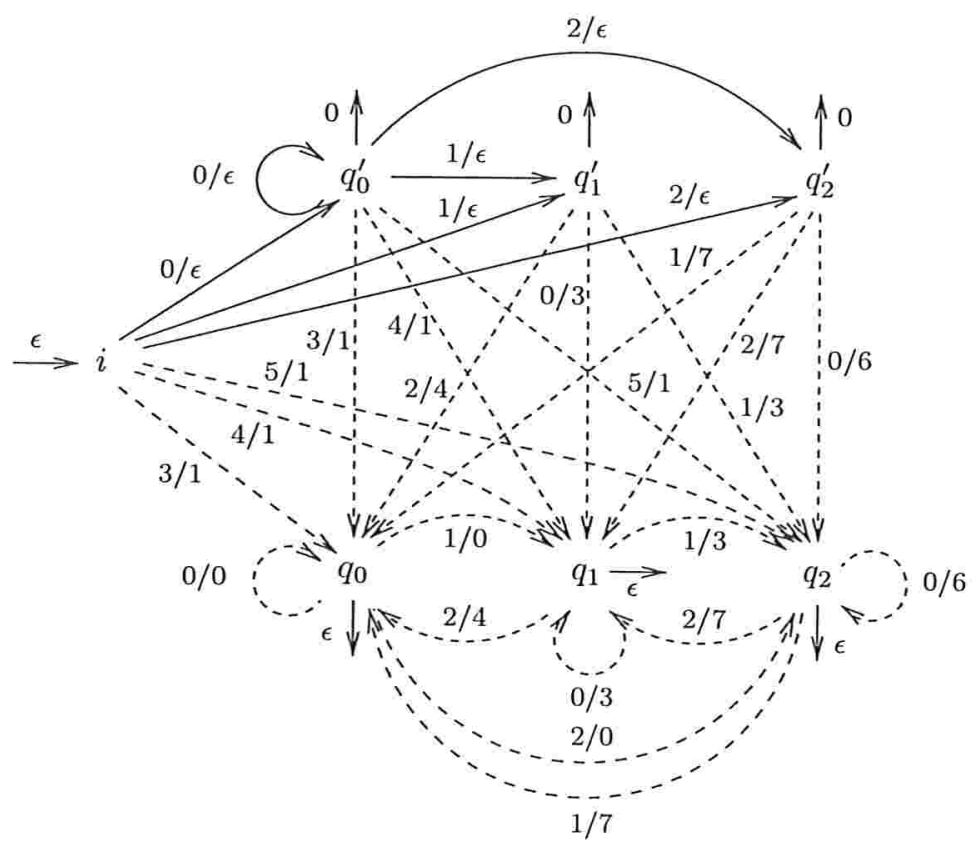

Nessa figura, cada linha tracejada representa um conjunto de transições. Esse conjunto é codificado no rótulo segundo a regra a seguir: um par $r / s$ representa o conjunto

$$
r+3 l / s+l, \forall l \geq 0 \text { tal que } r+3 l<10 .
$$

Por exemplo, o par $0 / 3$ representa as transições

$$
0 / 3,3 / 4,6 / 5 \text { e } 9 / 6
$$

Também observamos que a função que associa a cada entrada $u$ o resto da divisão de $\langle u\rangle$ por $c$ é realizada pelo mesmo transdutor após a substituição das emissões finais para

$$
q_{r} \rho=q_{r}^{\prime} \rho=r, \forall r, 0 \leq r<c,
$$


e da emissão de todas as transições para a palavra vazia. No entanto, em geral, um transdutor subseqüencial com um número menor de estados pode ser construído para essa função. Um exemplo é o transdutor subseqüencial exibido no Exemplo 4.2.6.

Exemplo 4.2.11 Sejam $\Sigma=\{\sigma, \gamma\}$ e $f: \Sigma^{*} \rightarrow \Sigma^{*}$ a função com domínio igual a $\{\gamma, \sigma \gamma\}$ e definida por

$$
\gamma f=\gamma \quad \text { e } \quad(\sigma \gamma) f=\sigma \gamma .
$$

Essa função é seqüencial à direita, e é realizada pelo transdutor seqüencial à direita $(\{i, p, q\}, i)$ definido por

$$
\gamma i=p, \gamma * i=\gamma, \sigma p=q, \sigma * p=\sigma .
$$

Essa funçao também é o comportamento do transdutor subseqüencial

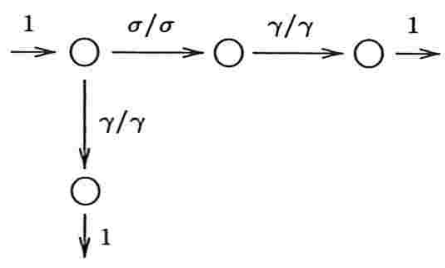

No entanto, essa função não é seqüencial à esquerda, pois não preserva prefixos.

Os exemplos 4.2.1, 4.2.5 e 4.2.11, mais o fato de que a função definida no Exemplo 4.2.1 não é subseqüencial (o que será provado na Seção 4.4), mostram que as classes das funções racionais, funções seqüenciais à esquerda, funções seqüenciais à direita e funções subseqüenciais, todas com imagem em um monóide livre, são relacionadas conforme a figura a seguir:

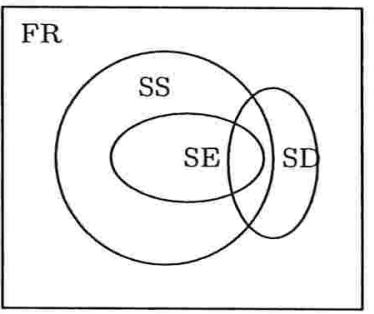

Nesse diagrama, cada uma das partes é não-vazia. As abreviações FR, SE, SD e SS representam as funções racionais, seqüenciais à esquerda, seqüenciais à direita e subseqüenciais, respectivamente.

O exemplo a seguir é o Exercício XI.4.2 de [Eil74].

Exemplo 4.2.12 O objetivo deste exemplo é caracterizar as mudanças de base de representação numérica que podem ser realizadas por transdutores seqüenciais. A notação introduzida no Exemplo 4.2 .3 será utilizada.

Sejam $k$ e $l$ inteiros, com $k, l>1$. Vamos provar que, se existir uma função

$$
f: \mathrm{k}^{*} \rightarrow \mathrm{l}^{*}
$$

que preserva prefixos e satisfaz

$$
\langle s f\rangle_{l}=\langle s\rangle_{k}, \forall s \in \mathrm{k}^{*}
$$


então $k=l^{x}$, para algum inteiro $x>0$.

Claramente, $1 f=0^{a} 1$ para algum inteiro $a \geq 0$. Como $f$ preserva prefixos, podemos escrever

$$
(10) f=\left(0^{a}\right) 1 d_{1} \ldots d_{r}
$$

onde $r$ é um inteiro positivo e cada $d_{i}, 1 \leq i \leq r$, é uma letra em 1. Da mesma forma,

$$
(100) f=\left(0^{a}\right) 1 d_{1} \ldots d_{r} d_{1}^{\prime} \ldots d_{r^{\prime}}^{\prime}
$$

onde $r^{\prime}$ é um inteiro positivo e cada $d_{i}^{\prime}, 1 \leq i \leq r^{\prime}$, é uma letra em 1 .

Vamos mostrar que $r=r^{\prime}$. Primeiro, observe que

$$
k=\langle 10\rangle_{k}=\left\langle 1 d_{1} \ldots d_{r}\right\rangle_{l}=l^{r}+\sum_{i=1}^{r} d_{i} l^{r-i} .
$$

Assim,

$$
l^{r+1}>k \geq l^{r}
$$

De (4.2.13), segue que $k^{2} \geq l^{2 r}$. Como $k^{2}=\langle 100\rangle_{k}$, podemos utilizar (4.2.11) e reescrever essa propriedade como

$$
l^{r+r^{\prime}}+\sum_{i=1}^{r+r^{\prime}} d_{i} l^{\left(r+r^{\prime}\right)-i} \geq l^{2 r},
$$

onde $d_{i}=d_{i-r}^{\prime}$ para todo índice $i>r$. Como

$$
\sum_{i=1}^{r+r^{\prime}} d_{i} l^{\left(r+r^{\prime}\right)-i}<l^{r+r^{\prime}}
$$

temos que

$$
l^{r+r^{\prime}+1}>l^{2 r},
$$

o que implica em $r^{\prime} \geq r$.

Em seguida, observe que

$$
\left\langle 1 d_{1} \ldots d_{r} d_{1}^{\prime} \ldots d_{r^{\prime}}^{\prime}\right\rangle_{l} \geq\left\langle 1 d_{1} \ldots d_{r}\right\rangle_{l} l^{r^{\prime}}
$$

Como $k=\left\langle 1 d_{1} \ldots d_{r}\right\rangle_{l}$ e $k^{2}=\left\langle 1 d_{1} \ldots d_{r} d^{\prime}{ }_{1} \ldots d^{\prime}{ }_{r^{\prime}}\right\rangle_{l}$, podemos reescrever essa propriedade como $k^{2} \geq k l^{r^{\prime}}$. Assim,

$$
k \geq l^{r^{\prime}} .
$$

De (4.2.13), segue então que $r^{\prime} \leq r$.

Vamos agora mostrar que

$$
d_{i}=0, \forall i, 1 \leq i \leq r
$$

Suponha que exista algum dígito $d_{i}$ diferente de 0 . Escolha $d_{h}$ como o dígito com essa propriedade cujo índice $h$ seja mínimo. 
Elevando ambos os lados de (4.2.12) ao quadrado, obtemos

$$
k^{2}=l^{2 r}+2 l^{r} \sum_{i=1}^{r} d_{i} l^{r-i}+\left(\sum_{i=1}^{r} d_{i} l^{r-i}\right)^{2}=l^{2 r}+\sum_{i=1}^{r}\left(2 d_{i}\right) l^{2 r-i}+\sum_{\substack{1 \leq i \leq r \\ 1 \leq j \leq r}} d_{i} d_{j} l^{2 r-(i+j)} .
$$

Utilizando a propriedade de que $r=r^{\prime}$, podemos expandir $\left\langle 1 d_{1} \ldots d_{r} d^{\prime}{ }_{1} \ldots d^{\prime}{ }_{r^{\prime}}\right\rangle_{l}$ como segue:

$$
l^{2 r}+\sum_{i=1}^{r} d_{i} l^{2 r-i}+\sum_{i=1}^{r} d_{i}^{\prime} l^{r-i}
$$

Como $k^{2}=\left\langle 1 d_{1} \ldots d_{r} d_{1}^{\prime} \ldots d_{r^{\prime}}^{\prime}\right\rangle_{l}$, podemos igualar (4.2.15) e (4.2.16). Como $d_{i}=0$ para todo $i<h$, essa igualdade se torna

$$
\sum_{i=h}^{r}\left(2 d_{i}\right) l^{2 r-i}+\sum_{\substack{h \leq i \leq r \\ h \leq j \leq r}} d_{i} d_{j} l^{2 r-(i+j)}=\sum_{i=h}^{r} d_{i} l^{2 r-i}+\sum_{i=1}^{r} d_{i}^{\prime} l^{r-i} .
$$

A soma no lado esquerdo dessa igualdade é maior ou igual a $2 d_{h} l^{2 r-h}$, e a do lado direito é estritamente menor que $d_{h} l^{2 r-h}+l^{2 r-h}=\left(d_{h}+1\right) l^{2 r-h}$. Mas, isto é impossível se $d_{h} \neq 0$, e chegamos portanto a uma contradição.

Concluímos então (4.2.14). Utilizando (4.2.12), obtemos

$$
k=l^{r} .
$$

Ademais, a função $f$ pode ser escolhida como um morfismo. Esse fato simples está demonstrado na Proposição V.2.1 de [Eil74].

Portanto, se uma mudança de base $\mathrm{k}^{*} \rightarrow \mathrm{l}^{*}$ puder ser realizada por um $\mathrm{k}$-l-transdutor seqüencial, então $k$ é uma potência de $l$. Por outro lado, se $k=l^{r}$, então a função $f: \mathbf{k}^{*} \rightarrow 1^{*}$, que associa toda palavra $s$ em $\mathrm{k}^{*}-0 \mathrm{k}^{*}$ à representação do inteiro $\langle s\rangle_{k}$ na base 1 , é o comportamento do k-1-transdutor seqüencial esquematizado a seguir:

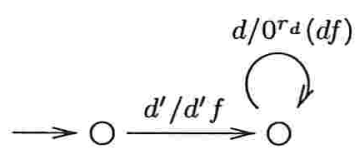

Nessa figura, $d^{\prime}$ representa os dígitos em $\mathrm{k}-0, d$ representa os dígitos em $\mathrm{k}$, e $r_{d}$ é o número de zeros que devem ser concatenados à esquerda de $d f$ para completar $r$ dígitos.

Eilenberg prova um resultado mais complexo nessa direção, mas para funções racionais (Proposição IX.9.3 de [Eil74]).

\subsection{Decomposição de funções racionais em funções seqüenciais}

Nem toda função racional pode ser realizada por um transdutor seqüencial. No entanto, uma conexão interessante entre o conceito de função racional e o de determinismo foi percebido por Schützenberger em 1961 [Sch61]. Schützenberger introduziu nesse ano o conceito de bimachines, 
que pode ser entendido como um transdutor que trabalha de maneira seqüencial à esquerda e à direita, alternadamente, e que realiza exatamente a classe das funções racionais palavra-palavra cuja imagem da palavra vazia é 1 .

O aprimoramento do entendimento das relações entre funções racionais e a noção de determinismo resultou no teorema de decomposição de Elgot e Mezei de 1965 [EM65], que mostra que toda função racional palavra-palavra cuja imagem da palavra vazia é 1 pode ser representada como uma composição de uma função seqüencial à esquerda com uma função seqüencial à direita.

Em essência, o resultado de Elgot e Mezei também mostra que a classe das funções realizadas pelas bimachines de Schützenberger é exatamente a classe das funções racionais palavra-palavra cuja imagem da palavra vazia é 1. Em seu livro, Eilenberg [Eil74] retoma o conceito de bimachines e apresenta uma demonstração rigorosa desse fato, mas não menciona o teorema de Elgot e Mezei. Berstel, em seu livro [Ber79], apresenta também uma demonstração desse fato, semelhante à de Eilenberg, e a utiliza para demonstrar o teorema de Elgot e Mezei. Em acréscimo, Sakarovitch mostrou em 1998 como esse resultado pode ser obtido utilizando apenas propriedades de representações matriciais de transdutores [Sak98].

Nossa intenção nesta seção é apresentar uma demonstração do teorema de Elgot e Mezei diretamente, sem utilizar as bimachines. Não tratamos desse formalismo porque os livros de Eilenberg e Berstel já contém discussões sobre todos os fatos relevantes relacionados a ele, e também porque utilizando funções seqüenciais, é possível construir facilmente uma bimachine.

A demonstração apresentada aqui é essencialmente a mesma que a do trabalho original de Elgot e Mezei. Dada uma função racional $f$, a decomposição de $f$ numa função seqüencial à esquerda e outra à direita consiste em considerar um transdutor $\mathcal{T}$ que realiza $f$, e construir um transdutor seqüencial à esquerda e outro à direita com o seguinte funcionamento. O transdutor seqüencial à esquerda lê uma entrada e a transforma em uma palavra representando todos os passeios com origem em um estado inicial e que soletram essa entrada em $\mathcal{T}$. Em seguida, o transdutor seqüencial à direita lê essa palavra da direita para a esquerda, e escreve as saídas presentes nas transições de um dos possíveis passeios bem-sucedidos, se houver algum. Esse segundo transdutor "escolhe" apenas um passeio bem-sucedido dentre todos os possíveis, para escrever a saída correspondente. Como $f$ é uma função, todos os passeios bem-sucedidos que soletram uma entrada têm a mesma saída. Portanto, o resultado da composição dessas funções é $f$.

Teorema 4.3.1 (Elgot e Mezei 1965) Sejam $\Sigma$ e $\Gamma$ alfabetos. Uma função $f: \Sigma^{*} \rightarrow \Gamma^{*}$ tal que $1 f=1$ é racional se, e somente se, existem um alfabeto $\Xi$, uma função seqüencial à esquerda $l: \Sigma^{*} \rightarrow \Xi^{*}$, e uma função seqüencial à direita $r: \Xi^{*} \rightarrow \Gamma^{*}$, tais que $f=l r$.

Demonstração. Se $f=l r$, então $f$ é a composição de duas funções racionais, e portanto é uma função racional.

Para demonstrar a recíproca, seja $\mathcal{T}=(Q, E, i, t)$ um $\Sigma$ - $\Gamma$-transdutor letra-palavra bi-acessível e normalizado que realiza a restrição de $f$ a $\Sigma^{+}$. Pela Proposição 3.2.4, existe um transdutor com essas propriedades. Vamos supor fixa uma ordem $<$ no alfabeto $E$ das transições de $\mathcal{T}$, e denotar a origem de uma transição $e$ por $e \iota$.

Sejam $\mathcal{A}$ o autômato determinístico acessível obtido aplicando-se a construção dos subconjuntos no autômato de entrada de $\mathcal{T}$, e $\Xi$ o conjunto de todos os subconjuntos não-vazios de $E$. A partir de $\mathcal{A}$, construímos um $\Sigma$ - $\Xi$-transdutor seqüencial à esquerda letra-letra $\mathcal{L}$ como segue. O conjunto de estados, o estado inicial e a função de transição de $\mathcal{L}$ são idênticos aos de $\mathcal{A}$. A função de saída 
é definida a seguir. Para todo $X$ em $2^{Q}$ e toda letra $\sigma$ em $\Sigma$ tais que $X \cdot \sigma \neq \varnothing$,

$$
X * \sigma=\{e \in E: e \iota \in X \text { e } e \alpha=\sigma\} .
$$

Vamos denotar o comportamento de $\mathcal{L}$ por $l$.

Vamos agora construir um $\Xi$ - $\Gamma$-transdutor seqüencial à direita $\mathcal{R}$. O conjunto de estados de $\mathcal{R}$ é $Q$, e seu estado inicial é $t$. Para toda letra $\xi$ em $\Xi$ e todo estado $p$ em $Q$, definimos

$$
\xi \cdot p=\varnothing
$$

se não existir nenhuma transição em $\xi$ com término igual a $p$. Caso contrário, seja $e$ a menor transição em $\xi$ pela ordem < com término igual a $p$. Definimos então

$$
\xi \cdot p=e \iota \quad \text { e } \quad \xi * p=e \beta .
$$

O comportamento de $\mathcal{R}$ será denotado por $r$.

Vamos mostrar que $f=l r$. Para isso, vamos primeiro estudar duas propriedades de $l$ e $r$.

Sejam

$$
u=\sigma_{1} \ldots \sigma_{n} \in \operatorname{dom} f \quad \text { e } \quad i * u=\xi_{1} \ldots \xi_{n},
$$

onde $n>0$, cada $\sigma_{j}$ é uma letra em $\Sigma$, e cada $\xi_{j}$ é uma letra em $\Xi$. Vamos primeiro provar que $\xi_{n}$ é o conjunto das últimas transições dos passeios em $\mathcal{T}$ com origem em $i$ e entrada $u$.

Seja $e_{1} \ldots e_{n}$ um passeio em $\mathcal{T}$ com origem em $i$ e entrada $u$, onde $e_{j}$ é uma transição para todo $j$. Como $\mathcal{L}$ é um transdutor letra-letra, segue de (4.2.2) que

$$
\xi_{n}=\left(i \cdot\left(\sigma_{1} \ldots \sigma_{n-1}\right)\right) * \sigma_{n} .
$$

Pela construção dos subconjuntos, a origem de $e_{n}$ pertence a $i \cdot\left(\sigma_{1} \ldots \sigma_{n-1}\right)$. Como $e_{n} \alpha=\sigma_{n}$, segue da definição da emissão de $\mathcal{L}$ que $e_{n}$ pertence a $\xi_{n}$.

Seja agora e uma transição em $\xi_{n}$. Então, pela definição da emissão de $\mathcal{L}$, e $\iota$ pertence a $i \cdot\left(\sigma_{1} \ldots \sigma_{n-1}\right)$. Pela construção dos subconjuntos, existe um passeio $c$ em $\mathcal{T}$ de $i$ a $e \iota$ com entrada $\sigma_{1} \ldots \sigma_{n-1}$. Assim, ce é um passeio em $\mathcal{T}$ com origem em $i$ e entrada $u$.

Temos então que $i \cdot u$ é exatamente o conjunto dos términos das transições em $\xi_{n}$.

Em seguida, vamos provar que um estado $q$ pertence a $i u$ se, e somente se, existe um passeio em $\mathcal{T}$ de $i$ a $q$ com entrada igual a $u$ e saída igual a $(i * u) * q$.

Se $c$ for um tal passeio, então o fato de que $q$ pertence a $i u$ segue da construção dos subconjuntos.

Para demonstrar a recíproca, vamos usar indução em $n=|u|$ :

- $n=1$ : seja $e$ a menor transição em $\xi_{1}$ pela ordem < com término igual a $q$. Segue da definição da emissão de $\mathcal{L}$ que $e \iota=i$. Pela escolha de $e$, segue da definição de $\mathcal{R}$ que $\xi_{1} * q=e \beta$. Assim, $e$ é um passeio como queríamos.

- $n>1$ : como $q \in i u$, segue da construção dos subconjuntos que existe um passeio de $i$ a $q$ com entrada $u$ em $\mathcal{T}$. Então, existe uma transição em $\xi_{n}$ com término igual a $q$. Seja $e$ a menor transição em $\xi_{n}$ pela ordem < com término igual a esse estado.

Pela construção dos subconjuntos, eı pertence a $i \cdot\left(\sigma_{1} \ldots \sigma_{n-1}\right)$. Pela hipótese de indução, existe um passeio $c$ em $\mathcal{T}$ de $i$ a eı com entrada igual a $\sigma_{1} \ldots \sigma_{n-1}$ e saída igual a $\left(\xi_{1} \ldots \xi_{n-1}\right) *$ $e \iota$. Pela escolha de $e$ e pela definição de $\mathcal{R}, \xi_{n} \cdot q=e \iota$ e $\xi_{n} * q=e \beta$. Utilizando (4.2.5b), obtemos então $(i * u) * q=\left(\left(\xi_{1} \ldots \xi_{n-1}\right) *\left(\xi_{n} \cdot q\right)\right)\left(\xi_{n} * q\right)=\left(\left(\xi_{1} \ldots \xi_{n-1}\right) * e \iota\right)(e \beta)=(c e) \beta$. Assim, ce é um passeio como queríamos. 
Como $1 f=1=1 l r$, basta mostrar que $f$ e $l r$ coincidem em $\Sigma^{+}$para concluir que $f=l r$.

Seja $u$ uma palavra em $\Sigma^{+}$. Conforme demonstrado, $t$ pertence a $i u$ se, e somente se, existe um passeio bem-sucedido em $\mathcal{T}$ com entrada $u$ e saída igual a $(i * u) * t$. Então, $u l r=(i * u) * t \neq \varnothing \mathrm{se}$, e somente se, $u$ pertence a $\operatorname{dom} f$, e, neste caso, $u l r$ é o rótulo de saída de um passeio bem-sucedido em $\mathcal{T}$ com entrada $u$. Como $f$ é funcional, segue que $u l r=u f$, o que conclui a demonstração.

Observamos que argumentos semelhantes (utilizando o reverso de $\mathcal{T}$ ) permitem concluir que toda função racional $f: \Sigma^{*} \rightarrow \Gamma^{*}$ tal que $1 f=1$ é a composição de uma função seqüencial à direita com uma função seqüencial à esquerda.

\subsection{Caracterização de funções seqüenciais e de funções subseqüen- ciais}

Em 1966, Ginsburg e Rose apresentaram a seguinte caracterização de funções seqüenciais palavra-palavra [GR66]:

Teorema 4.4.1 (Ginsburg e Rose 1966) Uma função $f: \Sigma^{*} \rightarrow \Gamma^{*}$ é seqüencial se, e somente se, as três condições a seguir são satisfeitas:

i. $f$ preserva prefixos;

ii. $A f^{-1} \in \operatorname{Rac}\left(\Sigma^{*}\right)$ para todo $A \in \operatorname{Rac}\left(\Gamma^{*}\right)$;

iii. existe um inteiro $K \geq 0$ tal que, para todo $u$ em $\Sigma^{*}$ e $\sigma$ em $\Sigma$, se $(u \sigma) f \neq \varnothing$, então $|(u \sigma) f|-$ $|u f| \leq K$.

Em 1978, Choffrut obteve, em sua tese de doutorado [Cho78, Cho79], uma caracterização de funções subseqüenciais palavra-palavra, que estende a de Ginsburg e Rose. Essa caracterização depende do seguinte conceito topológico:

Definição 4.4.1 Dizemos que uma função $f: \Sigma^{*} \rightarrow \Gamma^{*}$ tem variação limitada se, para todo inteiro $k \geq 0$, existe um inteiro $K \geq 0$ tal que, para todo $u$ e $v$ em $\operatorname{dom} f$, se $\|u, v\| \leq k$ então $\|u f, v f\| \leq K$.

Não é difícil demonstrar que toda função subseqüencial tem variação limitada. A recíproca não é verdadeira, mas, com uma condição adicional, temos a caracterização de Choffrut das funções subseqüenciais:

Teorema 4.4.2 (Choffrut 1978) Uma função $f: \Sigma^{*} \rightarrow \Gamma^{*}$ é subseqüencial se, e somente se, as duas condições a seguir são satisfeitas:

i. $A f^{-1} \in \operatorname{Rac}\left(\Sigma^{*}\right)$ para todo $A \in \operatorname{Rac}\left(\Gamma^{*}\right)$;

ii. $f$ tem variação limitada.

Corolário 4.4.1 Uma função racional $f: \Sigma^{*} \rightarrow \Gamma^{*}$ é subseqüencial se, e somente se, tem variação limitada. 
Demonstração. O corolário segue do Teorema 4.4.2 e do fato de que $f^{-1}$ é uma relação racional, e portanto preserva conjuntos racionais.

O teorema de Ginsburg e Rose é uma conseqüência do teorema de Choffrut:

Demonstração do Teorema 4.4.1. Se $f$ for uma função seqüencial, a prova de que $f$ satisfaz as condições $i$, ii e iii do Teorema 4.4.1 é trivial, e não utiliza o Teorema 4.4.2.

A condição $i$ foi observada na Seção 4.2 (Proposição 4.2.2), e a condição $i i$ é uma conseqüência do fato de que $f^{-1}$ é uma relação racional e da Proposição 2.2.2. A condição iii é obtida considerandose um transdutor seqüencial que realiza $f$, e definindo $K$ como o máximo dentre os comprimentos das emissões desse transdutor.

Suponha agora que $f$ satisfaça as condições $i, i i$ e $i i i$ do Teorema 4.4.1. Sejam $k \geq 0$ um inteiro, e $u$ e $v$ palavras tais que

$$
\|u, v\| \leq k .
$$

Defina $w=u \wedge v$, e $u^{\prime}$ e $v^{\prime}$ como as palavras tais que $u=w u^{\prime}$ e $v=w v^{\prime}$. Então,

$$
\left|u^{\prime}\right|+\left|v^{\prime}\right| \leq k .
$$

Como $f$ preserva prefixos, podemos escrever

$$
u f=(w f)\left(u^{\prime} f\right) \quad \text { e } \quad v f=(w f)\left(v^{\prime} f\right) .
$$

Assim,

Por $i i i$, temos que

$$
\|u f, v f\| \leq\left|u^{\prime} f\right|+\left|v^{\prime} f\right|
$$

$$
\left|u^{\prime} f\right|+\left|v^{\prime} f\right| \leq K\left(\left|u^{\prime}\right|+\left|v^{\prime}\right|\right) \leq K k .
$$

Como $K$ é fixo e $K k$ só depende de $k$, concluímos que $f$ tem variação limitada.

Então, $f$ satisfaz as condições $i$ e $i i$ do Teorema 4.4.2. Portanto, $f$ é subseqüencial. Como $f$ preserva prefixos, segue da Proposição 4.2 .8 que $f$ é seqüencial.

Uma prova do Teorema 4.4.2, diferente da de Choffrut, foi apresentada por Reutenauer [Reu90] em 1990. Nessa prova, Reutenauer utiliza uma generalização do conceito de diferencial de uma função, definido por Eilenberg na sua demonstração do teorema de Ginsburg e Rose [Eil74].

No entanto, a demonstração de Reutenauer continha algumas incorreções. Uma versão correta dessa demonstração foi apresentada por Reutenauer e Bruyère em 1999 [BR99].

A caracterização de Choffrut é um resultado muito forte. Com ela, pode-se verificar facilmente se uma função racional é ou não subseqüencial. Os algoritmos para a decidibilidade de se uma função racional é subseqüencial, e para a construção de um transdutor subseqüencial a partir de um transdutor letra-palavra que realiza uma função subseqüencial, estudados nas seções 4.5 e 4.6 , dependem explicitamente desse resultado.

Nesta seção, vamos demonstrar o teorema de Choffrut. Nossa demonstração segue a de Reutenauer e Bruyère.

Começamos com algumas definições.

Definição 4.4.2 Seja $f: \Sigma^{*} \rightarrow \Gamma^{*}$ uma função. Dizemos que $f$ é fechada por prefixos se, para toda palavra $w \sigma$, onde $\sigma$ é uma letra, se $w \sigma \in \operatorname{dom} f$, então $w \in \operatorname{dom} f$. 
Note que, se $f$ for uma função fechada por prefixos não-vazia, então $1 f \neq \varnothing$.

Definição 4.4.3 Seja $f: \Sigma^{*} \rightarrow \Gamma^{*}$ uma função fechada por prefixos.

$O$ diferencial de $f$ é a função parcial $\varphi: \Sigma^{+} \rightarrow \Gamma^{(*)}$ definida por

$$
(u \sigma) \varphi=(u f)^{-1}((u \sigma) f), \quad \forall u \in \Sigma^{*}, \forall \sigma \in \Sigma .
$$

Dada uma palavra u em $\Sigma^{*}$, o diferencial de $f$ com relação a $u$ é a função parcial $\varphi_{u}: \Sigma^{+} \rightarrow \Gamma^{(*)}$ definida indutivamente como segue. Para toda letra $\sigma$ em $\Sigma$ tal que $(u \sigma) f \neq \varnothing$,

$$
\sigma \varphi_{u}=(u \sigma) \varphi
$$

Para toda palavra $v$ em $\Sigma^{+}$e toda letra $\sigma$ em $\Sigma$ tais que $(u v \sigma) f \neq \varnothing$,

$$
(v \sigma) \varphi_{u}=\left(v \varphi_{u}\right)((u v \sigma) \varphi) .
$$

Da definição de $\varphi$, segue que

$$
\begin{aligned}
& \operatorname{dom} f \cap \Sigma^{+}=\operatorname{dom} \varphi ; \\
& (u \sigma) \varphi \in\left(\Gamma^{(*)}-\Gamma^{+}\right) \Gamma^{*}, \forall u \in \Sigma^{*}, \forall \sigma \in \Sigma, \text { tais que } u \sigma \in \operatorname{dom} \varphi ; \\
& (u \sigma) f=(u f)((u \sigma) \varphi)=(u f)\left(\sigma \varphi_{u}\right), \forall u \in \Sigma^{*}, \forall \sigma \in \operatorname{dom} \varphi_{u} .
\end{aligned}
$$

A propriedade (4.4.1b) significa que toda palavra $(u \sigma) \varphi$ na forma reduzida é igual a um produto $x^{-1} y$, onde $x$ e $y$ são palavras em $\Gamma^{*}$. A propriedade (4.4.1c) significa que $(u \sigma) f$ pode ser fatorado como $(u f) x^{-1} y$. Assim, podemos construir a imagem $(u \sigma) f$ a partir de $u f$ da seguinte forma. Primeiro, o sufixo $x$ é apagado de $u f$. Em seguida, $y$ é concatenada à direita da palavra obtida.

Dada uma palavra $s$ em $\Sigma^{*}$ e uma palavra $x=\sigma_{1} \ldots \sigma_{n}$ tal que $u=s x \in \operatorname{dom} f$, onde cada $\sigma_{i}$ é uma letra, a seguinte propriedade pode ser demonstrada por indução em $n$ :

$$
\left(\left(s \sigma_{1}\right) \varphi\right) \ldots\left(\left(s \sigma_{1} \ldots \sigma_{n}\right) \varphi\right)=(s f)^{-1}((s x) f) .
$$

O produto $\left(\left(s \sigma_{1}\right) \varphi\right) \ldots\left(\left(s \sigma_{1} \ldots \sigma_{n}\right) \varphi\right)$ é uma palavra no grupo livre $\Gamma^{(*)}$. O lado direito de (4.4.2) mostra que essa palavra pode ser escrita na forma reduzida como $y^{-1} z$, onde $y$ e $z$ são palavras em $\Gamma^{*}$. Portanto,

$$
(s x) f=(s f) y^{-1} z .
$$

Assim, para construir a imagem $(s x) f$, primeiro o sufixo $y$ é apagado de $s f$, e, em seguida, $z$ é concatenada à direita da palavra obtida.

Pela definição de $\varphi_{u}$, também podemos escrever (4.4.3) como

$$
(s x) f=(s f)\left(x \varphi_{s}\right) .
$$

Uma conseqüência trivial de (4.4.3) é a propriedade a seguir:

$$
s f \wedge(s x) f=(s f) y^{-1} .
$$


A demonstração do Teorema 4.4.2 utiliza o lema a seguir. Nesse lema, o resto da divisão de um inteiro $i$ por um inteiro $j$ será denotado por $i \bmod j$, e $i \equiv j(\bmod k)$ indica que $i$ e $j$ são equivalentes na relação em $\mathbb{Z}$ definida por

$$
i \equiv j \text { se, e somente se, } i \bmod k=j \bmod k \text {, }
$$

onde $k$ é um inteiro fixo.

Lema 4.4.1 Seja $f: \Sigma^{*} \rightarrow \Gamma^{*}$ uma função fechada por prefixos. Se $f^{-1}$ preservar conjuntos racionais, e existir um inteiro positivo $k$ tal que, para toda palavra $w \sigma$ em $\operatorname{dom} f$, onde $\sigma$ é uma letra,

$$
\|w f,(w \sigma) f\| \leq k
$$

então $f$ é subseqüencial.

Demonstração. Inicialmente, vamos mostrar que, dada uma palavra reduzida $u^{-1} v$ em $\operatorname{im} \varphi$, $\left(u^{-1} v\right) \varphi^{-1}$ é um subconjunto racional de $\Sigma^{*}$.

Para cada $i$ entre 0 e $2 k$, e cada palavra $h$ em $\Gamma^{k}$, defina uma linguagem em $\Gamma^{*}$ como segue:

$$
A_{i, h}=\left\{x \in \Gamma^{*}:|x| \equiv i \quad(\bmod (2 k+1)) \text { e } x \in \Gamma^{*} h \text { ou } h \in \Gamma^{*} x\right\} .
$$

A condição $x \in \Gamma^{*} h$ ou $h \in \Gamma^{*} x$ significa que $h$ é um sufixo de todas as palavras em $A_{i, h}$ de comprimento maior ou igual $k$, e que pode haver no máximo uma palavra de comprimento menor que $k$ nessa linguagem, e essa palavra é um sufixo de $h$.

Claramente, essas linguagens são racionais. Como $f^{-1}$ preserva conjuntos racionais,

$$
B=\bigcup_{\substack{0 \leq i \leq 2 k \\ h \in \Gamma^{k}}}\left(A_{i, h} v\right) f^{-1} \cap\left(\left(A_{i, h} u\right) f^{-1} \Sigma\right) .
$$

é um subconjunto racional de $\Sigma^{+}$.

Vamos provar que $\left(u^{-1} v\right) \varphi^{-1}=B$.

Seja $x \sigma$ uma palavra em $\left(u^{-1} v\right) \varphi^{-1}$, onde $\sigma$ é uma letra. Então, existe uma palavra $y$ em $\Gamma^{*}$ tal que $x f=y u$ e $(x \sigma) f=y v$. Sejam $i=|y| \bmod (2 k+1)$, e $h$ a palavra definida como segue. Se $|y| \geq k$, então $h$ é o sufixo de comprimento $k$ de $y$. Senão, $h$ é qualquer palavra de comprimento $k$ que possa ser fatorada como $h^{\prime} y$. Então, $x \sigma \in\left(A_{i, h} v\right) f^{-1}$ e $x \in\left(A_{i, h} u\right) f^{-1}$, ou seja, $x \sigma \in\left(A_{i, h} v\right) f^{-1} \cap\left(\left(A_{i, h} u\right) f^{-1} \Sigma\right)$. Portanto, $x \sigma \in B$. Obtemos assim

$$
\left(u^{-1} v\right) \varphi^{-1} \subseteq B
$$

Seja agora $x \sigma$ uma palavra em $B$, onde $\sigma$ é uma letra. Então, $x \sigma \in\left(A_{i, h} v\right) f^{-1}$ e $x \in\left(A_{i, h} u\right) f^{-1}$, para algum índice $i$ entre 0 e $2 k$, e alguma palavra $h$ em $\Gamma^{k}$. Portanto, existem palavras $y$ e $z$ em $A_{i, h}$ tais que $(x \sigma) f=y v$ e $x f=z u$.

Afirmamos que $|y|=|z|$. Para provar essa afirmação, note primeiro que, dada uma palavra $w \sigma$ em $\left(u^{-1} v\right) \varphi^{-1}$, onde $\sigma$ é uma letra, a condição (4.4.5) é equivalente a

$$
\left|u^{-1} v\right| \leq k
$$


Como $\|z u|-| y v\| \leq\|x f,(x \sigma) f\|$, segue de (4.4.5) que $\|z u|-| y v\| \leq k$. Como $u^{-1} v$ é uma palavra reduzida, (4.4.6) implica em $|u| \leq k$ e $|v| \leq k$. Portanto, $\| z|-| y|| \leq 2 k$. Como $|z| \equiv|y|$ $(\bmod (2 k+1))$, obtemos $|y|=|z|$.

Agora, afirmamos que $y=z$. De fato, se $|y|<k$, então, pela definição de $A_{i, h}, y$ e $z$ são iguais ao sufixo de comprimento $|y|$ de $h$. Senão, essas palavras podem ser fatoradas como $y=y^{\prime} h$ e $z=z^{\prime} h$. Se $y^{\prime} \neq z^{\prime}$, então $\|x f,(x \sigma) f\|=\left\|z^{\prime} h u, y^{\prime} h v\right\|>|h|=k$, o que contradiz (4.4.5). Concluímos então que $y=z$.

Assim, $(x \sigma) \varphi=(x f)^{-1}((x \sigma) f)=u^{-1} y^{-1} y v=u^{-1} v$, ou seja, $x \sigma \in\left(u^{-1} v\right) \varphi^{-1}$. Obtemos então

$$
B \subseteq\left(u^{-1} v\right) \varphi^{-1} \text {. }
$$

Logo, $\left(u^{-1} v\right) \varphi^{-1}$ é um subconjunto racional de $\Sigma^{*}$.

Agora, vamos construir um $\Sigma$ - $\Gamma$-transdutor subseqüencial $\mathcal{T}$ que realiza $f$. Primeiro, vamos definir o autômato de entrada desse transdutor.

Como observado em (4.4.6), o comprimento de toda palavra reduzida em $\operatorname{im} \varphi$ é limitado por $k$. Portanto, im $\varphi$ é finito. Vamos enumerar as palavras nesse conjunto como $r_{1}, \ldots, r_{n}$.

Conforme acabamos de demonstrar, para cada palavra $r_{l}, r_{l} \varphi^{-1}$ é um conjunto racional. Então, pelo Teorema de Kleene, esse conjunto é reconhecível. Seja $\mathcal{A}_{l}=\left(Q_{l}, i_{l}, T_{l}\right)$ um $\Sigma$-autômato determinístico completo e acessível que reconhece $r_{l} \varphi^{-1}$.

Seja $\mathcal{A}=(Q, i, T)$ a parte bi-acessível do produto $\mathcal{A}_{1} \times \cdots \times \mathcal{A}_{n}$, considerando como conjunto de estados finais a união de $\left(i_{1}, \ldots, i_{n}\right)$ com toda $n$-upla $\left(q_{1}, \ldots, q_{n}\right)$ tal que pelo menos uma das coordenadas seja um estado final. Definimos o autômato de entrada de $\mathcal{T}$ como $\mathcal{A}$.

É conveniente observar as seguintes propriedades de $\mathcal{A}$ :

(4.4.7a) $|\mathcal{A}|=\operatorname{dom} f$.

(4.4.7b) $T=Q$.

(4.4.7c) Para todo par de palavras $s$ e $t$ em $\Sigma^{+}$, se $i s=i t$, então $s \varphi=t \varphi$.

(4.4.7d) Para toda palavra $x$ em $\operatorname{dom} f \cap \Sigma^{+}, i x \neq i$. segue:

A propriedade (4.4.7a) segue da definição dos estados finais de $\mathcal{A}$, e pode ser demonstrada como

$$
|\mathcal{A}| \stackrel{(1.6 .4)}{=} \bigcup_{1 \leq l \leq n}\left|\mathcal{A}_{l}\right| \cup 1=\operatorname{dom} \varphi \cup 1 \stackrel{(4.4 .1 \mathrm{a})}{=} \operatorname{dom} f \text {. }
$$

A propriedade (4.4.7b) é uma conseqüência direta de (4.4.7a), e do fato de que $\mathcal{A}$ é bi-acessível e $f$ é fechada por prefixos.

Para provar (4.4.7c), observe que os conjuntos $\left|\mathcal{A}_{l}\right|$ são dois a dois disjuntos porque $\varphi$ é uma função. Assim, em cada estado $\left(q_{1}, \ldots, q_{n}\right)$ de $\mathcal{A}$, no máximo uma coordenada é um estado final. Temos então que, se $i s=i t$, então essas palavras pertencem ao comportamento de um único autômato $\mathcal{A}_{l}$. Portanto, $s \varphi=t \varphi$.

Para provar (4.4.7d), seja $x \in \operatorname{dom} f \cap \Sigma^{+}$. Por (4.4.1a), $x \in \operatorname{dom} \varphi$. Então, $x$ pertence ao comportamento de algum autômato $\mathcal{A}_{l}$. Como a palavra vazia não pertence a dom $\varphi$, também não pertence a esse comportamento. Portanto, $i_{l} x \neq i_{l}$. Assim, $i x=\left(i_{1} x, \ldots, i_{n} x\right) \neq\left(i_{1}, \ldots, i_{n}\right)$.

Para definir as emissões de $\mathcal{T}$, precisamos do seguinte formalismo. Para toda palavra $s$ em $\operatorname{dom} f$, definimos

$$
\hat{s}=\wedge\{(s x) f: s x \in \operatorname{dom} f\} .
$$


Vamos demonstrar que, dadas palavras distintas $s$ e $t$ em $\Sigma^{*}$ tais que $i s=i t$,

$$
\begin{aligned}
& \hat{s}^{-1}(s f)=\hat{t}^{-1}(t f), \quad \text { e } \\
& \hat{s}^{-1}(\hat{s \sigma})=\hat{t}^{-1}(\hat{t \sigma}), \quad \forall \sigma \in \Sigma \text { tal que } s \sigma \in \operatorname{dom} \varphi .
\end{aligned}
$$

Primeiro, observe que, como $s$ e $t$ são distintas, pelo menos uma dessas palavras deve ser diferente da palavra vazia. Como is $=i t$, segue de (4.4.7d) que ambas as palavras são diferentes da palavra vazia.

Para demonstrar (4.4.8a), seja $x$ uma palavra em $\Sigma^{*}$ tal que $s x \in \operatorname{dom} \varphi$. Afirmamos que existe uma palavra reduzida $y_{x}^{-1} z_{x}$ no grupo livre $\Gamma^{(*)}$ tal que

$$
(s f)^{-1}((s x) f)=(t f)^{-1}((t x) f)=y_{x}^{-1} z_{x} .
$$

Se $x=1$, isso é evidente.

Senão, escreva $x$ como $\sigma_{1} \ldots \sigma_{l}$, onde todo $\sigma_{j}$ é uma letra. Como is $=i t$, temos que, para todo índice $j, i\left(s \sigma_{1} \ldots \sigma_{j}\right)=i\left(t \sigma_{1} \ldots \sigma_{j}\right)$. De (4.4.7c) segue então que $\left(s \sigma_{1} \ldots \sigma_{j}\right) \varphi=\left(t \sigma_{1} \ldots \sigma_{j}\right) \varphi$. Assim,

$$
\left(\left(s \sigma_{1}\right) \varphi\right) \ldots\left(\left(s \sigma_{1} \ldots \sigma_{l}\right) \varphi\right)=\left(\left(t \sigma_{1}\right) \varphi\right) \ldots\left(\left(t \sigma_{1} \ldots \sigma_{l}\right) \varphi\right) .
$$

Por (4.4.2), o lado esquerdo dessa igualdade é igual a $(s f)^{-1}((s x) f)$, e o lado direito igual a $(t f)^{-1}((t x) f)$, o que prova (4.4.9).

Seja $u$ uma palavra em $\Sigma^{*}$ tal que $s u \in \operatorname{dom} f$ e $\left|y_{u}\right|$ seja máximo. Então, segue de (4.4.4) que $(s f) y_{u}^{-1}$ e $(t f) y_{u}^{-1}$ são as menores palavras dos conjuntos $\left\{s f \wedge(s x) f: x \in \Sigma^{*}\right.$ e $\left.s x \in \operatorname{dom} f\right\}$ e $\left\{t f \wedge(t x) f: x \in \Sigma^{*}\right.$ e $\left.t x \in \operatorname{dom} f\right\}$, respectivamente. Portanto,

$$
\hat{s}=(s f) y_{u}^{-1} \text { e } \hat{t}=(t f) y_{u}^{-1} .
$$

Assim, $\hat{s}^{-1}(s f)=y_{u}=\hat{t}^{-1}(t f)$, o que prova (4.4.8a).

A discussão acima também é útil para provar a propriedade (4.4.8b).

Seja $\sigma$ uma letra tal que $s \sigma \in \operatorname{dom} \varphi$. Afirmamos que, para todo $x$ em $\Sigma^{*}$ tal que $s \sigma x \in \operatorname{dom} \varphi$, existe uma palavra $v_{x}$ de $\Gamma^{*}$ tal que

$$
(s \sigma x) f=\hat{s} v_{x} \text { e }(t \sigma x) f=\hat{t} v_{x} .
$$

De fato, de (4.4.10) segue que $s f$ pode ser fatorado como $\hat{s} y_{u}$. Assim, $(s \sigma x) f=\hat{s} y_{u} y_{\sigma x}^{-1} z_{\sigma x}$. Pela maximalidade de $\left|y_{u}\right|, y_{\sigma x}$ deve ser um sufixo de $y_{u}$. Portanto, $(s \sigma x) f$ pode ser fatorado como $\hat{s} y z_{\sigma x}$, onde $y=y_{u} y_{\sigma x}^{-1}$ é uma palavra em $\Gamma^{*}$. Da mesma forma, $(t \sigma x) f$ pode ser fatorado como $\hat{t} y z_{\sigma x}$. Podemos então definir $v_{x}=y z_{\sigma x}$.

Utilizando essas palavras, podemos escrever

$$
\{(s \sigma x) f: s \sigma x \in \operatorname{dom} f\}=\hat{s}\left\{v_{x}: s \sigma x \in \operatorname{dom} f\right\}
$$

$\mathrm{e}$

$$
\{(t \sigma x) f: t \sigma x \in \operatorname{dom} f\}=\hat{t}\left\{v_{x}: s \sigma x \in \operatorname{dom} f\right\} .
$$

Seja $v=\wedge\left\{v_{x}: s \sigma x \in \operatorname{dom} f\right\}$. Claramente, $\hat{s \sigma}=\hat{s} v$ e $\hat{t} \sigma=\hat{t} v$. Assim, $\hat{s}^{-1}(\hat{s} \sigma)=\hat{t}^{-1}(\hat{t \sigma})=v$, o que prova (4.4.8b).

Vamos agora completar a definição de $\mathcal{T}$. 
Para toda palavra $s$ em $\Sigma^{*}$ tal que $i s \neq \varnothing$, a emissão final do estado is é

$$
\text { (is) } \rho=\hat{s}^{-1}(s f) \text {. }
$$

Por (4.4.8a), essa emissão está bem definida.

Dadas uma palavra $s$ em $\Sigma^{*}$ e uma letra $\sigma$ tais que $i(s \sigma) \neq \varnothing$, a emissão do estado $(i s)$ com $\sigma$ é definida por

$$
(i s) * \sigma=\hat{s}^{-1}(\hat{s \sigma}) \text {. }
$$

Por (4.4.8b), essa emissão também está bem definida.

A emissão inicial de $\mathcal{T}$ é

$$
i \lambda=\hat{1} .
$$

Vamos demonstrar que $|\mathcal{T}|=f$.

Primeiro, observe que $\operatorname{dom}|\mathcal{T}|=|\mathcal{A}|=\operatorname{dom} f$. Seja $s$ uma palavra nesse domínio. Vamos mostrar, por indução em $|s|$, que

$$
(i \lambda)(i * s)=\hat{s} .
$$

- $|s|=0$ : então $(i \lambda)(i * s)=i \lambda=\hat{1}$.

- $|s|>0$ : fatore $s$ como to, onde $\sigma$ é uma letra. Então,

$$
(i \lambda)(i * s)=((i \lambda)(i * t))((i t) * \sigma)=\hat{t}\left(\hat{t}^{-1}(\hat{t \sigma})\right)=\hat{s} .
$$

A hipótese de indução e a definição de $*$ foram utilizadas na segunda igualdade.

Utilizando essa propriedade, obtemos

$$
s|\mathcal{T}|=(i \lambda)(i * s)((i s) \rho)=\hat{s}\left(\hat{s}^{-1}(s f)\right)=s f, \quad \forall s \in \Sigma^{*} .
$$

Portanto, $|\mathcal{T}|=f$, e $f$ é uma função subseqüencial.

Demonstração do Teorema 4.4.2. Suponha que $f$ seja uma função subseqüencial.

Como $f^{-1}$ é uma relação racional, essa inversa preserva conjuntos racionais.

Vamos mostrar que $f$ tem variação limitada. Seja $\mathcal{T}=(Q, i, \lambda, \rho)$ um transdutor subseqüencial que realiza $f$. Denote por $M$ o máximo dos comprimentos das emissões $q * \sigma$, para todo estado $q$ de $\mathcal{T}$ e toda letra $\sigma$ tal que $q \sigma \neq \varnothing$, e por $N$ o máximo dos comprimentos das emissões $q \rho$, para todo estado final $q$ de $\mathcal{T}$.

Sejam $k$ um inteiro não-negativo, e $u$ e $v$ palavras em $\operatorname{dom} f$ tais que $\|u, v\| \leq k$. Existem então fatorações $u=x u^{\prime}$ e $v=x v^{\prime}$ tais que $\left|u^{\prime}\right|+\left|v^{\prime}\right| \leq k$.

Como $u f=(i \lambda)(i * x)\left(i x * u^{\prime}\right)((i u) \rho)$ e $v f=(i \lambda)(i * x)\left(i x * v^{\prime}\right)((i v) \rho)$, temos que

$$
\|u f, v f\|=\left\|\left(i x * u^{\prime}\right)((i u) \rho),\left(i x * v^{\prime}\right)((i v) \rho)\right\| \leq\left(\left|u^{\prime}\right|+\left|v^{\prime}\right|\right) M+2 N \leq k M+2 N .
$$

Assim, $K=k M+2 N$ é um inteiro que depende apenas de $k$ e das constantes $M$ e $N$. Portanto, $f$ tem variação limitada.

Suponha agora que a inversa $f^{-1}$ preserve conjuntos racionais, e que $f$ tenha variação limitada. Vamos mostrar que $f$ é uma função subseqüencial. 
Pela primeira hipótese, $\Gamma^{*} f^{-1}=\operatorname{dom} f$ é um subconjunto racional de $\Sigma^{*}$. Pelo Teorema de Kleene, esse conjunto é reconhecível. Seja $\mathcal{A}=(Q, i, T)$ um $\Sigma$-autômato determinístico bi-acessível que reconhece $\operatorname{dom} f$.

Para cada estado $q$ desse autômato, fixe uma palavra $x_{q}$ tal que $q x_{q}$ seja um estado final. Se $q$ for um estado final, fixe $x_{q}=1$. Seja

$$
M=\max \left\{\left|x_{q}\right|: q \in Q\right\}
$$

Seja $g: \Sigma^{*} \rightarrow \Gamma^{*}$ a função definida como segue. O domínio de $g$ é o conjunto dos prefixos de todas as palavras de $\operatorname{dom} f$. Para cada palavra $u$ nesse conjunto, defina

$$
u g=\left(u x_{i u}\right) f
$$

Então, $g$ é uma função fechada por prefixos, e $f=g \mid \operatorname{dom} f$.

Vamos mostrar que a função $g$ tem variação limitada, e que $g^{-1}$ preserva conjuntos racionais.

Seja $k$ um inteiro positivo, e palavras $u$ e $v$ em dom $g$ tais que $\|u, v\| \leq k$. Então, $\left\|u x_{i u}, v x_{i v}\right\| \leq$ $k+2 M$. Como $u x_{i u}$ e $v x_{i v}$ são palavras em $\operatorname{dom} f$, e $f$ tem variação limitada, existe um inteiro $K$ tal que $\left\|\left(u x_{i u}\right) f,\left(v x_{i v}\right) f\right\| \leq K$. Assim, $\|u g, v g\| \leq K$. Como $K$ depende apenas de $k$ e da constante $M$, concluímos que $g$ tem variação limitada.

Seja $A$ um subconjunto racional de $\Gamma^{*}$. Da definição de $g$, segue facilmente que

$$
A g^{-1}=\bigcup_{q \in Q} \mu_{i q} \cap\left(A f^{-1}\right) x_{q}^{-1}
$$

onde $\mu_{i q}$ é o conjunto dos rótulos dos passeios de $i$ a $q$ em $\mathcal{A}$. Como $f^{-1}$ preserva conjuntos racionais, esse conjunto é racional. Portanto, $g^{-1}$ preserva conjuntos racionais.

Como $g$ tem variação limitada, existe um inteiro $k$ tal que, para toda palavra $w$ e toda letra $\sigma$ tal que $w \sigma \in \operatorname{dom} g,\|w g,(w \sigma) g\| \leq k$. Assim, pelo Lema 4.4.1, $g$ é subseqüencial. Da Proposição 4.2.11, segue então que $f=g \mid \operatorname{dom} f$ é subseqüencial.

Uma aplicação interessante do Teorema de Choffrut é na demonstração de que a composição de duas funções subseqüenciais é uma função subseqüencial.

Proposição 4.4.1 Sejam $\Sigma, \Gamma$ e $\Omega$ alfabetos, e $f: \Sigma^{*} \rightarrow \Gamma^{*}$ e $g: \Gamma^{*} \rightarrow \Omega^{*}$ funções subseqüenciais. Então, a composiçãa $\mathrm{fg}: \Sigma^{*} \rightarrow \Omega^{*}$ é uma função subseqüencial.

Demonstração. Pelo Teorema 2.5.1, $f g$ é uma função racional. Portanto, $(f g)^{-1}$ preserva conjuntos racionais.

Vamos mostrar que $f g$ tem variação limitada.

Seja $k$ um inteiro não-negativo. Como $f$ tem variação limitada, existe um inteiro $K$ tal que, para todo par de palavras $u$ e $v$ em $\operatorname{dom} f$ tal que $\|u, v\| \leq k,\|u f, v f\| \leq K$. Como $g$ tem variação limitada, existe um inteiro $L$ tal que, para todo par de palavras $x$ e $y$ em $\operatorname{dom} g$ tal que $\|x, y\| \leq K$, $\|x g, y g\| \leq L$.

Sejam $u$ e $v$ um par de palavras em dom $f g$ tal que $\|u, v\| \leq k$. Então, $\|u f g, v f g\| \leq L$. Como $k$ é arbitrário, temos que $f g$ tem variação limitada.

Assim, pelo Teorema 4.4.2, $f g$ é uma função subseqüencial. 
Corolário 4.4.2 Sejam $\Sigma, \Gamma$ e $\Omega$ alfabetos, e $f: \Sigma^{*} \rightarrow \Gamma^{*}$ e $g: \Gamma^{*} \rightarrow \Omega^{*}$ funções seqüenciais. Então, a composição $\mathrm{fg}: \Sigma^{*} \rightarrow \Omega^{*}$ é uma função seqüencial.

Demonstração. Pela Proposição 4.4.1, $f g$ é uma função subseqüencial. Notando que $f g$ preserva prefixos, segue da Proposição 4.2 .8 que $f g$ é seqüencial.

Outras aplicações desse teorema serão vistas nas seções seguintes.

Exemplo 4.4.1 Toda função $\Sigma^{*} \rightarrow M$ com domínio finito é subseqüencial.

Exemplo 4.4.2 Do Corolário 4.4.1, segue que toda função racional com imagem finita é subseqüencial.

Exemplo 4.4.3 Considere a função racional $\tau$ definida no Exemplo 3.1.5.

Para todo inteiro positivo $n,\left\|\sigma^{n}, \sigma^{n+1}\right\|=1$, mas $\left\|\sigma^{n} \tau, \sigma^{n+1} \tau\right\|=2 n+1$, o que mostra que $\tau$ não tem variação limitada. Portanto, essa função não é subseqüencial.

No entanto, demonstra-se facilmente que a inversa $\tau^{-1}$ é uma função subseqüencial.

Exemplo 4.4.4 Seja $\Sigma=\{\sigma\}$. Vamos identificar o monóide aditivo $\mathbb{N}$ com $\Sigma^{*}$.

Seja $\tau: \mathbb{N} \rightarrow \mathbb{N}$ a função racional

$$
\sigma^{n} \tau= \begin{cases}\sigma^{n} & \text { se } n \text { for par } \\ 1 & \text { se } n \text { for ímpar }\end{cases}
$$

O gráfico de $\tau$ é $\left(\sigma^{2}, \sigma^{2}\right)^{*} \cup(\sigma, 1)\left(\sigma^{2}, 1\right)^{*}$. Portanto, essa função é racional.

Mas, $\tau$ não é uma função subseqüencial porque, para todo número natural $n$ par, $\left\|\sigma^{n}, \sigma^{n+1}\right\|=1$ e $\left\|\sigma^{n} \tau, \sigma^{n+1} \tau\right\|=n$.

Exemplo 4.4.5 Considere a função $f$ definida no Exemplo 4.2.1. Conforme observado, essa função é seqüencial à direita.

No entanto, $f$ não tem variação limitada, pois, para todo $x$ em $\Sigma^{*}$,

$$
\|x \sigma, x \gamma\|=2 \text { e }\|(x \sigma) f,(x \gamma) f\|=2(|x|+1) .
$$

Portanto, $f$ não é subseqüencial.

Exemplo 4.4.6 Considere o transdutor letra-palavra

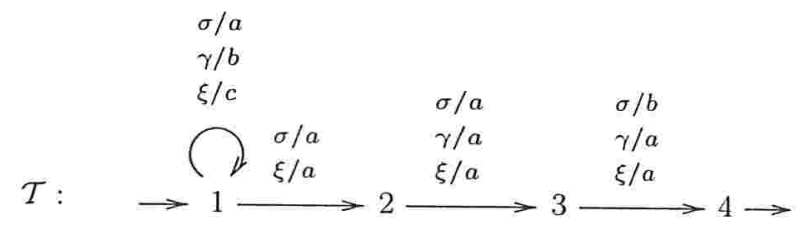


Esse transdutor não é subseqüencial, mas realiza uma função racional $f$. Vamos mostrar que $f$ é uma função subseqüencial.

Primeiro, observe que todas as palavras no domínio de $f$ têm comprimento maior ou igual a 3. Ademais, para toda palavra $x=u \sigma_{1} \sigma_{2} \sigma_{3}$ nesse domínio, onde cada $\sigma_{i}$ é uma letra, podemos escrever

$$
x f=(u g) x^{\prime},
$$

onde $g:\{\sigma, \gamma, \xi\} \rightarrow\{a, b, c\}$ é o morfismo muito fino gerado por

$$
\sigma g=a, \gamma g=b \text { e } \xi g=c
$$

e $x^{\prime}$ é uma palavra de comprimento igual a 3 .

Vamos provar que $f$ tem variação limitada. Dado um inteiro $k \geq 0$, sejam $x=u \sigma_{1} \sigma_{2} \sigma_{3} \mathrm{e}$ $y=v \gamma_{1} \gamma_{2} \gamma_{3}$ palavras em $\operatorname{dom} f$, onde cada $\sigma_{i}$ e cada $\gamma_{i}$ é uma letra, tais que $\|x, y\| \leq k$. Podemos também escrever $y f=(v g) y^{\prime}$. Vamos considerar dois casos para $w=x \wedge y$ :

- $|w|<|u|$ e $|w|<|v|$ : então

$$
\|x f, y f\|=\left|\left(\left(w^{-1} u\right) g\right) x^{\prime}\right|+\left|\left(\left(w^{-1} v\right) g\right) y^{\prime}\right|=\left|w^{-1} u\right|+\left|x^{\prime}\right|+\left|w^{-1} v\right|+\left|y^{\prime}\right| \leq k .
$$

- $|w| \geq|u|$ ou $|w| \geq|v|$ : suponha que $|u| \leq|v|$. Então, $|w| \geq|u|$. Assim, $u \succ v$. Observe que, como $\|x, y\| \leq k,\left|u^{-1} v\right| \leq k$ necessariamente. Temos então que

$$
|| x f, y f|| \leq\left|x^{\prime}\right|+\left|\left(u^{-1} v\right) g\right|+\left|y^{\prime}\right|=\left|x^{\prime}\right|+\left|u^{-1} v\right|+\left|y^{\prime}\right| \leq k+6 .
$$

O caso $|v|<|u|$ é análogo.

Portanto, $f$ tem variação limitada. Utilizando o Corolário 4.4.1, concluímos que $f$ é uma função subseqüencial.

Exemplo 4.4.7 O objetivo deste exemplo é mostrar que a variação limitada é essencial na caracterização das funções subseqüenciais, e que a condição iii do Teorema 4.4.1 é essencial na caracterização das funções seqüenciais. O resto da divisão de um inteiro $a$ por um inteiro $b$ será denotado por $a \bmod b$, e o monóide $\mathbb{N}$ com a operação de adição será identificado com o monóide livre gerado por uma única letra $\sigma$.

Conforme discutido no Exemplo 2.2.3, a função $\tau: \mathbb{N} \rightarrow \mathbb{N}$ que associa cada número natural ao seu quadrado não é racional. Ademais, essa função não tem variação limitada, nem satisfaz a condição iii do Teorema 4.4.1.

Claramente, $\tau$ preserva prefixos. Vamos mostrar que a inversa $\tau^{-1}$ preserva conjuntos reconhecíveis. Pelo Teorema de Kleene, esse fato implica que $\tau^{-1}$ preserva conjuntos racionais.

Primeiro, recordamos a propriedade simples de que todo subconjunto reconhecível de $\mathbb{N}$ é uma união finita de progressões aritméticas. Uma demonstração desse fato pode ser vista no livro de Eilenberg [Eil74]. Assim, é suficiente mostrar que a imagem inversa de uma progressão aritmética por $\tau$ é uma união finita de progressões aritméticas.

Seja $A=\{a+r k: k \geq 0\}$ uma progressão aritmética, onde $a$ e $r$ são números naturais. $\mathrm{O}$ caso em que $r=0$ é trivial, portanto vamos supor que $r>0$. 
Seja $X$ o subconjunto de $\{0, \ldots, r-1\}$ definido como segue. Um inteiro $i$ está em $X$ se, e somente se, existe um inteiro positivo $m$ tal que $m^{2} \in A$, e $m \equiv i(\bmod r)$. Denotamos por $m_{i}$ o menor $m$ com essa propriedade.

Afirmamos que

$$
A \tau^{-1}=\bigcup_{i \in X}\left\{m_{i}+r k: k \geq 0\right\} .
$$

Sejam $x$ um inteiro em $A \tau^{-1}$, e $i=x \bmod r$. Então, $i \in X$. Como $m_{i} \bmod r=i$, temos que $\left(x-m_{i}\right) \bmod r=0$, ou seja, existe um inteiro $k$ tal que $x=m_{i}+r k$. Obtemos assim

$$
A \tau^{-1} \subseteq \bigcup_{i \in X}\left\{m_{i}+r k: k \geq 0\right\}
$$

Seja agora $x$ um inteiro da forma $m_{i}+r k$, para algum $i$ em $X$ e algum inteiro $k \geq 0$. Como $x^{2}=m_{i}{ }^{2}+2 m_{i} r k+r^{2} k^{2}$, temos que $x^{2} \equiv m_{i}{ }^{2}(\bmod r)$, ou seja, existe um inteiro $p$ tal que $x^{2}-m_{i}^{2}=p r$.

Como $m_{i}{ }^{2} \in A$, existe um inteiro $l$ tal que $m_{i}{ }^{2}=a+r l$. Portanto, $x^{2}=a+r(p+l)$, o que mostra que $x^{2} \in A$. Obtemos então

$$
\bigcup_{i \in X}\left\{m_{i}+r k: k \geq 0\right\} \subseteq A \tau^{-1} .
$$

Assim, $\tau$ é uma função que não é racional, mas satisfaz as condições $i$ e $i i$ do Teorema 4.4.1, e a condição $i$ do Teorema 4.4.2.

\subsection{Decidibilidade de se uma função racional é subseqüencial}

A decidibilidade de se um transdutor funcional realiza uma função subseqüencial foi demonstrada por Choffrut em 1977 [Cho77, Ber79]. Choffrut estudou uma propriedade estrutural nesses transdutores equivalente ao comportamento ter variação limitada, e mostrou como essa propriedade pode ser verificada algoritmicamente. Seu algoritmo tem complexidade de tempo exponencial.

Um algoritmo com complexidades de tempo e espaço polinomiais para verificar essa propriedade foi apresentado por Weber e Klemm em 1995 [WK95]. Um algoritmo mais simples com complexidades polinomiais foi publicado por Béal, Carton, Prieur e Sakarovitch em 2003 [BCPS03]. De acordo com esses autores, ambos os algoritmos têm complexidades de tempo e espaço semelhantes.

O algoritmo de Béal, Carton, Prieur e Sakarovitch utiliza a construção do quadrado de um transdutor, e verifica propriedades nessa construção que são, essencialmente, uma reformulação da propriedade estudada por Choffrut. Nesta seção, vamos descrever esse algoritmo.

Fixamos um $\Sigma$ - $\Gamma$-transdutor letra-palavra bi-acessível funcional $\mathcal{T}$, e denotamos por $M$ o máximo dentre os comprimentos das saídas de $\mathcal{T}$, por $n$ o número de estados de $\mathcal{T}$, e por $m$ o número de transições de $\mathcal{T}$. Vamos numerar os estados de $\mathcal{T}$ de 1 a $n$.

O formalismo introduzido na Seção 4.1 será novamente utilizado. Recordamos que, nessa seção, a parte bi-acessível do quadrado foi analisada para decidir se um transdutor realiza uma função racional. Para decidir se um transdutor realiza uma função subseqüencial, a parte acessível será considerada. 
Dizemos que um estado $(p, q)$ é limitado se para todo par $(x, y)$ em $\Delta_{p q}$ e todo passeio fechado $(p, q) \stackrel{s /(u, v)}{\longrightarrow}(p, q)$

$$
(x, y) \cdot(u, v)=(x, y) .
$$

O teorema a seguir mostra a relação do conceito de variação limitada, que é uma caracterização intrínseca da subseqüencialidade, com o de estado limitado, que é uma caracterização efetiva (no sentido de que pode ser verificada algoritmicamente). Choffrut chama de twins os estados de um par limitado, e estuda essa propriedade no transdutor original, sem construir o quadrado [Cho78].

Teorema 4.5.1 (Choffrut 1978) Seja $\mathcal{T}$ um $\Sigma$-Г-transdutor funcional letra-palavra bi-acessível. As condições seguintes são equivalentes:

i. Todo estado acessivel de $\mathcal{T} \times \mathcal{T}$, que pertence a um passeio fechado com saída diferente de $(1,1)$, é limitado.

ii. Para todo estado acessivel $(p, q)$,

$$
|x|,|y| \leq 2 M\left(\begin{array}{l}
n \\
2
\end{array}\right), \forall(x, y) \in \Delta_{p q} .
$$

iii. Para todo estado acessivel $(p, q)$,

$$
\operatorname{card}\left(\Delta_{p q}\right)<\infty
$$

iv. Para todo estado $(p, q)$ acessivel e pertencente a um passeio fechado com saída diferente de $(1,1)$,

$$
\operatorname{card}\left(\Delta_{p q}\right)<\infty
$$

v. $|\mathcal{T}|$ é uma função subseqüencial.

Demonstração. Vamos primeiro provar $i \Rightarrow i i \Rightarrow i i i \Rightarrow v \Rightarrow i$.

- $i \Rightarrow i i$ : Sejam $(x, y)$ uma diferença em $\Delta_{p q}$, e $c$ um passeio de comprimento mínimo de um estado inicial a $(p, q)$ tal que $(x, y)=(c \beta) \Delta$. Afirmamos que $c$ é um caminho.

Suponha o contrário. Então, podemos fatorar $c$ como $c_{1} c_{2} c_{3}$, onde $c_{2}$ é um passeio fechado não-trivial. Seja $(r, s)$ a origem de $c_{2}$. Se a saída de $c_{2}$ for igual a $(1,1)$, então $\left(c_{1} \beta\right) \Delta \cdot\left(c_{2} \beta\right)=$ $\left(c_{1} \beta\right) \Delta$. Senão, por hipótese, esse estado é limitado. Portanto, nesse caso, temos também que $\left(c_{1} \beta\right) \Delta \cdot\left(c_{2} \beta\right)=\left(c_{1} \beta\right) \Delta$. Em ambos os casos,

$$
(x, y) \stackrel{(3.6 .9 \mathrm{~b})}{=}\left(c_{1} \beta\right) \Delta \cdot\left(c_{2} \beta\right) \cdot\left(c_{3} \beta\right)=\left(c_{1} \beta\right) \Delta \cdot\left(c_{3} \beta\right) \stackrel{(3.6 .9 \mathrm{~b})}{=}\left(\left(c_{1} \beta\right)\left(c_{3} \beta\right)\right) \Delta=\left(\left(c_{1} c_{3}\right) \beta\right) \Delta .
$$

Como $c_{1} c_{3}$ é um passeio mais curto que $c$, chegamos a uma contradição.

Considere a fatoração

$$
c=\left(i, i^{\prime}\right) \stackrel{s /(u, v)}{\longrightarrow}\left(p^{\prime}, q^{\prime}\right) \stackrel{s^{\prime} /\left(u^{\prime}, v^{\prime}\right)}{\longrightarrow}(p, q)
$$

definida como segue. Se existir algum estado em $c$ com as duas coordenadas idênticas, então $\left(p^{\prime}, q^{\prime}\right)$ é o último estado em $c$ com essa propriedade. Caso contrário, $\left(p^{\prime}, q^{\prime}\right)$ é $\left(i, i^{\prime}\right)$. 
Afirmamos que

$$
(u, v) \Delta=(1,1) .
$$

O caso $\left(p^{\prime}, q^{\prime}\right)=\left(i, i^{\prime}\right)$ é trivial. No caso em que $p=q, u=v$ (porque $\mathcal{T}$ é funcional), e temos também (4.5.3). Suponha então que $p^{\prime}=q^{\prime}$, e $p \neq q$.

Pela definição de $\mathcal{T} \times \mathcal{T}$, existem passeios $d: i \stackrel{s / u}{\longrightarrow} p^{\prime}$ e $d^{\prime}: i^{\prime} \stackrel{s / v}{\longrightarrow} p^{\prime}$ em $\mathcal{T}$. Como $\mathcal{T}$ é bi-acessível, existe um passeio $f: p^{\prime} \stackrel{z / w}{\longrightarrow} t$ tal que $t$ é um estado final. Então, $d f$ e $d^{\prime} f$ são passeios bem-sucedidos com entrada $s z$ e saídas $u w$ e $v w$, respectivamente. Como $\mathcal{T}$ é funcional, $u w=v w$, o que implica em (4.5.3).

Seja $e$ o segundo caminho na fatoração (4.5.2). Por $(3.6 .9 \mathrm{~b}),(x, y)=(1,1) \cdot\left(u^{\prime}, v^{\prime}\right)=$ $\left(u^{\prime}, v^{\prime}\right) \Delta$. Então,

$$
|x|,|y| \leq \max \left\{\left|u^{\prime}\right|,\left|v^{\prime}\right|\right\} \leq M\|e\| .
$$

Pela escolha de $\left(p^{\prime}, q^{\prime}\right)$, todo estado em $e$ após $\left(p^{\prime}, q^{\prime}\right)$ tem coordenadas distintas. Como $e$ não passa duas vezes por um mesmo estado,

$$
\|e\| \leq n^{2}-n
$$

o que conclui a demonstração de (4.5.1).

- $i i \Rightarrow i i i$ : Evidente.

- $i i i \Rightarrow v$ : Seja $N$ o máximo dentre os comprimentos das palavras que aparecem nas diferenças dos estados acessíveis de $\mathcal{T} \times \mathcal{T}$. Afirmamos que, para todo par $u x$ e $u y \operatorname{em} \operatorname{dom}|\mathcal{T}|$ tal que $u x \wedge u y=u$,

$$
\|(u x)|\mathcal{T}|,(u y)|\mathcal{T}|\| \leq 2 N+(|x|+|y|) M .
$$

De fato, considere passeios bem-sucedidos

$$
i \stackrel{u / v}{\longrightarrow} p \stackrel{x / w}{\longrightarrow} t \quad \text { e } \quad i^{\prime} \stackrel{u / v^{\prime}}{\longrightarrow} q \stackrel{y / w^{\prime}}{\longrightarrow} t^{\prime}
$$

Seja $z=v \wedge v^{\prime}$. Então,

$$
\| v w, v^{\prime} w^{\prime}|| \leq\left|\left(z^{-1} v\right)\right|+\left|\left(z^{-1} v^{\prime}\right)\right|+|w|+\left|w^{\prime}\right|
$$

(recorde a notação definida em (1.3.1)). Pela definição da operação $\Delta$, temos que $\left(z^{-1} v, z^{-1} v^{\prime}\right)$ é uma diferença em $\Delta_{p q}$. Então, $\left|\left(z^{-1} v\right)\right|+\left|\left(z^{-1} v^{\prime}\right)\right| \leq 2 N$. Observando que $|w| \leq|x| M$ e $\left|w^{\prime}\right| \leq|y| M$, obtemos (4.5.4).

A propriedade (4.5.4) mostra que $|\mathcal{T}|$ tem variação limitada. Pelo Corolário 4.4.1, $|\mathcal{T}|$ é uma função subseqüencial.

- $v \Rightarrow i$ : Sejam $(p, q)$ um estado acessível, $(x, y)$ uma diferença em $\Delta_{p q},(p, q) \stackrel{v /(z, w)}{\longrightarrow}(p, q)$ um passeio fechado, e $\left(i, i^{\prime}\right) \stackrel{u /\left(x^{\prime}, y^{\prime}\right)}{\longrightarrow}(p, q)$ um passeio tal que $i$ e $i^{\prime}$ são estados iniciais e $(x, y)=\left(x^{\prime}, y^{\prime}\right) \Delta$.

Como $\mathcal{T}$ é bi-acessível, existem passeios $p \stackrel{s / r}{\longrightarrow} t$ e $q \stackrel{s^{\prime} / r^{\prime}}{\longrightarrow} t^{\prime}$ tais que $t$ e $t^{\prime}$ são estados finais. Temos então a situação ilustrada na figura a seguir: 


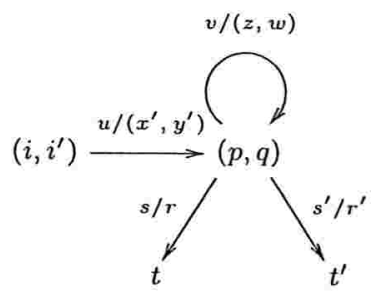

Para todo $j \geq 0,\left\|u v^{j} s, u v^{j} s^{\prime}||=|| s, s^{\prime}\right\| \leq|s|+\left|s^{\prime}\right|$. Como $|\mathcal{T}|$ tem variação limitada, existe um inteiro $K$ tal que

$$
\left\|x^{\prime} z^{j} r, y^{\prime} w^{j} r^{\prime}\right\| \leq K, \quad \forall j \geq 0 .
$$

Assim, por (3.6.9c), o conjunto $\left\{\left(x^{\prime} z^{j} r, y^{\prime} w^{j} r^{\prime}\right) \Delta: j \geq 0\right\}$ é finito. Por (3.6.9b), esse conjunto é igual a $\left\{\left(x^{\prime}, y^{\prime}\right) \Delta \cdot(z, w)^{j} \cdot\left(r, r^{\prime}\right): j \geq 0\right\}$. A Proposição 3.6 .3 implica então em $\left(x^{\prime}, y^{\prime}\right) \Delta$. $(z, w)=\left(x^{\prime}, y^{\prime}\right) \Delta$. Portanto, $(p, q)$ é limitado.

Em seguida, vamos provar que $i i i \Rightarrow i v \Rightarrow i$.

- $i i i \Rightarrow i v$ : Evidente.

- $i v \Rightarrow i$ : Sejam $(p, q)$ um estado acessível, $(x, y)$ uma diferença em $\Delta_{p q}$ e $(p, q) \stackrel{v /(z, w)}{\longrightarrow}(p, q)$ um passeio fechado. Então, para todo $j \geq 0$,

$$
(x, y) \cdot(z, w)^{j} \in \Delta_{p q}
$$

Por hipótese, o conjunto $\left\{(x, y) \cdot(z, w)^{j}: j \geq 0\right\}$ deve ser finito. A Proposição 3.6.3 implica então em $(x, y) \cdot(z, w)=(x, y)$. Portanto, $(p, q)$ é limitado.

O algoritmo testa a condição $i v$ do Teorema 4.5.1. A eficiência desse teste depende do resultado a seguir.

Proposição 4.5.1 Seja $(p, q)$ um estado acessivel e que seja co-acessivel a um estado limitado $e$ pertencente a um passeio fechado com saída diferente de $(1,1)$. Então, se $\mathcal{T}$ for subseqüencial, as duas propriedades a seguir são válidas:

$$
\begin{aligned}
& \Delta_{p q} \subseteq\left(1 \times \Gamma^{*}\right) \cup\left(\Gamma^{*} \times 1\right) \\
& \text { para quaisquer }(x, y),\left(x^{\prime}, y^{\prime}\right) \in \Delta_{p q}, \text { os pares } x, x^{\prime} \text { e } y, y^{\prime} \text { são comparáveis por prefixo. }
\end{aligned}
$$

Demonstração. Sejam $(x, y)$ e $\left(x^{\prime}, y^{\prime}\right)$ pares em $\Delta_{p q}$, e

$$
c:(p, q) \stackrel{s /(u, v)}{\longrightarrow}\left(p^{\prime}, q^{\prime}\right)
$$


um passeio tal que $\left(p^{\prime}, q^{\prime}\right)$ é um estado limitado pertencente a um passeio fechado

$$
d:\left(p^{\prime}, q^{\prime}\right) \stackrel{s^{\prime} /\left(u^{\prime}, v^{\prime}\right)}{\longrightarrow}\left(p^{\prime}, q^{\prime}\right)
$$

$\operatorname{com}\left(u^{\prime}, v^{\prime}\right) \neq(1,1)$.

Vamos provar (4.5.5a).

Como $(x, y) \cdot(u, v) \in \Delta_{p^{\prime} q^{\prime}}$ e $\left(p^{\prime}, q^{\prime}\right)$ é limitado,

$$
((x, y) \cdot(u, v)) \cdot\left(u^{\prime}, v^{\prime}\right)=(x, y) \cdot(u, v) .
$$

Pela Proposição 3.6.2,

$$
(x, y) \cdot(u, v) \in\left(\Gamma^{*} \times 1\right) \cup\left(1 \times \Gamma^{*}\right) .
$$

De $(3.6 .9 \mathrm{~g})$, segue então que $(x, y)$ pertence a $\left(\Gamma^{*} \times 1\right) \cup\left(1 \times \Gamma^{*}\right)$.

Vamos provar (4.5.5b).

Suponha que $x \neq 1$ e $x^{\prime} \neq 1$. Então, por (4.5.5a), $y=1$. Assim, $(x, y) \cdot(u, v)=(x u, v) \Delta$. De (4.5.7), segue que $x$ e $v$ devem ser comparáveis pelo prefixo. Da mesma forma, $x^{\prime}$ e $v$ devem ser comparáveis pelo prefixo.

Vamos estudar duas possibilidades para as relações de prefixação de $x, x^{\prime}$ e $v$ :

- $x \succ v$ ou $x^{\prime} \succ v$ : se $x \succ v$, então, independentemente de se $x^{\prime} \succ v$ ou $v \succ x^{\prime}, x$ e $x^{\prime}$ são comparáveis pelo prefixo. O caso $x^{\prime} \succ v$ é análogo.

- $v \succ x$ e $v \succ x^{\prime}$ : então $(x, y) \cdot(u, v)=\left(\left(v^{-1} x\right) u, 1\right)$. De (4.5.6) e da Proposição 3.6.2, segue que existem palavras $r$ e $s$ e um inteiro $k$ tais que

$$
v^{\prime}=r s \quad \text { e } \quad\left(v^{-1} x\right) u=v^{\prime k} r .
$$

Da mesma forma, existem palavras $r^{\prime}$ e $s^{\prime}$ e um inteiro $k^{\prime}$ tais que

$$
v^{\prime}=r^{\prime} s^{\prime} \quad \text { e } \quad\left(v^{-1} x^{\prime}\right) u=v^{\prime k^{\prime}} r^{\prime}
$$

Assim, podemos fazer as fatorações $v^{-1} x=v^{\prime l} w$ e $v^{-1} x^{\prime}=v^{\prime l^{\prime}} w^{\prime}$, onde $w$ e $w^{\prime}$ são prefixos de $v^{\prime}$. Essas fatorações mostram que $x$ e $x^{\prime}$ são comparáveis pelo prefixo.

O caso em que $y \neq 1$ e $y^{\prime} \neq 1$ é análogo. Obtemos assim (4.5.5b).

Os estados acessíveis e que são co-acessíveis a algum estado que pertence a um passeio fechado com saída diferente de $(1,1)$ serão denominados especiais.

Para encontrar esses estados, o passo principal é marcar os estados que pertencem a um passeio fechado com saída diferente de $(1,1)$. Uma possibilidade para determinar esses estados é como segue. Primeiro, os componentes fortemente conexos do grafo da parte acessível de $\mathcal{T} \times \mathcal{T}$ são construídos. Em seguida, o algoritmo visita as transições de $\mathcal{T} \times \mathcal{T}$. Para cada transição com saída diferente de $(1,1)$, cuja origem e término pertencem ao mesmo componente, o algoritmo marca todos os estados desse componente. A corretude desse processo é uma conseqüência da propriedade de que um estado $(p, q)$ pertence a um passeio fechado com saída diferente de $(1,1)$ se, e somente se, existe uma transição com saída diferente de $(1,1)$ no componente de $(p, q)$. 
O algoritmo de Béal, Carton, Prieur e Sakarovitch pode ser visto como um algoritmo de busca, que visita os vértices do grafo dirigido $\mathcal{G}_{\mathcal{T}}$ definido como segue. O conjunto de vértices desse grafo é

$$
R=\bigcup_{(p, q) \text { especial }}\left((p, q) \times \Delta_{p q}\right) .
$$

Esse grafo tem um arco

$$
((p, q),(x, y)) \rightarrow\left(\left(p^{\prime}, q^{\prime}\right),\left(x^{\prime}, y^{\prime}\right)\right)
$$

para cada transição

$$
(p, q) \stackrel{w /(u, v)}{\longrightarrow}\left(p^{\prime}, q^{\prime}\right)
$$

em $\mathcal{T} \times \mathcal{T}$ tal que $\left(p^{\prime}, q^{\prime}\right)$ é um estado especial e

$$
\left(x^{\prime}, y^{\prime}\right)=(x, y) \cdot(u, v) .
$$

O conjunto dos arcos de $\mathcal{G}_{\mathcal{T}}$ será denotado por $F$.

Esse grafo tem um passeio

$$
c:((i, j),(1,1)) \longrightarrow((p, q),(x, y))
$$

onde $i$ e $j$ são estados iniciais se, e somente se, existe um passeio

$$
c^{\prime}:(i, j) \stackrel{w /(u, v)}{\longrightarrow}(p, q)
$$

em $\mathcal{T} \times \mathcal{T}$ tal que $(x, y)=(u, v) \Delta$ e $(p, q)$ é um estado especial. Essa propriedade pode ser demonstrada por indução em $|c|$, utilizando (3.6.9b). Dela, segue que $\mathcal{G}_{\mathcal{T}}$ é acessível a partir dos vértices da forma $((i, j),(1,1))$.

Da definição do conjunto de vértices de $\mathcal{G}_{\mathcal{T}}$ e da condição iv do Teorema 4.5.1, segue que $|\mathcal{T}|$ é subseqüencial se, e somente se, $\mathcal{G}_{\mathcal{T}}$ é finito.

Vamos novamente utilizar a notação $\mathcal{G}_{\mathcal{T}}[X]$ para representar a restrição de $\mathcal{G}_{\mathcal{T}}$ a um conjunto de $\operatorname{arcos} X$. Se $\mathcal{G}_{\mathcal{T}}[X]$ for acessível a partir dos vértices da forma $((i, j),(1,1))$ nessa restrição, onde $i$ e $j$ são estados iniciais, vamos dizer simplesmente que $\mathcal{G}_{\mathcal{T}}[X]$ é acessível.

$\mathrm{O}$ algoritmo executa uma seqüência de iterações. Cada iteração começa com um subconjunto $X$ de arcos de $F$ e um subconjunto $S$ de vértices de $R$. A primeira iteração começa com $X=\varnothing$ e

$$
S=\bigcup_{\substack{\left(i, i^{\prime}\right) \\ i, i^{\prime} \in I}}\left(\left(i, i^{\prime}\right) \times(1,1)\right) .
$$

Cada iteração consiste no seguinte:

Caso $1 X=F$

Pare e responda sim.

Caso $2 \quad X \neq F$

Seja $e:((p, q),(x, y)) \rightarrow\left(\left(p^{\prime}, q^{\prime}\right),\left(x^{\prime}, y^{\prime}\right)\right)$ um arco em $F-X$ tal que $((p, q),(x, y)) \in S$.

Se alguma das condições a seguir ocorrer, pare e responda não. Senão, insira $\left(\left(p^{\prime}, q^{\prime}\right),\left(x^{\prime}, y^{\prime}\right)\right)$ em $S$ e $e$ em $X$. 
- $\left(x^{\prime}, y^{\prime}\right) \notin\left(1 \times \Gamma^{*}\right) \cup\left(\Gamma^{*} \times 1\right)$;

- $\left|x^{\prime}\right|>M\left(n^{2}-n\right)$ ou $\left|y^{\prime}\right|>M\left(n^{2}-n\right)$;

- existe um vértice $\left(\left(p^{\prime}, q^{\prime}\right),(z, w)\right)$ em $S$ tal que $x^{\prime}$ e $z$ não são comparáveis pelo prefixo, ou $y^{\prime}$ e $w$ não são comparáveis pelo prefixo.

O algoritmo mantém o seguinte invariante:

Invariante 4.5.1 A restrição $\mathcal{G}_{\mathcal{T}}[X]$ é acessivel, e $S$ é exatamente a união dos vértices desse grafo com os vértices

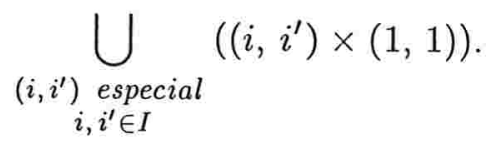

O algoritmo sempre pára, pois são explorados apenas os vértices $((p, q),(x, y))$ tais que $|x|,|y| \leq$ $M\left(n^{2}-n\right)$.

Se o algoritmo responder não, então ou alguma das condições da Proposição 4.5.1 ou a condição ii do Teorema 4.5.1 não é satisfeita. Em ambos os casos, $|\mathcal{T}|$ não é subseqüencial.

Se o algoritmo reponder sim, então $X=F$, e segue do Invariante 4.5 .1 que todos os vértices de $\mathcal{G}_{\mathcal{T}}$ são explorados. Pela definição desses vértices, temos neste caso que, para todo estado $(p, q)$ acessível e pertencente a um passeio fechado com saída diferente de $(1,1), \Delta_{p q}<\infty$. Pela condição $i v$ do Teorema 4.5.1, esta propriedade é equivalente a $|\mathcal{T}|$ ser subseqüencial. Essa discussão mostra que o algoritmo é correto.

Uma melhoria pode ser feita utilizando a propriedade de que se existir um passeio

$$
c:((p, q),(x, y)) \rightarrow\left(\left(p^{\prime}, q^{\prime}\right),\left(x^{\prime}, y^{\prime}\right)\right)
$$

em $\mathcal{G}_{\mathcal{T}}$, então existe um passeio simétrico

$$
c^{\prime}:((q, p),(y, x)) \rightarrow\left(\left(q^{\prime}, p^{\prime}\right),\left(y^{\prime}, x^{\prime}\right)\right)
$$

nesse grafo. Portanto, para todo vértice

$$
((p, q),(x, y))
$$

em $\mathcal{G}_{\mathcal{T}}$, existe um vértice simétrico

$$
((q, p),(y, x))
$$

nesse grafo.

O novo algoritmo visita apenas os vértices $((p, q),(x, y))$ em $R$ tais que $p \leq q$, e insere em $X$ apenas os arcos cujo vértice de origem possua essa propriedade. Vamos denotar o conjunto desses arcos por $G$, e dizer que os vértices que possuem essa propriedade são essenciais. Também vamos dizer que um estado $(p, q)$ é essencial se for especial e $p \leq q$.

O algoritmo começa com $X=\varnothing \mathrm{e}$

$$
S=\bigcup_{\substack{\left(i, i^{\prime}\right) \text { especial } \\ i, i^{\prime} \in I \\ i \leq i^{\prime}}}\left(\left(i, i^{\prime}\right) \times(1,1)\right) .
$$

Cada iteração é como segue: 
Caso $1 X=G$

Pare e responda sim.

Caso $2 \quad X \neq G$

Seja $e:((p, q),(x, y)) \rightarrow\left(\left(p^{\prime}, q^{\prime}\right),\left(x^{\prime}, y^{\prime}\right)\right)$ um arco em $G-X$ tal que $((p, q),(x, y)) \in S$.

Caso 2a $p^{\prime} \leq q^{\prime}$

Se alguma das condições a seguir ocorrer, pare e responda não. Senão, insira $\left(\left(p^{\prime}, q^{\prime}\right),\left(x^{\prime}, y^{\prime}\right)\right)$ em $S$ e $e$ em $X$.

- $\left(x^{\prime}, y^{\prime}\right) \notin\left(1 \times \Gamma^{*}\right) \cup\left(\Gamma^{*} \times 1\right)$;

- $\left|x^{\prime}\right|>M\left(n^{2}-n\right)$ ou $\left|y^{\prime}\right|>M\left(n^{2}-n\right)$;

- existe um vértice $\left(\left(p^{\prime}, q^{\prime}\right),(z, w)\right)$ em $S$ tal que $x^{\prime}$ e $z$ não são comparáveis pelo prefixo, ou $y^{\prime}$ e $w$ não são comparáveis pelo prefixo.

Caso $2 \mathrm{~b} p^{\prime}>q^{\prime}$

Se alguma das condições a seguir ocorrer, pare e responda não. Senão, insira $\left(\left(q^{\prime}, p^{\prime}\right),\left(y^{\prime}, x^{\prime}\right)\right)$ em $S$ e $e$ em $X$.

- $\left(x^{\prime}, y^{\prime}\right) \notin\left(1 \times \Gamma^{*}\right) \cup\left(\Gamma^{*} \times 1\right)$;

- $\left|x^{\prime}\right|>M\left(n^{2}-n\right)$ ou $\left|y^{\prime}\right|>M\left(n^{2}-n\right)$;

- existe um vértice $\left(\left(q^{\prime}, p^{\prime}\right),(w, z)\right)$ em $S$ tal que $x^{\prime}$ e $z$ não são comparáveis pelo prefixo, ou $y^{\prime}$ e $w$ não são comparáveis pelo prefixo.

O invariante do novo algoritmo está enunciado a seguir. As seguintes notações são utilizadas: dado um conjunto de $\operatorname{arcos} X, X^{\prime}$ representa o conjunto dos simétricos dos arcos em $X$; dado um conjunto $S$ de vértices, $S^{\prime}$ representa o conjunto dos simétricos dos vértices em $S$.

Invariante 4.5.2 A restrição $\mathcal{G}_{\mathcal{T}}\left[X \cup X^{\prime}\right]$ é acessivel, e $S \cup S^{\prime}$ é exatamente a união dos vértices desse grafo com os vértices

$$
\bigcup_{\substack {\left(i, i^{\prime}\right) \\
\begin{subarray}{c}{\text { especial } \\
i, i^{\prime} \in I{ ( i , i ^ { \prime } ) \\
\begin{subarray} { c } { \text { especial } \\
i , i ^ { \prime } \in I } }\end{subarray}}\left(\left(i, i^{\prime}\right) \times(1,1)\right) .
$$

A demonstração da corretude desse algoritmo é análoga à do anterior.

Uma descrição mais detalhada do algoritmo, bem como as análises das complexidades de tempo e espaço, dependem de como os vértices de $\mathcal{G}_{\mathcal{T}}$ são armazenados. Vamos mostrar uma possibilidade (sugerida em [BCPS03]), que utiliza as propriedades da Proposição 4.5.1.

Sejam $b: \Gamma \rightarrow \Gamma^{\prime}$ uma cópia de $\Gamma$, e $f:\left(\Gamma \cup \Gamma^{\prime}\right)^{*} \rightarrow \Gamma^{*}$ o morfismo gerado por

$$
\sigma f= \begin{cases}\sigma & \text { se } \sigma \in \Gamma \\ \sigma b^{-1} & \text { se } \sigma \in \Gamma^{\prime}\end{cases}
$$

O algoritmo mantém um conjunto $\delta_{p q}^{0}$ e duas palavras $\delta_{p q}^{1}$ e $\delta_{p q}^{2}$ em $\left(\Gamma \cup \Gamma^{\prime}\right)^{*}$ para cada estado essencial $(p, q)$. Essas informações representam os vértices em $S$ segundo as regras a seguir: 
- se não existir um vértice da forma $((p, q),(x, 1)) \operatorname{com} x \neq 1$ em $S$, então $\delta_{p q}^{1}=1$. Senão, seja $\left((p, q),\left(x_{1}, 1\right)\right), \ldots,\left((p, q),\left(x_{k}, 1\right)\right)$ a ordenação dos vértices em $S$ da forma $((p, q),(x, 1))$, com $x \neq 1$, que satisfaz

$$
x_{1} \succ \cdots \succ x_{k}
$$

Para cada palavra $x_{i}$, seja $x_{i}=u_{i} \gamma_{i}$ a fatoração dessa palavra, onde $\gamma_{i}$ é uma letra. Então,

$$
\delta_{p q}^{1}=\left(u_{1}\right)\left(\gamma_{1} b\right)\left(x_{1}^{-1} u_{2}\right)\left(\gamma_{2} b\right) \ldots\left(x_{k-1}^{-1} u_{k}\right)\left(\gamma_{k} b\right) .
$$

- se não existir um vértice da forma $((p, q),(1, y))$ com $y \neq 1$ em $S$, então $\delta_{p q}^{2}=1$. Senão, seja $\left((p, q),\left(1, y_{1}\right)\right), \ldots,\left((p, q),\left(1, y_{l}\right)\right)$ a ordenação dos vértices em $S$ da forma $((p, q),(1, y))$, com $y \neq 1$, que satisfaz

$$
y_{1} \succ \cdots \succ y_{l}
$$

Para cada palavra $y_{i}$, seja $y_{i}=v_{i} \xi_{i}$ a fatoração dessa palavra, onde $\xi_{i}$ é uma letra. Então,

$$
\delta_{p q}^{2}=\left(v_{1}\right)\left(\xi_{1} b\right)\left(y_{1}^{-1} v_{2}\right)\left(\xi_{2} b\right) \ldots\left(y_{l-1}^{-1} v_{l}\right)\left(\xi_{l} b\right) .
$$

- $\delta_{p q}^{0} \neq \varnothing$ se, e somente se, $((p, q),(1,1)) \in S$. Se $((p, q),(1,1)) \in S$, então $\delta_{p q}^{0}=\{1\}$.

As ordenações (4.5.8) e (4.5.9) são possíveis devido à Proposição 4.5.1.

Utilizando essas informações, um vértice

$$
((p, q),(x, y)) \text { em } S
$$

pode ser armazenado como

$$
((p, q),(|x|,|y|)) \text {. }
$$

Assim, dado um par $((p, q),(k, l))$, onde $k$ e $l$ são inteiros, correspondente a um vértice $((p, q),(x, y))$, as palavras $x$ e $y$ podem ser obtidas a partir de $\delta_{p q}^{1}$ e $\delta_{p q}^{2}$ como segue:

$$
x=\left(\delta_{p q}^{1}[1 \ldots k]\right) f \quad \text { e } \quad y=\left(\delta_{p q}^{2}[1 \ldots l]\right) f .
$$

Uma descrição detalhada pode ser vista no Algoritmo 4.5.1. Nessa descrição, o algoritmo mantém um conjunto $P$ com os vértices de $S$ que são a origem de algum arco ainda não visitado. A cada iteração, o algoritmo seleciona um vértice $((p, q),(k, l))$ em $P$, e todos os arcos da forma

$$
e:((p, q),(x, y)) \rightarrow\left(\left(p^{\prime}, q^{\prime}\right),\left(x^{\prime}, y^{\prime}\right)\right), \operatorname{com} x=\left(\delta_{p q}^{1}[1 \ldots k]\right) f \text { e } y=\left(\delta_{p q}^{2}[1 \ldots l]\right) f,
$$

são analisados.

As complexidades de tempo e espaço para determinar o conjunto dos estados essenciais de $\mathcal{T} \times \mathcal{T}$ é $O\left(n^{2}+m^{2}\right)$. De fato, os algoritmos para a construção da parte acessível e dos componentes fortemente conexos de $\mathcal{T} \times \mathcal{T}$ têm complexidades de tempo e espaço lineares no número de estados e transições desse transdutor.

Vamos fazer um estudo mais detalhado das complexidades de tempo e espaço do algoritmo que só percorre os vértices essenciais. 


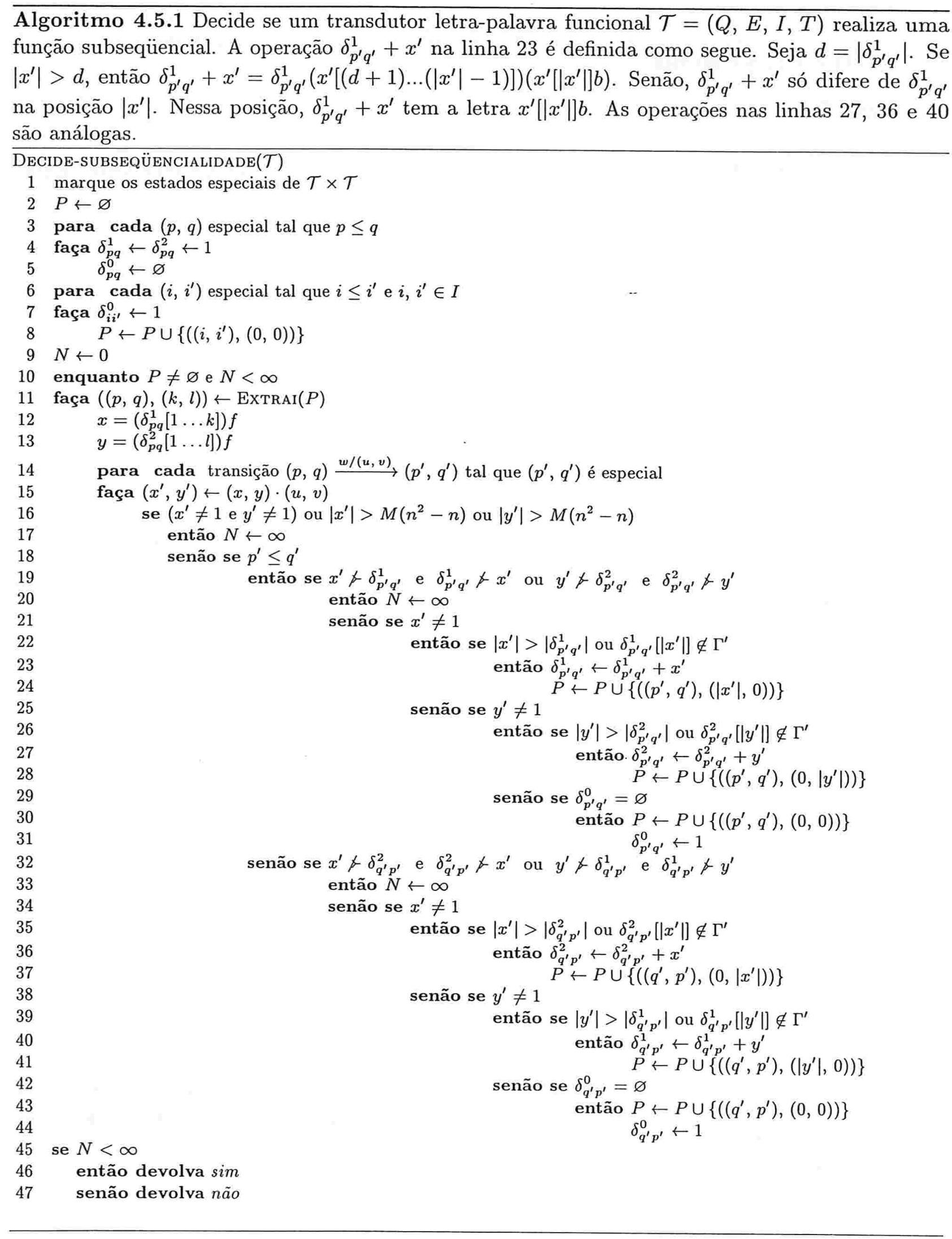


Conforme observado na Seção 4.1, o número de transições de $\mathcal{T} \times \mathcal{T}$ cuja origem $(p, q)$ satisfaz $p \leq q$ é no máximo $m(m+1) / 2$. Para cada transição $(p, q) \rightarrow\left(p^{\prime}, q^{\prime}\right)$ com essa propriedade, o número de arcos de $\mathcal{G}_{\mathcal{T}}$ da forma $((p, q),(x, y)) \rightarrow\left(\left(p^{\prime}, q^{\prime}\right),\left(x^{\prime}, y^{\prime}\right)\right)$ que são explorados pelo algoritmo é no máximo $2 M\left(n^{2}-n\right)+1$ (pois apenas estados com $|x|$ e $|y|$ limitados por $M\left(n^{2}-n\right)$ são explorados). Portanto, o número máximo de arcos explorados pelo algoritmo é

$$
\frac{m(m+1)\left(2 M\left(n^{2}-n\right)+1\right)}{2}
$$

A cada arco $((p, q),(x, y)) \rightarrow\left(\left(p^{\prime}, q^{\prime}\right),\left(x^{\prime}, y^{\prime}\right)\right)$ explorado, o número de operações realizadas é $O\left(\left|x^{\prime}\right|+\left|y^{\prime}\right|\right)=O\left(M\left(n^{2}-n\right)\right)$. Portanto, a complexidade de tempo do algoritmo é, no pior caso, proporcional a

$$
\frac{m(m+1)\left(2 M\left(n^{2}-n\right)+1\right)\left(M\left(n^{2}-n\right)\right)}{2} .
$$

A única informação armazenada pelo algoritmo é o conjunto dos vértices visitados. Portanto, a complexidade de espaço depende apenas da soma dos comprimentos das palavras $\delta_{p q}^{1}$ e $\delta_{p q}^{2}$, bem como do conjunto $\delta_{p q}^{0}$. O tamanho total dessas informações é

$$
\frac{n(n+1)\left(2 M\left(n^{2}-n\right)+1\right)}{2} \text {. }
$$

Exemplo 4.5.1 Os transdutores da família definida no Exemplo 4.1.2 são subseqüenciais, pois, para todo estado $(p, q), \Delta_{p q}$ é unitário.

Exemplo 4.5.2 Neste exemplo, vamos definir uma família de transdutores cujos quadrados contêm uma diferença que atinge o comprimento máximo provado em (4.5.1). Este exemplo é uma modificação do Exemplo 4.1.2.

Sejam $n$ e $M$ inteiros positivos, com $n>2$. Vamos denotar $\left(\begin{array}{l}n \\ 2\end{array}\right)$ por $k$. Seja

$$
\mathcal{E}=\left(p_{1}, q_{1}\right), \ldots,\left(p_{k}, q_{k}\right)
$$

uma enumeração dos pares $(p, q)$ em $[n] \times[n]$ tais que $p<q$. Vamos fazer a convenção de que $\left(p_{0}, q_{0}\right)=(1,1)$.

Para cada par $\left(p_{i}, q_{i}\right)$ em $\mathcal{E}$ tal que $0<i<k$, defina duas letras $\sigma_{i}$ e $\gamma_{i}$. Sejam

$$
\Sigma=\left\{\sigma_{0}, \sigma_{1}, \ldots, \sigma_{k-1}, \gamma_{1}, \ldots, \gamma_{k-1}, \gamma_{k}\right\} \quad \text { e } \Gamma=\{a\}
$$

onde as letras $\sigma_{0}, \gamma_{k}$ e $a$ são novas.

Utilizando esses dados, definimos um $\Sigma$ - $\Gamma$-transdutor $\mathcal{T}$ como segue. $\mathrm{O}$ conjunto de estados de $\mathcal{T}$ é $[n]$. O único estado inicial é 1 , e esse estado também é o único final. Para cada par $\left(p_{i}, q_{i}\right)$ em $\mathcal{E}$ tal que $0<i<k, \mathcal{T}$ tem as transições

$$
p_{i} \stackrel{\sigma_{i} / 1}{\longrightarrow} q_{i+1}, \quad q_{i} \stackrel{\sigma_{i} / a^{M}}{\longrightarrow} p_{i+1}, \quad p_{i} \stackrel{\gamma_{i} / a^{M}}{\longrightarrow} q_{i} \text { e } q_{i} \stackrel{\gamma_{i} / 1}{\longrightarrow} p_{i} .
$$

As únicas transições de $\mathcal{T}$ além dessas são

$$
1 \stackrel{\sigma_{0} / a^{M}}{\longrightarrow} p_{1}, \quad 1 \stackrel{\sigma_{0} / 1}{\longrightarrow} q_{1}, p_{k} \stackrel{\gamma_{k} / a^{M}}{\longrightarrow} q_{k} \text { e } q_{k} \stackrel{\gamma_{k} / 1}{\longrightarrow} p_{k} .
$$


Para provar que $\mathcal{T}$ é bi-acessível, seja $q$ um estado diferente de 1 e $\left(p_{i}, q_{i}\right)$ o par $(1, q)$. Da definição das transições de $\mathcal{T}$ segue que existe um passeio da forma

$$
1 \stackrel{\sigma_{0} / 1}{\longrightarrow} q_{1} \stackrel{\gamma_{1} / 1}{\longrightarrow} p_{1} \ldots p_{i-1} \stackrel{\sigma_{i-1} / 1}{\longrightarrow} q_{i} \stackrel{\gamma_{i} / 1}{\longrightarrow} 1 .
$$

As seguintes propriedades de $\mathcal{T} \times \mathcal{T}$ são uma conseqüência imediata dessa definição:

i. As únicas transições com origem em um par $\left(p_{i}, q_{i}\right)$ em $\mathcal{E} \operatorname{com} 0<i<k$ são

$$
\left(p_{i}, q_{i}\right) \stackrel{\gamma_{i} /\left(a^{M}, 1\right)}{\longrightarrow}\left(q_{i}, p_{i}\right) \text { e }\left(p_{i}, q_{i}\right) \stackrel{\sigma_{i} /\left(1, a^{M}\right)}{\longrightarrow}\left(q_{i+1}, p_{i+1}\right) .
$$

Ademais, há uma única transição com origem em $\left(p_{k}, q_{k}\right)$, a saber,

$$
\left(p_{k}, q_{k}\right) \stackrel{\gamma_{k} /\left(a^{M}, 1\right)}{\longrightarrow}\left(q_{k}, p_{k}\right) .
$$

ii. As únicas transições com origens e entradas idênticas são $1 \stackrel{\sigma_{0} / a^{M}}{\longrightarrow} p_{1}$ e $1 \stackrel{\sigma_{0} / 1}{\longrightarrow} q_{1}$. Assim, para todo estado $p$ diferente de $1, \mathcal{T} \times \mathcal{T}$ tem uma transição da forma

$$
(p, p) \stackrel{\sigma /\left(x, x^{\prime}\right)}{\longrightarrow}\left(q, q^{\prime}\right)
$$

se, e somente se, $q=q^{\prime}, x=x^{\prime}$ e $\mathcal{T}$ tem uma transição da forma

$$
p \stackrel{\sigma / x}{\longrightarrow} q
$$

A mesma propriedade é válida para $p=1$ com toda letra $\sigma$ em $\Sigma-\sigma_{0}$. Ademais, há quatro transições em $\mathcal{T} \times \mathcal{T}$ com origem em $(1,1)$ e entrada $\sigma_{0}$, ilustradas a seguir:

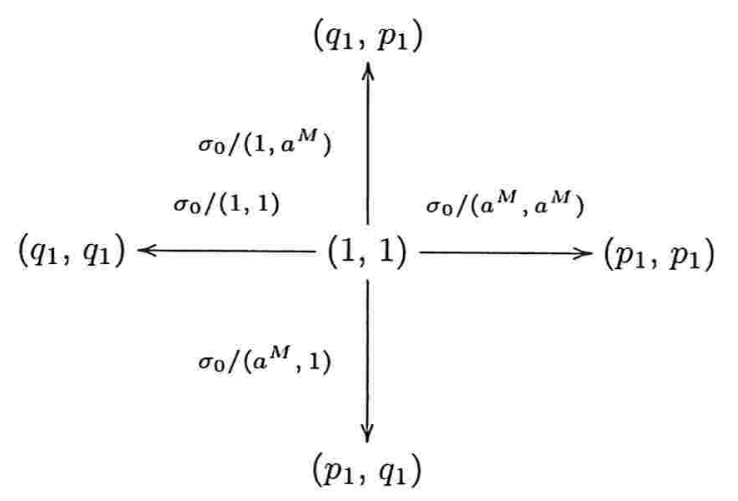

A figura abaixo ilustra uma parte de $\mathcal{T} \times \mathcal{T}$. O restante desse transdutor contém os estados da forma $(p, p)$, as transições com origem ou término nesses estados, e as transições simétricas às transições cuja entrada é uma letra $\sigma_{i}, 1 \leq i \leq k$.

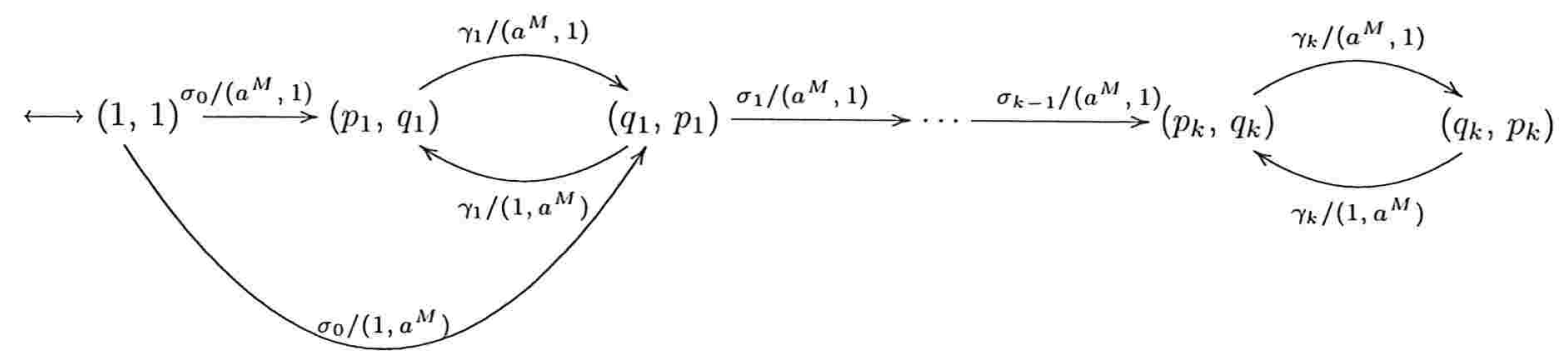


Essa discussão deixa claro que $\mathcal{T} \times \mathcal{T}$ é acessível, que nenhum estado $(p, q)$ tal que $p \neq q$ é co-acessível, e que

$$
\Delta_{q q}=\{(1,1)\}, \quad \forall q \in Q .
$$

Por (4.1.2), segue dessa propriedade que $\mathcal{T}$ é um transdutor funcional.

Todos os passeios fechados que passam por algum estado $(p, q)$ tal que $p \neq q$ são concatenações de circuitos da forma

$$
\left(p_{i}, q_{i}\right) \stackrel{\gamma_{i} /\left(a^{M}, 1\right)}{\longrightarrow}\left(q_{i}, p_{i}\right) \stackrel{\gamma_{i} /\left(1, a^{M}\right)}{\longrightarrow}\left(p_{i}, q_{i}\right)
$$

ou

$$
\left(q_{i}, p_{i}\right) \stackrel{\gamma_{i} /\left(1, a^{M}\right)}{\longrightarrow}\left(p_{i}, q_{i}\right) \stackrel{\gamma_{i} /\left(a^{M}, 1\right)}{\longrightarrow}\left(p_{i}, q_{i}\right) .
$$

Ademais, a saída de toda transição com origem ou término em um estado da forma $(p, p)$ é um múltiplo de $(a, a)$. Portanto, a saída de todo passeio fechado é um múltiplo de $(a, a)$. Essa propriedade mostra que todo estado $(p, q)$ é limitado, e segue então do Teorema 4.5 .1 que $|\mathcal{T}|$ é uma função subseqüencial.

A figura também mostra que

$$
\left(a^{K}, 1\right) \in \Delta_{p_{k} q_{k}}, \text { onde } K=M\left(n^{2}-n\right) .
$$

Ou seja, os comprimentos máximos para as diferenças provados no Teorema 4.5.1 ocorrem nos estados $\left(p_{k}, q_{k}\right)$ e $\left(q_{k}, p_{k}\right)$.

Exemplo 4.5.3 Vamos simular o Algoritmo 4.5.1 em um dos transdutores definidos no Exemplo 4.5.2.

O transdutor para $n=3$ e a enumeração

$$
(1,2),(1,3),(2,3)
$$

é

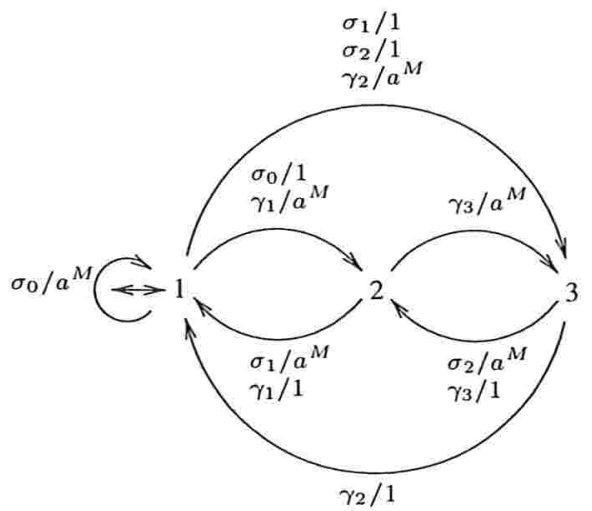

O quadrado desse transdutor está ilustrado na Figura 4.5.1. As transições contínuas têm saída $\left(a^{M}, 1\right)$, e as pontilhadas têm saída $\left(1, a^{M}\right)$. Os rótulos das transições da restrição de $\mathcal{T} \times \mathcal{T}$ aos estados $(1,1),(2,2)$ e $(3,3)$ não estão indicados, mas podem ser obtidos a partir de $\mathcal{T}$.

A simulação começa na página 148. Apenas os estados essenciais são considerados. Os pares representados pelas palavras $\delta_{p q}^{1}$ e $\delta_{p q}^{2}$ são escritos explicitamente. Cada nova diferença descoberta 


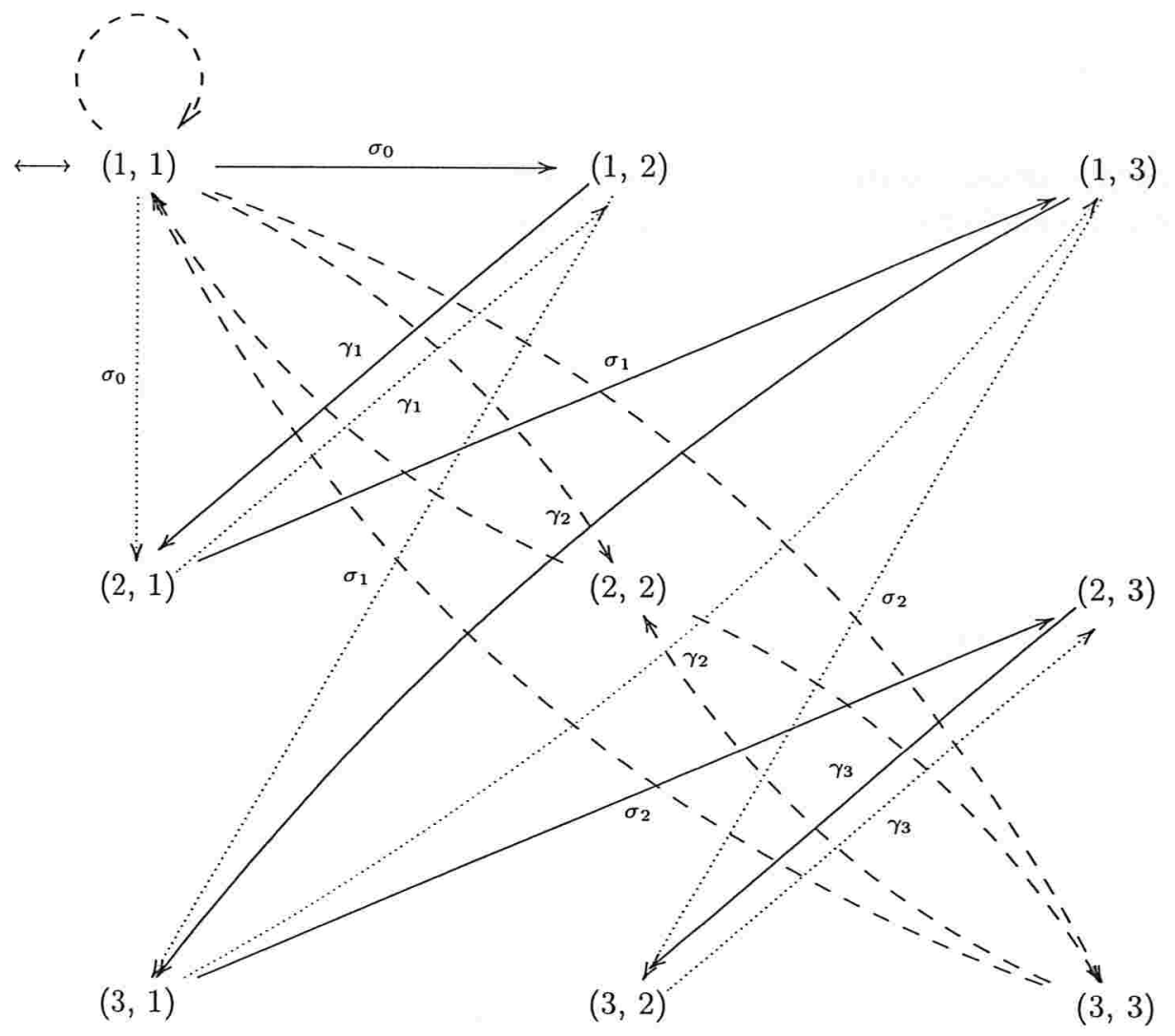

Figura 4.5.1: Quadrado do transdutor do Exemplo 4.5.3

\begin{tabular}{|c|c|}
\hline$P$ & $\Delta$ \\
\hline$((1,1),(0,0))$ & $\Delta_{11}:(1,1)$ \\
\hline$\underline{((1,2),(M, 0))}, \underline{((2,2),(0,0))}$ & $\begin{array}{l}\Delta_{11}:(1,1) \\
\Delta_{12}:\left(a^{M}, 1\right) \\
\Delta_{22}: \underline{(1,1)}\end{array}$ \\
\hline$((2,2),(0,0)), \underline{((1,2),(0,2 M))}, \underline{((1,3),(0,0))}$ & $\begin{array}{l}\Delta_{11}:(1,1) \\
\Delta_{12}:\left(a^{M}, 1\right), \underline{\left(1, a^{2 M}\right)} \\
\Delta_{22}:(1,1) \\
\Delta_{13}: \underline{(1,1)}\end{array}$ \\
\hline$((1,2),(0,2 M)),((1,3),(0,0)), \underline{((3,3),(0,0))}$ & $\begin{array}{l}\triangle_{11}:(1,1) \\
\Delta_{12}:\left(a^{M}, 1\right),\left(1, a^{2 M}\right) \\
\triangle_{22}:(1,1) \\
\Delta_{13}:(1,1) \\
\Delta_{33}:(1,1)\end{array}$ \\
\hline
\end{tabular}




\begin{tabular}{|c|c|}
\hline$((1,3),(0,0)),((3,3),(0,0)), \underline{((1,3),(3 M, 0))}$ & $\begin{aligned} & \Delta_{11}:(1,1) \\
& \Delta_{12}:\left(a^{M}, 1\right),\left(1, a^{2 M}\right) \\
& \Delta_{22}:(1,1) \\
& \Delta_{13}:(1,1),\left(a^{3 M}, 1\right) \\
& \Delta_{33}:(1,1)\end{aligned}$ \\
\hline $\begin{array}{l}((3,3),(0,0)),((1,3),(3 M, 0)), \underline{((1,3),(0, M))} \\
((2,3),(M, 0))\end{array}$ & $\begin{aligned} & \Delta_{11}:(1,1) \\
& \Delta_{12}:\left(a^{M}, 1\right),\left(1, a^{2 M}\right) \\
& \Delta_{22}:(1,1) \\
& \Delta_{13}:(1,1),\left(a^{3 M}, 1\right), \underline{\left(1, a^{M}\right)} \\
& \Delta_{33}:(1,1) \\
& \Delta_{23}:\left(a^{M}, 1\right)\end{aligned}$ \\
\hline$((1,3),(3 M, 0)),((1,3),(0, M)),((2,3),(M, 0))$ & $\begin{aligned} & \Delta_{11}:(1,1) \\
& \Delta_{12}:\left(a^{M}, 1\right),\left(1, a^{2 M}\right) \\
& \Delta_{22}:(1,1) \\
& \Delta_{13}:(1,1),\left(a^{3 M}, 1\right),\left(1, a^{M}\right) \\
& \Delta_{33}:(1,1) \\
& \Delta_{23}:\left(a^{M}, 1\right)\end{aligned}$ \\
\hline $\begin{array}{l}((1,3),(0, M)),((2,3),(M, 0)), \underline{((1,3),(0,4 M))}, \\
\underline{((2,3),(0,2 M))}\end{array}$ & $\begin{aligned} & \Delta_{11}:(1,1) \\
& \Delta_{12}:\left(a^{M}, 1\right),\left(1, a^{2 M}\right) \\
& \Delta_{22}:(1,1) \\
& \Delta_{13}:(1,1),\left(a^{3 M}, 1\right),\left(1, a^{M}\right), \underline{\left(1, a^{4 M}\right)} \\
& \Delta_{33}:(1,1) \\
& \Delta_{23}:\left(a^{M}, 1\right),\left(1, a^{2 M}\right)\end{aligned}$ \\
\hline $\begin{array}{l}((2,3),(M, 0)),((1,3),(0,4 M)),((2,3),(0,2 M)), \\
\underline{((2,3),(2 M, 0))}\end{array}$ & $\begin{array}{l}\Delta_{11}:(1,1) \\
\Delta_{12}:\left(a^{M}, 1\right),\left(1, a^{2 M}\right) \\
\Delta_{22}:(1,1) \\
\Delta_{13}:(1,1),\left(a^{3 M}, 1\right),\left(1, a^{M}\right),\left(1, a^{4 M}\right) \\
\Delta_{33}:(1,1) \\
\Delta_{23}:\left(a^{M}, 1\right),\left(1, a^{2 M}\right), \underline{\left(a^{2 M}, 1\right)}\end{array}$ \\
\hline$((1,3),(0,4 M)),((2,3),(0,2 M)),((2,3),(2 M, 0))$ & $\begin{array}{l}\triangle_{11}:(1,1) \\
\triangle_{12}:\left(a^{M}, 1\right),\left(1, a^{2 M}\right) \\
\triangle_{22}:(1,1) \\
\triangle_{13}:(1,1),\left(a^{3 M}, 1\right),\left(1, a^{M}\right),\left(1, a^{4 M}\right) \\
\triangle_{33}:(1,1) \\
\triangle_{23}:\left(a^{M}, 1\right),\left(1, a^{2 M}\right),\left(a^{2 M}, 1\right)\end{array}$ \\
\hline$((2,3),(0,2 M)),((2,3),(2 M, 0)), \underline{((2,3),(5 M, 0))}$ & $\begin{array}{l}\Delta_{11}:(1,1) \\
\Delta_{12}:\left(a^{M}, 1\right),\left(1, a^{2 M}\right) \\
\Delta_{22}:(1,1) \\
\Delta_{13}:(1,1),\left(a^{3 M}, 1\right),\left(1, a^{M}\right),\left(1, a^{4 M}\right) \\
\Delta_{33}:(1,1) \\
\Delta_{23}:\left(a^{M}, 1\right),\left(1, a^{2 M}\right),\left(a^{2 M}, 1\right),\left(a^{5 M}, 1\right)\end{array}$ \\
\hline$((2,3),(2 M, 0)),((2,3),(5 M, 0))$ & $\begin{array}{l}\Delta_{11}:(1,1) \\
\Delta_{12}:\left(a^{M}, 1\right),\left(1, a^{2 M}\right) \\
\Delta_{22}:(1,1) \\
\Delta_{13}:(1,1),\left(a^{3 M}, 1\right),\left(1, a^{M}\right),\left(1, a^{4 M}\right) \\
\Delta_{33}:(1,1) \\
\Delta_{23}:\left(a^{M}, 1\right),\left(1, a^{2 M}\right),\left(a^{2 M}, 1\right),\left(a^{5 M}, 1\right)\end{array}$ \\
\hline
\end{tabular}




\begin{tabular}{l|l}
\hline & $\Delta_{11}:(1,1)$ \\
& $\Delta_{12}:\left(a^{M}, 1\right),\left(1, a^{2 M}\right)$ \\
& $\Delta_{22}:(1,1)$ \\
& $\Delta_{13}:(1,1),\left(a^{3 M}, 1\right),\left(1, a^{M}\right),\left(1, a^{4 M}\right)$ \\
& $\Delta_{33}:(1,1)$ \\
& $\Delta_{23}:\left(a^{M}, 1\right),\left(1, a^{2 M}\right),\left(a^{2 M}, 1\right),\left(a^{5 M}, 1\right)$, \\
& $\left(1, a^{3 M}\right)$ \\
\hline & $\Delta_{11}:(1,1)$ \\
& $\Delta_{12}:\left(a^{M}, 1\right),\left(1, a^{2 M}\right)$ \\
& $\Delta_{22}:(1,1)$ \\
& $\Delta_{13}:(1,1),\left(a^{3 M}, 1\right),\left(1, a^{M}\right),\left(1, a^{4 M}\right)$ \\
& $\Delta_{33}:(1,1)$ \\
& $\Delta_{23}:\left(a^{M}, 1\right),\left(1, a^{2 M}\right),\left(a^{2 M}, 1\right),\left(a^{5 M}, 1\right)$, \\
& $\left(1, a^{3 M}\right),\left(1, a^{6 M}\right)$ \\
\hline & $\Delta_{11}:(1,1)$ \\
& $\Delta_{12}:\left(a^{M}, 1\right),\left(1, a^{2 M}\right)$ \\
& $\Delta_{22}:(1,1)$ \\
& $\Delta_{13}:(1,1),\left(a^{3 M}, 1\right),\left(1, a^{M}\right),\left(1, a^{4 M}\right)$ \\
& $\Delta_{33}:(1,1)$ \\
& $\Delta_{23}:\left(a^{M}, 1\right),\left(1, a^{2 M}\right),\left(a^{2 M}, 1\right),\left(a^{5 M}, 1\right)$, \\
& $\left(1, a^{3 M}\right),\left(1, a^{6 M}\right)$ \\
\hline & $\Delta_{11}:(1,1)$ \\
& $\Delta_{12}:\left(a^{M}, 1\right),\left(1, a^{2 M}\right)$ \\
& $\Delta_{22}:(1,1)$ \\
& $\Delta_{13}:(1,1),\left(a^{3 M}, 1\right),\left(1, a^{M}\right),\left(1, a^{4 M}\right)$ \\
& $\Delta_{33}:(1,1)$ \\
& $\Delta_{23}:\left(a^{M}, 1\right),\left(1, a^{2 M}\right),\left(a^{2 M}, 1\right),\left(a^{5 M}, 1\right)$, \\
& $\left(1, a^{3 M}\right),\left(1, a^{6 M}\right)$ \\
\hline &
\end{tabular}

é grifada. A cada iteração, o par mais à esquerda de $P$ é analisado. Observe que todos os estados são especiais.

Os exemplos 4.5.4, 4.5.5 e 4.5.6 foram extraídos de [BCPS03]. Vamos fixar $\Sigma=\{\sigma\}$ e $\Gamma=\{a, b\}$.

Exemplo 4.5.4 A função $f: \Sigma^{+} \rightarrow \Gamma^{*}$ definida por

$$
\sigma^{n} f= \begin{cases}a^{2 n} & \text { se } n \text { for par } \\ a^{n} & \text { se } n \text { for ímpar }\end{cases}
$$

é o comportamento do transdutor letra-palavra

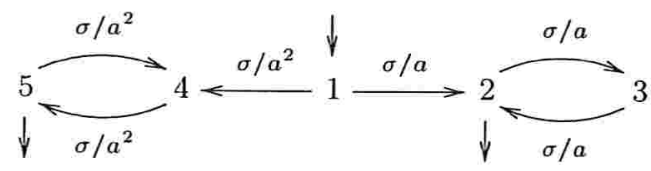


Os estados acessíveis em $\mathcal{T} \times \mathcal{T}$ são $(1,1),(2,2),(3,3),(4,4),(5,5),(2,4),(4,2),(3,5)$ e $(5,3)$. Todos são especiais.

$\mathrm{O}$ estado $(2,4)$ pertence ao passeio fechado

$$
(2,4) \stackrel{\sigma /\left(a, a^{2}\right)}{\longrightarrow}(3,5) \stackrel{\sigma /\left(a, a^{2}\right)}{\longrightarrow}(2,4) .
$$

A saída desse passeio é $\left(a^{2}, a^{4}\right)$, e, para toda diferença $(u, v)$ em $\Delta_{24}$,

$$
(u, v) \cdot\left(a^{2}, a^{4}\right) \neq(u, v) .
$$

Portanto, $(2,4)$ não é limitado, e $f$ não é subseqüencial.

Exemplo 4.5.5 A função $f: \Sigma^{+} \rightarrow \Gamma^{*}$ definida por

$$
\sigma^{n} f= \begin{cases}b a^{n} & \text { se } n \text { for par } \\ a^{n} & \text { se } n \text { for ímpar }\end{cases}
$$

é o comportamento do transdutor letra-palavra

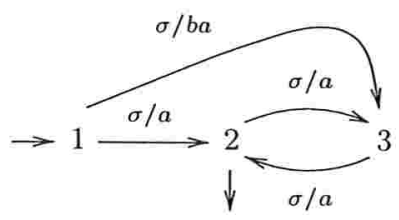

A parte acessível do quadrado desse transdutor é

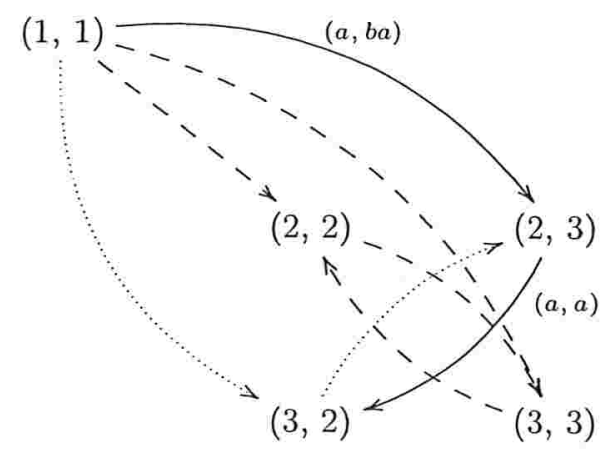

A entrada de todas as transições é igual a $\sigma$. As transições pontilhadas não são visitadas pelo Algoritmo 4.5.1. Todos os estados acessíveis são especiais.

$\mathrm{O}$ estado $(2,3)$ pertence ao passeio fechado

$$
(2,3) \stackrel{\sigma /(a, a)}{\longrightarrow}(3,2) \stackrel{\sigma /(a, a)}{\longrightarrow}(2,3) .
$$

A saída desse passeio é $\left(a^{2}, a^{2}\right)$. Mas, $(a, b a)$ pertence a $\Delta_{23}$, e

$$
(a, b a) \cdot\left(a^{2}, a^{2}\right) \neq(a, b a) .
$$

Logo, $(2,3)$ não é limitado. Portanto, $f$ não é uma função subseqüencial.

Exemplo 4.5.6 Considere o transdutor 


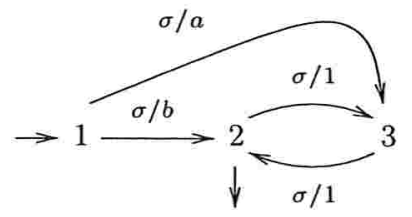

A parte acessível do quadrado desse transdutor é

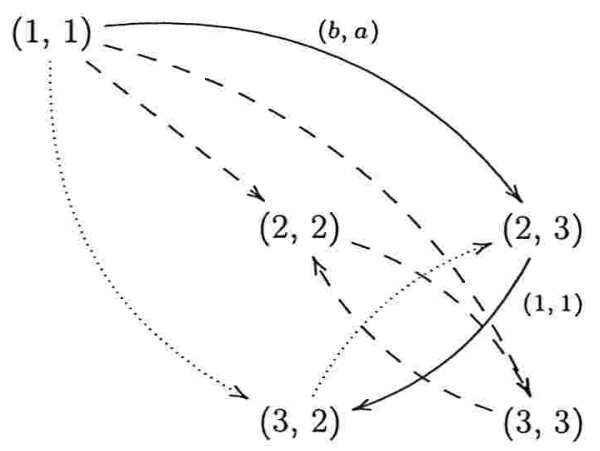

Nenhum estado é especial. Portanto, o comportamento desse transdutor é uma função subseqüencial.

\subsection{Determinização de um transdutor}

Nesta seção, apresentamos um algoritmo de determinização de transdutores funcionais.

Determinizar um transdutor funcional significa construir um transdutor subseqüencial equivalente. Portanto, nem todo transdutor pode ser determinizado. O algoritmo recebe como entrada um transdutor funcional letra-palavra, não necessariamente subseqüencial, mas que realiza uma função subseqüencial, e devolve um transdutor subseqüencial equivalente.

Esse algoritmo pode ser visto como uma extensão da construção dos subconjuntos para a determinização de autômatos finitos, e depende explicitamente do Teorema 4.5.1. O algoritmo está descrito apenas em algumas referências recentes [RS97, BC00b, Moh95].

Na construção dos subconjuntos, cada estado do autômato determinístico construído é um conjunto de estados acessíveis com uma determinada entrada no autômato original. No caso dos transdutores, o processo é semelhante, mas considera também as saídas. Informalmente, cada estado do transdutor subseqüencial construído representa um conjunto de estados acessíveis com uma determinada entrada no trandutor original, e também uma informação sobre a saída que ainda não foi emitida para cada estado nesse subconjunto.

Inicialmente, vamos descrever formalmente um transdutor subseqüencial equivalente a um $\Sigma$ $\Gamma$-transdutor letra-palavra $\mathcal{T}=(Q, E, I, T)$ que realiza uma função subseqüencial. Em seguida, vamos discutir o algoritmo, que realiza a construção da parte acessível desse transdutor.

Começamos com algumas notações. Seja

$$
R=2^{Q \times \Gamma^{*}} .
$$

Dados um conjunto $X$ em $R$ e uma palavra $u$ em $\Sigma^{*}$, denotamos por $P(X, u)$ o conjunto dos passeios em $\mathcal{T}$ com origem em um estado pertencente a algum par em $X$ e entrada $u$. Se $P(X, u) \neq \varnothing$, 
definimos

$$
\hat{P}(X, u)=\wedge\left(\bigcup_{\substack{(p, x) \in X \\ u / y \\ p \longrightarrow p(X, u)}} x y\right) .
$$

Vamos usar uma ação

$$
R \circ \Sigma^{*} \rightarrow R
$$

definida como segue. Para todo conjunto $X$ em $R$ e toda letra $\sigma$ em $\Sigma$,

$$
X \circ \sigma=\bigcup_{\substack{(p, x) \in X \\ \sigma / y \\ p \stackrel{-}{q} \in P(X, \sigma)}}\left(q, \hat{P}(X, \sigma)^{-1}(x y)\right) .
$$

Vamos demonstrar uma propriedade da extensão de o. Para todo conjunto $X$ em $R$ e toda palavra $u$ em $\Sigma^{+}$,

$$
X \circ u=\bigcup_{\substack{(p, x) \in X \\ u / y \\ p \stackrel{q}{\longrightarrow} \in P(X, u)}}\left(q, \hat{P}(X, u)^{-1}(x y)\right) .
$$

Vamos usar indução em $|u|$ :

- $|u|=1$ : este caso corresponde à definição de 0 .

- $|u|>1$ : fatore $u$ como $u^{\prime} \sigma$, onde $\sigma$ é uma letra. Seja $Y=X \circ u^{\prime}$.

Primeiro, vamos provar que

$$
\hat{P}\left(X, u^{\prime}\right) \hat{P}(Y, \sigma)=\hat{P}(X, u) .
$$

Começamos estudando $\hat{P}(Y, \sigma)$ como segue:

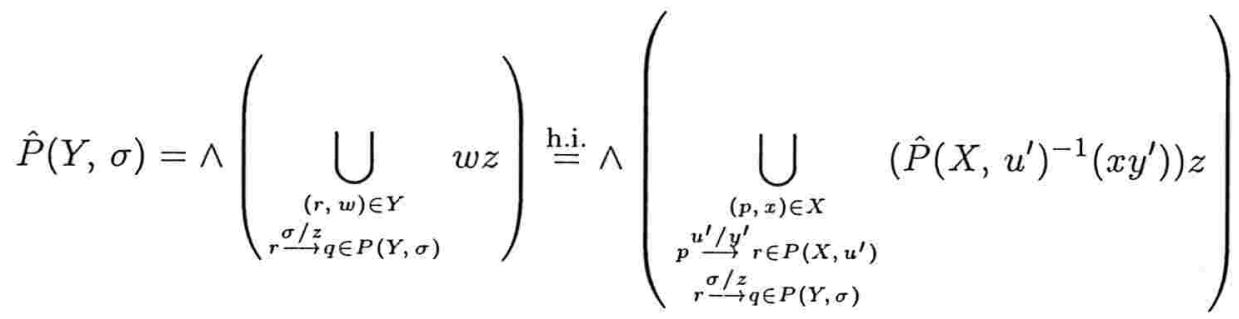

$$
\begin{aligned}
& =\wedge\left(\bigcup_{\substack{(p, x) \in X \\
u / y \\
p \stackrel{y}{\longrightarrow} q \in P(X, u)}} \hat{P}\left(X, u^{\prime}\right)^{-1}(x y)\right) .
\end{aligned}
$$

Para completar a prova, vamos precisar da seguinte propriedade. Dados um subconjunto não-vazio $S$ de $\Gamma^{*}$ e uma palavra $s$ em $\Gamma^{*}$,

$$
s(\wedge S)=\wedge(s S)
$$


Essa propriedade será demonstrada na Seção 4.7, em um contexto mais geral (Proposição 4.7.3). Dessa propriedade, segue que, concatenando $\hat{P}\left(X, u^{\prime}\right)$ à esquerda do lado direito da última igualdade de (4.6.5), obtemos $\hat{P}(X, u)$, o que conclui a demonstração de (4.6.4).

Em seguida, estudamos $X$ o $u$ como segue:

(4.6.6) $X \circ u=\left(X \circ u^{\prime}\right) \circ \sigma \stackrel{\text { h.i. }}{=}\left(\bigcup_{\substack{(p, x) \in X \\ u^{\prime} / \Psi^{\prime} \\ q \in P\left(X, u^{\prime}\right) \\(4.6 .2)}}\left(q, \hat{P}\left(X, u^{\prime}\right)^{-1}\left(x y^{\prime}\right)\right)\right) \circ \sigma$

A propriedade

$$
\hat{P}(Y, \sigma)^{-1}\left(\left(\hat{P}\left(X, u^{\prime}\right)^{-1}\left(x y^{\prime}\right)\right) z\right)=\left(\hat{P}\left(X, u^{\prime}\right) \hat{P}(Y, \sigma)\right)^{-1}\left(x y^{\prime} z\right)
$$

é de fácil verificação, e também está enunciada em (4.7.4a) (Seção 4.7) em um contexto mais geral. Utilizando essa propriedade, podemos aplicar (4.6.4) em (4.6.6), o que resulta em (4.6.3).

Teorema 4.6.1 Dado um $\Sigma$ - $\Gamma$-transdutor funcional letra-palavra bi-acessível $\mathcal{T}=(Q, E, I, T)$ que realiza uma função subseqüencial, um $\Sigma$-Г-transdutor subseqüencial equivalente pode ser construído efetivamente.

Demonstração. Sejam $M$ o máximo dentre os comprimentos das saídas das transições de $\mathcal{T}$, $n=\operatorname{card}(Q)$ e $l=M\left(n^{2}-n\right)$. Vamos definir um transdutor subseqüencial $\mathcal{T}^{\text {det }}=\left(Q^{\prime}, i, \lambda, \rho\right)$, e mostrar que ele é equivalente a $\mathcal{T}$.

O conjunto de estados, o estado inicial e a emissão inicial de $\mathcal{T}^{\text {det }}$ são

$$
\begin{aligned}
& Q^{\prime}=2^{(Q \times \Gamma \leq l)} \\
& i=I \times 1 \\
& i \lambda=1
\end{aligned}
$$

Um estado $X$ de $\mathcal{T}^{\text {det }}$ é final se, e somente se, existe algum par $(t, x)$ em $X$ tal que $t$ é um estado final de $\mathcal{T}$ e, para todo par $\left(t^{\prime}, x^{\prime}\right)$ em $X$ tal que $t^{\prime}$ é um estado final, $x=x^{\prime}$. Neste caso, definimos

$$
X \rho=x .
$$

Para toda letra $\sigma$, uma transição $X \cdot \sigma$ está definida se, e somente se, $P(X, \sigma) \neq \varnothing$ e, para todo $\operatorname{par}(q, x)$ em $X \circ \sigma,|x| \leq l$. Nesse caso, definimos

$$
\begin{aligned}
& X \cdot \sigma=X \circ \sigma \\
& X * \sigma=\hat{P}(X, \sigma) .
\end{aligned}
$$


Antes de provar que $\left|\mathcal{T}^{\text {det }}\right|=|\mathcal{T}|$, vamos estudar algumas propriedades de $\mathcal{T}^{\text {det }}$. Dada uma palavra $u$ em $\Sigma^{+}$,

$$
\begin{aligned}
& X * u=\hat{P}(X, u) \text {; } \\
& \text { para todo passeio } p \stackrel{u / x}{\longrightarrow} q \text { em } P(i, u),\left|\hat{P}(i, u)^{-1} x\right| \leq l \text {; } \\
& i \cdot u=i \circ u=\bigcup\left(q, \hat{P}(i, u)^{-1} x\right) \text {; } \\
& p \stackrel{u / x}{\longrightarrow} q \in P(i, u) \\
& \text { se }(t, x) \text { e }\left(t^{\prime}, x^{\prime}\right) \in i \cdot u \operatorname{com} t, t^{\prime} \text { em } T \text {, então } x=x^{\prime}
\end{aligned}
$$

Vamos provar (4.6.10a) por indução em $|u|$ :

- $|u|=1$ : este caso corresponde à definição de *.

- $|u|>1$ : fatore $u$ como $u^{\prime} \sigma$, onde $\sigma$ é uma letra. Seja $Y=X \cdot u^{\prime}$. Por (4.2.2), $X * u=\left(X * u^{\prime}\right)(Y *$ $\sigma)$. Pela hipótese de indução, $X * u^{\prime}=\hat{P}\left(X, u^{\prime}\right)$, e pela definição de $*,(Y * \sigma)=\hat{P}(Y, \sigma)$. Utilizando (4.6.4), obtemos $X * u=\hat{P}(X, u)$.

Vamos provar (4.6.10b). Seja $p \stackrel{u / x}{\longrightarrow} q$ um passeio em $P(i, u)$. Pela definição de $i$ em (4.6.7b), $\hat{P}(i, u)$ é o prefixo do conjunto

$$
A=\bigcup_{p \stackrel{u / z}{\longrightarrow} q \in P(i, u)} z .
$$

Pela definição de $\wedge$, deve haver uma palavra $y$ em $A$ tal que $\hat{P}(i, u)=x \wedge y$. Pela definição de $A$, existe um passeio da forma $p^{\prime} \stackrel{u / y}{\longrightarrow} q^{\prime}$ em $P(i, u)$. Assim, $\left(p, p^{\prime}\right) \stackrel{u /(x, y)}{\longrightarrow}\left(q, q^{\prime}\right)$ é um passeio em $\mathcal{T} \times \mathcal{T}$. Como $p$ e $p^{\prime}$ são estados iniciais, $\left(\hat{P}(i, u)^{-1} x, \hat{P}(i, u)^{-1} y\right)$ é uma diferença em $\Delta_{q q^{\prime}}$. Pelo Teorema 4.5.1, temos então que $\left|\hat{P}(i, u)^{-1} x\right| \leq l$.

Antes de provar (4.6.10c), observamos que essa propriedade significa que $i \cdot u \neq \varnothing$ sempre que houver pelo menos um passeio em $\mathcal{T}$ com origem em um estado inicial e entrada $u$. Para prová-la, vamos utilizar indução em $|u|$. O caso $u=1$ é evidente, e vamos considerar que $|u|>0$.

- $|u|=1:$ por (4.6.10b), para toda transição $p \stackrel{u / x}{\longrightarrow} q$ em $\mathcal{T}$ tal que $p$ seja um estado inicial, $\left|\hat{P}(i, u)^{-1} x\right| \leq l$. Assim, pela definição de $\cdot$ e do estado $i, i \cdot u=i \circ u$.

- $|u|>1$ : fatore $u$ como $u^{\prime} \sigma$, onde $\sigma$ é uma letra. Suponha que exista um passeio $p \stackrel{u / x}{\longrightarrow} q$ em $\mathcal{T}$ tal que $p$ seja um estado inicial. Pela hipótese de indução, $i \cdot u^{\prime}=i \circ u^{\prime} \neq \varnothing$. Por (4.6.3)

$$
\left(i \circ u^{\prime}\right) \circ \sigma=i \circ u=\bigcup_{p \stackrel{u / x}{\longrightarrow} q \in P(i, u)}\left(q, \hat{P}(i, u)^{-1} x\right) .
$$

Por (4.6.10b), para todo par $(q, x)$ em $\left(i \circ u^{\prime}\right) \circ \sigma,|x| \leq l$. Pela definição de $\cdot$, concluímos que $\left(i \cdot u^{\prime}\right) \cdot \sigma$ está definido, e é igual a $i \circ u$.

Para provar (4.6.10d), considere passeios bem-sucedidos $p \stackrel{u / y}{\longrightarrow} t$ e $p^{\prime} \stackrel{u / y^{\prime}}{\longrightarrow} t^{\prime}$ em $P(i, u)$ tais que $x=\hat{P}(i, u)^{-1} y$ e $x^{\prime}=\hat{P}(i, u)^{-1} y^{\prime}$. Como $\mathcal{T}$ é funcional, $x=x^{\prime}$. 
Vamos agora provar que $|\mathcal{T}|=\left|\mathcal{T}^{\text {det }}\right|$.

Por construção, para toda palavra $u$ em $\Sigma^{*}$, se existir um par $(t, x)$ em $i \cdot u$ tal que $t$ é um estado final, então existe um passeio bem-sucedido $p \stackrel{u / v}{\longrightarrow} t$ em $\mathcal{T}$. Pela definição dos estados finais de $\mathcal{T}^{\text {det }}$, temos então que $\operatorname{dom}\left|\mathcal{T}^{\text {det }}\right| \subseteq \operatorname{dom}|\mathcal{T}|$.

Por (4.6.10c), para cada passeio bem-sucedido $p \stackrel{u / v}{\longrightarrow} t$ em $\mathcal{T}$, existe um par $\left(t, \hat{P}(i, u)^{-1} v\right)$ em $i \cdot u$. Por (4.6.10d) e pela definição dos estados finais de $\mathcal{T}^{\text {det }}, i \cdot u$ é um estado final. Portanto, $\operatorname{dom}|\mathcal{T}| \subseteq \operatorname{dom}\left|\mathcal{T}^{\text {det }}\right|$.

Ademais, a emissão final de $i \cdot u$ é $\hat{P}(i, u)^{-1} v$. Utilizando (4.6.10a) e a definição de $\lambda$, obtemos $u\left|\mathcal{T}^{\operatorname{det}}\right|=(i \lambda)(i * u)(i \cdot u) \rho=v=u|\mathcal{T}|$, o que conclui a demonstração.

A construção da parte acessível de $\mathcal{T}^{\text {det }}$ a partir de $\mathcal{T}$ é elementar. O algoritmo mantém uma lista $L$ de estados de $\mathcal{T}^{\text {det }}$, e executa uma seqüência de iterações. A lista começa com $i$. A cada iteração, o algoritmo retira um estado $X$ de $L$ e, para cada letra $\sigma \in \Sigma$ tal que $P(X, \sigma) \neq \varnothing$, contrói o conjunto $X \cdot \sigma$ e a saída $X * \sigma$. Isso é feito visitando-se, para cada par $(p, x)$ em $X$, as transições de $\mathcal{T}$ com origem em $p$ e entrada $\sigma$. Se $X \cdot \sigma \neq \varnothing$ e $X \cdot \sigma$ é um estado novo, o algoritmo insere $X \cdot \sigma$ em $L$. $\mathrm{O}$ algoritmo pára quando $L=\varnothing$.

O Algoritmo 4.6.1 realiza essa construção. Nesse algoritmo, $\mathcal{T}^{\text {det }}$ representa a parte acessível do transdutor descrito na demonstração do Teorema 4.6.1. Para simplificar a descrição, utilizamos um elemento 0 que não pertence a $\Sigma^{*}$, e estendemos a operação $\wedge$ para $\Sigma^{*} \cup 0$ como segue:

$$
x \wedge 0=0 \wedge x=x, \quad \forall x \in \Sigma^{*} \cup 0 .
$$

Para analizar as complexidade de tempo e espaço, utilizamos a seguinte observação sobre os estados acessíveis de $\mathcal{T}^{\text {det }}$. Sejam $u$ uma palavra em $\Sigma^{*}$, e $(q, x)$ e $\left(q^{\prime}, x^{\prime}\right)$ pares em $i \cdot u$. Se $q=q^{\prime}$, então $x=x^{\prime}$.

De fato, considere passeios $c: p \stackrel{u / y}{\longrightarrow} q$ e $c^{\prime}: p^{\prime} \stackrel{u / y^{\prime}}{\longrightarrow} q$ em $P(i, u)$ tais que $x=\hat{P}(i, u)^{-1} y$ e $x^{\prime}=\hat{P}(i, u)^{-1} y^{\prime}$. Como $\mathcal{T}$ é bi-acessível, existe um passeio $d: q \stackrel{v / w}{\rightarrow} t$ em $\mathcal{T}$, onde $t$ é um estado final. Então, $c d$ e $c^{\prime} d$ são passeios bem-sucedidos com entrada $u v$ e saídas $\hat{P}(i, u) x w$ e $\hat{P}(i, u) x^{\prime} w$, respectivamente. Como $\mathcal{T}$ é funcional, $x=x^{\prime}$.

Portanto, todo estado na parte acessível de $\mathcal{T}^{\text {det }}$ pode ser visto como uma função parcial de $Q$ em $\Gamma \leq l$. O número dessas funções é

$$
\left(\operatorname{card}\left(\Gamma^{\leq l}\right)+1\right)^{n}=\left(\frac{\operatorname{card}(\Gamma)^{l+1}-1}{\operatorname{card}(\Gamma)-1}+1\right)^{n} .
$$

Temos então que

$$
\operatorname{card}\left(Q^{\prime}\right)=O\left(\operatorname{card}(\Gamma)^{l n}\right) .
$$

Os rótulos das transições de $\mathcal{T}^{\text {det }}$ têm comprimento no máximo $l+M$. Ademais, há no máximo card $(\Sigma)$ transições com origem em cada estado desse transdutor. Um limitante para a complexidade de espaço do algoritmo é então

$$
O\left(\operatorname{card}(\Sigma) \operatorname{card}(\Gamma)^{l n} l\right)
$$

Para cada estado retirado de $L$, as seguintes operações são realizadas:

- construção de $Y: O(\ln )$ 
- teste de se $Y$ pertence a $Q^{\prime}: O\left(\operatorname{card}\left(Q^{\prime}\right) n l\right)$

Assim, um limitante para a complexidade de tempo é

$$
O\left(\operatorname{card}(\Sigma) \operatorname{card}(\Gamma)^{2 \ln } \ln \right)
$$

Afirmamos sem demonstração que existe um morfismo de autômatos (veja uma definição na Seção 4.12) do autômato de entrada de $\mathcal{T}^{\text {det }}$ no autômato determinístico $\mathcal{A}$ obtido com a construção dos subconjuntos para o autômato de entrada de $\mathcal{T}$. Portanto, o número de estados de $\mathcal{T}$ det é maior ou igual ao número de estados de $\mathcal{A}$.

Existem famílias de autômatos não-determinísticos cujo autômato determinístico minimal equivalente tem tamanho exponencial no número de estados $n$ do original. Utilizando essas famílias, podemos construir transdutores com a propriedade de que o tamanho de um transdutor subseqüencial equivalente é obrigatoriamente $\Omega\left(2^{n}\right)$.

Em (4.6.13), o tamanho do alfabeto $\Gamma$ aparece na expressão da complexidade de tempo devido à possibilidade de um mesmo subconjunto de estados aparecer mais de uma vez (como domínio de mais de uma função do conjunto de estados do transdutor original em $\Gamma^{\leq l}$ ) no conjunto de estados do transdutor subseqüencial.

Exemplo 4.6.1 Sejam $\Sigma=\{\sigma, \gamma\}$ e $\Gamma=\{\gamma\}$. Considere a função $f: \Sigma^{*} \rightarrow \Gamma^{*}$ definida por

$$
x f=\left\{\begin{array}{ll}
1 & \text { se } x \in\left(\sigma^{2}\right)^{*} \\
\gamma^{|x|_{\gamma}} & \text { se } x \in\left(\Sigma^{3}\right)^{*} \\
\varnothing & \text { caso contrário }
\end{array} \quad \forall x \in \Sigma^{*} .\right.
$$

Essa função é realizada pelo transdutor letra-palavra ilustrado a seguir:
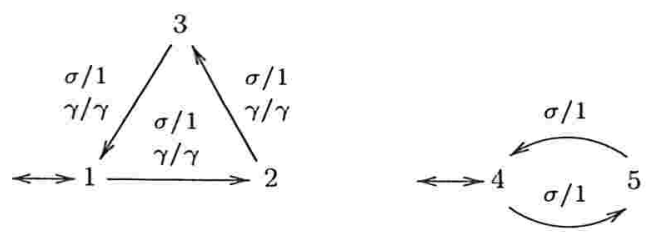

Note que este transdutor é ambíguo.

Aplicando a construção dos subconjuntos, obtemos o transdutor subseqüencial ilustrado abaixo. Como todos os pares nos subconjuntos têm a palavra vazia na segunda coordenada, indicamos apenas os números dos estados.

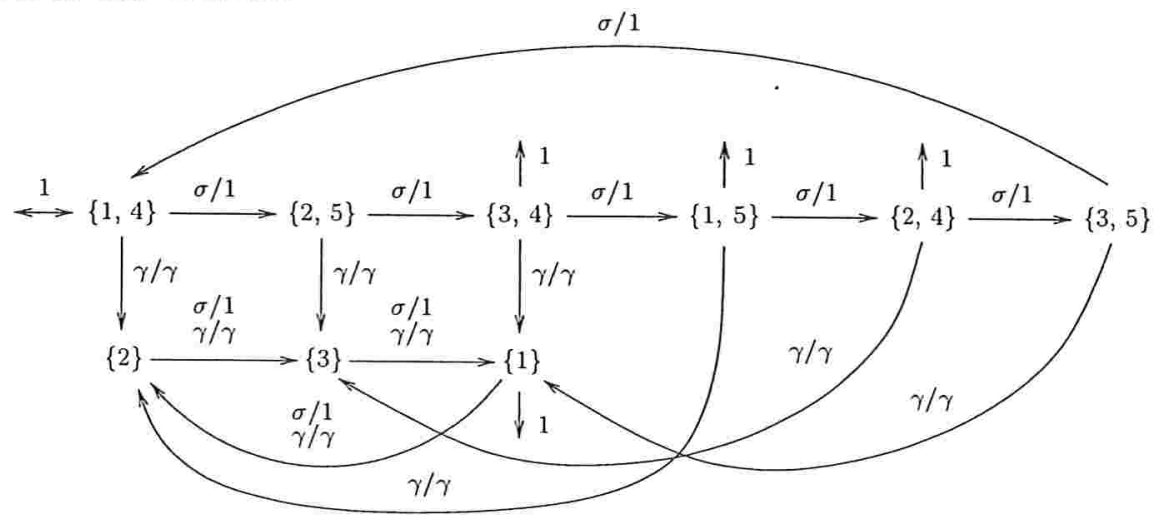




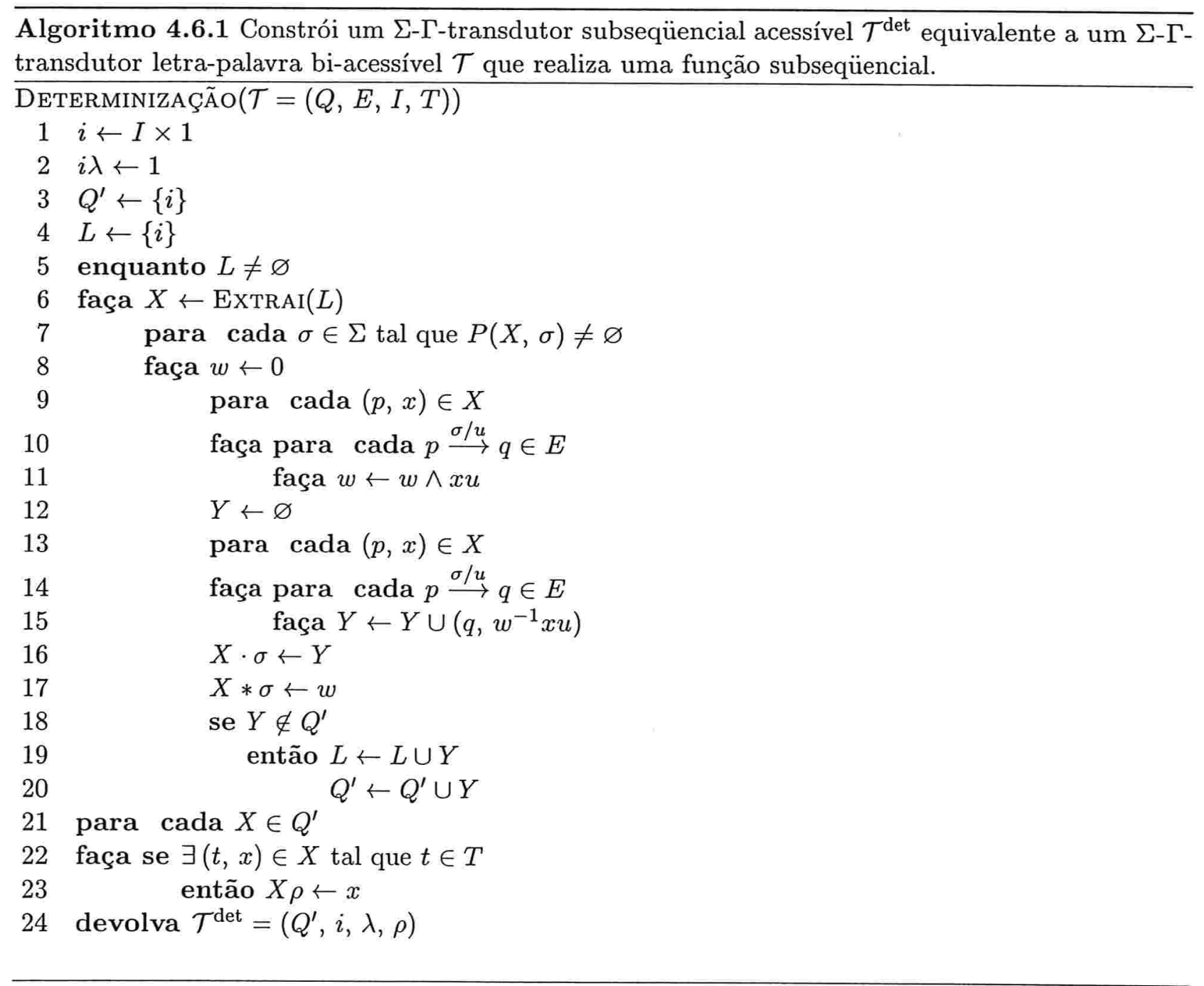


Exemplo 4.6.2 Sejam $\Sigma=\{\sigma, \gamma\}$ e $\Gamma=\{\sigma\}$. Considere o transdutor

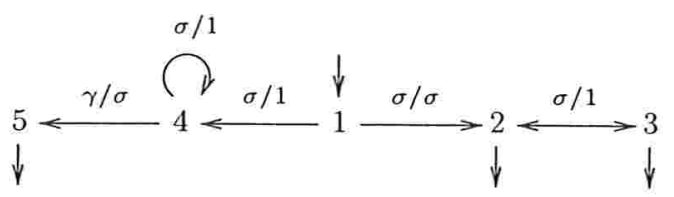

Esse transdutor é não-ambíguo, e realiza uma função subseqüencial. Aplicando a construção dos subconjuntos, obtemos o transdutor subseqüencial equivalente ilustrado a seguir. A palavra vazia é indicada por $\epsilon$.

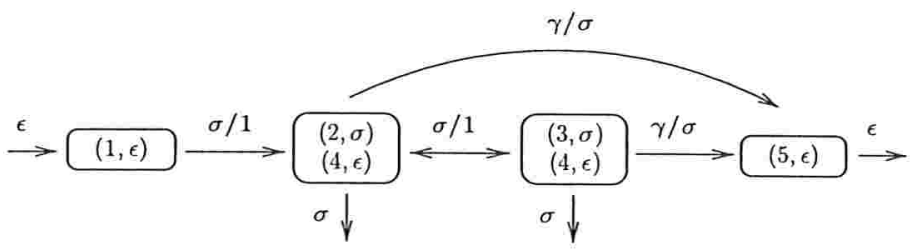

Exemplo 4.6.3 Considere o seguinte transdutor letra-palavra funcional:

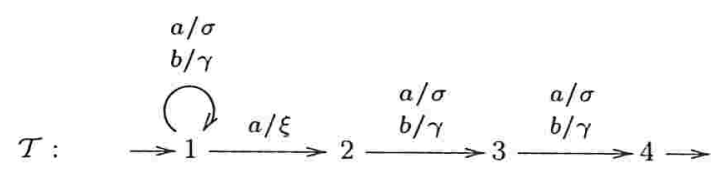

O transdutor subseqüencial equivalente construído pela construção dos subconjuntos está ilustrado abaixo. A palavra vazia é representada por $\epsilon$. 


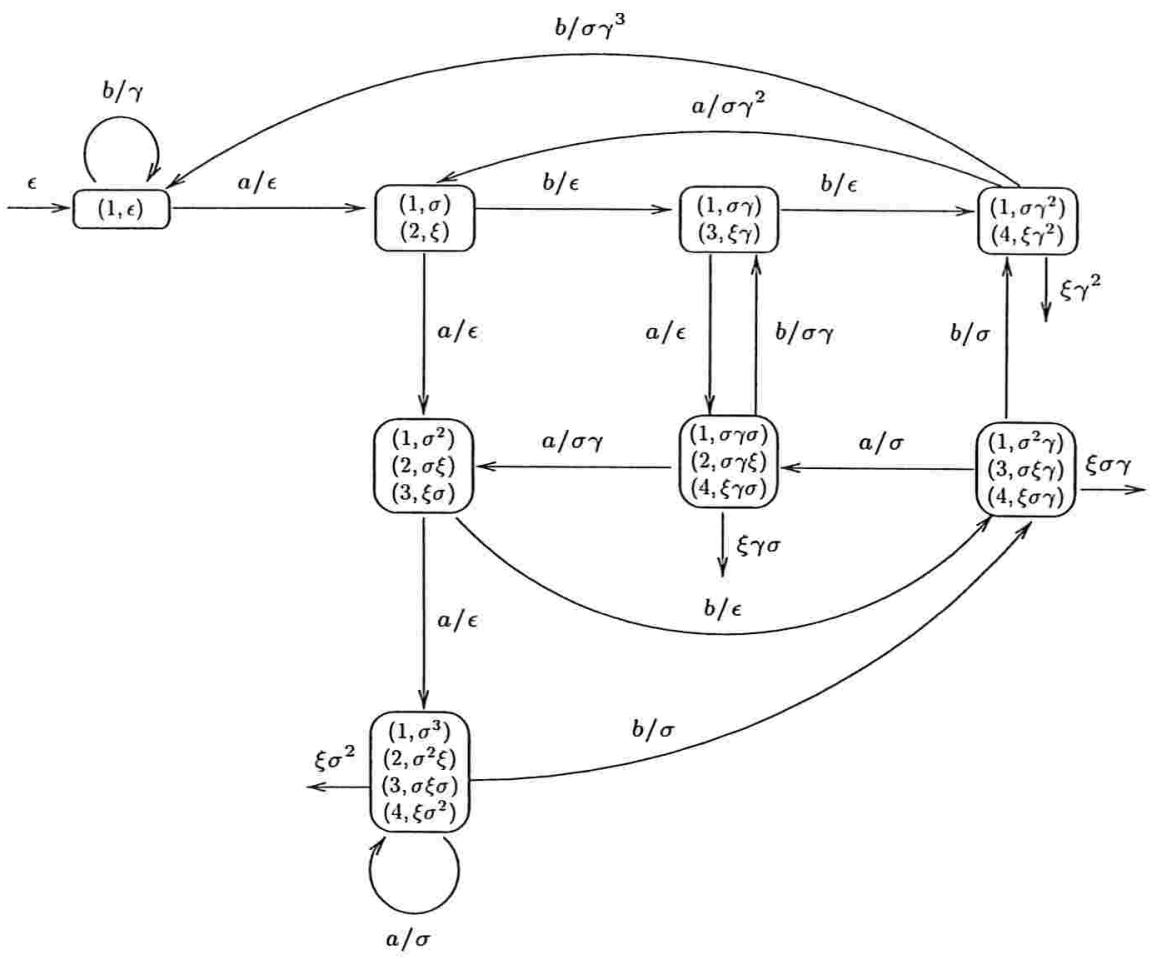

Cada estado desse transdutor pode ser visto como uma função de um subconjunto de $\{1,2,3,4\}$ em $\Gamma \leq l$. Todos esses subconjuntos contém 1. Ademais, cada um desses subconjuntos é o domínio de exatamente uma função.

Esse exemplo pode ser generalizado, associando-se a todo inteiro $n \geq 3$ o transdutor

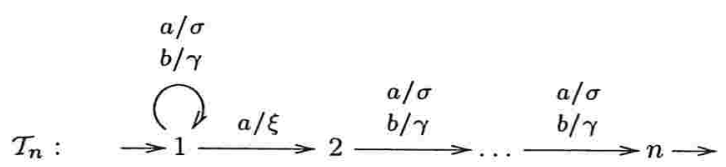

Observamos sem demonstração que, para todo $n$, o autômato de entrada do transdutor subseqüencial equivalente a $\mathcal{T}_{n}$ construído pelo algoritmo é isomorfo ao autômato determinístico minimal equivalente ao autômato de entrada de $\mathcal{T}_{n}$, e esse fato independe das saídas das transições de $\mathcal{T}_{n}$ (veja a Seção 4.12 para as definições de autômato minimal e isomorfismo de autômatos). Cada estado desse autômato minimal é um subconjunto de $[n]$ que contém 1 , e todos os subconjuntos que contém 1 são acessíveis e co-acessíveis. Portanto, o número de estados de $\mathcal{T}_{n}^{\text {det }}$ é $2^{n-1}$.

Exemplo 4.6.4 Modifique o exemplo anterior como segue:

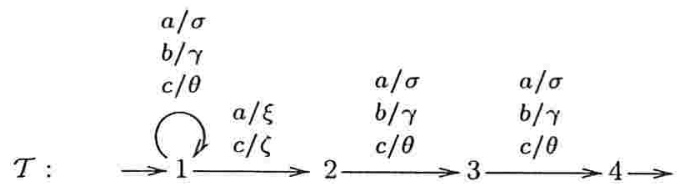

O transdutor subseqüencial equivalente construído pelo algoritmo está ilustrado na Figura 4.6.1. A palavra vazia é representada por $\epsilon$. Como no exemplo anterior, cada estado de $\mathcal{T}^{\text {det }}$ é uma função 
de um subconjunto de $\{1,2,3,4\}$ que contém $1 \mathrm{em} \Gamma^{\leq l}$. No entanto, um mesmo subconjunto pode ser o domínio de mais de uma função.

\subsection{Monóides com mdc}

Nesta seção, vamos definir e estudar algumas propriedades de um conceito de máximo divisor comum para monóides. A importância desse conceito é que ele abstrai algumas propriedades de monóides relevantes para a teoria da minimização de transdutores, que será desenvolvida nas próximas seções. As idéias presentes nesta seção também podem ser vistas em [Hef02].

Seja M um monóide. Dados elementos $a$ e $b$ em M, escrevemos

$$
a \mid b
$$

se existir um elemento $b^{\prime}$ em $M$ tal que

$$
b=a b^{\prime} .
$$

Nesse caso, dizemos que $a$ divide $b$, ou que $a$ é um divisor de $b$. A relação de divisibilidade é transitiva, reflexiva, mas não necessariamente anti-simétrica (considere o caso de um grupo).

Se $a$ não for um divisor de $b$, escrevemos $a \nmid b$.

Definição 4.7.1 Seja M um monóide. Dado um subconjunto não-vazio $S$ de $\mathrm{M}$, dizemos que um elemento $m$ em $\mathrm{M}$ é um máximo divisor comum, ou $\mathrm{mdc}$, de $S$, se

- $m \mid a$, para todo a em $S$, e

- para cada $n$ em $\mathrm{M}$, se $n \mid a$, para todo a em $S$, então $n \mid m$.

Definição 4.7.2 Dizemos que $\mathrm{M}$ é um monóide com $\mathrm{mdc}$ se todo subconjunto não-vazio de $\mathrm{M}$ tiver pelo menos um mdc.

Segue diretamente dessa definição que, dados subconjuntos $S$ de $\mathrm{M}$ e $X$ de $S$,

$$
\forall s \in \operatorname{mdc} S, \forall x \in \operatorname{mdc} X, s \mid x .
$$

O conjunto dos mdc's de um subconjunto $S$ será denotado por mdc $S$, e vamos convencionar que $\operatorname{mdc} \varnothing=\varnothing$.

É possível não existir nenhum mdc de um subconjunto não-vazio $S$, ou existir mais de um, conforme demonstrado nos exemplos a seguir.

Exemplo 4.7.1 Seja $\mathbb{Q}$ o monóide aditivo dos números racionais. A seguinte propriedade segue diretamente da definição de divisibilidade:

$$
a \mid b \text { se, e somente se, } a \leq b, \forall a, b \in \mathbb{Q} \text {. }
$$

Portanto, para todo subconjunto $S$ de $\mathbb{Q}$, um número racional $d$ é um mdc de $S$ se, e somente se,

- $d \leq a$, para todo $a$ em $S$; 


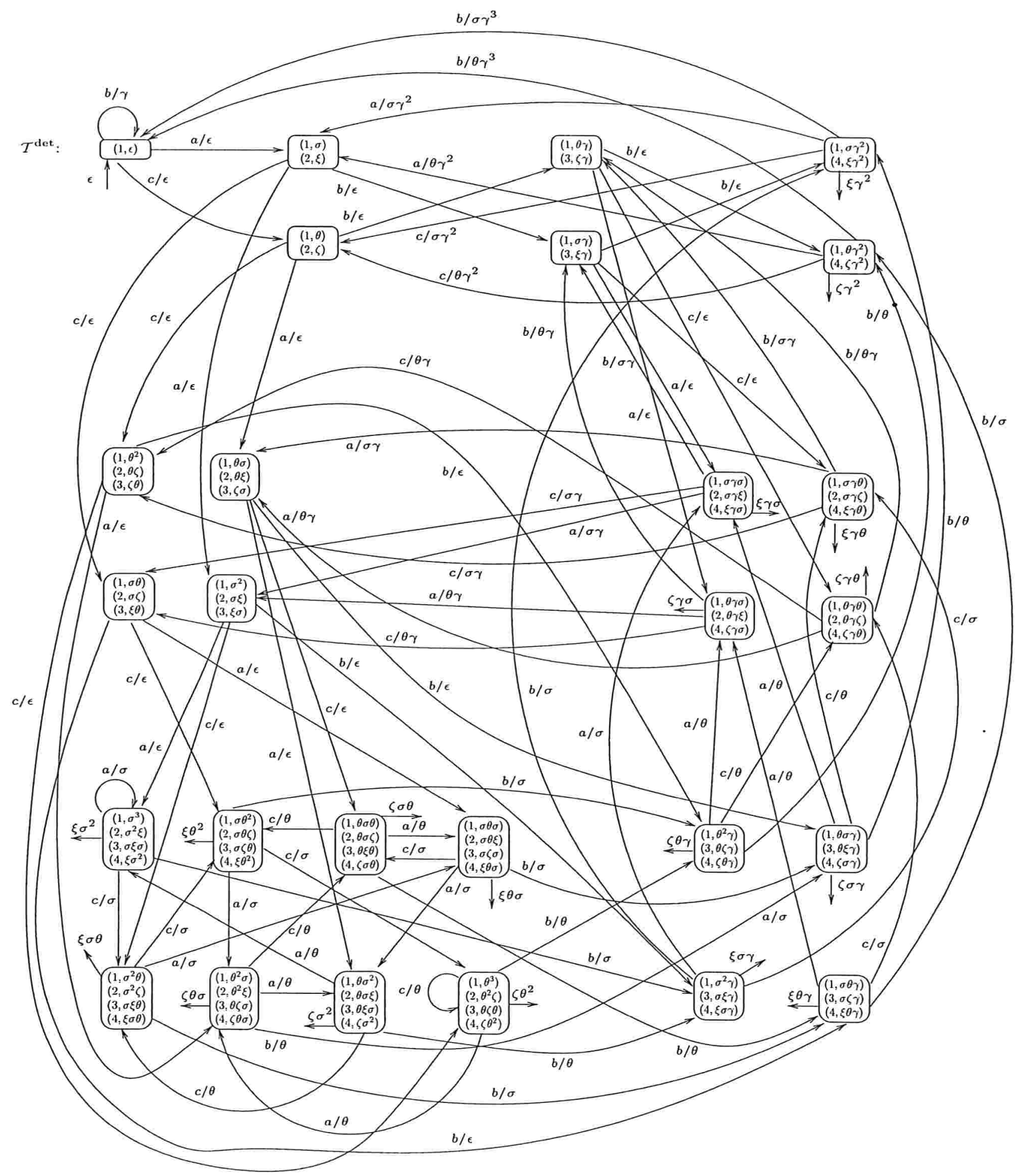

Figura 4.6.1: Transdutor subseqüencial resultante da construção dos subconjuntos para o transdutor do Exemplo 4.6.4. 
- se $d^{\prime} \leq a$, para todo $a$ em $S$, então $d^{\prime} \leq d$.

Ou seja, se existir um mdc de $S$, então esse mdc é único, e é igual ao ínfimo desse conjunto.

O conjunto

$$
S=\{a \in \mathbb{Q}: a>\sqrt{2}\}
$$

não tem ínfimo em $\mathbb{Q}$. Portanto, $\mathbb{Q}$ não é um monóide com mdc.

Exemplo 4.7.2 Seja $\mathrm{M}=(\{0,1\} \times \mathbb{N}) \cup 1$ o monóide definido como segue. A identidade de $\mathrm{M}$ é 1 , e sua operação é completamente definida por

$$
\left(i, i^{\prime}\right)\left(j, j^{\prime}\right)= \begin{cases}\left(i, i^{\prime}\right) & \text { se } i^{\prime} \geq j^{\prime} \\ \left(j, j^{\prime}\right) & \text { caso contrário, }\end{cases}
$$

para todo par de elementos $\left(i, i^{\prime}\right)$ e $\left(j, j^{\prime}\right)$ em $\{0,1\} \times \mathbb{N}$.

Segue diretamente dessa definição que o conjunto dos divisores de um elemento $\left(i, i^{\prime}\right)$ em M é

$$
\operatorname{div}\left\{\left(i, i^{\prime}\right)\right\}=\left(i, i^{\prime}\right) \cup\left\{\left(j, j^{\prime}\right): j^{\prime}<i^{\prime}\right\} .
$$

Ademais, nenhum elemento divide a identidade.

Seja

$$
S=\{(0,1),(1,1)\}
$$

O conjunto dos divisores desse conjunto é

$$
\operatorname{div} S=\{1,(0,0),(1,0)\} .
$$

As propriedades

$$
(0,0) \backslash 1, \quad(0,0) \chi(1,0) \text { e }(1,0) \chi(0,0)
$$

mostram que $S$ não tem mdc. Portanto, $M$ não é um monóide com mdc.

Exemplo 4.7.3 Seja $\mathrm{M}=2^{\mathbb{N}}$ o monóide dos subconjuntos de números naturais, cuja operação é obtida estendendo a operação de adição em $\mathbb{N}$ como segue:

$$
A B=\{a+b: a \in A, b \in B\}, \quad \forall A, B \in \mathrm{M} .
$$

Sejam $A_{1}=\{10,15,20,25,30\}, A_{2}=\{15,20,25,30\}$ e

$$
S=\left\{A_{1}, A_{2}\right\} \text {. }
$$

O conjunto $\operatorname{div} S$ dos elementos em $\mathrm{M}$ que dividem $A_{1}$ e $A_{2}$ é

$$
D_{0}=\{0\}, D_{1}=\{5\}, D_{2}=\{0,5\}, D_{3}=\{10\}, D_{4}=\{0,10\}, D_{5}=\{5,10\} \text { e } D_{6}=\{0,5,10\} .
$$

Verifica-se facilmente que, para todo $D_{i}$ em $\operatorname{div} S$, existe outro conjunto $D_{j}$ em $\operatorname{div} S$ tal que $D_{j}$ não divide $D_{i}$. Portanto, $S$ não tem nenhum mdc, e M não é um monóide com mdc.

Exemplo 4.7.4 Alguns monóides com mdc estão listados a seguir. 
1. Todos os grupos são monóides com mdc. Ademais, todo elemento de um grupo G é um mdc de qualquer subconjunto não-vazio de G;

2. Monóides livres;

3. Monóides livres comutativos;

4. O monóide $(\mathbb{Z}, \cdot)$ dos inteiros positivos, com a operação de produto;

5. O monóide $\left(\mathbb{R}_{+},+\right)$dos números reais não-negativos, com a operação de adição;

6. O monóide trivial $\{1\}$;

7. Dado um conjunto não-vazio $X$, o monóide das funções parciais injetoras $X \rightarrow X$ com a operação de composição de funções é um monóide com mdc ;

8. Dado um monóide com mdc $(\mathrm{M}, \cdot)$, o monóide $\mathrm{M}^{m \times n}$ das matrizes sobre $\mathrm{M}$ de dimensão $m \times n$ e com a operação - aplicada em cada entrada é um monóide com mdc .

Vamos mostrar que o monóide descrito em 7 é um monóide com mdc. Vamos chamar esse monóide de $\mathrm{M}$, e a função identidade $X \rightarrow X$ de $1_{X}$. Vamos denotar a operação de composição de funções por $\cdot$.

Sejam $S$ um conjunto não-vazio de funções parciais injetoras $X \rightarrow X$, e

$$
m=\left.1_{X}\right|_{f \in S} \operatorname{dom} f .
$$

Então, para toda função $f$ em $S$,

$$
m \cdot f=f .
$$

Portanto, $m$ divide todas as funções de $S$. Seja agora $m^{\prime}$ uma função que divide todas as funções de $S$. Defina

$$
n=m^{\prime-1} \cdot m
$$

Como $m^{\prime}$ é injetora, $m^{\prime-1}$ também é uma função, e $n$ é uma função injetora. Verifica-se facilmente que

$$
m=m^{\prime} \cdot n,
$$

ou seja, $m^{\prime} \mid m$. Portanto, $m \in \operatorname{mdc} S$.

Enfatizamos o fato de que, no caso dos monóides livres, o mdc de um conjunto é o seu prefixo comum de maior comprimento, e no caso dos números reais, é o ínfimo desse conjunto.

Vamos estar particularmente interessados no caso em que $\mathrm{M}$ é um monóide com mdc e com a propriedade de o mdc de todo subconjunto não-vazio de $\mathrm{M}$ ser único. Dizemos nesse caso que $\mathrm{M}$ é um monóide com mdc único. No Exemplo 4.7.4, os monóides listados nos itens 2 a 6 são monóides com mdc único. Os monóides 1, 7 e 8 não são necessariamente monóides com mdc único.

Uma consequiência dessa hipótese é que nenhum elemento de $M$ diferente de 1 é inversível. De fato, se $a$ for um elemento inversível, então 1 e $a$ são mdc's de $\{a\}$. Assim, a relação de divisibilidade induz uma ordem parcial em $\mathrm{M}$.

A seguir, vamos demonstrar algumas propriedades do conceito de mdc. Precisamos primeiramente de duas definições. 
Definição 4.7.3 Dizemos que dois elementos a e b de um monóide $\mathrm{M}$ são associados se existir um elemento inversivel $u$ em $\mathrm{M}$ tal que $a=b u$.

A relação ser associado é uma relação de equivalência em M.

Definição 4.7.4 Dizemos que um monóide $\mathrm{M}$ é cancelativo se toda igualdade $u x=u y$ ou $x u=y u$, onde $u, x$ e y são elementos de $\mathrm{M}$, implicar em $x=y$.

No Exemplo 4.7.4, os monóides de 1 a 6 são cancelativos. Os monóides 7 e 8 não são necessariamente cancelativos.

Observe que nenhum monóide cancelativo tem um zero, ou seja, um elemento 0 cuja multiplicação à direita ou à esquerda com qualquer elemento do monóide resulta em 0 . Também é verdade que, em um monóide cancelativo, nenhum elemento diferente de 1 é idempotente.

Proposição 4.7.1 Sejam M um monóide cancelativo, e $S$ um subconjunto não-vazio de M.

Se a e $a^{\prime}$ forem mdc's distintos de $S$, então esses elementos são associados.

Se $m$ for um mdc de $S$, então todo elemento associado a $m$ é um mdc de $S$.

Demonstração. Vamos mostrar a primeira afirmação. Pela definição de mdc, $a \mid a^{\prime}$ e $a^{\prime} \mid a$. Assim, podemos fazer as fatorações $a^{\prime}=a b$ e $a=a^{\prime} b^{\prime}$. Então, $a^{\prime}=a^{\prime} b^{\prime} b$ e $a=a b b^{\prime}$, e, pelo cancelamento, obtemos $b^{\prime} b=b b^{\prime}=1$. Portanto, $b$ é inversível, e $a$ e $a^{\prime}$ são associados.

Vamos provar a segunda afirmação. Seja $m b$ um elemento associado a $m$, onde $b$ é um elemento inversível de M. Dado um elemento $a$ em $S$, podemos fatorar $a=m a^{\prime}=m b\left(b^{-1} a^{\prime}\right)$. Portanto, $m b \mid a$. Como todos os elementos de $\mathrm{M}$ que dividem os elementos de $S$ devem dividir $m$, e $m \mid m b$, temos que esses elementos também dividem $m b$. Concluímos então que $m b$ é um mdc de $S$.

Proposição 4.7.2 Sejam M um monóide cancelativo e com mdc, $S$ um subconjunto não-vazio de $\mathrm{M}$, e d um elemento de $\mathrm{M}$. Se $d^{\prime}$ for um $\mathrm{mdc}$ de $S$, então $d d^{\prime}$ é um mdc de $d S$.

Demonstração. Seja $m$ um mdc de $d S$. Como $d^{\prime}$ divide todos os elementos de $S$, temos que $d d^{\prime}$ divide todos os elementos de $d S$. Portanto, $d d^{\prime} \mid m$, e para algum $m^{\prime}$ em M,

$$
m=d d^{\prime} m^{\prime} \text {. }
$$

Seja $s$ um elemento qualquer de $S$. Como $d s$ pertence a $d S$, temos que $m \mid d s$. Portanto, $d d^{\prime} m^{\prime} \mid d s$, e, pelo cancelamento, obtemos

$$
d^{\prime} m^{\prime} \mid s .
$$

Como $s$ é um elemento arbitrário de $S$, e $d^{\prime}$ é um mdc de $S$, temos que $d^{\prime} m^{\prime} \mid d^{\prime}$. Logo, para algum $m^{\prime \prime}$ em $M$,

$$
d^{\prime}=d^{\prime} m^{\prime} m^{\prime \prime} .
$$

Multiplicando ambos os lados por $m^{\prime}$, obtemos também

$$
\left(d^{\prime} m^{\prime}\right)=\left(d^{\prime} m^{\prime}\right) m^{\prime \prime} m^{\prime} .
$$

Pelo cancelamento, obtemos $m^{\prime} m^{\prime \prime}=m^{\prime \prime} m^{\prime}=1$. Assim, $m^{\prime}$ é inversível, e, por (4.7.2), temos que $d d^{\prime}$ e $m$ são associados. Segue então da Proposição 4.7 .1 que $d d^{\prime}$ é um mdc de $d S$. 
Proposição 4.7.3 Sejam M um monóide cancelativo e com mdc, $s$ um elemento de $\mathrm{M}$, e $S$ um subconjunto não-vazio de M. Então,

$$
\operatorname{mdc}(s S)=s(\operatorname{mdc} S)
$$

Demonstração. Seja $x$ um mdc de $s S$.

Seja $m$ um mdc de $S$. Como $s m$ divide todos os elementos de $s S, s m$ deve dividir $x$. Assim, $x=s m x^{\prime}$ para algum $x^{\prime}$ em M. Como $x$ divide todos os elementos de $s S$, segue do cancelamento que $m x^{\prime}$ deve dividir todos os elementos de $S$. Assim, $m x^{\prime}$ deve dividir $m$. Logo, $m=m x^{\prime} x^{\prime \prime}$ para algum $x^{\prime \prime}$ em M. Pelo cancelamento, obtemos $x^{\prime} x^{\prime \prime}=1$. Multiplicando ambos os lados por $x^{\prime}$, obtemos $x^{\prime} x^{\prime \prime} x^{\prime}=x^{\prime}$, e do cancelamento segue que $x^{\prime \prime} x^{\prime}=1$. Portanto, $x^{\prime}$ é inversível, e $m$ e $m x^{\prime}$ são associados. Pela Proposição 4.7.1, temos que $m x^{\prime}$ é um mdc de $S$. Assim, $x=s m x^{\prime} \in s(\operatorname{mdc} S)$. Obtemos então que

$$
\operatorname{mdc}(s S) \subseteq s(\operatorname{mdc} S)
$$

A inclusão

$$
s(\operatorname{mdc} S) \subseteq \operatorname{mdc}(s S)
$$

é uma conseqüência da Proposição 4.7.2.

Proposição 4.7.4 Sejam M um monóide com mdc, $S$ um subconjunto não-vazio de $\mathrm{M}$, e $\mathcal{P}$ uma família de subconjuntos não-vazios de $S$ tal que $S=\bigcup_{X \in \mathcal{P}} X$. Então,

$$
\operatorname{mdc} S=\operatorname{mdc}\left(\bigcup_{X \in \mathcal{P}} \operatorname{mdc} X\right) \text {. }
$$

Demonstração. Seja $d$ um mdc de $S$. Então, $d$ divide todos os elementos de todos os conjuntos em $\mathcal{P}$. Portanto, $d$ divide os mdc's desses conjuntos, ou seja, divide todos os elementos em $\bigcup_{X \in \mathcal{P}} \operatorname{mdc} X$.

Seja $d^{\prime}$ um elemento que divide todos os elementos em $\bigcup_{X \in \mathcal{P}} \operatorname{mdc} X$. Então, para cada $X$ em $\mathcal{P}, d^{\prime}$ divide todos os elementos em $X$. Como $S=\bigcup_{X \in \mathcal{P}} X$, temos que $d^{\prime}$ divide todos os elementos de $S$. Como $d$ é um mdc de $S$, temos que $d^{\prime}$ divide $d$. Portanto, $d$ é um mdc de $\bigcup_{X \in \mathcal{P}} \operatorname{mdc} X$. Assim,

$$
\operatorname{mdc} S \subseteq \operatorname{mdc}\left(\bigcup_{X \in \mathcal{P}} \operatorname{mdc} X\right) .
$$

Seja agora $d$ um mdc de $\bigcup_{X \in \mathcal{P}} \operatorname{mdc} X$. Como $d$ divide os mdc's de todos os subconjuntos em $\mathcal{P}$, temos que divide todos os elementos nesses subconjuntos. Como $S=\bigcup_{X \in \mathcal{P}} X$, temos então que $d$ divide todos os elementos em $S$.

Seja $d^{\prime}$ um elemento que divide todos os elementos em $S$. Então, $d^{\prime}$ divide todos os elementos em $X$, para todo $X$ em $\mathcal{P}$. Assim, $d^{\prime}$ divide todos os elementos em $\bigcup_{X \in \mathcal{P}} \operatorname{mdc} X$. Como $d$ é um mdc de $\bigcup_{X \in \mathcal{P}} \operatorname{mdc} X$, temos então que $d^{\prime}$ divide $d$. Portanto, $d$ é um mdc de $S$. Assim,

$$
\operatorname{mdc}\left(\bigcup_{X \in \mathcal{P}} \operatorname{mdc} X\right) \subseteq \operatorname{mdc} S \text {. }
$$


Concluímos a seção estudando uma extensão da notação definida em (1.3.1) para monóides cancelativos.

Suponha que M seja um monóide cancelativo. Sejam $u$ e $v$ elementos de $\mathrm{M}$ tais que $u \mid v$. Então, existe um único elemento $v^{\prime}$ tal que $v=u v^{\prime}$. Definimos

$$
u^{-1} v=v^{\prime}
$$

Por conveniência, se $u$ não dividir $v$, definimos $u^{-1} v=\varnothing$.

As seguintes propriedades são de fácil verificação:

$$
\begin{aligned}
& (x y)^{-1} z=y^{-1} x^{-1} z . \\
& \left(x^{-1} y\right) z=x^{-1}(y z), \text { se } x \mid y . \\
& x^{-1}(x y)=y .
\end{aligned}
$$

Podemos estender (4.7.3) para conjuntos como segue. Dado um subconjunto $A$ de $\mathrm{M}$,

$$
u^{-1} A=\left\{u^{-1} x: x \in A\right\}
$$

Suponha agora que M seja um monóide cancelativo e com mdc único. Sejam $A$ um subconjunto não-vazio de $\mathrm{M}$ e $u$ um elemento tal que $u \mid x$ para todo $x \mathrm{em} A$. Afirmamos que

$$
\operatorname{mdc}\left(u^{-1} A\right)=u^{-1}(\operatorname{mdc} A) \text {. }
$$

De fato, como $u \mid x$ para todo $x$ em $A$,

$$
u\left(u^{-1} A\right)=A .
$$

Determinando o mdc em ambos os lados dessa igualdade e aplicando a Proposição 4.7.3 no lado esquerdo, obtemos

$$
u\left(\operatorname{mdc}\left(u^{-1} A\right)\right)=\operatorname{mdc} A .
$$

Aplicando a operação $u^{-1}$ em ambos os lados dessa igualdade, obtemos (4.7.6).

\subsection{Forma prefixo e morfismos de transdutores subseqüenciais}

Nossa intenção nesta seção é definir uma noção de morfismo entre transdutores subseqüenciais. Essa ferramenta permitirá comparar os conjuntos de passeios bem-sucedidos de transdutores equivalentes, e será utilizado na próxima seção para a definição formal de um transdutor subseqüencial minimal.

A noção de morfismo entre máquinas consta no livro de Eilenberg [Eil74] na formalização dos conceitos de autômatos e máquinas seqüenciais minimais. Essa noção foi estendida para transdutores subseqüenciais palavra-palavra por Choffrut [Cho03]. Nossa definição não é exatamente a de Choffrut, mas permite descrever os seus resultados.

Na definição de morfismo de Eilenberg, as máquinas envolvidas não são necessariamente acessíveis ou co-acessíveis. Em nossa definição, vamos considerar somente transdutores bi-acessíveis. Essa restrição facilita a exposição, e não altera em nada os resultados que queremos apresentar. No entanto, esta seção segue as idéias de Eilenberg. 
Estamos interessados no caso de funções subseqüenciais $f: \Sigma^{*} \rightarrow \mathrm{M}$, onde $\Sigma$ é um alfabeto, e $\mathrm{M}$ é um monóide cancelativo com mdc único. Todas as funções consideradas são não-vazias. Observamos de antemão que todo o desenvolvimento desta seção pode ser feito de maneira idêntica para funções que não são subseqüenciais, se permitirmos transdutores infinitos.

Um pré-requisito para o nosso estudo de morfismos é a definição de uma forma normalizada para transdutores subseqüenciais, que faremos a seguir. Essa forma normalizada começou a ser estudada por Choffrut em seus trabalhos sobre a caracterização de funções subseqüenciais, e é também útil no estudo da minimização de transdutores subseqüenciais.

Seja $\mathcal{T}=(Q, i, \lambda, \rho)$ um $\Sigma$-M-transdutor subseqüencial bi-acessível. Definimos o mdc de um estado $q$ de $\mathcal{T}$ como

$$
\operatorname{mdc} q=\operatorname{mdc}\left\{(q * x)((q x) \rho): x \in \Sigma^{*} \mathrm{e}(q x) \rho \neq \varnothing\right\} .
$$

Como $q$ é co-acessível, o conjunto de emissões nessa definição não é vazio. Portanto, mdc $q$ está bem-definido.

Definição 4.8.1 Dizemos que um $\Sigma$-M-transdutor subseqüencial $\mathcal{T}$ é prefixo, ou está na forma prefixo, se, para todo estado co-acessivel $q$ de $\mathcal{T}$, $\operatorname{mdc} q=1$.

O teorema a seguir constrói um transdutor prefixo equivalente a um transdutor subseqüencial. Nessa construção, apenas as emissões do transdutor original são alteradas. Intuitivamente, essa alteração consiste em deslocar o prefixo de cada estado em direção ao estado inicial. Essa construção é devida a Choffrut [Cho79].

Teorema 4.8.1 Sejam $\Sigma$ um alfabeto e $\mathrm{M}$ um monóide cancelativo com mdc único. Toda função subseqüencial $f: \Sigma^{*} \rightarrow \mathrm{M}$ pode ser realizada por um $\Sigma$-M-transdutor subseqüencial na forma prefixo.

Demonstração. Dado um $\Sigma$-M-transdutor subseqüencial bi-acessível $\mathcal{T}=(Q, i, \lambda, \rho)$ que realiza $f$, vamos construir um $\Sigma$-M-transdutor subseqüencial $\hat{\mathcal{T}}=(Q, i, \hat{\lambda}, \hat{\rho})$ equivalente na forma prefixo. Esse transdutor difere de $\mathcal{T}$ apenas nas emissões das transições e estados.

Denotando a função de emissão de $\mathcal{T}$ por $*$, e a de $\hat{\mathcal{T}}$ por $\star$, definimos

$$
\begin{aligned}
& i \hat{\lambda}=(i \lambda)(\operatorname{mdc} i) ; \\
& \forall q \in Q, q \hat{\rho}= \begin{cases}(\operatorname{mdc} q)^{-1}(q \rho), & \text { se } q \rho \neq \varnothing \\
\varnothing, & \text { caso contrário; }\end{cases} \\
& q \star \sigma=(\operatorname{mdc} q)^{-1}((q * \sigma) \operatorname{mdc}(q \sigma)), \forall q \in Q, \forall \sigma \in \Sigma \text { tal que } q \sigma \neq \varnothing .
\end{aligned}
$$

Os mdc's dos estados são obtidos em $\mathcal{T}$.

Para mostrar que $\hat{\mathcal{T}}$ está bem definido, precisamos mostrar que (4.8.2b) e (4.8.2c) estão bem definidos.

Em (4.8.2b), a emissão $q \rho$ pertence ao conjunto de emissões na definição (4.8.1). Portanto,

$$
(\operatorname{mdc} q) \mid q \rho,
$$

o que mostra que $\hat{\rho}$ está bem definida.

Vamos mostrar que a função de emissão * está bem definida. 
Sejam $q \in Q$ e $\sigma \in \Sigma$ tais que $q \sigma \neq \varnothing$, e $X=(q * \sigma)\left\{(q \sigma * u)((q \sigma u) \rho): u \in \Sigma^{*}\right.$ e $\left.(q \sigma u) \rho \neq \varnothing\right\}$. Como $\mathcal{T}$ é bi-acessível, $q \sigma$ é co-acessível, e portanto o conjunto $X$ não é vazio.

Como $X \subseteq\left\{(q * u)((q u) \rho): u \in \Sigma^{*}\right.$ e $\left.(q u) \rho \neq \varnothing\right\}$, segue da propriedade (4.7.1) que $(\operatorname{mdc} q) \mid \operatorname{mdc} X$. Aplicando a Proposição $4.7 .3 \mathrm{em} \operatorname{mdc} X$, obtemos

$$
(\operatorname{mdc} q) \mid(q * \sigma) \operatorname{mdc}(q \sigma),
$$

o que mostra que (4.8.2c) está bem definido.

Vamos agora mostrar que $\hat{\mathcal{T}}$ está na forma prefixo. Para isso, precisamos da seguinte propriedade. Para toda palavra $u$ em $\Sigma^{+}$, e todo estado $q$ tal que $q u \neq \varnothing$,

$$
(q \star u)=(\operatorname{mdc} q)^{-1}((q * u) \operatorname{mdc}(q u)),
$$

onde mdc $q$ e mdc $(q u)$ são os mdc's desses estados no transdutor $\mathcal{T}$.

Vamos usar indução em $|u|$.

- $|u|=1$ : segue diretamente da definição.

- $|u|>1$ : fatore $u$ como $v \sigma$, onde $\sigma$ é uma letra. Então,

$$
(q \star u)=(q \star v)((q v) \star \sigma) \stackrel{\text { h.i. }}{=}(\operatorname{mdc} q)^{-1}((q * v) \operatorname{mdc}(q v))(\operatorname{mdc}(q v))^{-1}((q v * \sigma) \operatorname{mdc}(q v \sigma)) .
$$

Utilizando as propriedades (4.7.4b) e (4.7.4c), e em seguida (4.2.2), obtemos (4.8.5).

Sejam $q$ um estado de $\hat{\mathcal{T}}$, e

$$
A=\left\{(q \star u)(q u) \hat{\rho}: u \in \Sigma^{*} \mathrm{e}(q u) \rho \neq \varnothing\right\} .
$$

Por definição, mdc $q=\operatorname{mdc} A$. Vamos mostrar que

$$
\operatorname{mdc} A=1 \text {. }
$$

Por (4.8.5), e pela definição de $\hat{\rho}$,

$$
\begin{aligned}
A & =\left\{\left((\operatorname{mdc} q)^{-1}((q * u) \operatorname{mdc}(q u))\right)\left((\operatorname{mdc}(q u))^{-1}((q u) \rho)\right): u \in \Sigma^{*} \text { e }(q u) \rho \neq \varnothing\right\} \\
& =\left\{(\operatorname{mdc} q)^{-1}((q * u)((q u) \rho)): u \in \Sigma^{*} \text { e }(q u) \rho \neq \varnothing\right\} .
\end{aligned}
$$

Multiplicando ambos os lados por mdc $q$, obtemos

$$
(\operatorname{mdc} q) A=\left\{(q * u)((q u) \rho): u \in \Sigma^{*} \mathrm{e}(q u) \rho \neq \varnothing\right\} .
$$

Calculando o mdc em ambos os lados dessa igualdade, obtemos

$$
\operatorname{mdc}((\operatorname{mdc} q) A)=\operatorname{mdc} q \text {. }
$$

Aplicando a Proposição 4.7.3 em mdc $((\operatorname{mdc} q) A)$, e em seguida o cancelamento, obtemos (4.8.6). Como $q$ é arbitrário, concluímos que $\hat{\mathcal{T}}$ está na forma prefixo.

Vamos agora mostrar que $|\hat{\mathcal{T}}|=|\mathcal{T}|$. 
Como os estados finais e as funções de transição de ambos os transdutores coincidem, dom $|\mathcal{T}|=$ $\operatorname{dom}|\hat{\mathcal{T}}|$. Seja $u$ uma palavra qualquer nesse domínio. Então,

$$
\begin{aligned}
u|\hat{\mathcal{T}}| & =(i \hat{\lambda})(i \star u)((i u) \hat{\rho})=((i \lambda)(\operatorname{mdc} i))\left((\operatorname{mdc} i)^{-1}((i * u) \operatorname{mdc}(i u))\right)\left((\operatorname{mdc}(i u))^{-1}((i u) \rho)\right) \\
& =(i \lambda)(i * u)((i u) \rho)=u|\mathcal{T}| .
\end{aligned}
$$

Para a segunda igualdade, utilizamos as definições de $\hat{\lambda}$ e $\hat{\rho}$, e a propriedade (4.8.5).

O transdutor $\hat{\mathcal{T}}$ será denominado prefixo de $\mathcal{T}$. Na Seção 4.11, estudamos um algoritmo eficiente para construir o prefixo de um $\Sigma$ - $\Gamma$-transdutor subsequencial.

Exemplo 4.8.1 Sejam $\Sigma=\{\sigma, \gamma\}$ e $\mathbb{R}_{+}$o monóide aditivo dos números reais não-negativos. O $\Sigma$ - $\mathbb{R}_{+}$-transdutor ilustrado a seguir não é prefixo:

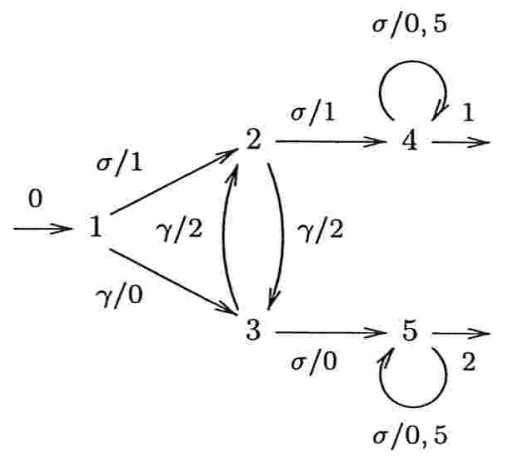

O mdc de cada estado está indicado a seguir. Observe que o mdc de um estado $q$ é o custo de um caminho de custo mínimo de $q$ a um estado final (incluindo a emissão final), no autômato de saída do transdutor.

$$
\operatorname{mdc} 1=2 ; \quad \operatorname{mdc} 2=2 ; \quad \operatorname{mdc} 3=2 ; \quad \operatorname{mdc} 4=1 ; \quad \operatorname{mdc} 5=2 .
$$

O prefixo desse transdutor é

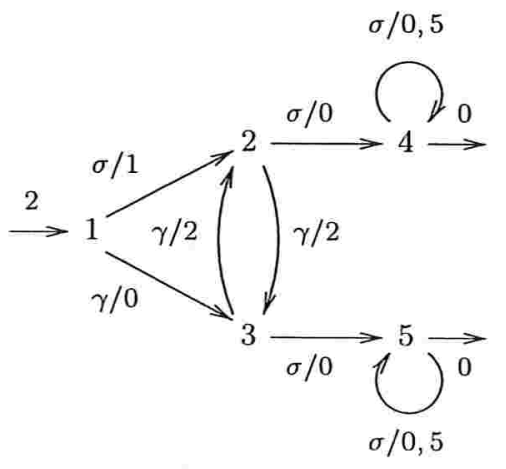

Passamos agora ao estudo de morfismos entre transdutores subseqüenciais. 
Definição 4.8.2 Sejam $\mathcal{T}=(Q, i, \lambda, \rho)$ e $\mathcal{T}^{\prime}=\left(Q^{\prime}, i^{\prime}, \lambda^{\prime}, \rho^{\prime}\right) \Sigma$-M-transdutores subseqüenciais bi-acessiveis, e $\hat{\mathcal{T}}=(Q, i, \hat{\lambda}, \hat{\rho})$ e $\hat{\mathcal{T}}^{\prime}=\left(Q^{\prime}, i^{\prime}, \hat{\lambda}^{\prime}, \hat{\rho}^{\prime}\right)$ os prefixos de $\mathcal{T}$ e $\mathcal{T}^{\prime}$, respectivamente. Um morfismo $\varphi: \mathcal{T} \rightarrow \mathcal{T}^{\prime}$ é uma função parcial $\varphi: Q \rightarrow Q^{\prime}$ que satisfaz

$$
\begin{aligned}
& i^{\prime}=i \varphi ; \\
& i \hat{\lambda}=i^{\prime} \hat{\lambda}^{\prime} ; \\
& (q \varphi) \sigma \subseteq(q \sigma) \varphi ; \\
& (q \varphi) \star \sigma \subseteq q \star \sigma ; \\
& (q \varphi) \hat{\rho}^{\prime} \subseteq q \hat{\rho},
\end{aligned}
$$

onde $q$ é um estado qualquer em $Q$, $\sigma$ é uma letra, $e \star$ denota as emissões dos transdutores $\hat{\mathcal{T}} e$ $\hat{\mathcal{T}}^{\prime}$.

As propriedades (4.8.7c) e (4.8.7d) podem ser estendidas para toda palavra $s$ em $\Sigma^{*}$ :

$$
\begin{aligned}
& (q \varphi) s \subseteq(q s) \varphi ; \\
& (q \varphi) \star s \subseteq q \star s .
\end{aligned}
$$

Essa extensão segue facilmente da definição, utilizando indução em $|s|$.

É conveniente ter em mente uma interpretação intuitiva de $\varphi$, entendendo o significado das propriedades (4.8.8a) e (4.8.8b). Dado um estado $q$ de $\mathcal{T}$ tal que $q \varphi \neq \varnothing$, a propriedade (4.8.8a) significa que todo passeio em $\mathcal{T}^{\prime}$ com origem em $q \varphi$ é a imagem por $\varphi$ de um passeio em $\mathcal{T}$ com mesma entrada. A propriedade (4.8.8b) diz que, nos transdutores $\hat{\mathcal{T}}$ e $\hat{\mathcal{T}}^{\prime}$, esses passeios têm a mesma saída.

As propriedades (4.8.8a) e (4.8.8b) também permitem relacionar os comportamentos de $\mathcal{T}$ e $\mathcal{T}^{\prime}$.

Como $i^{\prime}=i \varphi$, todo passeio bem-sucedido de $\mathcal{T}^{\prime}$ é a imagem de um passeio bem-sucedido de $\mathcal{T}$, e esses passeios têm a mesma saída nos transdutores $\hat{\mathcal{T}}$ e $\hat{\mathcal{T}}^{\prime}$. Utilizando (4.8.7b) e (4.8.7e), temos então que, para todo $u$ em $\operatorname{dom}\left|\mathcal{T}^{\prime}\right|$,

$$
u\left|\mathcal{T}^{\prime}\right|=u\left|\hat{\mathcal{T}}^{\prime}\right|=\left(i^{\prime} \hat{\lambda}^{\prime}\right)\left(i^{\prime} \star u\right)\left(\left(i^{\prime} u\right) \hat{\rho}^{\prime}\right)=(i \hat{\lambda})(i \star u)((i u) \hat{\rho})=u|\hat{\mathcal{T}}|=u|\mathcal{T}| .
$$

Portanto,

$$
\#\left|\mathcal{T}^{\prime}\right| \subseteq \#|\mathcal{T}|
$$

Exemplo 4.8.2 Considere os alfabetos $\Sigma=\{\sigma, \gamma\}, \Gamma=\{a, b, c\}$, e os $\Sigma$ - $\Gamma$-transdutores ilustrados abaixo.
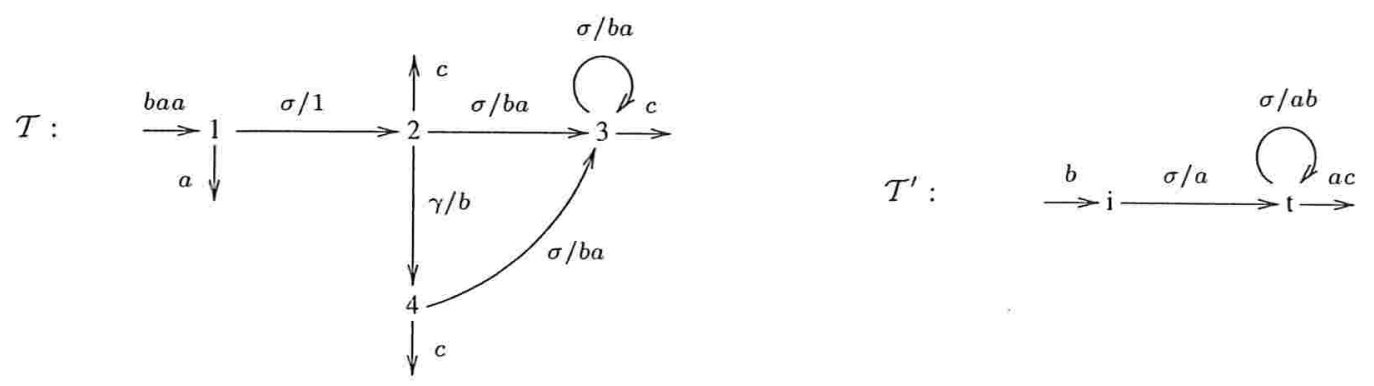
O transdutor $\mathcal{T}$ está na forma prefixo. A forma prefixo do transdutor $\mathcal{T}^{\prime}$ é

$$
\hat{\mathcal{T}}^{\prime}: \quad \stackrel{b a a}{\longrightarrow} \mathrm{i} \stackrel{\sigma / 1}{\longrightarrow \mathrm{i} \longrightarrow \overbrace{c}}
$$

A função $\varphi: Q \rightarrow Q^{\prime}$ dada por

$$
1 \varphi=i, 2 \varphi=3 \varphi=t
$$

é um morfismo $\varphi: \mathcal{T} \rightarrow \mathcal{T}^{\prime}$. Outro morfismo $\varphi^{\prime}: \mathcal{T} \rightarrow \mathcal{T}^{\prime}$ é obtido mantendo-se as imagens de 1,2 e 3 , e definindo $4 \varphi^{\prime}=t$.

Dizemos que um morfismo $\varphi$ é próprio se as seguintes propriedades forem satisfeitas:

$$
\begin{aligned}
& q \sigma \neq \varnothing \text { implica em }(q \varphi) \sigma \neq \varnothing ; \\
& q \hat{\rho} \subseteq(q \varphi) \hat{\rho}^{\prime},
\end{aligned}
$$

para todo $q$ em $Q$ e todo $\sigma$ em $\Sigma$.

Nesse caso, $\varphi$ associa todo passeio bem-sucedido de $\mathcal{T}$ a um passeio bem-sucedido de $\mathcal{T}^{\prime}$, o que implica em

$$
\#\left|\mathcal{T}^{\prime}\right|=\#|\mathcal{T}|
$$

Um morfismo próprio importante é o isomorfismo. Dizemos que um morfismo próprio $\varphi: \mathcal{T} \rightarrow$ $\mathcal{T}^{\prime}$ é um isomorfismo se $\varphi$ for uma função bijetora, e

$$
q * \sigma=(q \varphi) * \sigma
$$

onde $q$ é um estado qualquer em $Q, \sigma$ é uma letra, e $*$ denota as emissões dos transdutores $\mathcal{T}$ e $\mathcal{T}^{\prime}$ (e não nos prefixos desses transdutores, como na definição de morfismo). Nesse caso, podemos dizer que os transdutores $\mathcal{T}$ e $\mathcal{T}^{\prime}$ são iguais a menos dos rótulos dos estados. Dizemos que esses transdutores são isomorfos.

Evidentemente, o morfismo inverso $\varphi^{-1}: \mathcal{T}^{\prime} \rightarrow \mathcal{T}$ de um isomorfismo $\varphi$ e a identidade $1: \mathcal{T} \rightarrow \mathcal{T}$ também são isomorfismos. Assim, a relação $\cong$ sobre o conjunto de todos os $\Sigma$-M-transdutores subseqüenciais bi-acessíveis definida por

$$
\mathcal{T} \cong \mathcal{T}^{\prime} \text { se, e somente se, existe um isomorfismo } \varphi: \mathcal{T} \rightarrow \mathcal{T}^{\prime},
$$

é uma relação de equivalência.

As duas proposições a seguir são de fácil demonstração, e serão apenas enunciadas. A segunda é uma conseqüência do fato de estarmos considerando apenas transdutores bi-acessíveis.

Proposição 4.8.1 Sejam $\mathcal{T}, \mathcal{T}^{\prime}$ e $\mathcal{T}^{\prime \prime} \Sigma$-M-transdutores subseqüenciais bi-acessíveis, e $\varphi: \mathcal{T} \rightarrow \mathcal{T}^{\prime}$ e $\varphi^{\prime}: \mathcal{T}^{\prime} \rightarrow \mathcal{T}^{\prime \prime}$ morfismos. Então, a composição $\varphi \varphi^{\prime}: \mathcal{T} \rightarrow \mathcal{T}^{\prime \prime}$ é um morfismo. Ademais, se $\varphi$ e $\varphi^{\prime}$ forem morfismos próprios, então $\varphi \varphi^{\prime}$ é um morfismo próprio.

Proposição 4.8.2 Sejam $\mathcal{T}$ e $\mathcal{T}^{\prime} \Sigma$-M-transdutores subseqüenciais bi-acessíveis.

Todo morfismo $\varphi: \mathcal{T} \rightarrow \mathcal{T}^{\prime}$ é sobrejetor, e, se $|\mathcal{T}|=\left|\mathcal{T}^{\prime}\right|$, então $\varphi$ é um morfismo próprio.

Há no máximo um morfismo próprio $\varphi: \mathcal{T} \rightarrow \mathcal{T}^{\prime}$. 
Na próxima seção, estudamos o conceito de transdutor minimal realizando uma função subseqüencial. A definição formal desse conceito depende de uma relação de ordem parcial definida entre transdutores, utilizando morfismos. Vamos concluir essa seção definindo essa ordem.

Dada uma função subseqüencial $f: \Sigma^{*} \rightarrow \mathrm{M}$, denotamos por $\mathrm{T}(f)$ o conjunto dos transdutores subseqüenciais bi-acessíveis que realizam $f$. A relação $\cong$ restrita a $\mathbf{T}(f)$ é uma relação de equivalência nesse conjunto.

Utilizando morfismos de transdutores, definimos uma ordem parcial $\leq$ no conjunto quociente $\mathbf{T}(f) / \cong$ como segue. Dadas classes $[\mathcal{T}]$ e $\left[\mathcal{T}^{\prime}\right]$ nesse quociente, $\left[\mathcal{T}^{\prime}\right] \leq[\mathcal{T}]$ se, e somente se, $\mathcal{T}^{\prime}$ é prefixo, e existe um morfismo próprio $\varphi: \mathcal{T} \rightarrow \mathcal{T}^{\prime}$.

Essa definição independe da escolha dos representantes. De fato, dados transdutores quaisquer $\mathcal{U}$ e $\mathcal{U}^{\prime}$ em $[\mathcal{T}]$ e $\left[\mathcal{T}^{\prime}\right]$, respectivamente, $\mathcal{U}^{\prime}$ é prefixo se, e somente se, $\mathcal{T}^{\prime}$ é prefixo (porque esses transdutores são isomorfos). Ademais, se existir um morfismo próprio $\varphi: \mathcal{T} \rightarrow \mathcal{T}^{\prime}$, então, pela Proposição 4.8.1, a composição $\varphi_{1} \varphi \varphi_{2}: \mathcal{U} \rightarrow \mathcal{U}^{\prime}$ é um morfismo próprio, onde $\varphi_{1}: \mathcal{U} \rightarrow \mathcal{T}$ e $\varphi_{2}: \mathcal{T}^{\prime} \rightarrow \mathcal{U}^{\prime}$ são isomorfismos.

A relação $\leq$ é transitiva, porque a composição de dois morfismos é um morfismo, e é também anti-simétrica. Para provar essa propriedade, suponha que $[\mathcal{T}] \leq\left[\mathcal{T}^{\prime}\right]$ e $\left[\mathcal{T}^{\prime}\right] \leq[\mathcal{T}]$. Então, $\mathcal{T}$ e $\mathcal{T}^{\prime}$ estão na forma prefixo, e existem morfismos próprios $\varphi: \mathcal{T} \rightarrow \mathcal{T}^{\prime}$ e $\varphi^{\prime}: \mathcal{T}^{\prime} \rightarrow \mathcal{T}$. Como as composições $\varphi \varphi^{\prime}: \mathcal{T} \rightarrow \mathcal{T}$ e $\varphi^{\prime} \varphi: \mathcal{T}^{\prime} \rightarrow \mathcal{T}^{\prime}$ são morfismos próprios, e há no máximo um morfismo próprio entre um dado par de transdutores, essas composições devem ser a identidade. Assim, $\varphi$ e $\varphi^{\prime}$ devem ser bijetores. Como $\mathcal{T}$ e $\mathcal{T}^{\prime}$ estão na forma prefixo, concluímos que esses morfismos são isomorfismos. Portanto, $[\mathcal{T}]=\left[\mathcal{T}^{\prime}\right]$.

Há uma classe minimal em $\mathbf{T}(f) / \cong$ de acordo com essa ordem, e o estudo dessa classe é o assunto da próxima seção.

\subsection{Transdutores subseqüenciais minimais}

Nesta seção, estudamos o conceito de transdutor minimal realizando uma função subseqüencial.

Os primeiros resultados sobre o assunto remontam ao fim da década de 1970, e apareceram implicitamente nos trabalhos de Choffrut sobre a caracterização das funções subseqüenciais [Cho78, Cho79].

Posteriormente, notou-se que algumas das idéias presentes nesses trabalhos poderiam ser aproveitadas para uma teoria de minimização. O primeiro autor a fazer esse estudo explicitamente foi Reutenauer, em 1990 [Reu90]. Em 2003, Choffrut publicou um artigo que apresenta os resultados de Reutenauer com mais detalhes [Cho03].

Nossa intenção é apresentar o conceito de transdutor subseqüencial minimal com rigor, utilizando para isso morfismos de transdutores subseqüenciais. Esse tratamento, utilizado por Eilenberg no estudo da minimização de autômatos e máquinas seqüenciais, aparece superficialmente no trabalho de Reutenauer, e explicitamente no trabalho de Choffrut de 2003. Nessa abordagem, o primeiro passo é definir o transdutor subseqüencial minimal associado a uma função, de maneira puramente algébrica. Em seguida, utilizando morfismos de transdutores subseqüenciais, demonstra-se a minimalidade desse transdutor.

Estamos interessados no estudo de máquinas minimais para funções subseqüenciais $f: \Sigma^{*} \rightarrow \mathrm{M}$, onde $\Sigma$ é um alfabeto, e $\mathrm{M}$ é um monóide cancelativo com mdc único. A teoria aqui desenvolvida é então uma extensão do trabalho de Choffrut de 2003, no qual o monóide de saída é sempre um monóide livre. Ademais, Choffrut define o transdutor minimal utilizando a congruência sintática 
de uma função. Nesta seção, fazemos uma construção direta do transdutor minimal a partir de $f$, seguindo as idéias desenvolvidas nas seções III.3 e XII.5 do livro de Eilenberg [Eil74].

Observamos que a teoria desenvolvida nesta seção contém a teoria da minimização de autômatos. Para isso, escolhemos $\mathrm{M}$ como um monóide que só contém a identidade, e uma linguagem $A$ é vista como uma função $f: \Sigma^{*} \rightarrow \mathrm{M}$ definida apenas nas palavras em $A$. Esse fato será desenvolvido na Seção 4.12 .

A justificativa dessa extensão para monóides com mdc está no interesse atual em aplicar transdutores subseqüenciais em problemas de Processamento de Linguagem Natural. Nessa área, são utilizadas tanto máquinas cujo monóide de saída é um monóide livre, quanto aquelas que possuem emissões em conjuntos numéricos, como números reais. No entanto, a teoria de minimização é essencialmente a mesma para todos esses tipos de máquinas. A função dos monóides cancelativos com mdc é então abstrair as propriedades tanto de monóides livres, quanto de outros monóides de interesse, que sejam essenciais para a definição de um transdutor minimal.

Algumas referências para as aplicações de transdutores subseqüenciais em Processamento de Linguagem Natural são os trabalhos de Mohri [Moh95, Moh97].

Dada uma função subseqüencial não-vazia $f: \Sigma^{*} \rightarrow \mathrm{M}$, vamos primeiro mostrar a construção de um transdutor subseqüencial minimal para essa função. Em seguida, demonstramos a minimalidade desse transdutor.

Precisamos de algumas definições preliminares. Dada uma palavra $s$ tal que o conjunto $\{(s x) f$ : $s x \in \operatorname{dom} f\}$ não seja vazio, definimos o mdc de $f$ com $s$ como

$$
\operatorname{mdc}(f, s)=\operatorname{mdc}\{(s x) f: s x \in \operatorname{dom} f\} .
$$

Se $s=1$, escrevemos simplesmente

$$
\operatorname{mdc} f=\operatorname{mdc}(f, 1)=\operatorname{mdc}\{x f: x \in \operatorname{dom} f\},
$$

e dizemos que $\operatorname{mdc} f$ é o mdc de $f$.

Definimos também $\hat{f}: \Sigma^{*} \rightarrow$ M como a função cujo domínio é igual a $\operatorname{dom} f$, e tal que

$$
s \hat{f}=(\operatorname{mdc} f)^{-1}(s f), \quad \forall s \in \operatorname{dom} f .
$$

Seja $Q^{0}$ o conjunto de todas as funções parciais não-vazias $g: \Sigma^{*} \rightarrow \mathrm{M}$ tais que $\operatorname{mdc} g=1$. O primeiro passo na construção de um transdutor subseqüencial minimal para $f$ é definir uma ação . de $\Sigma^{*}$ sobre $Q^{0}$, e uma emissão $*$ de $\Sigma^{*}$ sobre $Q^{0}$ em M. As restrições de $\cdot$ e * a uma parte especial de $Q^{0}$ serão a função de transição e a de emissão do transdutor minimal.

Vamos primeiro definir as imagens de $\cdot \mathrm{e} *$ nas letras de $\Sigma$, e depois estender essas definições para $\Sigma^{*}$.

Sejam $g$ uma função em $Q^{0}$, e $\sigma$ uma letra. Se o conjunto $\sigma^{-1}(\operatorname{dom} g$ ) for vazio (recorde a notação definida em (4.7.5)), então $g \cdot \sigma$ é indefinido. Senão, $g \cdot \sigma$ é a função com domínio igual a $\sigma^{-1}($ dom $g)$ e definida por

$$
u(g \cdot \sigma)=(\operatorname{mdc}(g, \sigma))^{-1}((\sigma u) g), \quad \forall u \in \sigma^{-1}(\operatorname{dom} g) .
$$

A emissão $*$ é definida como

$$
g * \sigma=\operatorname{mdc}(g, \sigma) .
$$


Para estender essa definição a uma ação de $\Sigma^{*}$ sobre $Q^{0}$, é preciso primeiro mostrar que $g \cdot \sigma$ é uma função em $Q^{0}$, sempre que $g \cdot \sigma \neq \varnothing$. Equivalentemente, precisamos mostrar que mdc $(g \cdot \sigma)=1$. Para isso, tomamos o mdc em ambos os lados da igualdade

$$
\{(\sigma x) g: \sigma x \in \operatorname{dom} g\}=\operatorname{mdc}(g, \sigma)\left\{\operatorname{mdc}(g, \sigma)^{-1}((\sigma x) g): \sigma x \in \operatorname{dom} g\right\},
$$

e aplicamos a Proposição 4.7.3 no lado direito, obtendo

$$
\operatorname{mdc}(g, \sigma)=\operatorname{mdc}(g, \sigma) \operatorname{mdc}\left\{\operatorname{mdc}(g, \sigma)^{-1}((\sigma x) g): \sigma x \in \operatorname{dom} g\right\} \stackrel{(4.9 .4)}{=} \operatorname{mdc}(g, \sigma) \operatorname{mdc}(g \cdot \sigma) \text {. }
$$

Aplicando o cancelamento, obtemos mdc $(g \cdot \sigma)=1$.

Vamos denotar por $T^{0}$ o subconjunto de $Q^{0}$ das funções cuja imagem da palavra vazia esteja definida.

Considere o $\Sigma$-M-transdutor subseqüencial infinito

$$
\mathcal{T}_{f}^{0}=\left(Q^{0}, \hat{f}, \lambda^{0}, \rho^{0}\right)
$$

com função de transição igual a $\cdot$, função de emissão igual a $*$, conjunto de estados finais igual a $T^{0}$, e emissões inicial e final definidas por

$$
\begin{aligned}
& \hat{f} \lambda^{0}=\operatorname{mdc} f \\
& g \rho^{0}=1 g, \forall g \in T^{0} .
\end{aligned}
$$

O transdutor subseqüencial minimal que realiza $f$ será definido a partir de $\mathcal{T}_{f}^{0}$. Antes de fazer essa definição, vamos demonstrar algumas propriedades de $\cdot$ e $*$.

Proposição 4.9.1 Seja g uma função em $Q^{0}$. Então,

$$
\begin{aligned}
& \operatorname{dom}(g \cdot s)=s^{-1}(\operatorname{dom} g), \forall s \in \Sigma^{*} ; \\
& u(g \cdot s)=(\operatorname{mdc}(g, s))^{-1}((s u) g), \forall u \in \operatorname{dom}(g \cdot s), \forall s \in \Sigma^{*} \text { tal que } g \cdot s \neq \varnothing ; \\
& g * s=\operatorname{mdc}(g, s), \forall s \in \Sigma^{*} \text { tal que } g \cdot s \neq \varnothing .
\end{aligned}
$$

Demonstração. O caso $s=1$ é evidente para as três propriedades.

Para as demais palavras, vamos usar indução em $|s|$. As três propriedades serão demonstradas simultaneamente.

- $|s|=1$ : este caso segue diretamente das definições de $\cdot \mathrm{e} *$ nas letras.

- $|s|>1$ : fatore $s$ como $t \sigma$, onde $\sigma$ é uma letra.

A demonstração do passo de indução para a primeira propriedade é simples:

$$
\operatorname{dom}(g \cdot s)=\operatorname{dom}((g \cdot t) \cdot \sigma) \stackrel{\text { h.i. }}{=} \sigma^{-1} t^{-1}(\operatorname{dom} g) \stackrel{(4.7 .4 \mathrm{a})}{=}(t \sigma)^{-1}(\operatorname{dom} g) .
$$

Vamos demonstrar a segunda propriedade. Dada uma palavra $u$ em dom $(g \cdot s)$,

$$
u(g \cdot s)=u((g \cdot t) \cdot \sigma) \stackrel{(4.9 .4)}{=}(\operatorname{mdc}(g \cdot t, \sigma))^{-1}((\sigma u)(g \cdot t)) .
$$


Pela hipótese de indução,

$$
(\sigma u)(g \cdot t)=(\operatorname{mdc}(g, t))^{-1}((t \sigma u) g) .
$$

Substituindo em (4.9.9), obtemos

$$
\begin{aligned}
u(g \cdot s) & =(\operatorname{mdc}(g \cdot t, \sigma))^{-1}(\operatorname{mdc}(g, t))^{-1}((t \sigma u) g) \\
& \stackrel{(4.7 .4 \mathrm{a})}{=}(\operatorname{mdc}(g, t) \operatorname{mdc}(g \cdot t, \sigma))^{-1}((s u) g) .
\end{aligned}
$$

Afirmamos que

$$
\operatorname{mdc}(g, t) \operatorname{mdc}(g \cdot t, \sigma)=\operatorname{mdc}(g, s) .
$$

De fato,

$$
\begin{aligned}
\operatorname{mdc}(g, t) \operatorname{mdc}(g \cdot t, \sigma) & =\operatorname{mdc}(g, t)(\operatorname{mdc}\{(\sigma x)(g \cdot t): \sigma x \in \operatorname{dom}(g \cdot t)\}) \\
& \stackrel{\text { h.i. }}{=} \operatorname{mdc}(g, t)\left(\operatorname{mdc}\left\{(\operatorname{mdc}(g, t))^{-1}((t \sigma x) g): t \sigma x \in \operatorname{dom} g\right\}\right) .
\end{aligned}
$$

Utilizando Proposição 4.7.3, o lado direito dessa igualdade se torna

$$
\begin{aligned}
\operatorname{mdc}\left(\operatorname{mdc}(g, t)\left\{\operatorname{mdc}(g, t)^{-1}(t \sigma x) g: t \sigma x \in \operatorname{dom} g\right\}\right) & =\operatorname{mdc}\{(t \sigma x) g: t \sigma x \in \operatorname{dom} g\} \\
& =\operatorname{mdc}(g, s) .
\end{aligned}
$$

Substituindo (4.9.11) em (4.9.10), obtemos

$$
u(g \cdot s)=(\operatorname{mdc}(g, s))^{-1}((s u) g) .
$$

O passo de indução para a terceira propriedade pode ser demonstrado como segue:

$$
g * s=(g * t)((g \cdot t) * \sigma) \stackrel{\text { h.i. }}{=} \operatorname{mdc}(g, t) \operatorname{mdc}(g \cdot t, \sigma) \stackrel{(4.9 .11)}{=} \operatorname{mdc}(g, s) .
$$

Com a propriedade (4.9.8a), podemos provar que o transdutor $\mathcal{T}_{f}^{0}$ é co-acessível. Seja $g$ uma função em $Q^{0}$. Então, pela definição desse conjunto, $g$ não é vazia. Seja $s$ uma palavra em dom $g$. Então, por (4.9.8a), $1 \in \operatorname{dom}(g \cdot s)$. Pela definição de $T^{0}$, temos então que $g \cdot s$ é um estado final. Portanto, $g$ é co-acessível.

A parte acessível de $\mathcal{T}_{f}^{0}$ é um transdutor subseqüencial bi-acessível que será denotado por $\mathcal{T}_{f}$. O conjunto de estados de $\mathcal{T}_{f}$ será denotado por $Q_{f}$. Pela definição de $\mathcal{T}_{f}{ }^{0}$,

$$
Q_{f}=\left\{\hat{f} \cdot s: s \in \Sigma^{*} \text { e } \hat{f} \cdot s \neq \varnothing\right\} .
$$

Por (4.9.8a), podemos também escrever

$$
Q_{f}=\left\{\hat{f} \cdot s: s \in \Sigma^{*} \text { e } s^{-1}(\operatorname{dom} \hat{f}) \neq \varnothing\right\} .
$$


Como $1 \in \operatorname{dom}(\hat{f} \cdot s)$ se, e somente se, $s \in \operatorname{dom} \hat{f}$, e $\operatorname{dom} f=\operatorname{dom} \hat{f}$, temos também que o conjunto dos estados finais de $\mathcal{T}_{f}$ é

$$
T_{f}=\{\hat{f} \cdot s: s \in \operatorname{dom} f\} .
$$

Assim,

$$
\mathcal{T}_{f}=\left(Q_{f}, \hat{f}, \lambda_{f}, \rho_{f}\right),
$$

onde $\lambda_{f}$ e $\rho_{f}$ são respectivamente as restrições de $\lambda^{0}$ e $\rho^{0}$ a $Q_{f}$.

As proposições a seguir mostram algumas propriedades de transdutores prefixo utilizando a notação introduzida nessa seção. Após essas propriedades, demonstramos que $\mathcal{T}_{f}$ é um transdutor subseqüencial minimal realizando $f$.

Proposição 4.9.2 Sejam $\mathcal{T}=(Q, i, \lambda, \rho)$ um $\Sigma$-M-transdutor subseqüencial bi-acessível e prefixo, e $f$ o seu comportamento. Então,

$$
\begin{aligned}
& i \lambda=\operatorname{mdc} f \\
& (i s) * t=\operatorname{mdc}(\hat{f} \cdot s, t), \forall s, t \in \Sigma^{*} \text { tais que } i(s t) \neq \varnothing ; \\
& (i s) \rho=1(\hat{f} \cdot s), \forall s \in \operatorname{dom} f ; \\
& \text { se is }=i t, \text { então } \hat{f} \cdot s=\hat{f} \cdot t, \forall s, t \in \Sigma^{*} .
\end{aligned}
$$

Demonstração. Para provar a primeira propriedade, considere a igualdade

$$
(i \lambda)\{(i * x)((i x) \rho): x \in \operatorname{dom} f\}=\{x f: x \in \operatorname{dom} f\} .
$$

Tomando o mdc em ambos os lados e utilizando a Proposição 4.7.3, obtemos

$$
(i \lambda)(\operatorname{mdc} i)=\operatorname{mdc} f \text {. }
$$

Como $\mathcal{T}$ é prefixo, mdc $i=1$. Obtemos assim $i \lambda=\operatorname{mdc} f$.

Para demonstrar a segunda propriedade, vamos mostrar primeiro que

$$
i * s=\operatorname{mdc}(\hat{f}, s) .
$$

Para isso, tome o mdc em ambos os lados da igualdade

$$
(i \lambda)(i * s)\{(i s * x)((i(s x)) \rho): s x \in \operatorname{dom} f\}=\{(s x) f: s x \in \operatorname{dom} f\}
$$

e utilize a Proposição 4.7.3, obtendo

$$
(i \lambda)(i * s)(\operatorname{mdc}(i s))=\operatorname{mdc}\{(s x) f: s x \in \operatorname{dom} f\} .
$$

Como $\mathcal{T}$ é prefixo, $\operatorname{mdc}(i s)=1$. Como $i \lambda=\operatorname{mdc} f$, podemos reescrever essa igualdade como

$$
(i * s)=(\operatorname{mdc} f)^{-1} \operatorname{mdc}(\{(s x) f: s x \in \operatorname{dom} f\}) .
$$

Aplicando a propriedade (4.7.6) no lado direito, obtemos

$$
i * s=\operatorname{mdc}\left((\operatorname{mdc} f)^{-1}\{(s x) f: s x \in \operatorname{dom} f\}\right) \stackrel{(4.9 .3)}{=} \operatorname{mdc}(\{(s x) \hat{f}: s x \in \operatorname{dom} f\})=\operatorname{mdc}(\hat{f}, s) .
$$


A propriedade (4.9.13b) pode agora ser obtida a partir das igualdades a seguir:

$$
i *(s t) \stackrel{(4.9 .14)}{=} \operatorname{mdc}(\hat{f}, s t) \stackrel{(4.9 .8 \mathrm{c})}{=} \hat{f} *(s t)=(\hat{f} * s)((\hat{f} \cdot s) * t) \stackrel{(4.9 .8 \mathrm{c})}{=} \operatorname{mdc}(\hat{f}, s) \operatorname{mdc}(\hat{f} \cdot s, t)
$$

e

$$
i *(s t)=(i * s)((i s) * t) \stackrel{(4.9 .14)}{=} \operatorname{mdc}(\hat{f}, s)((i s) * t) .
$$

Igualando e cancelando, obtemos $(i s) * t=\operatorname{mdc}(\hat{f} \cdot s, t)$.

Para demonstrar a propriedade (4.9.13c), utilizamos (4.9.13a) e (4.9.14) para escrever

$$
s f=(i \lambda)(i * s)((i s) \rho)=(\operatorname{mdc} f)(\operatorname{mdc}(\hat{f}, s))((i s) \rho) .
$$

Logo,

$$
(i s) \rho=(\operatorname{mdc}(\hat{f}, s))^{-1}(\operatorname{mdc} f)^{-1}(s f) \stackrel{(4.9 .3)}{=}(\operatorname{mdc}(\hat{f}, s))^{-1}(s \hat{f}) .
$$

Por $(4.9 .8 \mathrm{~b}),(\operatorname{mdc}(\hat{f}, s))^{-1}(s \hat{f})=1(\hat{f} \cdot s)$, o que conclui a demonstração de $(4.9 .13 \mathrm{c})$.

Para demonstrar a propriedade (4.9.13d), vamos demonstrar primeiro que

$$
\text { se } i s=i t, \text { então } \operatorname{dom}(\hat{f} \cdot s)=\operatorname{dom}(\hat{f} \cdot t)
$$

$$
((i s) * x)((i(s x)) \rho)=x(\hat{f} \cdot s), \forall s, x \in \Sigma^{*} \text { tais que }(i(s x)) \rho \neq \varnothing .
$$

Para demonstrar (4.9.15), note que

$$
s^{-1}(\operatorname{dom} f)=\{x:(i(s x)) \rho \neq \varnothing\} \stackrel{i s \equiv i t}{=}\{x:(i(t x)) \rho \neq \varnothing\}=t^{-1}(\operatorname{dom} f) .
$$

Pela definição de $\hat{f}, \operatorname{dom} \hat{f}=\operatorname{dom} f$. Da igualdade acima, segue então que $s^{-1}(\operatorname{dom} \hat{f})=t^{-1}(\operatorname{dom} \hat{f})$. Utilizando (4.9.8a), obtemos $\operatorname{dom}(\hat{f} \cdot s)=\operatorname{dom}(\hat{f} \cdot t)$.

Para demonstrar (4.9.16), utilizamos a propriedade (4.9.8b) para escrever

$$
\operatorname{mdc}(\hat{f}, s x)(1(\hat{f} \cdot(s x)))=(s x) \hat{f} .
$$

Utilizando (4.9.13b) e (4.9.13c), obtemos

$$
(i *(s x))((i(s x)) \rho)=(s x) \hat{f} .
$$

Escrevendo $(i *(s x))$ como $(i * s)(i s * x)$, e usando (4.9.14), obtemos

$$
\operatorname{mdc}(\hat{f}, s)(i s * x)((i(s x)) \rho)=(s x) \hat{f},
$$

ou seja,

$$
(i s * x)((i(s x)) \rho)=(\operatorname{mdc}(\hat{f}, s))^{-1}((s x) \hat{f}) \stackrel{(4.9 .8 \mathrm{~b})}{=} x(\hat{f} \cdot s),
$$

o que conclui a demonstração de (4.9.16).

Vamos demonstrar (4.9.13d). Como $\operatorname{dom}(\hat{f} \cdot s)=\operatorname{dom}(\hat{f} \cdot t)$, basta demonstrar que $x(\hat{f} \cdot s)=$ $x(\hat{f} \cdot t)$ para todo $x$ nesse domínio. Como is $=i t,((i s) * x)((i(s x)) \rho)=((i t) * x)((i(t x)) \rho)$. De (4.9.16), segue que $x(\hat{f} \cdot s)=x(\hat{f} \cdot t)$. 
Proposição 4.9.3 Sejam $f: \Sigma^{*} \rightarrow \mathrm{M}$ uma função subseqüencial, e $\mathcal{T}=(Q, i, \lambda, \rho)$ um $\Sigma$-Mtransdutor subseqüencial bi-acessivel que realiza $f$. Então, existe um morfismo próprio $\varphi: \mathcal{T} \rightarrow \mathcal{T}_{f}$.

Demonstração. Basta considerar o caso em que $\mathcal{T}$ é prefixo.

Afirmamos que a função $\varphi: Q \rightarrow Q_{f}$ dada por

$$
(i s) \varphi=\hat{f} \cdot s, \forall s \in \operatorname{dom} f
$$

é um morfismo próprio. Pela propriedade (4.9.13d), essa função está bem definida.

A definição de $\varphi$ garante automaticamente as seguintes propriedades:

$$
\begin{aligned}
& i \varphi=\hat{f} \\
& \text { e } \quad(q \varphi) \cdot \sigma=(q \sigma) \varphi, \forall q \in Q, \forall \sigma \in \Sigma .
\end{aligned}
$$

Resta então demonstrar que

$$
\begin{aligned}
& i \lambda=\hat{f} \lambda^{0}, \\
& q \rho=(q \varphi) \rho^{0}, \forall q \in Q \\
& \text { e } \quad q * \sigma=(q \varphi) * \sigma, \forall q \in Q, \forall \sigma \in \Sigma \text { tais que } q \cdot \sigma \neq \varnothing .
\end{aligned}
$$

Essas propriedades são uma conseqüência direta de (4.9.13a), (4.9.13b) e (4.9.13c), das definições de $\lambda^{0}, \rho^{0}$, e da emissão $*$ sobre $Q^{0}$.

Teorema 4.9.1 Seja $f: \Sigma^{*} \rightarrow \mathrm{M}$ uma função subseqüencial, onde M é um monóide cancelativo com mdc único. Então, $\mathcal{T}_{f}$ é um $\Sigma$-M-transdutor subseqüencial minimal que realiza $f$.

Demonstração. A existência do morfismo próprio $\varphi$ demonstrada na Proposição 4.9.3 mostra que

$$
\left|\mathcal{T}_{f}\right|=f .
$$

Vamos demonstrar que a classe $\left[\mathcal{T}_{f}\right]$ é minimal na ordem $\leq$ definida no final da seção anterior. Parte dessa demonstração é a Proposição (4.9.3). Resta demonstrar que $\mathcal{T}_{f}$ é prefixo.

Seja $g$ um estado desse transdutor. Então, o mdc desse estado é

$$
\operatorname{mdc}\left\{(g * x)\left((g \cdot x) \rho^{0}\right):(g \cdot x) \rho^{0} \neq \varnothing\right\} .
$$

Pela propriedade (4.9.8c) e pela definição de $\rho^{0}$, esse mdc é igual a

$$
\operatorname{mdc}\{(\operatorname{mdc}(g, x))(1(g \cdot x)): 1(g \cdot x) \neq \varnothing\} \stackrel{(4.9 .8 \mathrm{~b})}{=} \operatorname{mdc}\{x g: x \in \operatorname{dom} g\}=\operatorname{mdc} g \text {. }
$$

Como $g \in Q^{0}, \operatorname{mdc} g=1$. Como $g$ é arbitrário, $\mathcal{T}_{f}$ é prefixo.

Como $\varphi: \mathcal{T} \rightarrow \mathcal{T}_{f}$ é uma função sobrejetora (pois é um morfismo próprio),

$$
\left|Q_{f}\right| \leq n,
$$


onde $n$ é o número de estados de $\mathcal{T}$. Essa propriedade não apenas mostra que $\mathcal{T}_{f}$ é finito, se $f$ for subseqüencial, mas também que $\mathcal{T}_{f}$ tem o menor número de estados dentre todos os transdutores que realizam $f$. Ademais, é fácil ver que, se $\mathcal{T}$ tiver o mesmo número de estados de $\mathcal{T}_{f}$ e for prefixo, então $\mathcal{T}$ e $\mathcal{T}_{f}$ são isomorfos.

Observamos que a teoria desenvolvida nesta seção pode ser feita para funções arbitrárias, se transdutores infinitos forem permitidos. De fato, a construção do transdutor minimal que apresentamos não depende em momento algum da hipótese de $f$ ser subseqüencial. Se $f$ não for subseqüencial, o transdutor $\mathcal{T}_{f}$ tem infinitos estados.

Essa observação fornece um critério para decidir se uma função é subseqüencial análogo ao Teorema XII.4.2 do livro de Eilenberg [Eil74].

Proposição 4.9.4 Uma função $f: \Sigma^{*} \rightarrow \mathrm{M}$ é subseqüencial se, e somente se, a família de funções $\left\{\hat{f} \cdot s: s \in \Sigma^{*}\right\}$ é finita.

Esse resultado aparece no trabalho de Reutenauer, para funções com imagem em um monóide livre [Reu90].

Na prática, o transdutor minimal pode ser completamente descrito a partir das seguintes informações:

$$
\begin{aligned}
& \operatorname{mdc} f \\
& \hat{f} \cdot s ; \\
& \operatorname{mdc}(\hat{f}, s), \quad \forall s \in \Sigma^{*} \text { tal que } s^{-1}(\operatorname{dom} f) \neq \varnothing .
\end{aligned}
$$

O conjunto $Q_{f}$, a emissão inicial, e as emissões finais são obtidos diretamente dessas informações. A transição de um estado $\hat{f} \cdot s$ com uma letra $\sigma$ é

$$
(\hat{f} \cdot s) \cdot \sigma=\hat{f} \cdot(s \sigma)
$$

e a emissão é

$$
(\hat{f} \cdot s) * \sigma \stackrel{(4.2 .2)}{=}(\hat{f} * s)^{-1}(\hat{f} *(s \sigma)) \stackrel{(4.9 .8 \mathrm{c})}{=}(\operatorname{mdc}(\hat{f}, s))^{-1} \operatorname{mdc}(\hat{f}, s \sigma)
$$

Vamos ilustrar a construção do transdutor minimal com dois exemplos. Outros exemplos serão vistos na próxima seção.

Exemplo 4.9.1 Seja $f: \Sigma^{*} \rightarrow \Gamma^{*}$ um morfismo de monóides. As seguintes propriedades seguem diretamente da definição de morfismo:

$$
\begin{aligned}
& \operatorname{mdc} f=1 \\
& \hat{f}=\hat{f} \cdot s=f \cdot s=f, \quad \forall s \in \Sigma^{*} \\
& \operatorname{mdc}(f, s)=s f, \quad \forall s \in \Sigma^{*} .
\end{aligned}
$$

Essas propriedades mostram que o transdutor minimal que realiza $f$ tem apenas o estado $f$. Esse transdutor está descrito no Exemplo 4.2.2.

Exemplo 4.9.2 Neste exemplo, vamos mostrar uma aplicação da Proposição 4.9.4. 
Vamos utilizar os monóides aditivos $\mathbb{N}$ e $\mathbb{R}_{+}$, onde $\mathbb{R}_{+}$é o conjunto dos números reais nãonegativos. O monóide $\mathbb{N}$ será identificado com o monóide livre gerado por uma única letra $\sigma$. Ou seja, um número natural $n$ será visto como a palavra $\sigma^{n}$.

Vamos mostrar que a função $f: \mathbb{N} \rightarrow \mathbb{R}_{+}$definida por

$$
n f=1+\frac{1}{2^{n}}, \quad \forall n \in \mathbb{N}
$$

não é subseqüencial, provando que o transdutor minimal $\mathcal{T}_{f}$ tem infinitos estados.

O mdc dessa função é

$$
\operatorname{mdc} f=\inf \left\{1+\frac{1}{2^{n}}: n \geq 0\right\}=1 \text {. }
$$

Portanto,

$$
n \hat{f}=n f-1=\frac{1}{2^{n}}, \quad \forall n \in \mathbb{N} .
$$

Para todo número natural $n$,

$$
\operatorname{mdc}(\hat{f}, n)=\operatorname{mdc}\{(n+k) \hat{f}: k \geq 0\}=\inf \left\{\frac{1}{2^{n+k}}: k \geq 0\right\}=0 .
$$

Utilizando essas informações, vamos mostrar que $\hat{f} \cdot n$ é a função definida por

$$
k(\hat{f} \cdot n)=\frac{1}{2^{n+k}}, \quad \forall k \in \mathbb{N} .
$$

Vamos usar indução em $n$ :

- $n=0$ : evidente, pois $\hat{f} \cdot n=\hat{f}$.

- $n>0$ : para todo natural $k$,

$$
k(\hat{f} \cdot n)=k(\hat{f} \cdot(n-1) \cdot 1) \stackrel{(4.9 .4)}{=}(1+k)(\hat{f} \cdot(n-1)) \stackrel{\text { h.i. }}{=} \frac{1}{2^{n+k}} .
$$

Assim, para quaisquer números naturais distintos $s$ e $t$, os estados $\hat{f} \cdot s$ e $\hat{f} \cdot t$ são distintos. Portanto, pela Proposição 4.9.4, $f$ não é subseqüencial.

Para ilustrar a independência da teoria de minimização desenvolvida nesta seção da hipótese de $f$ ser subseqüencial, vamos exibir o transdutor minimal que realiza $f$.

Utilizando (4.9.17), temos que

$$
(\hat{f} \cdot n) * 1=0, \quad \forall n \in \mathbb{N} .
$$

Como $(\hat{f} \cdot n) \cdot 1=\hat{f} \cdot(n+1), \mathcal{T}_{f}$ é o $\Sigma-\mathbb{R}_{+}$-transdutor subseqüencial infinito esquematizado abaixo:

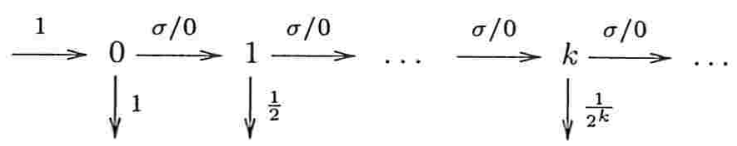

Observe que $f$ não é nem mesmo racional. De fato, a imagem de $f$ é o conjunto $\left\{1+1 / 2^{n}: n \geq\right.$ $0\}$, que não está contido em nenhum submonóide finitamente gerado de $\mathbb{R}_{+}$. Da Proposição 1.5.8, segue que esse conjunto não é racional. Pela Proposição 2.2.1, temos que $f$ não é uma função racional. 


\subsection{Congruência de uma função e redução}

Esta seção tem um duplo objetivo. O primeiro, é definir e discutir a congruência de uma função entre monóides. Essa congruência estende o conceito de congruência à direita, ou congruência de Nerode, de uma linguagem. O segundo, é mostrar um conceito de equivalência de estados para transdutores subseqüenciais, que estende o conceito correspondente para autômatos finitos. Com essa idéia, é possível construir um transdutor minimal a partir de uma partição do conjunto de estados de um dado transdutor subseqüencial.

O conceito da congruência à direita de uma função apareceu no trabalho fundamental de Schützenberger sobre funções subseqüenciais [Sch77]. Os conceitos de estados equivalentes e transdutor quociente estão no trabalho de Reutenauer [Reu90].

O conteúdo desta seção é fortemente relacionado com o da Seção 4.9. A ação · e a emissão * introduzidas naquela seção serão utilizadas novamente.

Vamos supor fixos um alfabeto $\Sigma$, um monóide cancelativo com mdc único $M$, e uma função parcial qualquer $f: \Sigma^{*} \rightarrow$ M.

Sejam $Q_{f}$ o conjunto de estados do transdutor minimal $\mathcal{T}_{f}$, e $\psi: \Sigma^{*} \rightarrow Q_{f} \cup \varnothing$ a função que associa cada palavra $s$ à função $\hat{f} \cdot s$. O símbolo $\varnothing$ representa a função vazia. A congruência à direita de $f$ é a relação de equivalência em $\Sigma^{*}$ definida pelo quociente gerado pela inversa $\psi^{-1}$. Explicitamente, para todo par de palavras $s$ e $t$ em $\Sigma^{*}$,

$$
s \sim_{f} t \text { se, e somente se, } \hat{f} \cdot s=\hat{f} \cdot t .
$$

A classe das palavras $s$ tais que $\hat{f} \cdot s=\varnothing$ será denotada por $\square$.

Para demonstrar que $\sim_{f}$ é uma congruência à direita, tome palavras equivalentes $s$ e $t$ e uma letra $\sigma$ em $\Sigma$. Então,

$$
\hat{f} \cdot(s \sigma)=(\hat{f} \cdot s) \cdot \sigma=(\hat{f} \cdot t) \cdot \sigma=\hat{f} \cdot(t \sigma) .
$$

Assim,

$$
s \sigma \sim_{f} t \sigma .
$$

Segue diretamente da definição de $\sim_{f}$ que a função

$$
\varphi:\left(\Sigma^{*} / \sim_{f}\right)-\square \longrightarrow Q_{f}
$$

definida por

$$
[s] \varphi=\hat{f} \cdot s, \forall[s] \neq \square,
$$

é uma bijeção. Portanto, $\Sigma^{*} / \sim_{f}$ é finito se, e somente se, $Q_{f}$ é finito. A Proposição 4.9 .4 implica então que $f$ ser subseqüencial é equivante a $\sim_{f}$ ter um número finito de classes de equivalência.

Uma demonstração mais construtiva desse fato, semelhante à demonstração clássica do resultado correspondente para autômatos finitos, é apresentada a seguir.

Teorema 4.10.1 Sejam $\Sigma$ um alfabeto, e M um monóide cancelativo com mdc único. Uma função $f: \Sigma^{*} \rightarrow \mathrm{M}$ é subseqüencial se, e somente se, o quociente $\Sigma^{*} / \sim_{f}$ é finito.

Demonstração. O caso em que $f$ é a função vazia é evidente. Vamos considerar que $f \neq \varnothing$. 
Suponha que $f$ seja subseqüencial, e seja $\mathcal{T}=(Q, i, \lambda, \rho)$ um $\Sigma$-M-transdutor subseqüencial bi-acessível e prefixo que realiza $f$. Utilizando esse transdutor, definimos uma congruência à direita $\sim_{\mathcal{T}}$ em $\Sigma^{*}$ por

$$
s \sim_{\mathcal{T}} t \text { se, e somente se, } i s=i t,
$$

para todo par de palavras $s$ e $t$. Essa definição considera o caso em que $i s=i t=\varnothing$. Observe que o número de classes no quociente $\Sigma^{*} / \sim \mathcal{T}$ é no máximo card $(Q+1)$.

Afirmamos que

$$
\text { se } s \sim_{\mathcal{T}} t \text {, então } \hat{f} \cdot s=\hat{f} \cdot t \text {. }
$$

De fato, seja $x$ uma palavra em $\Sigma^{*}$. Como $\mathcal{T}$ é prefixo, podemos escrever

$$
\begin{aligned}
& x(\hat{f} \cdot s)=\operatorname{mdc}(\hat{f} \cdot s, x)(\operatorname{mdc}(\hat{f} \cdot s, x))^{-1}(x(\hat{f} \cdot s)) \\
& \stackrel{(4.9 .8 \mathrm{~b})}{=} \operatorname{mdc}(\hat{f} \cdot s, x)(1((\hat{f} \cdot s) \cdot x))=\operatorname{mdc}(\hat{f} \cdot s, x)(1(\hat{f} \cdot(s x))) \stackrel{(4.9 .13)}{=}(i s * x)((i(s x)) \rho) .
\end{aligned}
$$

Da mesma forma, $x(\hat{f} \cdot t)=(i t * x)((i(t x)) \rho)$. Como $i s=i t$, e $x$ é arbitrário, obtemos (4.10.3).

A propriedade (4.10.3) mostra que o quociente $\Sigma^{*} / \sim_{\mathcal{T}}$ é um refinamento do quociente $\Sigma^{*} / \sim_{f}$. Assim, como $\Sigma^{*} / \sim_{\mathcal{T}}$ é finito, $\Sigma^{*} / \sim_{f}$ também é finito.

Suponha que $\Sigma^{*} / \sim_{f}$ seja finito. A partir dessa congruência, construímos um $\Sigma$-M-transdutor subseqüencial $\mathcal{T}$ como segue. $O$ conjunto de estados de $\mathcal{T}$ é o quociente $\Sigma^{*} / \sim_{f}$, excetuando-se a classe $\square$ (se houver). O estado inicial é $[1]$, e $[s]$ é um estado final se, e somente se, $s \in \operatorname{dom} f$. As transições e emissões desse transdutor são

$$
\begin{aligned}
& {[1] \lambda=\operatorname{mdc} f \text {, }} \\
& {[s] \cdot \sigma=[s \sigma],} \\
& {[s] * \sigma=\operatorname{mdc}(\hat{f} \cdot s, \sigma)} \\
& \text { e }[s] \rho=1(\hat{f} \cdot s) \text {, }
\end{aligned}
$$

para todo estado $[s]$ e toda letra $\sigma$ tal que $[s \sigma] \neq \square$.

Segue diretamente dessa construção que a função $\varphi$ definida em (4.10.2) é um isomorfismo do transdutor $\mathcal{T}$ no transdutor minimal $\mathcal{T}_{f}$. Portanto, $\mathcal{T}$ é um transdutor subseqüencial que realiza $f$. Assim, $f$ é subseqüencial.

A congruência $\sim_{f}$ tem como conseqüência um procedimento de construção do transdutor minimal $\mathcal{T}_{f}$ a partir de um transdutor subseqüencial bi-acessível e prefixo $\mathcal{T}=(Q, i, \lambda, \rho)$. Esse procedimento é análogo ao procedimento de redução de um autômato finito $\mathcal{A}$ no autômato minimal correspondente.

O procedimento baseia-se na definição de uma relação de equivalência $\equiv$ sobre o conjunto de estados de $\mathcal{T}$. Para definir essa relação, definimos primeiro, para cada estado $q$, uma função $q^{-1} \rho: \Sigma^{*} \rightarrow \mathrm{M}$ como segue:

$$
x\left(q^{-1} \rho\right)=(q * x)((q x) \rho), \quad \forall x \in \Sigma^{*} .
$$

Definimos $\equiv$ como

$$
p \equiv q \text { se, e somente se, } p^{-1} \rho=q^{-1} \rho \text {. }
$$


A redução de $\mathcal{T}$ é a construção do transdutor quociente $\mathcal{T} / \equiv=\left(Q_{\equiv}, i_{\equiv}, \lambda_{\equiv}, \rho_{\equiv}\right)$ definido a seguir:

$$
\begin{array}{ll} 
& Q_{\equiv}=Q / \equiv, \\
& i_{\equiv}=[i], \\
& i_{\equiv} \lambda_{\equiv}=i \lambda, \\
& {[q] \sigma=[q \sigma],} \\
& {[q] * \sigma=q * \sigma} \\
\text { e }[q] \rho_{\equiv}=q \rho,
\end{array}
$$

onde $q$ é um estado em $Q$ e $\sigma$ é uma letra.

Vamos mostrar que (4.10.7d), (4.10.7e) e (4.10.7f) não dependem de escolha do representante.

Primeiro, observe que, dados um estado $q$ e uma palavra $s$ tal que $i s=q$, segue de (4.10.4) que

$$
q^{-1} \rho=\hat{f} \cdot s .
$$

Sejam $p$ e $q$ estados equivalentes e $s$ e $t$ palavras tais que $i s=p$ e $i t=q$. Então, da definição de $\equiv$

$$
i s \equiv i t \quad \text { se, e somente se, } \quad \hat{f} \cdot s=\hat{f} \cdot t .
$$

Dessa propriedade segue que, para toda palavra $x$,

$$
p x \neq \varnothing \text { se, e somente se, } q x \neq \varnothing .
$$

No caso de $p x \neq \varnothing$, temos também as propriedades

$$
\begin{aligned}
& p x \equiv q x \\
& {[p] * x=[q] * x \stackrel{(4.9 .13 \mathrm{~b})}{=} \operatorname{mdc}(\hat{f} \cdot s, x) } \\
\mathrm{e} & {[p] \rho_{\equiv}=[q] \rho_{\equiv} \stackrel{(4.9 .13 \mathrm{c})}{=} 1(\hat{f} \cdot s) . }
\end{aligned}
$$

A primeira propriedade utiliza simplesmente o fato de que $(\hat{f} \cdot s) \cdot x=\hat{f} \cdot(s x)$ e $(\hat{f} \cdot t) \cdot x=\hat{f} \cdot(t x)$. Observe que a hipótese de $\mathcal{T}$ ser prefixo é necessária para que (4.9.13b) e (4.9.13c) possam ser usados da demonstração das outras propriedades.

De (4.10.10), segue que a definição de $\mathcal{T} / \equiv$ não depende de escolha de representantes.

Teorema 4.10.2 Seja $\mathcal{T}=(Q, i, \lambda, \rho)$ um $\Sigma$-M-transdutor subseqüencial bi-acessivel e prefixo que realiza uma função subseqüencial $f: \Sigma^{*} \rightarrow$ M. Então,

$$
\mathcal{T} / \equiv \cong \mathcal{T}_{f}
$$

Demonstração. Afirmamos que a função $\varphi: Q_{\equiv} \rightarrow Q_{f}$ definida como

$$
[i s] \varphi=\hat{f} \cdot s, \quad \forall s \in \Sigma^{*}
$$


é um isomorfismo. De fato, de (4.9.12) e (4.10.8) segue que $\varphi$ é uma bijeção. As demais propriedades necessárias para que $\varphi$ seja um isomorfismo são uma conseqüência da definição de $\equiv$, da Proposição 4.9.2, e do fato de que $\mathcal{T}$ e $\mathcal{T}_{f}$ são bi-acessíveis e prefixo.

Dizemos que um transdutor $\mathcal{T}$ é reduzido se dois estados distintos quaisquer não forem equivalentes por $\equiv$. Do Teorema 4.10.2 segue que

Corolário 4.10.1 Um $\Sigma$-M-transdutor subseqüencial é minimal se, e somente se, é bi-acessível, prefixo e reduzido.

Com base nessa discussão, a construção do transdutor minimal a partir de um transdutor subseqüencial bi-acessível $\mathcal{T}$ pode ser feita em dois passos. O primeiro é a construção do prefixo $\hat{\mathcal{T}}$. Em seguida, a relação $\equiv$ é determinada, e estados equivalentes são unidos.

Evidentemente, para que um tal procedimento seja efetivo, é preciso que o monóide de saída $\mathrm{M}$ possa ser implementado de modo que tanto a operação desse monóide, quando o cálculo de mdc 's, possam ser feitos efetivamente.

Nas seções 4.11 e 4.12, um algoritmo completo de construção do transdutor minimal será estudado para o caso em que $\mathrm{M}=\Gamma^{*}$. Exemplos dessa construção podem ser vistos na Seção 4.13.

Suponha agora que $\mathrm{M}$ seja um monóide cancelativo com mdc, sem necessariamente a propriedade de o mdc de cada conjunto ser único. É possível definir uma congruência à direita em $\Sigma^{*}$ para uma função $f: \Sigma^{*} \rightarrow M$, de maneira bem semelhante ao caso em que M é um monóide com mdc único. Concluímos a seção definindo essa congruência, e mostrando que ela caracteriza as funções subseqüenciais $\Sigma^{*} \rightarrow \mathrm{M}$.

A congruência à direita de $f$ será novamente denotada por $\sim_{f}$. Para quaisquer palavras $u$ e $v$ em $\Sigma^{*}$, definimos que $u \sim_{f} v$ se, e somente se, existem elementos $\bar{u}$ e $\bar{v}$ em $\mathrm{M}$ e uma função $h: \Sigma^{*} \rightarrow$ M tais que

$$
(u x) f=\bar{u}(x h) \quad \text { e } \quad(v x) f=\bar{v}(x h), \quad \forall x \in \Sigma^{*} .
$$

Essa definição fica mais concisa com duas notações. O símbolo · será utilizado deste ponto em diante para representá-las. A primeira, é uma ação à esquerda de $M$ sobre o conjunto $X$ de todas as funções $\Sigma^{*} \rightarrow$ M. Dados uma função $h$ em $X$ e um elemento $\alpha$ em $\mathrm{M}, \alpha \cdot h$ é a função definida por

$$
x(\alpha \cdot h)=\alpha(x h), \quad \forall x \in \Sigma^{*} .
$$

A segunda, é uma ação à direita de $\Sigma^{*}$ sobre $X$. Dados uma função $h$ em $X$ e uma palavra $s$ em $\Sigma^{*}, h \cdot s$ é a função definida por

$$
x(h \cdot s)=(s x) h, \quad \forall x \in \Sigma^{*} .
$$

É fácil ver que essas definições de fato são ações, e que satisfazem

$$
\alpha \cdot(h \cdot s)=(\alpha \cdot h) \cdot s .
$$

Com essas notações, (4.10.11) pode ser reescrito como

$$
f \cdot u=\bar{u} \cdot h \quad \text { e } \quad f \cdot v=\bar{v} \cdot h .
$$


Para mostrar que $\sim_{f}$ é uma congruência, será útil observar que toda função não-vazia $r: \Sigma^{*} \rightarrow$ M pode ser escrita como $\alpha \cdot h$, onde $h$ é uma função tal que 1 é um mdc de im $h$. De fato, seja $\alpha$ um mdc de $\operatorname{im} r$. Então, cada elemento $y \operatorname{em} \operatorname{im} r$ pode ser fatorado como $\alpha y^{\prime}$. Fixe uma fatoração, e denote o elemento $y^{\prime}$ por $y_{\alpha}$. Seja $h$ a função definida por

$$
x h=(x r)_{\alpha}, \quad \forall x \in \Sigma^{*} .
$$

Uma simples verificação mostra que

$$
r=\alpha \cdot h .
$$

Ademais, $\operatorname{im} r=\alpha(\operatorname{im} h)$. Pela Proposição 4.7.3, $\operatorname{mdc}(\alpha(\operatorname{im} h))=\alpha(\operatorname{mdc}(\operatorname{im} h))$. Como $\alpha \in \operatorname{im} r$, e $M$ é cancelativo, 1 deve ser um mdc de im $h$.

Dizemos que uma função $h$ tal que 1 é um mdc de im $h$ é uma função normalizada.

Podemos então escrever (4.10.12) como

$$
f \cdot u=(\bar{u} \alpha) \cdot h^{\prime} \quad \text { e } f \cdot v=(\bar{v} \alpha) \cdot h^{\prime},
$$

onde $h^{\prime}$ é uma função normalizada, $\alpha$ um elemento de $\mathrm{M}$, e $h=\alpha \cdot h^{\prime}$.

A relação $\sim_{f}$ é evidentemente reflexiva e simétrica. A essência da demonstração de que é transitiva é a proposição a seguir, que também será aplicada posteriormente.

Proposição 4.10.1 Sejam $r$ e $s$ funções $\Sigma^{*} \rightarrow$ M normalizadas. Se existirem elementos $\alpha$ e $\beta$ em $\mathrm{M}$ tais que $\alpha \cdot r=\beta \cdot s$, então existe um elemento inversível $t$ em $\mathrm{M}$ tal que $r=t \cdot s$.

Demonstração. Claramente, $\operatorname{im}(\alpha \cdot r)=\alpha(\operatorname{im} r)$. Como 1 é um mdc de im $r$, segue da Proposição 4.7.2, que $\alpha$ é um mdc de im $(\alpha \cdot r)$. Da mesma forma, $\beta$ é um mdc de $\operatorname{im}(\beta \cdot s)$. Como $\operatorname{im}(\alpha \cdot r)=\operatorname{im}(\beta \cdot s)$, segue da Proposição 4.7 .1 que existe um elemento inversível $t$ tal que $\beta=\alpha t$. Então, $\alpha \cdot r=(\alpha t) \cdot s$. Utilizando o cancelamento, obtemos $r=t \cdot s$.

Vamos mostrar que $\sim_{f}$ é transitiva. Sejam $u$, $v$ e $w$ palavras tais que $u \sim_{f} v$ e $v \sim_{f} w$. Existem então elementos $\bar{u}$ e $\bar{v}$ em $\mathrm{M}$ e uma função normalizada $r: \Sigma^{*} \rightarrow \mathrm{M}$ tais que

$$
f \cdot u=\bar{u} \cdot r \quad \text { e } \quad f \cdot v=\bar{v} \cdot r,
$$

e elementos $\hat{v}$ e $\hat{w}$ em $\mathrm{M}$ e uma função normalizada $s: \Sigma^{*} \rightarrow \mathrm{M}$ tais que

$$
f \cdot v=\hat{v} \cdot s \quad \text { e } \quad f \cdot w=\hat{w} \cdot s .
$$

Como $\bar{v} \cdot r=\hat{v} \cdot s$, podemos aplicar a Proposição 4.10 .1 nessas funções. Seja $t$ um inversível tal que $r=t \cdot s$. Então, $f \cdot u=(\bar{u} t) \cdot s$. Portanto, $u \sim_{f} w$.

Resta mostrar que $\sim_{f}$ é uma congruência à direta. Seja $z$ uma palavra em $\Sigma^{*}$. Então,

$$
f \cdot(u z)=(f \cdot u) \cdot z=(\bar{u} \cdot r) \cdot z=\bar{u} \cdot(r \cdot z) .
$$

Da mesma forma,

$$
f \cdot(v z)=\bar{v} \cdot(r \cdot z)
$$

Portanto, $u z \sim_{f} v z$.

Novamente, $\sim_{f}$ tem no máximo uma classe $[u]$ tal que $(u x) f=\varnothing$ para toda palavra $x$. Vamos denotar essa classe por $\square$.

O teorema a seguir caracteriza as funções subseqüenciais $\Sigma^{*} \rightarrow \mathrm{M}$. 
Teorema 4.10.3 Sejam $\Sigma$ um alfabeto, e M um monóide cancelativo com mdc. Uma função $f: \Sigma^{*} \rightarrow \mathrm{M}$ é subseqüencial se, e somente se, o quociente $\Sigma^{*} / \sim_{f}$ é finito.

Demonstração. Podemos supor que $f \neq \varnothing$.

Suponha que $f$ seja subseqüencial, e seja $\mathcal{T}=(Q, i, \lambda, \rho)$ um $\Sigma$-M-transdutor subseqüencial bi-acessível que realiza $f$. Utilizando esse transdutor, definimos uma congruência à direita $\sim_{\mathcal{T}}$ em $\Sigma^{*}$ por

$$
s \sim \mathcal{T} t \text { se, e somente se, } i s=i t,
$$

para todo par de palavras $s$ e $t$. Como no Teorema 4.10.1, card $\left(\Sigma / \sim_{\mathcal{T}}\right)$ é no máximo card $(Q+1)$.

Afirmamos que

$$
\text { se } s \sim_{\mathcal{T}} t \text {, então } s \sim_{f} t .
$$

Para provar essa afirmação, basta considerar o caso em que $i s$ e it estejam definidos. Sejam $\bar{s}=(i \lambda)(i * s), \bar{t}=(i \lambda)(i * t)$, e $h: \Sigma^{*} \rightarrow \mathrm{M}$ a função definida por

$$
x h=((i s) * x)((i(s x)) \rho), \quad \forall x \in \Sigma^{*} .
$$

Se $s \sim \mathcal{T} t$, então

$$
f \cdot s=\bar{s} \cdot h \quad \text { e } \quad f \cdot t=\bar{t} \cdot h
$$

ou seja, $s \sim_{f} t$.

Assim, o quociente $\Sigma^{*} / \sim_{\mathcal{T}}$ é um refinamento do quociente $\Sigma^{*} / \sim_{f}$. Como $\Sigma^{*} / \sim_{\mathcal{T}}$ é finito, segue que $\Sigma^{*} / \sim_{f}$ também é finito.

Suponha que $\Sigma^{*} / \sim_{f}$ seja finito. Enumere os elementos desse quociente como $\left\{c_{1}, \ldots, c_{n}\right\}$. Para cada classe $c_{i}$ diferente de $\square$, fixe um representante $u_{i}$, uma função normalizada $h_{i}: \Sigma^{*} \rightarrow \mathrm{M}$, e um elemento $\overline{u_{i}}$ de $M$ tais que

$$
f \cdot u_{i}=\overline{u_{i}} \cdot h_{i} .
$$

Utilizando esses dados, vamos construir um transdutor subseqüencial $\mathcal{T}$ que realiza $f$.

Primeiro, vamos mostrar que, para toda palavra $s$ equivalente a $u_{i}$, existe um elemento $\bar{s}$ de $\mathrm{M}$ tal que

$$
f \cdot s=\bar{s} \cdot h_{i} .
$$

De fato, como $s \sim_{f} u_{i}$, existem elementos $\alpha$ e $\beta$ de M e uma função normalizada $r$ tais que $f \cdot s=\alpha \cdot r$ e $f \cdot u_{i}=\beta \cdot r$. Como $\overline{u_{i}} \cdot h_{i}=\beta \cdot r$, a Proposição 4.10 .1 fornece um elemento inversível $t$ tal que $r=t \cdot h_{i}$. Assim, $f \cdot s=\bar{s} \cdot h_{i}$, onde $\bar{s}=\alpha t$.

O conjunto de estados de $\mathcal{T}$ é $Q=\left(\Sigma^{*} / \sim_{f}\right)-\square$. A função de transição é descrita por

$$
\left[u_{i}\right] \sigma=\left[u_{i} \sigma\right], \quad \forall\left[u_{i}\right] \in Q, \forall \sigma \in \Sigma \text { tais que }\left[u_{i} \sigma\right] \neq \square .
$$

O estado inicial é $i=[1]$, e um estado $\left[u_{i}\right]$ é final se, e somente se, $u_{i} \in \operatorname{dom} f$. Essa propriedade é equivalente a $1\left(f \cdot u_{i}\right) \neq \varnothing$, ou ainda, a $1 h_{i} \neq \varnothing$.

Antes de descrever as emissões, vamos estudar algumas propriedades desse transdutor. Primeiro, observe que

$$
\begin{gathered}
i s \neq \varnothing \quad \text { se, e somente se, }[s] \neq \square \\
\text { e } i s=[s], \quad \forall s \in \Sigma^{*} \text { tal que }[s] \neq \square .
\end{gathered}
$$


A demonstração dessas propriedades é simples, e pode ser feita por indução em $|s|$. Pela definição dos estados finais, temos então que

$$
\operatorname{dom}|\mathcal{T}|=\operatorname{dom} f .
$$

De (4.10.15b) e da definição de $Q$, segue que $\mathcal{T}$ é acessível. Para provar que é co-acessível, seja is um estado desse transdutor. Então, $f \cdot s \neq \varnothing$. Seja $x$ uma palavra tal que $x(f \cdot s) \neq \varnothing$. Então, $(s x) f \neq \varnothing$, ou seja, $1(f \cdot(s x)) \neq \varnothing$. Portanto, $[s x] \neq \square$, e $[s x]$ é um estado final. Basta agora observar que de (4.10.15b) segue que $[s] x=(i s) x=i(s x)=[s x]$. Concluímos então que $\mathcal{T}$ é bi-acessível.

Sejam $c_{i}=[s]$ uma classe diferente de $\square, \sigma$ uma letra tal que $[s \sigma] \neq \varnothing$, e $c_{j}$ a classe $[s \sigma]$. A definição da emissão $[s] * \sigma$ depende de algumas manipulações. Primeiro, escreva $f \cdot(s \sigma)$ como

$$
f \cdot(s \sigma)=(f \cdot s) \cdot \sigma=\left(\bar{s} \cdot h_{i}\right) \cdot \sigma=\bar{s} \cdot\left(h_{i} \cdot \sigma\right) .
$$

Então,

$$
\bar{s} \cdot\left(h_{i} \cdot \sigma\right)=\overline{s \sigma} \cdot h_{j} .
$$

Sejam $\alpha_{i, \sigma}$ um elemento em M e $h_{i, \sigma}$ uma função normalizada tais que $h_{i} \cdot \sigma=\alpha_{i, \sigma} \cdot h_{i, \sigma}$. Então, (4.10.16) se torna

$$
\left(\bar{s} \alpha_{i, \sigma}\right) \cdot h_{i, \sigma}=\overline{s \sigma} \cdot h_{j} .
$$

Pela Proposição 4.10.1, existe um inversível $t_{i, \sigma}$ de $\mathrm{M}$ tal que

$$
h_{i, \sigma}=t_{i, \sigma} \cdot h_{j} .
$$

Definimos então

$$
[s] * \sigma=\alpha_{i, \sigma} t_{i, \sigma} .
$$

A emissão inicial é $\overline{1}$, e a emissão de um estado final $c_{i}=[s]$ é $1 h_{i}$.

Afirmamos que

$$
\overline{1}(i * t)=\bar{t}, \quad \forall t \in \operatorname{dom} f .
$$

Para mostrar esse fato, usamos indução em $|t|$ :

- $|t|=0$ : evidente.

- $|t|>0$ : fatore $t$ como $s \sigma$, onde $\sigma$ é uma letra. Então, $\overline{1}(i * t)=\overline{1}(i * s)((i s) * \sigma)$. Pela hipótese de indução, $\overline{1}(i * s)=\bar{s}$. Sejam $c_{i}=[s]$, e $c_{j}=[s \sigma]$. Então, $(i s) * \sigma=\alpha_{i, \sigma} t_{i, \sigma}$. Assim, $\overline{1}(i * t)=\bar{s} \alpha_{i, \sigma} t_{i, \sigma}$.

Substituindo (4.10.18) em (4.10.17), obtemos

$$
\left(\bar{s} \alpha_{i, \sigma} t_{i, \sigma}\right) \cdot h_{j}=\overline{s \sigma} \cdot h_{j} .
$$

Pelo cancelamento, $\bar{s} \alpha_{i, \sigma} t_{i, \sigma}=\overline{s \sigma}$. Portanto, $\overline{1}(i * t)=\bar{t}$.

Assim, para toda palavra $t$ em $\operatorname{dom} f$,

$$
t|\mathcal{T}|=\overline{1}(i * t)([t] \rho)=\bar{t}\left(1 h_{i}\right)=1\left(\bar{t} \cdot h_{i}\right)=1(f \cdot t)=t f,
$$


onde $c_{i}=[t]$. Concluímos então que $|\mathcal{T}|=f$, e $f$ é subseqüencial.

Vamos concluir esta seção com um exemplo da redução de um transdutor, e dois exemplos da construção do transdutor minimal. Nos dois últimos exemplos, seguimos a construção descrita na Seção 4.9 , mas a congruência $\sim_{f}$ é utilizada. Outros exemplo da redução de um transdutor podem ser vistos na Seção 4.13.

Exemplo 4.10.1 Neste exemplo, vamos utilizar a função definida em (4.10.5).

Sejam $\Sigma=\{\sigma, \gamma\}, \Gamma=\{a, b\}$, e $\mathcal{T}$ o $\Sigma$ - $\Gamma$-transdutor

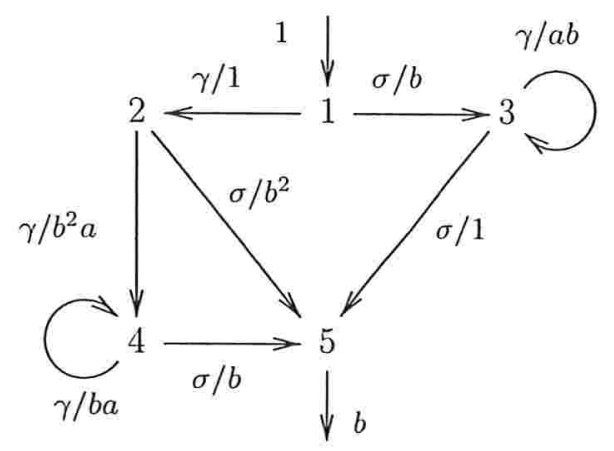

Nesse transdutor, não é possível identificar nenhum par de estados, utilizando a relação de equivalência definida em (4.10.6). No entanto, observe que

$$
\operatorname{dom}\left(2^{-1} \rho\right)=\operatorname{dom}\left(3^{-1} \rho\right)=\operatorname{dom}\left(4^{-1} \rho\right)=\gamma^{*} \sigma,
$$

e, para toda palavra $\gamma^{i} \sigma$,

$$
\left(\gamma^{i} \sigma\right)\left(2^{-1} \rho\right)=b\left(\left(\gamma^{i} \sigma\right)\left(4^{-1} \rho\right)\right)=b^{2}\left(\left(\gamma^{i} \sigma\right)\left(3^{-1} \rho\right)\right) .
$$

Portanto, as funções $2^{-1} \rho, 3^{-1} \rho$ e $4^{-1} \rho$ são iguais a menos de um prefixo.

Os mdc's dos estados de $\mathcal{T}$ são

$$
\operatorname{mdc} 1=b ; \operatorname{mdc} 2=b^{2} ; \operatorname{mdc} 3=1 ; \operatorname{mdc} 4=b ; \operatorname{mdc} 5=b .
$$

Portanto, $\mathcal{T}$ não é prefixo. Construindo o prefixo de $\mathcal{T}$, obtemos o transdutor ilustrado a seguir. Nesse transdutor, os estados 2, 3 e 4 são equivalentes:

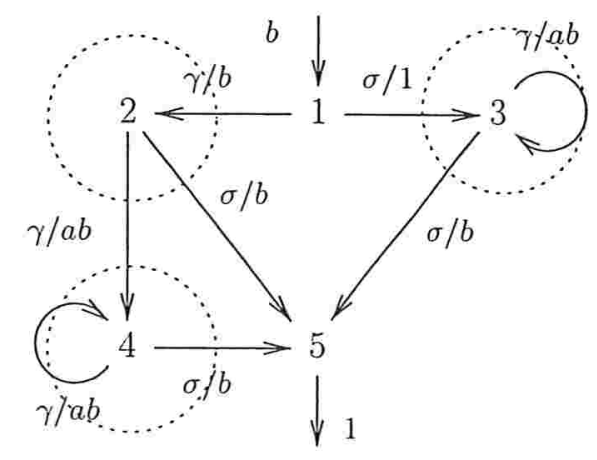


Unindo esses estados, obtemos o transdutor ilustrado a seguir, que é o transdutor minimal equivalente a $\mathcal{T}$ :

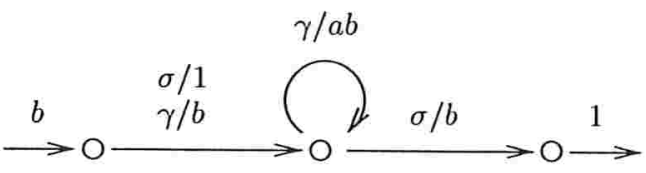

Exemplo 4.10.2 Seja $\Sigma$ um alfabeto. O conjunto das letras de $\Sigma$ que ocorrem em uma palavra $u$ sobre esse alfabeto será denotado por $|u|_{\Sigma}$. Vamos utilizar o monóide $\mathrm{M}=\mathbb{Z}_{+}-0$ dos inteiros positivos com a operação de produto. Observe que esse monóide é cancelativo e com mdc único, e que o resultado da operação $x^{-1} y$, quando definido, é o inteiro $y / x$.

Sejam $g: \Sigma \rightarrow$ M uma função total e $f: \Sigma^{*} \rightarrow \mathrm{M}$ a função parcial com domínio igual ao conjunto das palavras que contenham pelo menos uma ocorrência de cada letra de $\Sigma$, e definida por

$$
s f=\prod_{\sigma \in \Sigma}(\sigma g)^{|s|_{\sigma}}, \quad \forall s \in \operatorname{dom} f .
$$

Vamos construir o transdutor subseqüencial minimal que realiza $f$. Primeiro, vamos provar que

$$
\operatorname{mdc} f=\prod_{\sigma \in \Sigma} \sigma g
$$

$$
\operatorname{mdc}(\hat{f}, s)=\prod_{\sigma \in|s|_{\Sigma}}(\sigma g)^{\left(|s|_{\sigma}-1\right)}, \quad \forall s \in \Sigma^{*} ;
$$

$$
x(\hat{f} \cdot s)=\left(\prod_{\sigma \in|s|_{\Sigma} \cap|x|_{\Sigma}}(\sigma g)^{|x|_{\sigma}}\right)\left(\prod_{\sigma \in|x|_{\Sigma}-|s|_{\Sigma}}(\sigma g)^{\left(|x|_{\sigma}-1\right)}\right), \forall s \in \Sigma^{*}, \forall x \in \operatorname{dom}(\hat{f} \cdot s) .
$$

Em (4.10.21b) e (4.10.21c), fazemos a convenção de que um produto cujo conjunto de índices seja vazio é igual a 1.

Vamos provar (4.10.21a). Seja

$$
s \in \Sigma^{+} \text {tal que }|s|_{\sigma}=1, \quad \forall \sigma \in \Sigma .
$$

Então, pela definição de $f$, temos que

$$
s \in \operatorname{dom} f \quad \text { e } \quad s f=\prod_{\sigma \in \Sigma} \sigma g .
$$

Ademais, para toda palavra $t \operatorname{em} \operatorname{dom} f$,

$$
(s f) \mid(t f),
$$


e (4.10.21a) segue da definição de mdc.

Para demonstrar (4.10.21b) e (4.10.21c), vamos utilizar

$$
u \hat{f}=\prod_{\sigma \in \Sigma}(\sigma g)^{\left(|u|_{\sigma}-1\right)}, \quad \forall u \in \operatorname{dom} f .
$$

Essa propriedade é obtida dividindo-se $u f$ por mdc $f$.

Vamos provar (4.10.21b). Basta considerar o caso $s \neq 1$. Seja

$$
p=\prod_{\sigma \in|s|_{\Sigma}}(\sigma g)^{\left(|s|_{\sigma}-1\right)} .
$$

De (4.10.22), segue que

$$
p \mid(s x) \hat{f}, \quad \forall x \in \Sigma^{*} \text { tal que } s x \in \operatorname{dom} \hat{f} .
$$

Seja $t$ uma palavra satisfazendo

$$
|t|_{\Sigma}=\Sigma-|s|_{\Sigma}, \quad \text { ou } \quad t=1 \text {, se }|s|_{\Sigma}=\Sigma .
$$

Então, st $\in \operatorname{dom} \hat{f}$, e por (4.10.22),

$$
(s t) \hat{f}=p .
$$

Essas duas observações mostram que $\operatorname{mdc}(\hat{f}, s)=p$.

Para provar (4.10.21c), escrevemos

$$
x(\hat{f} \cdot s) \stackrel{(4.9 .8 \mathrm{~b})}{=}(\operatorname{mdc}(\hat{f}, s))^{-1}((s x) \hat{f})=\left(\prod_{\sigma \in|s|_{\Sigma}}(\sigma g)^{\left(|s|_{\sigma}-1\right)}\right)^{-1}\left(\prod_{\sigma \in \Sigma}(\sigma g)^{\left(|s x|_{\sigma}-1\right)}\right) .
$$

Na segunda igualdade, as propriedades $(4.10 .21 \mathrm{~b})$ e (4.10.22) foram utilizadas. Fazendo a manipulação

$$
\prod_{\sigma \in \Sigma}(\sigma g)^{\left(|s x|_{\sigma}-1\right)}=\left(\prod_{\sigma \in|s|_{\Sigma}}(\sigma g)^{\left(|s|_{\sigma}-1\right)}\right)\left(\prod_{\sigma \in|s|_{\Sigma} \cap|x|_{\Sigma}}(\sigma g)^{|x|_{\sigma}}\right)\left(\prod_{\sigma \in|x|_{\Sigma}-|s|_{\Sigma}}(\sigma g)^{\left(|x|_{\sigma}-1\right)}\right)
$$

e substituindo em (4.10.23), obtemos (4.10.21c).

A seguinte propriedade servirá para caracterizar os estados do transdutor $\mathcal{T}_{f}$. Dadas palavras $u$ e $v$ em $\Sigma^{*}$,

$$
u \sim_{f} v \text { se, e somente se, }|u|_{\Sigma}=|v|_{\Sigma}
$$

Suponha que $u \sim_{f} v$. Então, $\hat{f} \cdot u=\hat{f} \cdot v$. Como $\operatorname{dom} \hat{f}=\operatorname{dom} f$, segue de (4.9.8a) que

$$
u^{-1}(\operatorname{dom} f)=v^{-1}(\operatorname{dom} f) .
$$

Seja $x$ uma palavra satisfazendo

$$
|x|_{\Sigma}=\Sigma-|u|_{\Sigma}, \quad \text { ou } \quad x=1, \text { se }|u|_{\Sigma}=\Sigma
$$


Então, $x \in u^{-1} \operatorname{dom} f$. Portanto, $x \in v^{-1}(\operatorname{dom} f)$, ou seja,

$$
v x \in \operatorname{dom} f .
$$

Pela definição do domínio de $f$, temos então que $\Sigma-|x|_{\Sigma} \subseteq|v|_{\Sigma}$. Ou seja,

$$
|u|_{\Sigma} \subseteq|v|_{\Sigma}
$$

A inclusão inversa se demonstra de maneira análoga.

Suponha agora que $|u|_{\Sigma}=|v|_{\Sigma}$. Então, para toda palavra $x,|u x|_{\Sigma}=|v x|_{\Sigma}$. Portanto, $u x \in$ $\operatorname{dom} f$ se, e somente se, $v x \in \operatorname{dom} f$. Utilizando (4.9.8a), temos então que

$$
\operatorname{dom}(\hat{f} \cdot u)=\operatorname{dom}(\hat{f} \cdot v)
$$

Como $|u|_{\Sigma}=|v|_{\Sigma}$, segue de (4.10.21c) que

$$
x(\hat{f} \cdot u)=x(\hat{f} \cdot v), \quad \forall x \in \operatorname{dom}(\hat{f} \cdot u) .
$$

Portanto, $\hat{f} \cdot u=\hat{f} \cdot v$, ou seja, $u \sim_{f} v$.

Por (4.10.24), cada estado $\hat{f} \cdot s$ pode ser identificado com o conjunto de letras $|s|_{\Sigma}$. Temos então os seguintes dados:

$$
\begin{aligned}
& \begin{array}{l}
Q_{f}=2^{\Sigma} ; \\
i=\varnothing ; \\
i \lambda=\prod_{\sigma \in \Sigma} \sigma g ; \\
q \sigma=q \cup \sigma, \quad \forall q \in 2^{\Sigma}, \forall \sigma \in \Sigma .
\end{array}
\end{aligned}
$$

A última propriedade mostra que $\mathcal{T}_{f}$ é completo.

Da definição de $\operatorname{dom} f$, segue diretamente que $1 \in s^{-1}(\operatorname{dom} f)$ se, e somente se, $|s|_{\Sigma}=\Sigma$. Equivalentemente, $1(\hat{f} \cdot s) \neq \varnothing$ se, e somente se, $|s|_{\Sigma}=\Sigma$. Portanto, $\Sigma$ é o único estado final de $\mathcal{T}_{f}$. Por (4.10.21c), a emissão desse estado é

$$
\Sigma \rho^{0}=1(\hat{f} \cdot s)=1
$$

A emissão de um estado $q=\hat{f} \cdot s$ com uma letra $\sigma$ é

$$
q * \sigma \stackrel{(4.9 .17)}{=} \operatorname{mdc}(\hat{f}, s)^{-1} \operatorname{mdc}(\hat{f}, s \sigma) \stackrel{(4.10 .21 \mathrm{~b})}{=} \begin{cases}\sigma g & \text { se } \sigma \in|s|_{\Sigma}, \\ 1 & \text { caso contrário. }\end{cases}
$$

O $\Sigma$-M-transdutor subseqüencial minimal para $\Sigma=\{\sigma, \gamma, \xi\}$ e a função $g$ definida por $\sigma g=3$, $\gamma g=5$ e $\xi g=7$ é 


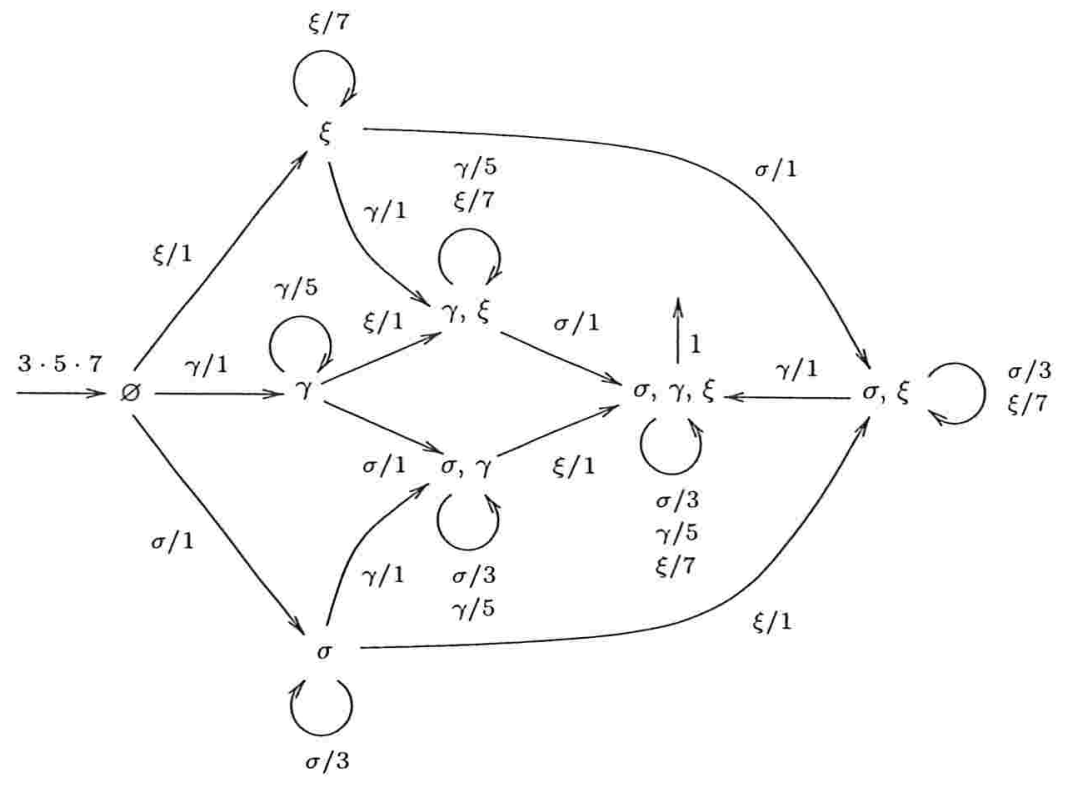

Exemplo 4.10.3 (Transdutor dos Sufixos) Vamos utilizar o monóide aditivo $\mathbb{N}$. Vamos fixar um alfabeto $\Sigma$ e uma palavra $t$ em $\Sigma^{+}$, que chamaremos de texto.

Seja $f: \Sigma^{*} \rightarrow \mathbb{N}$ a função parcial com domínio igual a $\operatorname{suf}(t)$, e definida por

$$
u f=|t|-|u| \text {. }
$$

Ou seja, $f$ associa a cada sufixo $u$ de $t$ à posição anterior ao início da última ocorrência de $u$ em $t$. Como o domínio de $f$ é finito, essa função é subseqüencial.

Crochemore chama o transdutor subseqüencial minimal que realiza $f$ de transdutor dos sufixos, e estuda algumas de suas propriedades e aplicações em [Cro86]. O autômato de entrada desse transdutor é o autômato minimal que reconhece o conjunto dos sufixos do texto, e é conhecido na literatura como autômato dos sufixos. Essas máquinas, e outras estruturas relacionadas, têm muitas aplicações em processamento de dados textuais.

A propriedade principal do transdutor dos sufixos é que o seu tamanho, entendido como o número de estados mais o número de transições, é linear no tamanho do texto. Nosso objetivo neste exemplo é fornecer uma demonstração rápida de um limitante superior para o número de estados desse transdutor em função do comprimento do texto, utilizando a construção de $\mathcal{T}_{f}$. Um estudo mais detalhado pode ser visto no trabalho de Crochemore.

Listamos abaixo sem demonstração algumas propriedades de $f$. Essas propriedades são obtidas facilmente da definição de $f$ com algumas manipulações.

(4.10.26a) $\operatorname{mdc} f=0$;

(4.10.26b) $\hat{f}=f$;

(4.10.26c) $\operatorname{dom}(f \cdot s)=\left\{x \in \Sigma^{*}: s x \in \operatorname{suf}(t)\right\}, \quad \forall s \in \Sigma^{*}$

(4.10.26d) $\operatorname{mdc}(f, s)=|t|-\max \{|x|: s x \in \operatorname{suf}(t)\}-|s|, \quad \forall s \in \Sigma^{*}$ tal que $f \cdot s \neq \varnothing$;

(4.10.26e) $z(f \cdot s)=\max \{|x|: s x \in \operatorname{suf}(t)\}-|z|, \quad \forall s \in \Sigma^{*}$ tal que $f \cdot s \neq \varnothing, \forall z \in \operatorname{dom}(f \cdot s)$. 
As propriedades a seguir são uma conseqüência de (4.10.26), e caracterizam completamente $\mathcal{T}_{f}$ :

$$
\begin{aligned}
& i=f ; \\
& i \lambda=0 ; \\
& f \cdot s \neq \varnothing \quad \text { se, e somente se, } \quad s \in \operatorname{fat}(t) \\
& (f \cdot s) * \sigma \stackrel{(4.9 .17)}{=} \max \{|x|: s x \in \operatorname{suf}(t)\}-\max \{|x|: s \sigma x \in \operatorname{suf}(t)\}-1 ; \\
& (f \cdot s) \rho=\max \{|x|: s x \in \operatorname{suf}(t)\} .
\end{aligned}
$$

Seja $n=\left|Q_{f}\right|$. Para demonstrar que $n$ é linear em $|t|$, vamos identificar cada estado de $\mathcal{T}_{f}$ com um conjunto especial de posições no texto.

Dado um fator $s$ de $t$, definimos o conjunto dos términos de $s$ em $t$ como

$$
\mathbf{t}(s)=\{|x s|: x s \in \operatorname{pref}(t)\}
$$

Sejam $u$ e $v$ dois fatores de $t$. Vamos provar que

$$
u \sim_{f} v \text { se, e somente se, } \mathbf{t}(u)=\mathbf{t}(v) .
$$

Se $f \cdot u=f \cdot v$, segue diretamente de (4.10.26c) que $\mathbf{t}(u)=\mathbf{t}(v)$. Se $\mathbf{t}(u)=\mathbf{t}(v)$, segue de $(4.10 .26 \mathrm{c})$ que dom $(f \cdot u)=\operatorname{dom}(f \cdot v)$, e de $(4.10 .26 \mathrm{e})$ que $z(f \cdot u)=z(f \cdot v)$, para toda palavra $z$ nesse domínio. Portanto, $f \cdot u=f \cdot v$.

Podemos então identificar cada estado $f \cdot s$ de $\mathcal{T}_{f}$ com o conjunto dos términos de $s$. Portanto, o número de estados desse transdutor é exatamente a cardinalidade da família $\{\mathbf{t}(s): s \in$ fat $(t)\}$.

Para avaliar essa cardinalidade, vamos utilizar um resultado puramente combinatório, que demonstramos a seguir.

Seja $S$ um conjunto não-vazio, Dizemos que uma família $\mathcal{F}$ de subconjuntos de $S$ é livre de intersecções se, para todo par $A$ e $B$ em $\mathcal{F}$, se $A \cap B \neq \varnothing$, então $A \subseteq B$ ou $B \subseteq A$. Assim, $A$ e $B$ ou têm uma relação de inclusão, ou têm intersecção vazia.

Considere o máximo

$$
n(S)=\max \left\{|\mathcal{F}|: \mathcal{F} \subseteq 2^{S} \text { e } \mathcal{F} \text { é livre de intersecções }\right\}
$$

Vamos mostrar que

$$
n(S) \leq 2|S|
$$

utilizando indução em $|S|$ :

- $|S|=1$ : trivial.

- $|S|>1: \operatorname{sejam} \mathcal{F}$ uma família livre de intersecções sobre $S, s$ um elemento qualquer em $S$, e $\mathcal{F}^{\prime}$ a família obtida de $\mathcal{F}$ retirando-se $s$ de todos os conjuntos de $\mathcal{F}$ que contenham esse elemento. Verifica-se facilmente que $\mathcal{F}^{\prime}$ é uma família livre de interseç̧ões sobre $S-s$.

Afirmamos que

$$
|\mathcal{F}| \leq\left|\mathcal{F}^{\prime}\right|+2 .
$$


De fato, suponha que $|\mathcal{F}|>\left|\mathcal{F}^{\prime}\right|+2$. Deve então existir três conjuntos dois a dois distintos $A, B$ e $C$ em $\mathcal{F}$ que contém $s$, e tais que $A-s, B-s$ e $C-s$ pertencem a $\mathcal{F}$. Como $A$, $B$ e $C$ têm o elemento $s$ em comum, devem ter dois a dois uma relação de inclusão. Como esses conjuntos são dois a dois distintos, essas inclusões devem ser próprias. Vamos supor que $A \subsetneq B \subsetneq C$. Sejam $x$ um elemento em $C-B$ e $y$ um elemento em $B-A$. Então, $x$ pertence a $(C-s)-B, s$ pertence a $B-(C-s)$, e $y$ pertence a $(C-s) \cap B$, o que contradiz o fato de que $\mathcal{F}$ é livre de intersecções. Obtemos então (4.10.29).

Pela hipótese de indução, $\left|\mathcal{F}^{\prime}\right| \leq 2|S|-2$. De (4.10.29) segue então que $|\mathcal{F}| \leq 2|S|$.

Como $\mathcal{F}$ é arbitrário, obtemos (4.10.28).

Afirmamos que $\{\mathbf{t}(s): s \in$ fat $(t)$ e $s \neq 1\}$ é uma família livre de intersecções sobre $[|t|]$.

Isso segue de duas observações. A primeira, é que, se $s$ for um fator de $t$ diferente da palavra vazia, então $\mathrm{t}(s)$ é um subconjunto de $\{1, \ldots,|t|\}$. A segunda, é que, se dois fatores $u$ e $v$ de $t$ tiverem algum término em comum, então $\mathrm{t}(u) \subseteq \mathrm{t}(v)$, ou $\mathrm{t}(v) \subseteq \mathrm{t}(u)$. De fato, segue de $\mathrm{t}(u) \cap \mathrm{t}(v) \neq$ $\varnothing$ que esses fatores são sufixos de algum prefixo de $t$. Nesse caso, $u$ é sufixo de $v$, ou $v$ é sufixo de $u$, o que implica nas inclusões.

O conjunto $Q_{f}-f$ corresponde assim a uma família livre de intersecções sobre $[|t|]$. Utilizando a (4.10.28), obtemos

$$
n \leq 2|t|+1
$$

\subsection{Construção do prefixo de um transdutor subseqüencial}

Nesta seção, descrevemos um algoritmo eficiente para a construção do prefixo de um transdutor subseqüencial com emissões em um monóide livre $\Gamma^{*}$.

O primeiro algoritmo para essa construção foi apresentado em 1994 por Mohri [Moh94, Moh00]. No entanto, o algoritmo desse autor não é correto para um caso particular, conforme observado por Choffrut [Cho03].

Em 2000, Béal e Carton apresentaram um algoritmo correto [BC00a]. Vamos descrever o algoritmo desses autores.

Em seu trabalho, Mohri observou que a construção do prefixo de um transdutor subseqüencial independe do conceito de transdutor, e pode ser formulado como um problema para autômatos. Isso se deve ao fato de que apenas as emissões das transições são consideradas nessa construção. Béal e Carton também utilizam essa observação. Assim, o algoritmo desses autores é uma construção para autômatos.

Seguindo essa abordagem, estruturamos esta seção como segue. Primeiro, formalizamos o conceito de prefixo para autômatos. Em seguida, descrevemos o algoritmo de Béal e Carton. Finalmente, mostramos como essa construção pode ser utilizada para a obtenção do prefixo de um transdutor subseqüencial.

A construção do prefixo de um autômato utiliza as mesmas idéias da demonstração do Teorema 4.8.1. Por isso, alguns argumentos dessa demonstração podem se repetir. Esses argumentos serão apenas indicados. 
Vamos supor fixo um alfabeto $\Sigma$. Dados uma palavra $s$ sobre $\Sigma$ e um inteiro $k \geq 0$, vamos denotar por $s_{k}$ a palavra definida como segue. Se $|s|>k$, então $s_{k}$ é o prefixo de comprimento $k$ de $s$. Senão, $s_{k}$ é $s$.

Nesta seção, vamos considerar $\Sigma^{*}$-autômatos, ou seja, autômatos com transições rotuladas por palavras em $\Sigma^{*}$. Transições múltiplas, ou seja, transições em que origem, término e rótulo coincidem, são permitidas. Supomos que todos os autômatos são bi-acessíveis.

Seja $\mathcal{A}=(Q, E, I, T)$ um $\Sigma^{*}$-autômato. Para cada estado $p$, denotamos por $\vec{p}$ o conjunto dos rótulos dos passeios com origem em $p$ e término em algum estado inicial ou final. Como $\mathcal{A}$ é bi-acessível, $\vec{p} \neq \varnothing$.

O prefixo de $p$, que denotamos por $\hat{p}$, é a palavra definida como

$$
\hat{p}=\wedge \vec{p} .
$$

Segue diretamente dessa definição que

$$
\hat{p}=1, \forall p \in I \cup T \text {. }
$$

Denotamos por $P_{\mathcal{A}}$ o máximo dentre os comprimentos dos prefixos dos estados de $\mathcal{A}$.

Dizemos que $\mathcal{A}$ é prefixo ou está na forma prefixo se o prefixo de todo estado for a palavra vazia.

O objetivo principal desta seção é a demonstração do teorema a seguir:

Teorema 4.11.1 Sejam A um subconjunto reconhecivel de $\Sigma^{*}$ e $\mathcal{A}$ um $\Sigma^{*}$-autômato bi-acessível que reconhece $A$. Então, existe um $\Sigma^{*}$-autômato prefixo $\hat{\mathcal{A}}$ com o mesmo grafo de $\mathcal{A}$ e que reconhece A.

A prova desse teorema é o algoritmo de Béal e Carton.

Para descrever formalmente o algoritmo, vamos definir uma ação

$$
\cdot: \mathbf{A} \times \mathbb{N}
$$

do monóide aditivo dos números naturais no conjunto $\mathbf{A}$ de todos os $\Sigma^{*}$-autômatos bi-acessíveis. Dados um $\Sigma^{*}$-autômato $\mathcal{A}=(Q, E, I, T)$ e um natural $k, \mathcal{A} \cdot k$ é um $\Sigma^{*}$-autômato com o mesmo grafo de $\mathcal{A}$, os mesmos conjuntos de estados iniciais e finais, e que difere de $\mathcal{A}$ apenas nos rótulos das transições. Para cada transição

$$
e: p \stackrel{s}{\rightarrow} q \in E,
$$

$\mathcal{A} \cdot k$ tem uma transição

$$
e^{\prime}: p \stackrel{\hat{p}_{k}^{-1}\left(s \hat{q}_{k}\right)}{\longrightarrow} q
$$

Vamos denotar $e^{\prime}$ por $e \cdot k$. Observe que $\mathcal{A} \cdot 0=\mathcal{A}$.

Para provar que $\mathcal{A} \cdot k$ está bem-definido, é preciso mostrar que $\hat{p}_{k} \succ s \hat{q}_{k}$. Essa propriedade é uma conseqüência de que $\hat{p} \succ s \hat{q}$, cuja prova é semelhante à demonstração de (4.8.4).

Para provar que · é de fato uma ação, vamos relacionar os rótulos dos passeios e os prefixos dos estados de $\mathcal{A}$ com os de $\mathcal{A} \cdot k$.

Sejam

$$
p \stackrel{s}{\rightarrow} q=e_{1} \ldots e_{n}, \quad e_{i} \in E, \quad \forall i, 1 \leq i \leq n,
$$


um passeio em $\mathcal{A}$, e

$$
p \stackrel{s^{\prime}}{\rightarrow} q=\left(e_{1} \cdot k\right) \ldots\left(e_{n} \cdot k\right)
$$

o passeio correspondente em $\mathcal{A} \cdot k$. Afirmamos que

$$
s^{\prime}=\hat{p}_{k}^{-1}\left(s \hat{q}_{k}\right) .
$$

A demonstração dessa propriedade é feita por indução no comprimento desses passeios, e é semelhante à demonstração de (4.8.5).

Sejam $p$ um estado e $y$ o prefixo de $p$ em $\mathcal{A} \cdot k$. Afirmamos que

$$
y=\hat{p}_{k}^{-1} \hat{p},
$$

onde $\hat{p}$ é o prefixo de $p$ em $\mathcal{A}$.

$\mathrm{O}$ caso em que $p$ é um estado inicial ou final é uma conseqüência direta de (4.11.2). Suponha que $p$ não seja inicial nem final. Sejam $X$ e $Y$ os conjuntos $\vec{p}$ em $\mathcal{A}$ e $\mathcal{A} \cdot k$, respectivamente. De (4.11.4) segue que (recorde a notação definida em (4.7.5))

$$
Y=\hat{p}_{k}^{-1} X \text {. }
$$

Ao aplicar (4.11.4), utilizamos o fato de que $\hat{q}_{k}=1$, já que, pela definição de $\vec{p}, q$ é um estado inicial ou final.

Multiplicando ambos os lados por $\hat{p}_{k}$ e tomando prefixos, obtemos

$$
\hat{p}_{k} y=\hat{p},
$$

o que implica em (4.11.5).

Seja $l$ um natural. Vamos provar que

$$
(\mathcal{A} \cdot k) \cdot l=\mathcal{A} \cdot(k+l) .
$$

Por (4.11.5), temos que o prefixo de $p \operatorname{em}(\mathcal{A} \cdot k) \cdot l$ é

$$
\hat{p}_{l}^{-1}\left(\hat{p}_{k}^{-1} \hat{p}\right) \stackrel{(4.7 .4 a)}{=}\left(\hat{p}_{k} \hat{p}_{l}\right)^{-1} \hat{p},
$$

onde o prefixo $\hat{p}_{l}$ é tomado em $\mathcal{A} \cdot k$. Dessa forma, $\hat{p}_{k} \hat{p}_{l}$ é o prefixo de comprimento $k+l$ de $\hat{p}$, se $|\hat{p}|>k+l$, ou $\hat{p}$, caso contrário. Ou seja,

$$
\hat{p}_{k} \hat{p}_{l}=\hat{p}_{k+l} .
$$

Seja $e: p \stackrel{s}{\rightarrow} q$ uma transição de $\mathcal{A}$. Temos então que

$$
|(e \cdot k) \cdot l|=\hat{p}_{l}^{-1}\left(\hat{p}_{k}^{-1}\left(s \hat{q}_{k}\right)\right) \hat{q}_{l} \stackrel{(4.7 .4 \mathrm{a})}{=}\left(\hat{p}_{k} \hat{p}_{l}\right)^{-1}\left(s\left(\hat{q}_{k} \hat{q}_{l}\right)\right) \stackrel{(4.11 .7)}{=} \hat{p}_{k+l}^{-1}\left(s \hat{q}_{k+l}\right)=|e \cdot(k+l)|,
$$

onde os prefixos $\hat{p}_{k}$ e $\hat{q}_{k}$ são tomados em $\mathcal{A}$, e $\hat{p}_{l}$ e $\hat{q}_{l}$ em $\mathcal{A} \cdot k$.

Assim,

$$
(e \cdot k) \cdot l=e \cdot(k+l),
$$

o que implica em (4.11.6). Portanto, · é uma ação.

Vamos denotar por $\hat{\mathcal{A}}$ o autômato $\mathcal{A} \cdot P_{\mathcal{A}}$. De (4.11.5) segue que $\hat{\mathcal{A}}$ é prefixo, e que $\hat{\mathcal{A}} \cdot k=\hat{\mathcal{A}}$, para todo $k \geq 0$.

Duas propriedades importantes de $\cdot$ são observadas a seguir. 
Proposição 4.11.1 Sejam $\mathcal{A}$ um $\Sigma^{*}$-autômato e $k \geq 0$ um inteiro. Então,

$$
|\mathcal{A} \cdot k|=|\mathcal{A}|
$$

Demonstração. Basta observar que, por (4.11.2) e (4.11.4), os rótulos de passeios bem-sucedidos correspondentes de $\mathcal{A}$ e $\mathcal{A} \cdot k$ são iguais.

A segunda propriedade utiliza a seguinte notação. Dado um $\Sigma^{*}$-autômato $\mathcal{A}$, vamos denotar por $\mathcal{A}_{0}$ o autômato com o mesmo conjunto de estados de $\mathcal{A}$, e que contém somente as transições de $\mathcal{A}$ rotuladas pela palavra vazia.

Proposição 4.11.2 Sejam $\mathcal{A}$ um $\Sigma^{*}$-autômato tal que $\mathcal{A}_{0}$ é acíclico e $k \geq 0$ um inteiro. Então, $(\mathcal{A} \cdot k)_{0}$ é acíclico.

Demonstração. Sejam $c$ um passeio fechado em $\mathcal{A}, s=|c|$, e $s^{\prime}$ o rótulo de $c \operatorname{em} \mathcal{A} \cdot k$. De (4.11.4), segue que $s^{\prime}$ é a palavra vazia se, e somente se, $s$ é a palavra vazia, o que conclui a demonstração.

Passamos à descrição do algoritmo de Béal e Carton. Como fizemos na Seção 4.6, vamos utilizar nessa descrição um elemento 0 que não pertence a $\Sigma^{*}$, e estender a operação $\wedge$ para $\Sigma^{*} \cup 0$ de modo que 0 seja uma identidade.

O algoritmo de Béal e Carton recebe um $\Sigma^{*}$-autômato $\mathcal{A}=(Q, E, I, T)$, e constrói $\hat{\mathcal{A}}$. Intuitivamente, o algoritmo executa uma seqüência de iterações, e em cada iteração puxa uma letra do prefixo de cada estado de $\mathcal{A}$. Assim, na $k$-ésima iteração, o algoritmo constrói $\mathcal{A} \cdot k$.

Vamos primeiro considerar o caso em que que $\mathcal{A}_{0}$ é acíclico, e em seguida utilizar esse caso para resolver o caso geral. O algoritmo PrefiXo-ACíCliCo contém uma descrição geral da construção de $\mathcal{A}$ nesse caso.

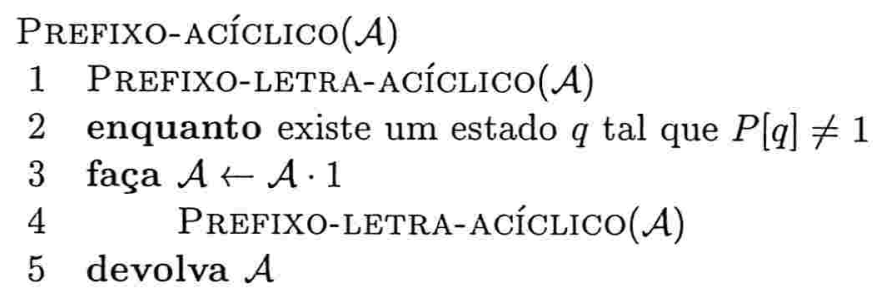

Nesse algoritmo, denotamos por $\mathrm{P}[q]$ o prefixo $\hat{q}_{1}$ de um estado $q$. O algoritmo PrefiXo-LETRAAcíclico calcula $\mathrm{P}[q]$ para cada estado $q$. Utilizando essa informação, a atualização $\mathcal{A} \leftarrow \mathcal{A} \cdot 1$ pode ser feita aplicando (4.11.3).

O algoritmo Prefixo-Letra-ACíclico simplesmente executa o algoritmo PrefiXo-LetraVISITA convenientemente, como descrito a seguir: 

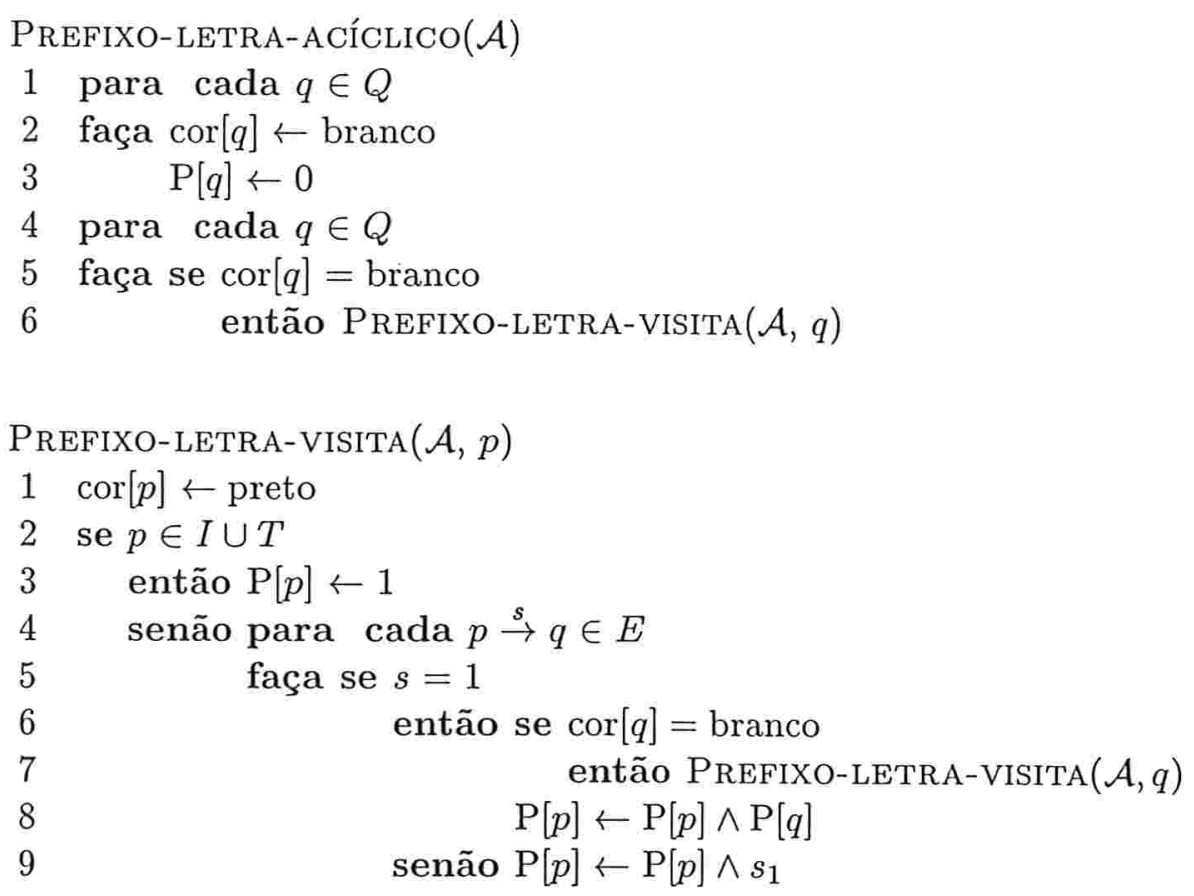

O algoritmo Prefixo-Letra-VISita calcula $\mathrm{P}[p]$, e funciona como segue. O caso em que $p$ é um estado inicial ou final é tratado diretamente. No caso contrário, o algoritmo utiliza o fato de que $\hat{p}_{1}$ é o prefixo do conjunto

$$
\left\{s_{1}: p \stackrel{s}{\rightarrow} q \in E, s \neq 1\right\} \cup\left\{\hat{q}_{1}: p \stackrel{1}{\rightarrow} q \in E\right\} .
$$

As letras no primeiro conjunto são obtidas diretamente das transições $p \stackrel{s}{\rightarrow} q$. Para o segundo conjunto, o algoritmo é executado recursivamente em cada estado $q$ tal que existe uma transição $p \stackrel{1}{\rightarrow} q$. Dessa forma, o algoritmo visita os estados como em uma busca em profundidade em $\mathcal{A}_{0}$ a partir de $p$.

Os estados são coloridos à medida que são visitados. O algoritmo tem o invariante de que, ao final de cada execução, para todo estado $q$ tal que cor $[q]=$ preto, $\mathrm{P}[q]$ já foi definido com uma palavra em $\Sigma^{*}$.

A corretude do algoritmo PrEFIXO-ACíClICO consiste na demonstração de que PrefiXo-LETRAVISITA constrói $\mathrm{P}[p]$ para cada estado $p$ corretamente. O caso em que $p$ é um estado inicial ou final é trivial. No caso contrário, vamos utilizar o invariante de que $\mathcal{A}_{0}$ é acíclico em cada iteração, conforme a Proposição 4.11.2, e a Proposição 1.4.1.

Recorde o conceito de numeração topológica, definido na Seção 1.4. Para cada execução de Prefixo-letra-VISiTA, como o grafo de $\mathcal{A}_{0}$ é acíclico, existe uma numeração topológica $\psi$ dos estados desse autômato. Vamos provar que o algoritmo define $\mathrm{P}[p] \operatorname{com} \hat{p}_{1}$, por indução em $p \psi$ :

- $p \psi=1$ : neste caso, não existe nenhuma transição da forma $p \stackrel{1}{\rightarrow} q$. Então, o algoritmo calcula $\mathrm{P}[p]$ diretamente a partir das transições da forma $p \stackrel{s}{\rightarrow} q, s \neq 1$.

- $p \psi>1$ : para toda transição $p \stackrel{1}{\rightarrow} q, q \psi<p \psi$. Pela hipótese de indução, o algoritmo define $\mathrm{P}[q]$ como $\hat{q}_{1}$. Assim, o segundo conjunto de (4.11.8) é calculado corretamente, o que conclui a demonstração. 
O algoritmo para o caso geral (isto é, $\mathcal{A}_{0}$ não é necessariamente acíclico) utiliza o algoritmo para o caso acíclico, e também uma partição de $Q$ nos componentes fortementes conexos de $\mathcal{A}_{0}$. Essa partição é útil devido à propriedade de que os prefixos dos estados em um mesmo componente são iguais.

Os componentes fortemente conexos de $\mathcal{A}_{0}$ são gerados pela relação $\sim$ sobre $Q$ definida por

$$
p \sim q \text { se, e somente se, existem passeios } p \stackrel{1}{\rightarrow} q \text { e } q \stackrel{1}{\rightarrow} p \text { em } \mathcal{A} .
$$

O componente a que pertence um estado $p$ será denotado por $C(p)$.

Veremos que as transições da forma $p \stackrel{1}{\rightarrow} q$ tais que $C(p)=C(q)$ são irrelevantes para a construção do prefixo. Vamos chamar essas transições de internas, e as demais de externas.

Utilizando $\sim$, definimos um $\Sigma^{*}$-autômato $\mathcal{A}^{\prime}=\left(Q^{\prime}, E^{\prime}, I^{\prime}, T^{\prime}\right)$ como segue:

- $Q^{\prime}=Q / \sim$;

- $I^{\prime}$ é o conjunto dos componentes que possuem algum estado inicial de $\mathcal{A}$;

- $T^{\prime}$ é o conjunto dos componentes que possuem algum estado final de $\mathcal{A}$;

- As transições de $\mathcal{A}^{\prime}$ relacionam-se com as transições externas de $\mathcal{A}$. Para cada transição externa $p \stackrel{s}{\rightarrow} q, \mathcal{A}^{\prime}$ tem uma transição

$$
C(p) \stackrel{s}{\rightarrow} C(q) .
$$

Relacionamos $\mathcal{A}$ e $\mathcal{A}^{\prime}$ com uma função

$$
f: E \rightarrow E^{\prime}
$$

que associa as transições externas de $\mathcal{A}$ às transições correspondentes de $\mathcal{A}^{\prime}$. Definindo ef $=1$ para toda transição interna $e$, podemos estender $f$ para um morfismo $E^{*} \rightarrow E^{\prime *}$ com as seguintes propriedades:

(4.11.9a) Se $s \in E^{*}$ for um passeio de um estado $p$ a um estado $q$ em $\mathcal{A}$, então $s f$ é um passeio de $C(p)$ a $C(q)$ em $\mathcal{A}^{\prime}$ com o mesmo rótulo.

Se $s \in E^{\prime *}$ for um passeio de um componente $C(p)$ a um componente $C(q)$ em $\mathcal{A}^{\prime}$, então existe um passeio $x \in E^{*}$ de $p$ a $q$ em $\mathcal{A}$ com o mesmo rótulo tal que $x f=s$.

Utilizando (4.11.9), podemos demonstrar as propriedades a seguir, onde $k$ é um natural qualquer, $p$ e $q$ são estados de $\mathcal{A}$, e $e$ é uma transição externa de $\mathcal{A}$ :

$$
\begin{aligned}
& \mathcal{A}_{0}^{\prime} \text { é acíclico; } \\
& \mathcal{A}^{\prime} \text { é bi-acessível; } \\
& \left|\mathcal{A}^{\prime}\right|=|\mathcal{A}| ; \\
& \hat{p}=C \hat{(p)} ; \\
& \text { se } p \sim q, \text { então } \hat{p}=\hat{q} ; \\
& |e \cdot k|=|(e f) \cdot k| .
\end{aligned}
$$


As três primeiras propriedades são triviais. Para demonstrar (4.11.10d), basta notar que (4.11.9) implica em

$$
\vec{p}=\overrightarrow{C(p)} .
$$

Finalmente, (4.11.10e) e (4.11.10f) são conseqüências de (4.11.10d).

Para construir $\mathcal{A}^{\prime}$, o primeiro passo é obter os componentes fortemente conexos do grafo de $\mathcal{A}_{0}$. $\mathrm{O}$ algoritmo clássico para calcular os componentes fortemente conexos de um grafo pode ser visto em [CLRS01].

Esse algoritmo também pode armazenar em cada vértice $q$ uma indicação do componente $C(q)$. Utilizando essa informação, as transições de $\mathcal{A}^{\prime}$ são obtidas percorrendo as transições de $\mathcal{A}$.

Utilizando o autômato $\mathcal{A}^{\prime}$, a construção de $\hat{\mathcal{A}}$ pode ser feita como segue:

$\operatorname{Prefixo}(\mathcal{A})$

$1 \mathcal{A}^{\prime} \leftarrow$ Constrói-Autômato-Componentes $(\mathcal{A})$

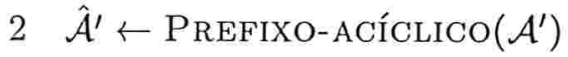

3 substitua o rótulo de cada transição externa $e$ de $\mathcal{A}$ por $\left|(e f) \cdot P_{\mathcal{A}^{\prime}}\right|$

Observe que esse algoritmo modifica $\mathcal{A}$. O autômato resultante é $\hat{\mathcal{A}}$, conforme demonstrado a seguir.

Seja $e: p \stackrel{s}{\rightarrow} q$ uma transição externa de $\mathcal{A}$. Após a execução do algoritmo Prefixo-ACíclico, temos que

$$
(e f) \cdot P_{\mathcal{A}^{\prime}}=C(p) \stackrel{\left.C \hat{(p)})^{-1}(s C \hat{q})\right)}{\longrightarrow} C(q) .
$$

Por $(4.11 .10 \mathrm{~d})$, o rótulo de $(e f) \cdot P_{\mathcal{A}^{\prime}}$ é igual a $\hat{p}^{-1}(s \hat{q})$. Portanto, os rótulos das transições externas do autômato resultante são corretos.

Ademais, a substituição dessas transições é suficiente para construir $\hat{\mathcal{A}}$. De fato, segue da propriedade (4.11.10e) que os rótulos das transições internas de $\mathcal{A}$ e $\hat{\mathcal{A}}$ são os mesmos.

Observamos que, ao longo da seqüência de iterações, é possível alterar diretamente os rótulos das transições externas de $\mathcal{A}$, ao invés das transições de $\mathcal{A}^{\prime}$.

Vamos agora mostrar como esse algoritmo pode ser utilizado para a construção do prefixo de um transdutor subseqüencial letra-palavra.

Sejam $\Gamma$ um alfabeto e $\mathcal{T}=(Q, i, \lambda, \rho)$ um $\Sigma$ - $\Gamma$-transdutor subseqüencial. Vamos fazer uma pequena modificação na definição de autômato de saída de um transdutor introduzida no Capítulo 2. $\mathrm{O}$ autômato de saída de $\mathcal{T}$ é o $\Gamma^{*}$-autômato $\mathcal{A}=\left(Q^{\prime}, E, i^{\prime}, t^{\prime}\right)$ definido como segue:

- $Q^{\prime}=Q \cup\left\{i^{\prime}, t^{\prime}\right\}$, onde os estados $i^{\prime}$ e $t^{\prime}$ não pertencem a $Q$;

- As transições de $\mathcal{A}$ são definidas como segue:

- para cada estado $q$ em $Q$ e cada letra $\sigma$ tais que $q \sigma \neq \varnothing, E$ tem uma transição

$$
e_{q, \sigma}: q \stackrel{q * \sigma}{\longrightarrow} q \sigma ;
$$

- para cada estado final $t$ em $Q, E$ tem uma transição

$$
e_{t}: t \stackrel{t \rho}{\rightarrow} t^{\prime}
$$


- $E$ tem uma transição

$$
e_{i}: i^{\prime} \stackrel{i \lambda}{\rightarrow} i
$$

Segue diretamente da definição de prefixo de um estado em (4.8.1) que, para todo estado $p$ em $Q$, o prefixo de $p$ em $\mathcal{T}$ é igual ao prefixo $\operatorname{de} p$ em $\mathcal{A}$.

Assim, para construir $\hat{\mathcal{T}}$, o primeiro passo é construir o prefixo de $\mathcal{A}$. Em seguida, utilizando os rótulos das transições de $\hat{\mathcal{A}}$, é possível alterar as saídas das transições, dos estados finais e do estado inicial de $\mathcal{T}$ segundo (4.8.2a), (4.8.2b) e (4.8.2c). Essas alterações estão descritas a seguir:

- para cada estado $p$ em $Q$ e cada letra $\sigma$ tal que $p \sigma \neq \varnothing$, a emissão $p * \sigma$ é alterada para $\left|e_{q, \sigma} \cdot P_{\mathcal{A}}\right|$

- a emissão inicial é alterada para $(i \lambda)\left|e_{i} \cdot P_{\mathcal{A}}\right|$;

- para cada estado final $t$, a emissão $t \rho$ é alterada para $\left|e_{t} \cdot P_{\mathcal{A}}\right|^{-1}(t \rho)$.

O transdutor resultante é $\hat{\mathcal{T}}$.

Concluímos a seção com uma análise das complexidades de tempo e espaço.

O algoritmo clássico para a construção dos componentes fortemente conexos de um grafo tem complexides de tempo e espaço $O(\operatorname{card}(Q)+\operatorname{card}(E))$.

Além de $\mathcal{A}^{\prime}$, as únicas estruturas auxiliares construídas são os prefixos $\mathrm{P}[q]$ e as colorações cor $[q]$. Portanto, a complexidade de espaço do algoritmo é

$$
O(\operatorname{card}(Q)+\operatorname{card}(E)) \text {. }
$$

No algoritmo Prefixo-Letra-ACÍclico, cada estado e cada transição são visitados uma vez. Portanto, a complexidade de tempo desse algoritmo é $O(\operatorname{card}(Q)+\operatorname{card}(E))$.

Cada iteração do algoritmo de Béal e Carton consiste em uma execução de Prefixo-LETRAACíclico, e a atualização $\mathcal{A} \leftarrow \mathcal{A} \cdot 1$. Nessa atualização, cada transição é visitada uma vez, e os rótulos devem ser atualizados em tempo constante. Uma possibilidade é armazenar esses rótulos como listas ligadas. Assim, tanto a remoção quanto a inserção de uma letra em uma ponta da lista podem ser feitas em tempo constante.

Portanto, a complexidade de tempo de cada iteração é $O(\operatorname{card}(Q)+\operatorname{card}(E))$. São feitas $P_{\mathcal{A}}$ iterações até a construção de $\hat{\mathcal{A}}$. Logo, a complexidade de tempo do algoritmo é

$$
O\left(P_{\mathcal{A}}(\operatorname{card}(Q)+\operatorname{card}(E))\right) .
$$

Exemplo 4.11.1 A execução do algoritmo para um caso simples em que $\mathcal{A}_{0}$ é acíclico está ilustrada na Figura 4.11.1.

Exemplo 4.11.2 Vamos mostrar a execução do algoritmo de Béal e Carton para o autômato ilustrado abaixo. Nesse autômato, $\mathcal{A}_{0}$ não é acíclico. A tabela à esquerda indica o prefixo de cada estado. 


\begin{tabular}{l|l}
1 & 1 \\
\hline 2 & $\gamma$ \\
\hline 3 & $\sigma$ \\
\hline 4 & 1
\end{tabular}

\begin{tabular}{l|l}
1 & 1 \\
\hline 2 & $\sigma$ \\
\hline 3 & $\gamma$ \\
\hline 4 & 1
\end{tabular}

\begin{tabular}{l|l}
1 & 1 \\
\hline 2 & 1 \\
\hline 3 & $\sigma$ \\
\hline 4 & 1
\end{tabular}

\begin{tabular}{l|l}
1 & 1 \\
\hline 2 & 1 \\
\hline 3 & 1 \\
\hline 4 & 1
\end{tabular}
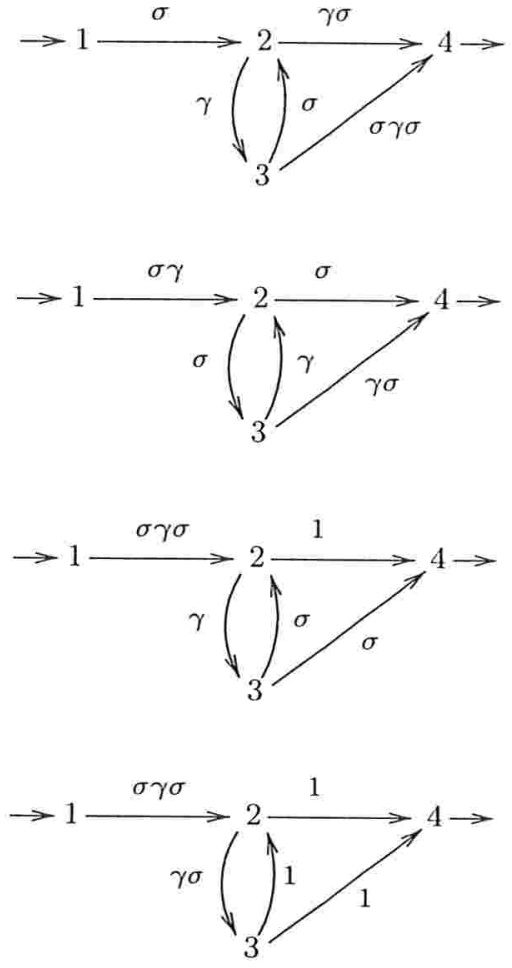

Figura 4.11.1: Construção do prefixo de $\mathcal{A}$ pelo algoritmo de Béal e Carton. A coluna da direita mostra $\mathcal{A} \cdot k$ em cada iteração do algoritmo, e a da esquerda o vetor $P$. A primeira linha contém $\mathcal{A}$.

\begin{tabular}{c|c}
$q$ & $\hat{q}$ \\
\hline$i$ & 1 \\
\hline 1 & $a$ \\
\hline 2 & $a$ \\
\hline 3 & $a$ \\
\hline 4 & $a b a$ \\
\hline 5 & $a b a$ \\
\hline 6 & $a b a$ \\
\hline 7 & $b a$ \\
\hline 8 & $a b a$ \\
\hline$t$ & 1
\end{tabular}

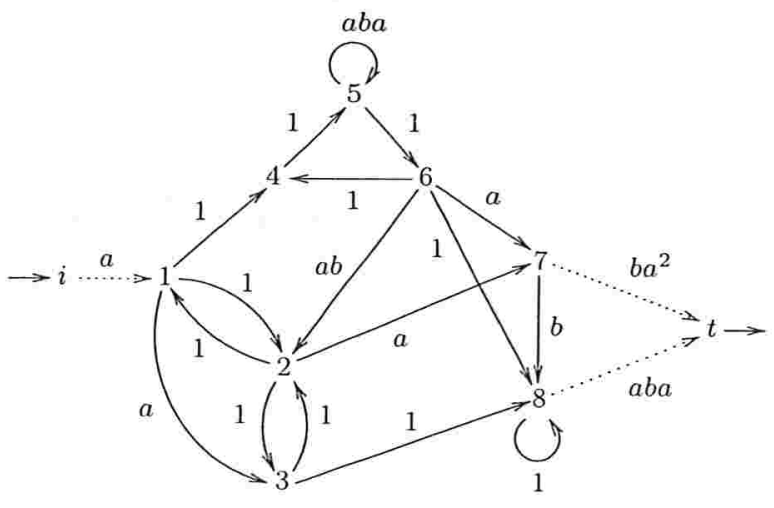

Os componentes fortemente conexos de $\mathcal{A}_{0}$ estão indicados a seguir: 


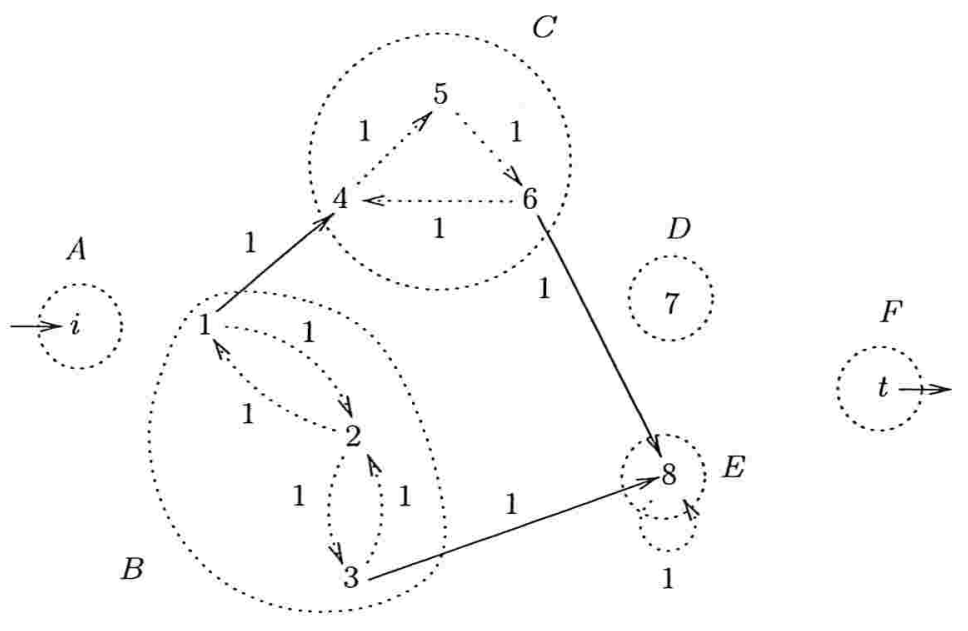

O autômato $\mathcal{A}^{\prime}$ está ilustrado a seguir. A tabela à esquerda indica o prefixo de cada estado.

\begin{tabular}{c|c}
$q$ & $\hat{q}$ \\
\hline$A$ & 1 \\
\hline$B$ & $a$ \\
\hline$C$ & $a b a$ \\
\hline$D$ & $b a$ \\
\hline$E$ & $a b a$ \\
\hline$F$ & 1
\end{tabular}

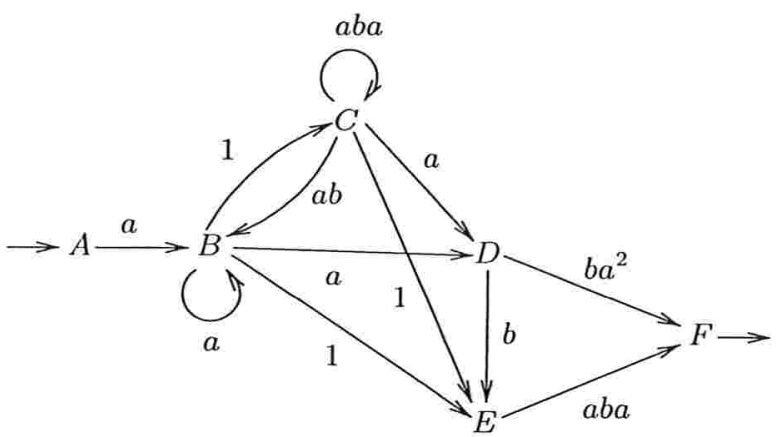

A execução do algoritmo PrefiXo-ACÍclico para $\mathcal{A}$ está ilustrada na Figura 4.11.2. Observe que, nessa simulação, alteramos diretamente as transições externas de $\mathcal{A}$, ao invés de utilizar $\mathcal{A}^{\prime}$.

\subsection{Construção do transdutor minimal}

Nesta seção, apresentamos um algoritmo para a construção do transdutor minimal equivalente a um transdutor subseqüencial.

Conforme observado por Mohri, esse problema pode ser reduzido ao problema de minimizar um autômato finito [Moh00]. Portanto, esta seção consiste na descrição de um algoritmo para a redução de um autômato, e na adaptação desse algoritmo para reduzir um transdutor.

Dentre os algoritmos de redução conhecidos, o mais eficiente foi descoberto por Hopcroft em 1971 [Hop71]. O artigo de Hopcroft é de difícil leitura, e uma nova descrição do seu algoritmo foi apresentada por Gries em 1973 [Gri73], com algumas pequenas modificações. Neste texto, baseamonos no trabalho de Gries, e quando escrevemos algoritmo de Hopcroft, estamos nos referindo à versão de Gries. 
\begin{tabular}{l|l}
$i$ & 1 \\
\hline 1 & $a$ \\
\hline 2 & $a$ \\
\hline 3 & $a$ \\
\hline 4 & $a$ \\
\hline 5 & $a$ \\
\hline 6 & $a$ \\
\hline 7 & $b$ \\
\hline 8 & $a$ \\
\hline$t$ & 1
\end{tabular}

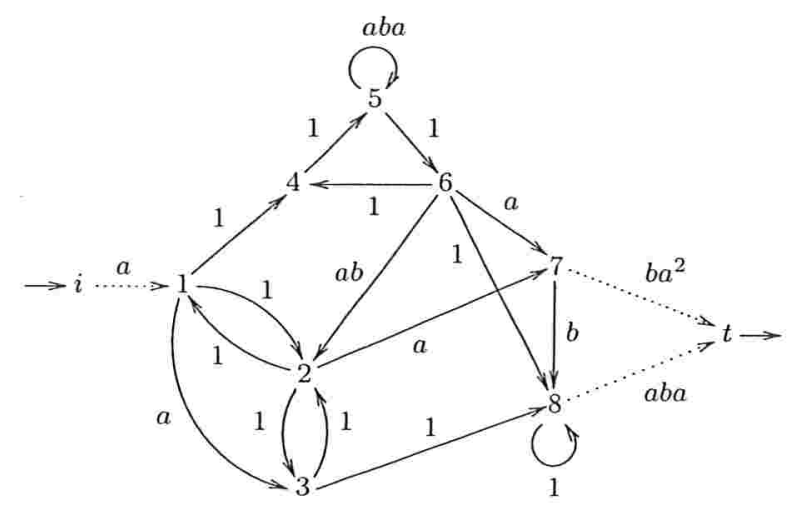

\begin{tabular}{c|c}
$i$ & 1 \\
\hline 1 & 1 \\
\hline 2 & 1 \\
\hline 3 & 1 \\
\hline 4 & $b$ \\
\hline 5 & $b$ \\
\hline 6 & $b$ \\
\hline 7 & $a$ \\
\hline 8 & $b$ \\
\hline$t$ & 1
\end{tabular}

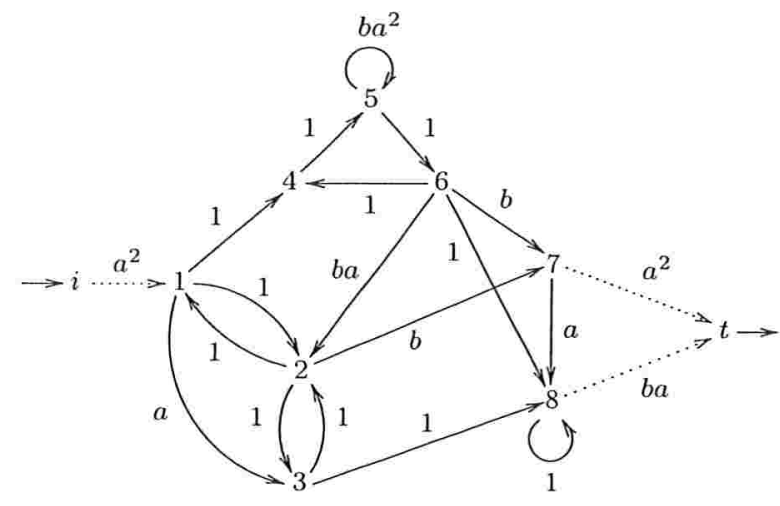

\begin{tabular}{l|l}
$i$ & 1 \\
\hline 1 & 1 \\
\hline 2 & 1 \\
\hline 3 & 1 \\
\hline 4 & $a$ \\
\hline 5 & $a$ \\
\hline 6 & $a$ \\
\hline 7 & 1 \\
\hline 8 & $a$ \\
\hline$t$ & 1
\end{tabular}

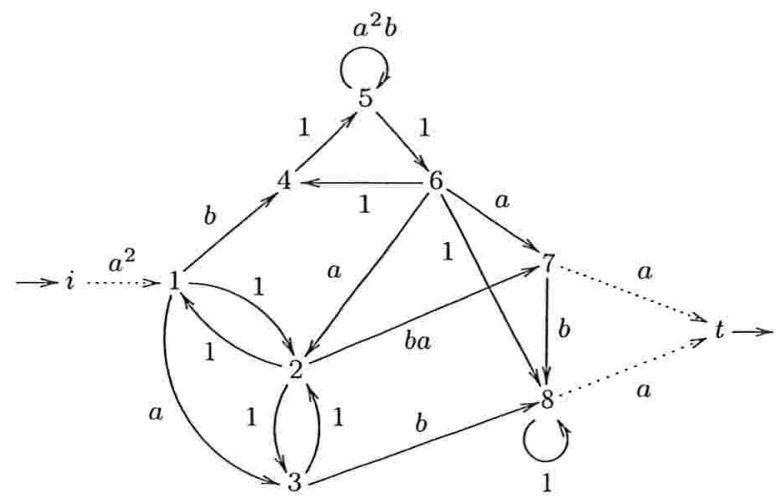

\begin{tabular}{c|c}
$i$ & 1 \\
\hline 1 & 1 \\
\hline 2 & 1 \\
\hline 3 & 1 \\
\hline 4 & 1 \\
\hline 5 & 1 \\
\hline 6 & 1 \\
\hline 7 & 1 \\
\hline 8 & 1 \\
\hline$t$ & 1
\end{tabular}

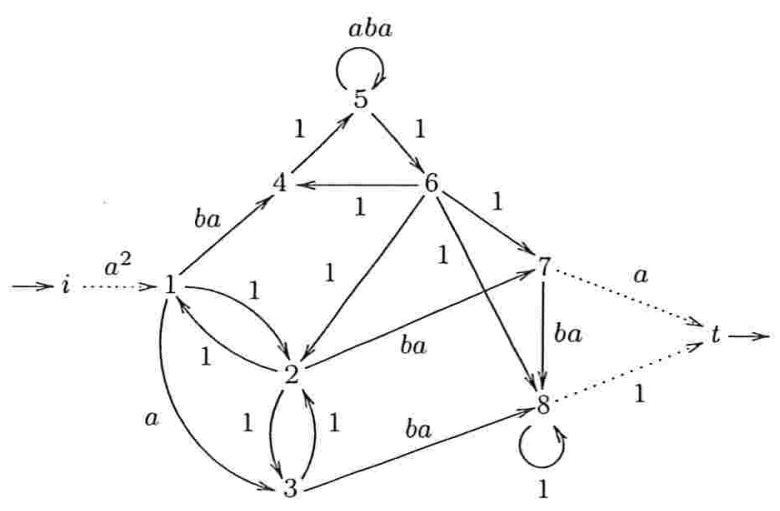

Figura 4.11.2: Construção do prefixo de um autômato pelo algoritmo de Béal e Carton. O exemplo é organizado como na Figura 4.11.1. 
Antes de descrever o algoritmo de Hopcroft, vamos deduzir alguns resultados da teoria da minimização de autômatos finitos a partir da teoria desenvolvida nas seções 4.10 e 4.9. As propriedades aqui descritas são iguais às desenvolvidas nas seções III.4, III.5 e III.6 do livro de Eilenberg [Eil74]. Essa relação entre as teorias de minimização de autômatos e de transdutores já havia sido observada por Reutenauer [Reu90], mas sem um desenvolvimento explícito.

Seja M um monóide que só contém a identidade. Vamos considerar uma linguagem $A$ sobre $\Sigma$ como uma função parcial

$$
A: \Sigma^{*} \rightarrow \mathrm{M}
$$

que satisfaz

$$
s A \neq \varnothing \text { se, e somente se, } s \in A, \quad \forall s \in \Sigma^{*} \text {. }
$$

Da mesma forma, um $\Sigma$-autômato determinístico pode ser visto com um $\Sigma$-M-transdutor subseqüencial (claramente prefixo). Portanto, um subconjunto $A$ de $\Sigma^{*}$ é reconhecível se, e somente se, é subseqüencial, quando visto como uma função.

Com base nessas observações, vamos definir um morfismo para autômatos finitos determinísticos, a partir do conceito correspondente para transdutores.

Sejam $\mathcal{A}=(Q, i, T)$ e $\mathcal{A}^{\prime}=\left(Q^{\prime}, i^{\prime}, T^{\prime}\right) \Sigma$-autômatos determinísticos bi-acessíveis. Adaptando a Definição 4.8.2, definimos um morfismo $\varphi: \mathcal{A} \rightarrow \mathcal{A}^{\prime}$ como uma função parcial $\varphi: Q \rightarrow Q^{\prime}$ que satisfaz

$$
\begin{aligned}
& i^{\prime}=i \varphi ; \\
& (q \varphi) \sigma \subseteq(q \sigma) \varphi ; \\
& T^{\prime} \varphi^{-1} \subseteq T,
\end{aligned}
$$

onde $q$ é um estado qualquer em $Q$ e $\sigma$ é uma letra. Adaptando (4.8.10), dizemos que $\varphi$ é um morfismo próprio se as propriedades a seguir forem satisfeitas:

$$
\begin{aligned}
& q \sigma \neq \varnothing \text { implica em }(q \varphi) \sigma \neq \varnothing, \quad \forall q \in Q, \forall \sigma \in \Sigma ; \\
& T \varphi \subseteq T^{\prime} .
\end{aligned}
$$

Segue de (4.8.9) que, se existir um morfismo

$$
\varphi: \mathcal{A} \rightarrow \mathcal{A}^{\prime}
$$

então

$$
\left|\mathcal{A}^{\prime}\right| \subseteq|\mathcal{A}| .
$$

Ademais, se $\varphi$ for um morfismo próprio, então $\left|\mathcal{A}^{\prime}\right|=|\mathcal{A}|$.

Dizemos que $\varphi$ é um isomorfismo se $\varphi$ for um morfismo próprio bijetor. Nesse caso, dizemos que $\mathcal{A}$ e $\mathcal{A}^{\prime}$ são isomorfos, e podemos dizer que esses autômatos são iguais a menos dos rótulos.

Para formalizar a noção de autômato minimal reconhecendo um conjunto, procedemos de maneira análoga à discussão feita no final da Seção 4.8 .

Vamos denotar por $\cong$ a relação de equivalência no conjunto $\mathbf{A}$ de todos os $\Sigma$-autômatos determinísticos bi-acessíveis definida por

$$
\mathcal{A} \cong \mathcal{A}^{\prime} \text { se, e somente se, } \mathcal{A} \text { e } \mathcal{A}^{\prime} \text { são isomorfos. }
$$


Em seguida, definimos uma ordem parcial $\leq$ no quociente $\mathbf{A} / \cong$ por

$$
\left[\mathcal{A}^{\prime}\right] \leq[\mathcal{A}] \text { se, e somente se, existe um morfismo próprio } \varphi: \mathcal{A} \rightarrow \mathcal{A}^{\prime} .
$$

Um autômato minimal reconhecendo um conjunto $A$ é um autômato na classe de isomorfismo minimal de $\leq$ restrita ao conjunto de todos os $\Sigma$-autômatos determinísticos bi-acessíveis que reconhecem $A$. Podemos dizer que um autômato minimal para um conjunto é único a menos de isomorfismo.

Esse autômato pode ser construído algebricamente a partir de $A$, de maneira semelhante à feita na Seção 4.9.

Primeiro, identificamos $Q^{0}$ com o conjunto de todos os subconjuntos não-vazios de $\Sigma^{*}$. A ação - definida em (4.9.4) torna-se então uma ação do monóide livre $\Sigma^{*}$ sobre $Q^{0}$ definida por

$$
X \cdot s=s^{-1} X, \forall X \in Q^{0}, \forall s \in \Sigma^{*} .
$$

Seguindo a construção do transdutor $\mathcal{T}_{f}^{0} \mathrm{em}$ (4.9.6), definimos um $\Sigma$-autômato determinístico infinito

$$
\mathcal{A}_{A}^{0}=\left(Q^{0}, A, T^{0}\right) \text {, }
$$

onde $T^{0}$ é o conjunto de todos os subconjuntos de $\Sigma^{*}$ que contêm a palavra vazia, e a função de transição é a ação definida acima.

A parte acessível desse autômato é um $\Sigma$-autômato bi-acessível que reconhece $A$, e que denotamos por $\mathcal{A}_{A}$. O conjunto de estados de $\mathcal{A}_{A}$ é

$$
\left\{s^{-1} A: s \in \Sigma^{*}\right\}
$$

e o conjunto de estados finais é

$$
\left\{s^{-1} A: s \in A\right\} .
$$

A Proposição 4.9.3 pode ser reescrita como segue:

Proposição 4.12.1 Sejam $A$ um conjunto reconhecível, e $\mathcal{A}$ um $\Sigma$-autômato determinístico biacessivel que reconhece $A$. Então, existe um morfismo próprio $\varphi: \mathcal{A} \rightarrow \mathcal{A}_{A}$.

Portanto, $\mathcal{A}_{A}$ é o autômato minimal reconhecendo $A$.

Agora, vamos discutir como $\mathcal{A}_{A}$ pode ser construído a partir de um $\Sigma$-autômato determinístico bi-acessível $\mathcal{A}=(Q, i, T)$. Essa construção é análoga à redução de um transdutor subseqüencial, descrita em (4.10.7).

Primeiro, definimos uma relação de equivalência $\equiv$ em $Q$ como

$$
p \equiv q \text { se, e somente se, } p^{-1} T=q^{-1} T,
$$

onde

$$
p^{-1} T=\{x: p x \in T\} .
$$

$\mathrm{O}$ autômato quociente de $\mathcal{A}$, ou autômato reduzido, é o $\Sigma$-autômato $\mathcal{A} / \equiv=\left(Q_{\equiv}, i_{\equiv}, T_{\equiv}\right)$ definido por

$$
\begin{aligned}
& Q_{\equiv}=Q / \equiv \\
& i_{\equiv}=[i], \\
& {[q] \sigma=[q \sigma],} \\
& {[q] \in T \equiv \text { se, e somente se, } q \in T,}
\end{aligned}
$$


onde $q$ é um estado em $Q$ e $\sigma$ é uma letra.

A proposição a seguir afirma que o autômato reduzido é o autômato minimal reconhecendo $A$. Esse resultado é uma conseqüência do Corolário 4.10.1.

Proposição 4.12.2 Um $\Sigma$-autômato é minimal se, e somente se, é bi-acessivel e reduzido.

Para facilitar a descrição do algoritmo de Hopcroft, vamos utilizar apenas autômatos acessíveis completos. Concluímos a discussão a respeito da teoria da minimização com algumas observações sobre esses autômatos.

Qualquer $\Sigma$-autômato determinístico bi-acessível não-completo $\mathcal{A}$ pode ser transformado em um $\Sigma$-autômato determinístico completo e acessível equivalente com a adição de um novo estado $\square$, e definindo

- $\square \sigma=\square$, para toda letra $\sigma$;

- $q \sigma=\square$, para toda letra $\sigma$ tal que $q \sigma=\varnothing$ em $\mathcal{A}$.

Vamos denominar esse autômato de completamento de $\mathcal{A}$, e denotá-lo por $\mathcal{A}^{\mathrm{c}}$. Esse autômato não é bi-acessível, porque $\square$ não é co-acessível.

Uma equivalência de estados para um $\Sigma$-autômato determinístico completo acessível, não necessariamente bi-acessível, pode ser definida de maneira análoga a (4.12.3). Utilizando essa equivalência, um autômato quociente pode ser construído da mesma forma que em (4.12.4). O autômato obtido também é acessível e completo.

A proposição a seguir mostra a relação entre o quociente de um autômato bi-acessível e o do seu completamento. Essa proposição é elementar, e não será demonstrada.

Proposição 4.12.3 Seja $\mathcal{A}$ um $\Sigma$-autômato determinístico bi-acessível. Se $\mathcal{A}$ não for completo, então $\mathcal{A} / \equiv$ é igual à parte bi-acessivel de $\mathcal{A}^{\mathrm{c}} / \equiv$.

Passamos agora à descrição do algoritmo de Hopcroft.

O algoritmo de Hopcroft recebe um autômato acessível completo, e constrói o quociente desse autômato. Para minimizar um autômato não-completo, primeiro o completamento desse autômato deve ser construído. $\mathrm{O}$ autômato minimal equivalente ao original pode então ser obtido eliminando a parte não-co-acessível do autômato minimal construído, conforme afirmado na Proposição 4.12.3.

Nossa descrição do algoritmo de Hopcroft depende de uma série de formalismos e notações, que introduzimos a seguir. Vamos supor fixo um $\Sigma$-autômato determinístico completo $\mathcal{A}=(Q, i, T)$.

A cardinalidade de $Q$ será denotada por $n$, e a de $\Sigma$ por $k$. Vamos supor uma ordenação $\left\{\sigma_{1}, \ldots, \sigma_{k}\right\}$ nesse alfabeto.

Dado um subconjunto $X$ de $Q$, vamos denotar por $[X]$ o inteiro

$$
[X]=\max \left\{i: \frac{n}{2^{i}} \geq \operatorname{card}(X)\right\} .
$$

O formalismo principal em nossa descrição é o de partição de $Q$. O termo partição será usado com o significado de partição de $Q$.

Os subconjuntos de uma partição serão denominados blocos. Dada uma partição, o bloco a que um estado $q$ pertence será denotado por $B(q)$. Essa nomenclatura será usada para conformidade com a literatura sobre o algoritmo de Hopcroft. 
Dizemos que um subconjunto $Z$ de $Q$ respeita uma partição $\mathcal{P}$ se $Z u$ for um subconjunto de algum bloco de $\mathcal{P}$ para toda palavra $u$ em $\Sigma \cup 1$.

Uma partição admissivel é uma partição $\mathcal{P}$ que satisfaz as condições a seguir:

$$
\begin{aligned}
& \text { para todo bloco } X \text {, ou } X \cap T=\varnothing \text {, ou } X \cap T=X ; \\
& Q / \equiv \text { é um refinamento de } \mathcal{P} \text {. }
\end{aligned}
$$

A condição (4.12.6b) é equivalente à afirmação

se dois estados forem equivalentes por $\equiv$, então pertencem a um mesmo bloco de $\mathcal{P}$.

Um exemplo simples de partição admissível é $Q / \equiv$.

Uma configuração é um par $(\mathcal{P}, \theta)$, onde $\mathcal{P}$ é uma partição admissível, e $\theta$ é uma função total $\theta: \mathcal{P} \rightarrow[k+1]$

Seja $C=(\mathcal{P}, \theta)$ uma configuração. Dizemos que um subconjunto $Z$ de $Q$ respeita $C$ se

$$
Z u \subseteq X \text { ou } Z u \subseteq Q-X, \quad \forall X \in \mathcal{P}, \forall u \in\left\{\sigma_{i}: X \theta \leq i \leq k\right\} \cup 1
$$

Sejam $X$ e $Y$ blocos de $C$, com $Y \theta \leq k$, e $i=Y \theta$. A divisão de $X$ com relação $a Y$, denotada por $X / Y$, é definida como segue. Se

$$
X \sigma_{i} \subseteq Y \text { ou } X \sigma_{i} \subseteq Q-Y \text {, }
$$

então

$$
X / Y=\{X\}
$$

Senão,

$$
X / Y=\{\bar{X}, \tilde{X}\}
$$

onde

$$
\bar{X}=\left\{q \in X: q \sigma_{i} \in Y\right\} \text { e } \widetilde{X}=\left\{q \in X: q \sigma_{i} \notin Y\right\} .
$$

Neste último caso, denotamos

$$
X^{<}=\bar{X} \quad \text { e } \quad X^{>}=\tilde{X}
$$

se

$$
\operatorname{card}(\bar{X}) \leq \operatorname{card}(\tilde{X})
$$

e

$$
X^{<}=\tilde{X} \quad \text { e } \quad X^{>}=\bar{X}
$$

caso contrário.

Seja $Y$ um bloco tal que $Y \theta \leq k$, e $i=Y \theta$. O refinamento de $C$ por $Y$ é a configuração $C^{\prime}=\left(\mathcal{P}^{\prime}, \theta^{\prime}\right)$ definida como segue:

- $\mathcal{P}^{\prime}$ é o refinamento de $\mathcal{P}$ definido por

$$
\mathcal{P}^{\prime}=\bigcup_{X \in \mathcal{P}} X / Y
$$


- Para todo bloco $X$ em $\mathcal{P}-\{Y\}$, se $X / Y=\{X\}$, então

$$
X \theta^{\prime}=X \theta .
$$

Senão,

$$
X^{<} \theta^{\prime}=1 \text { e } X^{>} \theta^{\prime}=X \theta .
$$

- Se $Y / Y=\{Y\}$, então

$$
Y \theta^{\prime}=Y \theta+1
$$

Senão,

$$
Y^{<} \theta^{\prime}=1 \text { e } Y^{>} \theta^{\prime}=Y \theta+1 .
$$

É preciso mostrar que $\mathcal{P}^{\prime}$ é uma partição admissível.

Como $\mathcal{P}^{\prime}$ é um refinamento de $\mathcal{P}$, temos que essa partição satisfaz (4.12.6a). Para mostrar que satisfaz (4.12.6b), basta provar que para todo bloco $X$ de $\mathcal{P}$ dividido em $\bar{X}$ e $\widetilde{X}$ e todo par de estados $p$ em $\bar{X}$ e $q$ em $\widetilde{X}, p \not \equiv q$. De fato, por construção, temos que $p \sigma \in Y$ e $q \sigma \notin Y$, ou vice-versa, para alguma letra $\sigma$. Como $\mathcal{P}$ satisfaz (4.12.6b), temos que $p \sigma \not \equiv q \sigma$. Então, (4.10.10a) implica em $p \not \equiv q$.

Uma propriedade útil de $\equiv$ utilizando essas notações está demonstrada a seguir.

Proposição 4.12.4 Seja $\mathcal{P}$ uma partição admissível. Então, $\mathcal{P}=Q / \equiv$ se, e somente se, para todo bloco $X$ em $\mathcal{P}$ e toda letra $\sigma$, existe um bloco $Y$ tal que

$$
X \sigma \subseteq Y \text {. }
$$

Demonstração. Suponha que $\mathcal{P}=Q / \equiv$. Então, (4.12.8) é uma conseqüência de (4.10.10a).

Suponha que $\mathcal{P}$ satisfaça (4.12.8). Por indução, temos que para todo bloco $X$ e toda palavra $s$,

$$
X s \subseteq Y \text {. }
$$

Portanto, dados estados $p$ e $q$ em $X$,

$$
p s \in T \text { se, e somente se, } q s \in T \text {. }
$$

Ou seja,

$$
p \equiv q
$$

Assim, $\mathcal{P}$ é um refinamento de $Q / \equiv$. Pela definição de partição admissível, temos então que $\mathcal{P}=Q / \equiv$.

Em linhas gerais, o algoritmo de Hopcroft recebe $\mathcal{A}$, e realiza uma seqüência de refinamentos de uma partição de $Q$, até a obtenção de $Q / \equiv$.

Mais precisamente, o algoritmo executa uma seqüência de iterações. Cada iteração começa com uma configuração $C=(\mathcal{P}, \theta)$. A primeira iteração começa com $C=(\{T, Q-T\}, \theta)$, onde

$$
\begin{aligned}
& T \theta= \begin{cases}1 & \text { se card }(T) \leq \operatorname{card}(Q-T) \\
k+1 & \text { caso contrário }\end{cases} \\
& (Q-T) \theta= \begin{cases}k+1 & \text { se card }(T) \leq \operatorname{card}(Q-T) \\
1 & \text { caso contrário }\end{cases}
\end{aligned}
$$

Cada iteração consiste no seguinte: 
Caso $1 X \theta=k+1$ para todo bloco $X$ : devolva $\mathcal{P}$ e pare;

Caso $2 Y \theta \leq k$ para algum bloco $Y$ : comece nova iteração com o refinamento de $C$ por $Y$ no papel de $C$.

O algoritmo pára, porque ao final de cada iteração ou $\mathcal{P}^{\prime}$ é um refinamento não-trivial de $\mathcal{P}$, ou $Y \theta^{\prime}=Y \theta+1$ para algum bloco $Y$.

A complexidade de tempo depende da complexidade da execução do refinamento de uma configuração. Antes de detalhar essa operação, bem como mostrar a corretude do algoritmo, vamos provar dois invariantes.

Invariante 4.12.1 Todo subconjunto de $Q$ que respeita a configuração $C$ no início de uma iteração respeita também a partição correspondente $\mathcal{P}$.

Demonstração. O invariante é claramente verdadeiro no início da primeira iteração.

Sejam $C=(\mathcal{P}, \theta)$ a configuração no início de uma iteração arbitrária, $Y$ o bloco escolhido pelo algoritmo, e $C^{\prime}=\left(\mathcal{P}^{\prime}, \theta^{\prime}\right)$ o refinamento de $C$ por $Y$. Supondo o invariante válido para $C$, vamos primeiro mostrar que, ao final dessa iteração, todo subconjunto de $Q$ que respeita $C^{\prime}$ respeita $C$.

Seja $Z$ um conjunto que respeita $C^{\prime}$. Como $\mathcal{P}^{\prime}$ é um refinamento de $\mathcal{P}, Z$ é um subconjunto de algum bloco em $\mathcal{P}$. Resta então provar que, para todo bloco $X$ em $\mathcal{P}$ e todo $i, X \theta \leq i \leq k$,

$$
Z \sigma_{i} \subseteq X \text { ou } Z \sigma_{i} \subseteq Q-X
$$

Essa propriedade é trivialmente verdadeira se $X \theta=k+1$. Suponha que $X \theta \leq k$. Vamos considerar quatro casos:

- $X \neq Y$ e $X / Y=\{X\}$ : Então $X \theta^{\prime}=X \theta$, e (4.12.9) segue do fato de que $Z$ respeita $C^{\prime}$.

- $X \neq Y$ e $X / Y=\left\{X^{>}, X^{<}\right\}$: Seja $i$ um inteiro entre $X \theta$ e $k$. Como $X^{<} \theta^{\prime}=1$ e $Z$ respeita $C^{\prime}$, ou $Z \sigma_{i} \subseteq X^{<}$, ou $Z \sigma_{i} \subseteq Q-X^{<}$. No primeiro caso, $Z \sigma_{i} \subseteq X$. Suponha o segundo caso. Como $X^{>} \theta^{\prime}=X \theta$, ou $Z \sigma_{i} \subseteq X^{>}$, ou $Z \sigma_{i} \subseteq Q-X^{>}$. No primeiro caso, $Z \sigma_{i} \subseteq X$, e no segundo, $Z \sigma_{i} \subseteq Q-X$. Logo, obtemos (4.12.9).

- $X=Y$ e $Y / Y=\{Y\}$ : Seja $i=Y \theta$. Como $Z$ é um subconjunto de algum bloco em $\mathcal{P}^{\prime}$, segue da definição de refinamento de $C$ por $Y$ que ou $Z \sigma_{i} \subseteq Y$, ou $Z \sigma_{i} \subseteq Q-Y$. Seja $j$ um inteiro tal que $Y \theta<j \leq k$. Como $Y \theta^{\prime}=Y \theta+1$ e $Z$ respeita $C^{\prime}$, ou $Z \sigma_{j} \subseteq Y$, ou $Z \sigma_{j} \subseteq Q-Y$. Obtemos assim (4.12.9).

- $X=Y$ e $Y / Y=\left\{Y^{>}, Y^{<}\right\}$: Seja $i=Y \theta$. Como no caso anterior, ou $Z \sigma_{i} \subseteq Y$, ou $Z \sigma_{i} \subseteq Q-Y$. Seja $j$ um inteiro tal que $Y \theta<j \leq k$. Como $Y^{<} \theta^{\prime}=1$ e $Z$ respeita $C^{\prime}$, ou $Z \sigma_{j} \subseteq Y^{<}$, ou $Z \sigma_{j} \subseteq Q-Y^{<}$. No primeiro caso, $Z \sigma_{j} \subseteq Y$. Suponha o segundo caso. Como $Y^{>} \theta^{\prime}=Y \theta+1$, ou $Z \sigma_{j} \subseteq Y^{>}$, ou $Z \sigma_{j} \subseteq Q-Y^{>}$. No primeiro caso, $Z \sigma_{j} \subseteq Y$, e no segundo, $Z \sigma_{j} \subseteq Q-Y$. Logo, obtemos (4.12.9).

Concluímos então que $Z$ respeita $C$.

Pela hipótese de que $C$ satisfaz o invariante, temos então que $Z$ respeita $\mathcal{P}$. Então, para cada letra $\sigma$, existe um bloco $X$ em $\mathcal{P}$ tal que $Z \sigma \subseteq X$. Se $X$ não for um bloco em $\mathcal{P}^{\prime}$, então 
$X / Y=\left\{X^{>}, X^{<}\right\}$, e esses blocos pertencem a $\mathcal{P}^{\prime}$. Como $X^{<} \theta=1$ e $Z$ respeita $C^{\prime}$, ou $Z \sigma \subseteq X^{<}$, ou $Z \sigma \subseteq Q-X^{<}$. Como $Z \sigma \subseteq X$, temos no segundo caso que $Z \sigma \subseteq X^{>}$. Portanto, $Z \sigma$ está contido em algum bloco em $\mathcal{P}^{\prime}$. Como $\sigma$ é arbitrário, concluímos que $Z$ respeita $\mathcal{P}^{\prime}$.

O próximo invariante utiliza uma seqüência de funções totais $Q \times \Sigma \rightarrow \mathbb{N}$ associada a uma execução do algoritmo. Cada função corresponde a uma iteração. Vamos denotar todas essas funções por uma mesma letra $V$, e descrever as alterações nas imagens de $V$ ao longo de uma execução. A imagem de um par $(q, \sigma)$ por $V$ será denotada por $V(q, \sigma)$.

Antes da execução do algoritmo, todas as imagens de $V$ são iguais a 0 . Essa função só é alterada nas execuções do Caso 2. A cada execução desse caso, $V\left(q, \sigma_{i}\right)$, onde $i=Y \theta$, é incrementado de 1 para cada $q$ em $Y$. Para os demais pares, a imagem por $V$ não se altera.

Invariante 4.12.2 No início de cada iteração, para todo estado q e toda letra $\sigma_{i}$,

$$
V\left(q, \sigma_{i}\right) \leq \begin{cases}{[B(q)]-1} & \text { se } i \geq B(q) \theta \\ {[B(q)]} & \text { se } i<B(q) \theta .\end{cases}
$$

Demonstração. O invariante é claramente verdadeiro no início da primeira iteração.

Sejam $C=(\mathcal{P}, \theta)$ a configuração no início de uma iteração arbitrária, $Y$ o bloco escolhido pelo algoritmo, $C^{\prime}=\left(\mathcal{P}^{\prime}, \theta^{\prime}\right)$ o refinamento de $C$ por $Y$, e $V^{\prime}$ a atualização de $V$ nessa iteração. Vamos denotar por $j$ o inteiro $Y \theta$, e por $X$ e $X^{\prime}$ os blocos a que $q$ pertence em $\mathcal{P}$ e $\mathcal{P}^{\prime}$, respectivamente. Fazendo a hipótese de que o invariante é válido para $V$, vamos mostrar que, ao final dessa iteração,

$$
V^{\prime}\left(q, \sigma_{i}\right) \leq \begin{cases}{\left[X^{\prime}\right]-1} & \text { se } i \geq X^{\prime} \theta^{\prime} \\ {\left[X^{\prime}\right]} & \text { se } i<X^{\prime} \theta^{\prime}\end{cases}
$$

Vamos considerar quatro casos:

- $X \neq Y$ e $X / Y=\{X\}$ : Como $X \neq Y$, segue da definição da atualização de $V$ que $V^{\prime}\left(q, \sigma_{i}\right)=$ $V\left(q, \sigma_{i}\right)$. Ademais, $X^{\prime}=X$ e $X^{\prime} \theta^{\prime}=X \theta$. Assim, (4.12.10) segue diretamente da hipótese.

- $X \neq Y$ e $X / Y=\left\{X^{>}, X^{<}\right\}$: Como $X \neq Y$, segue da definição da atualização de $V$ que $V^{\prime}\left(q, \sigma_{i}\right)=V\left(q, \sigma_{i}\right)$. Se $X^{\prime}=X^{>}$, então $\left[X^{\prime}\right] \geq[X]$ e $X^{\prime} \theta^{\prime}=X \theta$. Utilizando a hipótese, obtemos (4.12.10). Suponha que $X^{\prime}=X^{<}$. Como $\operatorname{card}\left(X^{<}\right) \leq \operatorname{card}(X) / 2,\left[X^{\prime}\right]>[X]$. Da hipótese, segue que $V^{\prime}\left(q, \sigma_{i}\right) \leq\left[X^{\prime}\right]-1$, para todo $i, 1 \leq i \leq k$. Obtemos então novamente (4.12.10).

- $X=Y$ e $Y / Y=\{Y\}$ : Então $\left[X^{\prime}\right]=[X]$ e $X \theta^{\prime}=Y \theta^{\prime}=j+1$. Se $i \neq j$, então, pela definição da atualização de $V, V^{\prime}\left(q, \sigma_{i}\right)=V\left(q, \sigma_{i}\right)$, e (4.12.10) segue diretamente da hipótese. Suponha que $i=j$. Então, $V^{\prime}\left(q, \sigma_{i}\right)=V\left(q, \sigma_{i}\right)+1$. Pela hipótese, $V\left(q, \sigma_{i}\right) \leq[X]-1$. Então, $V^{\prime}\left(q, \sigma_{i}\right) \leq\left[X^{\prime}\right]$. Como $i<X \theta^{\prime}$, obtemos novamente (4.12.10).

- $X=Y$ e $Y / Y=\left\{Y^{>}, Y^{<}\right\}$: Suponha que $X^{\prime}=Y^{>}$. Então, $\left[X^{\prime}\right] \geq[X]$ e $X^{\prime} \theta^{\prime}=j+1$. O restante da demonstração é análoga ao caso anterior.

Suponha que $X^{\prime}=Y^{<}$. Como $\operatorname{card}\left(Y^{<}\right) \leq \operatorname{card}(Y) / 2,\left[X^{\prime}\right]>[X]$. Suponha que $i \neq j$. Então, $V^{\prime}\left(q, \sigma_{i}\right)=V\left(q, \sigma_{i}\right)$. Da hipótese, segue que $V^{\prime}\left(q, \sigma_{i}\right) \leq\left[X^{\prime}\right]-1$, tanto no caso $i>j$ 
quanto no $i<j$. Obtemos então (4.12.10). Suponha que $i=j$. Então, $V^{\prime}\left(q, \sigma_{i}\right)=V\left(q, \sigma_{i}\right)+$ 1. Pela hipótese, $V\left(q, \sigma_{i}\right) \leq[X]-1$. Então, $V\left(q, \sigma_{i}\right) \leq\left[X^{\prime}\right]-2$, ou seja, $V^{\prime}\left(q, \sigma_{i}\right) \leq\left[X^{\prime}\right]-1$. Obtemos então novamente (4.12.10).

Utilizando os invariantes, vamos demonstrar que o algoritmo é correto, e analisar a complexidade de tempo.

Seja $C=(\mathcal{P}, \theta)$ a configuração devolvida pelo algoritmo. Então, a imagem de todos os blocos por $\theta$ é $k+1$. Assim, todos os blocos de $\mathcal{P}$ respeitam trivialmente $C$. Pelo Invariante 4.12.1, todos os blocos respeitam $\mathcal{P}$. Da Proposição 4.12.4, segue que $\mathcal{P}=Q / \equiv_{\mathcal{A}}$, o que mostra que o algoritmo é correto.

Para analisar a complexidade de tempo, vamos primeiro detalhar o refinamento de uma configuração $C=(\mathcal{P}, \theta)$ por um bloco $Y$. Essa operação depende fortemente das estruturas de dados utilizadas, e uma possibilidade de implementação é descrita a seguir.

Uma configuração é implementada como uma lista ligada de blocos. Um bloco $X$ é uma estrutura composta pelas seguintes informações:

- a cardinalidade de $X$;

- o valor de $X \theta$;

- um estado $q$ em $X$;

- um inteiro card'.

O significado do inteiro card' será explicado posteriormente.

O conjunto dos estados de $X$ é implementado como uma lista duplamente ligada. As seguintes informações são armazenadas em cada estado $q$ :

- apontadores para os estados anterior e seguinte na lista do bloco a que $q$ pertence;

- um apontador para o bloco a que $q$ pertence.

Assim, a remoção e inserção de um estado em um bloco podem ser feitas com complexidade de tempo constante. Ademais, a lista dos estados em $X$ pode ser acessada a partir do estado $q$ armazenado nesse bloco.

O algoritmo também deve construir de antemão uma estrutura que permita obter eficientemente o conjunto

$$
D_{p, \sigma}=\{q: q \sigma=p\},
$$

para cada estado $p$ e cada letra $\sigma$. Essa estrutura pode ser implementada como uma tabela $\Delta$, indexada por $Q \times \Sigma$, que armazena em cada posição $\Delta[p, \sigma]$ uma lista ligada com os estados em $D_{p, \sigma}$. Essa tabela pode ser construída com uma única passagem pelo conjunto de transições de $\mathcal{A}$.

Com essas estruturas, o refinamento de $C$ por $Y$ pode ser feito em duas etapas.

Seja $i=Y \theta$. A primeira etapa é a construção do conjunto

$$
D=\bigcup_{p \in Y} D_{p, \sigma_{i}}
$$


realizando uma única passagem por $Y$ e utilizando a tabela $\Delta$. Note que, como $\mathcal{A}$ é determinístico, os conjuntos $D_{p, \sigma_{i}}$ são dois a dois disjuntos.

A segunda etapa consiste em percorrer $D$ para determinar as divisões de blocos necessárias. $\mathrm{O}$ algoritmo realiza essas divisões retirando os estado em $D$ dos blocos que se dividem. Mais precisamente, primeiro o algoritmo determina a cardinalidade do conjunto

$$
X \cap D,
$$

para cada bloco $X$ tal que $X \cap D \neq \varnothing$. Essa cardinalidade é armazenada na variável auxiliar card ' de $X$. Para tal, o algoritmo realiza uma única passagem por $D$. A cada estado $q$ visitado, o valor de card' de $B(q)$ é incrementado.

Utilizando essa informação, o algoritmo constrói os blocos

$$
X \cap D \quad \text { e } \quad X-D
$$

a partir de cada bloco $X$ tal que

$$
X \cap D \neq \varnothing \quad \text { e } \quad X-D \neq \varnothing .
$$

Esses conjuntos são exatamente os blocos $\bar{X}$ e $\tilde{X}$, respectivamente, descritos em (4.12.7). Se $X-D=\varnothing$ ou $X \cap D=\varnothing$, então $X$ não é dividido.

Essas divisões podem ser feitas percorrendo novamente $D$. Para cada estado $q$ visitado, pode-se decidir se $B(q)$ é um bloco sendo dividido comparando card $(B(q))$ com a variável card' desse bloco. Em caso afirmativo, $q$ é retirado da lista de estados de $B(q)$, e inserido em um novo bloco para o conjunto $X \cap D$.

Nesse processo, as cardinalidades dos blocos e os valores de $B(q)$ para cada estado em $D$ devem ser atualizadas convenientemente.

A atualização de $\theta$ pode ser feita comparando-se as cardinalidades de $X$ e $X \cap D$, para cada bloco $X$ dividido. Com essa comparação, pode-se decidir qual subconjunto é $X^{>}$e qual é $X^{<}$.

Cada novo bloco $X \cap D$ deve ser adicionado a $C$. A configuração resultante é exatamente $C^{\prime}$.

A complexidade de tempo total desse processo é

$$
\operatorname{card}(Y)+\operatorname{card}(D) \text {. }
$$

A cada iteração, o algoritmo deve escolher um bloco $Y$ tal que $Y \theta \leq k$ em tempo constante. Para tal, o algoritmo mantém uma lista $L$ com os blocos que satisfazem essa condição. Essa lista deve ser atualizada convenientemente durante um refinamento.

O Algoritmo 4.12.1 é um detalhamento do algoritmo de Hopcroft, utilizando as estruturas descritas.

Concluímos então que a complexidade de tempo de uma iteração é proporcional a (4.12.11). Portanto, a complexidade de tempo total é proporcional à soma dessa expressão para cada iteração do algoritmo.

Para estimar essa soma, vamos contar o número de vezes que cada estado é visitado. Primeiro, vamos considerar apenas as visitas realizadas aos estados nos blocos $Y$. A partir desse número, vamos deduzir o número de visitas feitas aos estados nos conjuntos $D$.

Sejam $q$ um estado e $\sigma$ uma letra. Segue diretamente da definição de $V$ que o número de vezes que $q$ é visitado em um refinamento de uma configuração $C=(\mathcal{P}, \theta)$ por um bloco $Y$ tal que 


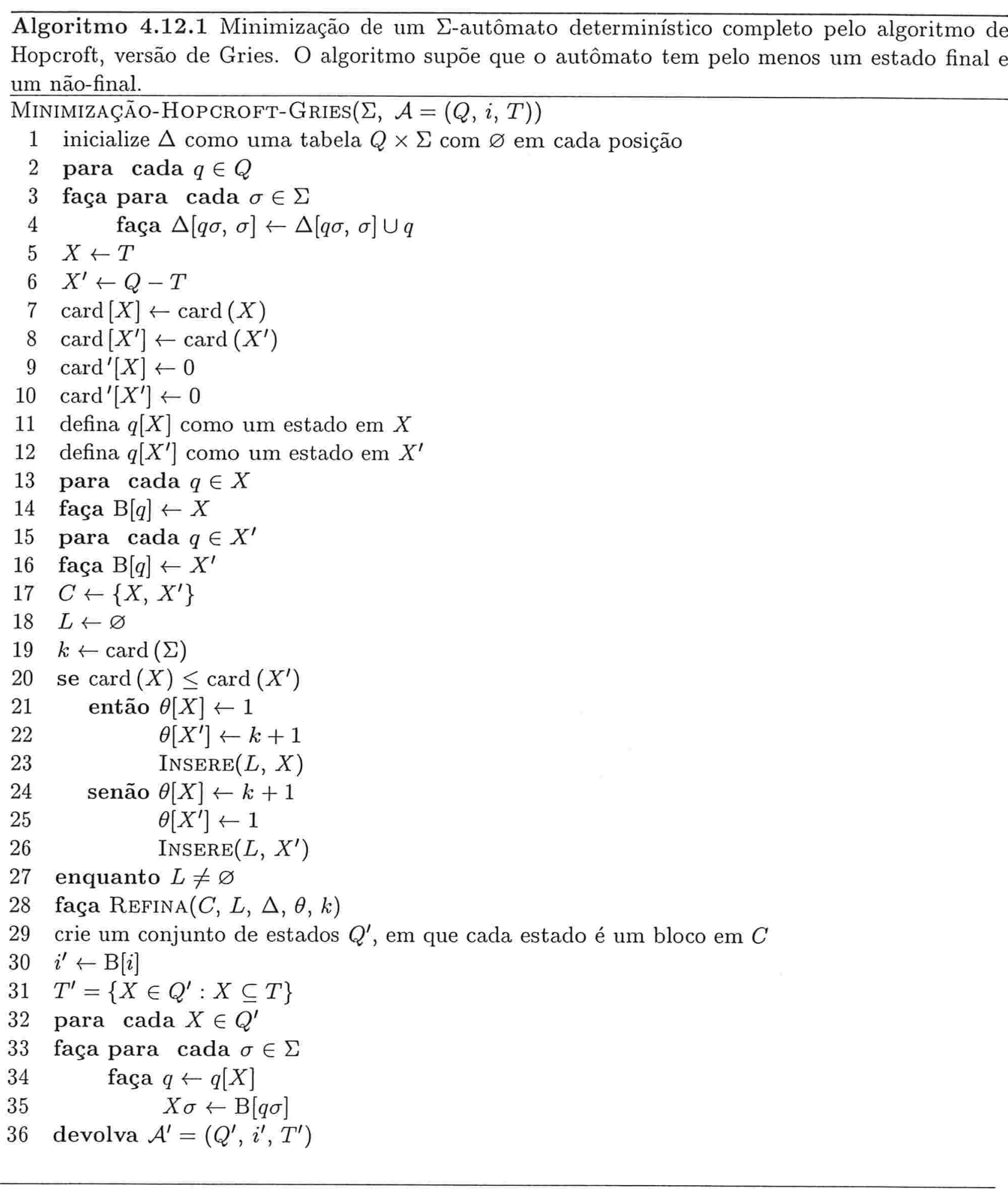


Algoritmo 4.12.2 Refinamento de uma configuração no algoritmo de Hopcroft-Gries. Recorde que a divisão de um bloco $X$ é feita se, e somente se, $X-D \neq \varnothing$ e $X \cap D \neq \varnothing$, o que é expresso pelo teste na linha 12. Na divisão de um bloco $X$ em blocos $\bar{X}$ e $\widetilde{X}$, apenas o bloco $\bar{X}=X \cap D$ é criado (linha 13). O bloco $\widetilde{X}$ é o bloco $X$ após a retirada dos estados em $X \cap D$. Esse fato é utilizado na atualização de $L$ como segue. No trecho entre as linhas 24 e 26 , o bloco $\bar{X}$ deve ser inserido em $L$, porque esse bloco é novo e $\bar{X} \theta=1$. Como $\widetilde{X} \theta=X \theta$, o bloco $\widetilde{X}$ deve pertencer a $L$ se, e somente se, $X$ pertence a $L$. Portanto, $L$ não precisa ser atualizada com $\tilde{X}$. No trecho entre as linhas 27 e 31 , se $X \theta \leq k$, então o bloco $\bar{X}$ deve ser inserido em $L$, porque esse bloco é novo e $\bar{X} \theta \leq k$ (linha 30). O bloco $\widetilde{X}$ não precisa ser inserido porque, como $X \in L$, esse bloco também pertence a $L$. Se $\bar{X} \theta>k$, então $\bar{X}$ não deve ser inserido em $L$. Mas, como $X \notin L$ e $\widetilde{X} \theta=1, \widetilde{X}$ deve ser inserido em $L$ (linha 31).

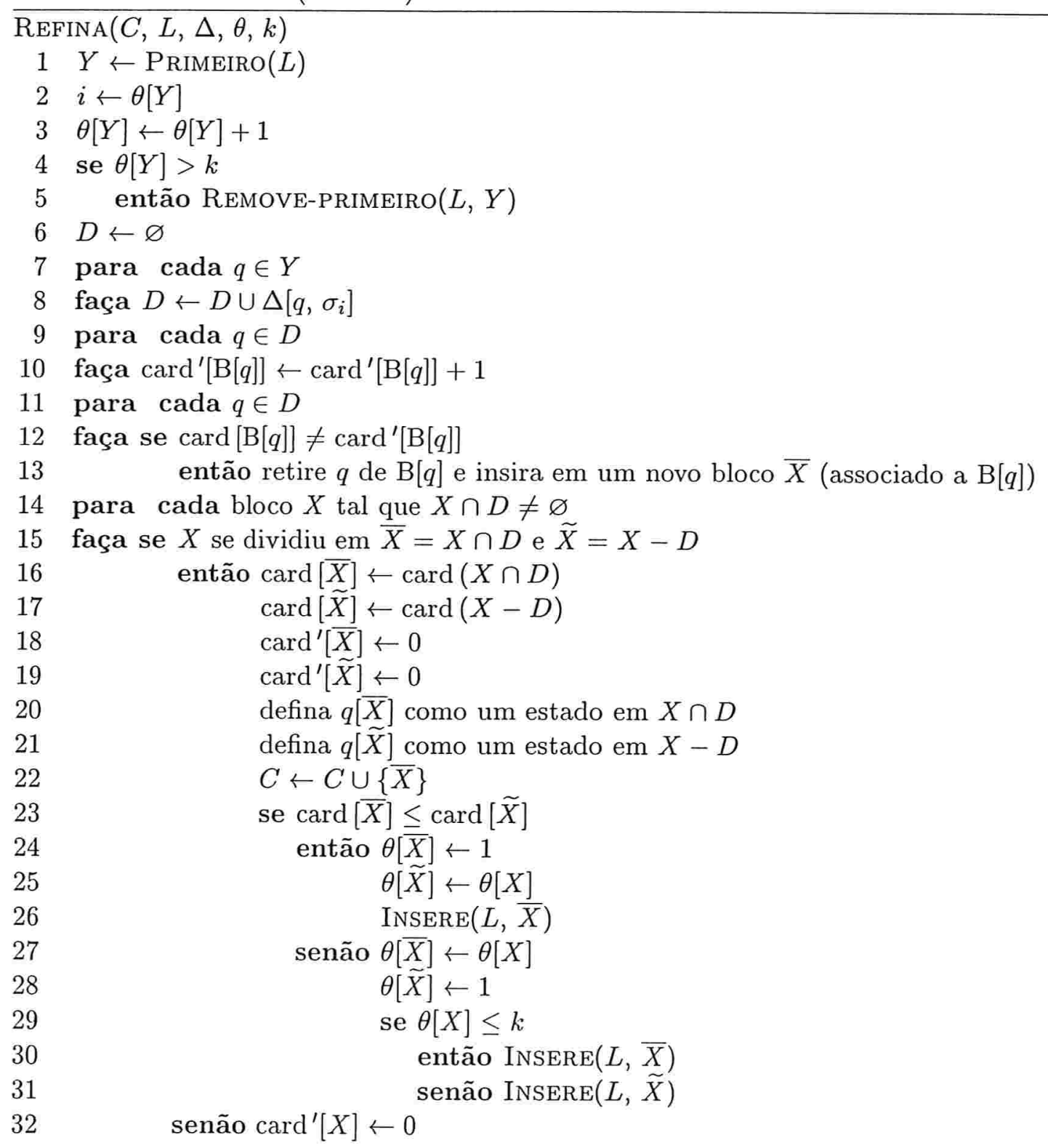


$\sigma=Y \theta$ e $q \in Y$ é proporcional a $V(q, \sigma)$. Pelo Invariante 4.12.2, esse número é proporcional a $[B(q)]$, onde $B(q)$ é o bloco a que $q$ pertence em $Q / \equiv_{\mathcal{A}}$ (pois este é o bloco a que $q$ pertence no início da última iteração).

Da definição de $[B(q)]$ em $(4.12 .5)$, segue que

$$
[B(q)]=O(\log n)
$$

onde $\log n$ é o logaritmo de $n$ na base 2 .

Somando o número de visitas feitas para cada letra $\sigma$, concluímos que o número total de visitas feitas a $q$ é

$$
O(\operatorname{card}(\Sigma) \log n)
$$

Portanto, o número total de visitas realizadas aos estados nos blocos $Y$ é

$$
O(\operatorname{card}(\Sigma) n \log n)
$$

Sejam $q$ um estado e $\sigma$ uma letra. Para analisar o número de vezes que o algoritmo visita $q$ em um conjunto $D$, considere que esse estado só entra em um tal conjunto quando $q \sigma$ entra em um bloco $Y$ no refinamento de uma configuração por $Y$. Portanto, o número de visitas a $q$ em um conjunto $D$ é proporcional ao número de visitas a $q \sigma$ em um conjunto $Y$.

Conforme discutido acima, esse número é proporcional a $O(\operatorname{card}(\Sigma) \log n)$. Portanto, o número total de visitas feitas aos estados nos conjuntos $D$ também é a indicada em (4.12.12).

Concluímos então que a complexidade de tempo do algoritmo é (4.12.12).

A complexidade de espaço consiste na soma dos tamanhos da tabela $\Delta$ e das estruturas para o armazenamento de $C$. Claramente, essas estruturas têm tamanho $O(n+m)$, onde $m=\operatorname{card}(E)$. A tabela $\Delta$ têm tamanho $O(\operatorname{card}(\Sigma) n)$, pois a soma dos tamanhos das listas armazenadas em todas as posições é card $(\Sigma) n$. Portanto, a complexidade de espaço do algoritmo é

$$
O(\operatorname{card}(\Sigma) n)
$$

Finalmente, vamos mostrar como o algoritmo de Hopcroft pode ser utilizado para construir um transdutor subseqüencial minimal.

Seja $\mathcal{T}=(Q, i, \lambda, \rho)$ um $\Sigma$ - $\Gamma$-transdutor subseqüencial bi-acessível e prefixo. Vamos definir um novo alfabeto $\Omega$, cujos símbolos são os rótulos das transições de $\mathcal{T}$, mais as emissões dos estados finais. Formalmente,

$$
\Omega=\{(\sigma, x): \exists q \in Q \text { tal que } q * \sigma=x\} \cup Q \rho .
$$

Vamos denotar por $\Omega_{1}$ o conjunto das letras que representam os pares, e por $\Omega_{2}$ o conjunto das letras que representam as emissões dos estados finais.

A seguir, vamos construir um $\Omega$-autômato determinístico bi-acessível $\mathcal{A}=\left(Q^{\prime}, i, t\right)$ a partir de $\mathcal{T}$. Vamos denominar esse autômato de autômato de $\mathcal{T}$.

- $Q^{\prime}=Q \cup t$, onde $t$ é um estado novo;

- As transições de $\mathcal{A}$ são definidas como segue:

- Para cada estado $q$ em $Q$ e cada letra $a=(\sigma, x)$ em $\Omega_{1}, q a \neq \varnothing$ em $\mathcal{A}$ se, e somente se, $q \sigma \neq \varnothing$ em $\mathcal{T}$ e $q * \sigma=x$. Nesse caso, $q a=q \sigma$. 
- Para cada estado $q$ em $Q$ e cada letra $x$ em $\Omega_{2}, q x \neq \varnothing$ se, e somente se, $q \rho=x$. Nesse caso, $q x=t$.

Algumas propriedades elementares dessa construção estão enunciadas a seguir.

(4.12.14a) Para todo estado $q$ em $Q$, toda palavra $w$ em $\Omega^{*}$ tal que $q w=t$ é da forma $v x$, onde $v$ é uma palavra em $\Omega_{1}{ }^{*}$ e $x$ é um letra em $\Omega_{2}$.

Sejam $q$ um estado em $Q, u=\sigma_{1} \ldots \sigma_{k}$ uma palavra em $\Sigma^{*}$, onde cada $\sigma_{i}$ é uma letra, e $v=\left(\sigma_{1}, x_{1}\right) \ldots\left(\sigma_{k}, x_{k}\right)$ uma palavra em $\Omega_{1}{ }^{*}$. Então,

$$
q v \neq \varnothing \text { se, e somente se, } q u \neq \varnothing \text { e } x_{i}=\left(q\left(\sigma_{1} \ldots \sigma_{i-1}\right)\right) * \sigma_{i} \quad \forall i, 1 \leq i \leq k .
$$

Ademais, se $q u \neq \varnothing$, então

$$
q u=q v \quad \mathrm{e} \quad q * u=x_{1} \ldots x_{k} .
$$

A segunda propriedade pode ser demonstrada facilmente por indução em $k$.

Observe que a emissão do estado inicial $i$ de $\mathcal{T}$ não é utilizada na definição do autômato desse transdutor.

Vamos denotar por $\equiv \mathcal{T}$ a relação de equivalência dos estados de $\mathcal{T}$ (definida em (4.10.6)), e por $\equiv_{\mathcal{A}}$ a relação de equivalência dos estados de $\mathcal{A}$ (definida em (4.12.3)). A proposição a seguir relaciona essas máquinas utilizando essas relações.

Proposição 4.12.5 Sejam $\mathcal{T}=(Q, i, \lambda, \rho)$ um $\Sigma$ - $\Gamma$-transdutor subseqüencial bi-acessível e prefixo, e $\mathcal{A}=(Q, i, t)$ o autômato de $\mathcal{T}$. Para todo par de estados $p, q$ em $Q$,

$$
p \equiv_{\mathcal{T}} q \text { se, e somente se, } p \equiv_{\mathcal{A}} q \text {. }
$$

Demonstração. Suponha que $p \equiv \mathcal{T} q$.

Seja $w=\left(\sigma_{1}, x_{1}\right) \ldots\left(\sigma_{k}, x_{k}\right) x$ uma palavra em $p^{-1} t$, onde cada $\left(\sigma_{i}, x_{i}\right)$ é uma letra em $\Omega_{1}$ e $x$ é um letra em $\Omega_{2}$. Então, por (4.12.14b) e pela construção de $\mathcal{A}, p\left(\sigma_{1} \ldots \sigma_{k}\right)$ é um estado final de $\mathcal{T}$ com emissão final igual a $x$.

Como $p \equiv_{\mathcal{T}} q$, segue de (4.10.10a) que

$$
p\left(\sigma_{1} \ldots \sigma_{i}\right) \equiv_{\mathcal{T}} q\left(\sigma_{1} \ldots \sigma_{i}\right), \quad \forall i, 1 \leq i \leq k .
$$

Em particular, $p\left(\sigma_{1} \ldots \sigma_{k}\right) \equiv \mathcal{T} q\left(\sigma_{1} \ldots \sigma_{k}\right)$. Então, segue de (4.10.10c) que $q\left(\sigma_{1} \ldots \sigma_{k}\right)$ também é um estado final de $\mathcal{T}$, com emissão final igual a $x$.

Por (4.10.10b),

$$
x_{i}=\left(p\left(\sigma_{1} \ldots \sigma_{i-1}\right)\right) * \sigma_{i}=\left(q\left(\sigma_{1} \ldots \sigma_{i-1}\right)\right) * \sigma_{i}, \quad \forall i, 1 \leq i \leq k .
$$

Então, por $(4.12 .14 \mathrm{~b}), q\left(\sigma_{1}, x_{1}\right) \ldots\left(\sigma_{k}, x_{k}\right)=q\left(\sigma_{1} \ldots \sigma_{k}\right)$. Pela construção de $\mathcal{A}, q w=t$. Ou seja, $w \in q^{-1} t$. 
Como $w$ é arbitrário, concluímos que $p^{-1} t \subseteq q^{-1} t$. De maneira análoga, demonstra-se a inclusão contrária. Portanto, $p \equiv_{\mathcal{A}} q$.

Suponha agora que $p \equiv_{\mathcal{A}} q$.

Seja $u=\sigma_{1} \ldots \sigma_{k}$ uma palavra tal que $p u$ é um estado final de $\mathcal{T}$. Sejam $x_{i}=\left(p\left(\sigma_{1} \ldots \sigma_{i-1}\right)\right) * \sigma_{i}$ para todo $i, 1 \leq i \leq k, x=(p u) \rho$ e $v=\left(\sigma_{1}, x_{1}\right) \ldots\left(\sigma_{k}, x_{k}\right)$.

Por (4.12.14b), $p v=p u$. Como $p u$ é um estado final de $\mathcal{T}$, segue da construção de $\mathcal{A}$ que $p(v x)=t$. Assim, $v x \in p^{-1} t$.

Como $p \equiv_{\mathcal{A}} q, v x \in q^{-1} t$. Ou seja, $q(v x)=t$. Então, pela construção de $\mathcal{A}$ e por $(4.12 .14 \mathrm{~b})$, $q * u=x_{1} \ldots x_{k}=p * u$, qu é um estado final de $\mathcal{T}$, e $(q u) \rho=x$. Então, $u\left(q^{-1} \rho\right)=x_{1} \ldots x_{k} x=$ $u\left(p^{-1} \rho\right)$, onde as funções $p^{-1} \rho$ e $q^{-1} \rho$ estão definidas em (4.10.5).

Da mesma forma, dada uma palavra $u$ tal que qu é um estado final em $\mathcal{T}$, demonstra-se que $u\left(p^{-1} \rho\right)=u\left(q^{-1} \rho\right)$. Portanto, $p^{-1} \rho=q^{-1} \rho$. Pela definição de $\equiv_{\mathcal{T}}$ em (4.10.6), concluímos que $p \equiv \mathcal{T} q$.

A construção algorítmica de $\mathcal{A}$ a partir de $\mathcal{T}$ pode ser feita eficientemente. A seguir, descrevemos uma possibilidade.

O primeiro passo é a construção do alfabeto $\Omega$. Para tal, utilizamos a estrutura de trie ou árvore digital, descrita por exemplo em [SM94]. Essa estrutura é um $\Sigma$-autômato determinístico, em forma de árvore, que reconhece um conjunto finito de palavras. Ademais, uma árvore digital é uma estrutura dinâmica, no sentido de que pode ser facilmente modificada para que novas palavras sejam adicionadas ao seu comportamento.

Não vamos descrever formalmente essa estrutura, nem possibilidades de implementação, para o que fazemos referência a [SM94]. Estamos interessados apenas na possibilidade da realização das duas operações seguintes em uma árvore digital:

- inserção de uma palavra;

- teste de se uma palavra pertence ao comportamento da árvore digital.

Ambas as operações têm complexidade de tempo proporcional ao comprimento da palavra.

A construção de $\Omega$ pode ser feita construindo-se primeiro estruturas auxiliares para os alfabetos $\Omega_{1}$ e $\Omega_{2}$ em separado, conforme descrito a seguir.

Para $\Omega_{1}$, contruímos um vetor $R$ indexado por $\Sigma$. Para cada letra $\sigma$, a posição $R[\sigma]$ contém uma árvore digital, cujo comportamento é o conjunto das saídas das transições de $\mathcal{T}$ com entrada igual a $\sigma$. Esse vetor pode ser construído com uma única passagem pelo conjunto de transições de $\mathcal{T}$. A cada transição visitada, a árvore digital correspondente à entrada dessa transição é atualizada com a saída dessa transição.

Para $\Omega_{2}$, construímos uma árvore digital reconhecendo o conjunto das emissões dos estados finais de $\mathcal{T}$. Essa árvore pode ser construída com uma única passagem pelo conjunto de estados de $\mathcal{T}$. A cada estado final visitado, a árvore é atualizada com sua emissão.

Finalmente, a cada estado final de cada uma dessas árvores digitais é associada uma letra. $\mathrm{O}$ conjunto dessas letras é exatamente $\Omega$.

Percorrendo-se novamente as transições e os estados finais de $\mathcal{T}$, as transições de $\mathcal{A}$ podem ser determinadas. Para isso, as emissões das transições e dos estados finais são soletradas nas árvores respectivas, para que a letra correspondente seja obtida.

A complexidade de tempo total dessa construção é proporcional a $n+m+S_{1}+S_{2}$, onde $m$ é o número de transições de $\mathcal{T}, S_{1}$ é a soma dos comprimentos das saídas das transições de $\mathcal{T}$ e $S_{2}$ é a 
soma dos comprimentos das emissões dos estados finais de $\mathcal{T}$. A complexidade de espaço consiste na soma dos tamanhos das árvores digitais construídas, sendo portanto proporcional a $n+S_{1}+S_{2}$.

Utilizando (4.12.12), temos então que a complexidade de tempo total da construção do transdutor minimal equivalente a $\mathcal{T}$ é

$$
O\left(\operatorname{card}(\Omega) n \log n+S_{1}+S_{2}\right)
$$

Note que $m$ não aparece nessa expressão porque $m \leq \operatorname{card}(\Omega) n$.

Utilizando (4.12.13), temos que a complexidade de espaço é

$$
O\left(\operatorname{card}(\Omega) n+S_{1}+S_{2}\right)
$$

Finalizamos observando que o tamanho de $\Omega$ pode ser proporcional ao número de transições de $\mathcal{T}$. Portanto, podemos também dizer que a complexidade de tempo é

$$
O\left(m n \log n+S_{1}+S_{2}\right)
$$

e a de espaço é

$$
O\left(m n+S_{1}+S_{2}\right)
$$

Exemplo 4.12.1 Seja $\Sigma=\{\sigma, \gamma\}$. Vamos simular o algoritmo de Hopcroft para o autômato

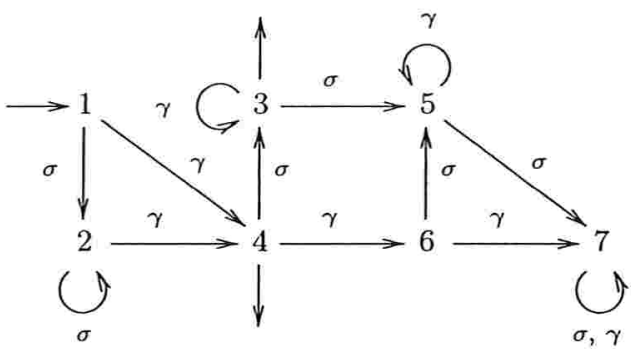

Vamos considerar a indexação $\sigma_{1}=\sigma$ e $\sigma_{2}=\gamma$. A simulação das 5 primeiras iterações está representada na Figura 4.12.1. Nenhuma outra divisão é feita até o final da execução do algoritmo. Os dados são anotados no início de cada iteração, antes de qualquer operação. O índice da letra utilizada no refinamento (ou seja, $Y \theta$ ) é representada por $i$.

A parte bi-acessível do autômato minimal é

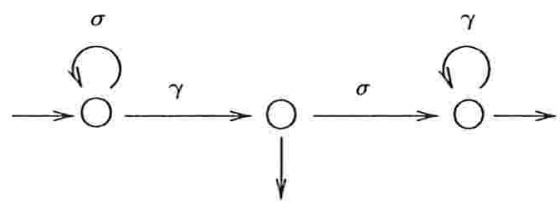



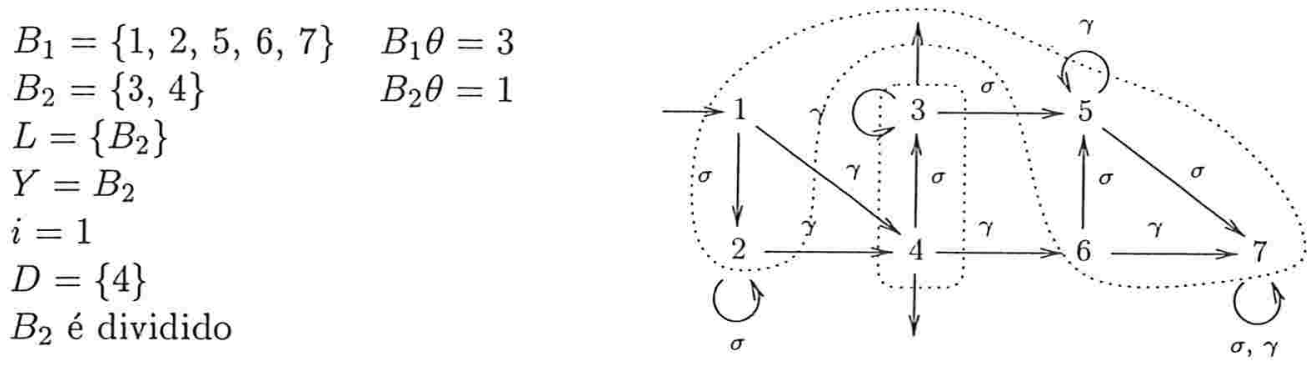

$$
\begin{array}{ll}
B_{1}=\{1,2,5,6,7\} & B_{1} \theta=3 \\
B_{2}=\{3\} & B_{2} \theta=2 \\
B_{3}=\{4\} & B_{3} \theta=1 \\
L=\left\{B_{2}, B_{3}\right\} & \\
Y=B_{2} & \\
i=2 & \\
D=\{3\} &
\end{array}
$$

Nenhuma divisão

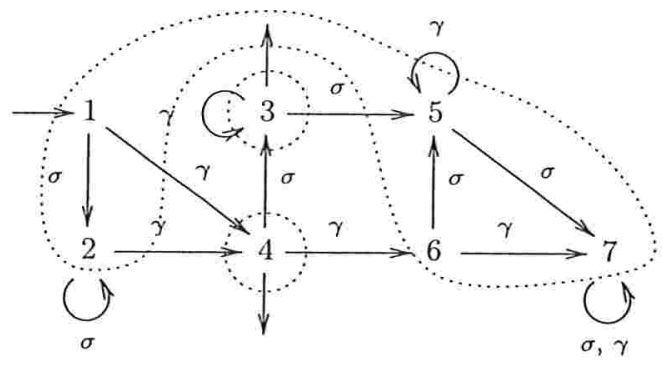

$$
\begin{array}{ll}
B_{1}=\{1,2,5,6,7\} & B_{1} \theta=3 \\
B_{2}=\{3\} & B_{2} \theta=3 \\
B_{3}=\{4\} & B_{3} \theta=1 \\
L=\left\{B_{3}\right\} & \\
Y=B_{3} & \\
i=1 & \\
D=\varnothing &
\end{array}
$$

Nenhuma divisão

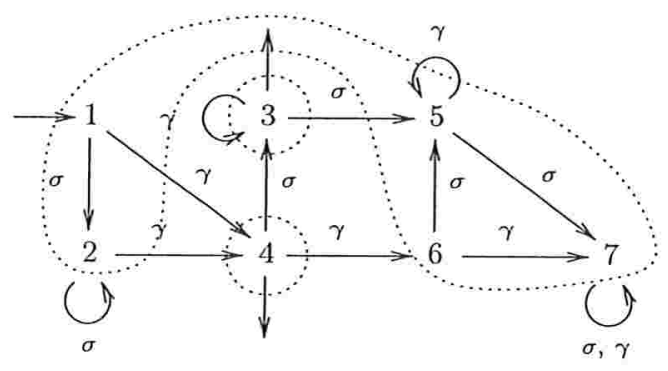

$$
\begin{array}{ll}
B_{1}=\{1,2,5,6,7\} & B_{1} \theta=3 \\
B_{2}=\{3\} & B_{2} \theta=3 \\
B_{3}=\{4\} & B_{3} \theta=2 \\
L=\left\{B_{3}\right\} & \\
Y=B_{3} & \\
i=2 & \\
D=\{1,2\} & \\
B_{1} \text { é dividido } &
\end{array}
$$

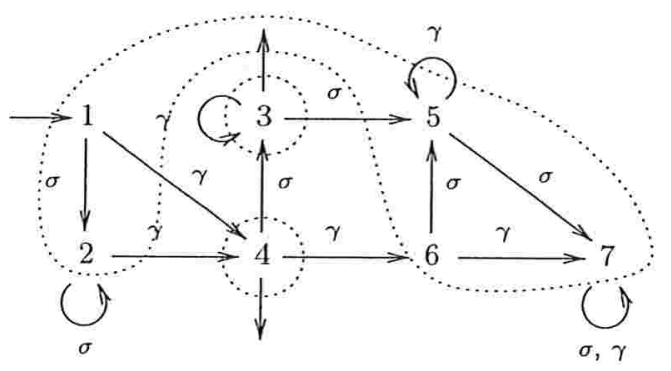

$$
\begin{array}{ll}
B_{1}=\{5,6,7\} & B_{1} \theta=3 \\
B_{2}=\{3\} & B_{2} \theta=3 \\
B_{3}=\{4\} & B_{3} \theta=3 \\
B_{4}=\{1,2\} & B_{4} \theta=1 \\
L=\left\{B_{4}\right\} & \\
Y=B_{4} & \\
i=1 & \\
D=\{1,2\} &
\end{array}
$$

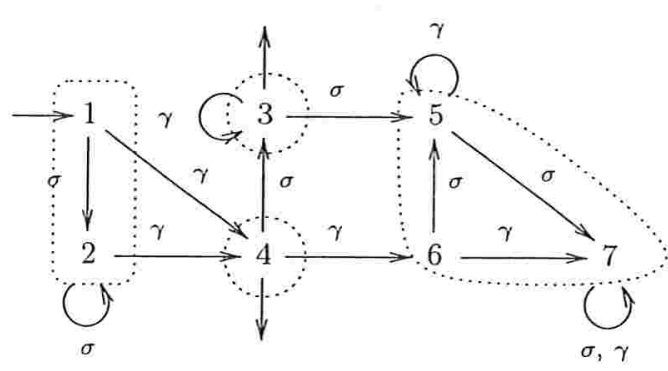

Nenhuma divisão

Figura 4.12.1: Simulação do algoritmo de Hopcroft para a minimização de um autômato. 


\subsection{Exemplos}

Esta seção contém alguns exemplos para as seções 4.6, 4.10 e 4.12 (determinização de um transdutor letra-palavra, redução de um transdutor subseqüencial prefixo, e construção do transdutor minimal).

Exemplo 4.13.1 O transdutor subseqüencial prefixo ilustrado no Exemplo 4.8.1 não é minimal. Os estados equivalentes estão indicados a seguir:

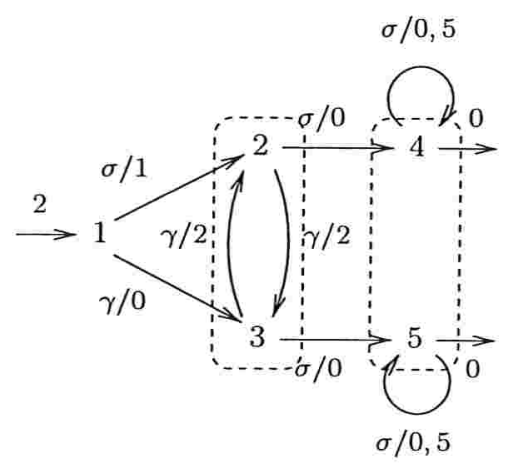

O $\Sigma-\mathbb{R}_{+}$-transdutor subseqüencial minimal equivalente é

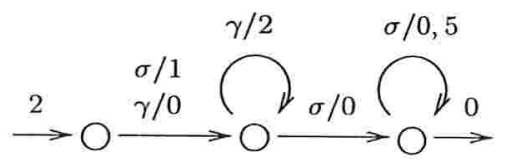

Exemplo 4.13.2 Nos exemplos 4.6.1 e 4.6.3, o transdutor subseqüencial resultante da construção dos subconjuntos já é minimal. Observe que os autômatos de entrada desses transdutores são reduzidos.

No Exemplo 4.6.4, uma inspeção mostra que o transdutor subseqüencial resultante da construção dos subconjuntos também já é minimal. No entanto, o autômato de entrada desse transdutor não é reduzido.

Exemplo 4.13.3 No Exemplo 4.6.2, o transdutor subseqüencial obtido com a construção dos subconjuntos não é prefixo. A forma prefixo equivalente é

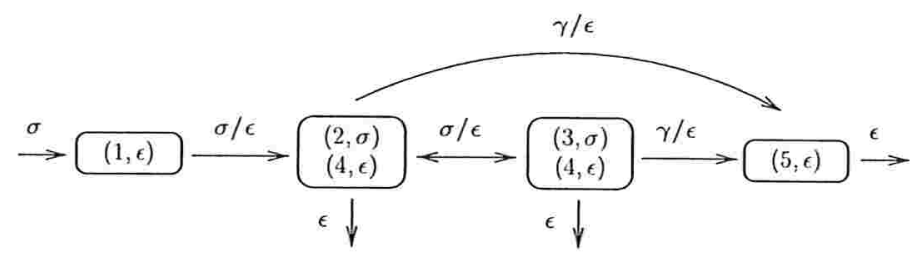

Esse transdutor não é minimal, pois os subconjuntos com dois elementos são equivalentes. O transdutor subseqüencial minimal equivalente é 


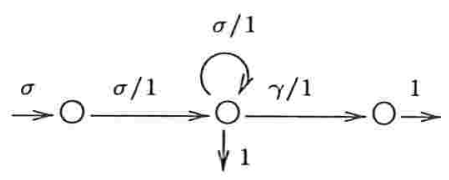

Exemplo 4.13.4 Aplicando a construção dos subconjuntos no transdutor letra-palavra

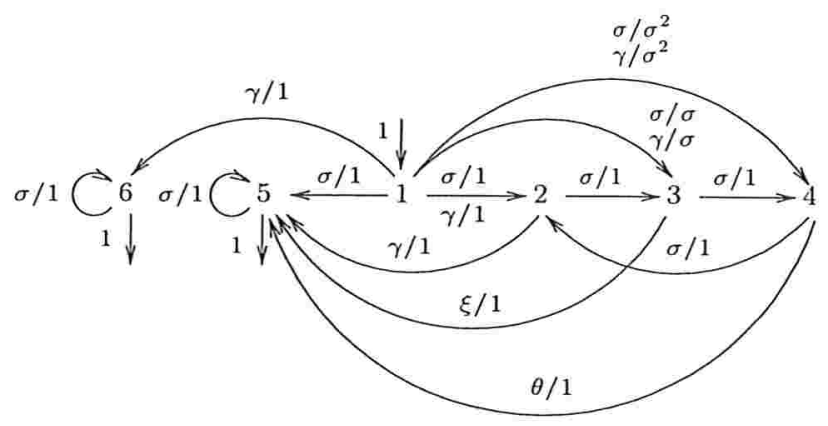

obtemos o transdutor subseqüencial ilustrado a seguir. A palavra vazia é representada por $\epsilon$.

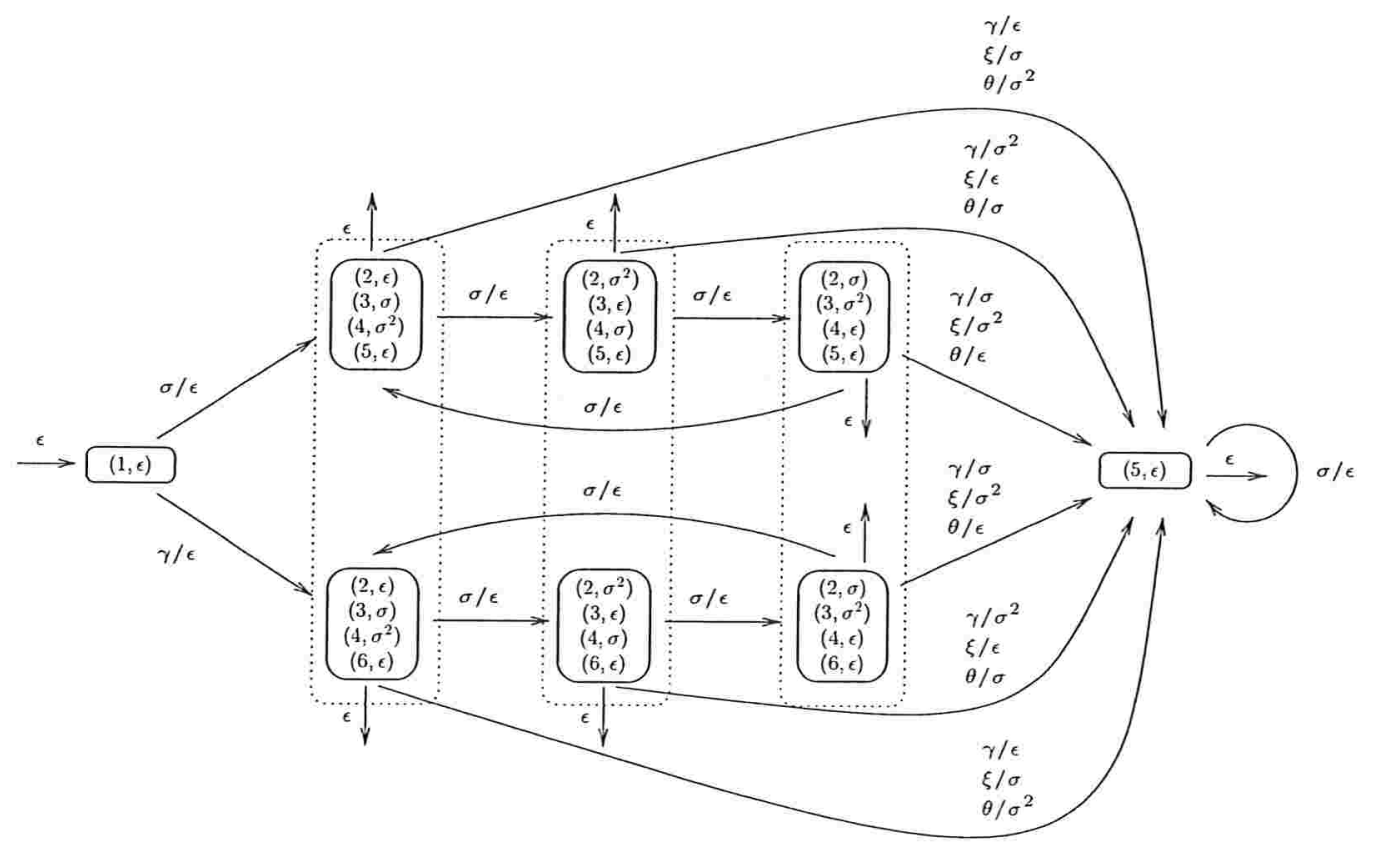

Esse transdutor é prefixo, mas não é minimal. Os estados equivalentes estão indicados na figura. O transdutor subseqüencial minimal equivalente é 


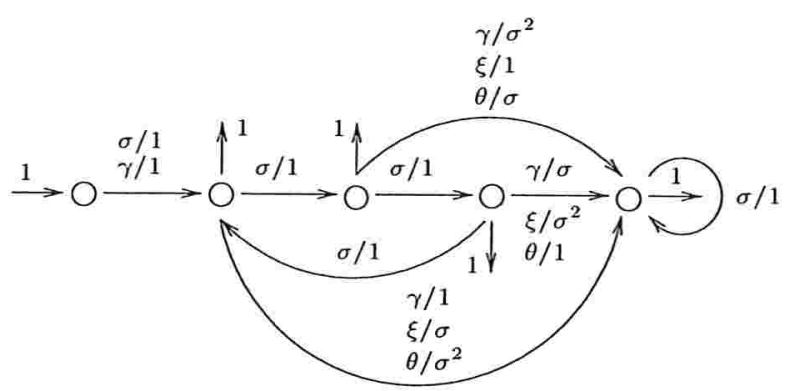

Exemplo 4.13.5 Vamos ilustrar os passos da minimização de um transdutor, construindo o transdutor dos sufixos para o texto abbabaab.

Um transdutor subseqüencial que realiza a função definida em (4.10.25) para este texto é

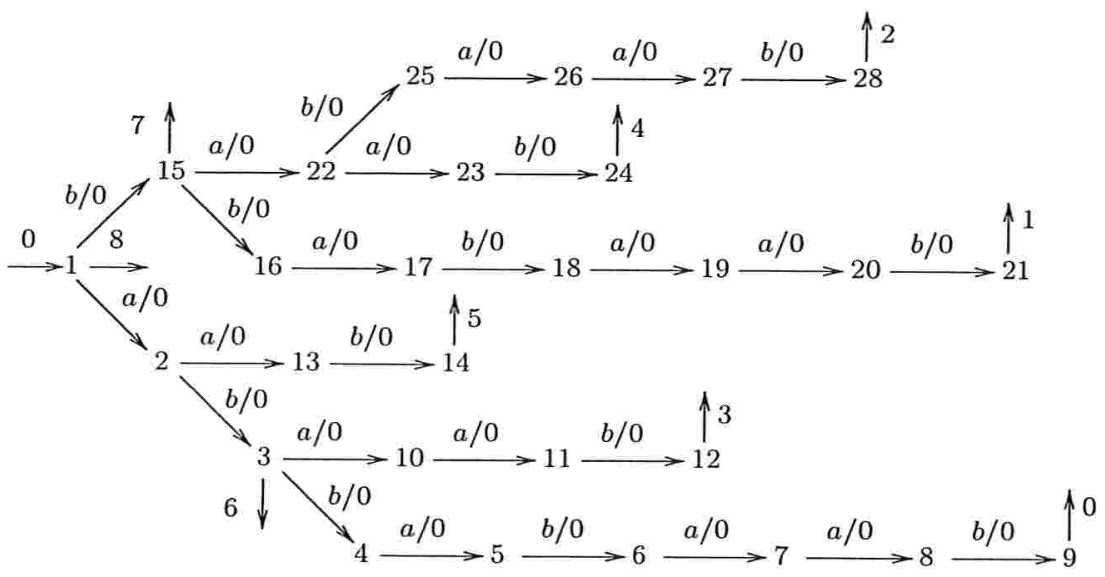

Calculando-se o prefixo, obtemos

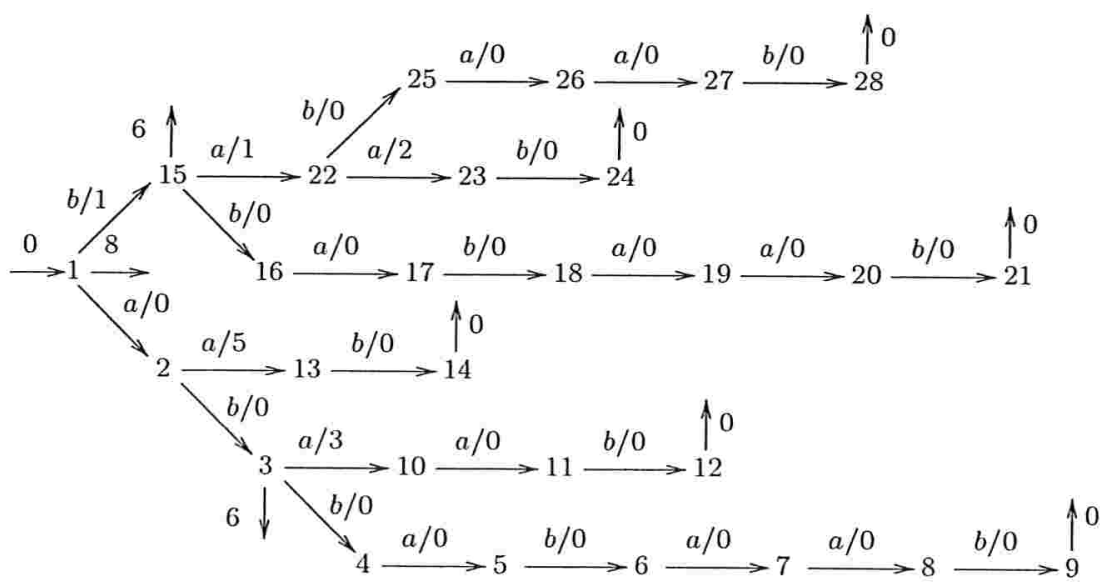

Para construir o autômato desse transdutor, um novo alfabeto $\Omega=\{A, B, C, D, E, F, G, H, I\}$ é criado. A correspondência entre as letras de $\Omega$ e os rótulos das transições e emissões dos estados 
finais do transdutor está indicada a seguir:

$$
A=a / 0 ; \quad B=b / 1 ; \quad C=a / 5 ; \quad D=b / 0 ; \quad E=a / 3 ; \quad F=a / 1 ; \quad G=a / 2 ; \quad H=8 ; \quad I=6 ; \quad J=0 .
$$

Substistituindo, obtemos o $\Omega$-autômato determinístico ilustrado abaixo. As transições correspondentes às emissões finais do transdutor estão indicadas em tracejado.

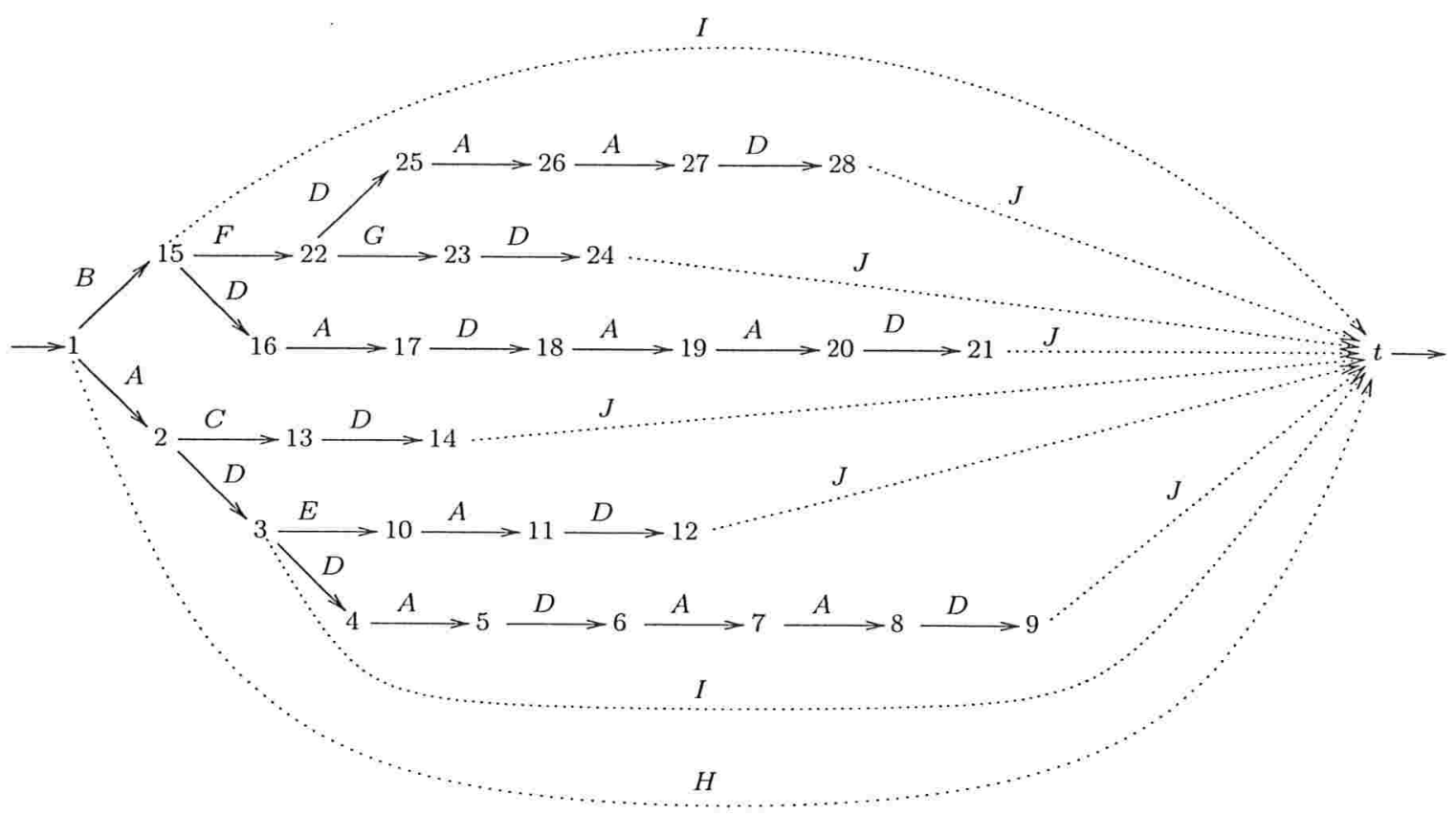

Como este autômato não é completo, o completamento deve ser feito antes da minimização, com a inclusão de um novo estado $\square$ e transições convenientes.

Os blocos calculados pelo algoritmo estão indicados a seguir:

\begin{tabular}{l|l}
$B_{0}$ & $\square$ \\
\hline$B_{1}$ & $t$ \\
\hline$B_{2}$ & 1 \\
\hline$B_{3}$ & 3 \\
\hline$B_{4}$ & $9,12,14,21,24,28$ \\
\hline$B_{5}$ & 2 \\
\hline$B_{6}$ & $8,11,13,20,23,27$ \\
\hline$B_{7}$ & $7,10,19,26$ \\
\hline$B_{8}$ & $6,18,25$ \\
\hline$B_{9}$ & 5,17 \\
\hline$B_{10}$ & 22 \\
\hline$B_{11}$ & 4,16 \\
\hline$B_{12}$ & 15
\end{tabular}

O autômato minimal está ilustrado a seguir, sem a indicação do estado $\square$. 


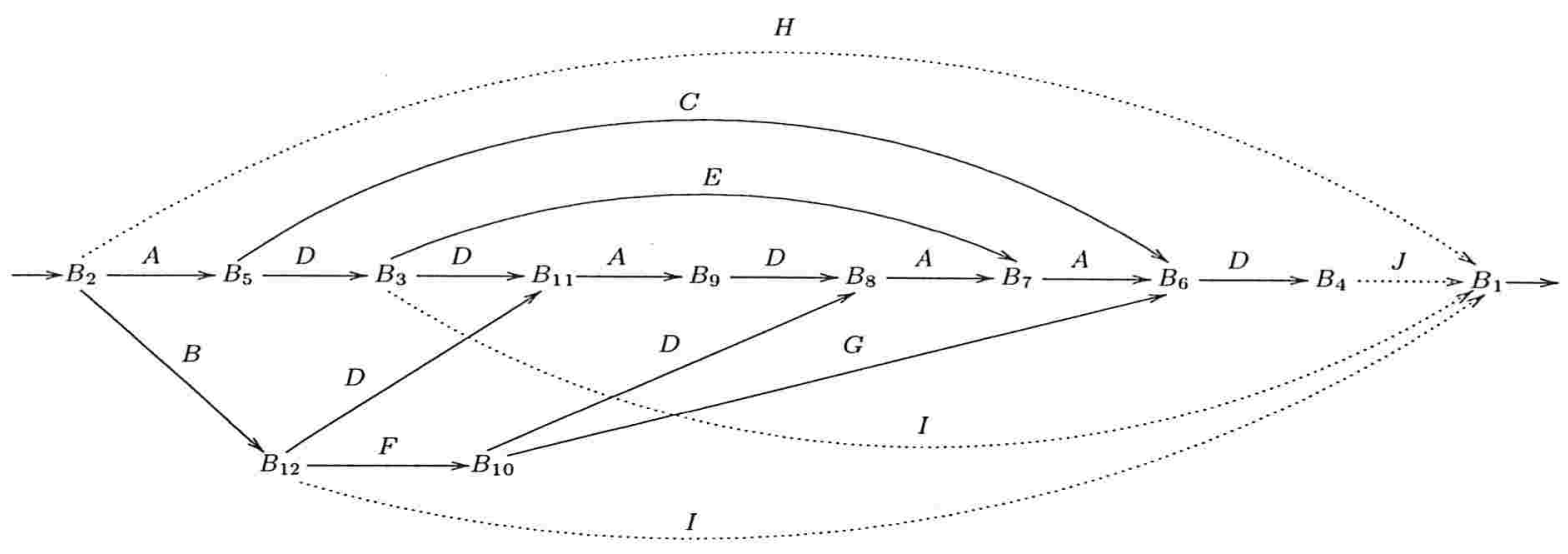

Substituindo as letras de $\Omega$, obtemos o transdutor minimal

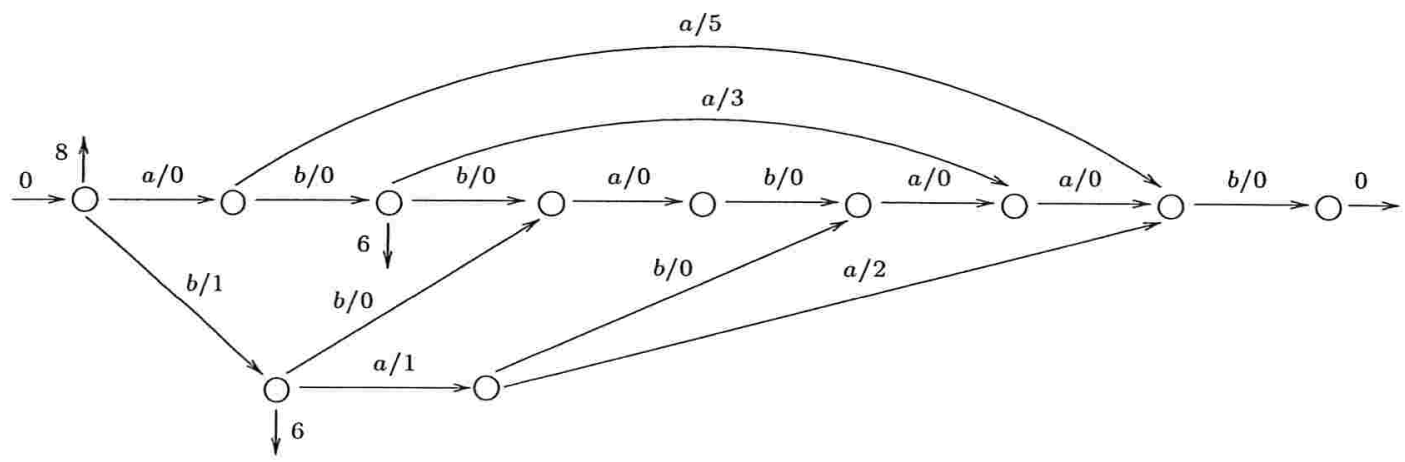

que é o transdutor dos sufixos para abbabaab.

Exemplo 4.13.6 Neste último exemplo, vamos ilustrar o fato de que uma máquina que realiza uma função $\Sigma^{*} \rightarrow \Gamma^{*}$ pode ser complexa, mesmo quando o domínio e a imagem dessa função são conjuntos simples (no sentido de que podem ser realizados por máquinas simples).

Sejam $\Sigma=\left\{\sigma_{1}, \ldots, \sigma_{l}\right\}$ e $\Gamma=\left\{\sigma_{1}, \ldots, \sigma_{l-1}\right\}$, com $l>2, f: \Sigma^{*} \rightarrow \Gamma^{*}$ o morfismo gerado por

$$
\sigma_{i} f= \begin{cases}\sigma_{i} & \text { se } i<l \\ 1 & \text { se } i=l\end{cases}
$$

e $\tau: \Sigma^{*} \rightarrow \Gamma^{*}$ a função definida por

$$
\forall x \in \Sigma^{*}, x \tau= \begin{cases}x f & \text { se existe uma letra } \sigma_{i} \text { que não ocorre em } x \\ (x f) \sigma_{1} & \text { caso contrário. }\end{cases}
$$

Vamos provar que $\tau$ é uma função subseqüencial, descrevendo um transdutor subseqüencial $\mathcal{T}$ tal que $|\mathcal{T}|=\tau$.

O conjunto de estados de $\mathcal{T}$ é $2^{\Sigma}$. O estado inicial é $i=\varnothing$, e a emissão inicial é a palavra vazia. Todos os estados são finais. A emissão final do estado $\Sigma$ é $\sigma_{1}$, e a emissão final dos demais estados é a palavra vazia. 
Esse transdutor é completo. Para cada subconjunto $X$ de $\Sigma$ e cada letra $\sigma$, definimos

$$
X \sigma=X \cup \sigma \quad \text { e } \quad X * \sigma=\sigma f .
$$

Vamos denotar por $|u|_{\Sigma}$ o conjunto das letras que ocorrem em uma palavra $u$. As seguintes propriedades são imediatas:

$$
X u=X \cup|u|_{\Sigma} \quad \text { e } \quad X * u=u f, \forall X \subseteq \Sigma .
$$

Dessas propriedades, segue que $|\mathcal{T}|=\tau$.

Afirmamos que $\mathcal{T}$ é prefixo. De fato, como $l>2$, existem letras $\sigma_{1}$ e $\sigma_{2}$ em $\Sigma$ tais que $\sigma_{1} f=\sigma_{1}$ e $\sigma_{2} f=\sigma_{2}$. Então, para todo estado $q$ de $\mathcal{T}$, existem duas transições com origem em $q$ e saídas $\sigma_{1}$ e $\sigma_{2}$. Portanto, o prefixo de todo estado desse autômato é a palavra vazia.

Afirmamos que $\mathcal{T}$ é minimal. De fato, sejam $X$ e $Y$ subconjuntos distintos de $\Sigma$. Sem perda de generalidade, podemos supor que $X-Y \neq \varnothing$. Seja $u$ uma palavra que contenha exatamente uma ocorrência de cada letra em $\Sigma-X$. Temos então as seguintes propriedades:

$$
\begin{gathered}
X u=\Sigma \quad \text { e } \quad(X * u)((X u) \rho)=(u f) \sigma_{1} \\
Y u \subsetneq \Sigma \quad \text { e } \quad(Y * u)((Y u) \rho)=(u f) .
\end{gathered}
$$

Assim, $X^{-1} \rho \neq Y^{-1} \rho$, o que mostra que $X$ e $Y$ não são equivalentes. Como esses estados são arbitrários, $\mathcal{T}$ é minimal.

Concluímos que o número de estados do transdutor minimal para $\tau$ é exponencial em card $(\Sigma)$.

Note agora que $\operatorname{dom} \tau=\Sigma^{*}$. O autômato de entrada de $\mathcal{T}$ é um $\Sigma$-autômato determinístico que reconhece esse conjunto. Mas, o autômato minimal que reconhece esse conjunto tem apenas um estado.

Temos também que $\operatorname{im} \tau=\Gamma^{*}$. Igualmente, o autômato minimal que reconhece esse conjunto tem um estado.

Assim, $\tau$ é um exemplo de função com a propriedade descrita no início do exemplo. 



\section{CONClusÃo}

Neste trabalho, estudamos algumas das principais propriedades de algumas classes de relações racionais. O conteúdo desse estudo está resumido a seguir:

- Relações racionais: fatoração de relações racionais, obtida por Nivat; fechamento da classe das relações racionais pela operação de composição, obtido por Elgot e Mezei.

- Relações racionais finitamente valoradas: algoritmo de complexidade de tempo polinomial para decidir se uma relação racional é finitamente valorada ; indecibilidade do problema da equivalência de relações racionais finitamente valoradas, obtida por Griffiths.

- Relações racionais $k$-valoradas: extensão de uma caracterização de Schützenberger das funções racionais para uma caracterização das relações racionais $k$-valoradas, para todo inteiro positivo $k$; demonstração da decomposição de uma relação racional $k$-valorada em uma união de $k$ funções racionais, resultado obtido por Weber; demonstração da decibilidade do problema da equivalência de relações racionais $k$-valoradas, resultado obtido por Culik e Karhumaki, e independentemente por Weber.

- Funções racionais: algoritmo de Sakarovitch et al. para decidir se uma relação racional finitamente valorada é uma função racional.

- Funções seqüenciais à esquerda e funções seqüenciais à direita: representação de uma função racional como uma composição de uma função seqüencial à esquerda com uma função seqüencial à direita, obtida por Elgot e Mezei; caracterização de Ginsburg e Rose das funções seqüenciais à esquerda.

- Funções subseqüenciais: caracterização de Choffrut das funções subseqüenciais; algoritmo de Sakarovitch et al. para decidir se uma função racional é uma função subseqüencial; algoritmo para a determinização de um transdutor letra-palavra; definição do transdutor subseqüencial minimal para uma função subseqüencial $\Sigma^{*} \rightarrow \mathrm{M}$, onde $\mathrm{M}$ é um monóide cancelativo com mdc único; caracterização das funções subseqüenciais $\Sigma^{*} \rightarrow M$, onde $M$ é um monóide cancelativo com mdc, utilizando a congruência sintática de uma função; algoritmo de Béal e Carton para a construção do prefixo de um transdutor subseqüencial; adaptação do algoritmo de minimização de autômatos finitos de Hopcroft, na versão de Gries, para a minimização de um transdutor subseqüencial prefixo. 
A seguir, apresentamos um diagrama relacionando as classes estudadas de relações racionais, entre monóides livres $\Sigma^{*}$ e $\Gamma^{*}$. As abreviações utilizadas nesse diagrama são as seguintes: SE, SD e SS representam as classes das funções seqüenciais à esquerda, funções seqüenciais à direita e funções subseqüenciais, respectivamente; FR representa a classe das funções racionais; FV representa a classe das funções racionais finitamente valoradas.

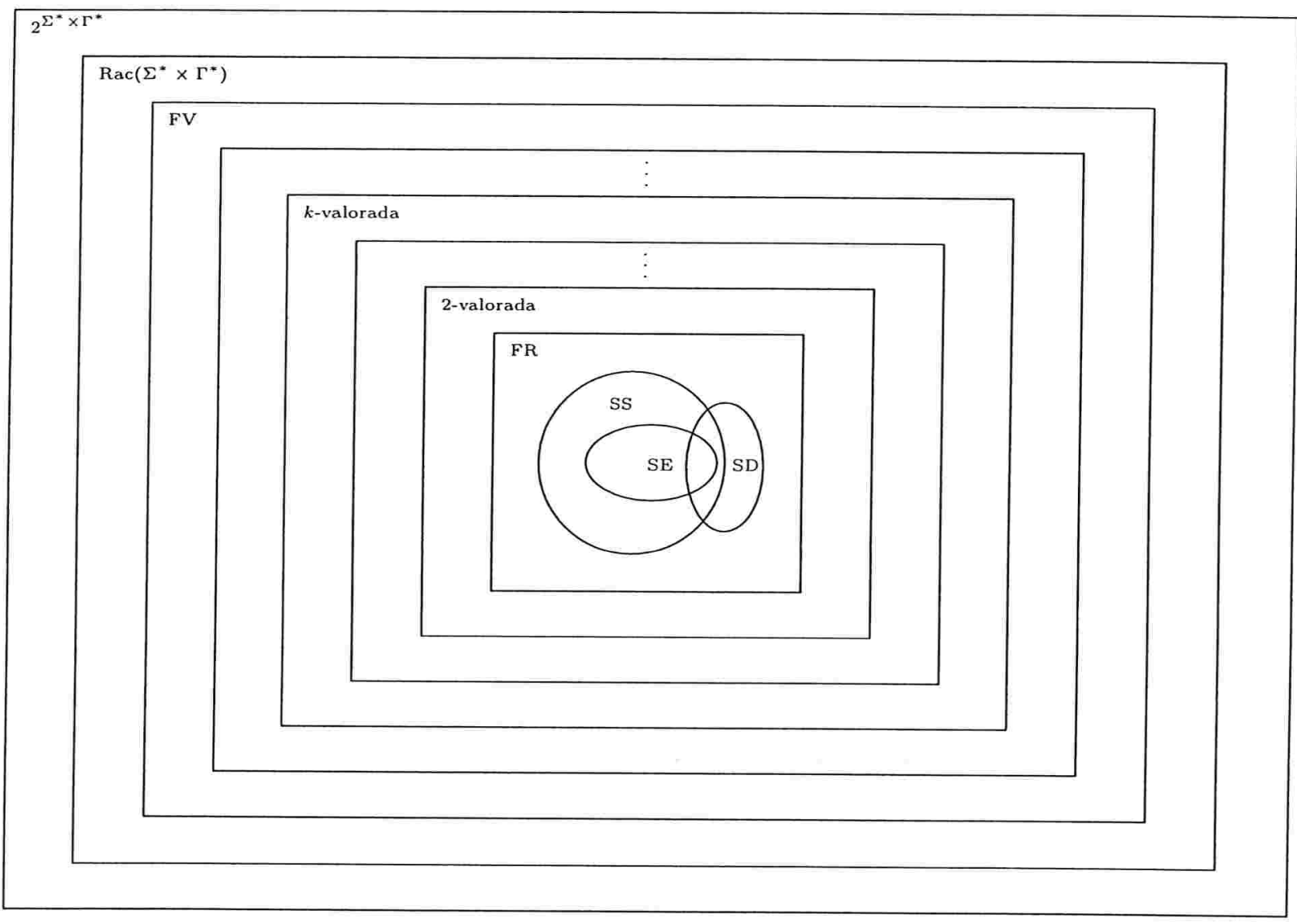

Julgamos interessante melhorar a demonstração da decomposição de uma relação racional $k$ valorada em $k$-funções racionais. Não sabemos se existe um limitante mais justo que $n^{k+1} M$ para os índices dos conjuntos utilizados no Teorema 3.7.2. Ademais, os transdutores que realizam as funções racionais obtidas com essa decomposição podem ter tamanho exponencial no transdutor original. Uma possibilidade de melhoria talvez surja com a investigação de relações entre as linguagens construídas com a aplicação do Teorema Cross-section.

Também gostaríamos de apresentar alguns exemplos de aplicação do Teorema 3.7.2. Neste trabalho, há um único exemplo da decomposição de uma relação racional $k$-valorada, que utilizamos para introduzir o resultado.

O algoritmo descrito na Seção 3.3 para construir um transdutor letra-saída equivalente é muito ingênuo, e certamente pode ser melhorado.

Julgamos interessante a pesquisa da extensão de alguns dos resultados sobre relações racionais palavra-palavra para outras famílias de monóides. Em particular, gostaríamos de investigar uma versão da caracterização de Choffrut das funções subseqüenciais para monóides de saída que não sejam um monóide livre. 
Outro próximo passo neste trabalho seria uma implementação completa e robusta dos algoritmos estudados. Iniciamos a implementação de alguns dos tipos de máquinas estudadas, a saber, $\Sigma$-autômatos, $\Sigma$-autômatos determinísticos, $\Sigma$ - $\Gamma$-transdutores palavra-palavra, $\Sigma$ - $\Gamma$-transdutores letra-palavra, e $\Sigma$ - $\Gamma$-transdutores subseqüenciais, utilizando a linguagem Java. Implementamos o algoritmo de determinização de transdutores, o algoritmo de Hopcroft (versão de Gries) para a minimização de autômatos, e a minimização de um transdutor subseqüencial prefixo utilizando o algoritmo de Hopcroft. Essas implementações foram utilizadas para a geração de alguns exemplos deste trabalho, mas alguns testes mais complexos revelaram a necessidade de melhorias.

A seguir, mencionamos alguns resultados importantes para as classes de relações racionais que consideramos, e que não foram estudados neste trabalho.

Um algoritmo de complexidade de tempo polinomial para decidir se uma função finitamente valorada é $k$-valorada, para um dado inteiro positivo $k$, é conhecido desde 1983 [GI83]. Os autores não fornecem uma descrição explícita desse algoritmo, mas sim uma redução em complexidade de tempo polinomial a outro problema, cuja solução em complexidade de tempo polinomial já era conhecida. Julgamos interessante o estudo de uma descrição mais precisa desse algoritmo.

Não estudamos também o problema de decidir se uma relação racional é $k$-valorada para algum inteiro positivo $k$. Weber [Web89] apresentou um algoritmo de complexidade de tempo polinomial para esse problema. No entanto, sua descrição desse algoritmo é complexa, e também julgamos interessante uma simplificação.

Recentemente, novos tipos de transdutores subseqüenciais têm sido estudados, como resultado de aplicações em problemas de processamento de linguagem natural. Um exemplo são os transdutores $p$-subseqüenciais, propostos por Mohri [Moh02]. Existe o esforço de estender para essa classe de transdutores alguns resultados importantes para transdutores subseqüenciais, como a caracterização de Choffrut.

Também não estudamos aplicações da teoria. Tínhamos o interesse de descrever algumas das aplicações recentes em Processamento de Linguagem Natural, e algumas aplicações na Teoria de Linguagens Formais, onde as relações racionais são uma ferramenta fundamental para o estudo de algumas classes de linguagens. 
. 


\section{REFERÊNCIAS BiBLIOGRÁfiCAS}

[BC00a] M.-P. Béal e O. Carton, Computing the prefix of an automaton, RAIRO - Theoretical Informatics and Applications 34 (2000), no. 6, 503-514. Citado na(s) página(s) vi, 195

[BC00b] _ Determinization of transducers over finite and infinite words, ICALP'2000 (U. Montanari et al., ed.), Lecture Notes in Computer Science, vol. 1853, 2000, pp. 561-570. Citado na(s) página(s) v, 152

[BCPS03] M.-P. Béal, O. Carton, C. Prieur, e J. Sakarovitch, Squaring transducers: An efficient procedure for deciding functionality and sequentiality, Theoretical Computer Science 292 (2003), 45-63. Citado na(s) página(s) iv, v, 70, 95, 101, 102, 135, 142, 150

[Ber79] J. Berstel, Transductions and context-free languages, B. G. Teubner, 1979. Citado na(s) página(s) $1,13,23,25,61,76,89,106,116,123,135$

[BP85] J. Berstel e D. Perrin, Theory of Codes, Pure and Applied Mathematics; 117, Academic Press, Orlando, Florida, 1985. Citado na(s) página(s) 91

[BR99] V. Bruyère e C. Reutenauer, A proof of Choffrut's theorem on subsequential functions, Theoretical Computer Science 215 (1999), no. 1-2, 329-335. Citado na(s) página(s) 126

[Cho77] C. Choffrut, Une caractérization des fonctions séquentielles et des fonctions sous-séquentielles en tant que relations rationnelles, Theoretical Computer Science 5 (1977), no. 3, 325-338. Citado na(s) página(s) 135

[Cho78] _ Contribution à l'étude de quelques families remarquables de fonctions rationelles, Thèse d'État, Université Paris VII, 1978. Citado na(s) página(s) iii, v, 69, 125, 136, 173

[Cho79] _ A generalization of Ginsburg and Rose's characterization of gsm mappings, ICALP'79, Lecture Notes in Computer Science, vol. 71, Springer-Verlag, 1979, pp. 88-103. Citado na(s) página(s) iii, v, 125, 168, 173

[Cho03] _ Minimizing subsequential transducers: A survey, Theoretical Computer Science 292 (2003), no. 1, 131-143. Citado na(s) página(s) v, 167, 173, 195

[CK86] K. Culik, II e J. Karhumaki, The equivalence of finite valued transducers (on HDTOL languages) is decidable, Theoretical Computer Science 47 (1986), no. 1, 71-84. Citado na(s) página(s) iv, 85

[CLRS01] T. H. Cormen, C. E. Leiserson, R. L. Rivest, e C. Stein, Introduction to algorithms, MIT Press, 2001. Citado na(s) página(s) 1, 12, 20, 21, 201

[CR94] M. Crochemore e W. Rytter, Text algorithms, Oxford University Press, 1994. Citado na(s) página(s) 21 
[Cro86] M. Crochemore, Transducers and repetitions, Theoretical Computer Science 45 (1986), no. 1, 63-86. Citado na(s) página(s) 193

[Eil74] S. Eilenberg, Automata, languages, and machines, vol. A, Academic Press, 1974. Citado na(s) página(s) iii, v, 1, 2, 15, 16, 23, 31, 39, 42, 76, 88, 105, 106, 107, 111, 120, 122, 123, 126, 134, 167, $174,180,206$

[EM65] C. C. Elgot e J. E. Mezei, On relations defined by generalized finite automata, IBM Journal of Research and Development 9 (1965), 47-68. Citado na(s) página(s) 42, 123

[GI83] E. Gurari e O. Ibarra, A note on finite-valued and finitely ambiguous transducers, Mathematical Systems Theory 16 (1983), 61-66. Citado na(s) página(s) 61, 65, 231

[GR66] S. Ginsburg e G. F. Rose, A characterization of machine mappings, Canadian Journal of Mathematics 18 (1966), 381-388. Citado na(s) página(s) v, 105, 125

[Gri68] T. V. Griffiths, The unsolvability of the equivalence problem for $\Lambda$-free nondeterministic generalized machines, Journal of the ACM 15 (1968), no. 3, 409-413. Citado na(s) página(s) iv, 65

[Gri73] D. Gries, Describing an algorithm by Hopcroft, Acta informatica 2 (1973), 97-109. Citado na(s) página(s) vi, 204

[Hef02] A. Hefez, Curso de Álgebra, vol. 1, IMPA, 2002. Citado na(s) página(s) 161

[HMU01] J. E. Hopcroft, R. Motwani, e J. D. Ullman, Introduction to automata theory, languages and computation, Addison-Wesley, 2001. Citado na(s) página(s) 15, 20, 65

[Hop71] J. E. Hopcroft, An $n \log n$ algorithm for minimizing the states in a finite-automaton, Theory of Machines and Computations (Z. Kohavi, ed.), Academic Press, 1971, pp. 189-196. Citado na(s) página(s) vi, 204

[How91] J. M. Howie, Automata and languages, Oxford Science Publications, 1991. Citado na(s) página(s) 15

[Iba78] O. Ibarra, The unsolvability of the equivalence problem for $\epsilon$-free NGSM's with unary input (output) alphabet and applications, SIAM Journal on Computing 7 (1978), no. 4, 524-532. Citado na(s) página(s) 65

[Leu98] H. Leung, Separating exponentially ambiguous finite automata from polynomially ambiguous finite automata, SIAM Journal on Computing 27 (1998), no. 4, 1073-1082. Citado na(s) página(s) 90, 91

[Lim03] E. L. Lima, Espaços métricos, IMPA, 2003. Citado na(s) página(s) 69

[Mea55] George H. Mealy, A method for synthesizing sequential circuits, Bell System Technical Journal 34 (1955), no. 5, 1045-1079. Citado na(s) página(s) 105

[MKS66] W. Magnus, A. Karrass, e D. Solitar, Combinatorial group theory, Interscience Publishers, 1966. Citado na(s) página(s) 10

[Moh94] M. Mohri, Minimization of sequential transducers, Lecture Notes in Computer Science 807 (1994), 151-163. Citado na(s) página(s) 195

[Moh95] — On some applications of finite-state automata theory to natural language processing, Journal of Natural Language Engineering 2 (1995), 1-20. Citado na(s) página(s) v, 113, 152, 174

[Moh97] _ Finite-state transducers in language and speech processing, Computational Linguistics 23 (1997), 269-311. Citado na(s) página(s) v, 113, 174 
[Moh00] Minimization algorithms for sequential transducers, Theoretical Computer Science 234 (2000), no. 1-2, 177-201. Citado na(s) página(s) 195, 204

[Moh02] _ plementation and Application of Automata (CIAA'2002) (Tours, France), 2002. Citado na(s) página(s) 231

[MR97] M. Mohri e M. Riley, Weighted determinization and minimization for large vocabulary speech recognition, Eurospeech'97, 1997. Citado na(s) página(s) 113

[Niv68] M. Nivat, Transductions des langages de Chomsky, Ann. Inst. Fourier 18 (1968), 339-455. Citado na(s) página(s) 26

[Reu90] C. Reutenauer, Subsquential functions: Characterizations, minimization, examples, International Meeting of Young Computer Scientists, Lecture Notes in Computer Science, no. 464, SpringerVerlag, 1990, pp. 62-79. Citado na(s) página(s) v, 116, 126, 173, 180, 182, 206

[RS97] E. Roche e Y. Schabes, Finite-state language processing, MIT Press, 1997. Citado na(s) página(s) $\mathrm{v}, 57,152$

[Sak98] J. Sakarovitch, A construction on finite automata that has remained hidden, Theoretical Computer Science 204 (1998), no. 1-2, 205-231. Citado na(s) página(s) 123

[Sch61] M. P. Schützenberger, A remark on finite transducers, Information and Control 4 (1961), 185-196. Citado na(s) página(s) 122

[Sch75] _ Sur les relations rationnelles, Automata Theory and Formal Languages, 2nd GI Conference, Lecture Notes in Computer Science, vol. 33, 1975, pp. 209-213. Citado na(s) página(s) 61

[Sch76] Sur les relations rationnelles entre monö̈des libres, Theoretical Computer Science 3 (1976), no. 2, 243-259. Citado na(s) página(s) 72

[Sch77] Sur une variante des fonctions séquentielles, Theoretical Computer Science 4 (1977), no. 1, 47-57. Citado na(s) página(s) v, 106, 112, 182

[Sch78] E. Schmidt, Succintness of descriptions of context-free, regular and finite languages, Ph.D. thesis, Cornell University, 1978. Citado na(s) página(s) 91

[SM94] J. L. Szwarcfiter e L. Markenzon, Estruturas de dados e seus algoritmos, Livros Técnicos e Científicos, 1994. Citado na(s) página(s) 219

[Web88] A. Weber, A decomposition theorem for finite-valued tranducers and an application to the equivalence problem, Mathematical Foundations of Computer Science 1988 (Carlsbad, Czechoslovakia) (Michael P. Chytil, Ladislav Janiga, e Václav Koubek, eds.), Lecture Notes in Computer Science, vol. 324, Springer, 1988, pp. 552-562. Citado na(s) página(s) iv, 85

[Web89] - On the valuedness of finite transducers, Acta Informatica 27 (1989), no. 8, 749-780. Citado na(s) página(s) 231

[Web92] _ Decomposing a k-valued transducer into $k$ unambiguous ones, Proceedings of Latin American Symposium on Theoretical Informatics (LATIN '92) (Imre Simon, ed.), Lecture Notes in Computer Science, vol. 583, Springer, 1992, pp. 503-515. Citado na(s) página(s) iv, 72, 88

[Web93] _ Decomposing finite-valued transducers and deciding their equivalence, SIAM Journal on Computing 22 (1993), no. 1, 175-202. Citado na(s) página(s) 72, 85

[Web96] _ Decomposing a k-valued transducer into $k$ unambiguous ones, RAIRO Informatique Théorique et Applications 30 (1996), no. 5, 379-413. Citado na(s) página(s) 72

[WK95] A. Weber e R. Klemm, Economy of description for single-valued transducers, Information and Computation 119 (1995), 327-340. Citado na(s) página(s) 135 



\title{
Índice Remissivo
}

\author{
Símbolos \\ $(x, y) \Delta, 68$ \\ $2^{A}, 1$ \\ $<_{l}, 7$ \\ $A / \sim, 4$ \\ $A^{+}, 5$ \\ $C(p), 11$ \\ $G[X], 11$ \\ $K^{n \times n}, 6$ \\ $L_{\infty}, 73$ \\ $L_{(f, g), \nu}, 73$ \\ $P_{\mathcal{A}}, 196$ \\ [a] (classe de equivalência), 4 \\ [k] (conjunto de inteiros), 1 \\ $\# \mathcal{A}, 19$ \\ $\# r, 3$ \\ $\Delta_{\Sigma^{*}}, 68$ \\ $\operatorname{Rac}(\mathrm{M}), 12$ \\ $\operatorname{Rec}(\mathrm{M}), 12$ \\ $\Sigma \leq l, 7$ \\ $\succ, \prec, 7$ \\ $\cong 172,206$ \\ dom, 2 \\ $\epsilon, 111,116,159,160$ \\ fat, 7 \\ $\hat{f}, 174$ \\ $\hat{p}, 196$ \\ im, 2 \\ $\langle s\rangle_{k}^{\varrho}, 111$ \\ $\langle s\rangle_{k}, 111$ \\ $\log , 217$ \\ $\mathbb{N}, \mathbb{Z}, \mathbb{Q}, \mathbb{R}, \mathbb{R}_{+}, 1$ \\ k, 111
}

$\mathcal{A} / \equiv, 207$

$\mathcal{T} / \equiv, 184$

$\mathcal{T} \times \mathcal{T}, 95$

card, 1

$\operatorname{mdc} f, 174$

$\operatorname{mdc} q, 168$

$\operatorname{mdc}(f, s), 174$

$\pi_{1}, \pi_{2}, 23$

pref, 7

$\sim_{f}, 182$

$\square, 182,186,208$

suf, 7

$\varnothing, 1$

$\varphi, 127,171$

$\varphi_{u}, 127$

$\wedge U, 67$

$q^{-1} \rho, 183$

$r \mid X, 3$

$r^{(\infty)}, 4$

$r^{(k)}, 3$

$r^{-1}, 3$

$u[i \ldots j], 7$

$u[i], 7$

$u^{e}, 7$

$\mathcal{T}_{f}, 176$

$\mathcal{T}_{f}^{0}, 175$

$|\mathcal{A}|, 16$

$|u|, 7$

$|u|_{\sigma}, 7$

$\|A\|_{g}, 70$

$\|u, v\|, 69$

$\|u, v\|_{g}, 69$ 
A

ação, 6

à direita, 6

à esquerda, 6

alfabeto, 7

cópia de, 9

de entrada, 24

de saída, 24

algoritmo

de Béal e Carton para a construção do prefixo de um autômato, 198-201

de Hopcroft-Gries para a minimização de um autômato, 215

de Sakarovitch et al. para decidir se uma função racional é subseqüencial, 144

de Sakarovitch et al. para decidir se uma relação racional é uma função, 100

para a determinização de um transdutor funcional, 158

arco, 10

árvore digital, 219

autômato

$\Sigma$-autômato, 16

M-autômato, 15

acessível, 16

bi-acessível, 16

co-acessível, 16

completamento de, 18, 208

completo, 18

comportamento de, 16

de entrada, 31

de saída, 31, 201

de um transdutor subseqüencial, 217

determinístico, 17

grafo de, 16

minimal, 18, 207

não-ambíguo, 88

normalizado, 16, 17

prefixo, 196

reduzido, 207

B

bijeção, 2

bimachine, 122-123

bimorfismo, 23 de um transdutor, 32

bloco, 208

C

caminho, 11

circuito, 11

classe de equivalência, 4

componente fortemente conexo, 11

comportamento

de um M-N-transdutor, 31

de um M-autômato, 16

composição de relações, $3,43-44,46-47,115$, 132-133

comprimento

de um passeio, 11

de uma palavra, 7

concatenação, 7

de passeios, 11

congruência, 6

à direita, 6

de uma função, 182

conjunto

cardinalidade de, 1

de entrada, 2

de saída, 2

gerador, 5

racional, 12

reconhecível, 12

construção

de um autômato não-ambíguo, 90

de um transdutor letra-palavra equivalente, $57-59$

de um transdutor não-ambíguo, 91-92

de uma cross-section, 88, 91

cross-section, 77,88

D

decidibilidade

da equivalência de relações racionais $k$ valoradas, 87

de se uma função racional é subseqüencial, 135

de se uma relação racional é $k$-valorada para um dado $k, 64-65$

de se uma relação racional é finitamente valorada, 57 
de se uma relação racional é uma função, 61,95

decomposição

de uma função racional em funções seqüenciais, 123-125

de uma relação racional $k$-valorada em $k$ funções racionais, $83-85,88$

determinização de um transdutor, 152

diferença

de um estado, 96

de um par de palavras, 68

diferencial, 127

divisor, 161

domínio de uma relação, 2

$\mathbf{E}$

elemento

associado, 165

inversível, 4

emissão

final, 113

inicial, 113

encaixamento, 62

esparsidade, 70

estado, 15

acessível, 16

co-acessível, 16

de uma representação matricial, 38

especial, 139

essencial, 141

final, 15,113

inicial, 15, 107, 108, 112

limitado, 136

estrela

de relações, 24, 48-49

de um conjunto, 5

expressão racional, 12

\section{$\mathrm{F}$}

fator, 7

fecho reflexivo e transitivo de uma relação, 4

função, 1

bijetora, 2

de emissão, 107

de transição, 18, 107

fechada por prefixos, 126 identidade, 3

injetora, 2

normalizada, 186

parcial, 1

que preserva prefixos, 108

que preserva sufixos, 109

racional, 45, 95

seqüencial, 107

composição de, 115, 133

intersecção de, 115

união de, 115

seqüencial à direita, 109

sobrejetora, 2

subseqüencial, 113

composição de, 115, 132

intersecção de, 115

inversa de, 133

restrição a um conjunto de, 116

união de, 115

total, 2

vazia, 2

G

generalized sequential machine, 105

gráfico de uma relação, 3

grafo, 10

acíclico, 11

de um autômato, 16

restrição de, 11

grupo, 5

livre, 10

gsm, 105

I

imagem

de um elemento, 2

imagem de uma relação, 2

indecidibilidade

da equivalência de relações racionais finitamente valoradas, 65-67

de se uma relação racional é exatamente $k$-valorada para um dado $k, 65$ isomorfismo

de transdutores subseqüenciais, 172

de autômatos determinísticos, 206 
L

Lema da Iteração, 20, 29-30

letra, 7

linguagem, 7

local, 18

M

máquina

de Mealy, 105

de Moore, 105

máximo divisor comum, 161

mdc, 161

de um estado, 168

de uma função, 174

monóide, 4

cancelativo, 165

com mdc , 161

com mdc único, 164

comutativo, 4

de entrada, 24

de saída, 24

finitamente gerado, 5

gerado por um conjunto, 5

livre, 7

produto, 23

morfismo

de autômatos determinísticos, 206

de entrada, 28

de monóides, 5

de saída, 28

de semigrupos, 5

de transdutores subseqüenciais, 171

desmembramento de, 23

fino, 8

muito fino, 8

próprio

de transdutores subseqüenciais, 172

de autômatos determinísticos, 206

$\mathrm{N}$

números

inteiros, 1

naturais, 1

racionais, 1

reais, 1

normalização de um autômato, 16

de um transdutor, 55

numeração topológica, 12

O

operações racionais, 12,24

ordem lexicográfica, 7

$\mathbf{P}$

palavra, 7

reduzida, 10

vazia, 7

partição, 1

refinamento de, 1

passeio, 11

bem-sucedido, 16

entrada de, 31

fechado, 11

origem, 11

rótulo de, 16

saída de, 31

simétrico, 98, 141

término, 11

vazio, 11

prefixo

de um autômato, 196

de um conjunto, 67

de um estado, 196

de um transdutor, 170

de uma palavra, 7

problema

da correspondência de Post, 20

decidível, 20

indecidível, 20

produto

cartesiano, 1

de autômatos, 19

de relações, 24, 47-49

de um semigrupo, 4

projeção, 23

Q

quadrado de um transdutor, 95

quociente de um conjunto por uma relação de equivalência, 4 
R

redução

de um autômato, 207

de um transdutor subseqüencial, 184

relação, 2

$k$-valorada, 45

composição de, 47

união de, 47

anti-simétrica, 3

composição de, 3

de equivalência, 4

de ordem, 4

estrela de, 24

exatamente $k$-valorada, 65

fecho reflexivo e transitivo de, 4

finitamente valorada, 45

composição de, 46-47

estrela de, 48-49

produto de, $47-49$

união de, 47

inversa de, 3

palavra-palavra, 24

produto de, 24

racional, 24

composição de, 43-44

fecho reflexivo e transitivo de, 41

inversa de, 40

restrição a um conjunto de, 41

reconhecível, 24

reflexiva, 3

restrição a um conjunto de, 3

simétrica, 3

sobre um conjunto, 2

transitiva, 3

união de, 3,24

representação matricial, $34-38,51-52$

normalizada, 39

bi-acessível, 39

de um transdutor, 38, 53-54

representante, 4

reverso de uma palavra, 7

$\mathrm{S}$

semi-anel, 5

semigrupo, 4 finitamente gerado, 5

gerado por um conjunto, 5

livre, 7

submonóide, 5

substituição, 8

sufixo, 7

$\mathrm{T}$

teorema

Cross-section, 76, 88-89

da composição de Elgot e Mezei, 43-44

da decomposição de Elgot e Mezei, 123125

de Choffrut, 125, 131-132

de Ginsburg e Rose, 125-126

de Kleene, 12

de Nivat, 26-28

de Weber, 83-84

território, 11

transdutor, 31

$\mathrm{M}-\mathrm{N}$-transdutor, 31

$k$-ambíguo, 88

bi-acessível, 31, 32

bimorfismo de, 32

comportamento de, 31

dos sufixos, 193

fino, 33

funcional, 52

letra-palavra, 52

letra-saída, 52

não-ambíguo, 88

normalizado, 31, 32, 55

reduzido, 185

representação matricial de, 38, 53-54

seqüencial, 106

seqüencial à direita, 108

seqüencial letra-letra, 107

subseqüencial, 112

acessível, 114

bi-acessível, 114

co-acessível, 114

completo, 114

prefixo, 168

transição, 15

$\mathrm{U}$ 
união de relações, $3,24,47,115$

V

vértice, 10

essencial, 141

variação limitada, 125

Z

zero de um monóide, 165 